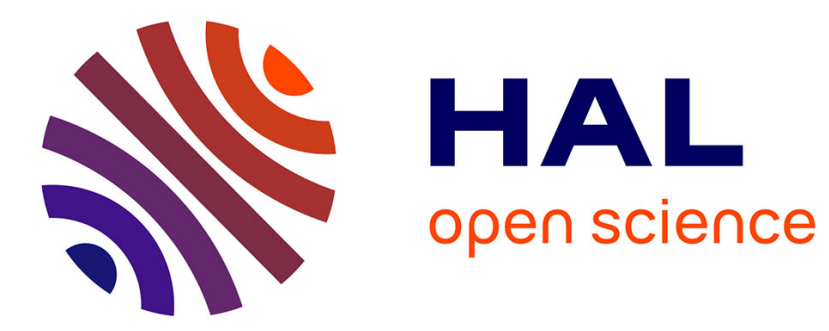

\title{
Foncteurs en grassmanniennes, filtration de Krull et cohomologie des foncteurs
}

\author{
Aurélien Djament
}

\section{To cite this version:}

Aurélien Djament. Foncteurs en grassmanniennes, filtration de Krull et cohomologie des foncteurs. Mémoires de la Société Mathématique de France, 2007, 111, xx+213 p. hal-00175492

\section{HAL Id: hal-00175492 \\ https://hal.science/hal-00175492}

Submitted on 28 Sep 2007

HAL is a multi-disciplinary open access archive for the deposit and dissemination of scientific research documents, whether they are published or not. The documents may come from teaching and research institutions in France or abroad, or from public or private research centers.
L'archive ouverte pluridisciplinaire HAL, est destinée au dépôt et à la diffusion de documents scientifiques de niveau recherche, publiés ou non, émanant des établissements d'enseignement et de recherche français ou étrangers, des laboratoires publics ou privés. 
Aurélien DJAMENT

FONCTEURS EN

GRASSMANNIENNES, FILTRATION DE KRULL ET COHOMOLOGIE DES FONCTEURS 


\section{A. DJAMENT}

LAGA, Institut Galilée, université Paris 13, 99 avenue J.-B. Clément, 93430 VILLETANEUSE (FRANCE).

E-mail : djament@math.univ-paris13.fr

Url : http://www.math.univ-paris13.fr/〜djament/

Classification mathématique par sujets (2000). - 16P60, 18A25, 18G15, 20C33. Secondaire : 16E20, 16P40, 18A40, 18C15, 18D15, 18E35, 18G05, 19D99, $55 \mathrm{~S} 10$.

Mots clefs. - Catégories de foncteurs, algèbre homologique, filtration de Krull, groupes linéaires sur les corps finis, grassmanniennes, objets noethériens, $K$-théorie stable, représentations modulaires, foncteur différence et filtration polynomiale, (co)monades.

L'auteur témoigne sa chaleureuse reconnaissance à Geoffrey Powell pour l'attention qu'il a portée à ce travail, tant sur le fond que sur la forme, durant toutes les étapes de sa réalisation. Il remercie également Lionel Schwartz et Christine Vespa pour leurs utiles conseils.

Cet article est une version fusionnée des prépublications [Dja06a], [Dja06b], qui reprennent la thèse de doctorat de l'auteur ([Dja06d]).

Décembre 2006 ; révisé en mai et septembre $200 \%$. 


\title{
FONCTEURS EN GRASSMANNIENNES, FILTRATION DE KRULL ET COHOMOLOGIE DES FONCTEURS
}

\author{
Aurélien DJAMENT
}

Résumé. — Soit $\mathcal{F}$ la catégorie des foncteurs entre espaces vectoriels sur un corps fini. Les catégories de foncteurs en grassmanniennes sont obtenues en remplaçant la source de cette catégorie par la catégorie des couples formés d'un espace vectoriel et d'un élément d'une de ses grassmanniennes. Ces catégories possèdent une très riche structure algébrique; nous étudions notamment leurs objets finis et leurs propriétés homologiques.

Nous établissons ainsi une propriété très générale d'annulation en cohomologie des foncteurs, que nous appliquons à la $K$-théorie stable des corps finis : nous obtenons une généralisation du théorème de Betley-Suslin exprimant des groupes d'extensions entre $G L_{\infty}$-modules en terme de cohomologie des foncteurs.

Notre seconde application des catégories de foncteurs en grassmanniennes a trait à la filtration de Krull de la catégorie $\mathcal{F}$. Nous en donnons une description conjecturale, dont nous examinons les conséquences, très puissantes, sur la structure de la catégorie $\mathcal{F}$. À l'aide d'outils dus à G. Powell, nous démontrons une forme faible de cette conjecture, dans le cas où le corps de base a deux éléments. Nous utilisons ce résultat pour établir le caractère noethérien de nouveaux foncteurs. 
Abstract (Grassmannian functors, Krull filtration and functor cohomology)

Let $\mathcal{F}$ be the category of functors between vector spaces over a finite field. The grassmannian functor categories are obtained by replacing the source of this category by the category of pairs formed by a vector space and an element of one of its grassmannians. These categories have a very rich algebraic structure; we study in particular their finite objects and their homological properties.

We give so a very general vanishing property in functor cohomology, which we apply to the stable $K$-theory of finite fields : we obtain a generalization of the theorem of Betley-Suslin which expresses certain extension groups of $G L_{\infty}$-modules in term of functor cohomology.

Our second application of the grassmannian functor categories concerns the Krull filtration of the category $\mathcal{F}$. We give a conjectural description of this filtration, of which we explore powerful implications. With the help of tools due to G. Powell, we show a weak form of this conjecture, in the case where the basis field has two elements. As a consequence, we establish the noetherian character of new functors. 


\section{TABLE DES MATIÈRES}

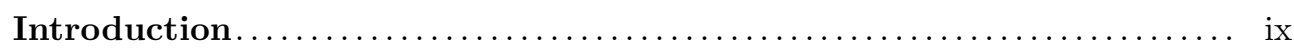

Les catégories de foncteurs en grassmanniennes : structures fondamentales . . xi Applications : algèbre homologique et filtration de Krull ............... xiv

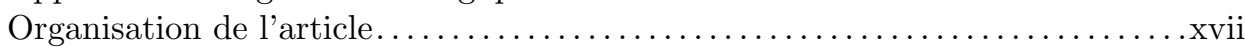

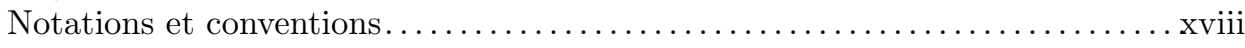

Partie I. Préliminaires.................................... 1

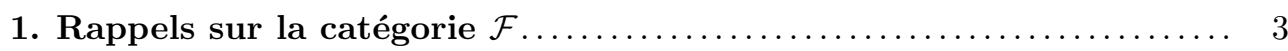

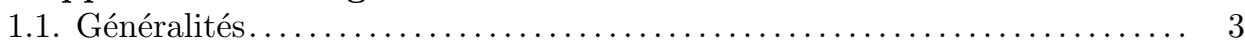

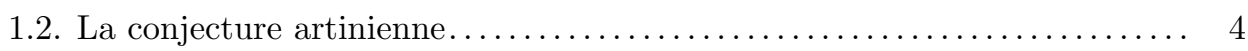

1.3. Le foncteur différence et les objets polynomiaux de $\mathcal{F}(\mathbb{k}) \ldots \ldots \ldots \ldots \ldots$. 5

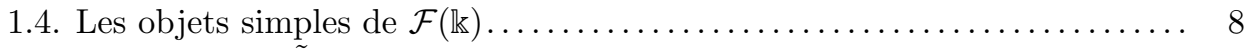

1.5. Les foncteurs $\tilde{\nabla}_{n}: \mathcal{F}\left(\mathbb{F}_{2}\right) \rightarrow \mathcal{F}\left(\mathbb{F}_{2}\right)$ de Powell................. 10

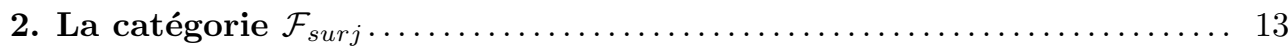

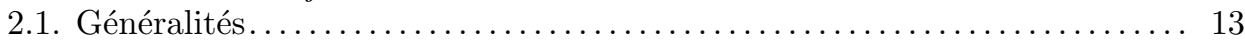

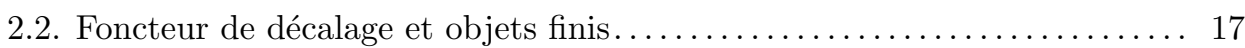

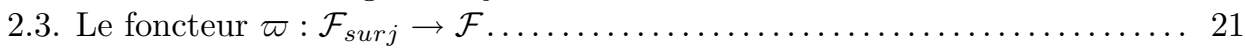

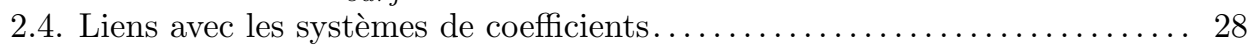

3. Catégories de comodules sur un foncteur en coalgèbres de Boole. . 33

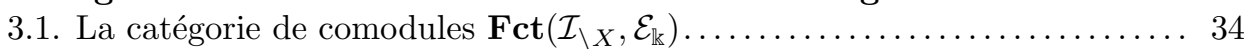

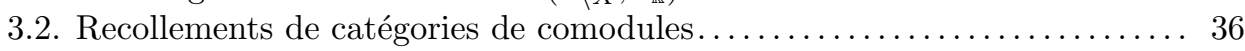

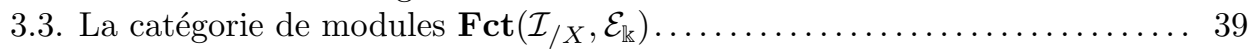

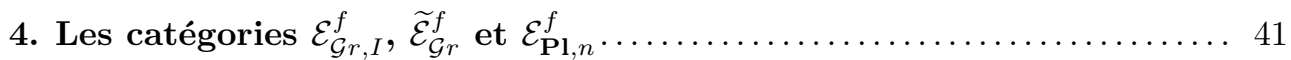

4.1. Définition des catégories et foncteurs utilisés................. 41

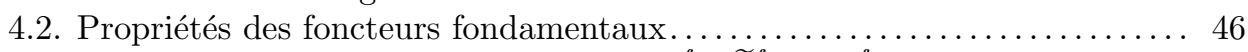

4.3. Propriétés de structure des catégories $\mathcal{E}_{\mathcal{G} r}^{f}, \widetilde{\mathcal{E}}_{\mathcal{G} r}^{f}$ et $\mathcal{E}_{\mathbf{P l}, n}^{f} \ldots \ldots \ldots \ldots \ldots 47$ 
Partie II. Les catégories de foncteurs en grassmanniennes......... 51

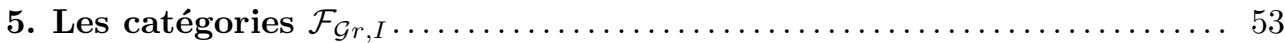

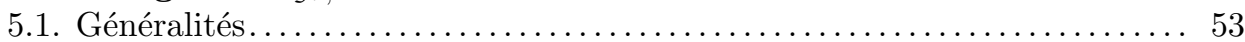

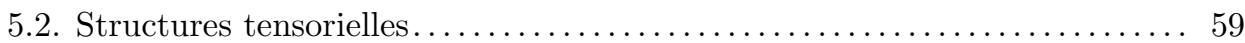

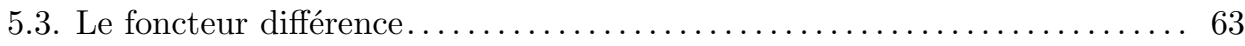

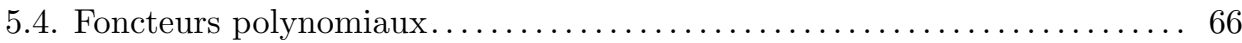

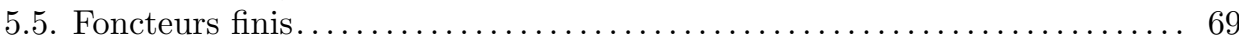

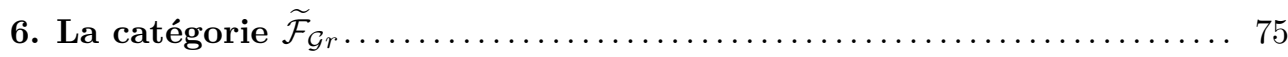

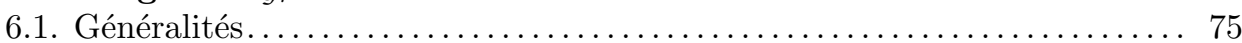

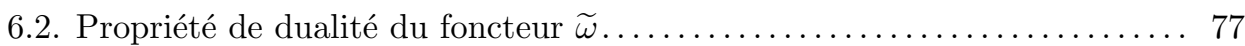

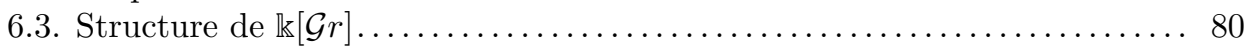

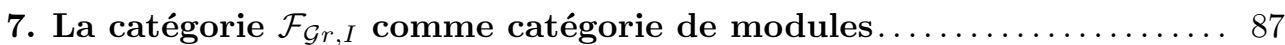

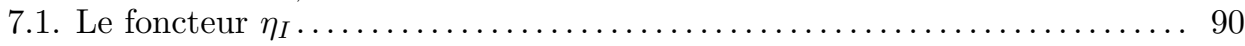

7.2. Résolution canonique et algèbre homologique monadique........... 92

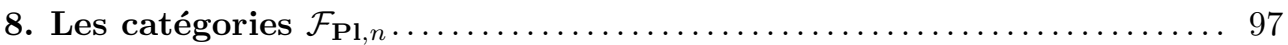

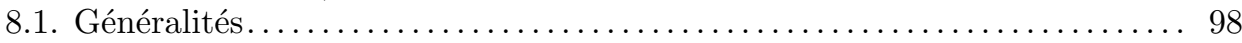

8.2. L'équivalence de catégories $\mathcal{F}_{\mathbf{P l}, n} \simeq \operatorname{Comod}_{I_{E_{n}}} \ldots \ldots \ldots \ldots \ldots \ldots \ldots \ldots 1$

9. Foncteurs hom internes et foncteurs de division dans $\mathcal{F}_{\mathcal{G} r, I} \ldots \ldots \ldots 109$

9.1. Comparaison entre les différentes catégories $\mathcal{F}_{\mathcal{G} r, I} \ldots \ldots \ldots \ldots \ldots \ldots \ldots$

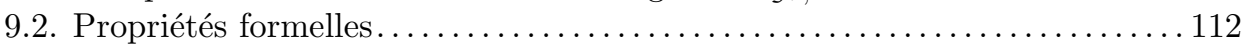

Partie III. Propriétés cohomologiques du foncteur $\omega$. Applications . . 117

10. Théorème d'annulation cohomologique..................... 119

10.1. Préliminaires................................................ 119

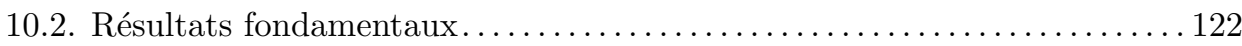

11. Foncteur $\omega$ et foncteurs hom internes....................... 125

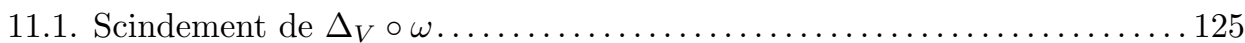

11.2. Le morphisme $h_{X, F}^{*}: \omega\left(\mathbf{E x t}_{\mathcal{G} r}^{*}(\iota(F), X)\right) \rightarrow \mathbf{E x t}_{\mathcal{F}}^{*}(F, \omega(X)) \ldots \ldots \ldots \ldots$

12. La filtration de Krull de la catégorie $\mathcal{F} \ldots \ldots \ldots \ldots \ldots \ldots \ldots \ldots \ldots \ldots$

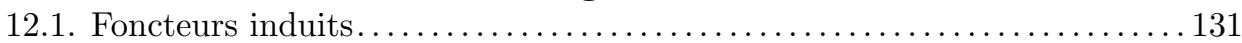

12.2. La conjecture artinienne extrêmement forte $\ldots \ldots \ldots \ldots \ldots \ldots \ldots \ldots \ldots 132$

12.3. Conséquences de la conjecture artinienne extrêmement forte......... 135

13. Résultats d'annulation cohomologique dans $\mathcal{F}_{i n j} \ldots \ldots \ldots \ldots \ldots \ldots 137$

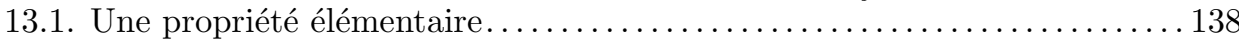

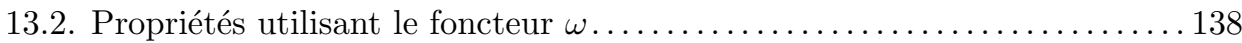

13.3. La filtration de Krull de la catégorie $\mathcal{F}_{i n j} \ldots \ldots \ldots \ldots \ldots \ldots \ldots \ldots \ldots \ldots \ldots \ldots \ldots$

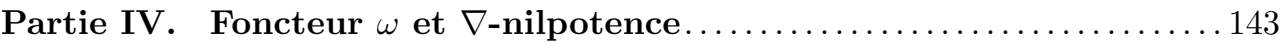




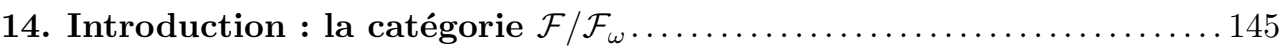

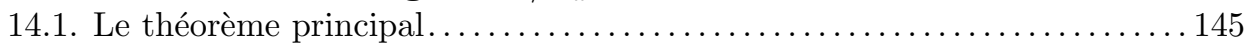

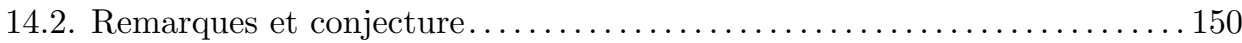

15. Préliminaires relatifs aux foncteurs $\omega$ et $\tilde{\nabla}_{n} \ldots \ldots \ldots \ldots \ldots \ldots \ldots \ldots$

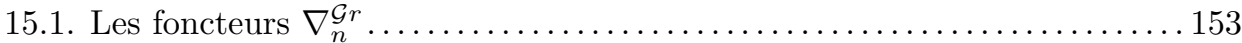

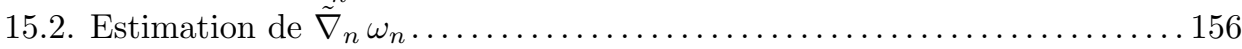

15.3. Théorème d'annulation cohomologique relatif à la $\nabla$-nilpotence....... 158

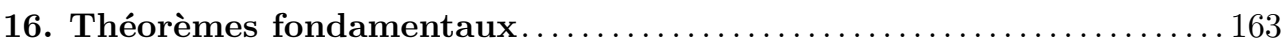
16.1. Le morphisme $\omega_{*}: G_{0}^{f}\left(\mathcal{F}_{\mathcal{G} r}\right) \rightarrow G_{0}^{t f}(\mathcal{F}) \rightarrow \widehat{G}_{0}^{f}(\mathcal{F}) \ldots \ldots \ldots \ldots \ldots \ldots 164$

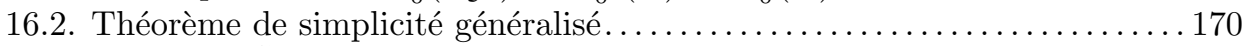

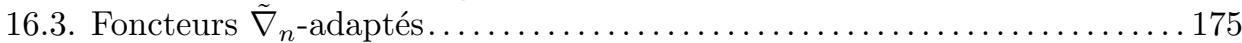

16.4. Structure de $P^{\otimes 2} \otimes F$ pour un foncteur fini $F \ldots \ldots \ldots \ldots \ldots \ldots \ldots \ldots$

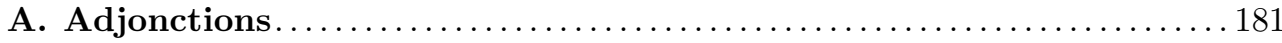

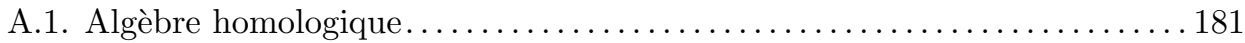

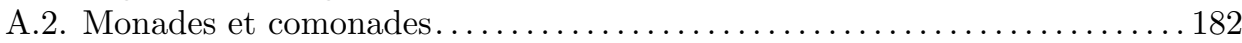

B. Propriétés de finitude dans les catégories abéliennes . . . . . . . . . 185

B.1. Définitions................................................. 185

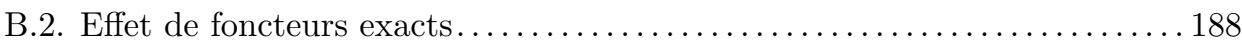

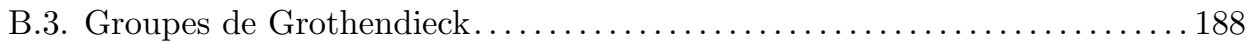

B.4. Filtration de Krull..................................... 189

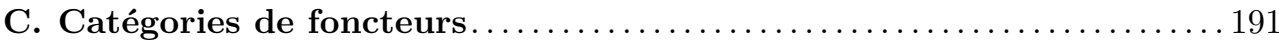

C.1. Généralités................................................... 191

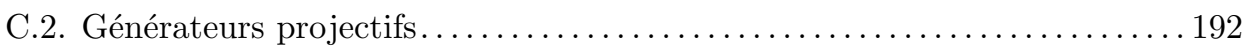

C.3. Foncteurs hom internes et foncteurs de division. ................. 194

C.4. Décomposition scalaire...................................... 194

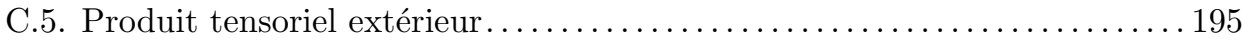

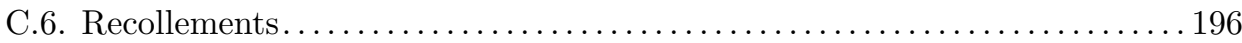

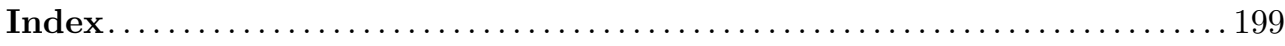

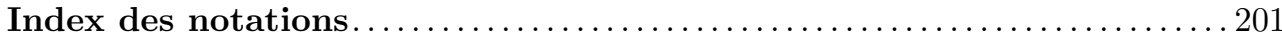

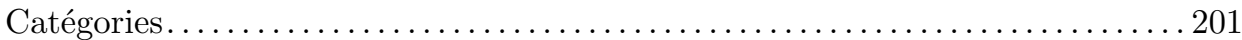

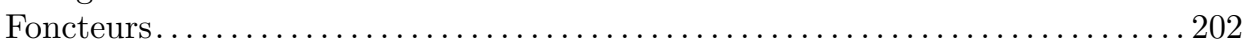

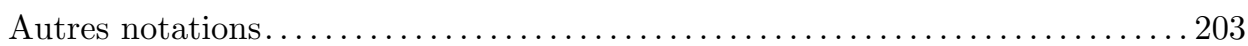

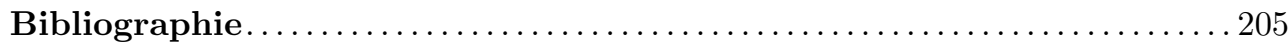





\section{INTRODUCTION}

Depuis plus de quarante ans, les algébristes utilisent les catégories de foncteurs pour étudier des catégories abéliennes, comme les catégories de modules, en particulier en théorie des représentations. Plusieurs notions de finitude interviennent dans ce contexte : la catégorie $D(R)$ associée à un anneau $R$, constituée des foncteurs de la catégorie des $R$-modules à droite de présentation finie vers les groupes abéliens, joue un rôle important ${ }^{(1)}$. Dans les catégories de foncteurs elles-mêmes apparaissent des notions de finitude. Ainsi, Auslander développe et applique la notion de foncteur cohérent dans l'article [Aus66] (la catégorie $C(R)$ des foncteurs cohérents de $D(R)$ est particulièrement importante), notion dont l'utilité n'a pas été démentie. (Comme texte plus récent exploitant et enrichissant ces idées, on pourra se reporter au mémoire ${ }^{(2)}$ [Kra01] de Krause, qui utilise en théorie des représentations les foncteurs cohérents et d'autres considérations sur les catégories de foncteurs.) Peu après les travaux inauguraux d'Auslander sur les foncteurs cohérents, la publication [Mit72] de Mitchell sur les anneaux à plusieurs objets systématise l'emploi des catégories de foncteurs : un anneau à plusieurs objets est une petite catégorie additive $\mathcal{C}$; un module à gauche $\operatorname{sur} \mathcal{C}$ est un foncteur additif de $\mathcal{C}$ vers les groupes abéliens. Mitchell vise autant à développer un point de vue et des outils nouveaux en théorie des anneaux et des modules qu'à généraliser celle-ci.

Si ces différents travaux ont motivé un intérêt intrinsèque pour les catégories de foncteurs, le sujet a connu un renouveau au début des années 1990, où de puissants calculs cohomologiques ont été menés et de nouvaux liens féconds entre les catégories de foncteurs, la topologie algébrique, les représentations modulaires des groupes finis et plusieurs théories cohomologiques établis. On pourra consulter l'article de Henn, Lannes et Schwartz [HLS93] et les articles [Kuh94a], [Kuh94b] et [Kuh95] de Kuhn pour les premiers développements à ce sujet; récemment, Powell a donné, dans l'article

\footnotetext{
(1) Par exemple, dans la publication [GJ81] de Gruson et Jenson, des investigations sur la catégorie $D(R)$ (où des notions variées de finitude sont utilisées) aboutissent à des résultats de nature cohomologique liés à l'annulation des foncteurs dérivés du foncteur limite.

${ }^{(2)} \mathrm{Sa}$ bibliographie donne également un aperçu de la richesse du développement des méthodes initiées, entre autres, par Auslander.
} 
[Pow06], une application significative des catégories de foncteurs à la cohomologie des espaces d'Eilenberg-Mac Lane. On pourra aussi se référer au chapitre 13 de l'ouvrage [Lod98] de Loday sur l'homologie cyclique et à la monographie [FFPS03] de Franjou, Friedlander, Pirashvili et Schwartz, qui constitue une introduction complète au domaine. Nous montrons dans le présent article comment progresser dans cette voie à l'aide de nouvelles catégories de foncteurs.

Nous obtenons ainsi des résultats nouveaux sur des catégories de foncteurs désormais classiques, notamment la catégorie $\mathcal{F}$ des foncteurs entre espaces vectoriels sur un corps fini (définie plus précisément ci-après). Nous démontrons en particulier un théorème d'annulation cohomologique très général, que l'on applique à la $K$-théorie stable des corps finis, et un résultat de structure global sur la catégorie $\mathcal{F}$, que l'on utilise pour établir le caractère noethérien de nouveaux foncteurs. Les deux principes intuitifs suivants guident notre travail : d'une part, la démonstration de propriétés d'annulation cohomologique dans une catégorie de foncteurs est souvent plus facile en transitant par une autre catégorie de foncteurs « plus grosse »; d'autre part, la structure des objets d'une catégorie de foncteurs peut souvent se ramener à celle d'objets « plus petits » (donc mieux compris) d'une autre catégorie de foncteurs. Ainsi donnerons-nous une description conjecturale de la filtration de Krull de la catégorie $\mathcal{F}$ à partir des objets localement finis d'autres catégories de foncteurs, dont le résultat de structure susmentionné constitue une forme partielle.

Dans tout cet article, $\mathbb{k}$ désigne un corps fini; on note $\mathcal{E}_{\mathbb{k}}$ la catégorie des espaces vectoriels sur $\mathbb{k}, \mathcal{E}_{\mathbb{k}}^{f}$ la sous-catégorie pleine des espaces de dimension finie et $\mathcal{F}(\mathbb{k})$ la catégorie des foncteurs de $\mathcal{E}_{\mathrm{k}}^{f}$ vers $\mathcal{E}_{\mathrm{k}}$ (la mention du corps $\mathbb{k}$ sera souvent omise dans les notations, par la suite). La catégorie $\mathcal{F}(\mathbb{k})$ est abélienne; elle possède suffisamment d'objets injectifs et projectifs - précisément, elle est engendrée par les foncteurs projectifs standard $P_{V}=\mathbb{k}\left[\operatorname{hom}_{\mathcal{E}^{f}}(V,-)\right]$. D'importants calculs cohomologiques ont été réalisés dans cette catégorie, notamment avec les travaux de Franjou, Lannes et Schwartz ([FLS94]) et de Franjou, Friedlander, Scorichenko et Suslin ([FFSS99]).

L'identification de la cohomologie de Mac Lane (définie dans [ML57]) comme un cas particulier de cohomologie des foncteurs par Jibladze et Pirashvili ([JP91]) illustre l'intérêt des calculs cohomologiques dans la catégorie $\mathcal{F}(\mathbb{k})$. L'isomorphisme entre la $K$-théorie stable de $\mathbb{k}$ et l'homologie dans $\mathcal{F}(\mathbb{k})$ pour des systèmes de coefficients polynomiaux, établi indépendamment par Betley (cf. [Bet99]) et Suslin (cf. appendice de [FFSS99]), a fourni une motivation majeure à l'étude des propriétés homologiques de $\mathcal{F}(\mathbb{k})$. Les articles de Pirashvili [Pir03], Pirashvili et Richter [PR02], et Pirashvili et Waldhausen [PW92] montrent comment aborder d'autres théories homologiques à l'aide de certaines catégories de foncteurs.

L'étude systématique de la catégorie $\mathcal{F}$ est menée depuis le début des années 1990 (cf. [Kuh94a], [Kuh94b] et [Kuh95]), à la suite des liens établis par Henn, Lannes et Schwartz dans [HLS93] (voir aussi l'ouvrage [Sch94] de Schwartz) entre les modules instables sur l'algèbre de Steenrod et cette catégorie; néanmoins, sa structure globale demeure fort mystérieuse. En effet, si la compréhension de ses objets de longueur finie peut se réduire à celle de modules sur des $\mathbb{k}$-algèbres de dimension finie, il en va bien différemment de ses objets de longueur infinie, dont l'étude se heurte à des 
problèmes profonds de compréhension générique des représentations modulaires (i.e. de compréhension des liens, notamment cohomologiques, entre les représentations de familles infinies de groupes) — la catégorie $\mathcal{F}(\mathbb{k})$ a d'ailleurs été nommée catégorie des représentations génériques des groupes linéaires sur k par Kuhn, qui a justifié cette terminologie dans les trois articles susmentionnés.

Ainsi, la conjecture suivante, proposée par Lannes et Schwartz, s'est révélée l'un des problèmes les plus difficiles à résoudre dans cette catégorie.

Conjecture 1 (Conjecture artinienne). — La catégorie $\mathcal{F}(\mathbb{k})$ est localement noethérienne.

La dénomination paradoxale de cette conjecture, dont une brève discussion est donnée dans la section 1.2, provient du contexte des modules instables sur l'algèbre de Steenrod, dans lequel il est plus naturel de formuler la question en termes duaux d'objets artiniens. La conjecture artinienne, qui a motivé nombre des travaux sur la catégorie $\mathcal{F}$, n'est qu'une illustration des questions qui demeurent ouvertes dans le domaine.

De fait, comme la catégorie $\mathcal{F}$ est une catégorie abélienne avec de bonnes propriétés de régularité, il s'impose de savoir si elle est localement noethérienne. Mais si cette question se trouve si ardue, c'est qu'on dispose de peu d'outils pour étudier les sousfoncteurs d'un foncteur donné. Ainsi le produit tensoriel, calculé au but, de deux foncteurs dont on connaît la structure peut-il s'avérer un objet très compliqué. Par

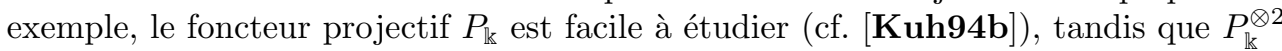
ne l'est nullement (cf.[Pow98a]).

\section{Les catégories de foncteurs en grassmanniennes : structures fondamentales}

Explicitons les catégories de foncteurs qui permettent de progresser dans l'étude de la conjecture artinienne. On note $\mathcal{G} r(V)$ la grassmannienne des sous-espaces vectoriels de $V$. On définit ainsi un foncteur $\mathcal{G} r$ de $\mathcal{E}_{\mathbb{k}}^{f}$ vers la catégorie des ensembles.

Définition 2. - Soit $\mathcal{E}_{\mathcal{G} r}^{f}(\mathbb{k})$ la catégorie des couples $(V, W)$, où $V$ est un $\mathbb{k}$-espace vectoriel de dimension finie et $W$ un élément de $\mathcal{G} r(V)$, et dont les morphismes $(V, W) \rightarrow\left(V^{\prime}, W^{\prime}\right)$ sont les applications linéaires $f: V \rightarrow V^{\prime}$ telles que $f(W)=W^{\prime}$. La catégorie de foncteurs en grassmanniennes $\mathcal{F}_{\mathcal{G} r}(\mathbb{k})$ est la catégorie des foncteurs de $\mathcal{E}_{\mathcal{G} r}^{f}(\mathbb{k})$ vers $\mathcal{E}_{\mathbb{k}}$.

Il est également naturel de s'intéresser à la catégorie, notée $\widetilde{\mathcal{E}}_{\mathcal{G} r}^{f}(\mathbb{k})$, qui a les mêmes objets que $\mathcal{E}_{\mathcal{G} r}^{f}(\mathbb{k})$, et dont les morphismes $(V, W) \rightarrow\left(V^{\prime}, W^{\prime}\right)$ sont les applications linéaires $f: V \rightarrow V^{\prime}$ telles que $f(W) \subset W^{\prime}$. La catégorie des foncteurs de $\widetilde{\mathcal{E}}_{\mathcal{G} r}^{f}(\mathbb{k})$ vers $\mathcal{E}$, notée $\widetilde{\mathcal{F}}_{\mathcal{G} r}(\mathbb{k})$, est également considérée dans cet article; elle constitue un adjuvant pour l'étude de la catégorie $\mathcal{F}_{\mathcal{G} r}(\mathbb{k})$.

Pour tout entier positif $n$, on note $\mathcal{F}_{\mathcal{G} r, n}$ la sous-catégorie pleine de $\mathcal{F}_{\mathcal{G} r}$ (rappelons que la mention du corps $\mathbb{k}$ est désormais sous-entendue) des foncteurs $X$ tels que $X(V, W)=0$ si $\operatorname{dim} W \neq n$. L'étude des catégories $\mathcal{F}_{\mathcal{G} r}$ et $\mathcal{F}_{\mathcal{G} r, n}$ et leur application à 
la catégorie $\mathcal{F}$ constituent le principal sujet de cet article; le lien entre $\mathcal{F}_{\mathcal{G} r}$ et les $\mathcal{F}_{\mathcal{G} r, n}$ provient de ce qu'il existe une stratification de $\mathcal{F}_{\mathcal{G} r}$ par des sous-catégories épaisses $\mathcal{F}_{\mathcal{G} r, \leq n}$, stratification dont les sous-quotients sont équivalents aux catégories $\mathcal{F}_{\mathcal{G} r, n}$.

On peut encore ramener l'étude des catégories $\mathcal{F}_{\mathcal{G} r, n}$, qui peuvent également se décrire comme des catégories de foncteurs, à celle de catégories plus simples : le groupe linéaire $G L_{n}(\mathbb{k})$ intervient naturellement dans la structure de $\mathcal{F}_{\mathcal{G} r, n}$, qui constitue une sorte de produit semi-direct tordu entre la catégorie des $\mathbb{k}\left[G L_{n}(\mathbb{k})\right]$-modules et la catégorie de foncteurs $\mathcal{F}_{\mathbf{P l}, n}$ définie comme suit. On note $\mathcal{E}_{\mathbf{P l}, n}^{f}$ la catégorie des objets $V$ de $\mathcal{E}^{f}$ munis d'un monomorphisme $\mathbb{k}^{n} \hookrightarrow V$. La catégorie $\mathcal{F}_{\mathbf{P l}, n}$ est la catégorie des foncteurs de $\mathcal{E}_{\mathrm{Pl}, n}^{f}$ vers $\mathcal{E}$. Outre ses liens avec $\mathcal{F}_{\mathcal{G} r, n}$, cette catégorie possède un intérêt intrinsèque, car elle est équivalente à la catégorie des comodules de la catégorie $\mathcal{F}$ sur le foncteur injectif standard associé à l'espace vectoriel $\mathbb{k}^{n}$.

Mentionnons une autre catégorie de foncteurs considérée dans cet article. Soit $\mathcal{E}_{\text {surj }}^{f}$ la sous-catégorie de $\mathcal{E}^{f}$ ayant les mêmes objets et dont les morphismes sont les épimorphismes de $\mathcal{E}^{f}$. La catégorie, notée $\mathcal{F}_{\text {surj }}$, des foncteurs de $\mathcal{E}_{\text {surj }}^{f}$ vers $\mathcal{E}$ joue un rôle important dans l'étude de la catégorie $\mathcal{F}_{\mathcal{G} r}$. En effet, si $f:(V, W) \rightarrow\left(V^{\prime}, W^{\prime}\right)$ est un morphisme de $\mathcal{E}_{\mathcal{G} r}^{f}$, alors $f$ induit un épimorphisme de $W$ sur $W^{\prime}$ : on obtient ainsi un foncteur $\mathcal{E}_{\mathcal{G} r}^{f} \rightarrow \mathcal{E}_{\text {surj }}^{f}$, donné sur les objets par $(V, W) \mapsto W$, puis par précomposition un foncteur fondamental $\mathcal{F}_{\text {surj }} \rightarrow \mathcal{F}_{\mathcal{G} r}$. Une catégorie analogue à $\mathcal{F}_{\text {surj }}$ s'obtient à partir des monomorphismes de $\mathcal{E}^{f}$; on la note $\mathcal{F}_{i n j}$. Elle possède des liens étroits avec les systèmes de coefficients introduits par Dwyer dans [Dwy80] et joue un rôle essentiel dans la comparaison entre la $K$-théorie stable de $\mathbb{k}$ et les groupes d'extension dans $\mathcal{F}(\mathbb{k})$ (théorème de Betley-Suslin susmentionné), comme l'a montré Scorichenko (qui a généralisé ce théorème sur un anneau quelconque).

Revenons à la catégorie $\mathcal{F}_{\mathcal{G} r}$. Le foncteur d'oubli $\mathcal{E}_{\mathcal{G} r}^{f} \rightarrow \mathcal{E}^{f}$ donné par $(V, W) \mapsto V$ induit par précomposition un foncteur $\iota: \mathcal{F} \rightarrow \mathcal{F}_{\mathcal{G} r}$. Il est adjoint à droite au foncteur d'intégrale en grassmanniennes $\omega: \mathcal{F}_{\mathcal{G} r} \rightarrow \mathcal{F}$ donné par

$$
\omega(X)(V)=\bigoplus_{W \in \mathcal{G} r(V)} X(V, W) .
$$

Ce foncteur constitue un outil très puissant pour relier les catégories $\mathcal{F}_{\mathcal{G} r}$ et $\mathcal{F}$. Son importance est d'abord illustrée par le résultat formel suivant, dans lequel le foncteur $\omega(\mathbb{k})=\mathbb{k}[\mathcal{G} r]$ de $\mathcal{F}$ est muni de la structure comultiplicative déduite du fait qu'il est la linéarisation d'un foncteur ensembliste.

Proposition 3. - Le foncteur $\omega: \mathcal{F}_{\mathcal{G} r} \rightarrow \mathcal{F}$ induit une équivalence entre $\mathcal{F}_{\mathcal{G} r}$ et la catégorie des $\mathbb{k}[\mathcal{G} r]$-comodules de $\mathcal{F}$.

On peut donner une description similaire des catégories $\mathcal{F}_{\mathcal{G} r, n}$ et $\mathcal{F}_{\mathcal{G} r, \leq n}$ en termes de comodules. Le cas le plus intuitif est celui de $\mathcal{F}_{\mathcal{G} r, \leq n}$, équivalente à la catégorie des comodules sur le sous-foncteur en coalgèbres $G(n)=\mathbb{k}\left[\mathcal{G} r_{\leq n}\right]$ de $\mathbb{k}[\mathcal{G} r]$ - ici $\mathcal{G} r_{\leq n}$ désigne le sous-foncteur ensembliste de $\mathcal{G} r$ donné par $\mathcal{G} r_{\leq n}(V)=\{W \in \mathcal{G} r(V) \mid \operatorname{dim} W \leq n\}$. Par dualité, on en déduit que la catégorie opposée à $\mathcal{F}_{\mathcal{G} r, n}$ est « presque » équivalente à la catégorie des modules sur un 
foncteur en algèbres $D(n)$ dual de $G(n)$. La notation provient de ce que le module instable nilfermé associé à $D(n)$ (cf. [HLS93]; on suppose ici le corps $\mathbb{k}$ premier) est exactement l'algèbre de Dickson $\mathbb{k}\left[x_{1}, \ldots, x_{n}\right]^{G L_{n}(\mathbb{k})}$. Le foncteur $D(n)$ (ainsi qu'un quotient remarquable $\bar{D}(n)$ qui apparaît plutôt dans l'étude de $\mathcal{F}_{\mathcal{G} r, n}$ ) joue un rôle significatif dans l'article [Pow98c] de Powell, qui a annoncé l'importance de la catégorie des modules sur $D(n)$ (ou $\bar{D}(n))$.

L'étude de la structure élémentaire de la catégorie $\mathcal{F}_{\mathcal{G} r}$ repose principalement sur le foncteur différence, analogue à l'endofoncteur du même nom dans $\mathcal{F}$. Ce foncteur, noté $\Delta^{\mathcal{G} r}$, est donné par le scindement canonique

$$
X(V \oplus \mathbb{k}, W) \simeq X(V, W) \oplus \Delta^{\mathcal{G} r}(X)(V, W) .
$$

Rappelons que le foncteur différence $\Delta$ de $\mathcal{F}$ est quant à lui caractérisé par le scindement canonique $F(V \oplus \mathbb{k}) \simeq F(V) \oplus \Delta(F)(V)$. Comme dans la catégorie $\mathcal{F}$, on introduit la définition suivante :

Définition 4. - Un objet $X$ de $\mathcal{F}_{\mathcal{G} r}$ est dit polynomial s'il existe un entier $n$ tel que $\left(\Delta^{\mathcal{G} r}\right)^{n}(X)=0$.

Contrairement au cas de la catégorie $\mathcal{F}$, les foncteurs polynomiaux de degré nul de $\mathcal{F}_{\mathcal{G} r}$ ne sont pas tous constants. Ces foncteurs sont appelés foncteurs pseudo-constants; ils forment une sous-catégorie de $\mathcal{F}_{\mathcal{G} r}$ équivalente à la catégorie $\mathcal{F}_{\text {surj }}$.

Dans ce qui suit, nous nommons fini un objet de longueur finie d'une catégorie abélienne. Un objet localement fini est un objet colimite d'objets finis.

Proposition 5. - Les foncteurs finis de la catégorie $\mathcal{F}_{\mathcal{G} r}$ sont polynomiaux.

Ce résultat permet de classifier et décrire explicitement les objets simples de la catégorie $\mathcal{F}_{\mathcal{G} r}$ à partir des objets simples de $\mathcal{F}$ et des représentations simples des groupes linéaires, par une méthode semblable à celle utilisée pour classifier les objets simples de $\mathcal{F}$ à partir des représentations simples des groupes symétriques.

Nombre des foncteurs entre la catégorie $\mathcal{F}_{\mathcal{G} r}$ et la catégorie $\mathcal{F}$, ou d'autres qui lui sont étroitement reliées, possèdent de bonnes propriétés de commutation au foncteur différence. Par exemple, il existe un isomorphisme canonique $\iota \circ \Delta \simeq \Delta^{\mathcal{G} r} \circ \iota$ de foncteurs $\mathcal{F} \rightarrow \mathcal{F}_{\mathcal{G} r}$. En revanche, le foncteur composé $\Delta \circ \omega: \mathcal{F}_{\mathcal{G} r} \rightarrow \mathcal{F}$ diffère du foncteur $\omega \circ \Delta^{\mathcal{G} r}$, qu'il contient comme facteur direct. Ainsi, l'image par le foncteur d'intégrale en grassmanniennes d'un objet fini de la catégorie $\mathcal{F}_{\mathcal{G} r}$ n'est généralement pas un objet localement fini de la catégorie $\mathcal{F}$. D'ailleurs, tous les objets projectifs de type fini de $\mathcal{F}$ sont l'image par le foncteur $\omega$ de foncteurs finis de $\mathcal{F}_{\mathcal{G} r}$.

L'image d'un objet fini de la catégorie $\mathcal{F}_{\mathcal{G} r}$ par le foncteur $\omega$ est toujours un objet de type fini de la catégorie $\mathcal{F}$. Le principe général pour comprendre $\mathcal{F}$ à partir de $\mathcal{F}_{\mathcal{G} r}$ consiste à ramener l'étude des objets de type fini de $\mathcal{F}$ à celle des objets finis de $\mathcal{F}_{\mathcal{G} r}$ grâce au foncteur $\omega$. La mise en œuvre de ce principe s'avère ardue; en effet, les objets de type fini de la catégorie $\mathcal{F}$ ne sont pas tous isomorphes à l'image par $\omega$ d'un objet fini de $\mathcal{F}_{\mathcal{G} r}$. Pour préciser les choses, nous avons introduit la notion de foncteur induit dans $\mathcal{F}$ (cf. section 12.1) : un foncteur induit s'obtient par un nombre fini d'opérations du type extension, noyau ou conoyau à partir des images par $\omega$ d'objets finis de $\mathcal{F}_{\mathcal{G} r}$ (cette terminologie est motivée par l'analogie entre $\omega$ et un foncteur d'induction). Un 
foncteur de $\mathcal{F}$ est induit de hauteur au plus $n$ si l'on peut choisir les objets finis de $\mathcal{F}_{\mathcal{G} r}$ qui interviennent dans la sous-catégorie $\mathcal{F}_{\mathcal{G} r, \leq n}$. La conjecture que nous émettons sur les objets de type fini de $\mathcal{F}$ peut s'énoncer ainsi :

Conjecture 6. - Pour tout entier n, la classe des foncteurs induits de hauteur au plus $n$ est stable par quotients.

La conjecture artinienne extrêmement forte (conjecture 9 présentée ci-dessous) est équivalente à cet énoncé, qui implique en particulier que tout foncteur de type fini de $\mathcal{F}$ est induit.

\section{Applications : algèbre homologique et filtration de Krull}

Venons-en maintenant à des propriétés profondes des catégories de foncteurs en grassmanniennes. Notre résultat d'annulation cohomologique principal est le suivant, dans lequel $\mathcal{I}$ désigne l'endofoncteur de $\mathcal{F}_{\mathcal{G} r}$ donné par $\mathcal{I}(X)(V, W)=\underset{B \in \mathcal{G} r(W)}{\bigoplus} X(V, B)$. Ce foncteur conserve les objets localement finis.

Théorème 7. - Soient $X$ et $Y$ des objets de $\mathcal{F}_{\mathcal{G}_{r}}, X$ étant supposé localement fini. Il existe un isomorphisme gradué naturel $\operatorname{Ext}_{\mathcal{F}}^{*}(\omega(X), \omega(Y)) \simeq \operatorname{Ext}_{\mathcal{F}_{\mathcal{G} r}}^{*}(X, \mathcal{I}(Y))$.

La démonstration de ce théorème, qui repose sur des arguments d'adjonction utilisant le foncteur différence $\Delta^{\mathcal{G} r}$, illustre en quoi il s'agit d'un résultat d'annulation cohomologique. Cet aspect d'annulation apparaît d'ailleurs très clairement dans le corollaire suivant, où $\omega_{n}: \mathcal{F}_{\mathcal{G} r, n} \rightarrow \mathcal{F}$ désigne le foncteur exact restriction du foncteur $\omega$ à la sous-catégorie $\mathcal{F}_{\mathcal{G} r, n}$ de $\mathcal{F}_{\mathcal{G} r}$.

Corollaire 8. - Soient $k$ et $n$ deux entiers naturels, $X$ un objet localement fini de $\mathcal{F}_{\mathcal{G} r, k}$ et $Y$ un objet de $\mathcal{F}_{\mathcal{G} r, n}$.

(1) Si $k<n$, alors $\operatorname{Ext}_{\mathcal{F}}^{*}\left(\omega_{k}(X), \omega_{n}(Y)\right)=0$.

(2) Si $k=n$, alors le morphisme naturel $\operatorname{Ext}_{\mathcal{F}_{\mathcal{G} r, n}}^{*}(X, Y) \rightarrow \operatorname{Ext}_{\mathcal{F}}^{*}\left(\omega_{n}(X), \omega_{n}(Y)\right)$ induit par $\omega_{n}$ est un isomorphisme.

Le théorème 7 et le corollaire 8 permettent d'une part de généraliser le théorème de Betley-Suslin sur la $K$-théorie stable de $\mathbb{k}$, d'autre part de mener de nombreux calculs cohomologiques dans la catégorie $\mathcal{F}$ utiles pour comprendre sa structure, comme le théorème de l'appendice de [Pow98a].

Un moyen efficace d'appréhender la structure globale d'une catégorie abélienne $\mathcal{A}$ consiste à étudier sa filtration de $\operatorname{Krull}$, notée $\left(\mathcal{K}_{n}(\mathcal{A})\right)$, qui est définie par $\mathcal{K}_{-1}(\mathcal{A})=$ $\{0\}$ et par le fait que, pour $n \geq 0, \mathcal{K}_{n}(\mathcal{A}) / \mathcal{K}_{n-1}(\mathcal{A})$ est la plus petite sous-catégorie épaisse et stable par colimites de $\mathcal{A} / \mathcal{K}_{n-1}(\mathcal{A})$ qui en contient les objets simples (cf. section B.4). Le corollaire 8, qui indique une hiérarchie dans la « taille $»$ des objets du type $\omega_{n}(X)$ selon la valeur de $n$, où $X$ est un objet localement fini de $\mathcal{F}_{\mathcal{G} r, n}$, suggère la description conjecturale suivante de la filtration de Krull de la catégorie $\mathcal{F}$. Celle-ci précise considérablement la conjecture artinienne (conjecture 1) et constitue une motivation essentielle à l'étude des catégories de foncteurs en grassmanniennes. 


\section{Conjecture 9 (Conjecture artinienne extrêmement forte)}

Pour tout entier positif $n$, le foncteur $\omega_{n}$ induit une équivalence entre la souscatégorie pleine $\mathcal{F}_{\mathcal{G} r, n}^{\text {lf }}$ des objets localement finis de $\mathcal{F}_{\mathcal{G} r, n}$ et la catégorie quotient $\mathcal{K}_{n}(\mathcal{F}) / \mathcal{K}_{n-1}(\mathcal{F})$.

Non seulement cet énoncé entraîne toutes les formes renforcées de la conjecture artinienne émises jusqu'à présent, mais il implique aussi un grand nombre d'autres résultats profonds sur la catégorie $\mathcal{F}$. L'une des illustrations les plus frappantes (corollaire 11 ci-dessous) se déduit du théorème suivant, où l'on se limite au corps $\mathbb{F}_{2}$ à deux éléments pour des raisons sur lesquelles nous reviendrons plus loin.

Théorème 10. - Soient $X$ et $Y$ deux objets finis de la catégorie $\mathcal{F}_{\mathcal{G}_{r}}\left(\mathbb{F}_{2}\right)$. Supposons que les objets $\omega(X)$ et $\omega(Y)$ de $\mathcal{F}\left(\mathbb{F}_{2}\right)$ ont les mêmes facteurs de composition. Alors $X$ et $Y$ ont les mêmes facteurs de composition.

Les facteurs de composition sont comptés avec multiplicité. On remarquera qu'un foncteur de type fini de $\mathcal{F}$ (ou plus généralement, prenant des valeurs de dimension finie) a en général un nombre infini de facteurs de composition, mais que chacun y a une multiplicité finie.

En admettant la conjecture artinienne extrêmement forte, le théorème 10 donne une description du groupe de Grothendieck $G_{0}^{t f}\left(\mathcal{F}\left(\mathbb{F}_{2}\right)\right)$ des objets de type fini de la catégorie $\mathcal{F}\left(\mathbb{F}_{2}\right)$, isomorphe via le foncteur $\omega$ au groupe de Grothendieck des objets finis de $\mathcal{F}_{\mathcal{G} r}\left(\mathbb{F}_{2}\right)$. Mais il procure aussi le résultat suivant, dans lequel les catégories de foncteurs en grassmanniennes n'interviennent pas.

Corollaire 11. - Si la conjecture artinienne extrêmement forte est vraie, alors la classe d'un objet de type fini de $\mathcal{F}\left(\mathbb{F}_{2}\right)$ dans le groupe de Grothendieck $G_{0}^{\text {tf }}\left(\mathcal{F}\left(\mathbb{F}_{2}\right)\right)$ est déterminée par ses facteurs de composition.

La démonstration du théorème 10 repose sur des méthodes de théorie des représentations (la représentation de Steinberg joue un rôle important) et sur l'étude conjointe des foncteurs $\omega: \mathcal{F}_{\mathcal{G} r} \rightarrow \mathcal{F}$ et $\tilde{\nabla}_{n}: \mathcal{F} \rightarrow \mathcal{F}$. Les foncteurs $\tilde{\nabla}_{n}$ ont été introduits par Powell dans [Pow98b]; ils forment une filtration décroissante de sous-foncteurs du foncteur différence $\Delta: \mathcal{F} \rightarrow \mathcal{F}$ obtenue à partir de la filtration polynomiale de l'injectif standard associé à $\mathbb{k}$. Certaines complications techniques apparaissant pour un corps fini quelconque, c'est pourquoi nous nous sommes placés dans le cas $\mathbb{k}=\mathbb{F}_{2}$ pour nos considérations utilisant les $\tilde{\nabla}_{n}$, comme dans plusieurs des articles de Powell à ce sujet.

Combiner les outils fournis d'une part par les catégories de foncteurs en grassmanniennes et d'autre part par les foncteurs $\tilde{\nabla}_{n}$ nous permet également d'établir une forme affaiblie de la conjecture artinienne extrêmement forte. Déjà, grâce aux $\tilde{\nabla}_{n}$, Powell a donné les premiers renseignements profonds connus sur la structure globale de la catégorie $\mathcal{F}\left(\mathbb{F}_{2}\right)$ : son théorème de simplicité (établi dans [Pow98c]) procure des informations sur tous les objets projectifs standard de la catégorie $\mathcal{F}\left(\mathbb{F}_{2}\right)$. De plus, Powell a déterminé la structure du foncteur projectif $P_{\mathbb{F}_{2}{ }^{2}}$, dont il a montré le caractère noethérien et l'appartenance à $\mathcal{K}_{2}(\mathcal{F})$, à l'aide du foncteur $\tilde{\nabla}_{2}$ (cf. [Pow98a]). La préservation des monomorphismes et des épimorphismes par les foncteurs $\tilde{\nabla}_{n}$ et le 
contrôle précis de leur effet sur les foncteurs simples de $\mathcal{F}\left(\mathbb{F}_{2}\right)$ permettent de fait de mener efficacement des raisonnements à la fois explicites (en termes d'éléments) et généraux dans la catégorie $\mathcal{F}\left(\mathbb{F}_{2}\right)$.

Le résultat suivant, où $\overline{\mathcal{N}}_{\tilde{\nabla}_{n}}$ désigne la plus petite sous-catégorie épaisse stable par colimites de $\mathcal{F}\left(\mathbb{F}_{2}\right)$ contenant les foncteurs $\tilde{\nabla}_{n}$-nilpotents, constitue l'avancée principale sur la structure de $\mathcal{F}\left(\mathbb{F}_{2}\right)$ présentée dans ce travail.

Théorème 12 (Théorème de simplicité généralisé). - Pour tout entier naturel $n$, le foncteur $\omega_{n}: \mathcal{F}_{\mathcal{G} r, n} \rightarrow \mathcal{F}$ induit une équivalence entre la sous-catégorie pleine des objets localement finis de $\mathcal{F}_{\mathcal{G} r, n}$ et une sous-catégorie épaisse de $\overline{\mathcal{N}}_{\tilde{\nabla}_{n+1}} / \overline{\mathcal{N}}_{\tilde{\nabla}_{n}}$.

Le théorème de simplicité de Powell énonce pour sa part que si $X$ est un objet simple pseudo-constant de $\mathcal{F}_{\mathcal{G} r, n}$, alors l'image de $\omega_{n}(X)$ dans la catégorie $\overline{\mathcal{N}}_{i} \tilde{\nabla}_{n+1} / \overline{\mathcal{N}}_{i} \tilde{\nabla}_{n}$ est simple. Nous gagnons donc essentiellement le passage à un objet simple quelconque de $\mathcal{F}_{\mathcal{G} r, n}$ et le contrôle des groupes d'extensions dans la catégorie quotient à partir des groupes d'extensions dans $\mathcal{F}_{\mathcal{G} r, n}$.

La démonstration du théorème 12 utilise deux types d'ingrédients. D'une part apparaissent des considérations explicites relatives aux foncteurs $\tilde{\nabla}_{n}$, qui procèdent des mêmes idées que le théorème de simplicité de Powell. D'autre part, des propriétés d'annulation cohomologique du foncteur $\omega$, fournies par le théorème 7 et une variante en termes des foncteurs $\tilde{\nabla}_{n}$ établie dans la section 15.3 , jouent un rôle significatif.

Une grande part de la riche structure de la catégorie de foncteurs en grassmanniennes $\mathcal{F}_{\mathcal{G} r}$ intervient dans le théorème de simplicité généralisé. Ainsi, la filtration par les sous-catégories $\mathcal{F}_{\mathcal{G} r, \leq n}$ est omniprésente; les propriétés cohomologiques du foncteur $\omega$ utilisées dans la démonstration reposent sur la description fonctorielle de ces catégories. On emploie également de manière décisive la description en termes de comodules des catégories de foncteurs en grassmanniennes, dans un argument de stabilisation qui constitue la partie la plus concrète de la démonstration. Cet argument généralise de manière conceptuelle des considérations déjà utilisées par Powell.

Comme conséquence du théorème de simplicité généralisé et des résultats de Powell sur les foncteurs annihilés par $\tilde{\nabla}_{2}$ (utilisés pour déterminer la structure de $P_{\mathbb{F}_{2}}{ }^{2}$ ), nous donnons la contribution suivante à la conjecture artinienne ${ }^{(3)}$ :

Théorème 13. - Pour tout foncteur fini $F$ de $\mathcal{F}$, le foncteur $P_{\mathbb{F}_{2}} \otimes F$ est noethérien et dans $\mathcal{K}_{2}(\mathcal{F})$.

Plus généralement, grâce au théorème 12, la conjecture artinienne extrêmement forte est ramenée à un problème plus concret : montrer que les quotients de la filtration par $\tilde{\nabla}_{n}$-nilpotence de certains foncteurs sont induits d'une certaine hauteur. Cette filtration est définie par le noyau de flèches entièrement explicites (cf. [Pow98b]); son étude se trouve étroitement liée à des questions fines de représentations des groupes symétriques ou linéaires $\left(\operatorname{sur} \mathbb{F}_{2}\right.$ ). En effet, les partitions associées aux facteurs de composition des quotients de cette filtration sont contrôlées, le problème consiste donc

${ }^{(3)}$ L'auteur a récemment généralisé le théorème 13, montrant que le foncteur $P_{\mathbb{F}_{2}}{ }^{3} \otimes F$ est noethérien et dans $\mathcal{K}_{3}(\mathcal{F})$ pour tout foncteur fini $F$ (voir $[\mathbf{D j a 0 7}]$ ). 
d'une certaine façon à maîtriser les extensions entre les différents facteurs de composition possibles. Ce problème dérive essentiellement de la théorie des représentations ; c'est une sorte de générisation de la question (beaucoup plus élémentaire) suivante : déterminer les sous-représentations maximales d'un produit tensoriel de puissances extérieures de la représentation régulière d'un groupe symétrique dont tous les facteurs de composition correspondent à des partitions de longueur inférieure à un entier fixé.

Une bonne partie des méthodes de cet article peuvent se généraliser : la définition, les constructions et propriétés de base de la catégorie $\mathcal{F}_{\mathcal{G} r}(\mathbb{k})$ se transposent sans changement si l'on remplace la catégorie source $\mathcal{E}_{\mathbb{k}}^{f}$ de $\mathcal{F}(\mathbb{k})$ par une catégorie abélienne essentiellement petite $\mathcal{A}$ dans laquelle les ensembles de morphismes et de sous-objets sont finis. En effet, dans cette situation, l'adjonction entre les foncteurs $\iota: \mathcal{F} \rightarrow \mathcal{F}_{\mathcal{G} r}$ et $\omega$ s'étend aussitôt, et l'on dispose de foncteurs différences. Des généralisations dans un cadre non abélien sont également envisageables. Les résultats combinant les catégories de foncteurs en grassmanniennes aux foncteurs $\tilde{\nabla}_{n}$, moins formels, sont plus délicats à généraliser. Cependant, le passage du corps $\mathbb{F}_{2}$ à un corps fini quelconque ne pose de problème réel que pour la conséquence concrète du théorème de simplicité généralisé donnée par le théorème 13 ; dans un cadre plus vaste que celui des espaces vectoriels sur les corps finis, c'est la description explicite de la filtration polynomiale du premier injectif standard non constant de $\mathcal{F}$ qu'il conviendrait d'étendre pour obtenir des analogues du théorème 12 .

\section{Organisation de l'article}

Le premier chapitre rappelle les rudiments nécessaires sur la catégorie $\mathcal{F}$, la conjecture artinienne et les foncteurs $\tilde{\nabla}_{n}$. Le deuxième traite des catégories $\mathcal{F}_{\text {surj }}$ et $\mathcal{F}_{\text {inj }}$, à la fois d'un point de vue intrinsèque et d'un point de vue préliminaire à l'étude des catégories de foncteurs en grassmanniennes et de la $K$-théorie stable de $\mathbb{k}$.

Les chapitres 3 et 4 s'attachent à des constructions catégoriques nécessaires à la deuxième partie. Celle-ci introduit les catégories de foncteurs en grassmanniennes $\mathcal{F}_{\mathcal{G} r}$, $\mathcal{F}_{\mathcal{G} r, n}, \mathcal{F}_{\mathbf{P l}, n}$ et $\widetilde{\mathcal{F}}_{\mathcal{G} r}$ et donne leurs propriétés de base : outre leur description fonctorielle, les trois premières d'entre elles sont identifiées comme catégories de comodules, et traitées de manière monadique, à l'aide du théorème de Beck. Les objets finis de la catégorie $\mathcal{F}_{\mathcal{G} r}(\mathbb{k})$, notamment, sont étudiés en détails. On établit ainsi la proposition 5 , dont on tire les conséquences, ainsi que les propriétés d'adjonction élémentaires entre les très nombreux foncteurs définis, qui font la richesse de la structure de ces catégories.

La troisième partie expose des applications de nature cohomologique des constructions des parties précédentes, à l'aide du foncteur d'intégrale en grassmanniennes $\omega: \mathcal{F}_{\mathcal{G} r}(\mathbb{k}) \rightarrow \mathcal{F}(\mathbb{k})$, dont on démontre, au chapitre 10, la propriété fondamentale, donnée par le théorème 7 et le corollaire 8 . Les premières applications à la catégorie $\mathcal{F}(\mathbb{k})$ (discussion de la conjecture artinienne extrêmement forte), données dans le chapitre 12 , constituent une introduction à la partie IV; le chapitre 11, qui tire des résultats du chapitre 10 des conséquences apparemment internes aux catégories de 
foncteurs en grassmanniennes, se révèle également utile dans $\mathcal{F}(\mathbb{k})$ puisqu'il sous-tend la démarche de $[\mathbf{D j a 0 6 c}]$. Enfin, le chapitre 13 est consacré à une propriété d'annulation cohomologique des systèmes de coefficients, déduite de celle du foncteur $\omega$, et qu'on applique à la $K$-théorie stable.

La dernière partie présente les résultats obtenus sur la catégorie $\mathcal{F}\left(\mathbb{F}_{2}\right)$ grâce aux foncteurs $\tilde{\nabla}_{n}$ et $\omega$. Le chapitre 14 a trait à la catégorie quotient de $\mathcal{F}$ par la sous-catégorie des foncteurs analytiques : elle expose une bonne partie du matériel nécessaire au théorème de simplicité généralisé avec des simplifications techniques appréciables provenant de l'identification du foncteur $\tilde{\nabla}_{1}$ au foncteur différence $\Delta$. Dans le chapitre 15 , nous étudions les foncteurs composés $\tilde{\nabla}_{n} \omega_{n}: \mathcal{F}_{\mathcal{G} r, n} \rightarrow \mathcal{F}$ à l'aide d'endofoncteurs $\nabla_{n}^{\mathcal{G} r}$ de $\mathcal{F}_{\mathcal{G} r}$. Cela permet, dans le chapitre 16, de démontrer les résultats principaux de la partie IV, les théorèmes 10, 12 et 13 .

Les appendices fixent nos notations et rappellent quelques résultats connus sur trois leitmotive de cet article : les adjonctions (à la base de plusieurs descriptions des catégories de foncteurs en grassmanniennes comme du théorème d'annulation cohomologique principal), les propriétés de finitude des catégories abéliennes (dans l'optique de la conjecture artinienne) et les catégories de foncteurs.

\section{Notations et conventions}

(1) fondamentales :

(a) Nous noterons $\mathcal{G} r(V)$ l'ensemble des sous-espaces vectoriels d'un espace vectoriel $V$. Si $I$ est une partie de $\mathbb{N}$, nous noterons $\mathcal{G} r_{I}(V)$ le sous-ensemble de $\mathcal{G} r(V)$ constitué des sous-espaces dont la dimension appartient à $I$.

(b) $\mathrm{Si} \mathcal{C}$ et $\mathcal{D}$ sont deux catégories, $\mathcal{C}$ étant essentiellement petite, nous noterons $\operatorname{Fct}(\mathcal{C}, \mathcal{D})$ la catégorie des foncteurs de $\mathcal{C}$ vers $\mathcal{D}$.

(2) couramment utilisées :

(a) La caractéristique du corps fini $\mathbb{k}$ sera notée $p ; q=p^{d}$ désignera son cardinal. Lorsqu'aucune confusion ne pourra en résulter, nous omettrons toute mention du corps $\mathbb{k}$ dans les notations.

(b) Nous noterons Ens la catégorie des ensembles, et $\mathbf{E n s}^{f}$ la sous-catégorie pleine des ensembles finis.

(c) Soit $E$ un ensemble. Nous noterons $\mathbb{k}[E]$ le $\mathbb{k}$-espace vectoriel somme directe de copies de $\mathbb{k}$ indexées par $E$. On peut voir l'association $E \mapsto \mathbb{k}[E]$ comme un foncteur de Ens vers $\mathcal{E}_{\mathbb{k}}$.

Nous noterons $[e]$ l'élément de la base canonique de $\mathbb{k}[E]$ associé à un élément $e$ de $E$.

(d) Nous désignerons par $\operatorname{Mod}_{A}$ la catégorie des modules à droite sur un anneau $A$ et ${ }_{A}$ Mod la catégorie des $A$-modules à gauche. Nous adopterons plus généralement ces notations lorsque $A$ est un objet d'une catégorie monoïdale symétrique muni d'une structure d'algèbre.

Dans le cas d'un objet $C$ muni d'une structure de coalgèbre, nous noterons $\operatorname{Comod}_{C}$ la catégorie des $C$-comodules à droite. 
Dans le cas d'une (co)algèbre (co)unitaire, les morphismes seront toujours censés préserver la (co)unité.

(e) Nous noterons $\mathrm{Ob} \mathcal{C}$ la classe des objets d'une catégorie $\mathcal{C}$. Si $X$ et $Y$ sont deux objets de $\mathcal{C}$, on note :

- $\operatorname{hom}_{\mathcal{C}}(X, Y)$ l'ensemble ${ }^{(4)}$ des morphismes de $X$ dans $Y$;

- $\operatorname{End}_{\mathcal{C}}(X)$ le monoïde $\operatorname{hom}_{\mathcal{C}}(X, X)$ des endomorphismes de $X$;

- $\operatorname{Aut}_{\mathcal{C}}(X)$ le groupe des automophismes de $X$;

- $\mathrm{Pl}_{\mathcal{C}}(X, Y)$ l'ensemble des monomorphismes de $X$ dans $Y$;

- $\operatorname{Epi}_{\mathcal{C}}(X, Y)$ l'ensemble des épimorphismes de $X$ vers $Y$;

- $\operatorname{Iso}_{\mathcal{C}}(X, Y)$ l'ensemble des isomorphismes de $X$ vers $Y$.

L'indice $\mathcal{C}$ sera omis quand aucune confusion ne peut en résulter.

Enfin, $\mathcal{C}^{o p}$ désignera la catégorie opposée de $\mathcal{C}$.

(f) On désigne par $\mathbb{N}$ l'ensemble des entiers positifs ou nuls, et par $\mathbb{N}^{*}$ l'ensemble des entiers strictement positifs.

Afin d'alléger les notations d'indices ou d'exposants où interviennent des parties de $\mathbb{N}$, on adoptera les abréviations suivantes :

(i) $n=\{n\}$,

(ii) $\leq n=\{i \in \mathbb{N} \mid i \leq n\}$,

(iii) $\geq n=\{i \in \mathbb{N} \mid i \geq n\}$.

(3) plus secondaires :

(a) Nous noterons $V^{*}$ le dual d'un espace vectoriel $V$, et $W^{\perp}$ l'orthogonal dans $V^{*}$ d'un sous-espace $W$ de $V$.

Plus généralement, si $V$ est une représentation d'un groupe, $V^{*}$ désignera la représentation contragrédiente.

(b) Soit $M$ un monoïde.

(i) Nous noterons $\mathbb{k}[M]$ l'algèbre de $M$ sur $\mathbb{k}$.

(ii) Nous désignerons par $\underline{M}$ la catégorie à un seul objet de monoïde d'endomorphismes $M$.

(iii) Pour toute catégorie $\mathcal{C}$, nous noterons $\mathcal{C}_{M}$ la catégorie $\boldsymbol{F} \operatorname{ct}(\underline{M}, \mathcal{C})$. C'est la catégorie des objets de $\mathcal{C}$ munis d'une action de $M$. En effet, les objets de $\mathcal{C}_{M}$ sont les objets $X$ de $\mathcal{C}$ munis d'un morphisme de monoïdes $M \rightarrow \operatorname{End}_{\mathcal{C}}(X)$. Nous noterons $O_{M}^{\mathcal{C}}: \mathcal{C}_{M} \rightarrow \mathcal{C}$ le foncteur d'oubli.

La catégorie $\left(\mathcal{E}_{\mathbb{k}}\right)_{M}$ est ainsi équivalente à ${ }_{\mathbb{k}[M]}$ Mod.

(c) Une catégorie $\mathbb{k}$-linéaire est une catégorie $\mathcal{I}$ telle que, pour tous objets $A$ et $B$ de $\mathcal{I}$, les ensembles $\operatorname{hom}_{\mathcal{I}}(A, B)$ sont munis d'une structure de $\mathbb{k}$-espace vectoriel, de sorte que la composition des morphismes soit $\mathbb{k}$-bilinéaire. On ne suppose pas $\mathcal{I}$ additive.

(d) Nous noterons $\Sigma_{n}$ le groupe symétrique sur $n$ lettres.

D'autres notations utilisées dans tout l'article sont introduites dans les appendices.

\footnotetext{
${ }^{(4)}$ Dans toutes les catégories que nous considérerons, la classe des morphismes entre deux objets sera un ensemble.
} 



\section{PARTIE I}

\section{PRÉLIMINAIRES}


Après avoir rappelé des propriétés de base de la catégorie $\mathcal{F}$, dont l'étude constitue l'une des principales motivations de cet article, nous traitons d'une catégorie analogue, notée $\mathcal{F}_{\text {surj }}$, avec plus de détail. Cette catégorie, qui possède un intérêt intrinsèque, s'avère essentielle autant pour la considération de la catégorie $\mathcal{F}$ que pour celle de la catégorie de foncteurs en grassmanniennes $\mathcal{F}_{\mathcal{G} r}$ que nous introduirons dans la partie II.

Les chapitres 3 et 4 constituent le soubassement des catégories de foncteurs en grassmanniennes, notamment $\mathcal{F}_{\mathcal{G} r}$ : le premier sous-tend sa description comme catégorie de comodules, le second sa description fonctorielle. 


\section{CHAPITRE 1}

\section{RAPPELS SUR LA CATÉGORIE $\mathcal{F}$}

Ce chapitre donne un résumé des propriétés de base de la catégorie $\mathcal{F}(\mathbb{k})$ dont nous aurons besoin par la suite. La plupart d'entre elles sont exposées dans le contexte général de ce travail, celui d'un corps fini $\mathbb{k}$ arbitraire. D'autres (notamment celles de la section 1.5, et la plupart de celles de la section 1.4) ne seront utilisées que dans la partie IV, c'est pourquoi elles ne sont traitées que dans le cas où le corps de base est le corps à deux éléments.

\subsection{Généralités}

Les résultats rappelés dans l'appendice $\mathrm{C}$ s'appliquent à la catégorie $\mathcal{F}(\mathbb{k})$; l'hypothèse C.2.9 est vérifiée car le corps $\mathbb{k}$ est fini. Le foncteur projectif standard $P_{V}^{\mathcal{E}_{\mathrm{k}}^{f}}$, où $V$ est un $\mathbb{k}$-espace vectoriel de dimension finie, sera simplement noté $P_{V}$; de même, l'injectif standard $I_{V}^{\mathcal{E}_{\mathrm{k}}^{f}}$ sera noté $I_{V}$.

La plupart des résultats de ce chapitre sont contenus dans [HLS93], [Kuh94a], [Sch94] ou [Pow98b] par exemple.

Comme le foncteur de dualité $\left(\mathcal{E}_{\mathbb{k}}^{f}\right)^{o p} \rightarrow \mathcal{E}_{\mathbb{k}}^{f}$ est une équivalence de catégories, la proposition/définition C.2.7 procure un foncteur $D: \mathcal{F}(\mathbb{k})^{o p} \rightarrow \mathcal{F}(\mathbb{k})$, donné sur les objets $\operatorname{par}(D F)(V)=F\left(V^{*}\right)^{*}$. Il induit une équivalence de catégories entre $\left(\mathcal{F}^{d f}(\mathbb{k})\right)^{o p}$ et $\mathcal{F}^{d f}(\mathbb{k})$, où l'on note $\mathcal{F}^{d f}(\mathbb{k})$ la sous-catégorie épaisse de $\mathcal{F}(\mathbb{k})$ constituée des foncteurs prenant des valeurs de dimension finie.

Définition 1.1.1. - Un objet $F$ de $\mathcal{F}$ est dit auto-dual s'il existe un isomorphisme $u: F \stackrel{\simeq}{\longrightarrow} D F$ invariant par l'isomorphisme d'adjonction $\operatorname{hom}_{\mathcal{F}}(F, D F) \stackrel{\simeq}{\longrightarrow}$ $\operatorname{hom}_{\mathcal{F}}(F, D F)$.

Exemple 1.1.2. - Les objets simples de $\mathcal{F}$ sont auto-duaux (cf. [Kuh94b]). 
Décomposition scalaire et tors de Frobenius. - Suivant la notation C.4.1, nous poserons $\mathcal{F}_{i}(\mathbb{k})=\left\{F \in \mathrm{Ob} \mathcal{F} \mid \forall \lambda \in \mathbb{k} \quad F(\lambda . i d)=\lambda^{i} . i d\right\}$. La catégorie $\mathcal{F}_{0}(\mathbb{k})$ est la sous-catégorie des foncteurs constants de $\mathcal{F}(\mathbb{k})$, elle est canoniquement équivalente à $\mathcal{E}_{\mathbb{k}}$; nous identifierons par la suite ces deux catégories. La décomposition scalaire d'un foncteur $F$ de $\mathcal{F}(\mathbb{k})$ (proposition/définition C.4.2) s'écrit donc $F \simeq F(0) \oplus F_{1} \oplus$ $\cdots \oplus F_{q-1}$. On notera $\bar{F}=F_{1} \oplus \cdots \oplus F_{q-1}$.

Si $V$ est un objet de $\mathcal{E}_{\mathbb{k}}^{f}$, nous noterons $P_{V, i}$ pour $\left(P_{V}\right)_{i}$. Pour $V=\mathbb{k}$, on obtient la décomposition de $P_{\mathbb{k}}$ en somme directe de projectifs indécomposables. Explicitement, $P_{\mathbb{k}, 0}$ est le foncteur constant $\mathbb{k}$, et pour $1 \leq i \leq q-1$, on peut voir $P_{\mathbb{k}, i}(V)$ comme le quotient de $P_{\mathbb{k}}(V)$ par l'espace vectoriel engendré par les $[\lambda v]-\lambda^{i}[v]$, pour $\lambda \in \mathbb{k}$ et $v \in V$.

Soit $\phi$ l'automorphisme de la catégorie $\mathcal{E}_{\mathbb{k}}^{f}$ obtenu en tordant l'action des scalaires par l'automorphisme de Frobenius de $\mathbb{k}$. Le foncteur de précomposition $\phi^{*}: \mathcal{F}(\mathbb{k}) \rightarrow$ $\mathcal{F}(\mathbb{k})$ est appelé tors de Frobenius. Il induit, pour tout entier naturel $i$, une équivalence de catégories entre $\mathcal{F}_{i}(\mathbb{k})$ et $\mathcal{F}_{p i}(\mathbb{k})$.

Changement de corps. - Soit $K$ une extension finie de $\mathbb{k}$. Les foncteurs d'extension des scalaires $t: \mathcal{E}_{\mathbb{k}} \rightarrow \mathcal{E}_{K}$ et de restriction des scalaires $\tau: \mathcal{E}_{K} \rightarrow \mathcal{E}_{\mathbb{k}}$ sont mutuellement adjoints (i.e. $t$ est adjoint à droite et à gauche à $\tau$ ), de même que leurs variantes entre $\mathcal{E}_{\mathbb{k}}^{f}$ et $\mathcal{E}_{K}^{f}$, que nous noterons encore $t$ et $\tau$ par abus. On en déduit que les foncteurs $\tau^{*} t_{*}: \mathcal{F}(\mathbb{k}) \rightarrow \mathcal{F}(K)$ et $\tau_{*} t^{*}: \mathcal{F}(K) \rightarrow \mathcal{F}(\mathbb{k})$ sont mutuellement adjoints. On rappelle que l'indice (resp. exposant) étoilé indique la postcomposition (resp. précomposition) — cf. appendice C. Pour plus de détails à ce sujet, voir [FFSS99], $\S 3$.

Le foncteur $\tau_{*} t^{*}: \mathcal{F}(K) \rightarrow \mathcal{F}(\mathbb{k})$ est appelé encore restriction des scalaires, et $\tau^{*} t_{*}: \mathcal{F}(\mathbb{k}) \rightarrow \mathcal{F}(K)$ induction.

\subsection{La conjecture artinienne}

La terminologie employée dans ce qui suit est rappelée dans l'appendice B.

Nous donnons différentes formulations équivalentes de la conjecture artinienne (conjecture 1 de l'introduction), qui constitue l'une des motivations essentielles aux constructions développées dans cet article. Pour la démonstration et plus de détails, voir [Pow00a] ou [Dja06d].

Proposition 1.2.1. — Les assertions suivantes sont équivalentes.

(1) La catégorie $\mathcal{F}$ est localement noethérienne.

(2) Les projectifs standard $P_{V}$ sont des objets noethériens de $\mathcal{F}$.

(3) La catégorie $\mathcal{F}$ est co-localement artinienne.

(4) Les injectifs standard $I_{V}$ sont des objets artiniens de $\mathcal{F}$.

(5) Le produit tensoriel de deux objets artiniens de $\mathcal{F}$ est artinien. 
(6) Le produit tensoriel de deux objets noethériens de $\mathcal{F}$ est noethérien.

(7) Le socle d'un objet de type fini de $\mathcal{F}$ est fini.

(8) Les objets injectifs de $\mathcal{F}_{\omega}$ restent injectifs dans $\mathcal{F}$.

(9) Pour tout foncteur de type fini $F$ de $\mathcal{F}$, l'ensemble des classes d'isomorphisme de foncteurs simples $S$ de $\mathcal{F}$ tels que $\operatorname{Ext}_{\mathcal{F}}^{1}(F, S) \neq 0$ est fini.

L'assertion 4 constitue la forme originelle de la conjecture (l'intérêt particulier pour les injectifs provenant des liens avec les modules instables sur l'algèbre de Steenrod — cf. [HLS93]), c'est pourquoi elle est appelée conjecture artinienne.

Les premières avancées significatives sur la conjecture artinienne remontent à Piriou ([Pir97]). Powell a accompli plusieurs progrès importants (cf. [Pow00b], [Pow98c] et $[\mathbf{P o w} \mathbf{9 8 a}])$. Dans $[\mathbf{D j a 0 6 c}]$, nous démontrons de nouveaux cas de cette conjecture; ceux-ci sont surpassés par les résultats déduits de l'étude des catégories de foncteurs en grassmanniennes que nous donnerons au chapitre 16 .

Il est naturel de poser la question du caractère localement noethérien ou colocalement artinien d'autres catégories de foncteurs $\operatorname{Fct}(\mathcal{I}, \mathcal{E})$, où $\mathcal{I}$ est une catégorie essentiellement petite vérifiant l'hypothèse C.2.9. On ne saurait obtenir une réponse affirmative en général, comme le montre l'exemple élémentaire suivant. Cependant, toutes les catégories de foncteurs que nous rencontrerons par la suite semblent « très probablement $»$ localement noethériennes et co-localement artiniennes.

Exemple 1.2.2. - Soit $\mathcal{I}$ la petite catégorie telle que $\mathrm{Ob} \mathcal{I}=\mathbb{N}$ et $\operatorname{hom}_{\mathcal{I}}(n, m)=*$ (ensemble à un élément) si $n=0$ ou $n=m, \varnothing$ sinon. Le projectif standard de type fini $P_{0}^{\mathcal{I}} \operatorname{de} \boldsymbol{F} \operatorname{ct}(\mathcal{I}, \mathcal{E})$ est le foncteur constant $\mathbb{k}$, puisque 0 est objet initial de $\mathcal{I}$. Ce n'est pas un objet noethérien. En effet, pour tout entier $n>0$, l'image $A_{n}$ du monomorphisme $\underset{0<i \leq n}{\bigoplus} P_{i}^{\mathcal{I}} \rightarrow P_{0}^{\mathcal{I}}$ dont chaque composante $P_{i}^{\mathcal{I}} \rightarrow P_{0}^{\mathcal{I}}$ est induite par le morphisme $0 \rightarrow i$ associe à l'objet $i$ l'espace vectoriel $\mathbb{k}$ si $0<i \leq n, 0$ sinon. Aussi $\left(A_{n}\right)_{n>0}$ est-elle une suite strictement croissante de sous-objets de $P_{0}^{\mathcal{I}}$.

Powell a émis la version renforcée suivante de la conjecture artinienne.

Conjecture 1.2.3 (Conjecture artinienne forte). - Pour tout $n \in \mathbb{N}$, le foncteur $P_{\mathbb{k} \oplus n}$ est noethérien de type $n$.

\subsection{Le foncteur différence et les objets polynomiaux de $\mathcal{F}(\mathbb{k})$}

Ces notions dépendent exclusivement du caractère additif de la catégorie source $\mathcal{E}_{\mathrm{k}}^{f}$. Si $V$ est un objet de cette catégorie, le foncteur de décalage par $V$ est l'endofoncteur de $\mathcal{F}(\mathbb{k})$, noté $\Delta_{V}$, de précomposition par $\oplus \nabla: \mathcal{E}_{\mathrm{k}}^{f} \rightarrow \mathcal{E}_{\mathrm{k}}^{f}$. Cette construction est fonctorielle en $V$; ainsi, du fait que la composition $0 \rightarrow \mathbb{k} \rightarrow 0$ est l'identité, et que $\Delta_{0} \simeq i d_{\mathcal{F}}$, on obtient un scindement $\Delta_{\mathrm{k}} \simeq i d \oplus \Delta$, où $\Delta$ est appelé foncteur différence de $\mathcal{F}(\mathbb{k})$. 
Le résultat classique suivant (cf. [Pow98b], appendice) est une application des propositions C.3.2 et C.3.3.

Proposition 1.3.1. - (1) $\quad$ (a) Le foncteur de décalage $\Delta_{V}$ est adjoint à droite $\grave{a} \cdot \otimes P_{V} ;$ cette adjonction est naturelle en $V$.

(b) Le foncteur différence $\Delta$ est adjoint à droite $\grave{a} \cdot \otimes \bar{P}_{\mathbb{k}}$.

(2) (a) Le foncteur de décalage $\Delta_{V}$ est adjoint à gauche $\grave{a} \cdot \otimes I_{V}$; cette adjonction est naturelle en $V$.

(b) Le foncteur différence $\Delta$ est adjoint à gauche $\grave{a} \cdot \otimes \bar{I}_{\mathrm{k}}$.

Notation 1.3.2. - Nous désignerons par $\mathcal{F}^{n}(\mathbb{k})$ la sous-catégorie épaisse de $\mathcal{F}(\mathbb{k})$ noyau du foncteur exact $\Delta^{n+1}$, où $n \in \mathbb{N}$. Pour $n$ entier strictement négatif ou $-\infty$, on convient que $\mathcal{F}^{n}(\mathbb{k})$ est la sous-catégorie réduite à 0 .

Définition 1.3.3. - Soit $F$ un foncteur de $\mathcal{F}(\mathbb{k})$.

(1) On dit que $F$ est polynomial s'il existe $n$ tel que $F \in \mathrm{Ob}^{n}(\mathbb{k})$. Le plus petit $n$ ayant cette propriété s'appelle alors le degré de $F$, et se note $\operatorname{deg} F$.

(2) On dit que $F$ est analytique s'il est colimite de ses sous-foncteurs polynomiaux. On note $\mathcal{F}_{\omega}(\mathbb{k})$ la sous-catégorie pleine de $\mathcal{F}(\mathbb{k})$ des foncteurs analytiques.

(3) On dit que $F$ est coanalytique s'il est limite de ses quotients polynomiaux.

La proposition suivante se trouve démontrée dans [Kuh94a] (§4), par exemple. Nous détaillons la démonstration du corollaire qui s'en déduit afin d'illustrer des raisonnements utiles dans d'autres catégories de foncteurs.

Proposition 1.3.4. - (1) Les foncteurs projectifs standard de $\mathcal{F}(\mathbb{k})$ sont coanalytiques.

(2) Les foncteurs injectifs standard de $\mathcal{F}(\mathbb{k})$ sont analytiques.

On rappelle (cf. section B.1) qu'un objet pf $f_{\infty}\left(\right.$ resp. co-pf $\left.f_{\infty}\right)$ est un objet qui admet une résolution projective (resp. injective) de type fini.

Corollaire 1.3.5. - (1) Un foncteur de $\mathcal{F}(\mathbb{k})$ est fini si et seulement s'il est polynomial et à valeurs de dimension finie.

(2) La sous-catégorie pleine $\mathcal{F}^{f}(\mathbb{k})$ des objets finis de $\mathcal{F}(\mathbb{k})$ est préservée par le produit tensoriel et par le foncteur différence.

(3) Les objets finis de $\mathcal{F}(\mathbb{k})$ sont $p f_{\infty}$ et co-pfo.

(4) La sous-catégorie $\mathcal{F}_{\omega}(\mathbb{k})$ de $\mathcal{F}(\mathbb{k})$ est épaisse.

(5) Un objet de $\mathcal{F}(\mathbb{k})$ est de co-type fini si et seulement s'il est analytique et de socle fini.

Démonstration. - Le foncteur $\left(\Delta, e v_{0}\right): \mathcal{F} \rightarrow \mathcal{F} \times \mathcal{E}$ (on rappelle que $e v_{0}$ désigne le foncteur d'évaluation en $0-$ cf. notation C.1.1) est exact et fidèle, car le noyau de $\Delta$ est constitué des foncteurs constants. La proposition B.2.3 montre alors qu'un foncteur $F$ tel que $\Delta F$ est fini et que $F(0)$ est un espace vectoriel de dimension finie 
est lui-même fini. Par récurrence, on en déduit qu'un foncteur polynomial à valeurs de dimension finie est fini. La réciproque résulte de la proposition 1.3.4, puisqu'un objet simple se plonge dans un injectif standard.

La deuxième assertion découle de la première, en raison de l'isomorphisme naturel $\Delta(F \otimes G) \simeq(\Delta F \otimes G) \oplus(F \otimes \Delta G) \oplus(\Delta F \otimes \Delta G)$.

Comme le foncteur exact et fidèle $\left(\Delta, e v_{0}\right)$ commute aux colimites, la proposition B.2.2 montre qu'un foncteur $F$ tel que $F(0)$ est de dimension finie et que $\Delta F$ est $\mathrm{pf}_{\infty}$ est lui-même $\mathrm{pf}_{\infty}$. On en déduit le caractère $\mathrm{pf}_{\infty}$ des objets finis; le cas co-pf $f_{\infty}$ est dual.

La quatrième assertion se déduit de la précédente via la proposition B.1.10.

Un objet de co-type fini est toujours de socle fini; il est aussi analytique, dans $\mathcal{F}$, parce que les injectifs standard le sont. La réciproque est donnée par la proposition B.1.13.

L'assertion 3 de ce corollaire, particulièrement importante, est due à L. Schwartz (cf. $[$ Sch94]).

Filtration polynomiale. - Le foncteur d'inclusion $\mathcal{F}^{n} \hookrightarrow \mathcal{F}$ admet un adjoint à droite noté $p_{n}: \mathcal{F} \rightarrow \mathcal{F}^{n}$; explicitement, $p_{n}(F)$ est le plus grand sous-foncteur polynomial de $F \in \mathrm{Ob} \mathcal{F}$ de degré au plus $n$. La suite croissante $\left(p_{n}(F)\right)_{n}$ de sousfoncteurs de $F$ s'appelle sa filtration polynomiale. Les considérations élémentaires que nous rappelons dans la suite de ce paragraphe sont détaillées dans [Pow98b], par exemple; elles interviendront dans la partie IV.

Concentrons-nous, par commodité, sur le cas où $\mathbb{k}=\mathbb{F}_{2}$ est le corps à deux éléments, qui sera le nôtre dans la partie IV. La filtration polynomiale du foncteur injectif standard $I_{\mathbb{F}_{2}}$ est explicite : si l'on note

$$
t_{n}=\sum_{l \in\left(\mathbb{F}_{2}^{n}\right)^{*}}[l] \in \mathbb{F}_{2}\left[\operatorname{hom}\left(\mathbb{F}_{2}^{n}, \mathbb{F}_{2}\right)\right]
$$

et $t_{n}^{*}: I_{\mathbb{F}_{2}} \rightarrow I_{\mathbb{F}_{2}{ }^{n}}$ le morphisme de $\mathcal{F}\left(\mathbb{F}_{2}\right)$ induit, la suite suivante est exacte :

$$
0 \rightarrow p_{n-1}\left(I_{\mathbb{F}_{2}}\right) \rightarrow I_{\mathbb{F}_{2}} \stackrel{t_{n}^{*}}{\longrightarrow} I_{\mathbb{F}_{2}} \text {. }
$$

Le résultat suivant joue un rôle fondamental dans le comportement des foncteurs $\tilde{\nabla}_{n}$ de Powell et des variantes que nous introduirons.

Lemme 1.3.6. - L'espace vectoriel $\left(I_{\mathbb{F}_{2}} / p_{n-1}\left(I_{\mathbb{F}_{2}}\right)\right)\left(\mathbb{F}_{2}{ }^{i}\right)$ est de dimension 0 si $i<n$ et 1 si $i=n$.

Dualement, le foncteur d'inclusion $\mathcal{F}^{n} \hookrightarrow \mathcal{F}$ admet un adjoint à gauche noté $q_{n}$ : $\mathcal{F} \rightarrow \mathcal{F}^{n}$; il envoie un foncteur sur son plus grand quotient polynomial de degré au plus $n$. Nous noterons $k_{n}(F)$, pour $F \in \mathrm{Ob} \mathcal{F}$, le noyau de l'épimorphisme canonique $F \rightarrow q_{n-1}(F)$. Le cas qui nous intéresse ici est dual de la filtration polynomiale de $I_{\mathbb{F}_{2}}$ : pour le projectif standard $P_{\mathbb{F}_{2}}$, on peut décrire $k_{n}\left(P_{\mathbb{F}_{2}}\right)$ de la façon suivante. 
Si $V$ est un objet de $\mathcal{E}_{\mathbb{F}_{2}}^{f}$ et $W$ un sous-espace de $V$, notons

$$
s_{W}=\sum_{v \in W}[v] \in P_{\mathbb{F}_{2}}(V) .
$$

Alors $k_{n}\left(P_{\mathbb{F}_{2}}\right)(V)$ est le sous-espace vectoriel de $P_{\mathbb{F}_{2}}(V)$ engendré par les éléments $s_{W}$, où $W$ parcourt les sous-espaces de dimension $n$ de $V$.

\subsection{Les objets simples de $\mathcal{F}(\mathbb{k})$}

La propriété suivante est une conséquence classique du caractère polynomial des foncteurs finis de $\mathcal{F}(\mathbb{k})$. Dans [Kuh02], on trouvera une démonstration de cet énoncé, sous une forme plus générale (qui ne suppose plus $\mathbb{k}$ premier).

Proposition 1.4.1. - On suppose $d=1$, i.e. le corps $\mathbb{k}$ premier. Soit $n$ un entier naturel.

Il existe un diagramme de recollement

$$
\mathcal{F}^{n-1}(\mathbb{k}) \underset{\longleftarrow}{\longleftarrow} \mathcal{F}^{n}(\mathbb{k}) \stackrel{\longleftarrow}{\longleftarrow} \operatorname{Mod}_{\mathbb{k}\left[\Sigma_{n}\right]} \text {. }
$$

Par la proposition B.3.5, on en déduit le résultat suivant.

Corollaire 1.4.2. - Supposons $d=1$. La décomposition par le degré polynomial induit un isomorphisme de groupes de Grothendieck

$$
G_{0}^{f}(\mathcal{F}(\mathbb{k})) \simeq \bigoplus_{n \in \mathbb{N}} G_{0}^{f}\left(\operatorname{Mod}_{\mathbb{F}_{p}\left[\Sigma_{n}\right]}\right)
$$

Une autre conséquence importante de la proposition 1.4.1, qui s'étend au corps d'un corps fini quelconque, est la suivante. On pourra encore se reporter à [Kuh02].

Proposition 1.4.3. - Les automorphismes des objets simples de $\mathcal{F}(\mathbb{k})$ sont réduits $\grave{a} \mathbb{k}: \mathbb{k}$ est un corps de décomposition de la catégorie $\mathcal{F}(\mathbb{k})$.

Comme le produit tensoriel est un bifoncteur exact en chaque variable, que son unité $\mathbb{k}$ est finie et qu'il préserve les objets finis, il induit une structure d'anneau sur $G_{0}^{f}(\mathcal{F}(\mathbb{k}))$.

Quelques propriétés des objets simples de $\mathcal{F}\left(\mathbb{F}_{2}\right)$. — Dans le cas où le corps $\mathbb{k}$ est premier, la théorie classique des représentations des groupes symétriques (cf. [Jam78]) permet de décrire les objets simples de $\mathcal{F}(\mathbb{k})$ à partir des partitions. Nous rappelons la terminologie et les résultats de base en la matière, en nous limitant au corps à deux éléments, le seul où les résultats de ce paragraphe interviendront (dans la partie IV).

Définition 1.4.4. - (1) Une partition est une suite décroissante d'entiers $\left(\lambda_{i}\right)_{i \in \mathbb{N}^{*}}$ qui stationne en 0. 
(2) La longueur $l(\lambda)$ d'une partition $\lambda$ est le plus grand entier tel que $\lambda_{l(\lambda)}>0$. Si $\lambda$ est identiquement nulle, on convient que $l(\lambda)=0$. Par la suite, on identifiera une partition $\lambda$ et le $n$-uplet $\left(\lambda_{1}, \ldots, \lambda_{n}\right)$ si $n \geq l(\lambda)$.

(3) Une partition $\lambda$ est dite 2 -régulière (ou simplement régulière) si $\lambda_{i}>\lambda_{i+1}$ pour $1 \leq i<l(\lambda)$.

Nous désignerons par $\mathfrak{p}$ l'ensemble des partitions régulières.

(4) Le degré d'une partition $\lambda$ est l'entier positif $|\lambda|=\sum_{i \in \mathbb{N}^{*}} \lambda_{i}$. Une partition de $n \in \mathbb{N}$ est par définition une partition de degré $n$.

(5) Si $\lambda$ et $\mu$ sont deux partitions de même degré, nous noterons $\lambda \leq \mu$ si

$$
\forall n \in \mathbb{N}^{*} \quad \sum_{i=1}^{n} \lambda_{i} \leq \sum_{i=1}^{n} \mu_{i} .
$$

On rappelle que les foncteurs simples de $\mathcal{F}\left(\mathbb{F}_{2}\right)$ peuvent se paramétriser par les partitions 2-régulières, à partir des représentations des groupes symétriques et de la proposition 1.4.1. Nous suivons ici les notations de [Pow00b], par exemple; une présentation détaillée des foncteurs simples est donnée dans [PS98], mais cet article indexe les foncteurs simples de manière différente (par la partition duale de celle que nous utilisons).

Notation 1.4.5. - On désigne par $S_{\lambda}$ le foncteur simple associé à une partition régulière $\lambda$. On note $I_{\lambda}$ l'enveloppe injective du foncteur $S_{\lambda}$.

Ainsi, $S_{\lambda}$ est un foncteur polynomial de degré $|\lambda|$.

Une conséquence formelle de la proposition 1.4.3 est qu'on peut calculer la multiplicité, notée $m_{\lambda}(F)$, d'un foncteur simple $S_{\lambda}$ dans un foncteur $F$, supposé à valeurs de dimension finie (pour assurer la finitude de cette multiplicité), par la formule

$$
m_{\lambda}(F)=\operatorname{dim}_{\mathbb{F}_{2}} \operatorname{hom}_{\mathcal{F}}\left(F, I_{\lambda}\right) .
$$

Notation 1.4.6. - Étant donnés une partition régulière $\lambda$ et un foncteur $F \in \mathrm{Ob} \mathcal{F}\left(\mathbb{F}_{2}\right)$, nous abrégerons l'assertion $S_{\lambda}$ est facteur de composition (i.e. sous-quotient) de $F$ en $\lambda \vdash F$.

Le théorème suivant résume les propriétés fondamentales des facteurs de composition du produit tensoriel, noté $\Lambda^{\lambda}$, des puissances extérieures $\Lambda^{\lambda_{i}}$. Il est l'analogue de propriétés classiques des modules de Specht dans le contexte des groupes symétriques (cf. [Jam78]) ; une démonstration dans le cadre de la catégorie $\mathcal{F}$ est donnée dans l'article [Kuh94b] de Kuhn (on prendra garde au fait que cet article emploie des conventions différentes des nôtres dans l'indexation des foncteurs simples).

Théorème 1.4.7. - Les facteurs de composition de $\Lambda^{\lambda}$, où $\lambda$ est une partition de longueur $r$ de $n \in \mathbb{N}$, sont :

- les $S_{\mu}$, où $\mu$ parcourt les partitions régulières de $n$ telles que $\mu \geq \lambda$,

- des simples du type $S_{\mu}$ avec $|\mu|<n, l(\mu)<r, \mu_{1} \geq \lambda_{1}$ et $\mu_{r-1} \leq \lambda_{r}$. 
En outre, $S_{\lambda}$ est facteur de composition unique de $\Lambda^{\lambda}$.

Nous rappelons maintenant un résultat fondamental dont on dispose sur les produits tensoriels.

Définition 1.4.8. - Soient $\lambda$ et $\mu$ deux partitions de longueurs respectives $n$ et $r$. Nous appellerons partition concaténée de $\lambda$ et $\mu$ la partition obtenue en réordonnant la suite d'entiers $\left(\lambda_{1}, \ldots, \lambda_{n}, \mu_{1}, \ldots, \mu_{r}\right)$; elle sera notée $(\lambda, \mu)$.

Proposition 1.4.9 (Kuhn). - Soient $\lambda$ et $\mu$ des partitions régulières telles que la partition $(\lambda, \mu)$ soit régulière. Alors $m_{(\lambda, \mu)}\left(S_{\lambda} \otimes S_{\mu}\right)=1$.

Cette proposition est le théorème 6.17.2 de [Kuh94b].

Objets simples de $\mathcal{F}$ et représentations des groupes linéaires. - On peut relier les objets simples de la catégorie $\mathcal{F}(\mathbb{k})$ aussi bien aux représentations des groupes symétriques qu'à celles des groupes linéaires $G L_{n}(\mathbb{k})$. L'approche utilisant les groupes linéaires est exploitée dans l'article de Kuhn [Kuh94b], où sont démontrés les résultats de ce paragraphe.

Proposition 1.4.10. - Si S est un objet simple de $\mathcal{F}$, alors $S\left(\mathbb{k}^{\oplus n}\right)$ est un $G L_{n}(\mathbb{k})$ module nul ou simple pour tout $n \in \mathbb{N}$.

Notation 1.4.11. - Soient $n \in \mathbb{N}$ et $\lambda$ une partition 2-régulière telle que $\lambda_{1}=n$. On désigne par $R_{\lambda}$ le $G L_{n}\left(\mathbb{F}_{2}\right)$-module $S_{\lambda}\left(\mathbb{F}_{2}{ }^{\oplus n}\right)$.

On peut préciser la proposition 1.4.10 comme suit.

Proposition 1.4.12. - Soient $\lambda$ une partition 2-régulière et $n \in \mathbb{N}$.

(1) Supposons $n=\lambda_{1}$. Alors $R_{\lambda}$ est un $\mathbb{F}_{2}\left[G L_{n}\left(\mathbb{F}_{2}\right)\right]$-module à gauche simple.

(2) Les $R_{\mu}$ forment un système complet de représentants des $\mathbb{F}_{2}\left[G L_{n}\left(\mathbb{F}_{2}\right)\right]$-modules simples lorsque $\mu$ parcourt les éléments de $\mathfrak{p}$ tels que $\mu_{1}=n$.

(3) Le $\mathbb{F}_{2}\left[G L_{n}\left(\mathbb{F}_{2}\right)\right]$-module $S_{\lambda}\left(\mathbb{F}_{2}{ }^{\oplus n}\right)$ est nul si $\lambda_{1}>n$, égal à $R_{\lambda}$ si $\lambda_{1}=n$ et isomorphe à $R_{\left(n, \lambda_{1}, \lambda_{1}, \ldots, \lambda_{r}\right)}$ (où $\left.r=l(\lambda)\right)$ si $\lambda_{1}<n$.

Notation 1.4.13. - Soient $n \in \mathbb{N}$ et $\lambda$ une partition 2-régulière telle que $\lambda_{1}=$ $n$. Nous noterons $m_{R_{\lambda}}(M)$ la multiplicité du $G L_{n}\left(\mathbb{F}_{2}\right)$-module simple $R_{\lambda}$ dans un $G L_{n}\left(\mathbb{F}_{2}\right)$-module à gauche $M$.

\subsection{Les foncteurs $\tilde{\nabla}_{n}: \mathcal{F}\left(\mathbb{F}_{2}\right) \rightarrow \mathcal{F}\left(\mathbb{F}_{2}\right)$ de Powell}

Cette section de rappels ne sera pas utilisée avant la partie IV. En particulier, on se limite au corps de base $\mathbb{F}_{2}$, bien que Powell ait définis les foncteurs $\tilde{\nabla}_{n}$ sur tout corps fini (cependant, il se restreint souvent à $\mathbb{F}_{2}$, ou au moins à un corps premier, pour des raisons techniques). 
On prendra garde que les foncteurs notés $\tilde{\nabla}_{n}$ sont les duaux de ceux introduits dans [Pow98b] par Powell, à qui sont dus tous les résultats de cette section : si l'on note $\tilde{\nabla}_{n}^{\text {Pow }}$ les foncteurs $\tilde{\nabla}_{n}$ de $[$ Pow $\mathbf{9 8 b}]$, nos foncteurs $\tilde{\nabla}_{n}$ satisfont des isomorphismes de dualité $D \circ \tilde{\nabla}_{n} \simeq \tilde{\nabla}_{n}^{\text {Pow }} \circ D$ et $D \circ \tilde{\nabla}_{n}^{\text {Pow }} \simeq \tilde{\nabla}_{n} \circ D$. Il n'en résultera aucune confusion par la suite, car nous n'utiliserons jamais les foncteurs $\tilde{\nabla}_{n}^{\text {Pow }}$, adaptés à l'étude de foncteurs analytiques, tandis que nous traiterons de foncteurs co-analytiques.

Définition 1.5.1. - Étant donné un entier $n>0$, on note $\tilde{\nabla}_{n}$ l'endofoncteur de $\mathcal{F}\left(\mathbb{F}_{2}\right)$ noyau de la transformation naturelle $\Delta_{\mathbb{F}_{2}} \simeq\left(-: I_{\mathbb{F}_{2}}\right) \rightarrow\left(-: p_{n-1}\left(I_{\mathbb{F}_{2}}\right)\right)$ induite par l'inclusion $p_{n-1}\left(I_{\mathbb{F}_{2}}\right) \hookrightarrow I_{\mathbb{F}_{2}}$.

Remarque 1.5.2. - Le foncteur $\tilde{\nabla}_{n}$ préserve les foncteurs à valeurs de dimension finie et les foncteurs de type fini.

Proposition 1.5.3. - (1) Le foncteur $\tilde{\nabla}_{n}$ est additif; il conserve les injections et les surjections.

(2) Deux foncteurs $\tilde{\nabla}_{n}$ et $\tilde{\nabla}_{m}$ (où $m \in \mathbb{N}$ ) commutent toujours (à isomorphisme naturel près); en particulier, le foncteur $\tilde{\nabla}_{n}$ commute au foncteur différence.

Proposition 1.5.4. - Il existe dans $\mathcal{F}\left(\mathbb{F}_{2}\right)$ un isomorphisme

$$
\tilde{\nabla}_{n}\left(P_{V}\right) \simeq k_{n}\left(P_{\mathbb{F}_{2}}\right)\left(V^{*}\right) \otimes P_{V}
$$

naturel en l'espace vectoriel de dimension finie V. En particulier, $\tilde{\nabla}_{n}\left(P_{\mathbb{F}_{2} \oplus n}\right) \simeq P_{\mathbb{F}_{2} \oplus n}$ et $\tilde{\nabla}_{n}\left(P_{\mathbb{F}_{2} \oplus k}\right)=0$ pour $k<n$.

Les propositions 1.5.3 et 1.5.4 sont démontrées dans [Pow98b].

Notation 1.5.5. - (1) Si $\lambda$ est une partition de longueur $r$, nous noterons $\lambda_{-}$la partition de longueur $\leq r$ définie $\operatorname{par}\left(\lambda_{-}\right)_{i}=\lambda_{i}-1$ pour $1 \leq i \leq r$.

(2) $\operatorname{Si} \alpha=\left(\alpha_{1}, \ldots, \alpha_{k}\right)$ est une suite finie d'entiers et $a \in \mathbb{N}$, on notera $\alpha_{+a}=$ $\left(\alpha_{1}+a, \ldots, \alpha_{k}+a\right)$. Cette notation est étendue au cas où $\alpha$ est une partition, en prenant $k=l(\alpha)$ (longueur de la partition $\alpha$ ).

L'un des grands intérêts des foncteurs $\tilde{\nabla}_{n}$, qui explique leur efficacité dans de nombreux calculs explicites sur les foncteurs simples, réside dans le résultat suivant, établi dans le $\S 4.3$ de [Pow98b].

Proposition 1.5.6. - Soit $\lambda$ une partition 2-régulière.

(1) Le foncteur $\tilde{\nabla}_{n}\left(S_{\lambda}\right)$ est nul si et seulement si $l(\lambda)<n$.

(2) Supposons $\lambda$ de longueur $n$. On a $\tilde{\nabla}_{n}\left(S_{\lambda}\right) \simeq S_{\lambda_{-}}$.

Proposition et définition 1.5.7. - La sous-catégorie pleine des foncteurs $\tilde{\nabla}_{n}$ nilpotents de $\mathcal{F}\left(\mathbb{F}_{2}\right)$ (i.e. des foncteurs $F$ tels qu'il existe $k \in \mathbb{N}$ tel que $\left(\tilde{\nabla}_{n}\right)^{k}(F)=0$ ) est épaisse. Nous la noterons $\mathcal{N} i l_{\tilde{\nabla}_{n}}$. 
Nous désignerons par $\overline{\mathcal{N i l}}_{\tilde{\nabla}_{n}}$ la plus petite sous-catégorie localisante de $\mathcal{F}$ contenant $\mathcal{N} i l_{\tilde{\nabla}_{n}}$.

C'est le théorème 4.2.3 de [Pow98b] (l'hypothèse d'analycité imposée par Powell aux foncteurs $\tilde{\nabla}_{n}$-nilpotents n'intervient pas dans la démonstration).

La conjecture suivante, discutée par Powell dans [Pow00a], §3, est une variante forte de la conjecture artinienne.

Conjecture 1.5.8. - Un objet de type fini de $\mathcal{F}\left(\mathbb{F}_{2}\right)$ est noethérien de type $n-1$ si et seulement s'il est $\tilde{\nabla}_{n}$-nilpotent.

Nous terminons ce paragraphe par un résultat concernant les produits tensoriels. Il est démontré dans [Pow01] (théorème 1), article qui précise en quoi la notion de $\tilde{\nabla}_{n}$-nilpotence fournit une « bonne » notion de dimension dans $\mathcal{F}\left(\mathbb{F}_{2}\right)$.

Proposition 1.5.9. - Le produit tensoriel d'un foncteur $\tilde{\nabla}_{n}$-nilpotent et d'un foncteur $\tilde{\nabla}_{m}$-nilpotent (où $m \in \mathbb{N}$ ) est $\tilde{\nabla}_{n+m-1}$-nilpotent. En particulier, le produit tensoriel d'un foncteur $\tilde{\nabla}_{n}$-nilpotent et d'un foncteur fini est $\tilde{\nabla}_{n}$-nilpotent. 


\section{CHAPITRE 2}

\section{LA CATÉGORIE $\mathcal{F}_{\text {surj }}$}

Notation 2.0.1. - Nous noterons $\mathcal{E}_{\text {surj }}^{f}(\mathbb{k})$ et $\mathcal{E}_{\text {inj }}^{f}(\mathbb{k})$ les sous-catégories de $\mathcal{E}_{\mathbb{k}}^{f}$ ayant les mêmes objets et dont les morphismes sont respectivement les épimorphismes et les monomorphismes de $\mathcal{E}_{\mathbb{k}}^{f}$.

Si $I$ est une partie de $\mathbb{N}$, nous noterons $\mathcal{E}_{\text {surj }}^{I}(\mathbb{k})$ la sous-catégorie pleine de $\mathcal{E}_{\text {sur } j}^{f}(\mathbb{k})$ dont les objets sont les espaces vectoriels dont la dimension appartient à $I$.

On introduit enfin les catégories de foncteurs

$$
\begin{gathered}
\mathcal{F}_{\text {surj }}(\mathbb{k})=\operatorname{Fct}\left(\mathcal{E}_{\text {surj }}^{f}(\mathbb{k}), \mathcal{E}_{\mathbb{k}}\right), \\
\mathcal{F}_{\text {inj }}(\mathbb{k})=\operatorname{Fct}\left(\mathcal{E}_{\text {inj }}^{f}(\mathbb{k}), \mathcal{E}_{\mathbb{k}}\right), \\
\mathcal{F}_{\text {surj }}^{I}(\mathbb{k})=\operatorname{Fct}\left(\mathcal{E}_{\text {surj }}^{I}(\mathbb{k}), \mathcal{E}_{\mathbb{k}}\right) .
\end{gathered}
$$

On rappelle que, conformément aux conventions générales, on omettra souvent toute référence au corps $\mathbb{k}$, parlant simplement de la catégorie $\mathcal{F}_{\text {surj }}$, par exemple.

L'idée d'étudier une catégorie de foncteurs en utilisant la catégorie de foncteurs auxiliaire obtenue en ne conservant comme morphismes à la source que les épimorphismes, implicite dans les travaux de Suslin, apparaît chez Scorichenko. Elle est exposée dans l'article «Stable K-theory is bifunctor homology » (§4) de Franjou et Pirashvili du volume [FFPS03].

Nombre des résultats présentés dans ce chapitre sont connus, mais ils ne semblent pas avoir fait l'objet d'une exposition systématique dans la littérature.

\subsection{Généralités}

Le foncteur de dualité $\left(\mathcal{E}^{f}\right)^{o p} \rightarrow \mathcal{E}^{f}$ induit une équivalence de catégories entre $\left(\mathcal{E}_{\text {surj }}^{f}\right)^{o p}$ et $\mathcal{E}_{\text {inj }}^{f}$. On en déduit une dualité entre les catégories $\mathcal{F}_{\text {surj }}$ et $\mathcal{F}_{\text {inj }}$ (cf. proposition/définition C.2.7). Par conséquent, nous nous bornerons souvent à énoncer 
les résultats relatifs à l'une des deux catégories, privilégiant, dans ce chapitre, la catégorie $\mathcal{F}_{\text {surj }}$.

La considération d'une seule de ces catégories n'aurait néanmoins pas suffit à nos investigations ultérieures : la catégorie $\mathcal{F}_{\text {surj }}$ intervient dans l'étude des catégories de foncteurs en grassmanniennes; en retour, celles-ci fournissent des renseignements non triviaux sur la catégorie $\mathcal{F}_{i n j}-$ cf. chapitre 13 .

Notation 2.1.1. - (1) Si $V$ est un espace vectoriel de dimension finie, nous noterons simplement $P_{V}^{\text {surj }}$ le projectif standard $P_{V}^{\mathcal{E}_{\text {surj }}^{f}}$, et $I_{V}^{\text {surj }}$ l'injectif standard $I_{V}^{\mathcal{E}_{\text {surj }}^{f}}$. Nous noterons de même $P_{V}^{i n j}$ et $I_{V}^{i n j}$ pour $P_{V}^{\mathcal{E}_{i n j}^{f}}$ et $I_{V}^{\mathcal{E}_{i n j}^{f}}$ respectivement.

(2) L'espace vectoriel $\mathbb{k}^{\oplus n}$ de dimension $n$ sera noté $E_{n}$.

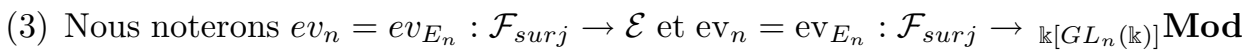
les foncteurs d'évaluation sur l'espace vectoriel $E_{n}$ (cf. notation C.1.1). Nous noterons aussi $e v_{n}$ et $\mathrm{ev}_{n}$ les foncteurs analogues de source $\mathcal{F}_{i n j}$.

Les considérations de la section C.6 fournissent le résultat suivant. La notation $\leq i$ en exposant désigne, selon nos conventions générales, la partie de $\mathbb{N}$ évidente.

Proposition 2.1.2. - Pour tout $n \in \mathbb{N}$, la sous-catégorie $\mathcal{E}_{\text {surj }}^{\leq n-1}$ est une souscatégorie complète à droite de $\mathcal{E}_{\text {surj }}^{\leq n}$ (cf. définition C.6.1). Par conséquent (cf. corollaire C.6.3), on a un diagramme de recollement

$$
\mathcal{F}_{\text {surj }}^{\leq n-1} \underset{\mathcal{R}}{\underset{\mathcal{P}}{\leftarrow}} \mathcal{F}_{\text {surj }}^{\leq n} \underset{\mathcal{P}}{\stackrel{\operatorname{ev}_{n} \rightrightarrows}{\gtrless}} \mathbb{k}\left[G L_{n}(\mathbb{k})\right] \text { Mod }
$$

où $\mathcal{R}$ est le foncteur de restriction et $\mathcal{P}$ le prolongement par zéro.

Remarque 2.1.3. - Vespa a étudié (cf. [Ves05], chapitre 3 et [Ves06], §4) une catégorie de foncteurs $\mathcal{F}_{\text {iso }}$ dont la source s'obtient à partir d'une catégorie d'espaces quadratiques dont toutes les flèches sont des monomorphismes, et de ce fait très ana$\operatorname{logue}$ à $\mathcal{E}_{i n j}^{f}$. Elle utilise une construction de foncteurs de Mackey qui permet d'éviter une partie des difficultés des catégories $\mathcal{F}_{\text {inj }}$ ou $\mathcal{F}_{\text {surj }}$ : le diagramme de recollement analogue à celui de la proposition 2.1.2 est trivial dans $\mathcal{F}_{\text {iso }}$ (i.e. la catégorie qui apparaît au centre du diagramme se scinde en le produit des deux autres catégories cf. [Ves06], théorème 4.2), contrairement à ce qui advient dans $\mathcal{F}_{\text {surj }}$ ou $\mathcal{F}_{i n j}$. Ce phénomène est à rapprocher de ce que $\mathcal{F}_{\text {iso }}$ possède un foncteur de dualité, alors que $\mathcal{F}_{i n j}$ a un comportement «fortement non auto-dual ». 
Notation 2.1.4. - Étant donné $n \in \mathbb{N}$, nous désignerons par $j_{*}^{n}:{ }_{\mathbb{k}[}\left[G L_{n}(\mathbb{k})\right] \operatorname{Mod} \rightarrow$ $\mathcal{F}_{\text {sur } j}(\mathbb{k})$ le foncteur de prolongement par zéro ${ }^{(1)}$ obtenu en considérant la souscatégorie relativement connexe $\mathcal{E}_{\text {surj }}^{n}(\mathbb{k}) \simeq \underline{G L_{n}(\mathbb{k})}$ de $\mathcal{E}_{\text {surj }}^{f}(\mathbb{k})$. On a ainsi un isomorphisme

$$
j_{*}^{n}(M)(V) \simeq \mathbb{k}\left[\operatorname{Iso}\left(E_{n}, V\right)\right] \underset{\mathbb{k}\left[G L_{n}(\mathbb{k})\right]}{\otimes} M
$$

naturel en $V \in \mathrm{Ob}_{\mathcal{E}_{\mathrm{k}}}^{f}$ et $M \in \mathrm{Ob}_{\mathbb{k}\left[G L_{n}(\mathbb{k})\right]}$ Mod.

Par abus, nous désignerons de la même façon les foncteurs analogues dans $\mathcal{F}_{\text {inj }}(\mathbb{k})$.

Nous noterons enfin $\operatorname{Is}_{n}$ l'objet $j_{*}^{n}\left(\mathbb{k}\left[G L_{n}(\mathbb{k})\right]\right)$ de $\mathcal{F}_{\text {surj }}(\mathbb{k})$, et $\mathbb{k}(n)=j_{*}^{n}(\mathbb{k})$.

Ainsi, $\operatorname{Is}_{n}(V)$ et $\mathbb{k}(n)(V)$ sont nuls si $V$ n'est pas de dimension $n$ et canoniquement isomorphes à $\mathbb{k}$ et $\mathbb{k}\left[\operatorname{Iso}\left(E_{n}, V\right)\right]$ respectivement pour $\operatorname{dim} V=n$.

On prendra garde que le foncteur $\mathbb{k}(n)$ était noté $S_{n}^{s u r j}$ dans les versions antérieures de ce travail (cf. [Dja06d] et [Dja06a]), tandis que $j_{*}^{n}$ était noté $i_{n}^{!}$.

Remarque 2.1.5. - Les endofoncteurs $j_{*}^{n} \circ \mathrm{ov}_{n}$ et $\cdot \otimes \mathbb{k}(n)$ de $\mathcal{F}_{\text {surj }}$ sont isomorphes. Ils sont idempotents et correspondent à une troncature.

Remarque 2.1.6. - Le foncteur $\mathrm{Is}_{n}$ n'est projectif que pour $n=0\left(\mathrm{Is}_{0} \simeq P_{0}^{\text {surj }}\right)$ et n'est jamais injectif. Les groupes d'extensions mettant en jeu les $\mathrm{Is}_{n}$ permettent en quelque sorte de mesurer la différence cohomologique entre la catégorie $\mathcal{F}_{\text {surj }}$ et le produit des catégories des $\mathbb{k}\left[G L_{n}(\mathbb{k})\right]$-modules (reliés par le foncteur exact et fidèle donné par les évaluations) — voir aussi le lemme 2.3.22 ci-après à ce sujet.

Décomposition scalaire et tors de Frobenius. - La catégorie $\mathcal{E}_{\text {surj }}^{f}$ n'est pas $\mathbb{k}$-linéaire, mais elle possède une action naturelle du groupe multiplicatif $\mathbb{k}^{\times}$. On en déduit, de façon analogue à la proposition C.4.2, l'énoncé suivant.

Proposition et définition 2.1.7. - Étant donné un entier $i$, notons $\mathcal{F}_{\text {surj }, i}(\mathbb{k})$ la sous-catégorie pleine de $\mathcal{F}_{\text {sur }}(\mathbb{k})$ formée des foncteurs $X$ tels que $X\left(\lambda . i d_{V}\right)=$ $\lambda^{i} . i d_{X(V)}$ pour tous $\lambda \in \mathbb{k}^{\times}$et $V \in \mathrm{Ob} \mathcal{E}_{\text {surj }}^{f}$. Les inclusions induisent une équivalence de catégories

$$
\mathcal{F}_{\text {surj }}(\mathbb{k}) \simeq \prod_{i=1}^{q-1} \mathcal{F}_{\text {surj }, i}(\mathbb{k}) .
$$

On notera $X \simeq \bigoplus_{i=1}^{q-1} X_{i}$ la décomposition naturelle d'un foncteur $X$ de $\mathcal{F}_{\text {surj } j}(\mathbb{k})$ qu'on en déduit, où $X_{i} \in \mathrm{Ob} \mathcal{F}_{\text {surj }, i}(\mathbb{k})$. On l'appelle décomposition scalaire de $X$.

Contrairement au cas d'une catégorie de foncteurs de source $\mathbb{k}$-linéaire, il n'y a pas de facteur correspondant à $i=0$ dans cette décomposition. Cela est à rapprocher de ce que la sous-catégorie pleine des foncteurs constants de $\mathcal{F}_{\text {surj }}$ n'est pas épaisse, contrairement à ce qui se produit dans $\mathcal{F}$ (rappelons que la sous-catégorie $\mathcal{F}_{0}$ obtenue

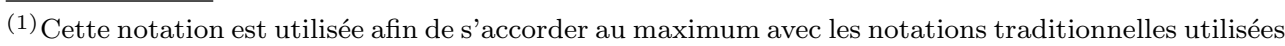
pour les recollements; l'étoile est ici sans rapport avec la notation utilisée pour la postcomposition. 
par la décomposition scalaire est celle des foncteurs constants de $\mathcal{F}$ ) - cf. aussi proposition 2.3.9.

L'automorphisme $\phi$ de la catégorie $\mathcal{E}_{\mathrm{k}}^{f}$ déduit du morphisme de Frobenius induit un automorphisme de la catégorie $\mathcal{E}_{\text {surj }}^{f}(\mathbb{k})$. La précomposition par ce foncteur procure un automorphisme de $\mathcal{F}_{\text {surj }}(\mathbb{k})$ que l'on appellera encore tors de Frobenius. Il induit une équivalence entre les catégories $\mathcal{F}_{\text {surj }, i}(\mathbb{k})$ et $\mathcal{F}_{\text {surj,pi }}(\mathbb{k})$.

On a des résultats analogues dans $\mathcal{F}_{\text {inj }}(\mathbb{k})$ et $\mathcal{F}_{\text {sur } j}^{I}$, pour $I \subset \mathbb{N}$. On obtient par exemple une variante de la proposition 2.1.2 en remplaçant $\mathcal{F}_{\text {surj }}^{\leq i}$ (pour $i=n, n-1$ ) par $\mathcal{F}_{\text {surj,q-1 }}^{\leq i}$ et $G L_{n}(\mathbb{k})$ par $P G L_{n}(\mathbb{k})$.

Changement de corps. - Si $K$ est une extension finie de $\mathbb{k}$, on dispose de foncteurs de restriction $\mathcal{F}_{\text {surj }}(K) \rightarrow \mathcal{F}_{\text {sur }}(\mathbb{k})$ et d'induction $\mathcal{F}_{\text {surj }}(\mathbb{k}) \rightarrow \mathcal{F}_{\text {surj }}(K)$, définis comme dans $\mathcal{F}$. Cependant, ces foncteurs ne sont plus adjoints : les foncteurs de restriction $\mathcal{E}_{K}^{f} \rightarrow \mathcal{E}_{\mathbb{k}}^{f}$ et $\mathcal{E}_{\mathrm{k}}^{f} \rightarrow \mathcal{E}_{K}^{f}$ se restreignent en des foncteurs $\mathcal{E}_{\text {surj }}^{f}(K) \rightarrow \mathcal{E}_{\text {surj }}^{f}(\mathbb{k})$, mais ces restrictions perdent les propriétés d'adjonction des foncteurs initiaux.

Produit tensoriel total. - Outre sa structure tensorielle usuelle, la catégorie $\mathcal{F}_{\text {surj }}$ possède une structure tensorielle qui possède l'avantage sur la structure usuelle de préserver les objets projectifs, et de bien se comporter à l'égard du foncteur fondamental $\varpi: \mathcal{F}_{\text {sur }} \rightarrow \mathcal{F}$ que nous introduirons dans la section 2.3 .

On rappelle que la notation $\mathcal{G} r$ désigne l'ensemble des sous-espaces d'un espace vectoriel.

Définition 2.1.8. - Étant donnés deux objets $F$ et $G$ de $\mathcal{F}_{\text {surj }}$, on appelle produit tensoriel total de $F$ et $G$ le foncteur noté $F \widetilde{\otimes} G$ et défini comme suit.

- Si $A$ est un objet de $\mathcal{E}_{\text {surj }}^{f}$, on pose

$$
(F \widetilde{\otimes} G)(A)=\bigoplus_{\substack{V, W \in \mathcal{G} r(A) \\ V+W=A}} F(V) \otimes G(W) .
$$

- Si $u: A \rightarrow A^{\prime}$ est un morphisme de $\mathcal{E}_{\text {surj }}^{f}$, le morphisme

$$
(F \widetilde{\otimes} G)(u):(F \widetilde{\otimes} G)(A) \rightarrow(F \widetilde{\otimes} G)\left(A^{\prime}\right)
$$

est défini comme la somme directe sur les sous-espaces $V$ et $W$ de $A$ tels que $V+W=A$ des morphismes

$$
F(V) \otimes G(W) \stackrel{F(u) \otimes G(u)}{\longrightarrow} F(u(V)) \otimes G(u(W)) \hookrightarrow \bigoplus_{\substack{V^{\prime}, W^{\prime} \in \mathcal{G} r\left(A^{\prime}\right) \\ V^{\prime}+W^{\prime}=A^{\prime}}} F\left(V^{\prime}\right) \otimes G\left(W^{\prime}\right)
$$

(où l'on note encore, par abus, $u$ pour les morphismes $V \rightarrow u(V)$ et $W \rightarrow u(W)$ induits par $u)$ - cette définition fait sens puisque $u(V)+u(W)=u(V+W)=$ $u(A)=A^{\prime}$. 
Proposition 2.1.9. - (1) Le produit tensoriel total définit sur $\mathcal{F}_{\text {surj }}$ une structure monoïdale symétrique exacte d'unité $\mathrm{Is}_{0}$.

(2) Il existe un monomorphisme naturel $F \otimes G \hookrightarrow F \widetilde{\otimes} G$ pour $F, G \in \mathrm{Ob} \mathcal{F}_{\text {surj }}$.

La première assertion se vérifie par inspection. Le monomorphisme de la seconde assertion est donné par l'inclusion du facteur direct $F(V) \otimes G(V)$ de $(F \widetilde{\otimes} G)(V)$ sur $V \in \mathrm{Ob} \mathcal{E}_{\text {surj }}^{f}$.

Par la suite, lorsque nous nous référerons à des notions dépendant d'une structure tensorielle sur $\mathcal{F}_{\text {surj }}$, nous utiliserons l'adjectif total lorsqu'il s'agira de la structure définie par $\widetilde{\otimes}$, l'absence de qualificatif signifiant qu'il s'agit de la structure tensorielle usuelle définie par $\otimes$.

Lemme 2.1.10. - Il existe un unique foncteur $\mathcal{E}_{\text {surj }}^{f} \times \mathcal{E}_{\text {surj }}^{f} \rightarrow \mathcal{E}_{\text {surj }}^{f}$ tel que le diagramme

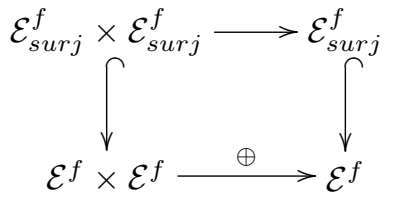

commute. Il sera encore noté $\oplus$ par abus.

Remarque 2.1.11. - Par dualité, on en déduit un résultat analogue dans la catégorie $\mathcal{E}_{i n j}^{f}$, où l'on peut aussi définir un produit tensoriel total.

Proposition 2.1.12. - Il existe un isomorphisme $P_{V}^{\text {surj }} \widetilde{\otimes} P_{W}^{s u r j} \simeq P_{V \oplus W}^{\text {surj }}$ naturel en les objets $V$ et $W$ de $\mathcal{E}_{\text {surj }}^{f}$.

Démonstration. - Cette proposition s'obtient en linéarisant l'isomorphisme ensembliste du lemme suivant.

Lemme 2.1.13. - Il existe une bijection

$$
\operatorname{Epi}_{\mathcal{E}}(A \oplus B, E) \simeq \coprod_{\substack{V, W \in \mathcal{G} r(E) \\ V+W=E}} \operatorname{Epi}_{\mathcal{E}}(A, V) \times \operatorname{Epi}_{\mathcal{E}}(B, W)
$$

naturelle en les objets $A, B$ et $E$ de $\mathcal{E}_{\text {surj }}^{f}$.

Corollaire 2.1.14. - Le produit tensoriel total de deux objets projectifs de $\mathcal{F}_{\text {surj }}$ est projectif.

\subsection{Foncteur de décalage et objets finis}

Nous étudions les objets finis de $\mathcal{F}_{\text {surj }}$ et $\mathcal{F}_{\text {inj }}$ à l'aide du foncteur de décalage, qui joue formellement le même rôle que le foncteur différence dans l'étude des objets finis de $\mathcal{F}$. 
Définition 2.2.1. - Étant donné un espace vectoriel de dimension finie $V$, on appelle foncteur de décalage par $V$ dans $\mathcal{F}_{\text {surj }}$ (resp. $\mathcal{F}_{\text {inj }}$ ) l'endofoncteur de précomposition par $\oplus \nabla$.

Notation 2.2.2. - Nous noterons $\delta_{V}^{\text {surj }}\left(\right.$ resp. $\delta_{V}^{\text {inj }}$ ) l'endofoncteur de décalage par un espace $V$ de la catégorie $\mathcal{F}_{\text {surj }}\left(\right.$ resp. $\mathcal{F}_{\text {inj }}$ ). Lorsque $V=\mathbb{k}$, ce foncteur sera simplement noté $\delta^{\text {surj }}$ (resp. $\delta^{\text {inj }}$ ).

De plus, l'exposant surj (resp. inj) sera omis lorsqu'aucune confusion ne pourra en résulter.

Ainsi, l'association $V \mapsto \delta_{V}$ est fonctorielle; si $V$ est de dimension $n$, les foncteurs $\delta_{V}$ et $\delta^{n}$ sont isomorphes.

Les foncteurs de décalage commutent aux limites, aux colimites et au produit tensoriel (usuel), puisque ce sont des foncteurs de précomposition.

Proposition 2.2.3. - Il existe un isomorphisme

$$
\operatorname{hom}_{\mathcal{F}_{\text {surj }}}\left(X \widetilde{\otimes} P_{A}^{\text {surj }}, Y\right) \simeq \operatorname{hom}_{\mathcal{F}_{\text {surj }}}\left(X, \delta_{A}^{\text {surj }}(Y)\right)
$$

naturel en les objets $X, Y$ de $\mathcal{F}_{\text {surj }}$ et $A$ de $\mathcal{E}_{\text {surj }}^{f}$.

Démonstration. - On définit un morphisme naturel $X \rightarrow \delta_{A}^{\text {surj }}\left(X \widetilde{\otimes} P_{A}^{\text {surj }}\right)$ par les inclusions

$$
X(V) \stackrel{X(V) \otimes\left[i d_{A}\right]}{\longrightarrow} X(V) \otimes P_{A}^{\text {surj }}(A) \hookrightarrow \bigoplus_{W_{1}+W_{2}=V \oplus A} X\left(W_{1}\right) \otimes P_{A}^{\text {surj }}\left(W_{2}\right) .
$$

On vérifie, grâce à la proposition 2.1.12, que l'application naturelle

$$
\operatorname{hom}_{\mathcal{F}_{\text {surj }}}\left(X \widetilde{\otimes} P_{A}^{\text {surj }}, Y\right) \rightarrow \operatorname{hom}_{\mathcal{F}_{\text {surj }}}\left(X, \delta_{A}^{\text {surj }}(Y)\right)
$$

que l'on en déduit est un isomorphisme lorque $X$ est un projectif standard. Le cas général s'en déduit par passage à la colimite.

Proposition 2.2.4. - Il existe dans $\mathcal{F}_{\text {surj }}$ un isomorphisme

$$
\delta_{A}\left(I_{B}^{\text {surj }}\right) \simeq \bigoplus_{W_{1}+W_{2}=B} I_{W_{1}}^{\text {surj }} \otimes I_{W_{2}}^{\text {surj }}(A)
$$

naturel en les objets $A$ et $B$ de $\mathcal{E}_{\text {surj }}^{f}$.

Démonstration. - Cette proposition s'obtient par linéarisation de la bijection du lemme 2.1.13 (ou à partir de la proposition précédente et du lemme de Yoneda).

Corollaire 2.2.5. - (1) Les foncteurs de décalage de $\mathcal{F}_{\text {surj }}$ préservent les objets injectifs et les objets de co-type fini.

(2) Les foncteurs de décalage de $\mathcal{F}_{\text {inj }}$ préservent les objets projectifs de type fini et les objets de type fini. 
Lemme 2.2.6. - (1) Soit $X$ un objet de $\mathcal{F}_{\text {surj }}$ tel que $X(0)$ est de dimension finie et $\delta(X)$ fini dans $\mathcal{F}_{\text {surj }}$. Alors $X$ est un objet fini de $\mathcal{F}_{\text {surj }}$.

(2) Soient $i \in \mathbb{N} \cup\{\infty\}$ et $X$ un objet de $\mathcal{F}_{i n j}$ tel que $X(0)$ est de dimension finie et $\delta(X) p f_{i}$ dans $\mathcal{F}_{\text {inj }}$. Alors $X$ est un objet $p f_{i}$ de $\mathcal{F}_{\text {inj }}$.

Démonstration. - Le foncteur $\left(\delta^{\text {surj }}, e v_{0}\right): \mathcal{F}_{\text {surj }} \rightarrow \mathcal{F}_{\text {surj }} \times \mathcal{E}$ est exact et fidèle. Le premier point résulte donc de la proposition B.2.3.

Pour le second, on utilise le foncteur $\left(\delta^{i n j}, e v_{0}\right): \mathcal{F}_{i n j} \rightarrow \mathcal{F}_{i n j} \times \mathcal{E}$, qui est exact et fidèle, commute aux colimites et conserve les objets projectifs de type fini (cf. corollaire 2.2.5). La conclusion est donc donnée par la proposition B.2.2.

Proposition 2.2.7 (Objets finis, de type fini de $\mathcal{F}_{\text {surj }}$ )

Soit $X$ un objet de $\mathcal{F}_{\text {surj }}$. Les assertions suivantes sont équivalentes :

(1) l'objet $X$ est fini;

(2) l'objet $X$ est de type fini;

(3) l'objet $X$ est à valeurs de dimension finie et nilpotent pour le foncteur décalage $\delta$.

La dernière condition de (3) signifie que $X(V)=0$ si $\operatorname{dim} V$ est assez grande.

Démonstration. - Il est clair que (1) implique (2).

Le foncteur projectif standard $P_{V}^{s u r j}$ associé à un espace $V$ de dimension $n$ est à valeurs de dimension finie et annihilé par le foncteur $\delta^{n+1}$, puisque Epi $(V, W)=0$ si $\operatorname{dim} W \geq n+1$. Cela montre que la deuxième assertion implique la troisième.

Enfin, la troisième implique la première par le lemme 2.2.6.

Dualement, on a le résultat suivant.

Proposition 2.2.8 (Objets finis, de co-type fini de $\mathcal{F}_{i n j}$ )

Soit $X$ un objet de $\mathcal{F}_{\text {inj }}$. Les assertions suivantes sont équivalentes :

(1) l'objet $X$ est fini;

(2) l'objet $X$ est de co-type fini;

(3) l'objet $X$ est à valeurs de dimension finie et nilpotent pour le foncteur décalage $\delta$.

Corollaire 2.2.9. - (1) Tout objet de $\mathcal{F}_{\text {surj }}$ est localement fini; en particulier, $\mathcal{F}_{\text {surj }}$ est une catégorie localement noethérienne.

(2) Tout objet de $\mathcal{F}_{i n j}$ est co-localement fini; en particulier, $\mathcal{F}_{\text {inj }}$ est une catégorie co-localement artinienne.

Corollaire 2.2.10. - (1) Les objets finis de $\mathcal{F}_{\text {inj }}$ sont $p f_{\infty}$.

(2) Les objets finis de $\mathcal{F}_{\text {surj }}$ sont co-pf $f_{\infty}$.

Démonstration. - La première assertion s'obtient en combinant le lemme 2.2.6 et la proposition 2.2.8. La seconde s'en déduit par dualité. 
Notation 2.2.11. - On note $\mathcal{F}_{i n j}^{l f}$ pour $\left(\mathcal{F}_{\text {inj }}\right)^{l f}$ la sous-catégorie des objets localement finis de $\mathcal{F}_{i n j}$. Celle-ci est épaisse parce que les objets finis de $\mathcal{F}_{i n j}$ sont de présentation finie, de sorte que l'on peut appliquer la proposition B.1.10.

Corollaire 2.2.12 (Filtration canonique dans $\mathcal{F}_{\text {surj }}$ ). - Étant donné $n \in \mathbb{N}$, notons $T_{n}$ l'endofoncteur de $\mathcal{F}_{\text {surj }}$ composé du foncteur de restriction $\mathcal{R}: \mathcal{F}_{\text {surj }} \rightarrow$ $\mathcal{F}_{\text {surj }}^{\leq n}$ et du prolongement par zéro $\mathcal{P}: \mathcal{F}_{\text {surj }}^{\leq n} \rightarrow \mathcal{F}_{\text {surj }}$; la coünité de l'adjonction entre $\mathcal{R}$ et $\mathcal{P}$ fournit un monomorphisme $T_{n} \hookrightarrow i d$.

Pour tout objet $F$ de $\mathcal{F}_{\text {surj }},\left(T_{n}(F)\right)_{n \in \mathbb{N}}$ est une suite croissante de sous-objets de $F$ de réunion $F$. Si $F$ est à valeurs de dimension finie, alors les $T_{n}(F)$ sont finis.

Cette filtration est similaire à la filtration polynomiale dans $\mathcal{F}$ (cf. par exemple [Pow98b], $\S 2$ à ce sujet). Notons $T_{n}^{\text {hom }}=T_{n} / T_{n-1}$; il existe un isomorphisme canonique $T_{n}^{\text {hom }}(X) \simeq X \otimes \mathbb{k}(n)$, où $\mathbb{k}(n)=j_{*}^{n}(\mathbb{k})$ est défini dans la notation 2.1.4. Contrairement à ce qui advient pour la filtration polynomiale dans $\mathcal{F}$, les foncteurs $T_{n}$ et $T_{n}^{\text {hom }}$ sont exacts.

Proposition 2.2.13 (Objets simples de $\mathcal{F}_{\text {surj }}$ ). - Un objet $S$ de $\mathcal{F}_{\text {surj }}$ est simple si et seulement s'il existe $n \in \mathbb{N}$ tel que $\operatorname{ev}_{n}(S)$ est un $\mathbb{k}\left[G L_{n}(\mathbb{k})\right]$-module simple et que $\operatorname{ev}_{k}(S)=0$ pour $k \neq n$. Il revient au même de dire que $S$ est isomorphe $\grave{a} j_{*}^{n}(R)$ pour un certain $n \in \mathbb{N}$ et un certain $\mathbb{k}\left[G L_{n}(\mathbb{k})\right]$-module simple $R$.

Démonstration. - Un objet simple dans une sous-catégorie épaisse $\mathcal{F}_{\text {surj }}^{\leq n}$ reste simple dans $\mathcal{F}_{\text {surj }}$; réciproquement, la proposition 2.2.7 montre qu'un objet simple de $\mathcal{F}_{\text {sur } j}$ appartient à une sous-catégorie $\mathcal{F}_{\text {surj }}^{\leq n}$. La conclusion s'obtient alors en combinant les propositions 2.1.2 et B.3.5.

Exemple 2.2.14. - Les objets $\mathbb{k}(n)$ sont simples dans $\mathcal{F}_{\text {surj }}$.

Remarque 2.2.15. - On peut formaliser ce raisonnement en notant que $\mathcal{E}_{\text {surj }}^{f}$ est la colimite filtrante (en un sens à préciser) des sous-catégories $\mathcal{E}_{\text {surj }}^{\leq n}$ et que, par conséquent, $\mathcal{F}_{\text {surj }}$ est la limite filtrante (id.) des $\mathcal{F}_{\text {surj }}^{\leq n}$. Kuhn détaille (dans le cadre analogue de la catégorie $\mathcal{F}$ ) cet argument dans [Kuh94b], $\S 2$.

Corollaire 2.2.16. - Dans $\mathcal{F}_{\text {surj }}$, le produit tensoriel total, donc a fortiori le produit tensoriel usuel, de deux objets finis, est fini. Cela munit le groupe de Grothendieck $G_{0}^{f}\left(\mathcal{F}_{\text {surj }}\right)$ d'une structure d'anneau commutatif sans unité (le foncteur constant $\mathbb{k}$ n'étant pas fini) via $\otimes$, et d'une structure d'anneau commutatif (unitaire, le foncteur $\mathrm{Is}_{0}$ étant fini) via $\widetilde{\otimes}$. Il est isomorphe, pour la première structure, à l'idéal

de l'anneau produit

$$
\bigoplus_{n \in \mathbb{N}} G_{0}^{f}\left(\mathbb{k}_{k}\left[G L_{n}(\mathbb{k})\right] \text { Mod }\right)
$$

$$
\prod_{n \in \mathbb{N}} G_{0}^{f}\left({ }_{k}\left[G L_{n}(\mathbb{k})\right] \text { Mod }\right)
$$


(chaque facteur étant muni de la structure d'anneau induite par le produit tensoriel sur $\mathbb{k})$.

\subsection{Le foncteur $\varpi: \mathcal{F}_{\text {surj }} \rightarrow \mathcal{F}$}

Nous établissons dans cette section les liens élémentaires entre les catégories $\mathcal{F}$ et $\mathcal{F}_{\text {surj }}$.

Le foncteur d'oubli $o: \mathcal{F} \rightarrow \mathcal{F}_{\text {surj }} \cdot-$

Notation 2.3.1. - Le foncteur de précomposition par le foncteur d'inclusion $\mathcal{E}_{\text {surj }}^{f} \rightarrow \mathcal{E}^{f}$ sera noté $o: \mathcal{F} \rightarrow \mathcal{F}_{\text {surj }}$

Ce foncteur est exact et fidèle.

Proposition 2.3.2. - L'image par le foncteur o: $\mathcal{F} \rightarrow \mathcal{F}_{\text {surj }}$ d'un objet simple de $\mathcal{F}$ est un objet unisériel ${ }^{(2)}$ de $\mathcal{F}_{\text {surj }}$, dont la filtration canonique est l'unique suite de composition.

Démonstration. - Soient $S$ un objet simple de $\mathcal{F}$ et $X$ un quotient de $o(S)$. Pour tout $n \in \mathbb{N}$, la projection $\pi_{n}: S\left(E_{n}\right) \rightarrow X\left(E_{n}\right)$ est soit nulle, soit un isomorphisme, par la proposition 1.4.10.

Comme $o(S)$ transforme les morphismes de $\mathcal{E}_{\text {surj }}$ en des épimorphismes, la commutation des diagrammes

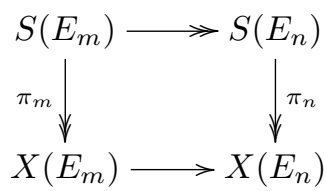

pour $m \geq n$ (induits par un épimorphisme arbitraire $\left.E_{m} \rightarrow E_{n}\right)$ montre que $X\left(E_{n}\right)=$ 0 si $X\left(E_{m}\right)=0$. Cela démontre la proposition.

Nous spécifions maintenant le cas particulier, immédiat mais important, du foncteur constant de $\mathcal{F}$.

Notation 2.3.3. - Pour tout $i \in \mathbb{N}$, on désigne par $\mathbb{k}^{\geq i}$ l'image du foncteur constant $\mathbb{k}$ de $\mathcal{F}_{\text {surj }}^{\geq i}$ par le foncteur de prolongement par zéro $\mathcal{P}: \mathcal{F}_{\text {surj }}^{\geq i} \rightarrow \mathcal{F}_{\text {sur } j}$.

Corollaire 2.3.4. - Pour tout $n \in \mathbb{N}$, il existe une suite exacte $0 \rightarrow \mathbb{k}(n) \rightarrow \mathbb{k}^{\geq n} \rightarrow$ $\mathbb{k}^{\geq n+1} \rightarrow 0$. De plus, $\mathbb{k}(n)$ est le socle de $\mathbb{k}^{\geq n}$.

$L$ 'objet $\mathbb{k}$ de $\mathcal{F}_{\text {surj }}$ est unisériel, de suite de composition $\mathbb{k}(0), \mathbb{k}(1), \ldots, \mathbb{k}(n), \ldots$

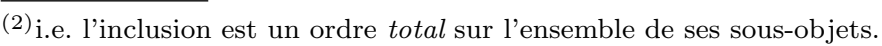


Ce résultat est à comparer avec le résultat fondamental sur la structure du foncteur $\bar{I}_{\mathbb{F}_{2}}$ de $\mathcal{F}\left(\mathbb{F}_{2}\right)$ : la filtration polynomiale de ce foncteur est son unique suite de composition, et son $n$-ième quotient est isomorphe au foncteur $n$-ième puissance extérieure $\Lambda^{n}$, qui correspond à la représentation triviale de $G L_{n}\left(\mathbb{F}_{2}\right)$. De plus, $\bar{I}_{\mathbb{F}_{2}}$ est le « plus petit $»$ injectif indécomposable non constant de $\mathcal{F}\left(\mathbb{F}_{2}\right)$. Le foncteur $\mathbb{k} \simeq I_{0}^{\text {surj }}$ est le « plus petit » injectif indécomposable de $\mathcal{F}_{\text {surj }}(\mathbb{k}) ;$ sa filtration canonique est son unique suite de composition, et son $n$-ième quotient est $T_{n}^{h o m}(\mathbb{k}) \simeq \mathbb{k}(n)$ (cf. corollaire 2.2.12), qui correspond à la représentation triviale de $G L_{n}(\mathbb{k})$ (noter qu'ici on a également un facteur pour $n=0$ ).

Le corollaire 2.3.4 interviendra de façon cruciale au chapitre 10.1.

\section{L'adjonction entre les foncteurs $o$ et $\varpi$. -}

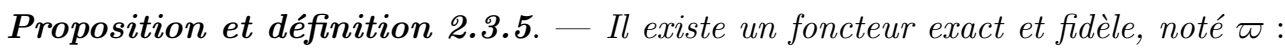
$\mathcal{F}_{\text {surj }} \rightarrow \mathcal{F}$, donné de la façon suivante.

- Si $X$ est un objet de $\mathcal{F}_{\text {surj }}$ et $V$ un objet de $\mathcal{E}^{f}$, on pose

$$
(\varpi(X))(V)=\bigoplus_{W \in \mathcal{G} r(V)} X(W) .
$$

- Si $X$ est un objet de $\mathcal{F}_{\text {surj }}$ et $V \stackrel{f}{\rightarrow} V^{\prime}$ une flèche de $\mathcal{E}^{f}$, et si $W$ et $W^{\prime}$ sont des sous-espaces respectifs de $V$ et $V^{\prime}$, la composante $X(W) \rightarrow X\left(W^{\prime}\right)$ de $(\varpi(X))(f)$ est égale à $X(g)$ si $f(W)=W^{\prime}$, où $g$ est l'épimorphisme $W \rightarrow W^{\prime}$ induit par $f$ et nulle sinon.

- Si $X \stackrel{u}{\rightarrow} Y$ est une flèche de $\mathcal{F}_{\text {surj }}$, le morphisme $\varpi(u): \varpi(X) \rightarrow \varpi(Y)$ de $\mathcal{F}$ est défini, sur l'espace vectoriel de dimension finie $V$, comme la somme directe sur les sous-espaces $W$ de $V$ des morphismes $u_{W}: X(W) \rightarrow Y(W)$.

Proposition 2.3.6. — (1) Le foncteur o est adjoint à droite au foncteur $\varpi$.

(2) Pour tout espace vectoriel $V$ de dimension finie, on a un isomorphisme $\varpi\left(P_{V}^{\text {surj }}\right) \simeq P_{V}$.

(3) Pour tout espace vectoriel $V$ de dimension finie, on a un isomorphisme $o\left(I_{V}\right) \simeq$ $\bigoplus I_{W}^{\text {surj }}$.

$W \in \mathcal{G} r(V)$

(4) Le foncteur o préserve les objets de co-type fini et, plus généralement, les objets co-pf, où $i \in \mathbb{N} \cup\{\infty\}$.

(5) Il existe un isomorphisme $\varpi(X \widetilde{\otimes} Y) \simeq \varpi(X) \otimes \varpi(Y)$ naturel en les objets $X$ et $Y$ de $\mathcal{F}_{\text {surj }}$.

Démonstration. - Soient $X$ un objet de $\mathcal{F}_{\text {surj }}$ et $F$ un objet de $\mathcal{F}$. Un morphisme $\varpi(X) \rightarrow F$ est la donnée, pour tout $V \in \mathrm{Ob} \mathcal{E}^{f}$ et tout sous-espace $W$ de $V$, d'une application linéaire $u_{V, W}: X(W) \rightarrow F(V)$ de sorte que, pour tout $f \in \operatorname{hom}_{\mathcal{E}^{f}}\left(V, V^{\prime}\right)$, 
le diagramme

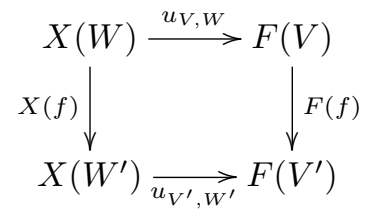

commute, où l'on a posé $W^{\prime}=f(W)$. Les applications linéaires $u_{V, V}$ fournissent en particulier un morphisme $X \rightarrow o(F)$ dans $\mathcal{F}_{\text {surj }}$.

Réciproquement, si $a: X \rightarrow o(F)$ est une flèche de $\mathcal{F}_{\text {surj }}$, définissons, pour $V \in \mathrm{Ob} \mathcal{E}^{f}$ et $W \in \mathcal{G} r(V)$, une application linéaire $u_{V, W}: X(W) \stackrel{a_{W}}{\longrightarrow} F(W) \hookrightarrow F(V)$, la dernière flèche étant induite par l'inclusion. Il est clair que les diagrammes (3) commutent, de sorte que $u$ fournit un morphisme $\varpi(X) \rightarrow F$ dans $\mathcal{F}$. On vérifie aussitôt que les deux applications naturelles entre $\operatorname{hom}_{\mathcal{F}_{\text {surj }}}(X, o(F))$ et $\operatorname{hom}_{\mathcal{F}}(\varpi(X), F)$ définies précédemment sont réciproques l'une de l'autre, ce qui établit le premier point.

Les assertions 2 et 3 s'obtiennent par adjonction à partir du lemme de Yoneda : on a des isomorphismes naturels

$$
\operatorname{hom}_{\mathcal{F}}\left(\varpi\left(P_{V}^{\text {surj }}\right), F\right) \simeq \operatorname{hom}_{\mathcal{F}_{\text {surj }}}\left(P_{V}^{\text {surj }}, o(F)\right) \simeq o(F)(V)=F(V) \simeq \operatorname{hom}_{\mathcal{F}}\left(P_{V}, F\right)
$$

et

$$
\begin{aligned}
\operatorname{hom}_{\mathcal{F}_{\text {surj }}}\left(X, o\left(I_{V}\right)\right) & \simeq \operatorname{hom}_{\mathcal{F}}\left(\varpi(X), I_{V}\right) \simeq \varpi(X)(V)=\bigoplus_{W \in \mathcal{G} r(V)} X(W) \\
& \simeq \operatorname{hom}_{\mathcal{F}_{\text {surj }}}\left(X, \bigoplus_{W \in \mathcal{G} r(V)} I_{W}^{\text {surj }}\right) .
\end{aligned}
$$

La quatrième assertion provient de la troisième et de l'exactitude de $o$.

La dernière s'obtient à partir de la décomposition ensembliste naturelle des couples de sous-espaces d'un espace vectoriel $V$ suivante :

$$
\{(A, B) \mid A, B \in \mathcal{G} r(V)\}=\coprod_{W \in \mathcal{G} r(V)}\{(A, B) \mid A, B \in \mathcal{G} r(W), A+B=W\} .
$$

Remarque 2.3.7. - L'isomorphisme de la dernière assertion est adjoint au monomorphisme canonique $F \widetilde{\otimes} G \hookrightarrow o(\varpi(F) \otimes \varpi(G))$ donné sur l'espace $A$ par l'injection du facteur direct

$$
\bigoplus_{V+W=A} F(V) \otimes G(W) \hookrightarrow \bigoplus_{V, W \in \mathcal{G} r(A)} F(V) \otimes G(W) .
$$

Proposition 2.3.8. - Soient $F$ un objet de $\mathcal{F}$ et $i \in \mathbb{N} \cup\{\infty\}$.

(1) Si $o(F)$ est artinien, alors $F$ est artinien.

(2) Si o $(F)$ est co-pfi, alors $F$ est co-pfi. 
Démonstration. - Le foncteur o est exact et fidèle, ce qui fournit la première assertion, en appliquant la proposition B.2.1. De plus, il commute aux limites et transforme les cogénérateurs injectifs de $\mathcal{F}_{\text {surj }}$ en des objets injectifs de co-type fini. La variante duale de la proposition B.2.2 donne donc la deuxième partie de la proposition.

La proposition suivante, que l'on vérifie par inspection, précise la compatibilité entre les foncteurs $o$ et $\varpi$ d'une part, et la décomposition scalaire et le tors de Frobenius d'autre part.

Proposition 2.3.9. - (1) Soit $F \in \mathrm{Ob} \mathcal{F}$. Il existe des isomorphismes naturels $o(F)_{i} \simeq o\left(F_{i}\right)$ pour $1 \leq i<q-1$ et $o(F)_{q-1} \simeq o\left(F_{q-1}\right) \oplus o(F(0))$.

(2) Soit $X \in \mathrm{Ob} \mathcal{F}_{\text {surj }}$. Il existe des isomorphismes naturels $\varpi(X)_{0} \simeq X(0)$, $\varpi(X)_{i} \simeq \varpi\left(X_{i}\right)$ pour $1 \leq i<q-1$ et $\varpi(X)_{q-1} \simeq \varpi\left(X_{q-1}\right) / X(0)$.

(3) Les foncteurs o et $\varpi$ commutent au tors de Frobenius, à isomorphisme naturel près.

De façon similaire, le foncteur $o$ commute aux foncteurs de restriction et d'induction associés à une extension finie du corps $\mathbb{k}$. En revanche, le comportement du foncteur $\varpi$ relativement aux changements de corps est délicat.

Nous donnons maintenant quelques traductions dans le cadre de la catégorie $\mathcal{F}_{i n j}$ de la proposition 2.3.6.

Proposition et définition 2.3.10. - (1) Nous noterons $o_{i n j}: \mathcal{F} \rightarrow \mathcal{F}_{\text {inj }}$ le foncteur d'oubli (précomposition par l'inclusion $\mathcal{E}_{i n j}^{f} \rightarrow \mathcal{E}^{f}$ ); il est exact et fidèle. De plus, il préserve les objets de type fini.

(2) Il existe un foncteur exact et fidèle $\varpi_{i n j}: \mathcal{F}_{i n j} \rightarrow \mathcal{F}$ donné sur les objets par

$$
\varpi_{i n j}(X)(V)=\bigoplus_{W \in \mathcal{G} r(V)} X(V / W)
$$

le morphisme $\varpi_{i n j}(X)(V) \rightarrow \varpi_{i n j}(X)\left(V^{\prime}\right)$ induit par une application linéaire $f:$ $V \rightarrow V^{\prime}$ ayant pour composante $X(V / W) \rightarrow X\left(V^{\prime} / W^{\prime}\right)$ l'application $X(\bar{f})$, où $\bar{f}$ est le monomorphisme $V / W \rightarrow V^{\prime} / W^{\prime}$ induit par $f$, si $W=f^{-1}\left(W^{\prime}\right)$, 0 sinon.

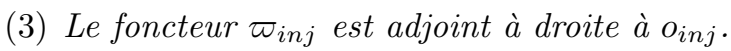

Nous avons noté $o_{i n j}$ le foncteur d'oubli de $\mathcal{F}$ vers $\mathcal{F}_{i n j}$, et simplement $o$ plutôt que $o_{\text {surj }}$ le foncteur d'oubli de $\mathcal{F}$ vers $\mathcal{F}_{\text {surj }}$ (qui est dual du précédent), car ce dernier interviendra beaucoup plus souvent.

Une propriété homologique du foncteur $\varpi$. — Le comportement homologique général de $\varpi$ semble délicat; dans le cas d'une action des groupes linéaires sur les foncteurs considérés, on dispose cependant de l'utile résultat suivant.

Proposition 2.3.11. - Soient $n$ un entier strictement positif et $Z$ un objet de $\mathcal{F}_{\text {surj }}$ vérifiant les propriétés suivantes : 
(1) l'action du groupe linéaire sur le $G L_{n+1}(\mathbb{k})$-module $M=\mathrm{ev}_{n+1}(Z)$ (resp. le $G L_{n}(\mathbb{k})$-module $\left.N=\mathrm{ev}_{n}(Z)\right)$ est triviale;

(2) le morphisme $u: M \rightarrow N$ induit par la projection $E_{n+1} \rightarrow E_{n}$ sur les $n$ premières coordonnées est injectif;

(3) on a $Z\left(E_{k}\right)=0$ si $k \notin\{n, n+1\}$.

On note $X=j_{*}^{n+1}(M)$ et $Y=j_{*}^{n}(N)$.

Alors l'extension

$$
0 \rightarrow \varpi(Y) \rightarrow \varpi(Z) \rightarrow \varpi(X) \rightarrow 0
$$

de $\mathcal{F}$ obtenue par application du foncteur exact $\varpi$ à la suite exacte $0 \rightarrow Y \rightarrow Z \rightarrow$ $X \rightarrow 0$ de $\mathcal{F}_{\text {surj }}$ est essentielle.

Démonstration. - Considérons un morphisme $f: \varpi(Z) \rightarrow F$ de $\mathcal{F}$ dont la restriction à $\varpi(Y)$ est injective. On établit que l'application linéaire $f_{V}: \varpi(Z)(V) \rightarrow F(V)$ est injective pour tout $V \in \mathrm{Ob}^{f}$ par récurrence sur $\operatorname{dim} V$. C'est clair si $\operatorname{dim} V \leq n$, nous supposerons donc $\operatorname{dim} V>n$.

Soit $a$ un élément de $k e r f_{V}$; si $W$ (resp. $B$ ) est un sous-espace de dimension $n+1$ (resp. $n$ ) de $V$, notons $x_{W}$ (resp. $\left.y_{B}\right)$ l'élément de $M$ (resp. $N$ ) correspondant à la composante de $a$ dans $X(W) \simeq M$ (resp. $Y(B) \simeq N)$. Pour tout élément $v$ non nul de $V$, l'hypothèse de récurrence montre que l'image de $a$ dans $\varpi(Z)(V / v)$ est nulle. Soient $H$ un sous-espace de dimension $n$ de $V / v$ et $W$ son image réciproque dans $V$ : la composante dans $Z(H)$ de cette image est

$$
u\left(x_{W}\right)+\sum_{\substack{B \in \mathcal{G} r_{n}(V) \\ W=B \oplus v}} y_{B}=0 .
$$

Étant donné un sous-espace $W$ de dimension $n+1$ de $V$, faisons la somme des relations ainsi obtenues pour $v \in W \backslash\{0\}$ : on obtient

$$
u\left(x_{W}\right)+\sum_{\substack{v \in W \backslash\{0\}, B \in \mathcal{G} r_{n}(W) \\ W=B \oplus v}} y_{B}=0,
$$

soit

$$
u\left(x_{W}\right)=\sum_{B \in \mathcal{G} r_{n}(W)} \operatorname{Card}(W \backslash B) y_{B} .
$$

Mais comme $n>0$, les cardinaux qui apparaissent dans cette somme, égaux à $q^{n+1}-q^{n}$, sont nuls dans $\mathbb{k}$, d'où $u\left(x_{W}\right)=0$.

Comme $u$ est supposé injectif, cela donne $x_{W}=0$; autrement dit, $a \in \varpi(Y)(V)$. Puisque la restriction à $\varpi(Y)(V)$ de $f_{V}$ est par hypothèse injective, il vient finalement $a=0$. Ainsi, l'extension de l'énoncé est bien essentielle. 
Foncteurs de Powell. - Les duaux des foncteurs définis ci-après ont été introduits par Powell dans [Pow98c] (où la catégorie $\mathcal{F}_{\text {surj }}$ n'apparaît pas explicitement) sous le nom de foncteurs co-Weyl; il en a montré l'intérêt dans l'étude de la conjecture artinienne.

Définition 2.3.12. - On appelle foncteur de Powell l'image par le foncteur $\varpi$ : $\mathcal{F}_{\text {sur } j} \rightarrow \mathcal{F}$ d'un objet simple de $\mathcal{F}_{\text {sur } j}$.

Nous nommerons filtration de Powell d'un foncteur $F$ de $\mathcal{F}$ toute filtration finie

$$
0=A_{0} \subset A_{1} \subset \cdots \subset A_{n}=F
$$

de $F$ dont les sous-quotients $A_{i} / A_{i-1}$ sont des foncteurs de Powell.

Notation 2.3.13. - Soient $n \in \mathbb{N}$ et $\lambda$ une partition 2-régulière telle que $\lambda_{1}=n$. On note $Q_{\lambda}$ le foncteur de Powell $\varpi\left(j_{*}^{n}\left(R_{\lambda}\right)\right)$ de $\mathcal{F}\left(\mathbb{F}_{2}\right)$.

On note également $\bar{G}(n)$ le foncteur de Powell $\varpi(\mathbb{k}(n))$ de $\mathcal{F}(\mathbb{k})$.

Dans [Pow98c], les foncteurs possédant une filtration de Powell sont appelés foncteurs $D J$-bons. De nombreuses propriétés en sont établies. Nous nous contenterons de démontrer la stabilité par produit tensoriel, particulièrement commode avec le formalisme de la catégorie $\mathcal{F}_{\text {surj }}$.

Proposition 2.3.14. - Si $X$ est un objet fini de $\mathcal{F}_{\text {surj }}$, alors $\varpi(X)$ admet une filtration de Powell.

Cette propriété résulte de l'exactitude du foncteur $\varpi$.

Exemple 2.3.15. - Les projectifs standard $P_{V} \simeq \varpi\left(P_{V}^{\text {surj }}\right)$ de $\mathcal{F}$ admettent une filtration de Powell.

Corollaire 2.3.16. - Le produit tensoriel de deux foncteurs de $\mathcal{F}$ possédant une filtration de Powell admet une filtration de Powell.

Démonstration. - Cela provient de l'isomorphisme $\omega(X) \otimes \omega(Y) \simeq \omega(X \widetilde{\otimes} Y)$ (dernière assertion de la proposition 2.3.6), de la stabilité des objets finis de $\mathcal{F}_{\text {surj }}$ par produit tensoriel total (cf. corollaire 2.2.16) et de l'exactitude du produit tensoriel de $\mathcal{F}$.

Ce corollaire, qui illustre l'intérêt du produit tensoriel total, est établi dans [Pow98c] à partir d'un critère homologique pour l'existence d'une filtration de Powell.

Signalons également le lien entre les objets simples de $\mathcal{F}$ et $\mathcal{F}_{\text {surj }}$ réalisé par les foncteurs de Powell, qui se déduit de [Pow98c], $§ 2.3$ (cf. aussi [Kuh94b]).

Proposition 2.3.17 (Kuhn-Powell). - Le cosocle d'un foncteur de Powell est simple. De plus, tout foncteur simple de $\mathcal{F}$ est le cosocle d'un unique foncteur de Powell, à isomorphisme près. 
Remarque 2.3.18. - Cette propriété procure un isomorphisme de groupes $G_{0}^{f}(\mathcal{F}) \simeq G_{0}^{f}\left(\mathcal{F}_{\text {surj }}\right)$. En revanche, les structures multiplicatives sur $G_{0}^{f}\left(\mathcal{F}_{\text {surj }}\right)$ et $G_{0}^{f}(\mathcal{F})$ semblent fort délicates à comparer.

Liens avec la conjecture artinienne. - $\grave{A}$ l'aune du corollaire 2.2.9, les problèmes de finitude dans $\mathcal{F}_{\text {surj }}$ se posent en les termes suivants.

Conjecture 2.3.19. — La catégorie $\mathcal{F}_{\text {surj }}$ est co-localement artinienne.

Par dualité, cette conjecture équivaut au caractère localement noethérien de $\mathcal{F}_{i n j}$.

Proposition 2.3.20. - La conjecture 2.3.19 implique la conjecture artinienne.

Démonstration. - Si la conjecture 2.3.19 est vérifiée, $o\left(I_{V}\right)$ est artinien pour tout $V \in \mathrm{Ob}^{f}$ (cf. proposition 2.3.6.3); la proposition 2.3.8 montre que les injectifs standard $I_{V}$ de $\mathcal{F}$ sont alors également artiniens.

Il ne semble en revanche exister aucun argument formel pour obtenir la réciproque de la proposition 2.3.20.

Notation 2.3.21. - Étant donné $n \in \mathbb{N}$, nous noterons $\widetilde{P}(n)$ l'objet $\varpi\left(\operatorname{Is}_{n}\right)$ de $\mathcal{F}$ (on rappelle que le foncteur $\mathrm{Is}_{n}$ est défini dans la notation 2.1.4).

On a ainsi un isomorphisme canonique $\widetilde{P}(n)(V) \simeq \mathbb{k}\left[\mathrm{Pl}_{\mathcal{E}^{f}}\left(E_{n}, V\right)\right]$ pour $V \in \mathrm{Ob} \mathcal{E}^{f}$ (on rappelle que $\mathrm{Pl}_{\mathcal{E}^{f}}\left(E_{n}, V\right)$ désigne l'ensemble des monomorphismes $E_{n} \hookrightarrow V$ dans la catégorie $\left.\mathcal{E}^{f}\right)$.

Lemme 2.3.22. - (1) Étant donné un objet $X$ de $\mathcal{F}_{\text {surj, }}$, les assertions suivantes sont équivalentes.

(a) L'objet $X$ est de co-type fini.

(b) L'ensemble

$$
\left\{n \in \mathbb{N} \mid \operatorname{hom}\left(\mathrm{Is}_{n}, X\right) \neq 0\right\}
$$

est fini et $X$ est à valeurs de dimension finie.

(c) Le socle de $X$ est fini.

(2) Étant donné un objet co-tf $X$ de $\mathcal{F}_{\text {surj, }}$ les assertions suivantes sont équivalentes.

(a) L'objet $X$ est co-pf.

(b) L'ensemble

$$
\left\{n \in \mathbb{N} \mid \operatorname{Ext}^{1}\left(\operatorname{Is}_{n}, X\right) \neq 0\right\}
$$

est fini.

Proposition 2.3.23. - La conjecture artinienne équivaut à l'assertion suivante : pour tout objet de co-type fini $F$ de $\mathcal{F}$, l'ensemble

$$
\left\{n \in \mathbb{N} \mid \operatorname{Ext}_{\mathcal{F}}^{1}(\widetilde{P}(n), F) \neq 0\right\}
$$


est fini.

Le lemme et la proposition précédents sont laissés au lecteur, qui pourra en trouver la démonstration dans $[\mathbf{D j a 0 6 d}], \S 5.4 .2$ (p. 126).

Powell a émis, à partir des résultats de [Pow98c], la conjecture suivante, dont nous proposerons une version renforcée ultérieurement.

\section{Conjecture 2.3.24 (Conjecture artinienne très forte)}

Pour tout objet simple $S$ de $\mathcal{F}_{\text {surj }}$ tel que ev $v_{n}(S) \neq 0$, le foncteur de Powell $\varpi(S)$ est simple noethérien de type $n$.

\subsection{Liens avec les systèmes de coefficients}

Nous rappelons la définition de Dwyer de la catégorie des systèmes de coefficients ([Dwy80], 2).

Convention 2.4.1. - Soient $m$ et $n$ des entiers tels que $0 \leq n \leq m$. Dans cette

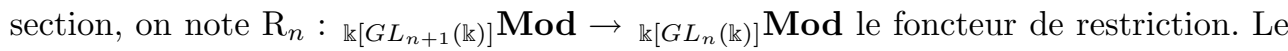
groupe $G L_{n}(\mathbb{k})$ est plongé dans $G L_{m}(\mathbb{k})$ par

$$
\alpha_{n, m}: A \mapsto\left(\begin{array}{cc}
A & 0 \\
0 & I_{m-n}
\end{array}\right) .
$$

On note $l_{n, m}: E_{n} \hookrightarrow E_{m}$ l'inclusion $x \mapsto(x, 0)$.

On note enfin $G L(m, n)$ le sous-groupe de $G L_{m}(\mathbb{k})$ des automorphismes $g$ de $E_{m}$ tels que $g \circ l_{n, m}=l_{n, m}$.

Remarque 2.4.2. - Comme le sous-groupe $G L_{n}(\mathbb{k})$ de $G L_{m}(\mathbb{k})$ normalise $G L(m, n)$, on peut voir le foncteur $M \mapsto M^{G L(m, n)}$ (invariants sous l'action de $G L(m, n))$ défini sur les $G L_{m}(\mathbb{k})$-modules comme un sous-foncteur de la restriction

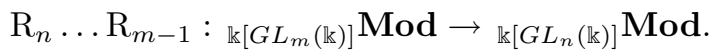

Définition 2.4.3. - On note $\mathcal{E}_{\text {coef }}^{f}$ la catégorie dont les objets sont les espaces $E_{n}$ pour $n \in \mathbb{N}$, les morphismes étant donnés par hom ${\mathcal{\mathcal { E } _ { \text { coef } } ^ { f }}}\left(E_{n}, E_{m}\right)=G L_{m}(\mathbb{k})$ si $n \leq m$, $\varnothing$ si $n>m$, et où la composition est définie comme suit. Si $0 \leq n \leq m \leq k$ sont des entiers, la composée $g \circ h$, où $g \in \operatorname{hom}_{\mathcal{E}_{\text {coef }}^{f}}\left(E_{m}, E_{k}\right)=G L_{k}(\mathbb{k})$ et $h \in$ $\operatorname{hom}_{\mathcal{E}_{\text {coef }}^{f}}\left(E_{n}, E_{m}\right)=G L_{m}(\mathbb{k})$, est l'élément $g . \alpha_{m, k}(h)$ de $G L_{k}(\mathbb{k})=\operatorname{hom}_{\mathcal{E}_{\text {coef }}^{f}}\left(E_{n}, E_{k}\right)$.

On note $\mathcal{C}$ oef la catégorie $\mathbf{F} \mathbf{c t}\left(\mathcal{E}_{\text {coef }}^{f}, \mathcal{E}\right)$; ses objets sont appelés systèmes de coefficients.

Remarque 2.4.4. - Dans [Dwy80], un système de coefficients est une suite $\left(M_{n}\right)_{n \in \mathbb{N}}$, où $M_{n}$ est un $G L_{n}(\mathbb{k})$-module à gauche, munie de morphismes de $G L_{n}(\mathbb{k})$ modules $r_{n}: M_{n} \rightarrow \mathrm{R}_{n}\left(M_{n+1}\right)$.

L'équivalence entre cette définition et la nôtre résulte de ce que la catégorie $\mathcal{E}_{\text {coef }}^{f}$ est engendrée (cf. [ML71], chapitre II, §8) par les éléments de $G L_{n}(\mathbb{k})=$ 
$\operatorname{hom}_{\mathcal{E}_{\text {coef }}^{f}}\left(E_{n}, E_{n}\right)$ et les morphismes $i_{n, m}: E_{n} \rightarrow E_{m}$ correspondant à $I_{m} \in G L_{m}(\mathbb{k})$, pour $n \leq m$, soumis aux relations $i_{n, n+1} g=\alpha_{n, n+1}(g) \in \operatorname{hom}_{\mathcal{E}_{\text {coef }}^{f}}\left(E_{n}, E_{n+1}\right)$ pour $n \in \mathbb{N}$ et $g \in G L_{n}(\mathbb{k})$.

Lemme 2.4.5. - On définit un foncteur plein et essentiellement surjectif $\mathcal{E}_{\text {coef }}^{f} \rightarrow$ $\mathcal{E}_{\text {inj }}^{f}$ de la façon suivante:

- on associe à l'objet $E_{n}$ de $\mathcal{E}_{\text {coef }}^{f}$ l'objet $E_{n}$ de $\mathcal{E}_{\text {inj }}^{f}$;

- si $0 \leq n \leq m$ sont des entiers, on associe à $g \in \operatorname{hom}_{\mathcal{E}_{\text {coef }}^{f}}\left(E_{n}, E_{m}\right)=G L_{m}(\mathbb{k})$ le monomorphisme $E_{n} \hookrightarrow E_{m}$ composé de l'inclusion $l_{n, m}: E_{n} \hookrightarrow E_{m}$ et de l'automorphisme $g: E_{m} \rightarrow E_{m}$.

Ce lemme se vérifie par inspection.

Notation 2.4.6. - On note $\mathrm{C}: \mathcal{F}_{i n j} \rightarrow \mathcal{C}$ oef le foncteur de précomposition par le foncteur du lemme 2.4.5.

On rappelle que la notion de sous-catégorie de Serre est introduite dans la définition B.1.9.

Proposition 2.4.7. - Le foncteur C est pleinement fidèle; son image est une souscatégorie de Serre de $\mathcal{C}$ oef.

Démonstration. - Ce résultat s'obtient en combinant le lemme 2.4.5 et la proposition C.1.4.

On peut préciser cette propriété comme suit.

Proposition 2.4.8. - L'image essentielle du foncteur $\mathrm{C}$ est constituée des systèmes de coefficients $\left(\left(M_{n}\right),\left(r_{n}\right)\right)$ tels que im $r_{m} r_{m-1} \ldots r_{n} \subset M_{m+1} G L(m+1, n)$ pour tous entiers $n$ et $m$ tels que $0 \leq n \leq m$, où l'on utilise les notations de la remarque 2.4.4 pour les systèmes de coefficients.

Démonstration. - C'est une conséquence formelle de l'observation suivante : le squelette de la catégorie $\mathcal{E}_{i n j}^{f}$ constitué des espaces $E_{n}$ est engendré par les inclusions $l_{n, n+1}$ et les éléments des différents $G L_{n}(\mathbb{k})$, soumis aux relations $\alpha_{n}(g) \circ l_{n}=l_{n} \circ g$ pour $g \in$ $G L_{n}(\mathbb{k}), g \circ h=g h$ pour $g, h \in G L_{n}(\mathbb{k})$ et $g \circ l_{n, m}=l_{n, m}$ pour $g \in G L(m+1, n)$.

Remarque 2.4.9. - En particulier, tous les systèmes de coefficients appartenant à l'image du foncteur C sont centraux au sens de [Dwy80], $§ 2$. 
Les catégories $\mathcal{C}$ oef $f^{l f}$ et $\mathcal{C}$ oef $/ \mathcal{C}$ oef $f^{l f}$ _ — Le foncteur de décalage $\delta^{i n j}: \mathcal{F}_{i n j} \rightarrow$ $\mathcal{F}_{i n j}$ admet un relèvement à $\mathcal{C}$ oef . Précisément, il existe un endofoncteur exact $\delta^{\text {coef }}$ de $\mathcal{C}$ oef tel que le diagramme

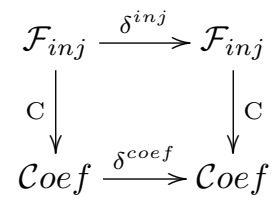

commute (à isomorphisme canonique près).

Le foncteur $\delta^{\text {coef }}$ s'obtient par précomposition par l'endofoncteur de $\mathcal{E}_{\text {coef }}^{f}$ donné sur les objets par $E_{n} \mapsto E_{n+1}$ et sur les morphismes par l'inclusion $G L_{n}(\mathbb{k}) \hookrightarrow$ $G L_{n+1}(\mathbb{k})$ donnée par

$$
A \mapsto\left(\begin{array}{cc}
1 & 0 \\
0 & A
\end{array}\right)
$$

( $\delta^{\text {coef }}$ est le foncteur $\Sigma$ de [Dwy80], $\left.\S 2\right)$.

On établit, par la même méthode que celle employée dans $\mathcal{F}_{i n j}$, le résultat suivant.

Proposition 2.4.10. - (1) Un objet de Coef est fini si et seulement s'il est nilpotent pour le foncteur $\delta^{\text {coef }}$ et à valeurs de dimension finie.

(2) Tout objet fini de $\mathcal{C}$ oef est $p f_{\infty}$. Par conséquent, la sous-catégorie pleine $\mathcal{C}$ oe $f^{l f}$ de $\mathcal{C}$ oef des systèmes de coefficients localement finis est épaisse.

Soit $G L(\mathbb{k})$ la colimite des groupes linéaires $G L_{n}(\mathbb{k})$ (relativement aux inclusions $\left.\alpha_{n, m}\right)$. On peut voir $G L(\mathbb{k})$ comme le groupe des automorphismes linéaires $g$ de l'espace vectoriel $E_{\infty}=\operatorname{colim}_{n \in \mathbb{N}} E_{n}$ tels que $\operatorname{ker}(g-i d)$ est de codimension finie.

On définit un foncteur $\mathrm{e}_{\text {coef }}: \mathcal{C o e f} \rightarrow \mathbb{k}[G L(\mathbb{k})] \operatorname{Mod}$ par $\mathrm{e}_{\text {coef }}(X)=\operatorname{colim}_{n \in \mathbb{N}} X\left(E_{n}\right)$, l'action du groupe linéaire $G L(\mathbb{k})$ provenant de l'action de $G L_{n}(\mathbb{k})$ sur $X\left(E_{n}\right)$.

Proposition 2.4.11. - (1) Le foncteur $\mathrm{e}_{\text {coef }}$ est adjoint à gauche au foncteur c : $\mathbb{k}_{\mathbb{k}[G L(\mathbb{k})]} \operatorname{Mod} \simeq \operatorname{Fct}(\underline{G L(\mathbb{k})}, \mathcal{E}) \rightarrow \mathcal{C}$ oef $=\operatorname{Fct}\left(\mathcal{E}_{\text {coef }}^{f}, \mathcal{E}\right)$ donné par la précomposition par le foncteur $\mathcal{E}_{\text {coef }}^{\rightarrow} \rightarrow \underline{G L(\mathbb{k})}$ obtenu en plongeant les ensembles de morphismes $G L_{n}(\mathbb{k})$ dans $G L(\mathbb{k})$.

(2) Le foncteur $\mathrm{e}_{\text {coef }}$ induit une équivalence entre les catégories $\mathcal{C}$ oef $/ \mathcal{C}$ oef $f^{l f}$ et $\mathbb{k}[G L(\mathbb{k})]$ Mod.

Démonstration. - Si $P$ est un générateur projectif standard de $\mathcal{C}$ oef $f$, il existe un isomorphisme canonique $\mathrm{e}_{\text {coef }}(P) \simeq \mathbb{k}[G L(\mathbb{k})]$. On en déduit, pour tout $G L(\mathbb{k})$-module $M$, un isomorphisme canonique

$$
\operatorname{hom}_{\mathbb{k}[G L(\mathbb{k})]}\left(\mathrm{e}_{\text {coef }}(P), M\right) \simeq M \simeq \operatorname{hom}_{\mathcal{C o e f}}(P, \mathrm{c}(M)) .
$$

Cela donne la première assertion, en écrivant un objet de $\mathcal{C}$ oef comme colimite de projectifs standard, puisque le foncteur $\mathrm{e}_{\text {coef }}$ commute aux colimites. 
La première partie de la proposition 2.4.10 implique que le noyau du foncteur exact $e_{\text {coef }}$ est égal à $\mathcal{C} o e f^{l f}$; il induit donc un foncteur exact et fidèle $\mathcal{C}$ oef $/ \mathcal{C}$ oef $f^{l f} \rightarrow$ $\mathbb{k}[G L(\mathbb{k})]$ Mod.

La conclusion s'obtient alors formellement à partir des deux observations suivantes :

(1) la coünité $\mathrm{e}_{c o e f} \mathrm{c} \rightarrow i d$ de l'adjonction est un isomorphisme;

(2) l'unité $i d \rightarrow \mathrm{ce}_{c o e f}$ induit un isomorphisme après application du foncteur $\mathrm{e}_{c o e f}$.

Remarque 2.4.12. - L'anneau $\mathbb{k}[G L(\mathbb{k})]$ n'est pas noethérien à gauche. En effet, si l'on note $A_{n}=\operatorname{ker}\left(\mathbb{k}[G L(\mathbb{k})] \rightarrow \mathbb{k}\left[G L(\mathbb{k}) / G L_{n}(\mathbb{k})\right]\right)$, les $A_{n}$ forment une suite strictement croissante d'idéaux à gauche de $\mathbb{k}[G L(\mathbb{k})]$. Par conséquent, la proposition 2.4.11 montre que la catégorie $\mathcal{C}$ oef n'est pas localement noethérienne.

Ce phénomène est à rapprocher de l'exemple 1.2.2 : dans cet exemple, l'obstruction au caractère noethérien de la catégorie de foncteurs considérée est de nature « combinatoire $\gg$ (la catégorie source n'a pas d'endomorphismes non identiques). Ici, l'obstruction vient plutôt de la théorie des groupes.

Notation 2.4.13. - Nous noterons e $: \mathcal{F}_{i n j}(\mathbb{k}) \rightarrow{ }_{\mathbb{k}[G L(\mathbb{k})]}$ Mod le foncteur composé

$$
\mathcal{F}_{i n j}(\mathbb{k}) \stackrel{\mathrm{C}}{\rightarrow} \text { Coef } \rightarrow \mathcal{C} \text { oef } / \mathcal{C} \text { oef } f^{l f} \simeq{ }_{\mathbb{k}[G L(\mathbb{k})]} \operatorname{Mod} .
$$

Explicitement, $\mathrm{e}(X)$ est la colimite des espaces vectoriels $X\left(E_{n}\right)$.

Exemple 2.4.14. - Le foncteur e annihile les foncteurs injectifs standard de $\mathcal{F}_{\text {inj }}$ (car ceux-ci sont finis), mais possède un comportement non trivial sur les projectifs : on a $\mathrm{e}\left(P_{V}^{i n j}\right) \simeq \mathbb{k}\left[\mathrm{Pl}_{\mathcal{E}}\left(V, E_{\infty}\right)\right]$. En particulier, $\mathrm{e}(\mathbb{k}) \simeq \mathbb{k}(\mathbb{k}[G L(\mathbb{k})]$-module constant $)$ et $\mathrm{e}\left(P_{\mathbb{F}_{2}}^{i n j}\right) \simeq \mathbb{F}_{2}\left[E_{\infty} \backslash\{0\}\right]$.

Remarque 2.4.15. - Le foncteur e est beaucoup moins élémentaire que le foncteur analogue $\mathcal{F}(\mathbb{k}) \rightarrow{ }_{k}[\mathcal{M}(\mathbb{k})]$ Mod, où $\mathcal{M}(\mathbb{k})$ désigne le monoïde sans unité colimite des $\mathcal{M}_{n}(\mathbb{k})$ relativement aux inclusions

$$
A \mapsto\left(\begin{array}{cc}
A & 0 \\
0 & 0
\end{array}\right) .
$$

Pour une discussion à ce sujet, voir $[$ Kuh94b], $\S 3$.

La proposition suivante précise l'image du foncteur e.

Proposition 2.4.16. - Le foncteur $\mathrm{C}: \mathcal{F}_{\text {inj }} \rightarrow$ Coef induit une équivalence entre la catégorie $\mathcal{F}_{\text {inj }} / \mathcal{F}_{\text {inj }}^{\text {lf }}$ et la sous-catégorie de Serre de $\operatorname{Coef} / \mathcal{C}_{\text {oef }}{ }^{l f} \simeq{ }_{\mathbb{k}[G L(\mathbb{k})]} \mathbf{M o d}$ des $G L(\mathbb{k})$-modules $M$ tels que $M=\operatorname{colim}_{n \in \mathbb{N}} M^{G L(\infty, n)}$, où $G L(\infty, n)$ désigne le sousgroupe de $G L(\mathbb{k})$ des automorphismes $g$ tels que $g(x)=x$ pour $x \in E_{n}$. 
Démonstration. - Le diagramme commutatif (4) montre qu'un objet $X$ de $\mathcal{F}_{\text {inj }}$ est fini si et seulement si son image par le foncteur $\mathrm{C}$ est finie. Comme ce foncteur commute aux colimites, un objet de $\mathcal{F}_{i n j}$ est localement fini si et seulement s'il en est de même pour son image par C. Ainsi, ce foncteur induit un foncteur exact et fidèle $\mathcal{F}_{\text {inj }} / \mathcal{F}_{\text {inj }}^{\text {lf }} \rightarrow$ Coef $/ \mathcal{C}_{\text {oef }}$ lf $^{l} \simeq \mathbb{k}[G L(\mathbb{k})]$ Mod.

D'autre part, le lemme de Yoneda montre que le foncteur e admet un adjoint à droite $r$ donné par

$$
r(M)=\operatorname{hom}_{G L(\mathbb{k})}\left(\mathbb{k}\left[\mathrm{Pl}_{\mathcal{E}}\left(V, E_{\infty}\right)\right], M\right) .
$$

Comme $\mathrm{Pl}_{\mathcal{E}}\left(E_{n}, E_{\infty}\right)$ est un $G L(\mathbb{k})$-ensemble transitif, et que le stabilisateur de l'inclusion canonique $E_{n} \hookrightarrow E_{\infty}$ est $G L(\infty, n)$, on a $r(M)\left(E_{n}\right) \simeq M^{G L(\infty, n)}$. En particulier, la coünité de l'adjonction er $\rightarrow i d$ est un monomorphisme. On en déduit formellement, comme dans la démonstration de la proposition 2.4.11, que le foncteur $\mathcal{F}_{i n j} / \mathcal{F}_{i n j}^{l f} \rightarrow \mathbb{k}[G L(\mathbb{k})]$ Mod qui nous intéresse induit une équivalence entre $\mathcal{F}_{i n j} / \mathcal{F}_{i n j}^{l f}$ et la sous-catégorie pleine de $\mathbb{k}_{\mathbb{k}}[G L(\mathbb{k})]$ Mod des $G L(\mathbb{k})$-modules $M$ tels que la coünité $\mathrm{e} r(M) \rightarrow M$ est un isomorphisme, d'où la proposition. 


\section{CHAPITRE 3}

\section{CATÉGORIES DE COMODULES SUR UN FONCTEUR EN COALGĖBRES DE BOOLE}

Ce chapitre est consacré à la description de certaines catégories de foncteurs comme catégories de comodules (ou de modules) dans d'autres catégories de foncteurs. Ces observations, qui constituent le point de départ des recherches sur les catégories de foncteurs en grassmanniennes, possèdent des implications qui dépassent largement les jeux assez formels d'adjonction qui sous-tendent les démonstrations des résultats de ce chapitre. De fait, bien que ceux-ci restent longtemps peu utilisés de manière explicite, ils jouent un rôle crucial dans la démonstration du théorème de simplicité généralisé établi dans le chapitre 16, théorème qui constitue l'un des résultats les plus significatifs de tout cet article. Précisément, une étape essentielle de cette démonstration, donnée dans la proposition 16.2.6, consiste à exhiber un sous-foncteur idoine $C^{\prime}$ d'un foncteur donné $C$ dans une catégorie de foncteurs en grassmanniennes, vue comme catégorie de comodules convenable de $\mathcal{F}$. Ce sous-foncteur $C^{\prime}$ est construit comme colimite d'une certaine suite de sous-foncteurs de $C$ dans $\mathcal{F}$ (ils n'ont pas de structure de comodule); on démontre ensuite que cette colimite $C^{\prime}$ est un sous-comodule de $C$.

Venons-on au contenu proprement dit du présent chapitre. On y expose des résultats formels généralisant, en termes de catégories de foncteurs, l'observation suivante. Soit $X$ un ensemble, munissons l'espace vectoriel $\mathbb{k}[X]$ de la structure de coalgèbre donnée par la diagonale $[x] \mapsto[x] \otimes[x]$ et la coünité $[x] \mapsto 1$. La catégorie des $\mathbb{k}$-espaces vectoriels $X$-gradués est naturellement équivalente à la sous-catégorie $\operatorname{Comod}_{\mathbb{k}[X]}$ des $\mathbb{k}[X]$-comodules de $\mathcal{E}_{\mathbb{k}}$. Une coalgèbre du type $\mathbb{k}[X]$ est appelée coalgèbre de Boole, car, lorsque $\mathbb{k}=\mathbb{F}_{2}$, la structure de coalgèbre sur $\mathbb{F}_{2}[X]$ est duale de la structure d'algèbre de Boole de $\mathbb{F}_{2}{ }^{X}$.

L'étude de catégories de modules ou de comodules sur des objets présentant une structure de type booléen dans un contexte proche des catégories de foncteurs remonte aux travaux des années 90 sur les modules instables sur l'algèbre de Steenrod.

Dans la deuxième partie de l'article [HLS93] (théorème 1.5), Henn, Lannes et Schwartz établissent un lien fondamental entre les foncteurs en algèbres de Boole de 
la catégorie $\mathcal{F}\left(\mathbb{F}_{p}\right)$ et les algèbres instables sur l'algèbre de Steenrod modulo $p$ (voir aussi [Lan92]). Ils utilisent dans [HLS95] des structures de module sur ces algèbres pour étudier la cohomologie équivariante modulo $p$. Des considérations analogues apparaissent dans les travaux de Lannes et Zarati (cf. [LZ95]).

Les idées sous-jacentes relatives aux algèbres instables booléennes présentes dans les articles [HLS93] et [LZ95] sont dues à J. Lannes, qui les a inaugurées dans l'appendice de [LZ87] (théorème A.2.2, connu sous le nom de principe de linéarisation).

Convention 3.0.1. - Dans toute ce chapitre, on se donne une catégorie essentiellement petite $\mathcal{I}$.

Remarque 3.0.2. - L'hypothèse de finitude du corps $\mathbb{k}$ n'interviendra pas dans ce chapitre.

\subsection{La catégorie de comodules $\operatorname{Fct}\left(\mathcal{I}_{\backslash X}, \mathcal{E}_{\mathbb{k}}\right)$}

Cette section a pour but d'identifier les catégories de comodules sur un foncteur obtenu par linéarisation d'un foncteur ensembliste à des catégories de foncteurs convenables.

Convention 3.1.1. - Dans cette section, $X$ désigne un foncteur de $\mathcal{I}$ vers Ens.

Notation 3.1.2. - On définit une catégorie $\mathcal{I}_{\backslash X}$ de la manière suivante.

- Les objets de $\mathcal{I}_{\backslash X}$ sont les couples $(E, x)$, où $E$ est un objet de $\mathcal{I}$ et $x$ un élément de $X(E)$.

- Les morphismes dans $\mathcal{I}_{\backslash X}$ sont donnés par

$$
\operatorname{hom}_{\mathcal{I}_{\backslash X}}((E, x),(F, y))=\left\{u \in \operatorname{hom}_{\mathcal{I}}(E, F) \mid X(u)(x)=y\right\} .
$$

- La composition des morphismes dans $\mathcal{I}_{\backslash X}$ est induite par celle de $\mathcal{I}$.

Le foncteur d'oubli $\mathcal{I}_{\backslash X} \rightarrow \mathcal{I}:(E, x) \mapsto E$ sera noté $\mathcal{O}_{\mathcal{I}, X}$.

Remarque 3.1.3. - Il s'agit d'un cas particulier de la construction catégorique classique étudiée dans [ML71], chapitre II, $\S 6$.

Notation 3.1.4. - Nous désignerons par $\Upsilon_{X}: \operatorname{Fct}\left(\mathcal{I}, \mathcal{E}_{\mathbb{k}}\right) \rightarrow \operatorname{Fct}\left(\mathcal{I}_{\backslash X}, \mathcal{E}_{\mathbb{k}}\right)$ le foncteur de précomposition par $\mathcal{O}_{\mathcal{I}, X}$.

Ainsi, $\Upsilon_{X}$ est un foncteur exact qui commute aux limites, aux colimites et au produit tensoriel (cf. proposition C.1.4). Dans le cas où $X$ prend ses valeurs dans les ensembles non vides, $\mathcal{O}_{\mathcal{I}, X}$ est essentiellement surjectif, donc $\Upsilon_{X}$ est fidèle.

Proposition et définition 3.1.5. - Il existe un foncteur exact et fidèle, appelé foncteur de $X$-intégrale et noté $\Omega_{X}: \mathbf{F} \operatorname{ct}\left(\mathcal{I}_{\backslash X}, \mathcal{E}_{\mathbb{k}}\right) \rightarrow \mathbf{F} \operatorname{ct}\left(\mathcal{I}, \mathcal{E}_{\mathbb{k}}\right)$, défini de la façon suivante. 
(1) Si F est un objet de $\boldsymbol{F} \operatorname{ct}\left(\mathcal{I}_{\backslash X}, \mathcal{E}_{\mathbb{k}}\right)$ et $E$ un objet de $\mathcal{I}$, on pose

$$
\left(\Omega_{X}(F)\right)(E)=\bigoplus_{x \in X(E)} F(E, x)
$$

(2) Si F est un objet de $\boldsymbol{F} \mathbf{c t}\left(\mathcal{I}_{\backslash X}, \mathcal{E}_{\mathbb{k}}\right)$ et $E \stackrel{f}{\rightarrow} E^{\prime}$ une flèche de $\mathcal{I}$, et si $x$ et $x^{\prime}$ sont des éléments respectifs de $X(E)$ et $X\left(E^{\prime}\right)$, la composante $F(E, x) \rightarrow F\left(E^{\prime}, x^{\prime}\right)$ de $\left(\Omega_{X}(F)\right)(f)$ est égale à $F(f)$ si $X(f)(x)=x^{\prime}$, et à 0 sinon.

(3) Si $F \stackrel{u}{\rightarrow} G$ est une flèche de $\operatorname{Fct}\left(\mathcal{I}_{\backslash X}, \mathcal{E}_{\mathbb{k}}\right)$, le morphisme $\Omega_{X}(u): \Omega_{X}(F) \rightarrow$ $\Omega_{X}(G)$ de $\boldsymbol{F} \operatorname{ct}\left(\mathcal{I}, \mathcal{E}_{\mathbb{k}}\right)$ est défini, sur l'objet $E$ de $\mathcal{I}$, comme la somme directe sur les éléments $x$ de $X(E)$ des morphismes $u(E, x): F(E, x) \rightarrow G(E, x)$.

Exemple 3.1.6. - On a $\Omega_{X}(\mathbb{k})=\mathbb{k}[X]$ (linéarisation du foncteur $X$ ) dans $\operatorname{Fct}\left(\mathcal{I}, \mathcal{E}_{\mathbb{k}}\right)$, où le foncteur $\mathbb{k} \in \operatorname{Ob} \mathbf{F} \operatorname{ct}\left(\mathcal{I}_{\backslash X}, \mathcal{E}_{\mathbb{k}}\right)$ du membre de gauche est le foncteur constant.

Proposition 3.1.7. - Le foncteur $\Omega_{X}$ est adjoint à gauche $\grave{a} \Upsilon_{X}$.

Cette proposition se vérifie de façon similaire à l'adjonction entre les foncteurs $o$ et $\varpi$ dans $\mathcal{F}_{\text {surj }}$.

Proposition 3.1.8. - (1) L'endofoncteur $\Omega_{X} \Upsilon_{X}$ de $\mathbf{F c t}\left(\mathcal{I}, \mathcal{E}_{\mathbb{k}}\right)$ est isomorphe à $\cdot \otimes \mathbb{k}[X]$. Plus généralement, on a un isomorphisme

$$
\Omega_{X}\left(A \otimes \Upsilon_{X}(F)\right) \simeq \Omega_{X}(A) \otimes F
$$

naturel en les objets $A$ de $\boldsymbol{F} \mathbf{c t}\left(\mathcal{I}_{\backslash X}, \mathcal{E}_{\mathbb{k}}\right)$ et $F \operatorname{de~} \boldsymbol{F} \mathbf{c t}\left(\mathcal{I}, \mathcal{E}_{\mathbb{k}}\right)$.

(2) La coünité $\Omega_{X} \Upsilon_{X} \rightarrow$ id de l'adjonction de la proposition 3.1 .7 s'identifie au produit tensoriel par le morphisme d'augmentation $\mathbb{k}[X] \rightarrow \mathbb{k}$ obtenu par linéarisation de l'unique transformation naturelle $X \rightarrow *$.

Proposition 3.1.9. - Les injections diagonales

$$
\bigoplus_{x \in X(E)} F(E, x) \otimes G(E, x) \hookrightarrow \bigoplus_{x, y \in X(E)} F(E, x) \otimes G(E, y)
$$

fournissent un monomorphisme $\Omega_{X}(F \otimes G) \hookrightarrow \Omega_{X}(F) \otimes \Omega_{X}(G)$ naturel en les objets $F$ et $G$ de $\operatorname{Fct}\left(\mathcal{I}_{\backslash X}, \mathcal{E}_{\mathbb{k}}\right)$.

Cette proposition se vérifie par inspection. Appliquée aux isomorphismes canoniques $F \stackrel{\simeq}{\longrightarrow} F \otimes \mathbb{k} \operatorname{de} \operatorname{Fct}\left(\mathcal{I}_{\backslash X}, \mathcal{E}_{\mathbb{k}}\right)$, elle donne le corollaire suivant.

Corollaire 3.1.10. - (1) Le foncteur $\mathbb{k}[X]=\Omega_{X}(\mathbb{k})$ est canoniquement une coalgèbre cocommutative dans la catégorie monoïdale symétrique $\mathbf{F} \mathbf{c t}\left(\mathcal{I}, \mathcal{E}_{\mathbb{k}}\right)$. 
(2) Pour tout objet $F$ de $\boldsymbol{F} \operatorname{ct}\left(\mathcal{I}_{\backslash X}, \mathcal{E}_{\mathbb{k}}\right), \Omega_{X}(F)$ est naturellement un $\mathbb{k}[X]$ comodule. Autrement dit, on peut compléter le diagramme suivant.

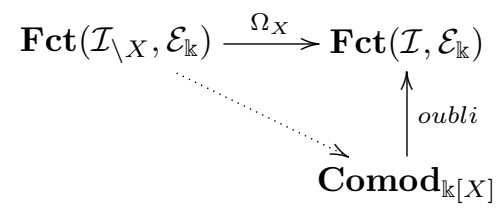

Remarque 3.1.11. - La structure de coalgèbre sur $\mathbb{k}[X]$ généralise celle de l'algèbre d'un groupe, puisque sa diagonale est donnée par les applications linéaires $[x] \mapsto$ $[x] \otimes[x]$.

Proposition 3.1.12. - Le foncteur $\Omega_{X}$ induit une équivalence de catégories entre $\operatorname{Fct}\left(\mathcal{I}_{\backslash X}, \mathcal{E}_{\mathbb{k}}\right)$ et $\operatorname{Comod}_{\mathbb{k}[X]}$.

Démonstration. - On applique la proposition A.2.6, de sorte qu'il suffit de constater qu'un $\mathbb{k}[X]$-comodule est un comodule sur la comonade déterminée par l'adjonction entre $\Omega_{X}$ et $\Upsilon_{X}$. Cela découle de la proposition 3.1.8 et du corollaire 3.1.10.

Remarque 3.1.13. - (1) Dans l'équivalence de catégories de la proposition, le produit tensoriel de $\boldsymbol{F} \boldsymbol{c t}\left(\mathcal{I}_{\backslash X}, \mathcal{E}_{\mathbb{k}}\right)$ correspond au produit cotensoriel de $\mathbb{k}[X]$ comodules.

(2) L'image du foncteur $\Upsilon_{X}$ correspond aux comodules libres.

Remarque 3.1.14. - Les considérations de cette section sont fonctorielles en $X$ en le sens suivant. Toute transformation naturelle de foncteurs ensemblistes $X \rightarrow X^{\prime}$ induit un foncteur $\mathcal{I}_{\backslash X} \rightarrow \mathcal{I}_{\backslash X^{\prime}}$, donc par précomposition un foncteur $i: \operatorname{Fct}\left(\mathcal{I}_{\backslash X^{\prime}}, \mathcal{E}_{\mathbb{k}}\right) \rightarrow \boldsymbol{F} \boldsymbol{c t}\left(\mathcal{I}_{\backslash X}, \mathcal{E}_{\mathbb{k}}\right)$ (qui généralise $\Upsilon_{X}$ ). Via l'équivalence de catégories de la proposition 3.1.12, ce foncteur correspond à la coïnduction : pour tous objets $F$ de $\boldsymbol{F} \operatorname{ct}\left(\mathcal{I}_{\backslash X^{\prime}}, \mathcal{E}_{\mathbb{k}}\right)$ et $E$ de $\mathcal{I}$, le $\mathbb{k}[X(E)]$-comodule $\Omega_{X}(i(F))(E)$ est le produit cotensoriel du $\mathbb{k}\left[X^{\prime}(E)\right]$-comodule $\Omega_{X^{\prime}}(F)(E)$ et de $\mathbb{k}[X(E)]$ (vu comme un $\mathbb{k}\left[X^{\prime}(E)\right]$-comodule par le morphisme de coalgèbres $\mathbb{k}[X(E)] \rightarrow \mathbb{k}\left[X^{\prime}(E)\right]$ déduit de la transformation naturelle $X \rightarrow X^{\prime}$ ).

\subsection{Recollements de catégories de comodules}

Cette section traite d'une variante non coünitaire des considérations précédentes, que l'on étudie commodément à partir d'un diagramme de recollement (proposition 3.2.3).

Convention 3.2.1. - Dans cette section, $X$ désigne un foncteur de $\mathcal{I}$ vers la catégorie Ens et $Y$ un sous-foncteur de $X$.

Nous désignerons par $\mathcal{I}_{X, Y}$ la sous-catégorie pleine de $\mathcal{I}_{\backslash X}$ dont les objets sont les couples $(E, x)$ pour lesquels $x \in X(E) \backslash Y(E)$. 
On prendra garde que $X \backslash Y$ n'est pas en général un foncteur ensembliste. Nous noterons cependant, par abus, $\mathbb{k}[X \backslash Y]$ le conoyau du monomorphisme $\mathbb{k}[Y] \hookrightarrow \mathbb{k}[X]$ $\operatorname{de} \boldsymbol{F} \operatorname{ct}\left(\mathcal{I}, E_{\mathbb{k}}\right)$ induit par l'inclusion $Y \hookrightarrow X$.

On a un scindement canonique

$$
\mathbb{k}[X / Y] \simeq \mathbb{k}[X \backslash Y] \oplus \mathbb{k}
$$

obtenu en constatant que le foncteur ensembliste $X / Y$ se relève canoniquement en un foncteur vers les ensembles pointés (on convient que $E / \varnothing=E \amalg\{*\}$ ).

Comme l'épimorphisme $\mathbb{k}[X / Y] \rightarrow \mathbb{k}$ est la coünité du foncteur en coalgèbres $\mathbb{k}[X / Y]$, le foncteur $\mathbb{k}[X \backslash Y]$ est une coalgèbre sans coünité dans $\mathbf{F} \operatorname{ct}\left(\mathcal{I}, \mathcal{E}_{\mathbb{k}}\right)$; nous y reviendrons à la proposition 3.2.8.

Dans le lemme suivant, nous nommons sous-catégorie complémentaire d'une souscatégorie pleine $\mathcal{B}$ d'une catégorie $\mathcal{A}$ la sous-catégorie pleine de $\mathcal{A}$ dont la classe d'objets est le complémentaire de celle de $\mathcal{B}$.

Lemme 3.2.2. - La catégorie $\mathcal{I}_{\backslash Y}$ s'identifie canoniquement à une sous-catégorie pleine complète à droite de $\mathcal{I}_{\backslash X}$, dont la catégorie complémentaire est $\mathcal{I}_{X, Y}$.

Démonstration. - Il suffit de remarquer que, si $f:(E, x) \rightarrow\left(E^{\prime}, x^{\prime}\right)$ est un morphisme de $\mathcal{I}_{\backslash X}$, où $x \in Y(E)$, alors on a $x^{\prime}=X(f)(x)=Y(f)(x) \in Y\left(E^{\prime}\right)$ puisque $Y$ est un sous-foncteur de $X$.

Appliquant le corollaire C.6.3, on en déduit le résultat suivant.

Proposition 3.2.3. - Il existe un diagramme de recollement

$$
\operatorname{Fct}\left(\mathcal{I}_{\backslash Y}, \mathcal{E}_{\mathbb{k}}\right) \underset{\mathcal{R}}{\longleftarrow} \underset{\mathcal{P} \rightarrow}{\leftarrow} \operatorname{Fct}\left(\mathcal{I}_{\backslash X}, \mathcal{E}_{\mathbb{k}}\right) \underset{\mathcal{P}}{\underset{\mathcal{R}}{\leftarrow}} \operatorname{Fct}\left(\mathcal{I}_{X, Y}, \mathcal{E}_{\mathbb{k}}\right)
$$

où $\mathcal{P}$ désigne le prolongement par zéro et $\mathcal{R}$ la restriction.

Nous adaptons maintenant certains résultats de la section précédente à la catégorie de foncteurs $\boldsymbol{F} \operatorname{ct}\left(\mathcal{I}_{X, Y}, \mathcal{E}_{\mathbb{k}}\right)$.

Notation 3.2.4. - Nous noterons $\Upsilon_{X, Y}$ le foncteur composé

$$
\operatorname{Fct}\left(\mathcal{I}, \mathcal{E}_{\mathbb{k}}\right) \stackrel{\Upsilon_{X}}{\longrightarrow} \operatorname{Fct}\left(\mathcal{I}_{\backslash X}, \mathcal{E}_{\mathbb{k}}\right) \stackrel{\mathcal{R}}{\longrightarrow} \operatorname{Fct}\left(\mathcal{I}_{X, Y}, \mathcal{E}_{\mathbb{k}}\right) .
$$

Autrement dit, $\Upsilon_{X, Y}$ est le foncteur de précomposition par le foncteur d'oubli $\mathcal{I}_{X, Y} \rightarrow \mathcal{I}$.

Définition 3.2.5. - Le foncteur de $X \backslash Y$-intégrale, noté $\Omega_{X, Y}$, est défini comme la composée

$$
\Omega_{X, Y}: \operatorname{Fct}\left(\mathcal{I}_{X, Y}, \mathcal{E}_{\mathbb{k}}\right) \stackrel{\mathcal{P}}{\longrightarrow} \operatorname{Fct}\left(\mathcal{I}_{\backslash X}, \mathcal{E}_{\mathbb{k}}\right) \stackrel{\Omega_{X}}{\longrightarrow} \operatorname{Fct}\left(\mathcal{I}, \mathcal{E}_{\mathbb{k}}\right)
$$


On a donc $\Omega_{X, Y}(F)(E)=\bigoplus_{x \in X(E) \backslash Y(E)} F(E, x)$ pour $F \in \operatorname{Ob} \mathbf{F c t}\left(\mathcal{I}_{X, Y}, \mathcal{E}_{\mathbb{k}}\right)$ et $E \in \mathrm{Ob} \mathcal{I}$. On prendra garde que les foncteurs $\Upsilon_{X, Y}$ et $\Omega_{X, Y}$ ne sont généralement pas adjoints.

Remarque 3.2.6. - (1) On a un isomorphisme canonique $\Omega_{X, Y}(\mathbb{k}) \simeq \mathbb{k}[X \backslash Y]$.

(2) Le foncteur $\Omega_{X, Y}$ est exact et fidèle.

La proposition 3.1.8 entraîne le résultat suivant, puisque les foncteurs de restriction et de prolongement par zéro commutent canoniquement au produit tensoriel.

Proposition 3.2.7. - L'endofoncteur $\Omega_{X, Y} \Upsilon_{X, Y}$ de $\mathbf{F c t}\left(\mathcal{I}, \mathcal{E}_{\mathbb{k}}\right)$ est isomorphe $\grave{a} \cdot \otimes$ $\mathbb{k}[X \backslash Y]$. Plus généralement, on a un isomorphisme

$$
\Omega_{X, Y}\left(A \otimes \Upsilon_{X, Y}(F)\right) \simeq \Omega_{X, Y}(A) \otimes F
$$

naturel en les objets $A$ de $\boldsymbol{F} \operatorname{ct}\left(\mathcal{I}_{X, Y}, \mathcal{E}_{\mathbb{k}}\right)$ et $F \operatorname{de} \boldsymbol{\operatorname { F c t }}\left(\mathcal{I}, \mathcal{E}_{\mathbb{k}}\right)$.

De même, la proposition 3.1.9 et le corollaire 3.1.10 procurent le résultat suivant.

Proposition 3.2.8. - (1) Les injections diagonales fournissent un monomorphisme $\Omega_{X, Y}(F \otimes G) \hookrightarrow \Omega_{X, Y}(F) \otimes \Omega_{X, Y}(G)$ naturel en les objets $F$ et $G$ de $\operatorname{Fct}\left(\mathcal{I}_{X, Y}, \mathcal{E}_{\mathbb{k}}\right)$. Celui-ci est compatible aux isomorphismes d'associativité et de commutativité du produit tensoriel.

(2) Le foncteur $\mathbb{k}[X \backslash Y]$ est canoniquement une coalgèbre cocommutative sans coünité dans la catégorie monö̈dale symétrique $\mathbf{F} \mathbf{c t}\left(\mathcal{I}, \mathcal{E}_{\mathbb{k}}\right)$; le morphisme canonique $\mathbb{k}[X] \rightarrow \mathbb{k}[X \backslash Y]$ est un morphisme de coalgèbres sans coünité.

(3) Pour tout objet $F$ de $\operatorname{Fct}\left(\mathcal{I}_{X, Y}, \mathcal{E}_{\mathbb{k}}\right), \Omega_{X, Y}(F)$ est naturellement un $\mathbb{k}[X \backslash Y]$ comodule.

Remarque 3.2.9. - Le scindement (5) (page 37) permet d'identifier les catégories de comodules sur $\mathbb{k}[X \backslash Y]$ et $\mathbb{k}[X / Y]$. On rappelle que les comodules sur une coalgèbre coünitaire, comme $\mathbb{k}[X / Y]$, sont supposés compatibles à la coünité.

Proposition 3.2.10. - Le foncteur $\Omega_{X, Y}$ induit une équivalence de catégories entre $\boldsymbol{F} \operatorname{ct}\left(\mathcal{I}_{X, Y}, \mathcal{E}_{\mathbb{k}}\right)$ et la sous-catégorie pleine de $\operatorname{Comod}_{\mathbb{k}[X \backslash Y]}$ formée des $\mathbb{k}[X \backslash Y]$ comodules à droite $\left(C, \psi_{C}\right)$ (que nous nommerons fidèles) tels que la comultiplication $\psi_{C}$ est injective, notée $\operatorname{Comod}_{\mathbb{k}[X \backslash Y]}^{\text {fid }}$.

Démonstration. - On utilise l'identification de la remarque précédente. Le foncteur de prolongement par zéro et la proposition 3.1.12 identifient alors $\operatorname{Fct}\left(\mathcal{I}_{X, Y}, \mathcal{E}_{\mathbb{k}}\right)$ à la sous-catégorie pleine de $\operatorname{Comod}_{\mathbb{k}[X \backslash Y]}$ des comodules $\left(C, \psi_{C}\right)$ tels que le produit fibré de l'inclusion $C \hookrightarrow C \oplus(C \otimes \mathbb{k}[X \backslash Y])$ et du morphisme $\left(i d_{C}, \psi_{C}\right): C \rightarrow$ $C \oplus(C \otimes \mathbb{k}[X \backslash Y])$ est nul. Ce produit fibré étant égal à ker $\psi_{C}$, cette condition équivaut à la fidélité de $\psi_{C}$, ce qui établit la proposition. 


\subsection{La catégorie de modules $\operatorname{Fct}\left(\mathcal{I}_{/ X}, \mathcal{E}_{\mathrm{k}}\right)$}

Nous présentons maintenant la situation duale de celle de la section 3.1. Toutes les propriétés énoncées se démontrent de façon analogue à celles de ladite section, c'est pourquoi nous les laissons au lecteur.

Convention 3.3.1. - Dans cette section, $X$ désigne un foncteur de la catégorie $\mathcal{I}^{o p}$ vers la catégorie $\mathbf{E n s}^{f}$ des ensembles finis.

Nous expliquerons en fin de section l'utilité de cette restriction aux ensembles finis.

Notation 3.3.2. - Nous noterons $\mathcal{I}_{/ X}$ la catégorie $\left(\left(\mathcal{I}^{o p}\right)_{\backslash X}\right)^{o p}$. Le foncteur d'oubli $\mathcal{I}_{/ X} \rightarrow \mathcal{I}$ sera noté $\mathcal{O}^{\mathcal{I}, X}$.

Explicitement, les objets de $\mathcal{I}_{/ X}$ sont les couples $(E, x)$ formés d'un objet $E$ de $\mathcal{I}$ et d'un élément $x$ de $X(E)$. On a $\mathcal{O}^{\mathcal{I}, X}(E, x)=E$ sur les objets.

Notation 3.3.3. - Nous désignerons par $\Upsilon^{X}: \operatorname{Fct}\left(\mathcal{I}, \mathcal{E}_{\mathrm{k}}\right) \rightarrow \operatorname{Fct}\left(\mathcal{I}_{/ X}, \mathcal{E}_{\mathrm{k}}\right)$ le foncteur de précomposition par $\mathcal{O}^{\mathcal{I}, X}$.

Proposition et définition 3.3.4. - Il existe un foncteur exact et fidèle $\Omega^{X}$ : $\boldsymbol{F} \operatorname{ct}\left(\mathcal{I}_{/ X}, \mathcal{E}_{\mathrm{k}}\right) \rightarrow \operatorname{Fct}\left(\mathcal{I}, \mathcal{E}_{\mathrm{k}}\right)$, appelé foncteur de $X$-intégrale, défini de la façon suivante.

(1) Si F est un objet de $\operatorname{Fct}\left(\mathcal{I}_{/ X}, \mathcal{E}_{\mathrm{k}}\right)$ et $E$ un objet de $\mathcal{I}$, on pose

$$
\left(\Omega^{X}(F)\right)(E)=\bigoplus_{x \in X(E)} F(E, x) .
$$

(2) Si $F$ est un objet de $\operatorname{Fct}\left(\mathcal{I}_{/ X}, \mathcal{E}_{\mathbb{k}}\right)$ et $E \stackrel{f}{\rightarrow} E^{\prime}$ une flèche de $\mathcal{I}$, et si $x$ et $x^{\prime}$ sont des éléments respectifs de $X(E)$ et $X\left(E^{\prime}\right)$, la composante $F(E, x) \rightarrow F\left(E^{\prime}, x^{\prime}\right)$ de $\left(\Omega^{X}(F)\right)(f)$ est égale à $F(f)$ si $X(f)\left(x^{\prime}\right)=x$, et à 0 sinon.

(3) Si $F \stackrel{u}{\rightarrow} G$ est une flèche de $\mathbf{F} \operatorname{ct}\left(\mathcal{I}_{/ X}, \mathcal{E}_{\mathbb{k}_{k}}\right)$, le morphisme $\Omega^{X}(u): \Omega^{X}(F) \rightarrow$ $\Omega^{X}(G)$ de $\operatorname{Fct}\left(\mathcal{I}, \mathcal{E}_{\mathrm{k}}\right)$ est défini, sur l'objet $E$ de $\mathcal{I}$, comme la somme directe sur les éléments $x$ de $X(E)$ des morphismes $u(E, x): F(E, x) \rightarrow G(E, x)$.

Exemple 3.3.5. - On a $\Omega^{X}(\mathbb{k})=\mathbb{k}^{X}$.

Proposition 3.3.6. - Le foncteur $\Omega^{X}$ est adjoint à droite à $\Upsilon^{X}$.

Proposition 3.3.7. - (1) L'endofoncteur $\Omega^{X} \Upsilon^{X} \operatorname{de} \mathbf{F c t}\left(\mathcal{I}, \mathcal{E}_{\mathbb{k}}\right)$ est isomorphe $\grave{a}$ $\cdot \otimes \mathbb{k}^{X}$. Plus généralement, on a un isomorphisme

$$
\Omega^{X}\left(A \otimes \Upsilon^{X}(F)\right) \simeq \Omega^{X}(A) \otimes F
$$

naturel en les objets $A \operatorname{de} \boldsymbol{\operatorname { F c t }}\left(\mathcal{I}_{/ X}, \mathcal{E}_{\mathrm{k}}\right)$ et $F \operatorname{de} \mathbf{F} \mathbf{c t}\left(\mathcal{I}, \mathcal{E}_{\mathbb{k}}\right)$.

(2) L'unité id $\rightarrow \Omega^{X} \Upsilon^{X}$ de l'adjonction de la proposition 3.3 .6 s'identifie au produit tensoriel par le morphisme $\mathbb{k} \rightarrow \mathbb{k}^{X}$ obtenu en appliquant cette unité au foncteur constant $\mathbb{k}$. 
Proposition 3.3.8. - Les projections canoniques

$$
\bigoplus_{x, y \in X(E)} F(E, x) \otimes G(E, y) \rightarrow \bigoplus_{x \in X(E)} F(E, x) \otimes G(E, x)
$$

fournissent un épimorphisme $\Omega^{X}(F) \otimes \Omega^{X}(G) \rightarrow \Omega^{X}(F \otimes G)$ naturel en les objets $F$ et $G$ de $\mathbf{F} \operatorname{ct}\left(\mathcal{I}_{/ X}, \mathcal{E}_{\mathbb{k}}\right)$.

Cette proposition, appliquée aux isomorphismes canoniques $F \otimes \mathbb{k} \stackrel{\simeq}{\longrightarrow} F$ de $\operatorname{Fct}\left(\mathcal{I}_{/ X}, \mathcal{E}_{\mathbb{k}}\right)$, procure le corollaire suivant.

Corollaire 3.3.9. - (1) Le foncteur $\mathbb{k}^{X}=\Omega^{X}(\mathbb{k})$ est canoniquement une algèbre commutative dans la catégorie monoïdale symétrique $\mathbf{F} \mathbf{c t}\left(\mathcal{I}, \mathcal{E}_{\mathbb{k}}\right)$.

(2) Pour tout objet $F$ de $\operatorname{Fct}\left(\mathcal{I}_{/ X}, \mathcal{E}_{\mathbb{k}}\right), \Omega^{X}(F)$ est naturellement un $\mathbb{k}^{X}$-module. Autrement dit, on peut compléter le diagramme suivant.

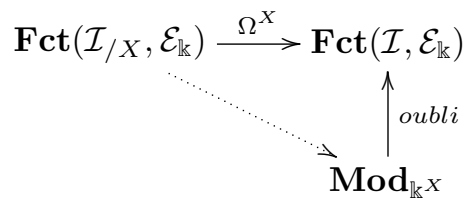

Proposition 3.3.10. - Le foncteur $\Omega^{X}$ induit une équivalence de catégories entre $\operatorname{Fct}\left(\mathcal{I}_{/ X}, \mathcal{E}_{\mathbb{k}}\right)$ et $\operatorname{Mod}_{\mathbb{k}} X$.

Remarque 3.3.11. - L'hypothèse de finitude des ensembles $X(E)$ permet d'assurer que, dans la définition de $\Omega^{X}$, on peut remplacer la somme directe par un produit, ce qui est nécessaire pour établir la proposition 3.3.6. Il est obligatoire de considérer une somme directe pour disposer des propriétés de commutation avec le produit tensoriel. 


\section{CHAPITRE 4}

\section{LES CATÉGORIES $\mathcal{E}_{\mathcal{G} r, I}^{f}, \widetilde{\mathcal{E}}_{\mathcal{G} r}^{f}$ ET $\mathcal{E}_{\mathrm{Pl}, n}^{f}$}

Dans ce chapitre, nous introduisons les catégories sources des catégories de foncteurs en grassmanniennes. Ces catégories sources, notées $\mathcal{E}_{\mathcal{G} r, I}^{f}(\mathbb{k}), \widetilde{\mathcal{E}}_{\mathcal{G} r}^{f}(\mathbb{k})$ et $\mathcal{E}_{\mathbf{P l}, n}^{f}(\mathbb{k})$, possèdent une riche structure : de nombreux foncteurs, avec des propriétés d'adjonction et de composition, apparaissent très naturellement. Toutes les propriétés s'établissent de façon directe, c'est pourquoi nous ne démontrons que certaines d'entre elles.

Une partie des définitions et des résultats de ce chapitre constituent des illustrations des constructions catégoriques utilisées dans le chapitre 3.

Convention 4.0.1. - Dans toute ce chapitre, $I$ désigne une partie de $\mathbb{N}$ et $n$ un entier naturel.

\subsection{Définition des catégories et foncteurs utilisés}

On rappelle que $\mathcal{G} r(V)$ désigne la grassmannienne des sous-espaces d'un sous-espace vectoriel $V$. Dans la suite, nous regarderons $\mathcal{G} r$ comme un foncteur $\mathcal{E}_{\mathbb{k}}^{f} \rightarrow$ Ens, en définissant $\mathcal{G} r(f): \mathcal{G} r(V) \rightarrow \mathcal{G} r\left(V^{\prime}\right)$, où $f: V \rightarrow V^{\prime}$ est un morphisme de $\mathcal{E}^{f}$, comme étant la fonction $W \mapsto f(W)$.

On rappelle également que, si $I$ est une partie de $\mathbb{N}, \mathcal{G} r_{I}(V)$ désigne le sous-ensemble de $\mathcal{G} r(V)$ formé des sous-espaces de $V$ dont la dimension appartient à $I$.

Remarque 4.1.1. - On notera que $\mathcal{G} r_{I}$ est un sous-foncteur de $\mathcal{G} r$ si et seulement si $I$ est de la forme $\leq n$ ou $\mathbb{N}$.

Définition 4.1.2 (Catégories $\mathcal{E}_{\mathcal{G} r, I}^{f}(\mathbb{k})$ et $\left.\widetilde{\mathcal{E}}_{\mathcal{G} r, I}^{f}(\mathbb{k})\right)$ - — $\quad(1)$ Nous désignerons $\operatorname{par} \widetilde{\mathcal{E}}_{\mathcal{G} r, I}^{f}(\mathbb{k})$ la catégorie donnée comme suit.

- Les objets de $\widetilde{\mathcal{E}}_{\mathcal{G} r, I}^{f}(\mathbb{k})$ sont les couples $(V, W)$, où $V$ est un $\mathbb{k}$-espace vectoriel de dimension finie et $W$ un élément de $\mathcal{G} r_{I}(V)$. 
- Les morphismes de $(V, W)$ vers $\left(V^{\prime}, W^{\prime}\right)$ dans $\widetilde{\mathcal{E}}_{\mathcal{G} r, I}^{f}(\mathbb{k})$ sont les applications linéaires $f: V \rightarrow V^{\prime}$ telles que $f(W) \subset W^{\prime}$.

- La composition des morphismes s'obtient par composition des applications linéaires sous-jacentes.

(2) Nous désignerons par $\mathcal{E}_{\mathcal{G} r, I}^{f}(\mathbb{k})$ la sous-catégorie de $\widetilde{\mathcal{E}}_{\mathcal{G} r, I}^{f}(\mathbb{k})$ ayant les mêmes objets et dont les morphismes sont donnés par

$$
\operatorname{hom}_{\mathcal{E}_{\mathcal{G} r, I}^{f}(\mathbb{k})}\left((V, W),\left(V^{\prime}, W^{\prime}\right)\right)=\left\{f \in \operatorname{hom}_{\widetilde{\mathcal{E}}_{\mathcal{G} r, I}^{f}(\mathbb{k})}\left((V, W),\left(V^{\prime}, W^{\prime}\right)\right) \mid f(W)=W^{\prime}\right\} .
$$

(3) Nous noterons $\widetilde{\text { incl }}_{I}$ le foncteur (fidèle et essentiellement surjectif) d'inclusion de $\mathcal{E}_{\mathcal{G} r, I}^{f}(\mathbb{k})$ dans $\widetilde{\mathcal{E}}_{\mathcal{G} r, I}^{f}(\mathbb{k})$.

La mention du corps $\mathbb{k}$ sera souvent omise.

De plus, lorsque la partie $I$ est égale à $\mathbb{N}$, nous omettrons l'indice $I$ dans la notation de ces catégories et des foncteurs où elles interviennent.

Par exemple, nous noterons $\widetilde{i n c l}: \mathcal{E}_{\mathcal{G} r}^{f} \rightarrow \widetilde{\mathcal{E}}_{\mathcal{G} r}^{f}$ pour $\widetilde{\text { incl }} \mathbb{N}: \mathcal{E}_{\mathcal{G} r, \mathbb{N}}^{f}(\mathbb{k}) \rightarrow \widetilde{\mathcal{E}}_{\mathcal{G} r, \mathbb{N}}^{f}(\mathbb{k})$.

Dans la suite, nous ne considérerons la catégorie $\widetilde{\mathcal{E}}_{\mathcal{G} r, I}^{f}(\mathbb{k})$ que lorsque $I=\mathbb{N}$. Nous ne donnerons donc la plupart des définitions et des propriétés des foncteurs où cette catégorie intervient que dans ce cadre, mais beaucoup d'entre elles se généralisent sans difficulté au cas de $\widetilde{\mathcal{E}}_{\mathcal{G} r, I}^{f}(\mathbb{k})$ pour $I \subset \mathbb{N}$ quelconque.

Notation 4.1.3 (Catégorie $\mathcal{E}_{\mathrm{Pl}, n}^{f}(\mathbb{k})$ ). - $\quad$ (1) Étant donné un objet $V$ de $\mathcal{E}_{\mathrm{k}}^{f}$, nous noterons $\mathbf{P l}_{n}(V)$ l'ensemble $\mathrm{Pl}_{\mathcal{E}_{\mathrm{k}}^{f}}\left(E_{n}, V\right)$ des monomorphismes $E_{n} \hookrightarrow V$.

(2) Nous désignerons par $\mathcal{E}_{\mathbf{P l}, n}^{f}(\mathbb{k})$ la catégorie définie ainsi.

- Les objets de $\mathcal{E}_{\mathbf{P l}, n}^{f}(\mathbb{k})$ sont les couples $(V, u)$ formés d'un objet $V$ de $\mathcal{E}_{\mathrm{k}}^{f}$ et d'un élément $u$ de $\mathbf{P l}_{n}(V)$.

- Les morphismes dans $\mathcal{E}_{\mathbf{P l}, n}^{f}(\mathbb{k})$ de $(V, u)$ vers $\left(V^{\prime}, u^{\prime}\right)$ sont les applications linéaires $f: V \rightarrow V^{\prime}$ faisant commuter le diagramme

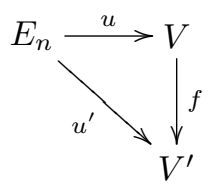

- La composition des morphismes est induite par la composition des applications linéaires.

Remarque 4.1.4. - (1) Si $I$ est une partie du type $\leq n$ ou $\mathbb{N}$, alors $\mathcal{E}_{\mathcal{G} r, I}^{f}(\mathbb{k})$ est la catégorie $\left(\mathcal{E}_{\mathbb{k}}^{f}\right) \backslash \mathcal{G} r_{I}$ (cf. notation 3.1.2).

(2) Avec les notations de la section 3.2, $\mathcal{E}_{\mathcal{G} r, n}^{f}$ est la catégorie $\mathcal{E}_{\mathcal{G} r_{\leq n}, \mathcal{G} r_{\leq n-1}}^{f}$.

(3) L'association $V \mapsto \mathbf{P l}_{n}(V)$ n'est pas fonctorielle, mais si l'on note hom $\leq i(E, V)$ le sous-ensemble de $\operatorname{hom}_{\mathcal{E}}(E, V)$ formé des applications linéaires de rang au plus $i$, 


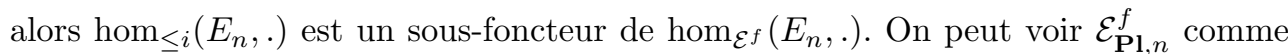
la catégorie $\mathcal{E}_{\operatorname{hom}_{\mathcal{E} f}\left(E_{n}, .\right), \operatorname{hom}_{\leq n-1}\left(E_{n}, .\right)}$.

(4) L'ensemble $\mathcal{G} r_{n}(V)$ s'identifie canoniquement au quotient de $\mathbf{P} \mathbf{l}_{n}(V)$ par l'action à droite libre du groupe $G L_{n}(\mathbb{k})$.

(5) Les catégories $\mathcal{E}_{\mathcal{G} r, 0}^{f}$ et $\mathcal{E}_{\mathrm{Pl}, 0}^{f}$ s'identifient canoniquement à $\mathcal{E}^{f}$.

(6) Les catégories $\mathcal{E}_{\mathcal{G} r, 1}^{f}\left(\mathbb{F}_{2}\right)$ et $\mathcal{E}_{\mathrm{Pl}, 1}^{f}\left(\mathbb{F}_{2}\right)$ sont isomorphes.

(7) Toutes les catégories introduites vérifient l'hypothèse C.2.9 (finitude des ensembles de morphismes), car $\mathbb{k}$ est fini.

Notation 4.1.5 (Foncteurs d'oubli).

(1) Soit $J$ une partie de $\mathbb{N}$ telle que $I \subset J$. On note $\operatorname{incl}_{I, J}: \mathcal{E}_{\mathcal{G} r, I}^{f} \rightarrow \mathcal{E}_{\mathcal{G} r, J}^{f}$ le foncteur (pleinement fidèle) d'oubli.

(2) Nous désignerons par $i n c_{n}^{\mathrm{Pl}}$ le foncteur (fidèle et essentiellement surjectif) d'oubli du plongement $\mathcal{E}_{\mathbf{P l}, n}^{f} \rightarrow \mathcal{E}_{\mathcal{G} r, n}^{f}$ associant à un objet $\left(V, E_{n} \stackrel{u}{\longrightarrow} V\right)$ de $\mathcal{E}_{\mathbf{P l}, n}^{f}$ l'objet $(V, i m u)$ de $\mathcal{E}_{\mathcal{G} r, n}^{f}$ et égal à l'inclusion évidente sur les morphismes.

Comme nous le verrons en fin de chapitre, les catégories introduites précédemment ne sont en général pas abéliennes. Une partie de la structure abélienne de la catégorie $\mathcal{E}^{f}$ s'y reflète cependant, grâce à la notion suivante.

Définition 4.1.6 (Foncteurs de translation). - On définit des foncteurs $\mathcal{E}^{f} \times$ $\mathcal{E}_{\mathcal{G} r, I}^{f} \rightarrow \mathcal{E}_{\mathcal{G} r, I}^{f}, \mathcal{E}^{f} \times \widetilde{\mathcal{E}}_{\mathcal{G} r}^{f} \rightarrow \widetilde{\mathcal{E}}_{\mathcal{G} r}^{f}$ et $\mathcal{E}^{f} \times \mathcal{E}_{\mathbf{P l}, n}^{f} \rightarrow \mathcal{E}_{\mathbf{P l}, n}^{f}$, appelés foncteurs de translation, et notés $\boxplus$, par $V \boxplus(A, B)=(V \oplus A, B)$ (dans les deux premiers cas) et $V \boxplus(A, u$ : $\left.E_{n} \hookrightarrow A\right)=\left(V \oplus A, E_{n} \stackrel{u}{\rightarrow} A \hookrightarrow V \oplus A\right)$ (dans le troisième) sur les objets, l'action sur les morphismes se déduisant de la fonctorialité de $\oplus: \mathcal{E}^{f} \times \mathcal{E}^{f} \rightarrow \mathcal{E}^{f}$.

Remarque 4.1.7. - On a des isomorphismes $0 \boxplus X \simeq X$ et $\left(V^{\prime} \oplus V\right) \boxplus X \simeq V^{\prime} \boxplus$ $(V \boxplus X)$ naturels en les objets $V, V^{\prime}$ de $\mathcal{E}^{f}$ et $X$ de $\mathcal{E}_{\mathcal{G} r, I}^{f}$ (resp. $\widetilde{\mathcal{E}}_{\mathcal{G} r}^{f}, \mathcal{E}_{\mathbf{P l}, n}^{f}$ ), vérifiant des propriétés de cohérence qu'on laisse au lecteur le soin d'expliciter. Ainsi, on peut voir $\boxplus$ comme un foncteur d'action de la catégorie additive $\mathcal{E}^{f}$ sur $\mathcal{E}_{\mathcal{G} r, I}^{f}$ (resp. $\widetilde{\mathcal{E}}_{\mathcal{G} r}^{f}$, $\left.\mathcal{E}_{\mathbf{P l}, n}^{f}\right)$.

Définition 4.1.8 (Foncteurs fondamentaux de source $\mathcal{E}_{\mathcal{G} r, I}^{f}, \widetilde{\mathcal{E}}_{\mathcal{G} r, I}^{f}$ ou $\mathcal{E}_{\mathbf{P l}, n}^{f}$ )

(1) Le foncteur d'oubli principal $\widetilde{\mathfrak{D}}_{I}: \widetilde{\mathcal{E}}_{\mathcal{G} r, I}^{f} \rightarrow \mathcal{E}^{f}$ est le foncteur associant à un objet $(V, W)$ de $\widetilde{\mathcal{E}}_{\mathcal{G} r, I}^{f}$ l'espace vectoriel $V$, et à un morphisme l'application linéaire sous-jacente.

(2) On appelle également foncteurs d'oubli principaux les foncteurs composés

$$
\mathfrak{O}_{I}: \mathcal{E}_{\mathcal{G} r, I}^{f} \stackrel{\widetilde{\text { incl }}}{\longrightarrow} \widetilde{\mathcal{E}}_{\mathcal{G} r, I}^{f} \stackrel{\widetilde{\mathfrak{D}}_{I}}{\longrightarrow} \mathcal{E}^{f}
$$

et

$$
\overline{\mathfrak{O}}_{n}: \mathcal{E}_{\mathbf{P l}, n}^{f} \stackrel{i n c_{n}^{\mathrm{Pl}}}{\longrightarrow} \mathcal{E}_{\mathcal{G} r, n}^{f} \stackrel{\mathfrak{O}_{n}}{\longrightarrow} \mathcal{E}^{f} .
$$


(3) Le foncteur base $\mathfrak{B}_{I}: \mathcal{E}_{\mathcal{G} r, I}^{f} \rightarrow \mathcal{E}_{\text {surj }}^{I}$ est défini sur les objets par $\mathfrak{B}_{I}(V, W)=W$, et associe à un morphisme $f:(V, W) \rightarrow\left(V^{\prime}, W^{\prime}\right)$ l'application linéaire (surjective par définition de $\left.\mathcal{E}_{\mathcal{G} r, I}^{f}\right) W \rightarrow W^{\prime}$ induite par $f$.

(4) Le foncteur d'oubli secondaire $\widetilde{\mathfrak{B}}: \widetilde{\mathcal{E}}_{\mathcal{G} r}^{f} \rightarrow \mathcal{E}^{f}$ associe à un objet $(V, W)$ l'espace vectoriel $W$ et à un morphisme $(V, W) \rightarrow\left(V^{\prime}, W^{\prime}\right)$ l'application linéaire induite $W \rightarrow$ $W^{\prime}$.

(5) Le foncteur de réduction $\widetilde{\mathfrak{K}}_{I}: \widetilde{\mathcal{E}}_{\mathcal{G} r, I}^{f} \rightarrow \mathcal{E}^{f}$ est donné par $(V, W) \mapsto V / W$ sur les objets et associe à un morphisme l'application linéaire induite.

(6) On appelle également foncteurs de réduction les foncteurs composés

$$
\mathfrak{K}_{I}: \mathcal{E}_{\mathcal{G} r, I}^{f} \stackrel{\widetilde{i n c l}_{I}}{\longrightarrow} \widetilde{\mathcal{E}}_{\mathcal{G} r}^{f} \stackrel{\widetilde{\mathfrak{K}}_{I}}{\longrightarrow} \mathcal{E}^{f}
$$

et

$$
\overline{\mathfrak{K}}_{n}: \mathcal{E}_{\mathbf{P l}, n}^{f} \stackrel{i n c_{n}^{\mathrm{Pl}}}{\longrightarrow} \mathcal{E}_{\mathcal{G} r, n}^{f} \stackrel{\mathfrak{K}_{n}}{\longrightarrow} \mathcal{E}^{f} .
$$

On rappelle que l'indice $I$ sera omis dans toutes ces notations lorsque $I=\mathbb{N}$.

Remarque 4.1.9. - (1) Soit $J$ une partie de $\mathbb{N}$ telle que $I \subset J$. Le foncteur composé $\mathcal{E}_{\mathcal{G} r, I}^{f} \stackrel{\operatorname{incl}_{I, J}}{\longrightarrow} \mathcal{E}_{\mathcal{G} r, J}^{f} \stackrel{\mathfrak{O}_{J}}{\longrightarrow} \mathcal{E}^{f}$ est égal à $\mathfrak{O}_{I}$. De même, le diagramme

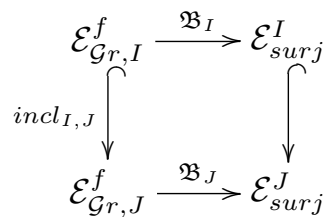

commute. On a d'autres propriétés analogues de compatibilité à l'extension de la partie $I$ avec les différents foncteurs introduits.

(2) Le diagramme

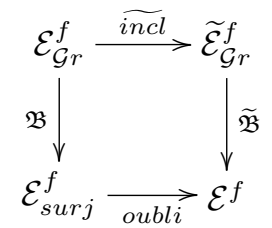

commute.

(3) Les foncteurs base ou d'oubli secondaire n'ont pas d'analogue non trivial en termes de la catégorie $\mathcal{E}_{\mathbf{P l}, n}^{f}$; le rôle de cette catégorie est justement de simplifier certaines des considérations relatives à $\mathcal{E}_{\mathcal{G} r, n}^{f}$ en « rendant la base canoniquement isomorphe à $E_{n} »$.

Remarque 4.1.10. - Le foncteur $\mathfrak{K}_{I}: \mathcal{E}_{\mathcal{G} r, I}^{f} \rightarrow \mathcal{E}^{f}$ est le conoyau de la transformation naturelle injective tautologique $\mathfrak{B}_{I} \rightarrow \mathfrak{O}_{I}$ (où l'on note par abus $\mathfrak{B}_{I}$ pour la 
composée de ce foncteur avec l'inclusion $\mathcal{E}_{\text {surj }}^{I} \rightarrow \mathcal{E}^{f}$ ); un constat analogue vaut pour $\widetilde{\mathfrak{K}}_{I}$ et $\overline{\mathfrak{K}}_{n}$.

La proposition suivante établit des liens entre les foncteurs de translation et les foncteurs introduits dans la définition 4.1.8.

Proposition 4.1.11. - Il existe des isomorphismes

$$
\begin{gathered}
\operatorname{hom}_{\mathcal{E}_{\mathcal{G} r, I}^{f}}(V \boxplus X, Y) \simeq \operatorname{hom}_{\mathcal{E}^{f}}\left(V, \mathfrak{O}_{I}(Y)\right) \times \operatorname{hom}_{\mathcal{E}_{\mathcal{G} r, I}^{f}}(X, Y) \text { et } \\
\operatorname{hom}_{\mathcal{E}_{\mathcal{G} r, I}^{f}}(X, V \boxplus Y) \simeq \operatorname{hom}_{\mathcal{E}^{f}}\left(\mathfrak{K}_{I}(X), V\right) \times \operatorname{hom}_{\mathcal{E}_{\mathcal{G} r, I}^{f}}(X, Y)
\end{gathered}
$$

naturels en les objets $V$ de $\mathcal{E}^{f}$ et $X, Y$ de $\mathcal{E}_{\mathcal{G} r, I}^{f}$.

On a des énoncés similaires dans $\widetilde{\mathcal{E}}_{\mathcal{G} r}^{f}$ et $\mathcal{E}_{\mathbf{P l}, n}^{f}$.

Démonstration. - Les deux isomorphismes étant très analogues, nous nous bornerons à montrer le second, qui s'obtient par la suite d'isomorphismes naturels

$$
\begin{aligned}
& \operatorname{hom}_{\mathcal{E}_{\mathcal{G} r, I}^{f}}(X, V \boxplus Y) \simeq\left\{f \in \operatorname{hom}_{\mathcal{E}^{f}}\left(\mathfrak{O}_{I}(X), V \oplus \mathfrak{O}_{I}(Y)\right) \mid f\left(\mathfrak{B}_{I}(X)\right)=\mathfrak{B}_{I}(Y)\right\} \simeq \\
&\left\{(a, b) \in \operatorname{hom}_{\mathcal{E}^{f}}\left(\mathfrak{O}_{I}(X), V\right) \times \operatorname{hom}_{\mathcal{E}^{f}}\left(\mathfrak{O}_{I}(X), \mathfrak{O}_{I}(Y)\right) \mid a\left(\mathfrak{B}_{I}(X)\right)=0 \text { et } b\left(\mathfrak{B}_{I}(X)\right)=\mathfrak{B}_{I}(Y)\right\} \\
& \simeq \operatorname{hom}_{\mathcal{E}^{f}}\left(\mathfrak{K}_{I}(X), V\right) \times \operatorname{hom}_{\mathcal{E}_{\mathcal{G}_{r, I}^{f}}^{f}(X, Y) .}
\end{aligned}
$$

Corollaire 4.1.12. - Supposons que I contient 0. Le foncteur d'inclusion incl ${ }_{0, I}$ : $\mathcal{E}^{f} \simeq \mathcal{E}_{\mathcal{G} r, 0}^{f} \hookrightarrow \mathcal{E}_{\mathcal{G} r, I}^{f}$ est adjoint à droite à $\mathfrak{K}_{I}$.

Définition 4.1.13 (Foncteurs fondamentaux de but $\mathcal{E}_{\mathcal{G} r, I}^{f}, \widetilde{\mathcal{E}}_{\mathcal{G} r}^{f}$ ou $\mathcal{E}_{\mathrm{Pl}, n}^{f}$ )

(1) Les foncteurs de plongement diagonal sont les foncteurs $\mathfrak{D}_{I}: \mathcal{E}_{\text {surj }}^{I} \rightarrow \mathcal{E}_{\mathcal{G} r, I}^{f}$ et $\widetilde{\mathfrak{D}}: \mathcal{E}^{f} \rightarrow \widetilde{\mathcal{E}}_{\mathcal{G} r}^{f}$ donnés sur les objets par $V \mapsto(V, V)$ et par le plongement évident sur les morphismes.

(2) Les foncteurs de plongement relatif sont les foncteurs composés

$$
\mathfrak{L}_{I}: \mathcal{E}^{f} \times \mathcal{E}_{\text {surj }}^{I} \stackrel{\mathcal{E}^{f} \times \mathfrak{D}_{I}}{\longrightarrow} \mathcal{E}^{f} \times \mathcal{E}_{\mathcal{G} r, I}^{f} \stackrel{\boxplus}{\longrightarrow} \mathcal{E}_{\mathcal{G} r, I}^{f}
$$

et

$$
\widetilde{\mathfrak{L}}: \mathcal{E}^{f} \times \mathcal{E}^{f} \stackrel{\mathcal{E}^{f} \times \widetilde{\mathfrak{D}}}{\longrightarrow} \mathcal{E}^{f} \times \widetilde{\mathcal{E}}_{\mathcal{G} r}^{f} \stackrel{\boxplus}{\longrightarrow} \widetilde{\mathcal{E}}_{\mathcal{G} r}^{f} .
$$

Ces foncteurs sont donc donnés sur les objets par $(A, B) \mapsto(A \oplus B, B)$.

(3) Le foncteur de décalage pointé $\mathfrak{S}_{n}: \mathcal{E}^{f} \rightarrow \mathcal{E}_{\mathrm{Pl}, n}^{f}$ associe à un objet $V$ de $\mathcal{E}^{f}$ l'objet $\left(V \oplus E_{n}, E_{n} \hookrightarrow V \oplus E_{n}\right)$ et à une application linéaire $u$ le morphisme $u \oplus E_{n}$. 
Remarque 4.1.14. -

(1) Le diagramme

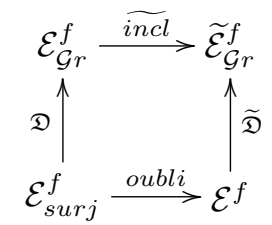

commute.

(2) Le diagramme

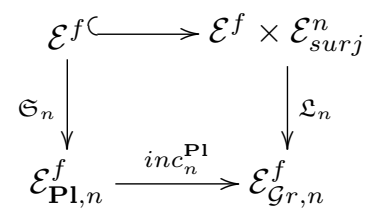

commute, où l'inclusion supérieure est donnée par $V \mapsto\left(V, E_{n}\right)$.

Remarque 4.1.15. - Le plongement évident $\underline{G L_{n}(\mathbb{k})} \rightarrow \mathcal{E}_{\text {surj }}^{n}(\mathbb{k})$ étant une équivalence de catégories, nous commettrons parfois des abus de notation consistant à l'assimiler à une égalité pour les foncteurs mettant en jeu $\mathcal{E}_{\text {surj }}^{n}(\mathbb{k})$.

\subsection{Propriétés des foncteurs fondamentaux}

Nous commençons par donner des propriétés d'adjonction entre les foncteurs introduits précédemment qui seront utilisées de façon intensive dans tout l'article.

Proposition 4.2.1. - (1) Le foncteur de plongement diagonal $\mathfrak{D}_{I}: \mathcal{E}_{\text {surj }}^{I} \rightarrow$ $\mathcal{E}_{\mathcal{G} r, I}^{f}$ est adjoint à gauche à $\mathfrak{B}_{I}: \mathcal{E}_{\mathcal{G} r, I}^{f} \rightarrow \mathcal{E}_{\text {surj }}^{I}$.

(2) Le foncteur de plongement diagonal $\widetilde{\mathfrak{D}}: \mathcal{E}^{f} \rightarrow \widetilde{\mathcal{E}}_{\mathcal{G} r}^{f}$ est adjoint à gauche $\grave{a}$ $\widetilde{\mathfrak{B}}: \widetilde{\mathcal{E}}_{\mathcal{G} r}^{f} \rightarrow \mathcal{E}^{f}$.

(3) Le foncteur de plongement relatif $\mathfrak{L}_{I}: \mathcal{E}^{f} \times \mathcal{E}_{\text {surj }}^{I} \rightarrow \mathcal{E}_{\mathcal{G} r, I}^{f}$ est adjoint à gauche au foncteur $\mathfrak{O}_{I} \times \mathfrak{B}_{I}: \mathcal{E}_{\mathcal{G} r, I}^{f} \rightarrow \mathcal{E}^{f} \times \mathcal{E}_{\text {surj }}^{I}$.

(4) Le foncteur de plongement relatif $\widetilde{\mathfrak{L}}: \mathcal{E}^{f} \times \mathcal{E}^{f} \rightarrow \widetilde{\mathcal{E}}_{\mathcal{G} r}^{f}$ est adjoint à gauche au foncteur $\widetilde{\mathfrak{D}} \times \widetilde{\mathfrak{B}}: \widetilde{\mathcal{E}}_{\mathcal{G} r}^{f} \rightarrow \mathcal{E}^{f} \times \mathcal{E}^{f}$.

(5) Le décalage pointé $\mathfrak{S}_{n}: \mathcal{E}^{f} \rightarrow \mathcal{E}_{\mathbf{P l}, n}^{f}$ est adjoint à gauche à $\overline{\mathfrak{O}}_{n}: \mathcal{E}_{\mathbf{P l}, n}^{f} \rightarrow \mathcal{E}^{f}$.

Démonstration. - Si $A$ est un objet de $\mathcal{E}_{\text {sur } j}^{I}$ et $(V, W)$ un objet de $\mathcal{E}_{\mathcal{G} r, I}^{f}$, on a un isomorphisme canonique

$$
\operatorname{hom}_{\mathcal{E}_{\mathcal{G}_{r, I}^{f}}}\left(\mathfrak{D}_{I}(A),(V, W)\right)=\left\{f \in \operatorname{hom}_{\mathcal{E}^{f}}(A, V) \mid f(A)=W\right\} \simeq \operatorname{Epi}_{\mathcal{E}^{f}}(A, W),
$$

ce qui démontre la première assertion. La troisième assertion s'en déduit en utilisant la proposition 4.1.11.

Les deuxième et quatrième points se traitent pareillement. 
Établissons le dernier : si $A$ est un objet de $\mathcal{E}^{f}$ et $\left(V, u: E_{n} \hookrightarrow V\right)$ un objet de $\mathcal{E}_{\mathrm{Pl}, n}^{f}$, on a un isomorphisme canonique

$$
\operatorname{hom}_{\mathcal{E}_{\mathbf{P}_{1}^{f}, n}^{f}}\left(\mathfrak{S}_{n}(A),(V, u)\right)=\left\{f \in \operatorname{hom}_{\mathcal{E}^{f}}\left(A \oplus E_{n}, V\right) \mid f_{\mid E_{n}}=u\right\} \simeq \operatorname{hom}_{\mathcal{E}^{f}}(A, V) .
$$

Cela achève la démonstration.

La proposition suivante, laissée au lecteur, jouera un rôle fondamental par la suite.

Proposition 4.2.2 (Compositions fondamentales). - Les foncteurs composés

$$
\mathcal{E}^{f} \times \mathcal{E}_{\text {surj }}^{I} \stackrel{\mathfrak{L}_{I}}{\longrightarrow} \mathcal{E}_{\mathcal{G} r, I}^{f} \stackrel{\mathfrak{K}_{I} \times \mathfrak{B}_{I}}{\longrightarrow} \mathcal{E}^{f} \times \mathcal{E}_{\text {surj }}^{I}
$$

et

$$
\mathcal{E}^{f} \times \mathcal{E}^{f} \stackrel{\widetilde{\mathfrak{S}}}{\longrightarrow} \widetilde{\mathcal{E}}_{\mathcal{G} r}^{f} \stackrel{\widetilde{\mathfrak{K}} \times \widetilde{\mathfrak{B}}}{\longrightarrow} \mathcal{E}^{f} \times \mathcal{E}^{f}
$$

sont canoniquement isomorphes aux foncteurs identités.

Proposition 4.2.3. - Supposons I non vide.

(1) Les foncteurs d'oubli principaux sont fidèles.

(2) Le foncteur $\overline{\mathfrak{D}}_{n}: \mathcal{E}_{\mathbf{P l}, n}^{f} \rightarrow \mathcal{E}^{f}$ induit un foncteur essentiellement surjectif $\mathcal{E}_{\mathbf{P l}, n}^{f} \rightarrow \mathcal{E}^{\geq n}$, où le but désigne la sous-catégorie pleine de $\mathcal{E}^{f}$ des espaces de dimension au moins $n$. Si I a pour plus petit élément $n, \mathfrak{O}_{I}: \mathcal{E}_{\mathcal{G} r, I}^{f} \rightarrow \mathcal{E}^{f}$ induit un foncteur essentiellement surjectif $\mathcal{E}_{\mathcal{G} r, I}^{f} \rightarrow \mathcal{E}^{\geq n}$. Le foncteur $\widetilde{\mathfrak{D}}: \widetilde{\mathcal{E}}_{\mathcal{G} r}^{f} \rightarrow \mathcal{E}^{f}$ est essentiellement surjectif.

(3) Les foncteurs de plongement relatif $\mathfrak{L}_{I}$ et $\widetilde{\mathfrak{L}}$ sont fidèles et essentiellement surjectifs. Il en est de même pour le décalage pointé $\mathfrak{S}_{n}$.

(4) Les foncteurs $\mathfrak{K}_{I} \times \mathfrak{B}_{I}$ (donc en particulier $\mathfrak{K}_{I}$ et $\mathfrak{B}_{I}$ ) et $\widetilde{\mathfrak{K}} \times \widetilde{\mathfrak{B}}$ (donc en particulier $\widetilde{\mathfrak{K}}$ et $\widetilde{\mathfrak{B}}$ ) sont pleins et essentiellement surjectifs.

(5) Les foncteurs de plongement diagonal $\mathfrak{D}_{I}$ et $\widetilde{\mathfrak{D}}$ sont pleinement fidèles.

Démonstration. - Les deux premières assertions s'établissent par inspection. L'essentielle surjectivité des foncteurs de plongement relatif et de décalage pointé découle de ce que tout sous-espace d'un espace vectoriel est facteur direct; leur fidélité est claire. La quatrième assertion s'obtient en combinant la proposition 4.2.2 et l'essentielle surjectivité des plongements relatifs. La dernière s'obtient à partir des deux premières adjonctions de la proposition 4.2.1 et du constat que leurs unités $i d \rightarrow \mathfrak{B}_{I} \mathfrak{D}_{I}$ et $i d \rightarrow \widetilde{\mathfrak{B}} \widetilde{\mathfrak{D}}$ sont des isomorphismes.

\subsection{Propriétés de structure des catégories $\mathcal{E}_{\mathcal{G} r}^{f}, \widetilde{\mathcal{E}}_{\mathcal{G} r}^{f}$ et $\mathcal{E}_{\mathrm{Pl}, n}^{f}$}

Les catégories qui nous intéresseront le plus par la suite sont les $\mathcal{E}_{\mathcal{G}_{r}, I}^{f}$. Les propriétés qui suivent montrent que, par certains côtés, les catégories $\widetilde{\mathcal{E}}_{\mathcal{G} r}^{f}$ de $\mathcal{E}_{\mathbf{P l}, n}^{f}$ ont une structure plus « régulière », c'est pourquoi nous serons parfois amenés à travailler dans ces catégories auxiliaires. 
Proposition 4.3.1. - La catégorie $\widetilde{\mathcal{E}}_{\mathcal{G} r}^{f}$ est additive et $\mathbb{k}$-linéaire. Les foncteurs d'oubli principal et secondaire sont additifs - autrement dit, on a un isomorphisme $(V, W) \oplus\left(V^{\prime}, W^{\prime}\right) \simeq\left(V \oplus V^{\prime}, W \oplus W^{\prime}\right)$ naturel en les objets $(V, W)$ et $\left(V^{\prime}, W^{\prime}\right)$ de $\widetilde{\mathcal{E}}_{\mathcal{G} r}^{f}$. En particulier, on a un isomorphisme $E \boxplus(V, W) \simeq(E, 0) \oplus(V, W)$ naturel en les objets $E$ de $\mathcal{E}^{f}$ et $(V, W)$ de $\widetilde{\mathcal{E}}_{\mathcal{G} r}^{f}$.

Remarque 4.3.2. - La catégorie $\widetilde{\mathcal{E}}_{\mathcal{G} r}^{f}$ n'est pas abélienne. En effet, on vérifie que pour tout $V \in \mathrm{Ob} \mathcal{E}^{f}$, le morphisme $(V, 0) \rightarrow(V, V)$ de $\widetilde{\mathcal{E}}_{\mathcal{G} r}^{f}$ dont l'application linéaire sous-jacente est l'identité est à la fois un monomorphisme et un épimorphisme. En revanche, ce n'est pas un isomorphisme, si $V$ est non nul.

Proposition et définition 4.3.3 (Dualité dans $\widetilde{\mathcal{E}}_{\mathcal{G} r}^{f}$ ). - Le foncteur de dualité $(\cdot)^{*}:\left(\mathcal{E}^{f}\right)^{o p} \rightarrow \mathcal{E}^{f}$ induit une équivalence de catégories $(\cdot)^{\vee}:\left(\widetilde{\mathcal{E}}_{\mathcal{G} r}^{f}\right)^{o p} \rightarrow \widetilde{\mathcal{E}}_{\mathcal{G} r}^{f}$ donnée sur les objets par $(V, W)^{\vee}=\left(V^{*}, W^{\perp}\right)$.

Proposition 4.3.4. - Soit $n \in \mathbb{N}$.

(1)

(a) La catégorie $\mathcal{E}_{\mathbf{P} \mathbf{1}, n}^{f}$ possède des sommes finies.

(b) Son objet initial est $\left(E_{n}, i d_{E_{n}}\right)$.

(c) La somme $(A, a) \amalg(B, b)$ de deux objets $(A, a)$ et $(B, b)$ de $\mathcal{E}_{\mathbf{P l}, n}^{f}$ s'obtient en formant le carré cocartésien d'inclusions

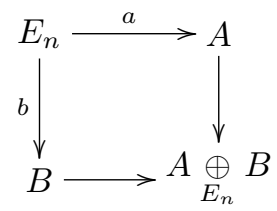

et en munissant l'espace vectoriel $A \underset{E_{n}}{\oplus} B$ du plongement donné par la diagonale du carré.

(2) Deux objets $(A, a)$ et $(B, b)$ de $\mathcal{E}_{\mathbf{P l}, n}^{f}$ possèdent toujours un produit. Il est donné par

$$
(A, a) \times(B, b)=\left(A \oplus B,(a, b): E_{n} \hookrightarrow A \oplus B\right) .
$$

(3) On a des isomorphismes

$$
\begin{gathered}
\mathfrak{S}_{n}(V) \amalg X \simeq V \boxplus X \quad \text { et } \\
\mathfrak{S}_{n}(V) \times X \simeq \mathfrak{S}_{n}\left(V \oplus \overline{\mathfrak{O}}_{n}(X)\right)
\end{gathered}
$$

naturels en les objets $V$ de $\mathcal{E}^{f}$ et $X$ de $\mathcal{E}_{\mathbf{P l}, n}^{f}$.

La démonstration des propositions 4.3.1, 4.3.3 et 4.3.4 est laissée au lecteur. La proposition 4.3 .4 ne sera d'ailleurs pas utilisée explicitement.

Remarque 4.3.5. - (1) Si $I$ contient 0 , alors $(0,0)$ est objet final de $\mathcal{E}_{\mathcal{G} r, I}^{f}$, et tout objet de $\mathcal{E}_{\mathcal{G} r, I}^{f}$ admet un produit avec $(V, 0)$ (où $V \in \mathrm{Ob}^{f}$ ), qui est donné par le foncteur $V \boxplus \cdot$. 
(2) En revanche, on vérifie facilement que deux objets $(V, W)$ et $\left(V^{\prime}, W^{\prime}\right)$ de $\mathcal{E}_{\mathcal{G} r, I}^{f}$ tels que $W$ et $W^{\prime}$ sont non nuls ne possèdent jamais de somme ni de produit.

Les propriétés suivantes fournissent un substitut à l'absence de sommes et de produits dans $\mathcal{E}_{\mathcal{G} r}^{f}$; elles reposent sur le lemme simple et très utile suivant.

Lemme 4.3.6. - Il existe une bijection

$$
\operatorname{hom}_{\mathcal{E}^{f}}\left(A, A^{\prime}\right) \simeq \coprod_{B^{\prime} \in \mathcal{G} r\left(A^{\prime}\right)} \operatorname{hom}_{\mathcal{E}_{\mathcal{G} r}^{f}}\left((A, B),\left(A^{\prime}, B^{\prime}\right)\right)
$$

naturelle en l'objet $(A, B)$ de $\mathcal{E}_{\mathcal{G} r}^{f}$ et l'objet $A^{\prime}$ de $\mathcal{E}^{f}$.

La fonctorialité doit être comprise dans le sens suivant :

- pour le terme de gauche, on considère le foncteur

$$
\left(\mathcal{E}_{\mathcal{G} r}^{f}\right)^{o p} \times \mathcal{E}^{f} \stackrel{(\mathcal{O})^{o p} \times \mathcal{E}^{f}}{\longrightarrow}\left(\mathcal{E}^{f}\right)^{o p} \times \mathcal{E}^{f} \stackrel{\text { hom }}{\longrightarrow} \text { Ens ; }
$$

- pour le terme de droite, la fonctorialité en $(A, B)$ provient de manière usuelle du foncteur hom; pour la fonctorialité en $A^{\prime}$, on fait correspondre à une application linéaire $u: A^{\prime} \rightarrow A^{\prime \prime}$ et à un élément $f$ de $\operatorname{hom}_{\mathcal{E}_{\mathcal{G}_{r}}^{f}}\left((A, B),\left(A^{\prime}, B^{\prime}\right)\right)$ (où $\left.B^{\prime} \in \mathcal{G} r\left(A^{\prime}\right)\right)$ la flèche $(A, B) \rightarrow\left(A^{\prime \prime}, B^{\prime \prime}\right)$, où $B^{\prime \prime}=u\left(B^{\prime}\right)$, donnée par $u \circ f: A \rightarrow A^{\prime \prime}$.

Démonstration. - Cette bijection s'obtient en faisant correspondre à une application linéaire $f: A \rightarrow A^{\prime}$ le sous-espace $B^{\prime}=f(B)$ de $A^{\prime}$ et le morphisme $(A, B) \rightarrow\left(A^{\prime}, B^{\prime}\right)$ de $\mathcal{E}_{\mathcal{G} r}^{f}$ induit par $f$.

Proposition 4.3.7. - Il existe une bijection

$$
\begin{gathered}
\coprod_{\substack{W_{1}, W_{2} \in \mathcal{G} r(W) \\
W_{1}+W_{2}=W}} \operatorname{hom}_{\mathcal{E}_{\mathcal{G} r}^{f}}\left(\left(A \oplus A^{\prime}, B \oplus B^{\prime}\right),(V, W)\right) \simeq \\
\text { naturelle en les objets }(A, B),\left(A^{\prime}, B^{\prime}\right) \text { et }(V, W) \text { de } \mathcal{E}_{\mathcal{G} r}^{f} .
\end{gathered}
$$

Cette proposition, comme la proposition 4.3 .9 ci-après, est laissée au lecteur. La fonctorialité doit être comprise dans le sens suivant :

- sur les ensembles hom, on utilise la fonctorialité usuelle;

- pour $\mathcal{E}_{\mathcal{G} r}^{f} \times \mathcal{E}_{\mathcal{G} r}^{f} \rightarrow \mathcal{E}_{\mathcal{G} r}^{f} \quad\left((A, B),\left(A^{\prime}, B^{\prime}\right)\right) \mapsto\left(A \oplus A^{\prime}, B \oplus B^{\prime}\right)$, on associe à un morphisme $(u, v)$ de $\mathcal{E}_{\mathcal{G} r}^{f} \times \mathcal{E}_{\mathcal{G} r}^{f}$ le morphisme $u \oplus v$ de $\mathcal{E}_{\mathcal{G} r}^{f}$;

- dans le terme de droite, la fonctorialité en $(V, W)$ s'obtient comme suit. Si $u:(V, W) \rightarrow\left(V^{\prime}, W^{\prime}\right)$ est un morphisme de $\mathcal{E}_{\mathcal{G} r}^{f}$ et $W_{1}, W_{2}$ deux sous-espaces de $W$ tels que $W_{1}+W_{2}=W$, on pose $W_{i}^{\prime}=f\left(W_{i}\right)(i \in\{1,2\})$, de sorte que $W_{1}^{\prime}+W_{2}^{\prime}=f(W)=W^{\prime}$. Le morphisme induit par $u$ s'obtient par somme sur les 
$\left(W_{1}, W_{2}\right)$ des morphismes $\operatorname{hom}_{\mathcal{E}_{\mathcal{G}_{r}}^{f}}\left((A, B),\left(V, W_{1}\right)\right) \times \operatorname{hom}_{\mathcal{E}_{\mathcal{G}_{r}}^{f}}\left(\left(A^{\prime}, B^{\prime}\right),\left(V, W_{2}\right)\right) \rightarrow$ $\operatorname{hom}_{\mathcal{E}_{\mathcal{G}_{r}}^{f}}\left((A, B),\left(V^{\prime}, W_{1}^{\prime}\right)\right) \times \operatorname{hom}_{\mathcal{E}_{\mathcal{G}_{r}}^{f}}\left(\left(A^{\prime}, B^{\prime}\right),\left(V^{\prime}, W_{2}^{\prime}\right)\right)$ induits par $u$.

Notation 4.3.8. - Soient $V$ et $W$ deux espaces vectoriels de dimension finie. Nous noterons $\operatorname{Gr}(V, W)$ le sous-ensemble de $\mathcal{G} r(V \oplus W)$ formé des sous-espaces $E$ de $V \oplus W$ tels que les morphismes $E \hookrightarrow V \oplus W \rightarrow V$ et $E \hookrightarrow V \oplus W \rightarrow W$ soient surjectifs.

On définit ainsi un foncteur $G r: \mathcal{E}_{\text {surj }}^{f} \times \mathcal{E}_{\text {surj }}^{f} \rightarrow$ Ens, l'action sur les morphismes étant obtenue par un biais analogue à celui détaillé précédemment.

Proposition 4.3.9. - Il existe une bijection

$$
\begin{gathered}
\operatorname{hom}_{\mathcal{E}_{\mathcal{G}_{r}}^{f}}((V, W),(A, B)) \times \operatorname{hom}_{\mathcal{E}_{\mathcal{G}_{r}}^{f}}\left((V, W),\left(A^{\prime}, B^{\prime}\right)\right) \\
\simeq \coprod_{C \in G r\left(B, B^{\prime}\right)} \operatorname{hom}_{\mathcal{E}_{\mathcal{G}_{r}}^{f}}\left((V, W),\left(A \oplus A^{\prime}, C\right)\right)
\end{gathered}
$$

naturelle en les objets $(A, B),\left(A^{\prime}, B^{\prime}\right)$ et $(V, W)$ de $\mathcal{E}_{\mathcal{G}_{r}}^{f}$.

La fonctorialité repose ici sur celle de $G r$ (d'une manière similaire à celle explicitée pour la proposition 4.3.7).

Remarque 4.3.10. - Les propositions 4.3.7 et 4.3.9 sont spécifiques à la catégorie « globale $» \mathcal{E}_{\mathcal{G} r}^{f}$; elles n'ont pas d'analogue dans $\mathcal{E}_{\mathcal{G} r, n}^{f}$, par exemple. 


\section{PARTIE II}

\section{LES CATÉGORIES DE FONCTEURS EN GRASSMANNIENNES}


Le principal sujet de cette partie réside dans l'étude de la catégorie $\mathcal{F}_{\mathcal{G} r}(\mathbb{k})$, appelée parfois catégorie de foncteurs en grassmanniennes globale, et de ses sous-catégories $\mathcal{F}_{\mathcal{G} r, n}(\mathbb{k})$, où $n \in \mathbb{N}$. Ces catégories possèdent un intérêt intrinsèque en raison de leur riche structure algébrique; elles s'interprètent notamment en termes de modules ou de comodules. De plus, nous verrons dans la partie III que le foncteur d'intégrale en grassmanniennes $\omega: \mathcal{F}_{\mathcal{G}_{r}}(\mathbb{k}) \rightarrow \mathcal{F}(\mathbb{k})$ constitue un outil puissant d'étude de la catégorie $\mathcal{F}(\mathbb{k})$.

Le chapitre 5 décrit la structure de base de la catégorie $\mathcal{F}_{\mathcal{G} r}$. Après avoir introduit les foncteurs fondamentaux reliant cette catégorie aux autres catégories de foncteurs que nous avons introduites, notamment $\mathcal{F}$ et $\mathcal{F}_{\text {surj }}$, il décrit les groupes de Grothendieck de ses objets finis et projectifs de type fini. Dans le chapitre 7, nous présentons une approche monadique de la catégorie $\mathcal{F}_{\mathcal{G} r}$, utilisée pour la détermination d'un adjoint et à des fins homologiques. Enfin, le chapitre 9 présente quelques propriétés générales des adjoints au produit tensoriel dans les catégories $\mathcal{F}_{\mathcal{G} r}$ et $\mathcal{F}_{\mathcal{G} r, n}$.

Les autres catégories de foncteurs en grassmanniennes que nous introduisons serviront surtout d'auxiliaires dans l'étude de la catégorie $\mathcal{F}_{\mathcal{G}_{r}}$. Dans le chapitre 6 , nous présentons quelques propriétés d'une catégorie notée $\widetilde{\mathcal{F}}_{\mathcal{G} r}(\mathbb{k})$ et étudions le foncteur $\mathbb{k}[\mathcal{G} r]$, dont une propriété importante, l'auto-dualité, constitue un cas particulier d'un résultat sur la catégorie $\widetilde{\mathcal{F}}_{\mathcal{G} r}(\mathbb{k})$. Le rôle fondamental du foncteur $\mathbb{k}[\mathcal{G} r]$ provient de ce que la catégorie de foncteurs en grassmanniennes globale $\mathcal{F}_{\mathcal{G} r}$ est équivalente à la catégorie des $\mathbb{k}[\mathcal{G} r]$-comodules.

Le chapitre 8 étudie une dernière famille de catégories de foncteurs en grassmanniennes, notées $\mathcal{F}_{\mathbf{P l}, n}(\mathbb{k})$, qui fournit un analogue des catégories $\mathcal{F}_{\mathcal{G} r, n}$ obtenu en trivialisant l'action du groupe linéaire $G L_{n}(\mathbb{k})$ (assertion qui sera précisée par la proposition 8.2.18). En outre, la descrition monadique de la catégorie $\mathcal{F}_{\mathbf{P l}, n}$ donne lieu à de nouvelles structures dans les catégories de foncteurs en grassmanniennes. 


\section{CHAPITRE 5}

\section{LES CATÉGORIES $\mathcal{F}_{\mathcal{G} r, I}$}

Ce chapitre est consacré à une première étude des catégories suivantes. Son principal objectif consiste à en comprendre les objets finis.

Définition 5.0.1. — Étant donnée une partie $I$ de $\mathbb{N}$, on introduit la catégorie de foncteurs

$$
\mathcal{F}_{\mathcal{G} r, I}(\mathbb{k})=\operatorname{Fct}\left(\mathcal{E}_{\mathcal{G} r, I}^{f}(\mathbb{k}), \mathcal{E}_{\mathbb{k}}\right) .
$$

Nous noterons simplement $\mathcal{F}_{\mathcal{G} r}(\mathbb{k})$, ou $\mathcal{F}_{\mathcal{G} r}$, pour $\mathcal{F}_{\mathcal{G} r, \mathbb{N}}(\mathbb{k})$.

Remarque 5.0.2. - Les cas les plus intéressants sont ceux où $I=\mathbb{N}, \leq n$ ou $n$.

\subsection{Généralités}

Nous introduisons les foncteurs obtenus par précomposition à partir de ceux de la section 4.1, et utilisons les propriétés établies dans la section 4.2 et le chapitre 3.

Notation 5.1.1. - (1) Soit $J$ une partie de $\mathbb{N}$. Nous abrégerons la notation des espaces vectoriels hom $_{\mathcal{F}_{\mathcal{G} r, J}}$ en hom $_{\mathcal{G} r, J}$, et utiliserons une convention analogue pour les groupes d'extensions ou les hom internes. De même, nous noterons les projectifs standard $P_{A}^{\mathcal{G} r, J}$ (où $A \in \mathrm{Ob} \mathcal{E}_{\mathcal{G} r, J}^{f}$ ) plutôt que $P_{A}^{\mathcal{E}_{\mathcal{G} r, J}^{f}}$, et les injectifs standard $I_{A}^{\mathcal{G} r, J}$ plutôt que $I_{A}^{\mathcal{E}_{\mathcal{G} r, J}^{f}}$ (cf. section C.2). L'exposant $J$ sera omis pour $J=\mathbb{N}$.

(2) Soient $I$ et $J$ deux parties de $\mathbb{N}$ telles que $J \subset I$. Nous noterons $\mathcal{R}_{I, J}: \mathcal{F}_{\mathcal{G} r, I} \rightarrow$ $\mathcal{F}_{\mathcal{G} r, J}$ le foncteur de restriction $\left(\text { incl }_{J, I}\right)^{*}$. Le foncteur de prolongement par zéro, lorsqu'il est défini (cf. section C.6), sera noté $\mathcal{P}_{J, I}: \mathcal{F}_{\mathcal{G} r, J} \rightarrow \mathcal{F}_{\mathcal{G} r, I}$. Les indices seront omis lorsqu'il n'y a pas d'ambiguïté.

La proposition 3.2.3 fournit le résultat suivant, dans lequel nous omettons les indices des foncteurs de restriction et de prolongement par zéro (de sorte que la notation $\mathcal{R}$ et $\mathcal{P}$ désigne à chaque fois deux foncteurs différents). 
Proposition 5.1.2. - Pour tout entier $n \geq 0$, il existe un diagramme de recollement

$$
\mathcal{F}_{\mathcal{G} r, \leq n-1} \underset{\mathcal{R}}{\underset{\mathcal{P} \rightarrow}{\leftarrow}} \mathcal{F}_{\mathcal{G} r, \leq n} \underset{\mathcal{P}}{\stackrel{\leftarrow}{\leftarrow} \rightarrow} \mathcal{F}_{\mathcal{G} r, n} .
$$

Nous introduisons maintenant les deux foncteurs fondamentaux de source $\mathcal{F}$ et de but $\mathcal{F}_{\mathcal{G} r, I}$.

Définition 5.1.3. - Soit $I$ une partie de $\mathbb{N}$.

(1) On définit le foncteur de plongement standard $\iota_{I}: \mathcal{F} \rightarrow \mathcal{F}_{\mathcal{G} r, I}$ comme le foncteur de précomposition par le foncteur d'oubli principal $\mathfrak{O}_{I}: \mathcal{E}_{\mathcal{G}_{r, I}}^{f} \rightarrow \mathcal{E}^{f}$.

(2) Le foncteur de plongement réduit $\kappa_{I}: \mathcal{F} \rightarrow \mathcal{F}_{\mathcal{G}_{r, I}}$ est le foncteur de précomposition par le foncteur de réduction $\mathfrak{K}_{I}: \mathcal{E}_{\mathcal{G} r, I}^{f} \rightarrow \mathcal{E}^{f}$.

Autrement dit, on a

$$
\iota_{I}(F)(V, W)=F(V)
$$

et

$$
\kappa_{I}(F)(V, W)=F(V / W)
$$

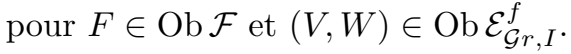

L'indice $I$ sera omis quand $I=\mathbb{N}$; des conventions analogues vaudront dans les notations suivantes.

Remarque 5.1.4. - Les foncteurs $\iota_{\leq n}$ et $\iota$ sont, avec la notation de la section 3.1, les foncteurs $\Upsilon_{\mathcal{G} r_{\leq n}}$ et $\Upsilon_{\mathcal{G} r}$ respectivement. De même, le foncteur $\iota_{n}$ est, selon la convention de la section 3.2 , le foncteur $\Upsilon_{\mathcal{G} r_{\leq n}, \mathcal{G} r_{\leq n-1}}$.

Grâce à la remarque précédente, nous pouvons considérer les foncteurs d'intégrale introduits dans les définitions 3.1.5 et 3.2.5. Il s'agit des seuls foncteurs de cette section qui ne soient pas des foncteurs de précomposition. Des foncteurs d'une catégorie de foncteurs en grassmanniennes vers la catégorie $\mathcal{F}$, ce sont les plus fondamentaux (cf. partie III).

\section{Définition 5.1.5 (Foncteurs d'intégrale en grassmanniennes)}

Soit $n \in \mathbb{N}$.

(1) Nous noterons $\omega_{\leq n}: \mathcal{F}_{\mathcal{G} r, \leq n} \rightarrow \mathcal{F}$ le foncteur $\Omega_{\mathcal{G} r_{\leq n}}$.

(2) Nous noterons $\omega: \mathcal{F}_{\mathcal{G} r} \rightarrow \mathcal{F}$ le foncteur $\Omega_{\mathcal{G} r}$.

(3) Nous désignerons par $\omega_{n}: \mathcal{F}_{\mathcal{G} r, n} \rightarrow \mathcal{F}$ le foncteur $\Omega_{\mathcal{G} r_{\leq n}, \mathcal{G} r_{\leq n-1}}$.

Ces foncteurs seront appelés foncteurs d'intégrale en grassmanniennes.

Explicitement, on a

$$
\omega(X)(V)=\bigoplus_{W \in \mathcal{G} r(W)} X(V, W)
$$

des formules analogues valent pour $\omega_{n}$ et $\omega_{\leq n}$. 
Remarque 5.1.6. - On a des isomorphismes canoniques $\omega_{\leq n} \simeq \omega \circ \mathcal{P}_{\leq n, \mathbb{N}}$ et $\omega_{n} \simeq$ $\omega_{\leq n} \circ \mathcal{P}_{n, \leq n}$.

Les résultats des sections 3.1 et 3.2 se traduisent par le résultat formel mais fondamental suivant.

Proposition 5.1.7. - Soit $n \in \mathbb{N}$.

(1) Le foncteur $\omega_{\leq n}$ est adjoint à gauche $\grave{a} \iota \leq n$. Il induit une équivalence de catégories entre $\mathcal{F}_{\mathcal{G} r, \leq n}$ et la sous-catégorie $\operatorname{Comod}_{\mathbb{k}\left[\mathcal{G}_{\leq n}\right]}$ de $\mathcal{F}$.

(2) Le foncteur $\omega$ est adjoint à gauche à $\iota$. Il induit une équivalence de catégories entre $\mathcal{F}_{\mathcal{G} \text { r }}$ et la sous-catégorie $\operatorname{Comod}_{\mathbb{k}[\mathcal{G} r]}$ de $\mathcal{F}$.

(3) Le foncteur $\omega_{n}$ induit une équivalence de catégories entre $\mathcal{F}_{\mathcal{G} r, n}$ et la souscatégorie $\operatorname{Comod}_{\bar{G}(n)}^{\text {fid }}$ de $\mathcal{F}$ des $\bar{G}(n)$-comodules fidèles.

(4) On a un isomorphisme

$$
\omega_{I}\left(X \otimes \iota_{I}(F)\right) \simeq \omega_{I}(X) \otimes F
$$

naturel en les objets $X$ de $\mathcal{F}_{\mathcal{G} r, I}$ et $F$ de $\mathcal{F}$, où $I=\mathbb{N}, \leq n$ ou $n$.

Démonstration. - Les deux premières assertions constituent des cas particuliers des propositions 3.1.7 et 3.1.12, la troisième de la proposition 3.2.10. Le dernier point résulte pour sa part des propositions 3.1.8 et 3.2.7.

Nous définissons maintenant les deux foncteurs fondamentaux entre les catégories $\mathcal{F}_{\mathcal{G} r, I}$ et $\mathcal{F}_{\text {sur } j}^{I}$.

Définition 5.1.8. - Soit $I$ une partie de $\mathbb{N}$.

(1) Le foncteur de plongement secondaire $\rho_{I}: \mathcal{F}_{\text {surj }}^{I} \rightarrow \mathcal{F}_{\mathcal{G} r, I}$ est le foncteur de précomposition par le foncteur base $\mathfrak{B}_{I}: \mathcal{E}_{\mathcal{G} r, I}^{f} \rightarrow \mathcal{E}_{\text {surj }}^{I}$.

(2) Le foncteur d'évaluation généralisée $\varepsilon_{I}: \mathcal{F}_{\mathcal{G} r, I} \rightarrow \mathcal{F}_{\text {surj }}^{I}$ est la précomposition par le foncteur de plongement diagonal $\mathfrak{D}_{I}: \mathcal{E}_{\text {surj }}^{I} \rightarrow \mathcal{E}_{\mathcal{G} r, I}^{f}$.

Autrement dit, on a

$$
\rho_{I}(F)(V, W)=F(W)
$$

pour $F \in \mathrm{Ob} \mathcal{F}_{\text {surj }}^{I}$ et $(V, W) \in \mathrm{Ob} \mathcal{E}_{\mathcal{G} r, I}^{f}$, et

$$
\varepsilon_{I}(X)(V)=X(V, V)
$$

pour $X \in \mathrm{Ob} \mathcal{F}_{\mathcal{G} r, I}$ et $V \in \mathrm{Ob} \mathcal{E}_{\text {surj }}^{I}$.

Remarque 5.1.9. - Avec l'abus de la remarque 4.1.15, le foncteur $\varepsilon_{n}: \mathcal{F}_{\mathcal{G} r, n} \rightarrow$ $\mathbb{k}\left[G L_{n}(\mathbb{k})\right]$ Mod s'identifie au foncteur d'évaluation $\operatorname{ev}_{\left(E_{n}, E_{n}\right)}$, ce qui justifie la terminologie employée.

On peut de même voir $\rho_{n}$ comme un foncteur ${ }_{\mathbb{k}\left[G L_{n}(\mathbb{k})\right]} \operatorname{Mod} \rightarrow \mathcal{F}_{\mathcal{G} r, n}$; il est donné par $\rho_{n}(X)(V, W)=\operatorname{Iso}\left(E_{n}, W\right) \underset{\mathbb{k}\left[G L_{n}(\mathbb{k})\right]}{\otimes} M$. 
On rappelle que l'on désigne par $\mathcal{F} \otimes \mathcal{F}_{\text {surj }}^{I}$ la catégorie de foncteurs $\mathbf{F} \mathbf{c t}\left(\mathcal{E}^{f} \times\right.$ $\left.\mathcal{E}_{\text {surj }}^{I}, \mathcal{E}\right)$, selon la notation C.5.1.

Définition 5.1.10 (Foncteurs fondamentaux entre $\mathcal{F}_{\mathcal{G} r, I}$ et $\mathcal{F} \otimes \mathcal{F}_{\text {surj }}^{I}$ )

Soit $I$ une partie de $\mathbb{N}$.

(1) Le foncteur de plongement complet $\xi_{I}: \mathcal{F} \otimes \mathcal{F}_{\text {surj }}^{I} \rightarrow \mathcal{F}_{\mathcal{G} r, I}$ est la précomposition par le foncteur $\mathfrak{O}_{I} \times \mathfrak{B}_{I}: \mathcal{E}_{\mathcal{G} r, I}^{f} \rightarrow \mathcal{E}^{f} \times \mathcal{E}_{\text {surj }}^{I}$.

(2) Le foncteur de plongement total $\theta_{I}: \mathcal{F} \otimes \mathcal{F}_{\text {sur } j}^{I} \rightarrow \mathcal{F}_{\mathcal{G} r, I}$ est la précomposition par le foncteur $\mathfrak{K}_{I} \times \mathfrak{B}_{I}: \mathcal{E}_{\mathcal{G} r, I}^{f} \rightarrow \mathcal{E}^{f} \times \mathcal{E}_{\text {surj }}^{I}$.

(3) On définit le foncteur de décalage en grassmanniennes $\sigma_{I}: \mathcal{F}_{\mathcal{G} r, I} \rightarrow \mathcal{F} \otimes \mathcal{F}_{\text {sur } j}^{I}$ comme le foncteur de précomposition par le foncteur de plongement relatif $\mathfrak{L}_{I}: \mathcal{E}^{f} \times$ $\mathcal{E}_{\text {surj }}^{I} \rightarrow \mathcal{E}_{\mathcal{G} r, I}^{f}$.

Ainsi, on a

$$
\begin{gathered}
\xi_{I}(F)(V, W)=F(V, W), \\
\theta_{I}(F)(V, W)=F(V / W, W)
\end{gathered}
$$

pour $F \in \mathrm{Ob} \mathcal{F} \otimes \mathcal{F}_{\text {surj }}^{I}$ et $(V, W) \in \mathrm{Ob} \mathcal{E}_{\mathcal{G} r, I}^{f}$, et

$$
\sigma_{I}(X)(A, B)=X(A \oplus B, B)
$$

pour $X \in \mathrm{Ob} \mathcal{F}_{\mathcal{G} r, I}, A \in \mathrm{Ob} \mathcal{E}^{f}$ et $B \in \mathrm{Ob} \mathcal{E}_{\text {surj }}^{I}$.

Intuitivement, la catégorie $\mathcal{F} \otimes \mathcal{F}_{\text {sur } j}^{I}$ ne doit pas être vue comme très différente de la catégorie $\mathcal{F}$. En effet, dans le cas où $I$ est réduite à un élément $n$, on a des équivalences de catégories canoniques $\mathcal{F} \otimes \mathcal{F}_{\text {surj }}^{n} \simeq \operatorname{Fct}\left(\mathcal{E}^{f} \times G L_{n}(\mathbb{k}), \mathcal{E}\right) \simeq$ $\boldsymbol{F} \mathbf{c t}\left(G L_{n}(\mathbb{k}), \boldsymbol{F} \operatorname{ct}\left(\mathcal{E}^{f}, \mathcal{E}\right)\right)=\mathcal{F}_{G L_{n}(\mathbb{k})}($ cf. notation $3 \mathrm{~b}$ donnée à la fin de l'introduction). Si $I$ est une partie quelconque de $\mathbb{N}$, la catégorie $\mathcal{F} \otimes \mathcal{F}_{\text {surj }}^{I}$ s'obtient par recollement de telles catégories (cf. proposition 2.1.2).

Remarque 5.1.11. - Compte-tenu des identifications que nous venons de mentionner, nous considérerons $\xi_{n}$ et $\theta_{n}$ comme des foncteurs $\mathcal{F}_{G L_{n}(\mathbb{k})} \rightarrow \mathcal{F}_{\mathcal{G} r, n}$ (et de même $\left.\sigma_{n}: \mathcal{F}_{\mathcal{G} r, n} \rightarrow \mathcal{F}_{G L_{n}(\mathbb{k})}\right)$

Premières propriétés. - Nous commençons par relier les foncteurs projectifs ou injectifs standard des catégories $\mathcal{F}_{\mathcal{G} r, I}, \mathcal{F}$ et $\mathcal{F}_{\text {surj }}^{I}$ à l'aide des foncteurs introduits plus haut.

Proposition 5.1.12. - Soit $J$ une partie de $\mathbb{N}$. On a des isomorphismes

$$
P_{\mathcal{L}_{J}(V, W)}^{\mathcal{G} r, J} \simeq \iota_{J}\left(P_{V}\right) \otimes \rho_{J}\left(P_{W}^{\mathcal{E}_{\text {surj }}^{J}}\right)
$$

$$
I_{\mathfrak{L}_{J}(V, W)}^{\mathcal{G} r, J} \simeq \kappa_{J}\left(I_{V}\right) \otimes I_{\mathfrak{D}_{J}(W)}^{\mathcal{G} r, J}
$$

naturels en les objets $V$ de $\mathcal{E}^{f}$ et $W$ de $\mathcal{E}_{\text {surj }}^{J}$. 
Démonstration. - Par la troisième adjonction de la proposition 4.2.1, on dispose d'un isomorphisme canonique

$$
\operatorname{hom}_{\mathcal{E}_{\mathcal{G} r, J}^{f}}\left(\mathfrak{L}_{J}(V, W), \cdot\right) \simeq\left(\operatorname{hom}_{\mathcal{E}^{f}}(V, \cdot) \circ \mathfrak{O}_{J}\right) \times\left(\operatorname{hom}_{\mathcal{E}_{\text {surj }}^{J}}(W, \cdot) \circ \mathfrak{B}_{J}\right) .
$$

On en déduit le premier isomorphisme, en linéarisant.

De même, on obtient formellement l'isomorphisme (7) à partir du second isomorphisme de la proposition 4.1.11.

Remarque 5.1.13. - (1) Comme le foncteur de plongement relatif $\mathfrak{L}_{J}: \mathcal{E}^{f} \times$ $\mathcal{E}_{\text {surj }}^{J} \rightarrow \mathcal{E}_{\mathcal{G} r, J}^{f}$ est essentiellement surjectif, cette proposition décrit tous les projectifs et tous les injectifs standard de $\mathcal{F}_{\mathcal{G} r, J}$.

(2) Les injectifs du type $I_{\mathfrak{D}_{J}(W)}^{\mathcal{G} r, J}=I_{(W, W)}^{\mathcal{G} r, J}$ ne se ramènent pas facilement à des injectifs de catégories plus simples. Illustrons-le pour $J=\mathbb{N}$, afin de simplifier les notations.

En effet, le comportement du foncteur $I_{(W, W)}^{\mathcal{G} r}$ se rapproche de l'injectif $I_{W}^{\text {surj }}$ de $\mathcal{F}_{\text {surj }}$ dans la mesure où il existe un isomorphisme canonique $\varepsilon_{J}\left(I_{(W, W}^{\mathcal{G} r}\right) \simeq I_{W}^{\text {surj }}$ (car $\left.\operatorname{hom}_{\mathcal{E}_{\mathcal{G} r}^{f}}((V, V),(W, W)) \simeq \operatorname{Epi}_{\mathcal{E}}(V, W)\right)$, et aussi parce que $I_{(W, W)}^{\mathcal{G} r}$ comme $I_{W}^{\text {surj }}$ ont un anneau d'endomorphismes isomorphe à $\mathbb{k}[G L(W)]$. Cependant, $I_{(W, W)}^{\mathcal{G} r}$ n'est pas isomorphe à l'image de $I_{W}^{\text {surj }}$ par le foncteur $\rho: \mathcal{F}_{\text {surj }} \rightarrow \mathcal{F}_{\mathcal{G} r}$.

La propriété suivante des foncteurs injectifs et projectifs standard de la catégorie $\mathcal{F}_{\mathcal{G} r}$ (qui n'a pas d'équivalent dans $\mathcal{F}_{\mathcal{G} r, I}$ pour $I \neq \mathbb{N}$ ) est analogue aux assertions 2 et 3 de la proposition 2.3.6.

Proposition 5.1.14. -

(1) Il existe un isomorphisme $\omega\left(P_{(V, W)}^{\mathcal{G} r}\right) \simeq P_{V}$ naturel en l'objet $(V, W)$ de $\mathcal{E}_{\mathcal{G} r}^{f}$.

(2) Il existe un isomorphisme $\iota\left(I_{V}\right) \simeq \bigoplus_{W \in \mathcal{G} r(V)} I_{(V, W)}^{\mathcal{G} r}$ naturel en l'objet $V$ de $\mathcal{E}^{f}$.

Démonstration. — Ces deux assertions proviennent, par linéarisation, du lemme 4.3.6.

La proposition qui suit donne les propriétés d'usage courant des foncteurs de précomposition introduits en début de section.

Proposition 5.1.15. - Soit I une partie non vide de $\mathbb{N}$.

(1) Les foncteurs $\iota_{I}: \mathcal{F} \rightarrow \mathcal{F}_{\mathcal{G} r, I}, \sigma_{I}: \mathcal{F}_{\mathcal{G} r, I} \rightarrow \mathcal{F} \otimes \mathcal{F}_{\text {surj }}^{I}, \xi_{I}: \mathcal{F} \otimes \mathcal{F}_{\text {surj }}^{I} \rightarrow \mathcal{F}_{\mathcal{G} r, I}$ et $\varepsilon_{I}: \mathcal{F}_{\mathcal{G} r, I} \rightarrow \mathcal{F}_{\text {surj }}^{I}$ sont exacts ; ils commutent au produit tensoriel, aux limites et aux colimites. De plus, les foncteurs $\iota_{I}, \sigma_{I}$ et $\xi_{I}$ sont fidèles.

(2) Les foncteurs $\kappa_{I}: \mathcal{F} \rightarrow \mathcal{F}_{\mathcal{G} r, I}, \rho_{I}: \mathcal{F}_{\text {surj }}^{I} \rightarrow \mathcal{F}_{\mathcal{G} r, I}$ et $\theta_{I}: \mathcal{F} \otimes \mathcal{F}_{\text {surj }}^{I} \rightarrow \mathcal{F}_{\mathcal{G} r, I}$ sont exacts et pleinement fidèles; ils commutent au produit tensoriel, aux limites et aux colimites. De surcroît, leurs images sont des sous-catégories de Serre de $\mathcal{F}_{\mathcal{G} r, I}$. 
Démonstration. - Tous les foncteurs de l'énoncé sont des foncteurs de précomposition, ils commutent donc aux limites, aux colimites et au produit tensoriel, par la proposition C.1.4.

Comme le foncteur $\mathfrak{L}_{I}$ est essentiellement surjectif, par la proposition 4.2.3, le foncteur de précomposition associé, $\sigma_{I}$, est fidèle (par la proposition C.1.4). La fidélité $\mathrm{du}$ foncteur $\iota_{I}$ s'établit de même, car l'inclusion $\mathcal{E}^{\leq n} \hookrightarrow \mathcal{E}^{f}$ (cf. la deuxième assertion de la proposition 4.2.3), où $n$ désigne le plus petit élément de $I$, induit par précomposition une équivalence de catégories $\mathcal{F}=\boldsymbol{F} \operatorname{ct}\left(\mathcal{E}^{f}, \mathcal{E}\right) \stackrel{\simeq}{\longrightarrow} \boldsymbol{F} \operatorname{ct}\left(\mathcal{E}^{\leq n}, \mathcal{E}\right)$. La fidélité de $\xi_{I}$ est analogue, en remarquant que tout objet de $\mathcal{E}^{f} \times \mathcal{E}_{\text {surj }}^{I}$ est rétracte d'un objet de l'image du foncteur $\mathfrak{O}_{I} \times \mathfrak{B}_{I}$.

Comme les foncteurs $\mathfrak{K}_{I}, \mathfrak{B}_{I}$ et $\mathfrak{K}_{I} \times \mathfrak{B}_{I}$ sont pleins et essentiellement surjectifs, par la proposition 4.2.3, la proposition C.1.4 montre qu'ils sont pleinement fidèles et que leurs images sont des sous-catégories de Serre de $\mathcal{F}_{\mathcal{G} r, I}$.

La partie de la proposition 5.1.15 relative au foncteur $\theta_{I}$, ainsi que la composition fondamentale donnée par la proposition suivante, apparaîtront sous un jour nouveau dans la section 7 .

Proposition 5.1.16. - Soit I une partie de $\mathbb{N}$. Le foncteur composé

$$
\mathcal{F} \otimes \mathcal{F}_{\text {surj }}^{I} \stackrel{\theta_{I}}{\longrightarrow} \mathcal{F}_{\mathcal{G} r, I} \stackrel{\sigma_{I}}{\longrightarrow} \mathcal{F} \otimes \mathcal{F}_{\text {sur } j}^{I}
$$

est canoniquement isomorphe au foncteur identique.

Cette propriété découle de la proposition 4.2.2.

Proposition 5.1.17. - Soit I une partie de $\mathbb{N}$. Le foncteur $\xi_{I}: \mathcal{F} \otimes \mathcal{F}_{\text {surj }}^{I} \rightarrow \mathcal{F}_{\mathcal{G} r, I}$ est adjoint à gauche au foncteur $\sigma_{I}$.

Ce résultat et le suivant s'obtiennent en combinant les propositions 4.2.1 et C.1.4.5.

Proposition 5.1.18. - Soit I une partie de $\mathbb{N}$. Le foncteur $\rho_{I}$ est adjoint à gauche au foncteur $\varepsilon_{I}$. De surcroît, l'unité $i d \rightarrow \varepsilon_{I} \rho_{I}$ de l'adjonction est un isomorphisme.

Le corollaire 4.1.12 et la proposition C.6.2 fournissent les adjonctions suivantes.

Proposition 5.1.19. - Soit I une partie de $\mathbb{N}$ contenant 0 . Le foncteur $\mathcal{R}_{I, 0}$ : $\mathcal{F}_{\mathcal{G} r, I} \rightarrow \mathcal{F}$ est adjoint à gauche à $\kappa_{I}$ et à droite à $\mathcal{P}_{0, I}$.

Nous terminons ce paragraphe par deux propriétés de compatibilité relatives aux foncteurs entre $\mathcal{F}, \mathcal{F}_{\text {sur } j}$ et $\mathcal{F}_{\mathcal{G} r}$.

Proposition 5.1.20. - Le foncteur composé $\mathcal{F} \stackrel{\iota}{\rightarrow} \mathcal{F}_{\mathcal{G} r} \stackrel{\varepsilon}{\rightarrow} \mathcal{F}_{\text {surj }}$ s'identifie canoniquement au foncteur d'oubli o.

Démonstration. - En effet, le foncteur composé $\mathcal{E}_{\text {surj }}^{f} \stackrel{\mathfrak{D}}{\longrightarrow} \mathcal{E}_{\mathcal{G} r}^{f} \stackrel{\mathfrak{O}}{\longrightarrow} \mathcal{E}^{f}$ s'identifie au foncteur d'inclusion. 
Par adjonction, on en déduit (cf. propositions 5.1.7, 5.1.18 et 2.3.6) le corollaire suivant, que l'on peut évidemment établir par une vérification directe.

Corollaire 5.1.21. - Le foncteur $\omega \circ \rho: \mathcal{F}_{\text {sur } j} \rightarrow \mathcal{F}$ est canoniquement isomorphe $\grave{a} \varpi$.

Décomposition scalaire et tors de Frobenius. - La catégorie $\mathcal{E}_{\mathcal{G} r, I}^{f}(\mathbb{k})$ est munie d'une action naturelle du groupe $\mathbb{k}^{\times}$. De façon analogue à la proposition/définition 2.1.7, on en déduit le résultat suivant.

Proposition et définition 5.1.22. - Étant donné un entier $i$, notons $\left(\mathcal{F}_{\mathcal{G} r, I}\right)_{i}(\mathbb{k})$ la sous-catégorie pleine de $\mathcal{F}_{\mathcal{G} r, I}(\mathbb{k})$ formée des foncteurs $X$ tels que $X\left(\lambda . i d_{V}\right)=$ $\lambda^{i} . i d_{X(V)}$ pour tous $\lambda \in \mathbb{k}^{\times}$et $V \in \mathrm{Ob} \mathcal{E}_{\mathcal{G} r, I}^{f}$. Les inclusions induisent une équivalence de catégories

$$
\mathcal{F}_{\mathcal{G} r, I}(\mathbb{k}) \simeq \prod_{i=1}^{q-1}\left(\mathcal{F}_{\mathcal{G} r, I}\right)_{i}(\mathbb{k})
$$

On notera $X \simeq \bigoplus_{i=1}^{q-1} X_{i}$ la décomposition naturelle d'un foncteur $X$ de $\mathcal{F}_{\mathcal{G} r, I}(\mathbb{k})$ qu'on en déduit, où $X_{i} \in \mathrm{Ob}\left(\mathcal{F}_{\mathcal{G} r, I}\right)_{i}(\mathbb{k})$. On l'appelle décomposition scalaire de $X$.

De même que $\mathcal{F}_{\text {surj }}(\mathbb{k})$, la catégorie $\mathcal{F}_{\mathcal{G} r, I}(\mathbb{k})$ possède un tors de Frobenius, parce que l'automorphisme $\phi$ de la catégorie $\mathcal{E}_{\mathrm{k}}^{f}$ déduit du morphisme de Frobenius se prolonge naturellement en un automorphisme de la catégorie $\mathcal{E}_{\mathcal{G} r, I}^{f}(\mathbb{k})$.

Les foncteurs introduits précédemment possèdent des propriétés de commutation vis-à-vis de la décomposition scalaire et du tors de Frobenius qu'on laisse au lecteur le soin d'expliciter (pour les foncteurs de précomposition, elles se lisent sur le foncteur d'origine entre les catégories sources).

Changement de corps. - Si $K$ est une extension finie de $\mathbb{k}$, les foncteurs d'induction et de restriction induisent des foncteurs $\mathcal{E}_{\mathcal{G} r, I}^{f}(\mathbb{k}) \rightarrow \mathcal{E}_{\mathcal{G} r, I}^{f}(K)$ et $\mathcal{E}_{\mathcal{G} r, I}^{f}(K) \rightarrow$ $\mathcal{E}_{\mathcal{G} r, I}^{f}(\mathbb{k})$, d'où l'on déduit, comme dans $\mathcal{F}$ et $\mathcal{F}_{\text {surj }}$, des foncteurs (encore dits d'induction et de restriction) $\mathcal{F}_{\mathcal{G} r, I}(\mathbb{k}) \rightarrow \mathcal{F}_{\mathcal{G} r, I}(K)$ et $\mathcal{F}_{\mathcal{G}_{r, I}}(K) \rightarrow \mathcal{F}_{\mathcal{G} r, I}(\mathbb{k})$. Néanmoins, comme dans le cas de $\mathcal{F}_{\text {surj }}$, on perd la propriété d'adjonction mutuelle entre induction et restriction.

Tous les foncteurs de précomposition introduits dans ce paragraphe commutent aux changements de corps. En revanche, ce n'est pas le cas du foncteur d'intégrale en grassmanniennes.

\subsection{Structures tensorielles}

Il est naturel, comme le montrera la proposition 5.2.5, d'introduire sur la catégorie de foncteurs en grassmanniennes globale $\mathcal{F}_{\mathcal{G} r}$, à côté de la structure tensorielle usuelle donnée par $\otimes$ (à laquelle nous nous référerons lorsque nous parlerons de structure 
tensorielle sans plus de précision) une seconde structure tensorielle, très analogue à celle donnée par le produit tensoriel total de $\mathcal{F}_{\text {surj }}$ (cf. section 2.1).

Définition 5.2.1 (Produit tensoriel total). - Étant donnés deux objets $X$ et $Y$ de $\mathcal{F}_{\mathcal{G} r}$, on appelle produit tensoriel total de $X$ et $Y$ le foncteur noté $X \widetilde{\otimes} Y$ et défini comme suit.

(1) $\operatorname{Si}(V, W)$ est un objet de $\mathcal{E}_{\mathcal{G} r}^{f}$, on pose

$$
(X \widetilde{\otimes} Y)(V, W)=\bigoplus_{\substack{A, B \in \mathcal{G} r(W) \\ A+B=W}} X(V, A) \otimes Y(V, B) .
$$

(2) Si $u:(V, W) \rightarrow\left(V^{\prime}, W^{\prime}\right)$ est un morphisme de $\mathcal{E}_{\mathcal{G} r}^{f}$, le morphisme $(X \widetilde{\otimes} Y)(u)$ : $(X \widetilde{\otimes} Y)(V, W) \rightarrow(X \widetilde{\otimes} Y)\left(V^{\prime}, W^{\prime}\right)$ est défini comme la somme directe sur les sousespaces $A$ et $B$ de $W$ tels que $A+B=W$ des morphismes

$$
\begin{gathered}
X(V, A) \otimes Y(V, B) \stackrel{X(u) \otimes Y(u)}{\longrightarrow} X\left(V^{\prime}, u(A)\right) \otimes Y\left(V^{\prime}, u(B)\right) \\
\hookrightarrow \bigoplus_{\substack{A^{\prime}, B^{\prime} \in \mathcal{G} r\left(W^{\prime}\right) \\
A^{\prime}+B^{\prime}=W^{\prime}}} X\left(V^{\prime}, A^{\prime}\right) \otimes Y\left(V^{\prime}, B^{\prime}\right)
\end{gathered}
$$

où l'on note encore, par abus, $u$ pour les morphismes $(V, A) \rightarrow\left(V^{\prime}, u(A)\right)$ et $(V, B) \rightarrow$ $\left(V^{\prime}, u(B)\right)$ induits par $u$.

Cette définition fait sens puisque $u(A)+u(B)=u(A+B)=u(W)=W^{\prime}$.

Avant de préciser les liens entre ce produit tensoriel total et celui défini dans $\mathcal{F}_{\text {surj }}$, définissons un produit tensoriel total dans la catégorie auxiliaire $\mathcal{F} \otimes \mathcal{F}_{\text {surj }}$.

Définition 5.2.2. - Le produit tensoriel total sur $\mathcal{F} \otimes \mathcal{F}_{\text {surj }}$ est le foncteur $\widetilde{\otimes}$ : $\left(\mathcal{F} \otimes \mathcal{F}_{\text {surj }}\right) \times\left(\mathcal{F} \otimes \mathcal{F}_{\text {surj }}\right) \rightarrow \mathcal{F} \otimes \mathcal{F}_{\text {surj }}$ donné, via l'isomorphisme $\mathcal{F} \otimes \mathcal{F}_{\text {surj }} \simeq$ $\operatorname{Fct}\left(\mathcal{E}^{f}, \mathcal{F}_{\text {sur } j}\right)$, par

$$
\operatorname{Fct}\left(\mathcal{E}^{f}, \mathcal{F}_{\text {surj }}\right) \times \mathbf{F} \operatorname{ct}\left(\mathcal{E}^{f}, \mathcal{F}_{\text {surj }}\right) \simeq \operatorname{Fct}\left(\mathcal{E}^{f}, \mathcal{F}_{\text {surj }} \times \mathcal{F}_{\text {surj }}\right) \stackrel{\widetilde{\otimes}_{*}}{\longrightarrow} \boldsymbol{F} \operatorname{ct}\left(\mathcal{E}^{f}, \mathcal{F}_{\text {surj }}\right) .
$$

Autrement dit,

$$
(X \widetilde{\otimes} Y)(A, B)=\bigoplus_{\substack{V, W \in \mathcal{G} r(B) \\ V+W=B}} X(A, V) \otimes Y(A, W) \quad\left(A \in \mathrm{Ob} \mathcal{E}^{f}, B \in \mathrm{Ob} \mathcal{E}_{\text {surj }}^{f}\right) .
$$

Proposition 5.2.3. - (1) Le produit tensoriel total définit sur $\mathcal{F}_{\mathcal{G} r}$ une structure monoïdale symétrique exacte d'unité $\rho\left(\mathrm{Is}_{0}\right)$.

(2) Il existe un monomorphisme $X \otimes Y \hookrightarrow X \widetilde{\otimes} Y$ naturel en les objets $X$ et $Y$ de $\mathcal{F}_{\mathcal{G} r}$. 
(3) Les produits tensoriels totaux sur les catégories $\mathcal{F}_{\mathcal{G} r}, \mathcal{F}_{\text {surj }}$ et $\mathcal{F} \otimes \mathcal{F}_{\text {surj }}$ vérifient les propriétés de compatibilité suivantes : on a des isomorphismes naturels

$$
\begin{array}{lll}
\rho(A \widetilde{\otimes} B) & \simeq \rho(A) \widetilde{\otimes} \rho(B) & \left(A, B \in \mathrm{Ob} \mathcal{F}_{\text {surj }}\right), \\
\xi(F \widetilde{\otimes} G) \simeq \xi(F) \widetilde{\otimes} \xi(G) & \left(F, G \in \mathrm{Ob} \mathcal{F} \otimes \mathcal{F}_{\text {surj }}\right) .
\end{array}
$$

Cette proposition se vérifie par inspection.

Remarque 5.2.4. - En revanche, les foncteurs $\varepsilon, \sigma$ et $\theta$ ne commutent pas au produit tensoriel total. Par exemple, on a

$$
\theta(F \widetilde{\otimes} G)(V, W) \simeq(F \widetilde{\otimes} G)(V / W, W)=\bigoplus_{\substack{A, B \in \mathcal{G} r(W) \\ A+B=W}} F(V / W, A) \otimes G(V / W, B)
$$

tandis que

$$
\begin{gathered}
(\theta(F) \widetilde{\otimes} \theta(G))(V, W)=\bigoplus_{\substack{A, B \in \mathcal{G} r(W) \\
A+B=W}} \theta(F)(V, A) \otimes \theta(G)(V, B) \\
\simeq \bigoplus_{\substack{A, B \in \mathcal{G} r(W) \\
A+B=W}} F(V / A, A) \otimes G(V / B, B)
\end{gathered}
$$

On voit cependant ainsi qu'il existe un épimorphisme canonique $\theta(F) \widetilde{\otimes} \theta(G) \rightarrow$ $\theta(F \widetilde{\otimes} G)$.

On dispose d'un foncteur $\mathcal{E}_{\mathcal{G} r}^{f} \times \mathcal{E}_{\mathcal{G} r}^{f} \rightarrow \mathcal{E}_{\mathcal{G} r}^{f}$ donné sur les objets par $\left((A, B),\left(A^{\prime}, B^{\prime}\right)\right) \mapsto$ $\left(A \oplus A^{\prime}, B \oplus B^{\prime}\right)$, dont l'action sur les morphismes se déduit de la fonctorialité de $\oplus: \mathcal{E}^{f} \times \mathcal{E}^{f} \rightarrow \mathcal{E}^{f}$. La proposition suivante montre l'utilité du produit tensoriel total à l'aide de ce foncteur.

Proposition 5.2.5. - Il existe dans $\mathcal{F}_{\mathcal{G} r}$ un isomorphisme $P_{(A, B)}^{\mathcal{G} r} \widetilde{\otimes} P_{\left(A^{\prime}, B^{\prime}\right)}^{\mathcal{G} r} \simeq$ $P_{\left(A \oplus A^{\prime}, B \oplus B^{\prime}\right)}^{\mathcal{G} r}$ naturel en les objets $(A, B)$ et $\left(A^{\prime}, B^{\prime}\right)$ de $\mathcal{E}_{\mathcal{G} r}^{f}$.

Démonstration. — Cet énoncé découle de la proposition 4.3.7.

Corollaire 5.2.6. - Le produit tensoriel total de $\mathcal{F}_{\mathcal{G} r}$ préserve les objets projectifs, les objets de type fini et les objets $p f_{n}$.

Remarque 5.2.7. - (1) Le produit tensoriel usuel de $\mathcal{F}_{\mathcal{G} r}$ préserve également les objets de type fini et $\mathrm{pf}_{n}$.

(2) Cependant, le produit tensoriel usuel de deux objets projectifs de $\mathcal{F}_{\mathcal{G} r}$ n'est généralement pas projectif, car il en est ainsi dans $\mathcal{F}_{\text {surj }}$ - par exemple, $P_{\mathbb{k}}^{\text {surj }} \otimes P_{E_{2}}^{\text {surj }}$ n'est pas projectif.

(3) Le produit tensoriel de deux objets projectifs d'une catégorie $\mathcal{F}_{\mathcal{G} r, n}$ est en revanche projectif. Cela provient de ce que le produit tensoriel d'un $\mathbb{k}\left[G L_{n}(\mathbb{k})\right]$-module projectif et d'un $\mathbb{k}\left[G L_{n}(\mathbb{k})\right]$-module fini est projectif, de ce que le produit tensoriel dans $\mathcal{F}$ de deux projectifs est projectif, et de l'isomorphisme (6) de la proposition 5.1.12. 
Contrairement à la situation que nous venons d'observer pour les projectifs, le produit tensoriel total possède un comportement déplaisant sur les injectifs. En revanche, le produit tensoriel ordinaire est adapté à leur étude.

On rappelle que le symbole $G r$ qui intervient dans la proposition suivante a été introduit dans la notation 4.3.8.

Proposition 5.2.8. - Il existe un isomorphisme

$$
I_{(A, B)}^{\mathcal{G} r} \otimes I_{\left(A^{\prime}, B^{\prime}\right)}^{\mathcal{G} r} \simeq \bigoplus_{C \in G r\left(B, B^{\prime}\right)} I_{\left(A \oplus A^{\prime}, C\right)}^{\mathcal{G} r}
$$

naturel en les objets $(A, B)$ et $\left(A^{\prime}, B^{\prime}\right)$ de $\mathcal{E}_{\mathcal{G} r}^{f}$.

Démonstration. — C'est la version linéarisée de la proposition 4.3.9.

Corollaire 5.2.9. - Le produit tensoriel de $\mathcal{F}_{\mathcal{G} r}$ préserve :

(1) les objets injectifs de co-type fini;

(2) les objets de co-type fini;

(3) les objets co-pf $f_{n}$.

Nous utiliserons également, dans la partie IV, la proposition 5.2.8 par l'intermédiaire du résultat suivant.

Corollaire 5.2.10. - Soient $(V, W)$ et $(A, B)$ deux objets de $\mathcal{E}_{\mathcal{G} r}^{f}$. Le morphisme $i_{(A, B)}: \mathbb{k} \rightarrow I_{(A, B)}^{\mathcal{G} r}$ correspondant à $1 \in \mathbb{k}$ par le lemme de Yoneda induit, par tensorisation par $I_{(V, W)}^{\mathcal{G} r}$, un morphisme

$$
I_{(V, W)}^{\mathcal{G} r} \rightarrow I_{(V, W)}^{\mathcal{G} r} \otimes I_{(A, B)}^{\mathcal{G} r} \simeq \bigoplus_{W^{\prime} \in G r(W, B)} I_{\left(V \oplus A, W^{\prime}\right)}^{\mathcal{G} r}
$$

(cf. proposition 5.2.8) dont les composantes $I_{(V, W)}^{\mathcal{G} r} \rightarrow I_{\left(V \oplus A, W^{\prime}\right)}^{\mathcal{G} r}$ sont induites par la projection $V \oplus A \rightarrow V$.

Démonstration. - La composante $I_{(V, W)}^{\mathcal{G} r} \rightarrow I_{\left(V \oplus A, W^{\prime}\right)}^{\mathcal{G} r}$ de $i_{(A, B)} \otimes I_{(V, W)}^{\mathcal{G} r}$ s'obtient par application du foncteur $E \mapsto \mathbb{k}^{E}: \mathbf{E n s}^{o p} \rightarrow \mathcal{E}$ à la transformation naturelle

$\operatorname{hom}_{\mathcal{E}_{\mathcal{G} r}^{f}}\left(\cdot,\left(V \oplus A, W^{\prime}\right)\right) \hookrightarrow \operatorname{hom}_{\mathcal{E}_{\mathcal{G} r}^{f}}(\cdot,(V, W)) \times \operatorname{hom}_{\mathcal{E}_{\mathcal{G} r}^{f}}(\cdot,(A, B)) \rightarrow \operatorname{hom}_{\mathcal{E}_{\mathcal{G} r}^{f}}(\cdot,(V, W))$, qui est induite par la projection $V \oplus A \rightarrow V$, d'où le corollaire.

La proposition suivante (qui généralise la dernière assertion de la proposition 2.3.6) constitue la principale motivation de l'introduction du produit tensoriel total dans $\mathcal{F}_{\mathcal{G} r}$.

Proposition 5.2.11. - Il existe dans $\mathcal{F}$ un isomorphisme

$$
\omega(X \widetilde{\otimes} Y) \simeq \omega(X) \otimes \omega(Y)
$$

naturel en les objets $X$ et $Y$ de $\mathcal{F}_{\mathcal{G} r}$. 
Démonstration. - Cela provient, par linéarisation, de la décomposition ensembliste

$$
\mathcal{G} r(V) \times \mathcal{G} r(V) \simeq \coprod_{W \in \mathcal{G} r(V)}\{(A, B) \in \mathcal{G} r(W) \times \mathcal{G} r(W) \mid A+B=W\}
$$

naturelle en l'objet $V$ de $\mathcal{E}^{f}$.

\subsection{Le foncteur différence}

Comme dans la catégorie $\mathcal{F}$, il existe dans les catégories $\mathcal{F}_{\mathcal{G} r, I}$ un foncteur différence, fondamental dans l'étude de ces catégories, notamment de leurs objets finis. L'analogie avec le cas de $\mathcal{F}$ est particulièrement étroite du fait que le foncteur différence des $\mathcal{F}_{\mathcal{G} r, I}$ « n'agit pas sur la base ».

Convention 5.3.1. - Dans toute cette section, $J$ désigne une partie de $\mathbb{N}$.

Définition 5.3.2 (Foncteurs de décalage et foncteur différence dans $\mathcal{F}_{\mathcal{G} r, J}$ )

(1) Soit $V$ un objet de $\mathcal{E}^{f}$. Le foncteur $\mathcal{F}_{\mathcal{G} r, J} \rightarrow \mathcal{F}_{\mathcal{G} r, J}$ de précomposition par le foncteur de translation $V \boxplus \cdot: \mathcal{E}_{\mathcal{G} r, J}^{f} \rightarrow \mathcal{E}_{\mathcal{G} r, J}^{f}$ (cf. définition 4.1.6) est appelé foncteur de décalage par $V$ et se note $\Delta_{V}^{\mathcal{G} r, J}$.

On a ainsi $\Delta_{V}^{\mathcal{G} r, J} X(A, B)=X(V \oplus A, B)$ pour $X \in \mathrm{Ob} \mathcal{F}_{\mathcal{G} r, J}$ et $(A, B) \in \operatorname{Ob} \mathcal{E}_{\mathcal{G} r, J}^{f}$.

La bifonctorialité de $\boxplus$ rend l'association $V \mapsto \Delta_{V}^{\mathcal{G} r, J}$ fonctorielle.

(2) Le foncteur différence $\Delta^{\mathcal{G} r, J}$ de $\mathcal{F}_{\mathcal{G} r, J}$ est le noyau de la transformation naturelle $\Delta_{\mathrm{k}}^{\mathcal{G} r, J} \rightarrow i d$ induite par le morphisme $\mathbb{k} \rightarrow 0$ de $\mathcal{E}^{f}$.

La proposition suivante, laissée au lecteur, montre que le comportement de ces foncteurs ne dépend guère de la partie $J$ de $\mathbb{N}$.

Proposition 5.3.3. - Si I est un sous-ensemble de J, le diagramme

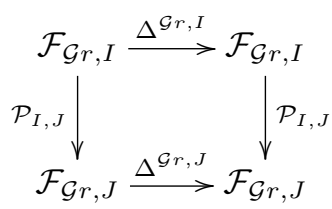

commute à isomorphisme canonique près, lorsque le prolongement par zéro $\mathcal{P}_{I, J}$ est défini. Il existe une propriété analogue relative au foncteur de restriction $\mathcal{R}_{J, I}$.

Une grande part des considérations relatives aux foncteurs de décalage et différence de $\mathcal{F}$ se transcrivent dans $\mathcal{F}_{\mathcal{G} r, J}$. Notons tout d'abord que la transformation naturelle $i d \rightarrow \Delta_{\mathbb{k}}^{\mathcal{G} r, J}$ procure un scindement canonique

$$
\Delta_{\mathrm{lk}}^{\mathcal{G} r, J} \simeq \Delta^{\mathcal{G} r, J} \oplus i d,
$$

de sorte que $\Delta^{\mathcal{G} r, J}$ commute aux limites et colimites, comme les foncteurs de décalage. 
Une autre propriété fondamentale, similaire à la proposition 1.3 .1 relative à $\mathcal{F}$, réside dans l'existence d'adjoints à gauche et à droite exacts et explicites aux foncteurs de décalage et différence.

Proposition 5.3.4. - Soit $V$ un objet de $\mathcal{E}^{f}$. Le foncteur $\Delta_{V}^{\mathcal{G} r, J}$ est adjoint :

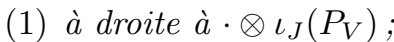

(2) à gauche $\grave{a} \cdot \otimes \kappa_{J}\left(I_{V}\right)$.

Ces adjonctions sont naturelles en $V$.

Démonstration. - On combine les propositions C.3.2 et C.3.3 avec les isomorphismes de la proposition 4.1.11.

Corollaire 5.3.5. - Le foncteur différence $\Delta^{\mathcal{G} r, J}$ est adjoint :

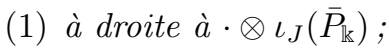

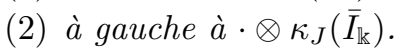

Corollaire 5.3.6. - Les foncteurs de décalage et le foncteur différence de $\mathcal{F}_{\mathcal{G} r, J}$ conservent les objets projectifs et les objets injectifs.

Démonstration. - C'est une conséquence formelle de la proposition et du corollaire précédents et de l'exactitude des adjoints.

Nous évaluons maintenant les foncteurs de décalage sur les objets projectifs et injectifs standard de la catégorie $\mathcal{F}_{\mathcal{G} r, J}$.

Proposition 5.3.7. - Il existe des isomorphismes

$$
\Delta_{V}^{\mathcal{G} r, J}\left(P_{(A, B)}^{\mathcal{G} r, J}\right) \simeq P_{A / B}(V) \otimes P_{(A, B)}^{\mathcal{G} r, J}
$$

et

$$
\Delta_{V}^{\mathcal{G} r, J}\left(I_{(A, B)}^{\mathcal{G} r, J}\right) \simeq I_{A}(V) \otimes I_{(A, B)}^{\mathcal{G} r, J}
$$

naturels en les objets $V$ de $\mathcal{E}^{f}$ et $(A, B)$ de $\mathcal{E}_{\mathcal{G} r, J}^{f}$.

Démonstration. — C'est une conséquence formelle de la proposition 4.1.11.

Comme dans le cas de $\mathcal{F}$, cette propriété a l'utile conséquence suivante.

Corollaire 5.3.8. - Les foncteurs de décalage et le foncteur différence de $\mathcal{F}_{\mathcal{G} r, J}$ conservent les objets de type fini, de co-type fini, $p f_{n}$ et co-pf $f_{n}$.

Démonstration. - Ce corollaire se déduit de la proposition 5.3.7 et de la commutation des foncteurs de décalage et différence aux limites et colimites.

La proposition suivante donne les propriétés de compatibilité entre les deux produits tensoriels de $\mathcal{F}_{\mathcal{G} \text { r }}$ et son foncteur différence. 
Proposition 5.3.9. - Soient $X$ et $Y$ deux objets de $\mathcal{F}_{\mathcal{G} r, J}$ et $V$ un objet de $\mathcal{E}^{f}$. Il existe des isomorphismes naturels

$$
\begin{gathered}
\Delta_{V}^{\mathcal{G} r}(X \otimes Y) \simeq \Delta_{V}^{\mathcal{G} r} X \otimes \Delta_{V}^{\mathcal{G} r} Y \\
\Delta^{\mathcal{G} r}(X \otimes Y) \simeq\left(\Delta^{\mathcal{G} r} X \otimes Y\right) \oplus\left(X \otimes \Delta^{\mathcal{G} r} Y\right) \oplus\left(\Delta^{\mathcal{G} r} X \otimes \Delta^{\mathcal{G} r} Y\right)
\end{gathered}
$$

et, dans le cas où $J=\mathbb{N}$,

$$
\begin{gathered}
\Delta_{V}^{\mathcal{G} r}(X \widetilde{\otimes} Y) \simeq \Delta_{V}^{\mathcal{G} r} X \widetilde{\otimes} \Delta_{V}^{\mathcal{G} r} Y, \\
\Delta^{\mathcal{G} r}(X \widetilde{\otimes} Y) \simeq\left(\Delta^{\mathcal{G} r} X \widetilde{\otimes} Y\right) \oplus\left(X \widetilde{\otimes} \Delta^{\mathcal{G} r} Y\right) \oplus\left(\Delta^{\mathcal{G} r} X \widetilde{\otimes} \Delta^{\mathcal{G} r} Y\right) .
\end{gathered}
$$

Démonstration. - Établissons la première assertion relative au produit tensoriel total : si $(A, B)$ est un objet de $\mathcal{E}_{\mathcal{G} r}^{f}$, on a

$$
\begin{gathered}
\Delta_{V}(X \widetilde{\otimes} Y)(A, B)=(X \widetilde{\otimes} Y)(V \oplus A, B)=\bigoplus_{\substack{W, W^{\prime} \in \mathcal{G} r(B) \\
W+W^{\prime}=B}} X(V \oplus A, W) \otimes Y\left(V \oplus A, W^{\prime}\right) \\
=\bigoplus_{\substack{W, W^{\prime} \in \mathcal{G} r(B) \\
W+W^{\prime}=B}} \Delta_{V}(X)(A, W) \otimes \Delta_{V}(Y)\left(A, W^{\prime}\right)=\Delta_{V}(X) \widetilde{\otimes} \Delta_{V}(Y)(A, B) .
\end{gathered}
$$

L'inclusion canonique

$$
\begin{gathered}
X \widetilde{\otimes} Y \hookrightarrow \Delta_{\mathrm{k}}^{\mathcal{G} r}(X \widetilde{\otimes} Y)=\Delta_{\mathrm{k}}^{\mathcal{G} r}(X) \widetilde{\otimes} \Delta_{\mathrm{k}}^{\mathcal{G} r}(Y) \simeq\left(X \oplus \Delta^{\mathcal{G} r}(X)\right) \widetilde{\otimes}\left(Y \oplus \Delta^{\mathcal{G} r}(Y)\right) \\
\simeq(X \widetilde{\otimes} Y) \oplus\left(X \widetilde{\otimes} \Delta^{\mathcal{G} r}(Y)\right) \oplus\left(\Delta^{\mathcal{G} r}(X) \widetilde{\otimes} Y\right) \oplus\left(\Delta^{\mathcal{G} r}(X) \widetilde{\otimes} \Delta^{\mathcal{G} r}(Y)\right)
\end{gathered}
$$

s'identifie à l'inclusion du facteur direct, d'où le dernier point.

La première assertion relative au produit tensoriel ordinaire résulte de ce que $\Delta_{V}^{\mathcal{G} r}$ est un foncteur de précomposition; la seconde s'en déduit comme dans le cas du produit tensoriel total.

Commutation des foncteurs fondamentaux aux foncteurs différences. Conformément à nos conventions générales (cf. notation C.1.1), l'endofoncteur $\Delta_{*}$ de $\mathcal{F} \otimes \mathcal{F}_{\text {surj }}^{J} \simeq \operatorname{F} \operatorname{ct}\left(\mathcal{E}_{\text {surj }}^{J}, \mathcal{F}\right)$ qui apparaît dans la proposition suivante est donné par la postcomposition par le foncteur différence $\Delta$ de $\mathcal{F}$.

Proposition 5.3.10. - (1) On a des isomorphismes canoniques $\Delta^{\mathcal{G} r, J} \circ \iota_{J} \simeq$ $\iota_{J} \circ \Delta$ et $\Delta^{\mathcal{G} r, J} \circ \kappa_{J} \simeq \kappa_{J} \circ \Delta$ de foncteurs $\mathcal{F} \rightarrow \mathcal{F}_{\mathcal{G} r, J}$.

(2) On a des isomorphismes canoniques $\Delta^{\mathcal{G} r, J} \circ \xi_{J} \simeq \xi_{J} \circ \Delta_{*}$ et $\Delta^{\mathcal{G} r, J} \circ \theta_{J} \simeq \theta_{J} \circ \Delta_{*}$ de foncteurs $\mathcal{F} \otimes \mathcal{F}_{\text {sur } j}^{J} \rightarrow \mathcal{F}_{\mathcal{G} r, J}$.

(3) On a un isomorphisme canonique $\Delta_{*} \circ \sigma_{J} \simeq \sigma_{J} \circ \Delta^{\mathcal{G} r, J}$ de foncteurs $\mathcal{F}_{\mathcal{G} r, J} \rightarrow$ $\mathcal{F} \otimes \mathcal{F}_{\text {surj }}^{J}$. 
Démonstration. - Établissons par exemple le dernier point, les autres se montrant de manière analogue. Le diagramme

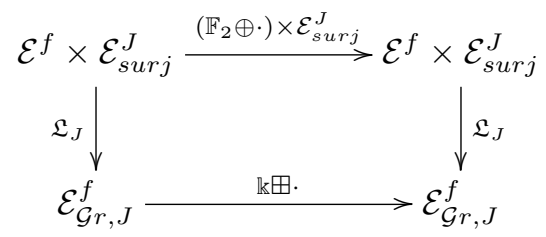

commute à isomorphisme canonique près, d'où un isomorphisme canonique $\widetilde{\Delta}_{*} \circ \sigma_{J} \simeq$ $\sigma_{J} \circ \Delta_{\mathrm{k}}^{\mathcal{G} r, J}$, puis $\Delta_{*} \circ \sigma_{J} \simeq \sigma_{J} \circ \Delta^{\mathcal{G} r, J}$.

\subsection{Foncteurs polynomiaux}

Le foncteur différence de $\mathcal{F}_{\mathcal{G} r, J}$ permet d'introduire les foncteurs polynomiaux de cette catégorie. On commence, à l'aide du lemme et de la proposition suivants, par identifier le noyau du foncteur différence, qui, contrairement au cas de la catégorie $\mathcal{F}$, ne se réduit pas aux foncteurs constants.

Lemme 5.4.1. - Le foncteur composé $\Delta^{\mathcal{G} r, J} \rho_{J}: \mathcal{F}_{\text {surj }}^{J} \rightarrow \mathcal{F}_{\mathcal{G} r, J}$ est nul.

Démonstration. - Pour tout $V \in \mathrm{Ob} \mathcal{E}^{f}$, l'injection naturelle $\rho_{J} \hookrightarrow \Delta_{V}^{\mathcal{G} r, J} \circ \rho_{J}$ est un isomorphisme, puisque le diagramme

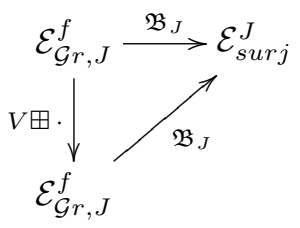

commute (à isomorphisme naturel près).

\section{Proposition et définition 5.4.2 (Foncteurs pseudo-constants)}

Soit $X$ un objet de $\mathcal{F}_{\mathcal{G} r, J}$. Les conditions suivantes sont équivalentes :

(1) le foncteur $\Delta^{\mathcal{G} r, J} X$ est nul;

(2) la coünité $\rho_{J} \varepsilon_{J}(X) \rightarrow X$ de l'adjonction de la proposition 5.1 .18 est un isomorphisme;

(3) il existe un objet $R$ de $\mathcal{F}_{\text {surj }}^{J}$ tel que $X$ est isomorphe à $\rho_{J}(R)$.

Lorsqu'elles sont vérifiées, nous dirons que $X$ est un foncteur pseudo-constant.

Démonstration. - La coünité $\rho_{J} \varepsilon_{J}(X) \rightarrow X$ est donnée sur l'objet $(V, W)$ de $\mathcal{E}_{\mathcal{G} r, J}^{f}$ par l'injection $X(W, W) \hookrightarrow X(V, W)$ induite par le morphisme canonique $(W, W) \rightarrow$ $(V, W)$ de $\mathcal{E}_{\mathcal{G} r, J}^{f}$. La première assertion signifie que le monomorphisme canonique $X(A, B) \hookrightarrow X(A \oplus \mathbb{k}, B)$ est un isomorphisme pour tout objet $(A, B)$ de $\mathcal{E}_{\mathcal{G} r, J}^{f}$, on en 
déduit donc que le morphisme précédent est un isomorphisme par récurrence sur la codimension de $W$ dans $V$. Ainsi, la première assertion implique la deuxième.

Il est clair que la deuxième implique la troisième. Enfin, la troisième entraîne la première par le lemme 5.4.1.

Nous introduisons maintenant la notion générale de foncteur polynomial et de foncteur analytique de $\mathcal{F}_{\mathcal{G} r, J}$, de la même manière que dans $\mathcal{F}$. Nous avons précédemment identifié les foncteurs polynomiaux de degré 0 .

\section{Définition 5.4.3 (Foncteurs polynomiaux, analytiques dans $\mathcal{F}_{\mathcal{G} r, J}$ )}

Un foncteur $X \in \mathrm{Ob} \mathcal{F}_{\mathcal{G} r, J}$ est dit :

(1) polynomial s'il existe un entier naturel $n$ tel que $\left(\Delta^{\mathcal{G} r, J}\right)^{n} X=0$;

(2) analytique s'il est colimite de ses sous-foncteurs polynomiaux.

Le degré d'un foncteur polynomial $X$ est le plus grand entier positif $n$ tel que $\left(\Delta^{\mathcal{G} r, J}\right)^{n} X \neq 0$ si $X$ est non nul, on le note $\operatorname{deg} X$; on pose également $\operatorname{deg} 0=-\infty$.

Notation 5.4.4. - Nous désignerons par $\mathcal{F}_{\mathcal{G} r, J}^{k}$ la sous-catégorie pleine de $\mathcal{F}_{\mathcal{G} r, J}$ formée des foncteurs polynomiaux de degré au plus $k$.

La proposition 5.3.9 a la conséquence suivante.

Proposition 5.4.5. - (1) Si $X$ et $Y$ sont deux foncteurs polynomiaux de $\mathcal{F}_{\mathcal{G} r, J}$, alors $X \otimes Y$ et $X \widetilde{\otimes} Y$ sont polynomiaux et $\operatorname{deg}(X \otimes Y)=\operatorname{deg}(X \widetilde{\otimes} Y)=\operatorname{deg} X+\operatorname{deg} Y$.

(2) Le produit tensoriel total (donc aussi usuel) de deux foncteurs analytiques de $\mathcal{F}_{\mathcal{G} r, J}$ est analytique.

(3) Un foncteur $X$ de $\mathcal{F}_{\mathcal{G} r, J}$ est polynomial si et seulement si $\Delta^{\mathcal{G} r, J} X$ est polynomial; dans ce cas $\operatorname{deg}\left(\Delta^{\mathcal{G} r, J} X\right)=\operatorname{deg} X-1$ si $X$ n'est pas pseudo-constant.

Afin de donner les propriétés de conservation des foncteurs polynomiaux ou analytiques par les foncteurs fondamentaux de source ou de but $\mathcal{F}_{\mathcal{G} r, J}$, nous avons besoin d'introduire la notion de foncteur polynomial ou analytique dans la catégorie $\mathcal{F} \otimes \mathcal{F}_{\text {surj }}^{J} \simeq \operatorname{Fct}\left(\mathcal{E}_{\text {surj }}^{J}, \mathcal{F}\right)$.

Proposition et définition 5.4.6. - Soient $X$ un objet de $\mathcal{F} \otimes \mathcal{F}_{\text {surj }}^{J}$ et $i \in \mathbb{N}$.

(1) Les assertions suivantes sont équivalentes.

(a) Le foncteur $\Delta_{*}^{i} X$ est nul.

(b) Le foncteur $X$ appartient à la sous-catégorie épaisse $\mathbf{F} \operatorname{ct}\left(\mathcal{E}_{\text {surj }}^{J}, \mathcal{F}^{i-1}\right)$ de $\operatorname{Fct}\left(\mathcal{E}_{\text {surj }}^{J}, \mathcal{F}\right)$.

Lorsque ces conditions sont vérifiées, nous dirons que $X$ est un foncteur polynomial de degré strictement inférieur à $i$.

(2) On dit que $X$ est analytique s'il est colimite de ses sous-foncteurs polynomiaux.

Démonstration. - C'est une application directe de la proposition C.1.3.

L'énoncé qui suit découle quant à lui de la proposition 5.3.10. 
Proposition 5.4.7. - (1) $\quad$ (a) Si F est un objet de $\mathcal{F}, F$ est polynomial si et seulement si $\iota_{J}(F)$ est un objet polynomial de $\mathcal{F}_{\mathcal{G} r, J}$. Ils ont alors même degré.

(b) Si F est un objet de $\mathcal{F}, F$ est polynomial si et seulement si $\kappa_{J}(F)$ est un objet polynomial de $\mathcal{F}_{\mathcal{G} r, J}$. Ils ont alors même degré.

(2) (a) Un objet $F$ de $\mathcal{F} \otimes \mathcal{F}_{\text {surj }}^{J}$ est polynomial si et seulement si l'objet $\xi_{J}(F)$ de $\mathcal{F}_{\mathcal{G} r, J}$ est polynomial. Ils ont alors même degré.

(b) Si $F$ est un objet de $\mathcal{F} \otimes \mathcal{F}_{\text {surj }}^{J}, F$ est polynomial si et seulement si $\theta_{J}(F)$ est un objet polynomial de $\mathcal{F}_{\mathcal{G} r, J}$. Ils ont alors même degré.

(3) Un objet $X$ de $\mathcal{F}_{\mathcal{G} r, J}$ est polynomial si et seulement si l'objet $\sigma_{J}(X)$ de $\mathcal{F} \otimes \mathcal{F}_{\text {sur }}^{J}$ est polynomial. Ils ont alors même degré.

Comme les foncteurs considérés commutent aux colimites, on en déduit le corollaire suivant.

Corollaire 5.4.8. - Les foncteurs $\iota_{J}, \kappa_{J}, \xi_{J}, \theta_{J}$ et $\sigma_{J}$ préservent les foncteurs analytiques.

Remarque 5.4.9. - En revanche, le foncteur d'intégrale en grassmanniennes $\omega$ : $\mathcal{F}_{\mathcal{G} r} \rightarrow \mathcal{F}$ ne préserve pas les foncteurs analytiques. Nous reviendrons en détail sur cette observation essentielle dans la partie III.

Quotients de la filtration polynomiale de $\mathcal{F}_{\mathcal{G}_{r, J}}$ - - Grâce à la proposition 5.4.2, la catégorie $\mathcal{F}_{\mathcal{G} r, J}^{0}$ est équivalente à la catégorie $\mathcal{F}_{\text {surj }}^{J}$. À l'aide des lemmes 5.4.10 et 5.4.12, nous allons généraliser ce résultat en identifiant les catégories quotients $\mathcal{F}_{\mathcal{G} r, J}^{k} / \mathcal{F}_{\mathcal{G} r, J}^{k-1}$ pour tout entier $k$. Dans la section suivante, nous appliquerons ces résultats à la description des objets simples de $\mathcal{F}_{\mathcal{G} r, J}$.

Lemme 5.4.10. - Soit $X$ un objet polynomial de degré $i \geq 0$ de $\mathcal{F}_{\mathcal{G} r, J}$. Le noyau de l'épimorphisme canonique $p_{X}: \xi_{J} \sigma_{J}(X) \rightarrow X$ (coünité de l'adjonction de la proposition 5.1.17) est de degré strictement inférieur à $i$.

Démonstration. - La proposition 5.3.10 montre que les endofoncteurs $\xi_{J} \sigma_{J}$ et $\Delta^{\mathcal{G} r, J}$ de $\mathcal{F}_{\mathcal{G} r, J}$ commutent à isomorphisme près; de plus, $\Delta^{\mathcal{G} r, J}\left(p_{X}\right) \simeq p_{\Delta \mathcal{G}_{r, J}{ }_{X}}$. Comme $p_{X}$ est un isomorphisme lorsque le foncteur $X$ est pseudo-constant, cela donne la conclusion.

Lemme 5.4.11. - Si $F$ est un objet polynomial de degré $i \geq 0$ de $\mathcal{F} \otimes \mathcal{F}_{\text {surj }}^{J}$, le noyau de l'épimorphisme canonique $\xi_{J}(F) \rightarrow \theta_{J}(F)$ est de degré strictement inférieur à $i$.

Démonstration. - Ce lemme s'obtient par application du précédent à l'objet $\theta_{J}(F)$ de $\mathcal{F}_{\mathcal{G} r, J}$, qui est de degré $i$ par la proposition 5.4.7, puisque $\sigma_{J} \circ \theta_{J} \simeq i d$ (proposition 5.1.16).

Le lemme suivant se démontre de la même façon que le lemme 5.4.10. 
Lemme 5.4.12. - Soit $F$ un objet polynomial de degré $i \geq 0$ de $\mathcal{F} \otimes \mathcal{F}_{\text {surj }}^{J}$. Le conoyau du monomorphisme canonique $F \hookrightarrow \sigma_{J} \xi_{J}(F)$ (unité de l'adjonction de la proposition 5.1.17) est de degré strictement inférieur à $i$.

Les lemmes 5.4.10 et 5.4.12 impliquent l'important résultat suivant.

Proposition 5.4.13. - (1) Le foncteur $\xi_{J}: \mathbf{F c t}\left(\mathcal{E}^{f} \times \mathcal{E}_{\text {surj }}^{J}, \mathcal{E}\right) \simeq \mathbf{F c t}\left(\mathcal{E}_{\text {surj }}^{J}, \mathcal{F}\right) \rightarrow$ $\mathcal{F}_{\mathcal{G} r, J}$ induit un foncteur $\mathbf{F} \operatorname{ct}\left(\mathcal{E}_{\text {surj }}^{J}, \mathcal{F}^{n} / \mathcal{F}^{n-1}\right) \rightarrow \mathcal{F}_{\mathcal{G} r, J}^{n} / \mathcal{F}_{\mathcal{G} r, J}^{n-1}$ pour tout $n \in \mathbb{N}$.

(2) Ce foncteur est une équivalence de catégories dont un inverse est donné par le foncteur $\mathcal{F}_{\mathcal{G} r, J}^{n} / \mathcal{F}_{\mathcal{G} r, J}^{n-1} \rightarrow \operatorname{Fct}\left(\mathcal{E}_{\text {surj }}^{J}, \mathcal{F}^{n} / \mathcal{F}^{n-1}\right)$ induit par $\sigma_{J}: \mathcal{F}_{\mathcal{G} r, J} \rightarrow \operatorname{Fct}\left(\mathcal{E}^{f} \times\right.$ $\left.\mathcal{E}_{\text {surj }}^{J}, \mathcal{E}\right) \simeq \operatorname{Fct}\left(\mathcal{E}_{\text {surj }}^{J}, \mathcal{F}\right)$.

Nous utiliserons également la description suivante de l'équivalence de catégories donnée par la proposition 5.4.13.

Proposition 5.4.14. - Le foncteur $\mathbf{F c t}\left(\mathcal{E}_{\text {surj }}^{J}, \mathcal{F}^{n} / \mathcal{F}^{n-1}\right) \rightarrow \mathcal{F}_{\mathcal{G} r, J}^{n} / \mathcal{F}_{\mathcal{G} r, J}^{n-1}$ induit par $\theta_{J}$ est le même que celui qu'induit $\xi_{J}$.

Démonstration. - Il s'agit d'une conséquence directe du lemme 5.4.11.

Les propositions 5.4.13 et 1.4.1 fournissent le corollaire suivant.

Corollaire 5.4.15. - On suppose $d=1$, i.e. le corps $\mathbb{k}$ premier.

Pour tout entier naturel $n$, les catégories $\mathcal{F}_{\mathcal{G} r, J}^{n} / \mathcal{F}_{\mathcal{G} r, J}^{n-1}$ et $\mathbf{F} \mathbf{c t}\left(\mathcal{E}_{\text {surj }}^{J}, \operatorname{Mod}_{\mathbb{k}\left[\Sigma_{n}\right]}\right)$ sont équivalentes.

\subsection{Foncteurs finis}

Les résultats de la section précédente permettent de décrire les objets simples et les objets finis des catégories $\mathcal{F}_{\mathcal{G} r, I}$ à partir de ceux de $\mathcal{F}$ et $\mathcal{F}_{\text {surj }}^{I}$. Comme pour la catégorie $\mathcal{F}$, l'un des résultats les plus importants réside dans le caractère polynomial des objets finis.

Convention 5.5.1. - Dans cette section, $I$ désigne une partie de $\mathbb{N}$.

Lemme 5.5.2. - Soit $X$ un objet de $\mathcal{F}_{\mathcal{G} r, I}$ tel que $\Delta^{\mathcal{G} r, I} X$ est un objet fini de $\mathcal{F}_{\mathcal{G} r, I}$ et $\varepsilon_{I}(X)$ un objet fini de $\mathcal{F}_{\text {surj }}^{I}$. Alors $X$ est fini.

Démonstration. - Le foncteur exact

$$
\left(\Delta^{\mathcal{G} r, I}, \varepsilon_{I}\right): \mathcal{F}_{\mathcal{G} r, I} \rightarrow \mathcal{F}_{\mathcal{G}_{r, I}} \times \mathcal{F}_{\text {surj }}^{I} .
$$

est fidèle : son noyau est constitué des foncteurs pseudo-constants $X$ tels que $\varepsilon_{I}(X)=$ 0 . Mais un tel foncteur $X$ est isomorphe à $\rho_{I} \varepsilon_{I}(X)=0$, par la proposition 5.4.2, d'où la fidélité annoncée. La proposition B.2.3 (page 188) donne donc la conclusion.

Remarque 5.5.3. - On a des résultats semblables en remplaçant dans cet énoncé fini par de type fini, $p f_{n}$ ou co-pf $f_{n}$. 
Définition 5.5.4 (Niveau). - On appelle niveau d'un objet $X$ de $\mathcal{F}_{\mathcal{G} r, I}$ l'élément

$$
\operatorname{niv}(X)=\sup \left\{\operatorname{dim} W \mid(V, W) \in \operatorname{Ob} \mathcal{E}_{\mathcal{G} r, I}^{f} X(V, W) \neq 0\right\}
$$

de $I \cup\{-\infty,+\infty\}$. On dit que $X$ est de niveau fini $\sin \operatorname{niv}(X)<+\infty$.

On définit de même le coniveau de $X$ comme l'élément

$$
\operatorname{coniv}(X)=\inf \left\{\operatorname{dim} W \mid(V, W) \in \mathrm{Ob} \mathcal{E}_{\mathcal{G} r, I}^{f} X(V, W) \neq 0\right\}
$$

de $I \cup\{+\infty\}$.

Ainsi, lorsque l'ensemble $I$ est fini, tous les foncteurs de $\mathcal{F}_{\mathcal{G} r, I}$ sont de niveau fini.

Proposition 5.5.5. - Un objet de $\mathcal{F}_{\mathcal{G} r, I}$ est fini si et seulement s'il est polynomial, à valeurs de dimension finie et de niveau fini.

Démonstration. - Soient $S$ un objet simple de $\mathcal{F}_{\mathcal{G} r, I}$ et $(A, B)$ un objet de $\mathcal{E}^{f} \times \mathcal{E}_{\text {surj }}^{I}$ tel que $S\left(\mathfrak{L}_{I}(A, B)\right) \neq 0$ (le foncteur $\mathfrak{L}_{I}$ est essentiellement surjectif - cf. proposition 4.2.3), de sorte qu'il existe un épimorphisme $P_{\mathfrak{L}_{I}(A, B)}^{\mathcal{G} r, I} \simeq \iota_{I}\left(P_{A}\right) \otimes \rho_{I}\left(P_{B}^{\mathcal{E}_{\text {surj }}^{I}}\right) \rightarrow$ $S$ (cf. proposition 5.1.12). Cela montre d'une part que $S$ est à valeurs de dimension finie, et que $S(V, W)=0$ pour $\operatorname{dim} W>\operatorname{dim} B$. D'autre part, comme l'objet $P_{A}$ de $\mathcal{F}$ est coanalytique (proposition 1.3.4), il existe un objet polynomial $F$ de $\mathcal{F}$ et un épimorphisme $\iota_{I}(F) \otimes \rho_{I}\left(P_{B}^{\text {surj }}\right) \rightarrow S$. La proposition 5.4.7 montre que $\iota_{I}(F) \otimes \rho_{I}\left(P_{B}^{s u r j}\right)$, donc $S$, est polynomial. On en déduit les mêmes propriétés pour les foncteurs finis par un argument d'épaisseur.

La réciproque résulte du lemme 5.5.2 et de la proposition 2.2.7 par récurrence sur le degré polynomial.

Cette proposition essentielle permet d'établir la préservation des foncteurs finis par les foncteurs $\xi_{I}, \theta_{I}$ et $\sigma_{I}$, donnée par le corollaire suivant.

Corollaire 5.5.6. - Soient $F$ un objet de $\mathcal{F}$ et $A$ un objet de $\mathcal{F}_{\text {sur } j}^{I}$.

(1) Si $F$ et $A$ sont finis, il en est de même pour $\iota_{I}(F) \otimes \rho_{I}(A)$. La réciproque est vraie si $A$ et $F$ sont non nuls.

(2) Si $F$ et $A$ sont finis, il en est de même pour $\kappa_{I}(F) \otimes \rho_{I}(A)$. La réciproque est vraie si $A$ et $F$ sont non nuls. fini.

(3) Un objet $X$ de $\mathcal{F}_{\mathcal{G} r, I}$ est fini si et seulement si l'objet $\sigma_{I}(X)$ de $\mathcal{F} \otimes \mathcal{F}_{\text {surj }}^{I}$ est

Démonstration. - Traitons le premier cas, en écartant le cas trivial où $F$ ou $A$ est nul. Le foncteur $\iota_{I}(F) \otimes \rho_{I}(A)=\xi_{I}(F \otimes A$ ) (on rappelle que le produit tensoriel extérieur $\triangle$ est introduit à la section C.5) est polynomial si et seulement s'il en est de même pour $F \otimes A$, par la proposition 5.4.7, ou encore de $F$, puisque $\Delta_{*}(F \otimes A)=\Delta(F) \otimes A$. Le foncteur $\iota_{I}(F) \otimes \rho_{I}(A)$ est à valeurs de dimension finie si et seulement s'il en est de même pour $F$ et $A$. Enfin, le foncteur $F \otimes A$ est de niveau fini si et seulement 
si $A\left(E_{n}\right)=0$ pour $n$ assez grand. Les propositions 5.5.5 et 2.2.7 fournissent donc la première assertion.

Les autres s'établissent de façon analogue.

Notation 5.5.7. - Nous désignerons par la suite par $\mathcal{F}_{\mathcal{G} r, I}^{l f}$ la sous-catégorie pleine de $\mathcal{F}_{\mathcal{G} r, I}$ formée des objets localement finis.

Le corollaire suivant donne les principales propriétés de régularité des objets finis et localement finis de $\mathcal{F}_{\mathcal{G} r, I}$.

Corollaire 5.5.8. - (1) Si $X$ est un objet fini de $\mathcal{F}_{\mathcal{G} r, I}$, il en est de même pour $\Delta^{\mathcal{G} r, I} X$.

(2) Le produit tensoriel total (donc, a fortiori, usuel) de deux objets finis de $\mathcal{F}_{\mathcal{G} r, I}$ est fini.

(3) Les objets finis de $\mathcal{F}_{\mathcal{G} r, I}$ sont $p f_{\infty}$ et co-pf $f_{\infty}$.

(4) La sous-catégorie $\mathcal{F}_{\mathcal{G} r, I}^{\text {lf }}$ de $\mathcal{F}_{\mathcal{G} r, I}$ est épaisse. Ses objets sont les foncteurs analytiques.

(5) Un objet de $\mathcal{F}_{\mathcal{G}_{r, I}}$ est de co-type fini si et seulement s'il est analytique et de socle fini.

Démonstration. - Elle est similaire à celle du corollaire 1.3.5. Pour le dernier point, on établit que les injectifs standard de $\mathcal{F}_{\mathcal{G} r, I}$ sont analytiques de la façon suivante : le corollaire 5.4.8 et la proposition 1.3.4 montrent que l'image par le foncteur $\iota_{I}$ d'un injectif standard de $\mathcal{F}$ est analytique. Comme tout injectif standard de $\mathcal{F}_{\mathcal{G} r, I}$ est facteur direct d'un tel foncteur, par la proposition 5.1.14, cela donne la conclusion.

Description des objets simples. - Les objets simples de la catégorie $\mathcal{F}_{\mathcal{G} r, I}$ seront ramenés à ceux de la catégorie $\mathcal{F} \otimes \mathcal{F}_{\text {surj }}^{I}$. Le lemme suivant les décrit à partir des objets simples de $\mathcal{F}$ et $\mathcal{F}_{\text {surj }}^{I}$, qui sont eux-mêmes assez bien compris (cf. chapitres 1 et 2).

Lemme 5.5.9. - Les objets simples de $\mathcal{F} \otimes \mathcal{F}_{\text {surj }}^{I}$ sont, à isomorphisme près, les $S \otimes R$, où $S$ est un objet simple de $\mathcal{F}$ et $R$ un objet simple de $\mathcal{F}_{\text {surj }}^{I}$. Le produit tensoriel extérieur induit de plus un isomorphisme d'anneaux (sans unité si $I$ est infini) $G_{0}^{f}\left(\mathcal{F} \otimes \mathcal{F}_{\text {surj }}^{I}\right) \simeq G_{0}^{f}(\mathcal{F}) \otimes G_{0}^{f}\left(\mathcal{F}_{\text {surj }}^{I}\right)$.

Démonstration. - Cela découle de la proposition C.5.2 (page 195) et de son corollaire C.5.3, dont les hypothèses sont vérifiées grâce à la proposition 1.4.3 (page 8).

Avec la proposition 5.5.5, le résultat suivant est le plus important de ce chapitre.

Proposition 5.5.10.

(1) Étant donné un objet $X$ de $\mathcal{F}_{\mathcal{G} r, I}$, les assertions suivantes sont équivalentes :

(a) l'objet $X$ de $\mathcal{F}_{\mathcal{G} r, I}$ est simple;

(b) l'objet $\sigma_{I}(X)$ de $\mathcal{F} \otimes \mathcal{F}_{\text {surj }}^{I}$ est simple; 
(c) il existe un objet simple $F$ de $\mathcal{F}$ et un objet simple $R$ de $\mathcal{F}_{\text {surj }}^{I}$ tel que $X$ est isomorphe à $\kappa_{I}(F) \otimes \rho_{I}(R)$.

(2) Les foncteurs exacts $\sigma_{I}$ et $\theta_{I}$ induisent des isomorphismes d'anneaux (sans unité si $I$ est infini $)$ entre $G_{0}^{f}\left(\mathcal{F}_{\mathcal{G} r, I}\right)$ et $G_{0}^{f}\left(\mathcal{F} \otimes \mathcal{F}_{\text {surj }}^{I}\right) \simeq G_{0}^{f}(\mathcal{F}) \otimes G_{0}^{f}\left(\mathcal{F}_{\text {surj }}^{I}\right)$ réciproques l'un de l'autre.

Démonstration. - La proposition 5.1.15.2 montre que le foncteur $\theta_{I}$ transforme un objet simple de $\mathcal{F} \otimes \mathcal{F}_{\text {surj }}^{I}$ en un objet simple de $\mathcal{F}_{\mathcal{G} r, I}$ et induit un monomorphisme de groupes abéliens, compatible au produit, $G_{0}^{f}\left(\mathcal{F} \otimes \mathcal{F}_{\text {surj }}^{I}\right) \hookrightarrow G_{0}^{f}\left(\mathcal{F}_{\mathcal{G} r, I}\right)$. Ces deux groupes sont naturellement gradués par le degré polynomial, qui est respecté par $\theta_{I}$ (cf. proposition 5.4.7), de sorte que ce monomorphisme s'identifie à la somme directe sur $k \in \mathbb{N}$ des morphismes

$$
G_{0}^{f}\left(\mathbf{F c t}\left(\mathcal{E}_{\text {surj }}^{I}, \mathcal{F}^{k} / \mathcal{F}^{k-1}\right)\right) \rightarrow \mathcal{F}_{\mathcal{G} r, J}^{k} / \mathcal{F}_{\mathcal{G} r, J}^{k-1}
$$

induits par $\theta_{I}$, lesquels sont des isomorphismes par la proposition 5.4.13. La compatibilité au produit tensoriel extérieur pour $I=\mathbb{N}$ provient de la proposition 5.2.3.

Cela démontre la seconde assertion de l'énoncé, et la première compte-tenu du lemme 5.5.9 et de ce que l'isomorphisme $G_{0}^{f}\left(\mathcal{F}_{\mathcal{G} r, I}\right) \stackrel{\simeq}{\longrightarrow} G_{0}^{f}\left(\mathcal{F} \otimes \mathcal{F}_{\text {sur } j}^{I}\right)$ inverse du précédent est induit par $\sigma_{I}$ (par les propositions 5.4 .7 et 5.4.14).

Corollaire 5.5.11. - Le foncteur exact $\xi_{I}$ induit un isomorphisme d'anneaux (sans unité si I est infini) entre $G_{0}^{f}(\mathcal{F}) \otimes G_{0}^{f}\left(\mathcal{F}_{\text {surj }}^{I}\right) \simeq G_{0}^{f}\left(\mathcal{F} \otimes \mathcal{F}_{\text {surj }}^{I}\right)$ et $G_{0}^{f}\left(\mathcal{F}_{\mathcal{G} r, I}\right)$.

Dans le cas où $I=\mathbb{N}$, ces foncteurs induisent également un isomorphisme d'anneaux pour la structure induite par le produit tensoriel total sur $\mathcal{F}_{\mathcal{G} r}$ et $\mathcal{F}_{\text {surj }}$ (et le produit tensoriel sur $\mathcal{F}$ ).

Démonstration. - Pour obtenir l'isomorphisme de groupes, il suffit de reprendre la démonstration de la proposition 5.5.10, compte-tenu de la proposition 5.4.14. La proposition 5.2.3 procure la compatibilité au produit tensoriel total.

Quelques conséquences. - Avant d'aborder la description des objets projectifs indécomposables de $\mathcal{F}_{\mathcal{G} r, I}$ (proposition 5.5.15), qui sera cependant moins explicite que celle de ses objets simples, nous tirons de la proposition 5.5.10 le corollaire suivant relatif aux cosocles - rappelons que cette notion est introduite dans la définition B.1.12.

Corollaire 5.5.12. - Pour tout objet $F$ de $\mathcal{F} \otimes \mathcal{F}_{\text {surj }}^{I}$, on a des isomorphismes naturels

$$
\operatorname{cosoc} \xi_{I}(F) \simeq \operatorname{cosoc} \theta_{I}(F) \simeq \theta_{I}(\operatorname{cosoc} F) .
$$

Le premier isomorphisme est induit par la projection canonique $\xi_{I}(F) \rightarrow \theta_{I}(F)$ et le second par $F \rightarrow \operatorname{cosoc} F$. 
Démonstration. - Grâce à la proposition 5.5.10, l'isomorphisme $\operatorname{cosoc} \xi_{I}(F) \simeq$ $\theta_{I}(\operatorname{cosoc} F)$ provient des isomorphismes naturels

$$
\operatorname{hom}_{\mathcal{G} r}\left(\xi_{I}(F), \theta_{I}(S)\right) \simeq \operatorname{hom}_{\mathcal{F} \otimes \mathcal{F}_{\text {surj }}^{I}}\left(F, \sigma_{I} \theta_{I}(S)\right) \simeq \operatorname{hom}_{\mathcal{F} \otimes \mathcal{F}_{\text {surj }}^{I}}(F, S)
$$

déduits des propositions 5.1.17 et 5.1.16.

L'isomorphisme $\operatorname{cosoc} \theta_{I}(F) \simeq \theta_{I}(\operatorname{cosoc} F)$ se déduit de la seconde assertion de la proposition 5.1.15.

Remarque 5.5.13. - Le premier isomorphisme du corollaire 5.5.12 implique, que pour tout objet de type fini $F$ de $\mathcal{F}$, l'épimorphisme canonique $\iota_{I}(F) \rightarrow \kappa_{I}(F)$ est essentiel. En effet, un épimorphisme entre objets co-localement finis et à valeurs de dimension finie de $\mathcal{F}_{\mathcal{G} r, I}$ qui induit un isomorphisme entre les cosocles est essentiel cet énoncé s'obtient, par dualité, à partir de l'observation que dans une catégorie de Grothendieck, un monomorphisme entre objets localement finis qui induit un isomorphisme entre les socles est essentiel.

Le comportement de l'épimorphisme canonique $\iota_{I} \rightarrow \kappa_{I}$ est totalement différent sur les objets de co-type fini ; ainsi, la proposition 5.1.14 montre que, dans le cas où $I=\mathbb{N}$, cet épimorphisme se scinde toujours lorsque $F$ est un injectif standard $I_{V}$ de $\mathcal{F}$ (alors que $\iota\left(I_{V}\right) \rightarrow \kappa\left(I_{V}\right)$ n'est un isomorphisme que pour $V=0$ ).

Rappelons que $K_{0}$ désigne le groupe de Grothendieck des classes d'objets projectifs indécomposables de type fini (cf. notation B.3.3).

Lemme 5.5.14. - (1) Le produit tensoriel extérieur induit un isomorphisme $K_{0}(\mathcal{F}) \otimes K_{0}\left(\mathcal{F}_{\text {surj }}^{I}\right) \stackrel{\simeq}{\longrightarrow} K_{0}\left(\mathcal{F} \otimes \mathcal{F}_{\text {surj }}^{I}\right)$.

(2) Si $\mathcal{I}$ est une catégorie essentiellement petite vérifiant l'hypothèse C.2.9 (page 193), on obtient des isomorphismes réciproques l'un de l'autre entre $G_{0}^{f}\left(\mathbf{F c t}\left(\mathcal{I}, \mathcal{E}_{\mathbb{k}}\right)\right)$ et $K_{0}\left(\mathbf{F} \operatorname{ct}\left(\mathcal{I}, \mathcal{E}_{\mathbb{k}}\right)\right)$ en associant à un objet simple sa couverture projective et à un objet projectif de type fini son cosocle.

Démonstration. - La première assertion se démontre de manière analogue au lemme 5.5.9.

La seconde est un résultat classique en théorie des représentations (cf. [CR90]); dans le cas général, on le déduit aisément en dualisant (à l'aide de l'hypothèse C.2.9) des propriétés des enveloppes injectives dans les catégories de Grothendieck (cf. $[$ Gab62 $]$ ) — on pourra se reporter à $[\mathbf{D j a 0 6 d}]$ pour une démonstration détaillée.

Proposition 5.5.15. - Le foncteur $\xi_{I}: \mathcal{F} \otimes \mathcal{F}_{\text {surj }}^{I} \rightarrow \mathcal{F}_{\mathcal{G} r, I}$ induit un isomorphisme de groupes abéliens $K_{0}\left(\mathcal{F} \otimes \mathcal{F}_{\text {surj }}^{I}\right) \stackrel{\simeq}{\longrightarrow} K_{0}\left(\mathcal{F}_{\mathcal{G} r, I}\right)$.

Ainsi, le produit tensoriel des morphismes induits par $\iota_{I}$ et $\rho_{I}$ fournit un isomorphisme $K_{0}(\mathcal{F}) \otimes K_{0}\left(\mathcal{F}_{\text {surj }}^{I}\right) \stackrel{\simeq}{\longrightarrow} K_{0}\left(\mathcal{F}_{\mathcal{G} r, I}\right)$. 
Dans le cas où $I=\mathbb{N}$, le produit tensoriel total sur $\mathcal{F}_{\mathcal{G} r}$ et $\mathcal{F}_{\text {surj }}$ et le produit tensoriel sur $\mathcal{F}$ munissent les deux membres de structures d'anneau commutatif; l'isomorphisme précédent est un isomorphisme d'anneaux pour ces structures.

Démonstration. - La proposition 5.1.12 montre que le foncteur exact $\xi_{I}$ conserve les objets projectifs de type fini, il induit donc un morphisme de groupes $K_{0}\left(\mathcal{F} \otimes \mathcal{F}_{\text {surj }}^{I}\right) \rightarrow$ $K_{0}\left(\mathcal{F}_{\mathcal{G} r, I}\right)$. Par le corollaire 5.5.12, le diagramme

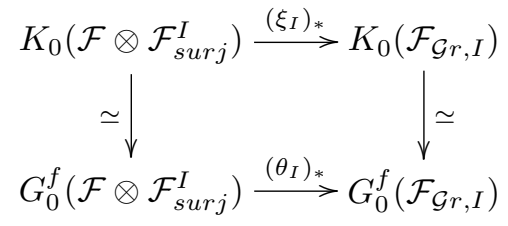

commute, où les flèches verticales, induites par le cosocle, sont des isomorphismes par la seconde assertion du lemme 5.5.14. Comme le morphisme $\left(\theta_{I}\right)_{*}$ est un isomorphisme par la proposition 5.5.10, il en est de même pour le morphisme $\left(\xi_{I}\right)_{*}$.

La première assertion du lemme 5.5.14 permet d'en déduire l'isomorphisme $K_{0}(\mathcal{F}) \otimes K_{0}\left(\mathcal{F}_{\text {sur } j}^{I}\right) \stackrel{\simeq}{\longrightarrow} K_{0}\left(\mathcal{F}_{\mathcal{G} r, I}\right)$.

Le corollaire 5.2.6 établit enfin la compatibilité au produit tensoriel extérieur.

Remarque 5.5.16. - L'inverse de l'isomorphisme de la proposition 5.5.15 ne se décrit pas simplement en termes des foncteurs fondamentaux depuis $\mathcal{F}_{\mathcal{G} r, I}$. 


\section{CHAPITRE 6}

\section{LA CATÉGORIE $\widetilde{\mathcal{F}}_{\mathcal{G} r}$}

Nous introduisons dans ce chapitre une catégorie de foncteurs $\widetilde{\mathcal{F}}_{\mathcal{G} r}(\mathbb{k})$, qui est intuitivement à $\mathcal{F}_{\mathcal{G} r}(\mathbb{k})$ ce que $\mathcal{F}(\mathbb{k})$ est à $\mathcal{F}_{\text {surj }}(\mathbb{k})$. Nous en étudions quelques propriétés dans la section 6.1 en vue d'applications à d'autres catégories. C'est la propriété de dualité démontrée dans la section 6.2 , qui n'a pas d'analogue dans $\mathcal{F}_{\mathcal{G} r}(\mathbb{k})$, qui nous permettra de compléter les renseignements sur la structure du foncteur $\mathbb{k}[\mathcal{G} r]$ déduits du chapitre 2 (section 6.3).

Cette propriété interviendra également dans la partie III, afin d'appliquer des propriétés du foncteur $\omega$ à la catégorie $\mathcal{F}_{i n j}(\mathbb{k})$.

Notation 6.0.1. - Nous noterons $\widetilde{\mathcal{F}}_{\mathcal{G} r}(\mathbb{k})$ la catégorie $\mathbf{F c t}\left(\widetilde{\mathcal{E}}_{\mathcal{G} r}^{f}(\mathbb{k}), \mathcal{E}_{\mathbb{k}}\right)$.

\subsection{Généralités}

La catégorie $\widetilde{\mathcal{F}}_{\mathcal{G} r}$ possède un comportement assez différent des catégories $\mathcal{F}_{\mathcal{G} r}$ ou $\mathcal{F}_{\mathcal{G} r, n}$ : elle n'entre pas dans le cadre étudié au chapitre 3 , de sorte qu'elle ne s'interprète pas clairement en termes de (co)modules.

Plutôt que de donner une description complète des foncteurs qui apparaissent naturellement, par précomposition, à partir des foncteurs étudiés dans le chapitre 4, nous nous focaliserons sur les liens entre $\widetilde{\mathcal{F}}_{\mathcal{G} r}$ et $\mathcal{F}_{\mathcal{G} r}$.

Notation 6.1.1. - Le foncteur de précomposition $\widetilde{\text { incl }}^{*}: \widetilde{\mathcal{F}}_{\mathcal{G} r} \rightarrow \mathcal{F}_{\mathcal{G} r}$ sera noté $\mathfrak{R}$.

Nous introduisons maintenant un foncteur très analogue au foncteur $\varpi$.

Proposition et définition 6.1.2. - Il existe un foncteur exact et fidèle $\mathfrak{J}: \mathcal{F}_{\mathcal{G} r} \rightarrow$

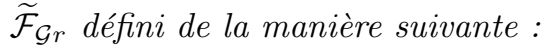

- action sur les objets :

$$
\mathfrak{J}(X)(V, B)=\bigoplus_{W \in \mathcal{G} r(B)} X(V, W)
$$


- action sur les morphismes : si $f:(V, B) \rightarrow\left(V^{\prime}, B^{\prime}\right)$ est un morphisme de $\widetilde{\mathcal{E}}_{\mathcal{G} r}^{f}, \mathfrak{J}(X)(f)$ a pour composante $X(V, W) \rightarrow X\left(V^{\prime}, W^{\prime}\right)$ (où $W \in \mathcal{G} r(B)$ et $W^{\prime} \in \mathcal{G} r\left(B^{\prime}\right)$ ) le morphisme induit par $f$ si $W^{\prime}=f(W)$ et 0 sinon,

- fonctorialité : si $t: X \rightarrow Y$ est un morphisme de $\mathcal{F}_{\mathcal{G} r}, \mathfrak{J}(t): \mathfrak{J}(X) \rightarrow \mathfrak{J}(Y)$

s'obtient sur l'objet $(V, B)$ par somme directe des $t(V, W)$ pour $W \in \mathcal{G} r(B)$.

Proposition 6.1.3. - Le foncteur $\mathfrak{J}: \mathcal{F}_{\mathcal{G} r} \rightarrow \widetilde{\mathcal{F}}_{\mathcal{G} r}$ est adjoint à gauche à $\mathfrak{R}$.

La vérification de ces propriétés, analogue à celle des propositions 2.3.5 et 2.3.6 (ou 3.1.5 et 3.1.7), est laissée au lecteur.

Notation 6.1.4. - L'endofoncteur $\mathfrak{R} \circ \mathfrak{J}$ de $\mathcal{F}_{\mathcal{G} r}$ sera noté $\mathcal{I}$.

Explicitement, on a

$$
\mathcal{I}(X)(V, W)=\bigoplus_{B \in \mathcal{G} r(W)} X(V, B)
$$

pour $X \in \mathrm{Ob} \mathcal{F}_{\mathcal{G} r}$ et $(V, W) \in \mathrm{Ob} \mathcal{E}_{\mathcal{G} r}^{f}$.

L'unité de l'adjonction de la proposition 6.1.3 procure une transformation naturelle injective $i d \rightarrow \mathcal{I}$. Elle est donnée sur un objet $X$ par l'inclusion $X(V, W) \hookrightarrow$ $\mathcal{I}(X)(V, W)$ correspondant au facteur direct de (8) obtenu pour $B=W$.

Notation 6.1.5. - Nous noterons $j^{\omega}: \mathcal{I} \rightarrow \iota \circ \omega$ la transformation naturelle donnée par les inclusions

$$
\bigoplus_{W \in \mathcal{G} r(B)} X(V, W) \hookrightarrow \bigoplus_{W \in \mathcal{G} r(V)} X(V, W) \quad\left(X \in \mathrm{Ob} \mathcal{F}_{\mathcal{G} r},(V, B) \in \mathrm{Ob} \mathcal{E}_{\mathcal{G} r}^{f}\right) .
$$

Cette transformation naturelle jouera un rôle essentiel dans la section 10.2.

Remarque 6.1.6. - Le diagramme suivant d'endofoncteurs de $\mathcal{F}_{\mathcal{G} r}$, dans lequel les flèches non spécifiées sont les unités des adjonctions des propositions 6.1.3 et 5.1.7.2, commute.

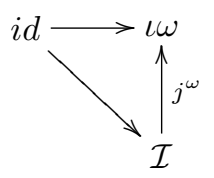

Nous introduisons à présent l'auto-dualité de la catégorie $\widetilde{\mathcal{F}}_{\mathcal{G} r}$ que l'on déduit de la proposition/définition 4.3.3, dont on conserve la notation $(\cdot)^{\vee}$.

Définition 6.1.7. - Le foncteur de dualité $\left(\widetilde{\mathcal{F}}_{\mathcal{G} r}\right)^{o p} \rightarrow \widetilde{\mathcal{F}}_{\mathcal{G} r}$, noté $D_{\mathcal{G} r}$, est la composée de la précomposition par $(\cdot)^{\vee}:\left(\widetilde{\mathcal{E}}_{\mathcal{G} r}^{f}\right)^{o p} \rightarrow \widetilde{\mathcal{E}}_{\mathcal{G} r}^{f}$ et de la postcomposition par $(\cdot)^{*}: \mathcal{E}^{o p} \rightarrow \mathcal{E}$.

On a ainsi $\left(D_{\mathcal{G} r} X\right)(V, W)=X\left((V, W)^{\vee}\right)^{*}$ pour tous $X \in$ Ob $\widetilde{\mathcal{F}}_{\mathcal{G} r}$ et $(V, W) \in$ $\operatorname{Ob} \widetilde{\mathcal{E}}_{\mathcal{G} r}^{f}$. 
La proposition suivante, que l'on déduit aussitôt de la proposition C.2.7, justifie l'appellation de dualité.

Proposition 6.1.8. - $\quad$ (1) Le foncteur $D_{\mathcal{G} r}:\left(\widetilde{\mathcal{F}}_{\mathcal{G} r}\right)^{o p} \rightarrow \widetilde{\mathcal{F}}_{\mathcal{G} r}$ est adjoint à droite $\grave{a} D_{\mathcal{G} r}^{o p}$.

(2) Le foncteur $D_{\mathcal{G} r}$ induit une équivalence entre la sous-catégorie pleine de $\widetilde{\mathcal{F}}_{\mathcal{G} r}$ des foncteurs à valeurs de dimension finie et sa catégorie opposée.

Cela permet de définir la notion d'objet auto-dual de $\widetilde{\mathcal{F}}_{\mathcal{G} r}$, de façon similaire à la définition 1.1.1.

Décomposition scalaire, tors de Frobenius et changement de corps. Comme la catégorie $\widetilde{\mathcal{E}}_{\mathcal{G} r}^{f}(\mathbb{k})$ est $\mathbb{k}$-linéaire, il existe dans $\widetilde{\mathcal{F}}_{\mathcal{G} r}$ une décomposition scalaire au sens de la proposition/définition C.4.2. On dispose par ailleurs, comme dans la catégorie $\mathcal{F}_{\mathcal{G} r}(\mathbb{k})$, d'un tors de Frobenius et de foncteurs de restriction et d'induction relativement à une extension finie de $\mathbb{k}$. On laisse au lecteur le soin d'écrire les détails et les propriétés de compatibilité de ces différents foncteurs.

Objets finis. - La catégorie $\widetilde{\mathcal{E}}^{f}$ étant additive, on dispose d'un endofoncteur de décalage $\Delta_{A}^{\widetilde{\mathcal{G} r}}$ dans $\widetilde{\mathcal{F}}_{\mathcal{G} r}$ associé à chaque objet $A$ de $\widetilde{\mathcal{E}}^{f}$, et l'on a des inclusions canoniques scindées $i d \hookrightarrow \Delta_{(\mathbb{k}, 0)}^{\widetilde{\mathcal{G} r}}$ et $i d \hookrightarrow \Delta_{(\mathbb{k}, \mathbb{k})}^{\widetilde{\mathcal{G} r}}$, de conoyaux notés $\bar{\Delta}_{(\mathbb{k}, 0)}^{\widetilde{\mathcal{G} r}}$ et $\left.\overline{\Delta_{(\mathbb{G},}} \widetilde{\mathrm{G} r}\right)$ respectivement. On peut alors montrer le résultat suivant.

Proposition 6.1.9. - Un objet de $\widetilde{\mathcal{F}}_{\mathcal{G} r}$ est fini si et seulement s'il est nilpotent pour les deux foncteurs $\overline{\Delta_{(\mathbb{k}, 0)}^{\widetilde{\mathcal{G} r}}}$ et $\overline{\Delta_{(\mathbb{k}, \mathbb{k})}^{\mathcal{G} r}}$ et à valeurs de dimension finie.

Esquissons la démonstration de cette proposition, que nous n'utiliserons pas :

(1) le foncteur $(\bar{\Delta} \underset{(\mathbb{\mathcal { G }}, 0)}{\widetilde{\mathcal{G} r}}, \bar{\Delta} \underset{(\mathbb{k} r, \mathbb{k})}{\widetilde{\mathcal{G} r}}): \widetilde{\mathcal{F}}_{\mathcal{G} r} \rightarrow \widetilde{\mathcal{F}}_{\mathcal{G} r} \times \widetilde{\mathcal{F}}_{\mathcal{G} r}$ est exact et son noyau se réduit aux foncteurs constants. On en déduit facilement qu'un foncteur de $\widetilde{\mathcal{F}}_{\mathcal{G} r}$ nilpotent pour $\overline{\Delta_{(\mathbb{k}, 0)}^{\widetilde{\mathcal{G}}}}$ et $\overline{\Delta_{(\mathbb{k}, \mathbb{k})}} \widetilde{\text { et }}$ à valeurs de dimension finie est fini ;

(2) à partir du caractère analytique des injectifs standard de $\mathcal{F}$, on obtient que les injectifs standard de $\widetilde{\mathcal{F}}_{\mathcal{G} r}$ sont colimite de foncteurs nilpotents pour $\bar{\Delta}_{(\mathbb{k}, 0)}^{\widetilde{\mathcal{G} r}}$ et $\bar{\Delta} \widetilde{\mathcal{G} r}$ et à valeurs de dimension finie, ce qui montre la réciproque.

Remarque 6.1.10. - On peut déduire de la proposition précédente un isomorphisme d'anneaux $G_{0}^{f}\left(\widetilde{\mathcal{F}}_{\mathcal{G} r}\right) \simeq G_{0}^{f}(\mathcal{F})^{\otimes 2}$, d'une manière analogue à la proposition 5.5.10.

\subsection{Propriété de dualité du foncteur $\widetilde{\omega}$}

Cette section est consacrée à l'étude d'une propriété de dualité liée au foncteur $\omega: \mathcal{F}_{\mathcal{G} r} \rightarrow \mathcal{F}$. Son cadre naturel est la catégorie $\widetilde{\mathcal{F}}_{\mathcal{G} r}$, par l'intermédiaire du foncteur 
introduit dans la notation suivante. En effet, la catégorie $\widetilde{\mathcal{F}}_{\mathcal{G} r}$ est étroitement liée à $\mathcal{F}_{\mathcal{G} r}$ et possède un foncteur dualité, contrairement à $\mathcal{F}_{\mathcal{G} r}$.

Notation 6.2.1. - Nous désignerons par $\widetilde{\omega}: \widetilde{\mathcal{F}}_{\mathcal{G} r} \rightarrow \mathcal{F}$ le foncteur composé

$$
\widetilde{\mathcal{F}}_{\mathcal{G} r} \stackrel{\Re}{\longrightarrow} \mathcal{F}_{\mathcal{G} r} \stackrel{\omega}{\longrightarrow} \mathcal{F}
$$

Le foncteur $\widetilde{\omega}$ est donné explicitement par $\widetilde{\omega}(X)(V)=\bigoplus_{B \in \mathcal{G} r(V)} X(V, B)$; un morphisme $f: V \rightarrow V^{\prime}$ de $\mathcal{E}^{f}$ induit l'application linéaire $\widetilde{\omega}(X)(V) \rightarrow \widetilde{\omega}\left(V^{\prime}\right)$ dont la composante $X(V, B) \rightarrow X\left(V^{\prime}, B^{\prime}\right)$ est le morphisme induit par $f$ si $B^{\prime}=f(B), 0$ sinon.

L'introduction de la variante suivante du foncteur $\widetilde{\omega}$ est motivée par les propositions 6.2 .3 et 6.2.4.

Notation 6.2.2. - On note $\widetilde{\omega}^{\prime}: \widetilde{\mathcal{F}}_{\mathcal{G} r} \rightarrow \mathcal{F}$ le foncteur défini par

$$
\widetilde{\omega}^{\prime}(X)(V)=\widetilde{\omega}(X)(V)=\bigoplus_{B \in \mathcal{G} r(V)} X(V, B)
$$

et tel que pour tout morphisme $f: V \rightarrow V^{\prime}$ de $\mathcal{E}^{f}$, la composante $X(V, B) \rightarrow$ $X\left(V^{\prime}, B^{\prime}\right)$ de $\widetilde{\omega}^{\prime}(X)(f)$ est l'application induite par le morphisme $(V, B) \rightarrow\left(V^{\prime}, B^{\prime}\right)$ induit par $f$ si $f^{-1}\left(B^{\prime}\right)=B, 0$ sinon.

La proposition suivante exprime que les foncteurs $\widetilde{\omega}$ et $\widetilde{\omega}^{\prime}$ sont duaux.

Proposition 6.2.3. - Il existe des isomorphismes $\widetilde{\omega} \circ D_{\mathcal{G} r} \simeq D \circ \widetilde{\omega}^{\prime}$ et $\widetilde{\omega}^{\prime} \circ D_{\mathcal{G} r} \simeq$ $D \circ \widetilde{\omega}$ de foncteurs contravariants de $\widetilde{\mathcal{F}}_{\mathcal{G} r}$ vers $\mathcal{F}$.

Démonstration. - Cela découle de l'observation suivante : si $f: V \rightarrow V^{\prime}$ est un morphisme de $\mathcal{E}^{f}, W$ un élément de $\mathcal{G} r(V)$ et $W^{\prime}$ un élément de $\mathcal{G} r\left(V^{\prime}\right)$, les conditions $W^{\perp}={ }^{t} f\left(W^{\prime \perp}\right)$ et $W=f^{-1}\left(W^{\prime}\right)$ sont équivalentes, où ${ }^{t} f: V^{*} \rightarrow V^{*}$ désigne la transposée de $f$.

La proposition suivante constitue le résultat principal de cette section.

Proposition 6.2.4. - Les foncteurs $\widetilde{\omega}$ et $\widetilde{\omega}^{\prime}$ sont isomorphes.

Démonstration. - Soit $u_{X, V}: \widetilde{\omega}(X)(V) \rightarrow \widetilde{\omega}^{\prime}(X)(V)$, pour $X \in$ Ob $\widetilde{\mathcal{F}}_{\mathcal{G} r}$ et $V \in$ Ob $\mathcal{E}^{f}$, l'application linéaire dont la composante $X(V, W) \rightarrow X(V, B)$ (où $W, B \in$ $\mathcal{G} r(V))$ est induite par l'inclusion $(V, W) \hookrightarrow(V, B)$ si $W \subset B, 0$ sinon. Alors $u_{X, V}$ est un isomorphisme d'espaces vectoriels, car si l'on munit $\mathcal{G} r(V)$ d'un ordre total $\leq$ tel que $W \subset B$ implique $W \leq B$, on obtient pour $u_{X, V}$ une matrice triangulaire par blocs, avec des blocs diagonaux égaux à l'identité. 
De plus, pour toute application linéaire $f: V \rightarrow V^{\prime}$, le diagramme

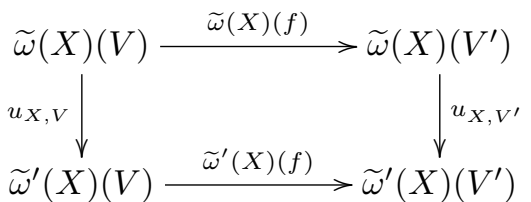

commute. En effet, la composante $X(V, W) \rightarrow X\left(V^{\prime}, B^{\prime}\right)$ de l'application $\widetilde{\omega}(X)(V) \rightarrow$ $\widetilde{\omega}^{\prime}(X)\left(V^{\prime}\right)$ obtenue en suivant la composée supérieure est la somme des applications induites par le morphisme $(V, W) \rightarrow\left(V^{\prime}, B^{\prime}\right)$ induit par $f$ indexée sur les $W^{\prime} \in \mathcal{G} r\left(V^{\prime}\right)$ tels que $f(W)=W^{\prime}$ et $W^{\prime} \subset B^{\prime}$. Autrement dit, cette composante est l'application induite par le morphisme $(V, W) \rightarrow\left(V^{\prime}, B^{\prime}\right)$ induit par $f$ si $f(W) \subset B^{\prime}$ et 0 sinon.

De même, la composante $X(V, W) \rightarrow X\left(V^{\prime}, B^{\prime}\right)$ de l'application $\widetilde{\omega}(X)(V) \rightarrow$ $\widetilde{\omega}^{\prime}(X)\left(V^{\prime}\right)$ obtenue en suivant la composée inférieure est l'application induite par le morphisme $(V, W) \rightarrow\left(V^{\prime}, B^{\prime}\right)$ induit par $f$ si $W \subset f^{-1}\left(B^{\prime}\right)$ et 0 sinon, d'où la commutativité recherchée.

Par conséquent, les applications linéaires $u_{X, V}$ définissent un isomorphisme $u_{X}$ : $\widetilde{\omega}(X) \stackrel{\simeq}{\longrightarrow} \widetilde{\omega}^{\prime}(X)$ de $\mathcal{F}$, qui est naturel en $X$, d'où un isomorphisme de foncteurs $u: \widetilde{\omega} \stackrel{\simeq}{\longrightarrow} \widetilde{\omega}^{\prime}$.

Les propositions 6.2.4 et 6.2.3 fournissent le corollaire suivant.

Corollaire 6.2.5. - Il existe un isomorphisme $\alpha: \widetilde{\omega} \circ D_{\mathcal{G} r} \cong D \circ \widetilde{\omega}$ tel que pour tout objet $X$ de $\widetilde{\mathcal{F}}_{\mathcal{G} r}$, le diagramme

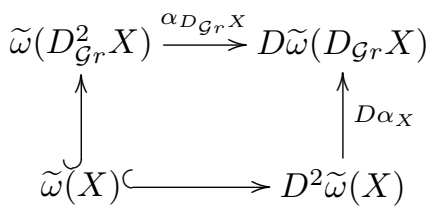

dont les monomorphismes non spécifiés sont les unités de l'adjonction commute.

Démonstration. - Soient $a: \widetilde{\omega} \circ D_{\mathcal{G} r} \stackrel{\simeq}{\longrightarrow} D \circ \widetilde{\omega}^{\prime}$ et $b: \widetilde{\omega}^{\prime} \circ D_{\mathcal{G} r} \stackrel{\simeq}{\rightarrow} D \circ \widetilde{\omega}$ les deux isomorphismes canoniques de la proposition 6.2.3, et $u: \widetilde{\omega} \stackrel{\simeq}{\longrightarrow} \widetilde{\omega}^{\prime}$ l'isomorphisme de la proposition 6.2.4. Les deux composées $\widetilde{\omega} \circ D_{\mathcal{G} r} \stackrel{a}{\longrightarrow} D \circ \widetilde{\omega}^{\prime} \stackrel{D u}{\longrightarrow} D \circ \widetilde{\omega}$ et $\widetilde{\omega} \circ D_{\mathcal{G} r} \stackrel{u_{D_{\mathcal{G}}}}{\longrightarrow}$ $\widetilde{\omega}^{\prime} \circ D_{\mathcal{G} r} \stackrel{b}{\rightarrow} D \circ \widetilde{\omega}$ coïncident, car si $W$ et $B$ sont deux sous-espaces de $V \in \mathrm{Ob}^{f}$, les conditions $W \subset B$ et $B^{\perp} \subset W^{\perp}$ sont équivalentes. Notons $\alpha$ l'isomorphisme donné par ces composées. La commutation du diagramme de l'énoncé se ramène à celle de

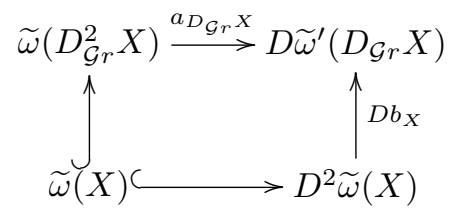


qui se vérifie par inspection.

Dans la section suivante, nous utiliserons le corollaire 6.2 .5 par le biais de sa conséquence directe suivante.

Corollaire 6.2.6. - Si X est un objet auto-dual de $\widetilde{\mathcal{F}}_{\mathcal{G} r}$, alors $\widetilde{\omega}(X)$ est un objet auto-dual de $\mathcal{F}$.

\subsection{Structure de $\mathbb{k}[\mathcal{G} r]$}

La proposition 5.1.7 identifiant la catégorie $\mathcal{F}_{\mathcal{G} r}$ à celle des $\mathbb{k}[\mathcal{G} r]$-comodules de $\mathcal{F}$ illustre l'importance de ce foncteur dans l'étude des catégories de foncteurs en grassmanniennes.

Structure fondamentale. - La proposition 2.3.9 montre que la décomposition scalaire de $\mathbb{k}[\mathcal{G} r]=\varpi(\mathbb{k})$ se réduit à $\mathbb{k}[\mathcal{G} r] \simeq \mathbb{k} \oplus \overline{\mathbb{k}[\mathcal{G} r]}$, où $\overline{\mathbb{k}[\mathcal{G} r]} \in \mathrm{Ob} \mathcal{F}_{q-1}$. La proposition 2.3.11 fournit quant à elle le résultat suivant.

Proposition 6.3.1. - Le foncteur $\mathbb{k}[\mathcal{G} r] \simeq \mathbb{k} \oplus \overline{\mathbb{k}[\mathcal{G} r]}$ est colimite filtrante des sousfoncteurs $\varpi\left(T_{n}(\mathbb{k})\right) \simeq \mathbb{k}\left[\mathcal{G} r_{\leq n}\right]$; on a des extensions essentielles

$$
0 \rightarrow \overline{\mathbb{k}\left[\mathcal{G} r_{\leq n-1}\right]} \rightarrow \overline{\mathbb{k}\left[\mathcal{G} r_{\leq n}\right]} \rightarrow \bar{G}(n) \rightarrow 0
$$

pour tout $n \in \mathbb{N}^{*}$. De plus, $\overline{\mathbb{k}\left[\mathcal{G} r_{\leq 1}\right]} \simeq \mathbb{k}\left[\mathcal{G} r_{1}\right] \simeq P_{\mathbb{k}, q-1}$.

Comme le foncteur $P_{\mathrm{k}, q-1}$ est indécomposable, on en déduit le corollaire suivant.

Corollaire 6.3.2. - Le foncteur $\overline{\mathbb{k}[\mathcal{G} r]}$ est indécomposable.

Appliqué au foncteur constant $\mathbb{k}$, le corollaire 6.2 .6 fournit l'important résultat suivant.

Proposition 6.3.3. - Le foncteur $\mathbb{k}[\mathcal{G} r]$ est auto-dual.

À partir de cette auto-dualité et de la structure de comodule de $\mathbb{k}[\mathcal{G} r]$, on obtient le corollaire suivant.

Corollaire 6.3.4. - Munissons le foncteur $\mathbb{k}[\mathcal{G} r]$ du produit et de l'unité obtenus en dualisant sa structure de coalgèbre de Boole.

Le foncteur $\mathbb{k}[\mathcal{G} r]$ devient ainsi un foncteur en algèbres de Hopf.

Démonstration. - En reprenant la démonstration de la proposition 6.2.4 et en notant que $B \subset W_{1}^{\perp}$ et $B \subset W_{2}^{\perp}$ équivaut à $B \subset\left(W_{1}+W_{2}\right)^{\perp}$, où $B, W_{1}$ et $W_{2}$ sont des sousespaces d'un espace vectoriel de dimension finie $V$, on voit que la structure d'algèbre sur $\mathbb{k}[\mathcal{G} r]$ est donnée sur $V$ par

$$
\mathbb{k}[\mathcal{G} r(V)] \otimes \mathbb{k}[\mathcal{G} r(V)] \rightarrow \mathbb{k}[\mathcal{G} r(V)] \quad\left[W_{1}\right] \otimes\left[W_{2}\right] \mapsto\left[W_{1}+W_{2}\right] .
$$


Ainsi, le produit de deux générateurs canoniques de $\mathbb{k}[\mathcal{G} r(V)]$ est encore un générateur canonique de $\mathbb{k}[\mathcal{G} r(V)]$, ce qui montre que les structures d'algèbre et de coalgèbre de Boole sur cet espace vectoriel sont compatibles : c'est une algèbre de Hopf.

À l'aide de la proposition 6.3.3 et du lemme suivant, laissé au lecteur, nous allons décrire les foncteurs $\operatorname{hom}_{\mathcal{F}}(\mathbb{k}[\mathcal{G} r], \cdot)$ et $\operatorname{Ext}_{\mathcal{F}}^{i}(\cdot, \mathbb{k}[\mathcal{G} r])$. On rappelle que $\operatorname{ev}_{n}: \mathcal{F}_{\text {surj }} \rightarrow$ $G L_{n}(\mathbb{k})$ Mod désigne le foncteur d'évaluation sur $E_{n}$.

Lemme 6.3.5. - Le foncteur $\operatorname{hom}_{\mathcal{F}_{\text {surj }}}(\mathbb{k}, \cdot)$ est isomorphe $\grave{a} \lim _{n \in \mathbb{N}} \operatorname{ev}_{n}^{G L_{n}(\mathbb{k})}$, où :

- l'on désigne par $\mathrm{ev}_{n}^{G L_{n}(\mathbb{k})}$ le foncteur obtenu en prenant les invariants de $\mathrm{ev}_{n}$ sous l'action naturelle de $G L_{n}(\mathbb{k})$;

- la limite est relative aux transformations naturelles $\mathrm{ev}_{n}^{G L_{n}(\mathbb{k})} \rightarrow \mathrm{ev}_{m}^{G L_{m}(\mathbb{k})}$ (pour $n \geq m$ ) induites par la projection $E_{n} \rightarrow E_{m}$ sur les $m$ premières coordonnées.

Proposition 6.3.6. - Soient $F \in \mathrm{Ob} \mathcal{F}$ et $i \in \mathbb{N}$. Il existe des isomorphismes naturels

$$
\operatorname{hom}_{\mathcal{F}}(\mathbb{k}[\mathcal{G} r], F) \simeq \lim _{n \in \mathbb{N}} F\left(E_{n}\right)^{G L_{n}(\mathbb{k})}
$$

et

$$
\operatorname{Ext}_{\mathcal{F}}^{i}(F, \mathbb{k}[\mathcal{G} r]) \simeq\left(\operatorname{colim}_{n \in \mathbb{N}} H_{i}\left(G L_{n}(\mathbb{k}), F\left(E_{n}\right)\right)\right)^{*}
$$

Démonstration. — Le lemme 6.3.5 et l'adjonction entre $\varpi$ et $o$ (cf. proposition 2.3.6) donnent le premier isomorphisme. Par auto-dualité de $\mathbb{k}[\mathcal{G} r]$ (proposition 6.3.3), on en déduit des isomorphismes naturels

$$
\operatorname{hom}_{\mathcal{F}}(F, \mathbb{k}[\mathcal{G} r]) \simeq \operatorname{hom}_{\mathcal{F}}(\mathbb{k}[\mathcal{G} r], D F) \simeq \lim _{n \in \mathbb{N}} D F\left(E_{n}\right)^{G L_{n}(\mathbb{k})} \simeq\left(\operatorname{colim}_{n \in \mathbb{N}} F\left(E_{n}\right)_{G L_{n}(\mathbb{k})}\right)^{*}
$$

L'intérêt de la dernière écriture est de remplacer la limite par une colimite filtrante, exacte. Cela permet d'en déduire le second isomorphisme, en dérivant les foncteurs considérés.

Anneau d'endomorphismes. - Nous poursuivons l'étude du foncteur $\mathbb{k}[\mathcal{G} r]$ par la détermination de son anneau d'endomorphisme, donnée par le corollaire 6.3.11.

Définition 6.3.7 (Algèbre en grassmanniennes). - On appelle algèbre en grassmanniennes, et l'on note $\mathrm{A}_{\mathcal{G} r}(\mathbb{k})$, la $\mathbb{k}$-algèbre $\operatorname{End}_{\mathcal{F}}(\mathbb{k}[\mathcal{G} r])$. L'algèbre en grassmanniennes réduite, notée $\overline{\mathrm{A}}_{\mathcal{G} r}(\mathbb{k})$, est définie comme $\operatorname{End}_{\mathcal{F}}(\overline{\mathbb{k}[\mathcal{G} r]})$.

Le scindement $\varpi(\mathbb{k}) \simeq \mathbb{k} \oplus \overline{\varpi(\mathbb{k})}$ fournit donc un isomorphisme d'algèbres $A_{\mathcal{G} r}(\mathbb{k}) \simeq$ $\mathbb{k} \oplus \overline{\mathrm{A}}_{\mathcal{G} r}(\mathbb{k})$.

Pour déterminer explicitement ces algèbres, nous emploierons le lemme combinatoire élémentaire suivant. On rappelle que $p$ désigne la caractéristique de $\mathbb{k}$ et $q$ son cardinal. 
Lemme 6.3.8. - Soient $l$ et $i$ deux entiers naturels.

(1) Soit a un élément non nul de l'espace vectoriel $E_{l}$. Les classes d'équivalences de la relation définie par $W \sim W^{\prime}$ si $W+a=W^{\prime}+a$ sur l'ensemble des éléments $W$ de $\mathcal{G} r_{i}\left(E_{l}\right)$ ne contenant pas a sont de cardinal $q^{i}$, donc multiple de $p$ si $i>0$.

(2) Si $i \leq l$, le cardinal de l'ensemble $\mathcal{G} r_{i}\left(E_{l}\right)$ est congru à 1 modulo $p$.

Démonstration. - Pour le premier point, on note que le groupe $G L(W \oplus a, a)$ des automorphismes $u$ de $W \oplus a$ tels que $u(a)=a$ opère transitivement sur la classe d'équivalence de $W$, le stabilisateur de $W$ étant $G L(W)$. Le cardinal de cette classe est donc celui de $G L(W \oplus a, a) / G L(W)$, soit $q^{i}$.

Pour la seconde assertion, on remarque que le cardinal de l'ensemble $\mathrm{Pl}_{\mathcal{E}}\left(E_{i}, E_{l}\right)$ est $\prod_{j=0}^{i-1}\left(q^{l}-q^{j}\right)$. Comme l'ensemble $\mathcal{G} r_{i}\left(E_{l}\right)$ s'identifie au quotient de $\operatorname{Pl}_{\mathcal{E}}\left(E_{i}, E_{l}\right)$ par l'action libre de $G L_{i}(\mathbb{k})$, on a pour $i \leq l$

$$
\operatorname{Card} \mathcal{G} r_{i}(l)=\frac{\operatorname{CardPl}_{\mathcal{E}}\left(E_{i}, E_{l}\right)}{\operatorname{Card} G L_{i}(\mathbb{k})}=\prod_{j=0}^{i-1} \frac{q^{l-j}-1}{q^{i-j}-1} \equiv 1 \quad(\bmod p) .
$$

La proposition suivante fournit une première description de l'algèbre en grassmanniennes.

Proposition 6.3.9. - La $\mathbb{k}$-algèbre $\mathrm{A}_{\mathcal{G} r}(\mathbb{k})$ est isomorphe au $\mathbb{k}$-espace vectoriel $\mathbb{k}^{\mathbb{N}}$ muni de loi multiplicative $*$ définie par

$$
(f * g)(n)=\sum_{\substack{i+j=n \\ i, j \geq 0}} f(i) g(j)+\sum_{\substack{i+j=n+1 \\ i, j>0}} f(i) g(j) .
$$

Démonstration. - Par la proposition 2.3.6, on a un isomorphisme (linéaire) d'adjonction $\mathrm{A}_{\mathcal{G} r}(\mathbb{k}) \simeq \operatorname{hom}_{\mathcal{F}_{\text {surj }}}(\mathbb{k}, o(\mathbb{k}[\mathcal{G} r]))$. Le $G L_{n}(\mathbb{k})$-module $\operatorname{ev}_{n}(o(\mathbb{k}[\mathcal{G} r]))$ est librement engendré comme $\mathbb{k}$-espace vectoriel par $\mathcal{G} r\left(E_{n}\right)$, sur lequel $G L_{n}(\mathbb{k})$ agit tautologiquement. Le sous-espace vectoriel $\operatorname{ev}_{n}(o(\mathbb{k}[\mathcal{G} r]))^{G L_{n}(\mathbb{k})}$ de $\mathbb{k}\left[\mathcal{G} r\left(E_{n}\right)\right]$ a pour base $s_{0}^{n}, s_{1}^{n}, \ldots, s_{n}^{n}$, où $s_{i}^{n}$ désigne la somme des générateurs canoniques $[B]$ associés à un sous-espace $B$ de dimension $i$ de $E_{n}$. L'application linéaire induite par la projection $E_{n+1} \rightarrow E_{n}$ envoie $s_{i}^{n+1}$ sur $s_{i-1}^{n}$ si $i>0$ et $s_{0}^{n+1}$ sur $s_{0}^{n}$, grâce à la première assertion du lemme 6.3.8.

Par le lemme 6.3.5, on en déduit une identification entre $\mathrm{A}_{\mathcal{G} r}(\mathbb{k})$ et la limite $L$ des espaces vectoriels $\mathbb{k}^{n+1}$ (dont nous continuerons à noter $s_{0}^{n}, s_{1}^{n}, \ldots, s_{n}^{n}$ une base privilégiée) relativement aux applications linéaires $f_{n}: \mathbb{k}^{n+1} \rightarrow \mathbb{k}^{n}$ données par $s_{i}^{n} \mapsto$ $s_{i-1}^{n-1}$ pour $i>0$ et $s_{0}^{n} \mapsto s_{0}^{n-1}$. 
Soit $l_{0}^{n}, l_{1}^{n}, \ldots, l_{n}^{n}$ la base duale de $s_{0}^{n}, s_{1}^{n}, \ldots, s_{n}^{n}$. L'application linéaire $a: L \rightarrow$ $\mathbb{k}^{\mathbb{N}} \quad\left(v_{n}\right)_{n \in \mathbb{N}} \mapsto\left(l_{0}^{n}\left(v_{n}\right)\right)_{n \in \mathbb{N}}$ est bijective; sa réciproque est donnée par

$$
b: \mathbb{k}^{\mathbb{N}} \rightarrow L \quad\left(t_{n}\right)_{n \in \mathbb{N}} \mapsto\left(t_{n} s_{0}^{n}+\sum_{i=1}^{n}\left(t_{n-i}+t_{n-i+1}\right) s_{i}^{n}\right)_{n \in \mathbb{N}} .
$$

En effet, $b$ prend bien ses valeurs dans $L$ parce que

$$
\begin{gathered}
f_{n}\left(t_{n} s_{0}^{n}+\sum_{i=1}^{n}\left(t_{n-i}+t_{n-i+1}\right) s_{i}^{n}\right)=t_{n} s_{0}^{n-1}+\sum_{i=1}^{n}\left(t_{n-i}+t_{n-i+1}\right) s_{i-1}^{n-1} \\
=t_{n-1} s_{0}^{n-1}+\sum_{j=1}^{n-1}\left(t_{n-1-j}+t_{n-j}\right) s_{j}^{n-1},
\end{gathered}
$$

l'égalité $a \circ b=i d$ est immédiate, et $b \circ a=i d$ se déduit facilement des remarques précécentes.

On a ainsi obtenu une identification de $\mathrm{A}_{\mathcal{G} r}(\mathbb{k})$ et $\mathbb{k}^{\mathbb{N}}$ comme espaces vectoriels; il reste à lire la structure multiplicative de $\mathrm{A}_{\mathcal{G}_{r}}(\mathbb{k})$ dans les isomorphismes précédents.

Soient $u$ un endomorphisme de $\mathbb{k}[\mathcal{G} r]$ et $\left(t_{n}\right)_{n \in \mathbb{N}}$ l'élément de $\mathbb{k}^{\mathbb{N}}$ correspondant. Par ce qui précède, pour tout sous-espace $W$ de $V \in \mathrm{Ob} \mathcal{E}^{f}$, le générateur $[W]$ de $\mathbb{k}[\mathcal{G} r(V)]$ est envoyé par $u(V)$ sur $t_{m} s_{0}(W)+\sum_{i=1}^{m}\left(t_{m-i}+t_{m-i+1}\right) s_{i}(W)$, où $m=\operatorname{dim} W$ et $s_{i}(W)$ est la somme des générateurs de $\mathbb{k}[\mathcal{G} r(V)]$ associés aux sous-espaces de dimension $i$ de $V$. On en déduit que $u_{E_{n}}$ envoie $s_{m}^{n} \operatorname{sur} t_{m} s_{0}^{n}+\sum_{i=1}^{m}\left(t_{m-i}+t_{m-i+1}\right) s_{i}^{n}$, puisque le coefficient d'un générateur $[B]$ (où $\operatorname{dim} B=i$ ) dans cette image est égal au cardinal de l'ensemble des sous-espaces $W$ de $E_{n}$ de dimension $m$ contenant $B$, multiplié par $t_{0}$ si $i=0, t_{m-i}+t_{m-i+1}$ si $1 \leq i \leq m$, et 0 sinon. Le cardinal en question n'est autre que celui des sous-espaces de dimension $m-i$ de $E_{n} / B$, égal à 1 dans $\mathbb{k}$ par la seconde assertion du lemme 6.3.8, d'où notre assertion.

Soient $u^{\prime}$ un autre endomorphisme de $\mathbb{k}[\mathcal{G} r]$ et $u^{\prime \prime}=u^{\prime} u$; notons $\left(t_{n}^{\prime}\right)_{n \in \mathbb{N}}$ et $\left(t_{n}^{\prime \prime}\right)_{n \in \mathbb{N}}$ les éléments de $\mathbb{k}^{\mathbb{N}}$ correspondant à $u^{\prime}$ et $u^{\prime \prime}$ respectivement. On a

$$
\begin{gathered}
u^{\prime \prime}\left(E_{n}\right)\left(\left[E_{n}\right]\right)=u^{\prime}\left(t_{n} s_{0}^{n}+\sum_{i=1}^{n}\left(t_{n-i}+t_{n-i+1}\right) s_{i}^{n}\right) \\
=t_{n} t_{0}^{\prime} s_{0}^{n}+\sum_{i=1}^{n}\left(t_{n-i}+t_{n-i+1}\right)\left(t_{i}^{\prime} s_{0}^{n}+\sum_{j=1}^{i}\left(t_{i-j}^{\prime}+t_{i-j+1}^{\prime}\right) s_{j}^{n}\right),
\end{gathered}
$$

d'où en identifiant le coefficient de $s_{0}^{n}$

$$
t_{n}^{\prime \prime}=t_{n} t_{0}^{\prime}+\sum_{i=1}^{n}\left(t_{n-i}+t_{n-i+1}\right) t_{i}^{\prime}=\sum_{\substack{i+j=n \\ i, j \geq 0}} t_{i} t_{j}^{\prime}+\sum_{\substack{i+j=n+1 \\ i, j>0}} t_{i} t_{j}^{\prime},
$$

ce qui achève la démonstration. 
Corollaire 6.3.10. — La loi * sur l'espace vectoriel $\mathbb{k}^{\mathbb{N}^{*}}$ définie par

$$
(f * g)(n)=\sum_{\substack{i+j=n \\ i, j \geq 1}} f(i) g(j)+\sum_{\substack{i+j=n+1 \\ i, j \geq 1}} f(i) g(j)
$$

fait de $\mathbb{k}^{\mathbb{N}^{*}}$ une $\mathbb{k}$-algèbre isomorphe à $\overline{\mathrm{A}}_{\mathcal{G} r}(\mathbb{k})$.

Démonstration. - Dans l'isomorphisme précédent, $\overline{\mathrm{A}}_{\mathcal{G} r}(\mathbb{k})$ correspond à l'idéal de $\mathbb{k}^{\mathbb{N}}$ des fonctions nulles en 0 .

Corollaire 6.3.11. — L'algèbre $\overline{\mathrm{A}}_{\mathcal{G} r}(\mathbb{k})$ est une algèbre de séries formelles sur l'élément $\tau$ donné par la fonction $\mathbb{N}^{*} \rightarrow \mathbb{k}$ associant 0 à 1 et 1 à $n \geq 2$.

Démonstration. - Pour tout $f \in \overline{\mathrm{A}}_{\mathcal{G} r}(\mathbb{k})$, on a $\tau f(n)=f(n-1)$ si $n \geq 2$ et $\tau f(1)=0$. On en déduit que $\tau^{k}$ est la fonction $n \mapsto 1$ si $n \geq k+1,0$ sinon. Cela fournit aussitôt le résultat.

Explicitement, l'isomorphisme est donné par $\sum_{i \in \mathbb{N}} a_{i} \tau^{i} \mapsto\left(\sum_{i=0}^{n-1} a_{i}\right)_{n \in \mathbb{N}^{*}}$.

Remarque 6.3.12. - (1) En reprenant la démonstration de la proposition 6.3.9, on voit que l'endomorphisme $\tau$ envoie un générateur canonique $[W]$ sur

$$
\sum_{\substack{B \in \mathcal{G} r(W) \\ \operatorname{codim}_{W} B=1}}[B] .
$$

(2) Malgré le corollaire 6.3.11, il pourra être utile de conserver la description de $\mathrm{A}_{\mathcal{G} r}(\mathbb{k})$ donnée à la proposition 6.3.9. Cela apparaîtra clairement à la remarque 6.3.14. 1 .

Nous terminons cette section par quelques considérations combinant l'auto-dualité de $\mathbb{k}[\mathcal{G} r]$ (proposition 6.3.3) et le corollaire 6.3.11.

Corollaire 6.3.13. — L'involution de l'algèbre $\mathrm{A}_{\mathcal{G} r}(\mathbb{k})$ induite par l'auto-dualité du foncteur $\mathbb{k}[\mathcal{G} r]$ est triviale.

Démonstration. - Par le corollaire 6.3.11, il suffit de le vérifier sur l'endomorphisme $\tau$ de $\mathbb{k}[\mathcal{G} r]$.

Pour cela, on note que la structure auto-duale de $\mathbb{k}[\mathcal{G} r]$ est donnée par les formes bilinéaires

$$
b_{V}: \mathbb{k}[\mathcal{G} r(V)] \times \mathbb{k}\left[\mathcal{G} r\left(V^{*}\right)\right] \rightarrow \mathbb{k} \quad([W],[H]) \mapsto 1 \text { si } H \subset W^{\perp}, 0 \text { sinon. }
$$

On calcule alors, pour $W \in \mathcal{G} r_{n}(V)$,

$$
b_{V}(\tau([W]),[H])=\sum_{B \in \mathcal{G} r_{n-1}(W)} b_{V}([B],[H])=
$$

$\operatorname{Card}\left\{B \in \mathcal{G} r_{n-1}(W) \mid H \subset B^{\perp}\right\}=\operatorname{Card} \mathcal{G} r_{n-1}\left(W \cap H^{\perp}\right)$. 
En utilisant le lemme 6.3 .8 , on voit que cet élément de $\mathbb{k}$ vaut 1 si $\operatorname{dim}\left(W \cap H^{\perp}\right) \geq$ $n-1$, i.e. $\operatorname{dim} W-\operatorname{dim}\left(W \cap H^{\perp}\right) \leq 1$, et 0 sinon. Comme cette condition est symétrique en $W$ et $H$ (modulo l'identification de $V$ à $V^{* *}$ ), cela donne la conclusion.

Remarque 6.3.14. - (1) On déduit de la structure d'algèbre de Hopf sur $\mathbb{k}[\mathcal{G} r]$ donnée par le corollaire 6.3.4 une nouvelle structure d'algèbre sur $\mathrm{A}_{\mathcal{G} r}(\mathbb{k})$, donnée comme suit. Si $u$ et $v$ sont deux éléments de $\mathrm{A}_{\mathcal{G} r}(\mathbb{k})$, leur produit $u . v$ est le morphisme composé

$$
\mathbb{k}[\mathcal{G} r] \rightarrow \mathbb{k}[\mathcal{G} r] \otimes \mathbb{k}[\mathcal{G} r] \stackrel{u \otimes v}{\longrightarrow} \mathbb{k}[\mathcal{G} r] \otimes \mathbb{k}[\mathcal{G} r] \rightarrow \mathbb{k}[\mathcal{G} r],
$$

où la première flèche est le coproduit et la dernière le produit (cf. [Kuh95], §5).

Dans l'isomorphisme $\mathrm{A}_{\mathcal{G} r}(\mathbb{k}) \simeq \mathbb{k}^{\mathbb{N}}$ de la proposition 6.3 .9 , le produit . est le produit usuel d'algèbre de Boole de l'anneau produit $\mathbb{k}^{\mathbb{N}}$. Pour le voir, il suffit de reprendre la démonstration de la proposition 6.3.9 et de constater que, en conservant ses notations, le produit de $s_{i}^{n}$ et de $s_{j}^{n}$ (pour la structure d'algèbre de Boole de $\mathbb{k}\left[\mathcal{G} r\left(E_{n}\right)\right]$ ) est $s_{0}^{n}$ si $i=j=0$, et que sinon le coefficient de $s_{0}^{n}$ dans sa décomposition dans la base $s_{0}^{n}, \ldots, s_{n}^{n}$ de $\mathbb{k}\left[\mathcal{G} r\left(E_{n}\right)\right]^{G L_{n}(\mathbb{k})}$ est nul (en fait, on a $s_{i}^{n} \cdot s_{j}^{n}=s_{\max (i, j)}^{n}$ ). Avec la description du corollaire 6.3.11, on a $\tau^{i} \cdot \tau^{j}=\tau^{\max (i, j)}$.

(2) Si $X$ est un objet de $\mathcal{F}_{\mathcal{G} r}$, on peut munir naturellement le groupe abélien $\operatorname{hom}_{\mathcal{G} r}(\omega(X), \mathbb{k}[\mathcal{G} r])$ d'une structure de module sur l'algèbre de Boole $\left(\mathrm{A}_{\mathcal{G} r}(\mathbb{k}),.\right)$, en utilisant la structure de $\mathbb{k}[\mathcal{G} r]$-comodule de $\omega(X)$ et en procédant comme au point précédent. Cela suggère une structure algèbrique très riche sur ces objets, qui sont également, comme tous les groupes $\operatorname{hom}_{\mathcal{G} r}(F, \mathbb{k}[\mathcal{G} r])$, des modules sur l'algèbre de séries formelles $\left(\mathrm{A}_{\mathcal{G} r}(\mathbb{k}), *\right) \simeq \mathbb{k} \oplus \mathbb{k}[[\tau]]$. 



\section{CHAPITRE 7}

\section{LA CATÉGORIE $\mathcal{F}_{\mathcal{G}_{r, I}}$ COMME CATÉGORIE DE MODULES}

L'équivalence entre la catégorie $\mathcal{F}_{\mathcal{G} r}$ et la catégorie des $\mathbb{k}[\mathcal{G} r]$-comodules repose sur l'adjonction entre les foncteurs $\iota$ et $\omega$. Nous donnons maintenant une équivalence entre la catégorie $\mathcal{F}_{\mathcal{G} r}$ (et plus généralement, toutes les catégories $\mathcal{F}_{\mathcal{G} r, I}$ ) et une catégorie de modules sur une monade explicite, à partir de l'adjonction entre les foncteurs $\xi$ et $\sigma$.

Cette équivalence permet d'obtenir aisément l'adjoint à gauche au foncteur $\theta$, noté $\eta$, introduit et étudié dans la section 7.1. Ce foncteur interviendra dans le chapitre 9. Quant à la section 7.2, elle indique comment utiliser les machineries simpliciales associées aux monades dans le contexte développé en début du présent chapitre; la résolution canonique ainsi obtenue (dont le début était déjà présent en filigrane dans le lemme 5.4.10) s'avère notamment adaptée à l'étude des foncteurs dérivés du foncteur $\eta$, qui est pratiquement le seul foncteur non exact que nous rencontrerons entre catégories de foncteurs en grassmanniennes.

Convention 7.0.1. - Dans ce chapitre, on se donne une partie $I$ de $\mathbb{N}$.

On rappelle que le foncteur $\xi_{I}: \mathcal{F} \otimes \mathcal{F}_{\text {surj }}^{I} \rightarrow \mathcal{F}_{\mathcal{G} r, I}$ est adjoint à gauche à $\sigma_{I}$ (proposition 5.1.17). Les transformations naturelles introduites dans la notation suivante seront identifiées dans la proposition 7.0.3.

Notation 7.0.2. - Nous désignerons par $\mathcal{T}_{\mathcal{G} r, I}=\left(\widetilde{\Delta}_{\text {surj }}^{I}, u_{\mathcal{G} r, I}, \mu_{\mathcal{G} r, I}\right)$ la monade sur $\mathcal{F} \otimes \mathcal{F}_{\text {surj }}^{I}$ associée à l'adjonction entre les foncteurs $\xi_{I}$ et $\sigma_{I}$ conformément à la proposition A.2.3. Ainsi :

(1) on a $\widetilde{\Delta}_{\text {surj }}^{I}=\sigma_{I} \circ \xi_{I}$, soit $\widetilde{\Delta}_{\text {surj }}^{I}(F)(A, B)=F(A \oplus B, B)$ sur les objets $(F \in$ $\left.\mathrm{Ob} \mathcal{F} \otimes \mathcal{F}_{\text {surj }}^{I}, A \in \mathrm{Ob}^{f}, B \in \mathrm{Ob}_{\text {surj }}^{I}\right)$.

(2) La transformation naturelle $u_{\mathcal{G} r, I}: i d \rightarrow \widetilde{\Delta}_{\text {surj }}^{I}$ est l'unité de l'adjonction.

(3) La transformation naturelle $\mu_{\mathcal{G} r, I}:\left(\widetilde{\Delta}_{\text {surj }}^{I}\right)^{2} \rightarrow \widetilde{\Delta}_{\text {surj }}^{I}$ est donnée par $\sigma_{I}\left(v_{\xi_{I}}\right)$, où $v$ désigne la coünité de l'adjonction. 
Proposition 7.0.3. - Soit $F$ un objet de $\mathcal{F} \otimes \mathcal{F}_{\text {surj }}^{I}$.

(1) L'unité $u_{\mathcal{G} r, I}: i d \hookrightarrow \widetilde{\Delta}_{\text {surj }}^{I}$ est la transformation naturelle injective telle que $\left(\left(u_{\mathcal{G} r, I}\right)_{F}\right)_{(A, B)}: F(A, B) \rightarrow F(A \oplus B, B)$ est induit par le monomorphisme canonique $(A, B) \hookrightarrow(A \oplus B, B)$ pour tous $F \in \mathrm{Ob} \mathcal{F} \otimes \mathcal{F}_{\text {surj }}^{I}, A \in \mathrm{Ob} \mathcal{E}^{f}$ et $B \in \mathrm{Ob} \mathcal{E}_{\text {surj }}^{I}$.

(2) La multiplication $\mu_{\mathcal{G} r, I}:\left(\widetilde{\Delta}_{\text {surj }}^{I}\right)^{2} \rightarrow \widetilde{\Delta}_{\text {surj }}^{I}$ est fournie par le morphisme $F(A \oplus$ $B \oplus B, B) \rightarrow F(A \oplus B, B)$ induit le morphisme $A \oplus B \oplus B \rightarrow A \oplus B$ somme directe de $i d_{A}$ et de la somme $B \oplus B \rightarrow B$, et par le morphisme identique $B \rightarrow B$.

(3) Il existe un scindement naturel

$$
F \stackrel{\left(u_{\mathcal{G} r, I}\right)_{F}}{\longrightarrow} \widetilde{\Delta}_{\text {surj }}^{I}(F) \stackrel{\left(p_{\mathcal{G} r, I}\right)_{F}}{\longrightarrow} F
$$

où $\left(p_{\mathcal{G} r, I}\right)_{F}$ est donné sur l'objet $(A, B)$ par le morphisme induit par l'épimorphisme canonique $(A \oplus B, B) \rightarrow(A, B)$, pour tous $F \in \mathrm{Ob} \mathcal{F} \otimes \mathcal{F}_{\text {surj }}^{I}, A \in \mathrm{Ob} \mathcal{E}^{f}$ et $B \in$ $\mathrm{Ob} \mathcal{E}_{\text {surj }}^{I}$.

De plus, $\left(F,\left(p_{\mathcal{G} r, I}\right)_{F}\right)$ est un module sur la monade $\mathcal{T}_{\mathcal{G} r, I}$.

Démonstration. - Analysons la monade associée à l'adjonction entre les foncteurs $\mathfrak{O}_{I} \times \mathfrak{B}_{I}: \mathcal{E}_{\mathcal{G} r, I}^{f} \rightarrow \mathcal{E}^{f} \times \mathcal{E}_{\text {surj }}^{I}$ et $\mathfrak{L}_{I}: \mathcal{E}^{f} \times \mathcal{E}_{\text {surj }}^{I} \rightarrow \mathcal{E}_{\mathcal{G} r, I}^{f}$ (cf. proposition 4.2.1). Son unité est la transformation naturelle $i d \rightarrow\left(\mathfrak{O}_{I} \times \mathfrak{B}_{I}\right) \mathfrak{L}_{I}$ donnée par l'inclusion $(A, B) \hookrightarrow(A \oplus B, B)$ (de composantes l'inclusion du facteur direct $A$ et $\left.i d_{B}\right)$. Sa multiplication est donnée par le morphisme $(A \oplus B \oplus B, B) \rightarrow(A \oplus B, B)$ induit le morphisme $A \oplus B \oplus B \rightarrow A \oplus B$ somme directe de $i d_{A}$ et de la somme $B \oplus B \rightarrow B$, et par le morphisme identique $B \rightarrow B$. En effet, la coünité de l'adjonction est donnée sur l'objet $(V, W)$ de $\mathcal{E}_{\mathcal{G} r, I}^{f}$ par le morphisme $(V \oplus W, W) \rightarrow(V, W)$ de composantes $i d_{V}$ et $W \hookrightarrow V$.

En utilisant la proposition C.1.4 (page 192), on en déduit les deux premières assertions. La dernière est immédiate.

Le dernier point de cette proposition conduit à donner la définition suivante, qui introduit une sorte de foncteur de différence dans $\mathcal{F} \otimes \mathcal{F}_{\text {surj }}^{I}$, distinct de l'endofoncteur $\Delta_{*}$ considéré à la fin de la section 5.3.

Définition 7.0.4. - Le noyau de $\left(p_{\mathcal{G} r, I}\right)_{F}$, qui s'identifie donc au conoyau de $\left(u_{\mathcal{G} r, I}\right)_{F}$, sera noté $\Delta_{\text {surj }}^{I}(F)$. On définit ainsi un endofoncteur exact $\Delta_{\text {surj }}^{I}$ de $\mathcal{F} \otimes \mathcal{F}_{\text {surj }}^{I}$.

Le reste de ce chapitre s'emploie à tirer les conséquences du résultat suivant.

Proposition 7.0.5. - La catégorie $\mathcal{F}_{\mathcal{G} r, I}$ est équivalente à la catégorie des modules sur la monade $\mathcal{T}_{\mathcal{G} r, I}$ de $\mathcal{F} \otimes \mathcal{F}_{\text {surj }}^{I}$.

Démonstration. - Il s'agit d'un cas particulier de la proposition A.2.7, page 183 (déduite du théorème de Beck). 
Convention 7.0.6. - Dans la suite de ce chapitre, nous identifierons la catégorie

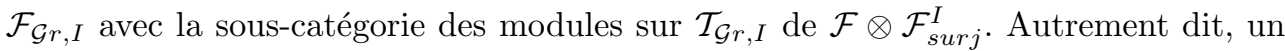
objet de $\mathcal{F}_{\mathcal{G} r, I}$ sera désormais un couple $\left(X, \widetilde{\Delta}_{\text {sur } j}^{I} X \stackrel{\tilde{m}_{X}}{\longrightarrow} X\right)$, où $X$ est un objet de $\mathcal{F} \otimes \mathcal{F}_{\text {sur } j}^{I}$ et $\tilde{m}_{X}$ un morphisme tel que :

(1) la composée $X \stackrel{\left(u_{\mathcal{G}_{r, I}}\right)_{X}}{\longrightarrow} \widetilde{\Delta}_{\text {surj }}^{I} X \stackrel{\tilde{m}_{X}}{\longrightarrow} X$ est le morphisme identique;

(2) les composées $\left(\widetilde{\Delta}_{\text {surj }}^{I}\right)^{2} X \stackrel{\left(\mu_{\left.\mathcal{G}_{r, I}\right)_{X}}\right.}{\longrightarrow} \widetilde{\Delta}_{\text {surj }}^{I} X \stackrel{\tilde{m}_{X}}{\longrightarrow} X$ et $\left(\widetilde{\Delta}_{\text {surj }}^{I}\right)^{2} X \stackrel{\widetilde{\Delta}_{\text {surj }}^{I} \tilde{m}_{X}}{\longrightarrow}$ $\widetilde{\Delta}_{\text {surj }}^{I} X \stackrel{\tilde{m}_{X}}{\longrightarrow} X$ coïncident.

Par abus, nous noterons souvent simplement $X$ pour $\left(X, \tilde{m}_{X}\right)$. Nous désignerons aussi par $m_{X}$ le morphisme $\Delta_{\text {surj }}^{I} X \hookrightarrow \widetilde{\Delta}_{\text {surj }}^{I} X \stackrel{\tilde{m}_{X}}{\longrightarrow} X$.

Avec ces conventions, les morphismes $\left(X, \tilde{m}_{X}\right) \rightarrow\left(Y, \tilde{m}_{Y}\right)$ de $\mathcal{F}_{\mathcal{G} r, I}$ sont les morphismes $f: X \rightarrow Y$ de $\mathcal{F} \otimes \mathcal{F}_{\text {surj }}^{I}$ tels que le diagramme

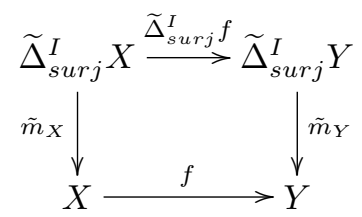

commute. Cette condition est équivalente à la commutation du diagramme analogue sans tilde.

Le lien avec la définition originelle de la catégorie $\mathcal{F}_{\mathcal{G} r, I}$ s'obtient à partir des remarques suivantes :

(1) le $\mathcal{T}_{\mathcal{G} r, I}$-module associé à un foncteur $X: \mathcal{E}_{\mathcal{G} r, I}^{f} \rightarrow \mathcal{E}$ est $\sigma_{I}(X)$ (muni de la multiplication dérivant de l'adjonction de la proposition 5.1.17);

(2) le foncteur $\mathcal{E}_{\mathcal{G}^{\prime}, I}^{f} \rightarrow \mathcal{E}$ associé à un $\mathcal{T}_{\mathcal{G} r, I}$-module $X$ est le coégalisateur de $\xi_{I}\left(\tilde{m}_{X}\right)$ et de la flèche canonique $\xi_{I}\left(\widetilde{\Delta}_{\text {surj }}^{I} X\right) \rightarrow \xi_{I}(X)$ (adjointe à $i d_{\widetilde{\Delta}_{\text {surj }}^{I} X}$ ).

Nous identifions maintenant le foncteur $\theta_{I}$ en termes de modules sur la monade $\mathcal{T}_{\mathcal{G} r, I}$.

Lemme 7.0.7. - Soient $F$ un objet de $\mathcal{F} \otimes \mathcal{F}_{\text {surj }}^{I}$, et $(A, B)$ un objet de $\mathcal{E}_{\mathcal{G} r, I}^{f}$. Notons $p_{A, B}: A \oplus B \rightarrow A$ et $\pi_{A, B}: A \rightarrow A / B$ les projections canoniques et $q_{A, B}: A \oplus B \rightarrow A$ le morphisme dont les composantes sont id $d_{A}$ et l'inclusion $B \hookrightarrow A$. La suite

$$
F(A \oplus B, B) \stackrel{F\left(p_{A, B}, i d_{B}\right)+F\left(q_{A, B}, i d_{B}\right)}{\longrightarrow} F(A, B) \stackrel{F\left(\pi_{A, B}, i d_{B}\right)}{\longrightarrow} F(A / B, B) \rightarrow 0
$$

de $\mathcal{E}$ est exacte.

Démonstration. - Notons $i: B \hookrightarrow A$ l'inclusion : on a $\pi_{A, B} \circ i=0$, donc $\pi_{A, B} \circ$ $q_{A, B}=\pi_{A, B} \circ p_{A, B}$, ce qui montre que la suite (9) est un complexe. La surjectivité de $F\left(\pi_{A, B}, i d_{B}\right)$ provient de ce que $\left(\pi_{A, B}, i d_{B}\right):(A, B) \rightarrow(A / B, B)$ admet une section. 
Pour établir l'exactitude en $F(A, B)$, considérons une rétraction $r: A \rightarrow B$ de $i$ et notons $u:(A, B) \rightarrow(A \oplus B, B)$ le morphisme $\left(i d_{A} \oplus r, i d_{B}\right)$. Alors $\left(p_{A, B}, i d_{B}\right) \circ u=$ $i d_{(A, B)}$, tandis que $\left(q_{A, B}, i d_{B}\right) \circ u$ est nul sur $(B, 0)$, donc se factorise par $\left(\pi_{A, B}, i d_{B}\right)$. Par conséquent, la restriction à $N=\operatorname{ker} F\left(\pi_{A, B}, i d_{B}\right)$ de $F(u)$ est une section du morphisme $F(A \oplus B, B) \rightarrow N$ induit par $F\left(p_{A, B}, i d_{B}\right)+F\left(q_{A, B}, i d_{B}\right)$, ce qui achève la démonstration.

Remarque 7.0.8. - Cette suite exacte est une partie d'une suite exacte longue dépendant d'une structure simpliciale (cf. proposition 7.2.1).

On déduit du lemme 7.0.7, compte-tenu des remarques précédentes sur le lien entre les deux descriptions de $\mathcal{F}_{\mathcal{G} r, I}$, la proposition suivante.

Proposition 7.0.9. - Le foncteur $\theta_{I}: \mathcal{F} \otimes \mathcal{F}_{\text {surj }}^{I} \rightarrow \mathcal{F}_{\mathcal{G} r, I}$ est donné par $\theta_{I}(F)=$ $\left(F,\left(p_{\mathcal{G} r, I}\right)_{F}: \widetilde{\Delta}_{\text {surj }}^{I} F \rightarrow F\right)$ sur les objets - cf. proposition 7.0.3. 3 - et par l'égalité $\operatorname{hom}_{\mathcal{G} r, I}\left(\theta_{I}(F), \theta_{I}(G)\right)=\operatorname{hom}_{\mathcal{F} \otimes \mathcal{F}_{\text {surj }}^{I}}(F, G)$ sur les morphismes.

Autrement dit, $\theta_{I}$ identifie $\mathcal{F} \otimes \mathcal{F}_{\text {surj }}^{I}$ à la sous-catégorie pleine de $\mathcal{F}_{\mathcal{G} r, I}$ formée des objets $X$ tels que $m_{X}=0$.

Remarque 7.0.10. - Ce résultat fournit une seconde démonstration de la pleine fidélité du foncteur $\theta_{I}$ et de ce que son image est une sous-catégorie de Serre de $\mathcal{F}_{\mathcal{G} r, I}$ (cf. proposition 5.1.15).

Exemple 7.0.11. - Considérons le cas où $I$ est réduit à l'entier 1 . On a $\Delta_{\text {surj }}^{1}=\Delta$; le foncteur $\omega_{1}: \mathcal{F}_{\mathcal{G} r, 1} \rightarrow \mathcal{F}$ associe à un objet $\left(X, \tilde{m}_{X}\right)$ le coégalisateur de la flèche canonique $\bar{P} \otimes \Delta X \rightarrow \bar{P} \otimes X$ (composée de $\bar{P} \otimes \Delta X \stackrel{j \otimes \Delta X}{\longrightarrow} \bar{P}^{\otimes 2} \otimes \Delta X$, où $j$ est le coproduit de $\bar{P}$, et de $\bar{P} \otimes 2 \otimes \Delta X \stackrel{\bar{P} \otimes f}{\longrightarrow} \bar{P} \otimes X$, où $f$ est la coünité de l'adjonction) et de $\bar{P} \otimes m_{X}$. Cette description de $\omega_{1}$ se déduit aisément de l'exactitude de ce foncteur de l'isomorphisme canonique $\omega_{1} \circ \iota_{1} \simeq \bar{P} \otimes \cdot$.

Par conséquent, la proposition 7.0.9 montre que, pour tout objet $F$ de $\mathcal{F}$, le foncteur $\omega_{1} \kappa_{1}(F)$ est canoniquement isomorphe au conoyau de l'application naturelle $\bar{P} \otimes$ $\Delta F \rightarrow \bar{P} \otimes F$.

\subsection{Le foncteur $\eta_{I}$}

Les considérations précédentes permettent de décrire très naturellement l'adjoint à gauche au foncteur $\theta_{I}$.

Définition 7.1.1. - On définit un foncteur $\eta_{I}: \mathcal{F}_{\mathcal{G} r, I} \rightarrow \mathcal{F} \otimes \mathcal{F}_{\text {surj }}^{I}$ par $\eta_{I}(X)=$ coker $m_{X}$ sur les objets; si $f: X \rightarrow Y$ est un morphisme de $\mathcal{T}_{\mathcal{G} r, I}$-modules, $\eta_{I}(f)$ : $\eta_{I}(X) \rightarrow \eta_{I}(Y)$ est le morphisme induit par $f$.

Ainsi, le foncteur $\eta_{I}: \mathcal{F}_{\mathcal{G} r, I} \rightarrow \mathcal{F} \otimes \mathcal{F}_{\text {surj }}^{I}$ est un quotient du foncteur $\sigma_{I}$. 
Lemme 7.1.2. - Pour tout foncteur $X$ de $\mathcal{F}_{\mathcal{G} r, I}$, il existe un épimorphisme naturel $X \rightarrow \theta_{I} \eta_{I}(X)$. De plus, $\theta_{I} \eta_{I}(X)$ est le plus grand quotient de $X$ appartenant à l'image du foncteur $\theta_{I}: \mathcal{F} \otimes \mathcal{F}_{\text {surj }}^{I} \rightarrow \mathcal{F}_{\mathcal{G} r, I}$.

Démonstration. - Le diagramme

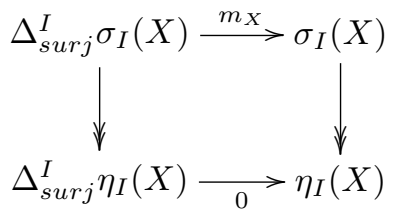

de $\mathcal{F} \otimes \mathcal{F}_{\text {surj }}^{I}$ commute, de sorte que la projection $\sigma_{I}(X) \rightarrow \eta_{I}(X)$ induit un épimorphisme $X \rightarrow \theta_{I} \eta_{I}(X)$ dans $\mathcal{F}_{\mathcal{G} r, I}$. La proposition 7.0.9 et la propriété universelle du conoyau montrent ensuite que tout épimorphisme de $X$ sur un foncteur dans l'image de $\theta_{I}$ se factorise par $X \rightarrow \theta_{I} \eta_{I}(X)$.

Le principal résultat de cette section est le suivant.

Proposition 7.1.3. - Le foncteur $\eta_{I}$ est adjoint à gauche au foncteur $\theta_{I}: \mathcal{F} \otimes$ $\mathcal{F}_{\text {surj }}^{I} \rightarrow \mathcal{F}_{\mathcal{G} r, I}$.

Démonstration. - C'est une conséquence directe du lemme précédent, puisque le foncteur $\theta_{I}$ est pleinement fidèle (cf. proposition 5.1.15).

Les deux énoncés suivants sont à rapprocher du corollaire 5.5.12 relatif aux cosocles.

Corollaire 7.1.4. - Il existe un isomorphisme

$$
\operatorname{cosoc} X \simeq \theta_{I}\left(\operatorname{cosoc} \eta_{I}(X)\right)
$$

naturel en l'objet $X$ de $\mathcal{F}_{\mathcal{G} r, I}$.

Démonstration. - Ce corollaire se déduit des propositions 5.5.10 et 7.1.3.

Proposition 7.1.5. - Il existe des isomorphismes d'endofoncteurs de $\mathcal{F} \otimes \mathcal{F}_{\text {sur } j}^{I}$

$$
\eta_{I} \circ \xi_{I} \simeq \eta_{I} \circ \theta_{I} \simeq i d .
$$

Démonstration. - L'isomorphisme $\eta_{I} \xi_{I} \simeq i d$ vient de ce que le foncteur $\eta_{I} \xi_{I}$ est adjoint à gauche à $\sigma_{I} \theta_{I} \simeq i d$ (proposition 5.1.16).

L'isomorphisme $\eta_{I} \theta_{I} \simeq i d$ découle de la proposition 7.0 .9 et de la définition de $\eta_{I}$.

Nous signalons dans la remarque qui suit un autre lien entre les foncteurs $\eta_{I}, \xi_{I}$, $\sigma_{I}$ et $\theta_{I}$. 
Remarque 7.1.6. - On vérifie qu'il existe un diagramme commutatif cocartésien

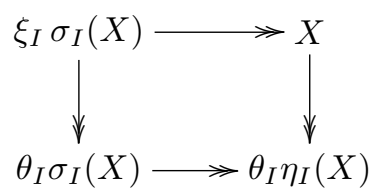

naturel en l'objet $X$ de $\mathcal{F}_{\mathcal{G} r, I}$.

La proposition suivante, qui ne découle pas formellement de l'adjonction entre les foncteurs $\eta_{I}$ et $\theta_{I}$, illustre l'utilité de la description monadique explicite de la catégorie $\mathcal{F}_{\mathcal{G} r, I}$

Proposition 7.1.7. - Il existe un isomorphisme

$$
\eta_{I}(X \otimes Y) \simeq \eta_{I}(X) \otimes \eta_{I}(Y)
$$

naturel en les objets $X$ et $Y$ de $\mathcal{F}_{\mathcal{G} r, I}$.

Démonstration. - La commutation du foncteur $\widetilde{\Delta}_{\text {surj }}^{I}$ au produit tensoriel procure un isomorphisme canonique $\Delta_{\text {surj }}^{I}(X \otimes Y) \simeq\left(\Delta_{\text {surj }}^{I} X \otimes Y\right) \oplus\left(X \otimes \Delta_{\text {surj }}^{I} Y\right) \oplus\left(\Delta_{\text {surj }}^{I} X \otimes\right.$ $\left.\Delta_{\text {surj }}^{I} Y\right)$, dans lequel $m_{X \otimes Y}$ se lit comme le morphisme de composantes $\Delta_{\text {surj }}^{I} X \otimes$ $Y \stackrel{m_{X} \otimes Y}{\longrightarrow} X \otimes Y, X \otimes \Delta_{\text {surj }}^{I} Y \stackrel{X \otimes m_{Y}}{\longrightarrow} X \otimes Y$ et $\Delta_{\text {surj }}^{I} X \otimes \Delta_{\text {surj }}^{I} Y \stackrel{m_{X} \otimes m_{Y}}{\longrightarrow} X \otimes Y$. Par conséquent, l'image de $m_{X \otimes Y}$ est la somme des sous-objets $i m\left(m_{X}\right) \otimes Y, X \otimes i m\left(m_{Y}\right)$ et $i m\left(m_{X}\right) \otimes i m\left(m_{Y}\right)$ de $X \otimes Y$, i.e. $i m\left(m_{X}\right) \otimes Y+X \otimes i m\left(m_{Y}\right)=\operatorname{ker}(X \otimes Y \rightarrow$ $\left.\eta_{I}(X) \otimes \eta_{I}(Y)\right)$. Ainsi, coker $m_{X \otimes Y}=\eta_{I}(X \otimes Y)$ s'identifie à $\eta_{I}(X) \otimes \eta_{I}(Y)$.

\subsection{Résolution canonique et algèbre homologique monadique}

L'intérêt majeur de l'adjonction entre les foncteurs $\xi_{I}: \mathcal{F} \otimes \mathcal{F}_{\text {surj }}^{I} \rightarrow \mathcal{F}_{\mathcal{G} r, I}$ et $\sigma_{I}$, qui sous-tend toute cette section, réside dans la possibilité de construire une résolution naturelle d'un objet de $\mathcal{F}_{\mathcal{G} r, I}$ par des foncteurs dans l'image du foncteur $\xi_{I}$. Cette résolution, donnée par la proposition suivante, permet de ramener le comportement homologique de la catégorie $\mathcal{F}_{\mathcal{G} r, I}$ à celui de la catégorie $\mathcal{F} \otimes \mathcal{F}_{\text {surj }}^{I}$.

Proposition 7.2.1. - Il existe une suite exacte

$$
\begin{gathered}
\cdots \rightarrow \xi_{I}\left(\Delta_{\text {surj }}^{I}\right)^{n} \sigma_{I}(X) \rightarrow \xi_{I}\left(\Delta_{\text {surj }}^{I}\right)^{n-1} \sigma_{I}(X) \rightarrow \cdots \\
\cdots \rightarrow \xi_{I} \Delta_{\text {surj }}^{I} \sigma_{I}(X) \rightarrow \xi_{I} \sigma_{I}(X) \rightarrow X \rightarrow 0
\end{gathered}
$$

naturelle en l'objet $X$ de $\mathcal{F}_{\mathcal{G} r, I}$.

Démonstration. - Nous revenons à la définition originelle de $\mathcal{F}_{\mathcal{G} r, I}$, et notons $\mathbf{E}$ la sous-catégorie pleine de $\mathbf{E n s}^{f}$ formée des ensembles finis non vides. 
On définit un foncteur $a: \mathcal{E}_{\mathcal{G} r, I}^{f} \times \mathbf{E}^{o p} \rightarrow \mathcal{E}_{\mathcal{G} r, I}^{f}$ en associant à un objet $(V, W)$ de $\mathcal{E}_{\mathcal{G} r, I}^{f}$ et à un ensemble fini non vide $E$ la somme amalgamée de $V$ et de $W^{E}$ relativement aux monomorphismes $W \hookrightarrow V$ (inclusion) et $W \hookrightarrow W^{E}$ (plongement diagonal - on utilise ici la non-vacuité de $E$ ) muni de la base $W$. L'action sur les morphismes se déduit de la fonctorialité de l'association $\mathcal{E}^{f} \times\left(\mathbf{E n s}^{f}\right)^{o p} \rightarrow \mathcal{E}^{f} \quad(V, E) \mapsto V^{E} \simeq$ $V \otimes \mathbb{k}^{E}$. On remarque que si $E$ est de cardinal $n$, l'endofoncteur $a(\cdot, E)$ de $\mathcal{E}_{\mathcal{G} r, I}^{f}$ est isomorphe à la $n-1$-ième itérée du foncteur $\mathfrak{L}_{I} \circ\left(\mathfrak{O}_{I} \times \mathfrak{B}_{I}\right)$.

Par restriction à la sous-catégorie simpliciale $\boldsymbol{\Delta}$ de $\mathbf{E}$ (squelette de la sous-catégorie des ensembles totalement ordonnés, les morphismes étant les applications croissantes), on en déduit un foncteur $\mathcal{E}_{\mathcal{G} r, I}^{f} \times \boldsymbol{\Delta}^{o p} \rightarrow \mathcal{E}_{\mathcal{G} r, I}^{f}$, puis $c_{\mathcal{G} r, I}^{\Delta}: \mathcal{E}_{\mathcal{G} r, I}^{f} \rightarrow \operatorname{Fct}\left(\boldsymbol{\Delta}^{o p}, \mathcal{E}_{\mathcal{G} r, I}^{f}\right)$. Ce foncteur vérifie les propriétés suivantes :

(1) en degré zéro, on a $\left(c_{\mathcal{G} r, I}^{\Delta}\right)_{0} \simeq i d$;

(2) en degré un, on a $\left(c_{\mathcal{G} r, I}^{\Delta}\right)_{1} \simeq \mathfrak{L}_{I} \circ\left(\mathfrak{O}_{I} \times \mathfrak{B}_{I}\right)$;

(3) plus généralement, en degré $n \in \mathbb{N}^{*}$, on a $\left(c_{\mathcal{G} r, I}^{\Delta}\right)_{n} \simeq \mathfrak{L}_{I} \circ\left(\left(\mathfrak{O}_{I} \times \mathfrak{B}_{I}\right) \mathfrak{L}_{I}\right)^{n-1}$ 。 $\left(\mathfrak{O}_{I} \times \mathfrak{B}_{I}\right)$.

Par précomposition, on en déduit un foncteur

$$
C_{\mathcal{G} r, I}^{\Delta}: \mathcal{F}_{\mathcal{G} r, I}=\boldsymbol{F} \operatorname{ct}\left(\mathcal{E}_{\mathcal{G} r, I}^{f}, \mathcal{E}\right) \rightarrow \boldsymbol{F} \operatorname{ct}\left(\mathcal{E}_{\mathcal{G} r, I}^{f} \times \boldsymbol{\Delta}^{o p}, \mathcal{E}\right) \simeq \operatorname{Fct}\left(\Delta^{o p}, \mathcal{F}_{\mathcal{G} r, I}\right)
$$

Les remarques précédentes montrent que $\left(C_{\mathcal{G} r, I}^{\Delta}\right)_{0} \simeq i d$ et $\left(C_{\mathcal{G} r, I}^{\Delta}\right)_{n} \simeq \xi_{I}\left(\widetilde{\Delta}_{\text {surj }}^{I}\right)^{n-1} \sigma_{I}$ pour $n>0$.

Le complexe de Moore associé à cet objet simplicial fournit dans $\mathcal{F}_{\mathcal{G} r, I}$ un complexe

$$
\begin{gathered}
\cdots \rightarrow \xi_{I}\left(\widetilde{\Delta}_{\text {surj }}^{I}\right)^{n} \sigma_{I}(X) \rightarrow \xi_{I}\left(\widetilde{\Delta}_{\text {surj }}^{I}\right)^{n-1} \sigma_{I}(X) \rightarrow \cdots \\
\cdots \rightarrow \xi_{I} \widetilde{\Delta}_{\text {surj }}^{I} \sigma_{I}(X) \rightarrow \xi_{I} \sigma_{I}(X) \rightarrow X \rightarrow 0
\end{gathered}
$$

naturel en $X$. Nous allons montrer qu'il est acyclique. Évaluée sur un objet $(V, W)$ de $\mathcal{E}_{\mathcal{G} r, I}^{f}$, sa différentielle $\partial_{n}:\left(C_{\mathcal{G} r, I}^{\boldsymbol{\Delta}}\right)_{n} \rightarrow\left(C_{\mathcal{G} r, I}^{\Delta}\right)_{n-1}$ est la somme alternée des $n+1$ morphismes induits par $\left[v, w_{0}, \ldots, w_{n}\right] \mapsto\left[v, w_{0}, \ldots, \hat{w}_{i}, \ldots, w_{n}\right]$ (le chapeau signifiant que le terme considéré doit être omis), où l'on désigne par $\left[v, w_{0}, \ldots, w_{n}\right]$ la classe dans $V \underset{W}{\oplus} W^{n+1}$ (base de $\left.\left(c_{\mathcal{G} r, I}^{\Delta}\right)_{n}(V, W)\right)$ de $\left(v, w_{0}, \ldots, w_{n}\right) \in V \oplus W^{n+1}$. On obtient donc une homotopie entre les endomorphismes nul et identique de ce complexe en considérant les morphismes induits par $\left[v, w_{0}, \ldots, w_{n}\right] \mapsto\left[v,-\pi(v), w_{0}, \ldots, w_{n}\right]$, où $\pi: V \rightarrow W$ est un projecteur.

La suite exacte de l'énoncé s'obtient en considérant le complexe normalisé associé à $C_{\mathcal{G} r, I}^{\Delta}$, qui est homotopiquement équivalent au complexe de Moore, donc également acyclique.

Remarque 7.2.2. - (1) La proposition 7.2.1 repose uniquement sur la proposition 7.0.3. 3 (relative au scindement naturel de la monade $\mathcal{T}_{\mathcal{G} r, I}$ ). Nous avons préféré 
en donner une démonstration directe car les objets simpliciaux de $\mathcal{E}_{\mathcal{G}_{r, I}}^{f}$ qui apparaissent sont particulièrement naturels, et plus parlants que la construction générale d'un objet simplicial canonique à partir d'une monade scindée.

(2) Les monades et les comonades (en particulier, celles qui proviennent d'adjonctions) fournissent un cadre général efficace pour faire de l'algèbre homologique, y compris dans un contexte non abélien; la proposition 7.2.1 et les quelques conséquences que nous développons en sont un cas particulier. On trouvera dans [BB69] une exposition systématique de ces notions.

On prendra garde au fait que la notion de résolution canonique dans ce cadre général est légèrement différente (on part d'une comonade pour obtenir une résolution homologique). En appliquant le foncteur $\sigma_{I}$ à la résolution de la proposition 7.2.1, on obtient la résolution canonique pour la comonade associée à l'adjonction entre $\xi_{I}$ et $\sigma_{I}$ qui sert de point de départ à [BB69]. La possibilité de « relever $»$ cette résolution dans $\mathcal{F} \otimes \mathcal{F}_{\text {surj }}^{I}$ en une résolution dans $\mathcal{F}_{\mathcal{G} r, I}$ provient du scindement de la monade $\mathcal{T}_{\mathcal{G} r, I} ;$ alors que la résolution initiale dans $\mathcal{F} \otimes \mathcal{F}_{\text {surj }}^{I}$ n'apporte essentiellement rien, la proposition 7.2.1 constitue un résultat important sur la structure de $\mathcal{F}_{\mathcal{G} r, I}$.

Définition 7.2.3 (Résolution canonique). - Le complexe concentré en degrés positifs

$$
\cdots \rightarrow \xi_{I}\left(\Delta_{\text {surj }}^{I}\right)^{n} \sigma_{I}(X) \rightarrow \xi_{I}\left(\Delta_{\text {surj }}^{I}\right)^{n-1} \sigma_{I}(X) \rightarrow \cdots \rightarrow \xi_{I} \Delta_{\text {surj }}^{I} \sigma_{I}(X) \rightarrow \xi_{I} \sigma_{I}(X)
$$

de la proposition 7.2.1 est appelé résolution canonique de l'objet $X$ de $\mathcal{F}_{\mathcal{G} r, I}$. Nous la noterons $\Re_{\bullet}^{\mathcal{G} r, I}(X)$. Ainsi, $\Re_{n}^{\mathcal{G} r, I}(X)=\xi_{I}\left(\Delta_{\text {surj }}^{I}\right)^{n} \sigma_{I}(X)$ si $n \geq 0,0$ sinon.

Remarque 7.2.4. - Le lemme 5.4.10, élémentaire mais fondamental pour classifier les objets finis de $\mathcal{F}_{\mathcal{G} r, I}$, se réinterprète de façon évidente à l'aide de la résolution canonique.

Dans le lemme 14.1.5 (assertion 4) que nous démontrerons dans la partie IV, c'est encore le début de la résolution canonique dans $\mathcal{F}_{\mathcal{G} r, 1}\left(\mathbb{F}_{2}\right)$ qui permettra de donner une estimation de degré essentielle à un argument de récurrence.

Ces observations illustrent le rôle de cette résolution canonique dans la structure de $\mathcal{F}_{\mathcal{G} r, I}$.

Le résultat suivant constitue l'une des conséquences les plus notables de la proposition 7.2.1.

Corollaire 7.2.5. - Il existe une suite spectrale du premier quadrant (donc convergente) naturelle en les objets $X$ et $Y$ de $\mathcal{F}_{\mathcal{G} r, I}$ dont le terme $E^{1}$ est donné par

$$
E_{p, q}^{1}=\operatorname{Ext}_{\mathcal{F} \otimes \mathcal{F}_{\text {surj }}^{I}}^{p}\left(\left(\Delta_{\text {surj }}^{I}\right)^{q} \sigma_{I}(X), \sigma_{I}(Y)\right)
$$

et dont l'aboutissement est $\operatorname{Ext}_{\mathcal{G} r, I}^{*}(X, Y)$. 
Démonstration. - On considère les deux suites spectrales associées au bicomplexe $\operatorname{hom}_{\mathcal{G} r, I}\left(\Re_{\bullet}^{\mathcal{G} r, I}(X), J^{*}(Y)\right)$ (cf. [ML63], chapitre XI, $\S 6$ ), où $J^{*}(Y)$ désigne une résolution injective de $Y$, que l'on peut choisir fonctorielle en $Y$, car la catégorie $\mathcal{F}_{\mathcal{G} r, I}$ possède un cogénérateur injectif. La suite spectrale obtenue en prenant d'abord la différentielle de $\Re_{\bullet}^{\mathcal{G} r, I}(X)$ dégénère au terme $E^{2}$, donné par $\operatorname{Ext}_{\mathcal{G} r, I}^{*}(X, Y)$, tandis que celle obtenue en considérant d'abord la différentielle de $J^{*}(Y)$ a le terme $E^{1}$ indiqué dans l'énoncé, par adjonction entre les foncteurs exacts $\xi_{I}$ et $\sigma_{I}$.

La proposition suivante montre que l'on peut ramener théoriquement le calcul des groupes d'extension dans $\mathcal{F}_{\mathcal{G} r, I}$ entre des foncteurs finis à un nombre fini de calculs de groupes d'extension entre foncteurs finis de $\mathcal{F} \otimes \mathcal{F}_{\text {surj }}^{I}$.

Proposition 7.2.6. — La résolution canonique d'un foncteur polynomial $X$ de $\mathcal{F}_{\mathcal{G} r, I}$ est finie; sa longueur est majorée par $\operatorname{deg} X+1$ si $X \neq 0$.

Démonstration. - Grâce à la proposition 5.4.7, il suffit de voir que l'endofoncteur $\Delta_{\text {surj }}^{I}$ de $\mathcal{F} \otimes \mathcal{F}_{\text {surj }}^{I}$ diminue strictement le degré des foncteurs polynomiaux non nuls. Ce fait est une traduction du lemme 5.4.12.

Nous appliquons maintenant la proposition 7.2.1 à des considérations relatives aux foncteurs dérivés gauches du foncteur $\eta_{I}$, qui est exact à droite par la proposition 7.1.3.

Notation 7.2.7. - Étant donné $n \in \mathbb{Z}$, nous noterons $h_{n}^{\mathcal{G} r, I}: \mathcal{F}_{\mathcal{G} r, I} \rightarrow \mathcal{F} \otimes \mathcal{F}_{\text {surj }}^{I}$ le $n$-ième foncteur dérivé gauche de $\eta_{I}$.

Remarque 7.2.8. - Les foncteurs dérivés de $\eta_{I}$ mesurent, intuitivement, le défaut d'essentielle surjectivité du foncteur $\theta_{I}$, donc la différence homologique entre les catégories $\mathcal{F} \otimes \mathcal{F}_{\text {surj }}^{I}$ et $\mathcal{F}_{\mathcal{G} r, I}$. Cela motive la notation employée pour ces foncteurs.

En effet, si l'on se restreint aux foncteurs finis, nous avons vu (proposition 5.5.10, page 71 ) que tout objet de $\mathcal{F}_{\mathcal{G} r, I}$ s'obtient par extensions successives de foncteurs appartenant à l'image du foncteur $\theta_{I}$. La description de ces objets équivaut donc essentiellement au calcul de groupes d'extensions entre deux objets de l'image de $\theta_{I}$. Ces calculs font naturellement intervenir les foncteurs $h_{n}^{\mathcal{G} r, I}$ : il existe une suite spectrale du premier quadrant

$$
E_{p, q}^{2}=\operatorname{Ext}_{\mathcal{F} \otimes \mathcal{F}_{\text {surj }}^{I}}^{p}\left(h_{q}^{\mathcal{G} r, I}(X), F\right) \Rightarrow \operatorname{Ext}_{\mathcal{G} r, I}^{*}\left(X, \theta_{I}(F)\right)
$$

naturelle en les objets $F$ de $\mathcal{F} \otimes \mathcal{F}_{\text {surj }}^{I}$ et $X$ de $\mathcal{F}_{\mathcal{G} r, I}$.

La proposition et le corollaire suivants établissent le lien entre le foncteur gradué $h_{*}^{\mathcal{G} r, I}$ et la résolution canonique.

Proposition 7.2.9. - Pour tout entier $n>0$ et tout objet $F$ de $\mathcal{F} \otimes \mathcal{F}_{\text {surj }}^{I}$, on a $h_{n}^{\mathcal{G} r, I}\left(\xi_{I}(F)\right)=0$. 
Démonstration. - Soit $P_{\bullet}$ une résolution projective de $F$. Comme le foncteur $\xi_{I}$ est exact et préserve les projectifs (son adjoint à droite $\sigma_{I}$ est exact), $\xi\left(P_{\bullet}\right)$ est une résolution projective de $\xi_{I}(F)$. La proposition 7.1 .5 montre que $\eta_{I} \xi_{I}\left(P_{\bullet}\right)$ s'identifie à $P_{\bullet}$. Ce complexe, dont l'homologie est (isomorphe à) $h_{*}^{\mathcal{G} r, I}\left(\xi_{I}(F)\right)$, est donc acyclique en degrés strictement positifs.

Corollaire 7.2.10. — Le foncteur gradué $h_{*}^{\mathcal{G} r, I}$ est canoniquement isomorphe à l'homologie du complexe $\eta_{I}\left(\Re_{\bullet}^{\mathcal{G} r, I}\right)$ de foncteurs $\mathcal{F}_{\mathcal{G} r, I} \rightarrow \mathcal{F} \otimes \mathcal{F}_{\text {surj }}^{I}$.

Nous illustrons à présent la proposition 7.2.9 par un calcul élémentaire.

Exemple 7.2.11 (Calcul sur les injectifs standard - cas $I=\mathbb{N}$ )

La proposition 5.1.14 permet un calcul rapide des objets $h_{*}^{\mathcal{G} r}\left(I_{(V, W)}^{\mathcal{G} r}\right)$. En effet, par les propositions 7.1.5 et 7.2.9, on a $h_{0}^{\mathcal{G} r}\left(\iota\left(I_{V}\right)\right) \simeq I_{V}$, où l'on plonge $\mathcal{F}$ dans $\mathcal{F} \otimes \mathcal{F}_{\text {surj }}$ par le foncteur $\cdot \nabla \mathbb{k}$, et $h_{n}^{\mathcal{G} r}\left(\iota\left(I_{V}\right)\right)=0$ si $n \neq 0$. Comme $\eta\left(I_{(V, 0)}\right) \simeq \eta\left(\kappa\left(I_{V}\right)\right) \simeq I_{V}$ (par les propositions 5.1.12 et 7.1.5), on a finalement :

(1) l'objet gradué $h_{*}^{\mathcal{G} r}\left(I_{(V, W)}^{\mathcal{G} r}\right)$ est nul si $\operatorname{dim} W>0$;

(2) l'objet gradué $h_{*}^{\mathcal{G} r}\left(I_{(V, 0)}^{\mathcal{G} r}\right)$ est concentré en degré 0 , où il est naturellement isomorphe à $I_{V}$.

La proposition 7.1.7 fournit la formule de Künneth suivante.

Proposition 7.2.12. - Il existe un isomorphisme d'objets gradués de $\mathcal{F} \otimes \mathcal{F}_{\text {surj }}^{I}$

$$
h_{*}^{\mathcal{G} r, I}(X \otimes Y) \simeq h_{*}^{\mathcal{G} r, I}(X) \otimes h_{*}^{\mathcal{G} r, I}(Y)
$$

naturel en les objets $X$ et $Y$ de $\mathcal{F}_{\mathcal{G} r, I}$.

Démonstration. - Comme le foncteur $\xi_{I}$ commute au produit tensoriel, la proposition 7.2.9 montre que le complexe total du produit tensoriel des résolutions canoniques

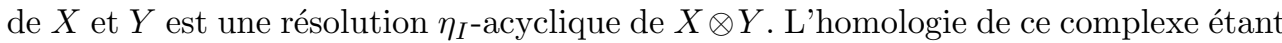
naturellement isomorphe au produit tensoriel de $h_{*}^{\mathcal{G} r, I}(X)$ et $h_{*}^{\mathcal{G} r}, I(Y)$, on en déduit la proposition. 


\section{CHAPITRE 8}

\section{LES CATÉGORIES $\mathcal{F}_{\mathrm{Pl}, n}$}

Le diagramme de recollement de la proposition 5.1.2 permet de dévisser la catégorie de foncteurs en grassmanniennes globale $\mathcal{F}_{\mathcal{G} r}(\mathbb{k})$ à l'aide des catégories $\mathcal{F}_{\mathcal{G} r, n}(\mathbb{k})$. Il est patent que l'on peut pousser plus loin ce dévissage, en raison de l'intervention du groupe linéaire $G L_{n}(\mathbb{k})$ dans un grand nombre de considérations relatives à cette catégorie. Nous étudions dans ce chapitre des catégories, notées $\mathcal{F}_{\mathbf{P l}, n}(\mathbb{k})$, réalisant un tel dévissage, dans un sens qui sera précisé et illustré à la fin de la section 8.2.

Nous traitons d'abord de généralités qui constituent le pendant dans ces catégories des propriétés de base des autres catégories de foncteurs en grassmanniennes, et qui s'expriment souvent plus simplement dans $\mathcal{F}_{\mathbf{P l}, n}$, parce que nous nous sommes « débarrassés » de l'action du groupe linéaire en « rigidifiant les bases ». Dans la section 8.2, nous nous attachons à des phénomènes spécifiques aux catégories $\mathcal{F}_{\mathbf{P l}, n}$ : nous en donnons une description en termes de comodules qui diffère de celles étudiées dans le cadre «booléen » du chapitre 3 - en particulier, le produit cotensoriel fournit une structure différente de la structure tensorielle usuelle de la catégorie de foncteurs $\mathcal{F}_{\mathbf{P l}, n}$. La fin de ce chapitre illustre l'intérêt de cette catégorie pour étudier $\mathcal{F}_{\mathcal{G}_{r, n}}$ : nous montrons comment la détermination d'un adjoint dans $\mathcal{F}_{\mathbf{P l}, n}$ procurée par la structure cotensorielle que nous venons d'évoquer permet de décrire un adjoint dans la catégorie $\mathcal{F}_{\mathcal{G} r, n}$. Cela constitue une motivation supplémentaire pour étudier les structures de comodules associées à $\mathcal{F}_{\mathbf{P l}, n}$, qui possèdent par ailleurs un intérêt intrinsèque.

Convention 8.0.1. - Dans toute ce chapitre, $n$ désigne un entier naturel. Nous noterons encore $E_{n}$, pour abréger, l'objet initial $\left(E_{n}, i d\right)$ de $\mathcal{E}_{\mathbf{P l}, n}^{f}(\mathbb{k})$.

Définition 8.0.2. - La catégorie de foncteurs en grassmanniennes $\mathcal{F}_{\mathbf{P l}, n}(\mathbb{k})$ est la catégorie définie par

$$
\mathcal{F}_{\mathbf{P l}, n}(\mathbb{k})=\operatorname{Fct}\left(\mathcal{E}_{\mathbf{P l}, n}^{f}(\mathbb{k}), \mathcal{E}_{\mathbb{k}}^{f}\right)
$$




\subsection{Généralités}

Nous introduisons maintenant des foncteurs analogues à ceux de la section 5.1 dans le cas $\mathcal{F}_{\mathbf{P l}, n}$ et en donnons les propriétés élémentaires. Les démonstrations, semblables à celles de ladite section, sont laissées au lecteur.

Notation 8.1.1. - Nous abrégerons respectivement en $\operatorname{hom}_{\mathrm{Pl}, n}, P_{X}^{\mathrm{Pl}, n}, I_{X}^{\mathrm{Pl}, n}$ les expressions $\operatorname{hom}_{\mathcal{F}_{\mathrm{Pl}, n}}, P_{X}^{\mathcal{E}_{\mathrm{Pl}, n}^{f}}$ et $I_{X}^{\mathcal{E}_{\mathrm{Pl}, n}^{f}}$.

Définition 8.1.2. - (1) Le foncteur de restriction sans plongement $\gamma_{n}: \mathcal{F}_{\mathcal{G} r, n} \rightarrow$ $\mathcal{F}_{\mathbf{P l}, n}$ est défini comme étant le foncteur de précomposition par le foncteur d'oubli du plongement $i n c_{n}^{\mathbf{P l}}: \mathcal{E}_{\mathbf{P l}, n}^{f} \rightarrow \mathcal{E}_{\mathcal{G} r, n}^{f}$.

(2) Le foncteur de plongement standard $\iota_{n}^{\mathbf{P l}}: \mathcal{F} \rightarrow \mathcal{F}_{\mathbf{P l}, n}$ est le foncteur de précomposition par le foncteur d'oubli principal $\overline{\mathfrak{O}}_{n}: \mathcal{E}_{\mathbf{P l}, n}^{f} \rightarrow \mathcal{E}^{f}$.

(3) Le foncteur de plongement réduit $\kappa_{n}^{\mathbf{P l}}: \mathcal{F} \rightarrow \mathcal{F}_{\mathbf{P l}, n}$ est le foncteur de précomposition par le foncteur de réduction $\overline{\mathfrak{K}}_{n}: \mathcal{E}_{\mathbf{P l}, n}^{f} \rightarrow \mathcal{E}^{f}$.

(4) On définit le foncteur de décalage en grassmanniennes $\sigma_{n}^{\mathbf{P l}}: \mathcal{F}_{\mathbf{P l}, n} \rightarrow \mathcal{F}$ comme le foncteur de précomposition par le foncteur de décalage pointé $\mathfrak{S}_{n}: \mathcal{E}^{f} \rightarrow \mathcal{E}_{\mathbf{P l}, n}^{f}$.

Explicitement, on a

$$
\begin{gathered}
\gamma_{n}(X)(V, u)=X(V, \text { im } u), \\
\iota_{n}^{\mathbf{P l}}(F)(V, u)=F(V), \\
\kappa_{n}^{\mathbf{P l}}(F)(V, u)=F(\text { coker } u), \\
\sigma_{n}^{\mathbf{P l}}(A)(W)=A\left(W \oplus E_{n}, E_{n} \hookrightarrow W \oplus E_{n}\right)
\end{gathered}
$$

pour $X \in \mathrm{Ob} \mathcal{F}_{\mathcal{G} r, n}, F \in \mathrm{Ob} \mathcal{F}, A \in \mathrm{Ob} \mathcal{F}_{\mathbf{P l}, n},\left(V, u: E_{n} \hookrightarrow V\right) \in \mathrm{Ob}_{\mathcal{E}_{\mathbf{P l}, n}^{f}}$ et $W \in \mathrm{Ob} \mathcal{E}^{f}$.

Remarque 8.1.3. - (1) On a $\iota_{n}^{\mathrm{Pl}}=\gamma_{n} \iota_{n}$ et $\kappa_{n}^{\mathrm{Pl}}=\gamma_{n} \kappa_{n}$.

(2) Nous ne donnons pas de notation pour les foncteurs d'intégrale $\mathcal{F}_{\mathbf{P l}, n} \rightarrow \mathcal{F}$, qui ne revêtent pas la même importance que les foncteurs d'intégrale en grassmanniennes des catégories de type $\mathcal{F}_{\mathcal{G} r, I}$.

La proposition suivante donne les principales variantes des propriétés de la section 5.1 en termes des foncteurs introduits précédemment.

Proposition 8.1.4. - (1) Les foncteurs $\gamma_{n}, \iota_{n}^{\mathrm{Pl}}, \sigma_{n}^{\mathrm{Pl}}$ et $\kappa_{n}^{\mathrm{Pl}}$ sont exacts et fidèles ; ils commutent au produit tensoriel, aux limites et aux colimites.

(2) Le foncteur $\kappa_{n}^{\mathrm{Pl}}$ est de plus plein, et son image est une sous-catégorie de Serre de $\mathcal{F}_{\mathbf{P l}, n}$.

(3) La composition $\mathcal{F} \stackrel{\kappa_{n}^{\mathrm{Pl}}}{\longrightarrow} \mathcal{F}_{\mathbf{P l}, n} \stackrel{\sigma_{n}^{\mathrm{Pl}}}{\longrightarrow} \mathcal{F}$ est canoniquement isomorphe au foncteur identique.

(4) Le foncteur $\iota_{n}^{\mathbf{P l}}$ est adjoint à gauche à $\sigma_{n}^{\mathbf{P l}}$. 
(5) On a des isomorphismes $P_{\mathfrak{S}_{n}(V)}^{\mathbf{P l}, n} \simeq \iota_{n}^{\mathbf{P l}}\left(P_{V}\right)$ et $I_{\mathfrak{S}_{n}(V)}^{\mathbf{P l}, n} \simeq \kappa_{n}^{\mathbf{P l}}\left(I_{V}\right) \otimes I_{E_{n}}^{\mathbf{P l}, n}$ naturels en l'objet $V$ de $\mathcal{E}^{f}$.

(6) Le foncteur composé $\mathbb{k}\left[G L_{n}(\mathbb{k})\right]$ Mod $\stackrel{\rho_{n}}{\longrightarrow} \mathcal{F}_{\mathcal{G} r, n} \stackrel{\gamma_{n}}{\longrightarrow} \mathcal{F}_{\mathbf{P l}, n}$ est canoniquement isomorphe $\grave{a}_{\mathbb{k}\left[G L_{n}(\mathbb{k})\right]} \operatorname{Mod} \rightarrow \mathcal{E} \rightarrow \mathcal{F}_{\mathbf{P l}, n}$, où la première flèche est le foncteur d'oubli de l'action de $G L_{n}(\mathbb{k})$ et le second le plongement canonique (donné par les foncteurs constants).

Décomposition scalaire, tors de Frobenius et changement de corps. Ces notions se définissent comme dans $\mathcal{F}_{\mathcal{G} r, n}$ et possèdent des propriétés tout-à-fait analogues.

Les objets polynomiaux et finis de la catégorie $\mathcal{F}_{\mathrm{Pl}, n}$. - L'endofoncteur de $\mathcal{F}_{\mathbf{P l}, n}$ donné par la précomposition par le foncteur de translation $V \boxplus \cdot: \mathcal{E}_{\mathbf{P l}, n}^{f} \rightarrow \mathcal{E}_{\mathbf{P l}, n}^{f}$ (où $V \in \mathrm{Ob} \mathcal{E}^{f}$ ) s'appelle foncteur de décalage et se note $\Delta_{V}^{\mathrm{Pl}, n}$; il existe un scindement canonique $\Delta_{\mathbb{k}}^{\mathbf{P l}, n} \simeq i d \oplus \Delta^{\mathbf{P l}, n}$, où $\Delta^{\mathbf{P l}, n}$ est le foncteur différence de $\mathcal{F}_{\mathbf{P l}, n}$.

Les foncteurs polynomiaux de $\mathcal{F}_{\mathbf{P l}, n}$ sont ses objets $\Delta^{\mathbf{P l}, n}$-nilpotents; on a une notion de degré. On note $\mathcal{F}_{\mathbf{P l}, n}^{k}$ la sous-catégorie épaisse de $\mathcal{F}_{\mathbf{P l}, n}$ des foncteurs polynomiaux de degré au plus $k$. La catégorie $\mathcal{F}_{\mathrm{Pl}, n}^{0}$ est réduite aux foncteurs constants, comme dans la catégorie $\mathcal{F}$ (contrairement à ce qui advient dans $\mathcal{F}_{\mathcal{G} r, n}$ ). Nous nous contentons d'énoncer les analogues les plus importants des propriétés des sections 5.4 et 5.5, qui s'adaptent sans difficulté à $\mathcal{F}_{\mathbf{P l}, n}$.

Le résultat suivant, similaire à la proposition 5.4 .13 identifie les quotients de la filtration polynomiale de $\mathcal{F}_{\mathbf{P l}, n}$.

Proposition 8.1.5. - Le foncteur $\sigma_{n}^{\mathbf{P l}}: \mathcal{F}_{\mathbf{P l}, n} \rightarrow \mathcal{F}$ induit pour tout $k \in \mathbb{N}$ une équivalence de catégories $\mathcal{F}_{\mathrm{Pl}, n}^{k} / \mathcal{F}_{\mathbf{P l}, n}^{k-1} \rightarrow \mathcal{F}^{k} / \mathcal{F}^{k-1}$.

Les foncteurs $\kappa_{n}^{\mathbf{P l}}$ et $\iota_{n}^{\mathbf{P l}}: \mathcal{F} \rightarrow \mathcal{F}_{\mathbf{P l}, n}$ induisent chacun une équivalence de catégories $\mathcal{F}^{k} / \mathcal{F}^{k-1} \rightarrow \mathcal{F}_{\mathbf{P l}, n}^{k} / \mathcal{F}_{\mathbf{P l}, n}^{k-1}$ inverse de la précédente.

L'analogue suivant de la proposition 5.5.5 sous-tend, avec la proposition précédente, la proposition 8.1 .7 ci-dessous.

Proposition 8.1.6. - Un foncteur de $\mathcal{F}_{\mathbf{P l}, n}$ est fini si et seulement s'il est polynomial et à valeurs de dimension finie.

Nous en venons maintenant à la description explicite des objets simples de la catégorie $\mathcal{F}_{\mathbf{P l}, n}$ (cf. proposition 5.5.10).

Proposition 8.1.7. - (1) Étant donné un objet $X$ de $\mathcal{F}_{\mathbf{P l}, n}$, les assertions suivantes sont équivalentes.

(a) L'objet $X$ de $\mathcal{F}_{\mathbf{P l}, n}$ est simple.

(b) L'objet $\sigma_{n}^{\mathrm{Pl}}(X)$ de $\mathcal{F}$ est simple.

(c) Il existe un objet simple $S$ de $\mathcal{F}$ tel que $X \simeq \kappa_{n}^{\mathrm{Pl}}(S)$. 
(2) Les foncteurs $\kappa_{n}^{\mathbf{P l}}$ et $\sigma_{n}^{\mathbf{P l}}$ induisent des isomorphismes d'anneaux inverses l'un de l'autre entre $G_{0}^{f}(\mathcal{F})$ et $G_{0}^{f}\left(\mathcal{F}_{\mathbf{P l}, n}\right)$.

Premiers liens entre les catégories $\mathcal{F}, \mathcal{F}_{\mathcal{G} r, n}$ et $\mathcal{F}_{\mathrm{Pl}, n}$ en termes de (co)modules. - L'un des thèmes de ce chapitre consiste à compléter l'équivalence de catégories fondamentale entre $\mathcal{F}_{\mathcal{G} r}$ et les $\mathbb{k}[\mathcal{G} r]$-comodules (cf. proposition 5.1.7) par l'identification d'autres catégories de modules ou de comodules à des catégories de foncteurs. Dans ce paragraphe, nous donnons deux telles propriétés, qui reposent sur le chapitre 3 .

Proposition 8.1.8. - La catégorie $\mathcal{F}_{\mathbf{P l}, n}$ est équivalente à la sous-catégorie $\operatorname{Comod}_{P_{\left(E_{n}, E_{n}\right)}^{\mathcal{G} r, n}}$ de $\mathcal{F}_{\mathcal{G} r, n}$.

Démonstration. - Le foncteur

$$
\left(\mathcal{E}_{\mathcal{G} r, n}^{f}\right) \backslash \operatorname{hom}\left(\left(E_{n}, E_{n}\right), .\right) \rightarrow \mathcal{E}_{\mathbf{P l}, n}^{f} \quad((V, W), u) \mapsto(V, u)
$$

est une équivalence de catégories, où la catégorie source dérive de la notation 3.1.2. La proposition est donc un cas particulier de la proposition 3.1.12.

Remarque 8.1.9. - Le foncteur $\operatorname{hom}_{\mathcal{G} r, n}\left(\left(E_{n}, E_{n}\right),.\right)$ est canoniquement isomorphe à la composée $\mathcal{F}_{\mathcal{G} r, n} \stackrel{\mathfrak{B}_{n}}{\longrightarrow} \mathcal{E}_{\text {surj }}^{n} \stackrel{\operatorname{hom}\left(E_{n}, .\right)}{\longrightarrow}$ Ens. Comme $\mathcal{E}_{\text {surj }}^{n} \simeq G L_{n}(\mathbb{k})$ est équivalente à sa catégorie opposée, on peut remplacer dans la démonstration précédente ce foncteur par un foncteur contravariant, et obtenir ainsi sur $P_{\left(E_{n}, E_{n}\right)}^{\mathcal{G} r, n}$ une structure d'algèbre telle que $\mathcal{F}_{\mathbf{P l}, n}$ est équivalente à la sous-catégorie $\operatorname{Mod}_{P_{\left(E_{n}, E_{n}\right)}^{\mathcal{G} r, n}}$ de $\mathcal{F}_{\mathcal{G} r, n}$. Ce phénomène est à rapprocher de la remarque suivante $: P_{\left(E_{n}, E_{n}\right)}^{\mathcal{G} r, n}$ s'identifie à $\rho_{n}\left(\mathbb{k}\left[G L_{n}(\mathbb{k})\right]\right)$, et $\mathbb{k}\left[G L_{n}(\mathbb{k})\right]$ est un objet auto-dual de $\mathbb{k}_{\mathbb{k}}\left[G L_{n}(\mathbb{k})\right]$ Mod (la dualité consistant à associer à une représentation linéaire la représentation contragrédiente).

Grâce au lemme suivant, nous établirons, à la proposition 8.1.11, un lien direct entre les catégories $\mathcal{F}$ et $\mathcal{F}_{\mathbf{P l}, n}$.

Lemme 8.1.10. - La catégorie $\left(\mathcal{E}_{\mathrm{Pl}, n}^{f}\right) / \operatorname{hom}_{\left(., E_{n}\right)}$ est équivalente à $\mathcal{E}^{f}$.

Démonstration. - On vérifie aussitôt que les deux foncteurs introduits ci-après sont des équivalences de catégories réciproques l'une de l'autre.

- On définit un foncteur $\mathcal{E}^{f} \rightarrow\left(\mathcal{E}_{\mathbf{P l}, n}^{f}\right) / \operatorname{hom}_{\left(., E_{n}\right)}$ en associant à $V \in \mathrm{Ob} \mathcal{E}^{f}$ l'objet $\mathfrak{S}_{n}(V)$ de $\mathcal{E}_{\mathbf{P l}, n}^{f}$ muni du morphisme vers $E_{n}$ donné par la projection $V \oplus E_{n} \rightarrow E_{n}$, et à une application linéaire $f: V \rightarrow W$ le morphisme $f \oplus E_{n}$.

- On définit un foncteur $\left(\mathcal{E}_{\mathbf{P l}, n}^{f}\right) / \operatorname{hom}\left(., E_{n}\right) \rightarrow \mathcal{E}^{f}$ en associant à un objet $((V, u$ : $\left.\left.E_{n} \hookrightarrow V\right), r: V \rightarrow E_{n}\right)$ de $\left(\mathcal{E}_{\mathrm{Pl}, n}^{f}\right) /$ hom $\left(., E_{n}\right)$ l'espace vectoriel coker $u \simeq k e r r$, et à un morphisme l'application linéaire induite. 
Proposition 8.1.11. - La catégorie $\mathcal{F}$ est équivalente à la sous-catégorie $\operatorname{Mod}_{I_{E_{n}}^{\mathrm{Pl}, n}}$ de $\mathcal{F}_{\mathbf{P l}, n}$.

Démonstration. - On combine le lemme précédent avec la proposition 3.3.10.

\subsection{L'équivalence de catégories $\mathcal{F}_{\mathrm{Pl}, n} \simeq \operatorname{Comod}_{I_{E_{n}}}$}

L'analogue des considérations du chapitre 7 dans les catégories $\mathcal{F}_{\mathbf{P l}, n}$ permet d'obtenir l'équivalence de catégories éponyme de cette section. Les structures que l'on en déduit constituent une justification essentielle à l'étude des catégories $\mathcal{F}_{\mathbf{P l}, n}$, dont nous verrons comment elle peut intervenir dans les catégories de type $\mathcal{F}_{\mathcal{G} r, I}$.

Le point de départ de cette section réside dans le fait que le foncteur $\iota_{n}^{\mathrm{Pl}}$ est adjoint à gauche au foncteur $\sigma_{n}^{\mathrm{Pl}}$ (proposition 8.1.4.4).

Proposition et définition 8.2.1. - La monade de $\mathcal{F}$ associée à l'adjonction entre les foncteurs $\iota_{n}^{\mathrm{Pl}}$ et $\sigma_{n}^{\mathrm{Pl}}$ conformément à la proposition A.2.3, que nous désignerons par $\mathcal{T}_{\mathbf{P l}, n}=\left(\Delta_{E_{n}}, u_{\mathbf{P l}, n}, \mu_{\mathbf{P l}, n}\right)$, est donnée comme suit.

- Le foncteur $\Delta_{E_{n}}$ est le foncteur de décalage de $\mathcal{F}$ (cf. chapitre 1).

- La transformation naturelle $u_{\mathrm{Pl}, n}: i d \rightarrow \Delta_{E_{n}}$ (unité de l'adjonction) est induite par l'application linéaire $0 \rightarrow E_{n}$, compte-tenu de l'identification entre id et $\Delta_{0}$.

- La multiplication $\mu_{\mathbf{P l}, n}:\left(\Delta_{E_{n}}\right)^{2} \simeq \Delta_{E_{n} \oplus E_{n}} \rightarrow \Delta_{E_{n}}$ est la transformation naturelle induite par la somme $E_{n} \oplus E_{n} \rightarrow E_{n}$.

En outre, il existe un scindement naturel id $\stackrel{u_{\mathbf{P l}, n}}{\longrightarrow} \Delta_{E_{n}} \stackrel{p_{\mathbf{P l}, n}}{\longrightarrow} i d$, où $p_{\mathbf{P l}, n}$ est induit par l'application linéaire $E_{n} \rightarrow 0$.

Proposition 8.2.2. - La catégorie $\mathcal{F}_{\mathbf{P l}, n}$ est équivalente à la catégorie des modules sur la monade $\mathcal{T}_{\mathbf{P l}, n}$ de $\mathcal{F}$.

Les deux propositions précédentes se démontrent comme les propositions 7.0.3 et 7.0.5.

Convention 8.2.3. - Dans la suite de cette section, nous identifierons la catégorie $\mathcal{F}_{\mathbf{P l}, n}$ avec la sous-catégorie des modules sur $\mathcal{T}_{\mathbf{P l}, n}$ de $\mathcal{F}$. Autrement dit, un objet de $\mathcal{F}_{\mathrm{Pl}, n}$ sera désormais un couple $\left(X, \Delta_{E_{n}} X \stackrel{m_{X}}{\longrightarrow} X\right)$, où $X$ est un objet de $\mathcal{F}$ et $m_{X}$ un morphisme tel que :

- la composée $X \stackrel{\left(u_{\mathbf{P l}, n}\right)_{X}}{\longrightarrow} \Delta_{E_{n}} X \stackrel{m_{X}}{\longrightarrow} X$ est le morphisme identique;

- les composées $\left(\Delta_{E_{n}}\right)^{2} X \stackrel{\left(\mu_{\mathrm{Pl}, n}\right)_{X}}{\longrightarrow} \Delta_{E_{n}} X \stackrel{m_{X}}{\longrightarrow} X$ et $\left(\Delta_{E_{n}}\right)^{2} X \stackrel{\Delta_{E_{n}} m_{X}}{\longrightarrow}$ $\Delta_{E_{n}} X \stackrel{m_{X}}{\longrightarrow} X$ coïncident.

Par abus, nous noterons parfois simplement $X$ pour $\left(X, m_{X}\right)$.

Notation 8.2.4. - Le morphisme $X \rightarrow X \otimes I_{E_{n}}$ adjoint à $m_{X}$ (cf. proposition 1.3.1) sera noté $\psi_{X}$ dans la suite de cette section. 
Nous indiquons, dans la proposition suivante, l'analogue du foncteur $\eta_{I}$. Nous omettons la démonstration, similaire à celle des propositions 7.1.3, 7.1.5 et 7.1.7.

Proposition et définition 8.2.5 (Foncteur $\eta_{n}^{\mathrm{Pl}}$ ).

(1) On définit le foncteur $\eta_{n}^{\mathbf{P l}}: \mathcal{F}_{\mathbf{P l}, n} \rightarrow \mathcal{F}$ comme le coégalisateur des deux transformations naturelles $p_{\mathbf{P l}, n}$ (cf. notation de la proposition 8.2.1) et $m: \Delta_{E_{n}} \sigma_{n}^{\mathbf{P l}} \rightarrow \sigma_{n}^{\mathbf{P l}}$.

(2) Le foncteur $\eta_{n}^{\mathbf{P l}}$ est adjoint à gauche au foncteur $\kappa_{n}^{\mathbf{P l}}$.

(3) Il existe des isomorphismes

$$
\eta_{n}^{\mathbf{P l}} \circ \kappa_{n}^{\mathrm{Pl}} \simeq \eta_{n}^{\mathbf{P l}} \circ \iota_{n}^{\mathbf{P l}} \simeq i d .
$$

(4) Le foncteur $\eta_{n}^{\mathbf{P l}}$ commute au produit tensoriel à isomorphisme naturel près.

On peut introduire, comme dans le chapitre 7 , une notion de résolution canonique dans $\mathcal{F}_{\mathbf{P l}, n}$, grâce à laquelle on peut calculer les foncteurs dérivés du foncteur $\eta_{n}^{\mathbf{P l}}$, et relier les groupes d'extension dans $\mathcal{F}_{\mathbf{P l}, n}$ à ceux de $\mathcal{F}$ par une suite spectrale. Plutôt que de détailler ces considérations, nous abordons des constructions plus spécifiques à la catégorie $\mathcal{F}_{\mathrm{Pl}, n}$.

Remarque 8.2.6. - Pour tout espace vectoriel $V$ de dimension finie, le foncteur $P_{V}$ est muni d'une structure naturelle d'algèbre commutative, dont la multiplication est le morphisme $P_{V} \otimes P_{V} \simeq P_{V \oplus V} \rightarrow P_{V}$ induit par la diagonale $V \rightarrow V \oplus V$ et l'unité $P_{V} \rightarrow \mathbb{k} \simeq P_{0}$ par $0 \rightarrow V$. Explicitement, la structure d'algèbre sur les espaces vectoriels $P_{V}(W)=\mathbb{k}[\operatorname{hom}(V, W)]$ qui s'en déduit est celle de l'algèbre du groupe abélien hom $(V, W)$.

En utilisant le foncteur de dualité $D$, on en déduit une structure naturelle de coalgèbre cocommutative sur les injectifs standard $I_{V}$ de $\mathcal{F}$.

La proposition fondamentale suivante repose sur l'idenfication de l'adjoint à droite au foncteur $\Delta_{E_{n}}$ de la monade $\mathcal{T}_{\mathbf{P l}, n}$. Elle n'a pas d'analogue dans les catégories $\mathcal{F}_{\mathcal{G} r, I}$, car l'adjoint à droite au foncteur $\Delta_{\text {surj }}^{I}$ (qui existe par le corollaire C.2.4) n'est généralement pas un foncteur qui admet une description simple.

Proposition 8.2.7. - Il existe une équivalence de catégories $\mathcal{F}_{\mathrm{Pl}, n} \stackrel{\simeq}{\longrightarrow} \operatorname{Comod}_{I_{E_{n}}}$ qui factorise le foncteur $\sigma_{n}^{\mathbf{P l}}: \mathcal{F}_{\mathbf{P l}, n} \rightarrow \mathcal{F}$ à travers le foncteur d'oubli $\operatorname{Comod}_{I_{E_{n}}} \rightarrow$ $\mathcal{F}$.

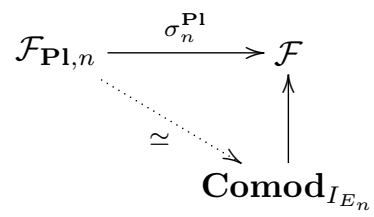

Démonstration. - La proposition découle de ce qu'une flèche $\Delta_{E_{n}} X \rightarrow X$ de $\mathcal{F}$ fait de $X$ un module sur $\mathcal{T}_{\mathbf{P l}, n}$ si et seulement si la flèche adjointe $X \rightarrow X \otimes I_{E_{n}}$ définit une structure de $I_{E_{n}}$-comodule sur $X$. En effet, la multiplication de $\mathcal{T}_{\mathbf{P l}, n}$ comme la 
comultiplication de $I_{E_{n}}$ sont induites par la somme $E_{n} \oplus E_{n} \rightarrow E_{n}$, et l'unité de $\mathcal{T}_{\mathbf{P l}, n}$ comme la coünité de $I_{E_{n}}$ proviennent du morphisme $0 \rightarrow E_{n}$.

Remarque 8.2.8. - La proposition 8.2.7 est à comparer avec l'équivalence de catégories $\mathcal{F}_{\mathbf{P l}, n} \stackrel{\simeq}{\longrightarrow} \operatorname{Comod}_{\widetilde{P}(n)}^{\text {fid }}$ (fournie par un foncteur d'intégrale) déduite de la proposition 3.2.10, où $\widetilde{P}(n)$ est le foncteur introduit dans la notation 2.3.21 (page 27).

Produit cotensoriel et foncteur $\chi_{n}^{\mathrm{Pl}}$. — Dans la suite de cette section, nous identifierons les catégories $\mathcal{F}_{\mathbf{P l}, n}$ et $\operatorname{Comod}_{I_{E_{n}}}$. En particulier, la coalgèbre $I_{E_{n}}$ étant cocommutative, on dispose dans $\mathcal{F}_{\mathbf{P l}, n}$ d'un produit cotensoriel $\underset{I_{E_{n}}}{\square}$, qui sera noté simplement $\square$ par la suite; c'est un bifoncteur exact à gauche.

Avant d'utiliser ce produit cotensoriel, nous avons besoin de décrire quelques foncteurs usuels de source ou de but $\mathcal{F}_{\mathbf{P l}, n}$ dans l'identification de $\mathcal{F}_{\mathbf{P l}, n}$ à $\operatorname{Comod}_{I_{E_{n}}}$.

Le foncteur $\sigma_{n}^{\mathbf{P l}}: \mathcal{F}_{\mathbf{P l}, n} \rightarrow \mathcal{F}$ correspond au foncteur d'oubli (cf. proposition 8.2.7).

Nous identifions, dans la proposition qui suit, le produit tensoriel de $\mathcal{F}_{\mathbf{P l}, n}$ en termes de $I_{E_{n}}$-comodules.

Proposition 8.2.9. - Le coproduit $\psi_{X \otimes Y}$ d'un produit tensoriel de deux objets $X$ et $Y$ de $\mathcal{F}_{\mathbf{P l}, n}$ est égal à la composée

$X \otimes Y \stackrel{\psi_{X} \otimes \psi_{Y}}{\longrightarrow}\left(X \otimes I_{E_{n}}\right) \otimes\left(Y \otimes I_{E_{n}}\right) \simeq(X \otimes Y) \otimes\left(I_{E_{n}} \otimes I_{E_{n}}\right) \stackrel{(X \otimes Y) \otimes a_{n}}{\longrightarrow}(X \otimes Y) \otimes I_{E_{n}}$ où $a_{n}: I_{E_{n}} \otimes I_{E_{n}} \rightarrow I_{E_{n}}$ est le produit du foncteur en algèbres de Boole $I_{E_{n}}$.

Ainsi, le produit tensoriel de la catégorie de comodules $\mathcal{F}_{\mathbf{P l}, n}$ provient de la structure d'algèbre sur $I_{E_{n}}$. Le fait que le produit tensoriel de deux $I_{E_{n}}$-comodules est naturellement un $I_{E_{n}}$-comodule provient de la compatibilité des structures d'algèbre et de coalgèbre sur $I_{E_{n}}$, qui est une algèbre de Hopf de la catégorie symétrique monoïdale $\mathcal{F}$.

Démonstration. - La propriété provient, par adjonction, de ce que le morphisme $m_{X \otimes Y}: \Delta_{E_{n}} \sigma_{n}^{\mathbf{P l}}(X \otimes Y) \rightarrow \sigma_{n}^{\mathbf{P l}}(X \otimes Y)$ s'identifie, modulo les isomorphismes canoniques $\sigma_{n}^{\mathbf{P l}}(X \otimes Y) \simeq \sigma_{n}^{\mathbf{P l}}(X) \otimes \sigma_{n}^{\mathbf{P l}}(Y)$ et $\Delta_{E_{n}} \sigma_{n}^{\mathbf{P l}}(X \otimes Y) \simeq \Delta_{E_{n}} \sigma_{n}^{\mathbf{P l}}(X) \otimes \Delta_{E_{n}} \sigma_{n}^{\mathbf{P l}}(Y)$, à $m_{X} \otimes m_{Y}: \Delta_{E_{n}} \sigma_{n}^{\mathbf{P l}}(X) \otimes \Delta_{E_{n}} \sigma_{n}^{\mathbf{P l}}(Y) \rightarrow \sigma_{n}^{\mathbf{P l}}(X) \otimes \sigma_{n}^{\mathbf{P l}}(Y)$.

Notation 8.2.10. - Dans cette section, nous noterons $j_{F}: F \hookrightarrow F \otimes I_{E_{n}}$, pour $F \in \mathrm{Ob} \mathcal{F}$, l'inclusion canonique déduite de $\mathbb{k} \hookrightarrow I_{E_{n}}$.

Nous identifions maintenant le foncteur $\kappa_{n}^{\mathrm{Pl}}$ en termes de $I_{E_{n}}$-comodules.

Proposition 8.2.11. - Le foncteur $\kappa_{n}^{\mathrm{Pl}}$ associe à un objet $F$ de $\mathcal{F}$ le $I_{E_{n}}$-comodule $\left(F, j_{F}\right)$.

Ainsi, le foncteur $\kappa_{n}^{\mathbf{P l}}$ identifie $\mathcal{F}$ à la sous-catégorie de Serre des $I_{E_{n}}$-comodules $X$ tels que $\psi_{X}=j_{X}$. 
Démonstration. - De manière semblable à la proposition 7.0 .9 , on voit que $\kappa_{n}^{\mathrm{Pl}}(F)$ est le $\mathcal{T}_{\mathbf{P l}, n}$-module $\left(F,\left(p_{\mathbf{P l}, n}\right)_{F}\right)$. La proposition se déduit alors de ce que le morphisme $\left(p_{\mathbf{P l}, n}\right)_{F}: \Delta_{E_{n}} F \rightarrow F$ est adjoint à $j_{F}$.

Corollaire 8.2.12. - Soient $F$ un objet de $\mathcal{F}$ et $X$ un objet de $\mathcal{F}_{\mathbf{P l}, n}$. Les morphismes $\psi_{\kappa_{n}^{\mathrm{Pl}}(F) \otimes X}$ et $F \otimes \psi_{X}: F \otimes \sigma_{n}^{\mathrm{Pl}}(X) \rightarrow F \otimes \sigma_{n}^{\mathrm{Pl}}(X) \otimes I_{E_{n}}$ de $\mathcal{F}$ sont égaux.

Démonstration. - C'est une conséquence directe des propositions 8.2.11 et 8.2.9.

Remarque 8.2.13. - Il existe un monomorphisme canonique $\sigma_{n}^{\mathrm{Pl}}(X \square Y) \hookrightarrow$ $\sigma_{n}^{\mathbf{P l}}(X \otimes Y) \simeq \sigma_{n}^{\mathbf{P l}}(X) \otimes \sigma_{n}^{\mathbf{P l}}(Y)$ dans $\mathcal{F}$; il ne provient pas d'un morphisme naturel $X \square Y \rightarrow X \otimes Y$ de $\mathcal{F}_{\mathbf{P l}, n}$. En revanche, le corollaire 8.2.12 montre qu'il est induit par un monomorphisme naturel $X \square Y \hookrightarrow \kappa_{n}^{\mathrm{Pl}} \sigma_{n}^{\mathbf{P l}}(X) \otimes Y$.

Nous introduisons à présent un nouveau foncteur déduit de la proposition 8.2.7.

Définition 8.2.14 (Foncteur $\chi_{n}^{\mathrm{Pl}}$ ). - On définit le foncteur $\chi_{n}^{\mathrm{Pl}}: \mathcal{F}_{\mathrm{Pl}, n} \rightarrow \mathcal{F}$ par la composition suivante.

$$
\chi_{n}^{\mathrm{Pl}}: \mathcal{F}_{\mathbf{P l}, n} \stackrel{\cdot \square \mathrm{k}}{\longrightarrow} \mathcal{F}_{\mathbf{P l}, n} \stackrel{\sigma_{n}^{\mathrm{Pl}}}{\longrightarrow} \mathcal{F}
$$

Ainsi, $\chi_{n}^{\mathbf{P l}}$ est l'égalisateur des transformations naturelles $\psi$ et $j_{\sigma_{n}^{\mathrm{Pl}}}: \sigma_{n}^{\mathbf{P l}} \rightarrow \sigma_{n}^{\mathbf{P l}} \otimes$ $I_{E_{n}}$.

La proposition suivante constitue le résultat principal de ce paragraphe.

Proposition 8.2.15. - Le foncteur $\chi_{n}^{\mathrm{Pl}}$ est adjoint à droite à $\kappa_{n}^{\mathrm{Pl}}: \mathcal{F} \rightarrow \mathcal{F}_{\mathrm{Pl}, n}$.

Démonstration. - Soient $F$ un objet de $\mathcal{F}$ et $X$ un $I_{E_{n}}$-comodule. Par la proposition 8.2.11, $\operatorname{hom}_{\mathbf{P l}, n}\left(\kappa_{n}^{\mathbf{P l}}(F), X\right)$ s'identifie à l'ensemble des morphismes $f: F \rightarrow$ $\sigma_{n}^{\mathrm{Pl}}(X)$ de $\mathcal{F}$ tels que le diagramme suivant commute.

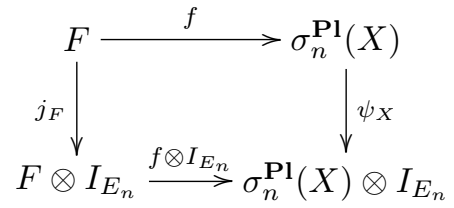

Comme le diagramme

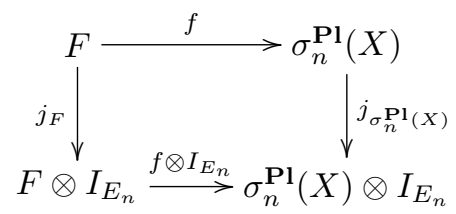

commute, la condition précédente revient à dire que le morphisme $f$ est à valeurs dans l'égalisateur des morphismes $j_{\sigma_{n}^{\mathrm{Pl}}(X)}$ et $\psi_{X}$, qui est $\chi_{n}^{\mathrm{Pl}}(X)$. Cela démontre la proposition. 
La proposition ci-après donne une propriété de compatibilité entre les foncteurs $\chi_{n}^{\mathbf{P l}}$ et $\kappa_{n}^{\mathbf{P l}}$.

Proposition 8.2.16. - Il existe des isomorphismes naturels $\chi_{n}^{\mathbf{P l}}\left(X \otimes \kappa_{n}^{\mathbf{P l}}(F)\right) \simeq$ $\chi_{n}^{\mathbf{P l}}(X) \otimes F$ et $X \square \kappa_{n}^{\mathbf{P l}}(F) \simeq \kappa_{n}^{\mathbf{P l}}\left(\chi_{n}^{\mathbf{P l}}(X) \otimes F\right)$ pour $X \in \mathrm{Ob} \mathcal{F}_{\mathbf{P l}, n}$ et $F \in \mathrm{Ob} \mathcal{F}$.

Démonstration. - Le foncteur $\chi_{n}^{\mathbf{P l}}\left(X \otimes \kappa_{n}^{\mathrm{Pl}}(F)\right)$ est l'égalisateur des flèches $\psi_{X \otimes \kappa_{n}^{\mathrm{Pl}}(F)}=\psi_{X} \otimes F\left(\right.$ par le corollaire 8.2.12) et $j_{\sigma_{n}^{\mathrm{Pl}}\left(X \otimes \kappa_{n}^{\mathrm{Pl}}(F)\right)}=j_{\sigma_{n}^{\mathrm{Pl}}(X)} \otimes F$, il s'identifie donc canoniquement au produit tensoriel de $\chi_{n}^{\mathbf{P l}}(X)$ et $F$.

Par ailleurs, $X \square \kappa_{n}^{\mathrm{Pl}}(F)$ est l'égalisateur des flèches $X \otimes F \stackrel{\psi_{X} \otimes F}{\longrightarrow} X \otimes I_{E_{n}} \otimes F$ et $X \otimes F \stackrel{X \otimes \psi_{\kappa_{n}^{\mathrm{Pl}}(F)}^{\longrightarrow}}{\longrightarrow} X \otimes F \otimes I_{E_{n}} \simeq X \otimes I_{E_{n}} \otimes F ;$ comme $X \otimes \psi_{\kappa_{n}^{\mathrm{Pl}}(F)}=X \otimes j_{F} \simeq$ $j_{\sigma_{n}^{\mathrm{Pl}}(X)} \otimes F$ (modulo l'isomorphisme d'échange des facteurs du produit tensoriel), ce foncteur s'identifie (dans $\mathcal{F}$ ) à $\chi_{n}^{\mathbf{P l}}(X) \otimes F$. Il reste à voir que sa structure de $I_{E_{n}}$-comodule est triviale, ce qui provient de l'inclusion $X \square \kappa_{n}^{\mathrm{Pl}}(F) \hookrightarrow \kappa_{n}^{\mathrm{Pl}} \sigma_{n}^{\mathrm{Pl}}(X) \otimes$ $\kappa_{n}^{\mathbf{P l}}(F) \simeq \kappa_{n}^{\mathbf{P l}}\left(\sigma_{n}^{\mathbf{P l}}(X) \otimes F\right)($ cf. remarque 8.2.13).

Remarque 8.2.17. - On peut expliciter l'équivalence de catégories de la proposition 8.1.11 à l'aide des foncteurs $\kappa_{n}^{\mathbf{P l}}$ et $\chi_{n}^{\mathbf{P l}}$. Ainsi, on vérifie que les foncteurs

$$
\mathcal{F} \stackrel{\kappa_{n}^{\mathrm{Pl}}}{\longrightarrow} \mathcal{F}_{\mathbf{P l}, n} \stackrel{I_{E_{n}}^{\mathrm{Pl}, n} \otimes}{\longrightarrow} \operatorname{Mod}_{I_{E_{n}}^{\mathrm{Pl}, n}}
$$

et

$$
\operatorname{Mod}_{I_{E_{n}}^{\mathrm{Pl}, n}} \stackrel{\text { oubli }}{\longrightarrow} \mathcal{F}_{\mathrm{Pl}, n} \stackrel{\chi_{n}^{\mathrm{Pl}}}{\longrightarrow} \mathcal{F}
$$

sont des équivalences réciproques l'une de l'autre.

Le foncteur $\chi_{n}: \mathcal{F}_{\mathcal{G} r, n} \rightarrow \mathcal{F}_{G L_{n}(\mathbb{k})}$. - Nous revenons maintenant à la catégorie $\mathcal{F}_{\mathcal{G} r, n}$, en montrant comment les résultats précédents peuvent être utilisés pour en étudier certaines propriétés. Nous commençons par préciser le lien entre $\mathcal{F}_{\mathbf{P l}, n}$ et $\mathcal{F}_{\mathcal{G} r, n}$.

Proposition et définition 8.2.18. - (1) Le groupe linéaire $G L_{n}(\mathbb{k})$ agit $\grave{a}$ droite sur la catégorie $\mathcal{E}_{\mathbf{P l}, n}^{f}:$ pour tout $g \in G L_{n}(\mathbb{k})$, on définit un foncteur $\tau_{g}: \mathcal{E}_{\mathbf{P l}, n}^{f} \rightarrow \mathcal{E}_{\mathbf{P l}, n}^{f}$ par $(V, u) \mapsto(V, u \circ g)$ sur les objets et par l'égalité $\operatorname{hom}_{\mathbf{P l}, n}\left((V, u),\left(V^{\prime}, u^{\prime}\right)\right)=\operatorname{hom}_{\mathbf{P l}, n}\left((V, u \circ g),\left(V^{\prime}, u^{\prime} \circ g\right)\right)$ sur les morphismes, et l'on $a \tau_{1}=i d$ et $\tau_{g} \circ \tau_{h}=\tau_{h g}$ pour tous $g, h \in G L_{n}(\mathbb{k})$.

(2) Par précomposition, on en déduit une action à gauche de $G L_{n}(\mathbb{k})$ sur $\mathcal{F}_{\mathbf{P l}, n}$ : les foncteurs $\tau_{g}^{*}: \mathcal{F}_{\mathbf{P l}, n} \rightarrow \mathcal{F}_{\mathbf{P l}, n}$ vérifient $\tau_{1}^{*}=i d$ et $\tau_{g}^{*} \circ \tau_{h}^{*}=\tau_{g h}^{*}$. 
(3) On appelle $G L_{n}(\mathbb{k})$-module dans $\mathcal{F}_{\mathbf{P l}, n}$ tout objet $X$ de $\mathcal{F}_{\mathbf{P l}, n}$ muni de flèches $\tau_{g}^{*}(X) \stackrel{t_{g}}{\longrightarrow} X$ (dites d'action de $\left.G L_{n}(\mathbb{k})\right)$ telles que le diagramme

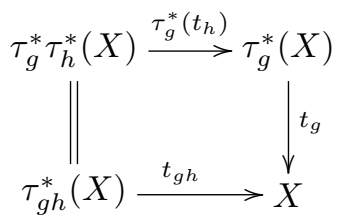

commute pour tous $g, h \in G L_{n}(\mathbb{k})$. Un morphisme de $G L_{n}(\mathbb{k})$-modules de $\mathcal{F}_{\mathbf{P l}, n}$ est un morphisme de $\mathcal{F}_{\mathbf{P l}, n}$ compatible aux morphismes d'action de $G L_{n}(\mathbb{k})$. On définit ainsi la sous-catégorie des $G L_{n}(\mathbb{k})$-modules de $\mathcal{F}_{\mathbf{P l}, n}$.

(4) Le foncteur $\gamma_{n}: \mathcal{F}_{\mathcal{G}_{r, n}} \rightarrow \mathcal{F}_{\mathbf{P l}, n}$ induit une équivalence de catégories entre $\mathcal{F}_{\mathcal{G} r, n}$ et la sous-catégorie des $G L_{n}(\mathbb{k})$-modules de $\mathcal{F}_{\mathbf{P l}, n}$.

(5) Le foncteur $\gamma_{n}$ admet un adjoint à gauche $\Phi_{n}: \mathcal{F}_{\mathbf{P l}, n} \rightarrow \mathcal{F}_{\mathcal{G} r, n}$ donné sur les objets par

$$
\Phi_{n}(X)(V, W)=\bigoplus_{u \in \operatorname{Iso} \mathcal{E}\left(E_{n}, W\right)} X\left(V, E_{n} \stackrel{u}{\rightarrow} W \hookrightarrow V\right) .
$$

Démonstration. - Les deux premières assertions sont claires. Le dernier point est un cas particulier de la proposition 3.1.7 (cf. démonstration de la proposition 8.1.8). Comme le foncteur $\gamma_{n}$ est exact et fidèle, la proposition A.2.7 montre que $\mathcal{F}_{\mathcal{G} r, n}$ est équivalente à la sous-catégorie de $\mathcal{F}_{\mathbf{P l}, n}$ des modules sur la monade associée à cette adjonction. Cette monade se décrit comme suit.

- L'endofoncteur $\gamma_{n} \Phi_{n}$ de $\mathcal{F}_{\mathbf{P l}, n}$ est $\bigoplus_{g \in G L_{n}(\mathbb{k})} \tau_{g}^{*}$.

- L'unité est l'inclusion $i d \hookrightarrow \bigoplus_{g \in G L_{n}(\mathbb{k})} \tau_{g}^{*} \mathrm{du}$ facteur direct correspondant à $g=1$.

- La multiplication est la transformation naturelle

$$
\bigoplus_{h, h^{\prime} \in G L_{n}(\mathbb{k})} \tau_{h}^{*} \tau_{h^{\prime}}^{*} \rightarrow \bigoplus_{g \in G L_{n}(\mathbb{k})} \tau_{g}^{*}
$$

dont la composante $\tau_{h}^{*} \tau_{h^{\prime}}^{*} \rightarrow \tau_{g}^{*}$ est l'identité si $h h^{\prime}=g, 0$ sinon.

La proposition en résulte.

Convention 8.2.19. - Dans la suite de ce paragraphe, on identifie $\mathcal{F}_{\mathcal{G} r, n}$ avec la catégorie des $G L_{n}(\mathbb{k})$-modules de $\mathcal{F}_{\mathbf{P l}, n}$.

Remarque 8.2.20. — Si $\mathcal{A}$ est une catégorie abélienne, on peut voir la catégorie $\mathcal{A}_{G L_{n}(\mathbb{k})}$ comme une catégorie de $G L_{n}(\mathbb{k})$-modules dans $\mathcal{A}$. La catégorie $\mathcal{F}_{\mathcal{G} r, n}$ est quant à elle une catégorie de $G L_{n}(\mathbb{k})$-modules «tordus » par l'action du groupe linéaire $\operatorname{sur} \mathcal{F}_{\mathrm{Pl}, n}$. 
Pour mener des raisonnements sur la catégorie $\mathcal{F}_{\mathcal{G} r, n}$ des $G L_{n}(\mathbb{k})$-modules tordus de $\mathcal{F}_{\mathbf{P l}, n}$ analogues à ceux relatifs aux $G L_{n}(\mathbb{k})$-modules ordinaires, nous sommes conduits à introduire la notion suivante.

Définition 8.2.21. - On appelle trivialisation sur $G L_{n}(\mathbb{k})$ d'un foncteur $\alpha$ de source $\mathcal{F}_{\mathbf{P l}, n}$ la donnée d'isomorphismes $T_{g}: \alpha \circ \tau_{g}^{*} \rightarrow \alpha$ pour tout $g \in G L_{n}(\mathbb{k})$ tels que le diagramme

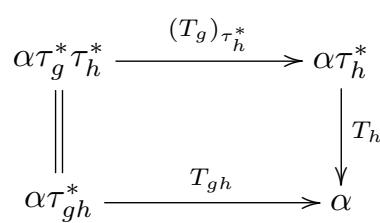

commute pour tous $g, h \in G L_{n}(\mathbb{k})$.

Proposition et définition 8.2.22. - Soient $\mathcal{A}$ une catégorie abélienne et $\alpha: \mathcal{F}_{\mathbf{P l}, n} \rightarrow \mathcal{A}$ un foncteur muni d'une trivialisation sur $G L_{n}(\mathbb{k})$. On définit un foncteur $\alpha_{G L_{n}}^{\mathcal{G} r}: \mathcal{F}_{\mathcal{G} r, n} \rightarrow \mathcal{A}_{G L_{n}(\mathbb{k})}$ comme suit.

- Sur les objets : si $X$ est un $G L_{n}(\mathbb{k})$-module de $\mathcal{F}_{\mathbf{P l}, n}$, alors $\alpha_{G L_{n}}^{\mathcal{G} r}(X)$ est l'objet $\alpha(X)$ de $\mathcal{A}$ muni de l'action de $G L_{n}(\mathbb{k})$ donnée par les flèches $\alpha(X) \simeq$ $\alpha\left(\tau_{g}^{*}(X)\right) \rightarrow \alpha(X)$ (pour $g \in G L_{n}(\mathbb{k})$ ), où la première flèche est la trivisalisation et la seconde est induite par la structure de $G L_{n}(\mathbb{k})$-module de $X$.

- La flèche qu'induit un morphisme de $G L_{n}(\mathbb{k})$-modules de $\mathcal{F}_{\mathbf{P l}, n}$ via $\alpha$ est un morphisme de $\mathcal{A}_{G L_{n}(\mathbb{k})}$, ce qui permet de déduire la fonctorialité de $\alpha_{G L_{n}}^{\mathcal{G} r}$ de celle de $\alpha$.

De plus, le diagramme suivant commute (à isomorphisme canonique près)

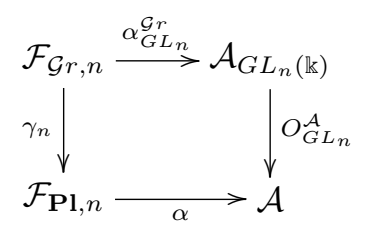

où $O_{G L_{n}}^{\mathcal{A}}$ désigne le foncteur d'oubli, conformément à la notation 3.1.2.

Nous illustrons cette construction élémentaire sur le foncteur $\chi_{n}^{\mathbf{P l}}: \mathcal{F}_{\mathbf{P l}, n} \rightarrow \mathcal{F}$, qui aboutit à une description explicite de l'adjoint à droite au foncteur $\theta_{n}: \mathcal{F}_{G L_{n}(\mathbb{k})} \rightarrow$ $\mathcal{F}_{\mathcal{G} r, n}$

Proposition et définition 8.2.23. - (1) Le foncteur $\sigma_{n}^{\mathrm{Pl}}: \mathcal{F}_{\mathbf{P l}, n} \rightarrow \mathcal{F}$ admet une trivialisation sur $G L_{n}(\mathbb{k})$ pour laquelle le foncteur $\left(\sigma_{n}^{\mathrm{Pl}}\right)_{G L_{n}}^{\mathcal{G} r}: \mathcal{F}_{\mathcal{G} r, n} \rightarrow \mathcal{F}_{G L_{n}(\mathbb{k})}$ s'identifie à $\sigma_{n}$.

(2) La trivialisation précédente induit, via le monomorphime canonique $\chi_{n}^{\mathbf{P l}} \hookrightarrow$ $\sigma_{n}^{\mathbf{P l}}$, une trivialisation sur $\chi_{n}^{\mathbf{P l}}$.

(3) Nous noterons $\chi_{n}: \mathcal{F}_{\mathcal{G} r, n} \rightarrow \mathcal{F}_{G L_{n}(\mathbb{k})}$ le foncteur $\left(\chi_{n}^{\mathbf{P l}}\right)_{G L_{n}}^{\mathcal{G} r}$. 
(4) Le foncteur $\chi_{n}$ est adjoint à droite $\grave{a} \theta_{n}$.

Démonstration. - La trivialisation canonique de $\sigma_{n}^{\mathbf{P l}}$ se lit sur le foncteur de décalage pointé $\mathfrak{S}_{n}$ : pour tout $g \in G L_{n}(\mathbb{k}), \tau_{g} \mathfrak{S}_{n}$ est donné sur un objet $V$ de $\mathcal{E}^{f}$ par $(V \oplus$ $E_{n}, E_{n} \stackrel{0 \oplus g}{\longrightarrow} V \oplus E_{n}$ ), et les transformations naturelles $\tau_{g} \mathfrak{S}_{n} \rightarrow \mathfrak{S}_{n}$ données par le diagramme commutatif suivant fournissent la trivialisation recherchée.

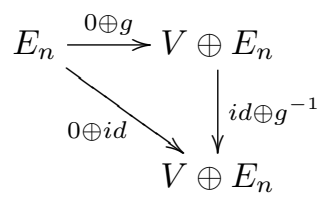

Pour en déduire le second point, on remarque que le diagramme

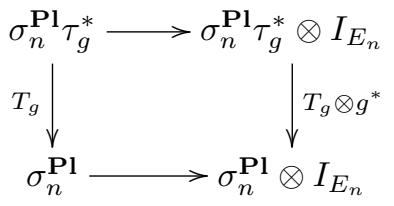

commute pour tout $g \in G L_{n}(\mathbb{k})$, où $T_{g}$ est l'isomorphisme de trivialisation et les flèches horizontales sont donnéees par la somme de $\psi$ et de l'inclusion canonique.

La dernière assertion s'obtient à partir des trois observations suivantes.

(1) La composée $\mathcal{F}_{\mathcal{G} r, n} \stackrel{\chi_{n}}{\longrightarrow} \mathcal{F}_{G L_{n}(\mathbb{k})} \stackrel{O_{G L_{n}}^{\mathcal{F}}}{\longrightarrow} \mathcal{F}$ est adjointe à droite à $\mathcal{F} \rightarrow$ $\mathcal{F}_{G L_{n}(\mathbb{k})} \stackrel{\theta_{n}}{\longrightarrow} \mathcal{F}_{\mathcal{G}_{r, n},}$, où la première flèche est la postcomposition par le foncteur $\mathcal{E} \rightarrow \mathbb{k}\left[G L_{n}(\mathbb{k})\right]$ Mod d'extension des scalaires. En effet, le diagramme commutatif de la proposition 8.2.22, la proposition 8.2.15 et la dernière assertion de la proposition 8.2.18 montrent que la première composée est adjointe à droite à $\mathcal{F} \stackrel{\kappa_{n}^{\mathrm{Pl}}}{\longrightarrow} \mathcal{F}_{\mathbf{P l}, n} \stackrel{\Phi_{n}}{\longrightarrow} \mathcal{F}_{\mathcal{G} r, n}$, qui coïncide avec $\kappa_{n} \otimes P_{\left(E_{n}, E_{n}\right)}^{\mathcal{G} r, n}$, flèche à laquelle s'identifie également $\mathcal{F} \rightarrow \mathcal{F}_{G L_{n}(\mathbb{k})} \stackrel{\theta_{n}}{\longrightarrow} \mathcal{F}_{\mathcal{G} r, n}$.

(2) Le foncteur $\chi_{n}$ est un sous-foncteur de $\sigma_{n}$. Cela découle de la première partie de la démonstration.

(3) Il existe un diagramme commutatif

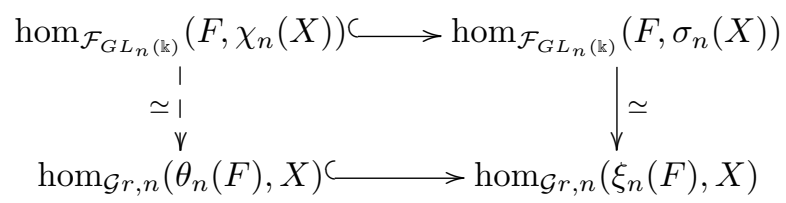

naturel en les objets $F$ de $\mathcal{F}_{G L_{n}(\mathbb{k})}$ et $X$ de $\mathcal{F}_{\mathcal{G} r, n}$, où la flèche verticale de droite provient de la proposition 5.1.17. L'isomorphisme en pointillé résulte des deux remarques précédentes lorsque $F$ appartient à l'image du foncteur $\mathcal{F} \rightarrow \mathcal{F}_{G L_{n}(\mathbb{k})}$ induit par l'extension des scalaires; le cas général s'en déduit par un argument de colimite. 


\section{CHAPITRE 9}

\section{FONCTEURS HOM INTERNES ET FONCTEURS DE DIVISION DANS $\mathcal{F}_{\mathcal{G} r, I}$}

Nous présentons dans ce chapitre des propriétés élémentaires des foncteurs hom internes et des foncteurs de division dans les catégories $\mathcal{F}_{\mathcal{G} r, I}$ (cf. proposition/définition C.3.1). Ces propriétés reposent sur les différentes adjonctions établies dans les sections 5.1 et 7.1 et la description explicite des projectifs et injectifs standard de $\mathcal{F}_{\mathcal{G} r, I}$.

Nous nous attacherons surtout aux foncteurs hom internes, notre but principal étant d'aboutir, dans la partie III, à la proposition 11.2.8 et ses corollaires, relatifs au comportement mutuel des foncteurs hom internes du foncteur $\omega$. Les foncteurs de division interviendront comme auxiliaires dans la section 11.1 et le chapitre 15 .

\subsection{Comparaison entre les différentes catégories $\mathcal{F}_{\mathcal{G} r, I}$}

Il est parfois commode de passer de la catégorie globale $\mathcal{F}_{\mathcal{G} r}$ aux catégories $\mathcal{F}_{\mathcal{G} r, n}$, par exemple, pour traiter des adjoints au produit tensoriel. En effet, si les foncteurs $\iota$ et $\omega$ sont adjoints, il n'en est pas de même des foncteurs $\iota_{n}$ et $\omega_{n}$, ce qui induit une difficulté dans $\mathcal{F}_{\mathcal{G} r, n}$ nouvelle par rapport à $\mathcal{F}_{\mathcal{G} r}$. En revanche, dès que l'on traite de foncteurs pseudo-constants, la catégorie globale se trouve beaucoup moins maniable que $\mathcal{F}_{\mathcal{G} r, n}$, puisque les adjoints au produit tensoriel entre $G L_{n}(\mathbb{k})$-modules sont faciles à décrire, contrairement à ce qui advient dans $\mathcal{F}_{\text {surj }}(\mathbb{k})$.

Convention 9.1.1. - Dans cette section, on se donne deux parties $I$ et $J$ de $\mathbb{N}$ telles que $I \subset J$.

On rappelle que $\mathcal{R}_{J, I}$ est le foncteur de restriction et $\mathcal{P}_{I, J}$ le foncteur de prolongement par zéro - défini sous certaines conditions sur $I$ et $J$ - introduits dans la notation 5.1.1. 
Proposition 9.1.2. - Supposons que tout élément de J supérieur ou égal à un élément de $I$ appartient à $I$. Alors il existe dans $\mathcal{F}_{\mathcal{G} r, J}$ un isomorphisme

$$
\operatorname{Hom}_{\mathcal{G} r, J}\left(X, \mathcal{P}_{I, J}(Y)\right) \simeq \mathcal{P}_{I, J}\left(\operatorname{Hom}_{\mathcal{G} r, I}\left(\mathcal{R}_{J, I}(X), Y\right)\right)
$$

naturel en les objets $X$ de $\mathcal{F}_{\mathcal{G} r, J}$ et $Y$ de $\mathcal{F}_{\mathcal{G} r, I}$, et dans $\mathcal{F}_{\mathcal{G} r, I}$ un isomorphisme naturel

$$
\mathcal{R}_{J, I}\left(\mathcal{P}_{I, J}(Y): X\right) \simeq\left(Y: \mathcal{R}_{J, I}(X)\right)
$$

si $X$ est à valeurs de dimension finie.

En particulier, pour tout $n \in \mathbb{N}$, on a dans $\mathcal{F}_{\mathcal{G} r, \leq n}$ un isomorphisme naturel

$$
\operatorname{Hom}_{\mathcal{G} r, \leq n}\left(X, \mathcal{P}_{n, \leq n}(Y)\right) \simeq \mathcal{P}_{n, \leq n}\left(\operatorname{Hom}_{\mathcal{G} r, n}\left(\mathcal{R}_{\leq n, n}(X), Y\right)\right)
$$

et, si $X$ est à valeurs de dimension finie, un isomorphisme naturel dans $\mathcal{F}_{\mathcal{G} r, n}$

$$
\mathcal{R}_{\leq n, n}\left(\mathcal{P}_{n, \leq n}(Y): X\right) \simeq\left(Y: \mathcal{R}_{\leq n, n}(X)\right) .
$$

Démonstration. - L'hypothèse assure que $\mathcal{E}_{\mathcal{G} r, I}^{f}$ est une sous-catégorie complète à gauche de $\mathcal{E}_{\mathcal{G} r, J}^{f}$. Par conséquent, le foncteur $\mathcal{P}_{I, J}$ est adjoint à droite à $\mathcal{R}_{J, I}$ (cf. proposition C.6.2). Le premier isomorphisme de la proposition provient alors de la commutation du foncteur de restriction au produit tensoriel, comme le montrent les isomorphismes naturels

$$
\begin{aligned}
& \operatorname{hom}_{\mathcal{G} r, J}(A\left.\operatorname{Hom}_{\mathcal{G} r, J}\left(X, \mathcal{P}_{I, J}(Y)\right)\right) \simeq \operatorname{hom}_{\mathcal{G} r, J}\left(A \otimes X, \mathcal{P}_{I, J}(Y)\right) \\
& \simeq \operatorname{hom}_{\mathcal{G} r, I}\left(\mathcal{R}_{J, I}(A \otimes X), Y\right) \simeq \operatorname{hom}_{\mathcal{G} r, I}\left(\mathcal{R}_{J, I}(A) \otimes \mathcal{R}_{J, I}(X), Y\right) \\
& \simeq \operatorname{hom}_{\mathcal{G} r, I}\left(\mathcal{R}_{J, I}(A), \operatorname{Hom}_{\mathcal{G} r, I}\left(\mathcal{R}_{J, I}(X), Y\right)\right) \\
& \simeq \operatorname{hom}_{\mathcal{G} r, J}\left(A, \mathcal{P}_{I, J}\left(\operatorname{Hom}_{\mathcal{G} r, I}\left(\mathcal{R}_{J, I}(X), Y\right)\right)\right)
\end{aligned}
$$

(où $A \in \mathrm{Ob} \mathcal{F}_{\mathcal{G} r, J}$ ) et le lemme de Yoneda. Le second se traite de façon analogue.

Nous donnons maintenant un résultat plus précis de commutation entre foncteurs hom internes et foncteurs de restriction ou de prolongement par zéro, dans le cas où la source du foncteur hom interne appartient à l'image du foncteur $\iota_{I}: \mathcal{F} \rightarrow \mathcal{F}_{\mathcal{G} r, I}$.

Proposition 9.1.3. - Il existe des isomorphismes naturels

$$
\operatorname{Hom}_{\mathcal{G} r, J}\left(\iota_{J}(F), \mathcal{P}_{I, J}(A)\right) \simeq \mathcal{P}_{I, J}\left(\operatorname{Hom}_{\mathcal{G} r, I}\left(\iota_{I}(F), A\right)\right)
$$

dans $\mathcal{F}_{\mathcal{G} r, J}$, si le prolongement par zéro est défini, et

$$
\operatorname{Hom}_{\mathcal{G} r, I}\left(\iota_{I}(F), \mathcal{R}_{J, I}(X)\right) \simeq \mathcal{R}_{J, I}\left(\operatorname{Hom}_{\mathcal{G} r, J}\left(\iota_{J}(F), X\right)\right)
$$

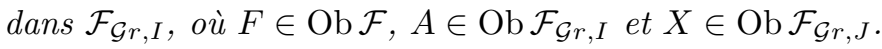

Démonstration. - Les morphismes naturels

$$
\mathcal{P}_{I, J}\left(\operatorname{Hom}_{\mathcal{G} r, I}\left(\iota_{I}(F), A\right)\right) \otimes \iota_{J}(F) \simeq \mathcal{P}_{I, J}\left(\operatorname{Hom}_{\mathcal{G} r, I}\left(\iota_{I}(F), A\right) \otimes \iota_{I}(F)\right) \rightarrow \mathcal{P}_{I, J}(A)
$$

(lorsque le prolongement par zéro est défini) et

$\mathcal{R}_{J, I}\left(\operatorname{Hom}_{\mathcal{G} r, J}\left(\iota_{J}(F), X\right)\right) \otimes \iota_{I}(F) \simeq \mathcal{R}_{J, I}\left(\operatorname{Hom}_{\mathcal{G} r, J}\left(\iota_{J}(F), X\right) \otimes \iota_{J}(F)\right) \rightarrow \mathcal{R}_{J, I}(X)$ 
dont les secondes flèches s'obtiennent par application du foncteur $\mathcal{P}_{I, J}$ ou $\mathcal{R}_{J, I}$ à la coünité de l'adjonction fournissent, par adjonction, des morphismes naturels $\mathcal{P}_{I, J}\left(\operatorname{Hom}_{\mathcal{G} r, I}\left(\iota_{I}(F), A\right)\right) \rightarrow \operatorname{Hom}_{\mathcal{G} r, J}\left(\iota_{J}(F), \mathcal{P}_{I, J}(A)\right)$ et $\mathcal{R}_{J, I}\left(\operatorname{Hom}_{\mathcal{G} r, J}\left(\iota_{J}(F), X\right)\right) \rightarrow$ $\operatorname{Hom}_{\mathcal{G} r, I}\left(\iota_{I}(F), \mathcal{R}_{J, I}(X)\right)$. Ce sont des isomorphismes dans le cas où $F$ est un projectif standard grâce aux propositions 5.3.4 et 5.3.3. Le cas général s'en déduit par passage à la colimite.

La notation ad hoc suivante n'interviendra que dans les deux lemmes techniques ci-dessous.

Notation 9.1.4. - Dans cette section, nous noterons $r: \mathcal{F}_{\mathcal{G}_{r, I}} \rightarrow \mathcal{F}_{\mathcal{G} r, J}$ le foncteur adjoint à gauche au foncteur $\mathcal{R}_{J, I}: \mathcal{F}_{\mathcal{G} r, J} \rightarrow \mathcal{F}_{\mathcal{G}_{r, I}}$, et $R: \mathcal{F}_{\text {surj }}^{I} \rightarrow \mathcal{F}_{\text {surj }}^{J}$ l'adjoint à gauche au foncteur de restriction $\mathcal{F}_{\text {surj }}^{J} \rightarrow \mathcal{F}_{\text {surj }}^{I}$ (de tels adjoints existent par le corollaire C.2.4).

Lemme 9.1.5. - Le diagramme

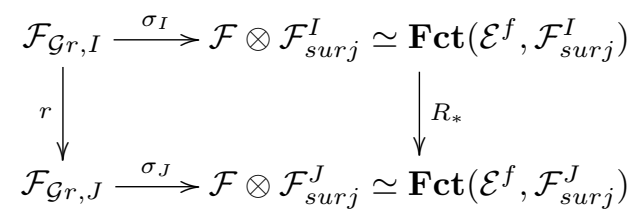

commute à isomorphisme naturel près.

Démonstration. - Soit $a: \mathcal{F} \otimes \mathcal{F}_{\text {surj }}^{J} \rightarrow \mathcal{F} \otimes \mathcal{F}_{\text {surj }}^{I}$ le foncteur de restriction. On définit une transformation naturelle $R_{*} \sigma_{I} \rightarrow \sigma_{J} r$ comme l'adjointe (cf. proposition C.1.3) à la flèche $\sigma_{I} \rightarrow a \sigma_{J} r \simeq \sigma_{I} \mathcal{R}_{J, I} r$ obtenue en composant $\sigma_{I}$ et l'unité $i d \rightarrow \mathcal{R}_{J, I} r$ de l'adjonction. Cette transformation naturelle est un isomorphisme, car elle induit un isomorphisme $\operatorname{hom}_{\mathcal{G} r, J}\left(\sigma_{J} r(X), I_{(A, B)}^{\mathcal{E}^{f} \times \mathcal{E}_{\text {surj }}^{J}}\right) \stackrel{\cong}{\leftrightarrows} \operatorname{hom}_{\mathcal{G} r, J}\left(R_{*} \sigma_{I}(X), I_{(A, B)}^{\mathcal{E}^{f} \times \mathcal{E}_{\text {surj }}^{J}}\right)$ pour tous $X \in \mathrm{Ob} \mathcal{F}_{\mathcal{G} r, I}$ et $(A, B) \in \mathrm{Ob} \mathcal{E}^{f} \times \mathcal{E}_{\text {surj }}^{J}$ grâce aux isomorphismes (7) (page 56).

Lemme 9.1.6. - Il existe dans $\mathcal{F}_{\mathcal{G} r, J}$ un isomorphisme $r\left(X \otimes \kappa_{I}(F)\right) \simeq r(X) \otimes$ $\kappa_{J}(F)$ naturel en les objets $X$ de $\mathcal{F}_{\mathcal{G} r, I}$ et $F$ de $\mathcal{F}$.

Démonstration. - On établit, à l'aide du lemme précédent, que la transformation naturelle $r\left(X \otimes \kappa_{I}(F)\right) \rightarrow r(X) \otimes \kappa_{J}(F)$ adjointe au morphisme $X \otimes \kappa_{I}(F) \rightarrow$ $\mathcal{R}_{J, I} r(X) \otimes \kappa_{I}(F) \simeq \mathcal{R}_{J, I}\left(r(X) \otimes \kappa_{J}(F)\right)$ obtenu en tensorisant par $\kappa_{I}(F)$ l'unité de l'adjonction procure un isomorphisme lorsqu'on lui applique $\sigma_{J}$. Cela provient du lemme 9.1.5 et des deux observations suivantes :

- il existe dans $\mathcal{F} \otimes \mathcal{F}_{\text {surj }}^{I}$ un isomorphisme canonique $\sigma_{I}\left(X \otimes \kappa_{I}(F)\right) \simeq \sigma_{I}(X) \otimes$ $(F \otimes \mathbb{k})$, que l'on peut encore voir comme l'image de $\sigma_{I}(X)$ par le foncteur $\mathcal{F} \otimes \mathcal{F}_{\text {surj }}^{I} \simeq \mathbf{F} \operatorname{ct}\left(\mathcal{E}_{\text {surj }}^{I}, \mathcal{F}\right) \stackrel{(\cdot \otimes F)_{*}}{\longrightarrow} \mathbf{F c t}\left(\mathcal{E}_{\text {surj }}^{I}, \mathcal{F}\right)$ 
- le diagramme

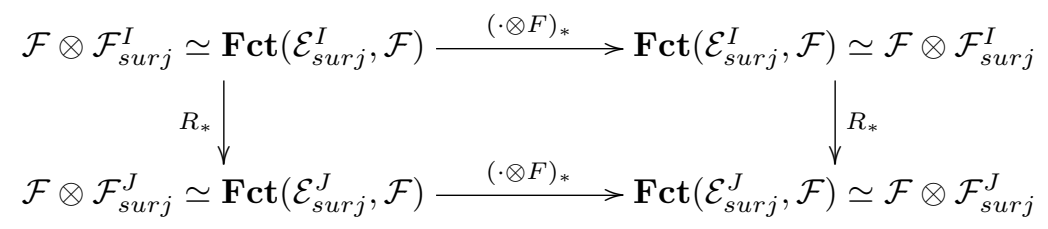

commute (à isomorphisme canonique près).

Comme le foncteur $\sigma_{J}$ est exact et fidèle, cela donne la conclusion.

Nous sommes désormais en mesure d'établir la commutation des foncteurs hom internes et des foncteurs de restriction, dans le cas où la source du foncteur hom interne appartient à l'image du foncteur $\kappa_{I}: \mathcal{F} \rightarrow \mathcal{F}_{\mathcal{G} r, I}$.

Proposition 9.1.7. - Il existe un isomorphisme

$$
\operatorname{Hom}_{\mathcal{G} r, I}\left(\kappa_{I}(F), \mathcal{R}_{J, I}(X)\right) \simeq \mathcal{R}_{J, I}\left(\operatorname{Hom}_{\mathcal{G} r, J}\left(\kappa_{J}(F), X\right)\right)
$$

naturel en les objets $F$ de $\mathcal{F}$ et $X$ de $\mathcal{F}_{\mathcal{G} r, J}$.

Démonstration. — Par le lemme 9.1.6 (dont on conserve la notation), on a des isomorphismes naturels

$$
\begin{gathered}
\operatorname{hom}_{\mathcal{G} r, I}\left(Y, \operatorname{Hom}_{\mathcal{G} r, I}\left(\kappa_{I}(F), \mathcal{R}_{J, I}(X)\right)\right) \simeq \operatorname{hom}_{\mathcal{G} r, I}\left(Y \otimes \kappa_{I}(F), \mathcal{R}_{J, I}(X)\right) \simeq \\
\operatorname{hom}_{\mathcal{G} r, J}\left(r\left(Y \otimes \kappa_{I}(F)\right), X\right) \simeq \operatorname{hom}_{\mathcal{G} r, J}\left(r(Y) \otimes \kappa_{J}(F), X\right) \simeq \\
\operatorname{hom}_{\mathcal{G} r, J}\left(r(Y), \operatorname{Hom}_{\mathcal{G} r, J}\left(\kappa_{J}(F), X\right)\right) \simeq \operatorname{hom}_{\mathcal{G} r, I}\left(Y, \mathcal{R}_{J, I}\left(\operatorname{Hom}_{\mathcal{G} r, J}\left(\kappa_{J}(F), X\right)\right)\right)
\end{gathered}
$$

(où $Y \in \mathrm{Ob} \mathcal{F}_{\mathcal{G} r, I}$ ), ce qui démontre la proposition.

\subsection{Propriétés formelles}

Nous étudions à présent le comportement mutuel des foncteurs de division ou hom internes et des foncteurs fondamentaux de la section 5.1.

Foncteurs de division. - C'est le cas des foncteurs de division par des objets injectifs de la catégorie $\mathcal{F}_{\mathcal{G} r}$ qui nous intéresse le plus (cf. proposition 9.2.3).

Nous donnons d'abord une propriété de compatibilité entre les foncteurs $\omega, \iota$ et les foncteurs de division.

Proposition 9.2.1. - Il existe dans $\mathcal{F}$ un isomorphisme $\omega(X: \iota(F)) \simeq(\omega(X): F)$ naturel en les objets $X$ de $\mathcal{F}_{\mathcal{G} r}$ et $F$ de $\mathcal{F}^{d f}$. On a un résultat analogue dans $\mathcal{F}_{\mathcal{G} r, \leq n}$. Démonstration. - Il s'agit d'une conséquence formelle de l'adjonction entre $\iota$ et $\omega$ (cf. proposition 5.1.7) et de la commutation de $\iota$ au produit tensoriel (cf. proposition 9.1.2).

La proposition élémentaire suivante interviendra dans la partie IV. 
Proposition 9.2.2. - Pour toute partie $I$ de $\mathbb{N}$, il existe dans $\mathcal{F}_{\mathcal{G} r, I}$ un isomorphisme canonique $\left(\rho_{I}(A): X\right) \simeq \rho_{I}\left(A: \varepsilon_{I}(X)\right)$ pour $A \in \mathrm{Ob} \mathcal{F}_{\text {surj }}^{I}$, et $X \in \mathrm{Ob} \mathcal{F}_{\mathcal{G} r, I}$ à valeurs de dimension finie.

Démonstration. - Cette proposition s'établit de la même manière que la précédente, à partir de la proposition 5.1.18 et de la commutation de $\varepsilon_{I}$ au produit tensoriel.

On rappelle que le symbole $G r$ qui apparaît dans l'importante proposition suivante a été introduit au notation 4.3.8.

Proposition 9.2.3. - Il existe un isomorphisme

$$
\left(X: \iota\left(I_{V}\right)\right)(A, B) \simeq \bigoplus_{\substack{C \in \mathcal{G} r(V \oplus A) \\ i m(C \hookrightarrow V \oplus A \rightarrow A)=B}} X(V \oplus A, C)
$$

naturel en les objets $V$ de $\mathcal{E}^{f},(A, B)$ de $\mathcal{E}_{\mathcal{G} r}^{f}$ et $X$ de $\mathcal{F}_{\mathcal{G} r}$.

De plus, pour tout $W \in \mathcal{G} r(V)$, le monomorphisme scindé naturel $\left(X: I_{(V, W)}^{\mathcal{G} r}\right) \hookrightarrow$ $\left(X: \iota\left(I_{V}\right)\right)$ induit par l'épimorphisme scindé $\iota\left(I_{V}\right) \rightarrow I_{(V, W)}^{\mathcal{G} r}$ fourni par la proposition 5.1.14 identifie $\left(X: I_{(V, W)}^{\mathcal{G} r}\right)(A, B)$ au sous-espace

$$
\bigoplus_{C \in G r(W, B)} X(V \oplus A, C)
$$

de $\left(X: \iota\left(I_{V}\right)\right)(A, B)$.

Démonstration. - On a des isomorphismes naturels

$$
\begin{gathered}
\left(\left(X: I_{(V, W)}^{\mathcal{G r} r}\right)(A, B)\right)^{*} \simeq \operatorname{hom}_{\mathcal{G} r}\left(\left(X: I_{(V, W)}^{\mathcal{G} r}\right), I_{(A, B)}^{\mathcal{G} r}\right) \simeq \operatorname{hom}_{\mathcal{G} r}\left(X, I_{(V, W)}^{\mathcal{G} r} \otimes I_{(A, B)}^{\mathcal{G} r}\right) \\
\simeq \bigoplus_{C \in G r(W, B)} \operatorname{hom}_{\mathcal{G} r}\left(X, I_{(V \oplus A, C)}^{\mathcal{G} r}\right) \simeq \bigoplus_{C \in G r(W, B)} X(V \oplus A, C)^{*}
\end{gathered}
$$

par la proposition 5.2.8, ce qui donne le résultat, pour $X$ à valeurs de dimension finie, en dualisant; le cas quelconque s'en déduit en écrivant $X$ comme colimite de sous-foncteurs à valeurs de dimension finie.

Foncteurs hom internes. - Nous ramenons dans ce paragraphe le calcul de nombreux foncteurs hom internes dans la catégorie $\mathcal{F}_{\mathcal{G} r, I}$ à celui de foncteurs hom internes des catégories $\mathcal{F}$ ou $\mathcal{F} \otimes \mathcal{F}_{\text {surj }}^{I}$.

Convention 9.2.4. - Dans ce paragraphe, $I$ désigne une partie de $\mathbb{N}$.

Le principal objectif de la proposition suivante est d'établir la commutation entre le foncteur $\kappa_{I}$ et les foncteurs hom internes (corollaire 9.2.7).

Proposition 9.2.5. - Il existe un isomorphisme

$$
\operatorname{Hom}_{\mathcal{G} r, I}\left(X, \theta_{I}(A)\right) \simeq \theta_{I}\left(\operatorname{Hom}_{\mathcal{F} \otimes \mathcal{F}_{\text {surj }}^{I}}\left(\eta_{I}(X), A\right)\right)
$$

naturel en les objets $X$ de $F_{\mathcal{G} r, I}$ et $A$ de $\mathcal{F} \otimes \mathcal{F}_{\text {surj }}^{I}$. 
Démonstration. - C'est une conséquence formelle de l'adjonction entre les foncteurs $\theta_{I}$ et $\eta_{I}$ (proposition 7.1.3) et de la commutation du foncteur $\eta_{I}$ au produit tensoriel (proposition 7.1.7).

Corollaire 9.2.6. - Il existe un isomorphisme

$$
\operatorname{Hom}_{\mathcal{G} r, I}\left(\theta_{I}(B), \theta_{I}(A)\right) \simeq \operatorname{Hom}_{\mathcal{G} r, I}\left(\xi_{I}(B), \theta_{I}(A)\right) \simeq \theta_{I}\left(\operatorname{Hom}_{\mathcal{F} \otimes \mathcal{F}_{\text {surj }}^{I}}(B, A)\right)
$$

naturel en les objets $A$ et $B$ de $\mathcal{F} \otimes \mathcal{F}_{\text {surj }}^{I}$.

Démonstration. — On combine les propositions 9.2.5 et 7.1.5.

Corollaire 9.2.7. - Il existe des isomorphismes

$$
\operatorname{Hom}_{\mathcal{G} r, I}\left(\kappa_{I}(F), \kappa_{I}(G)\right) \simeq \operatorname{Hom}_{\mathcal{G} r, I}\left(\iota_{I}(F), \kappa_{I}(G)\right) \simeq \kappa_{I}\left(\operatorname{Hom}_{\mathcal{F}}(F, G)\right)
$$

naturels en les objets $F$ et $G$ de $\mathcal{F}$.

Démonstration. — C'est le cas particulier $A=G \otimes \mathbb{k}$ et $B=F \otimes \mathbb{k}$ du corollaire précédent.

Remarque 9.2.8. - Une approche alternative de ce corollaire consiste à traiter d'abord le cas où la partie $I$ contient 0 à l'aide de la proposition 5.1 .19 , puis d'en déduire le cas général grâce à la proposition 9.1.7.

Nous traitons à présent de la commutation du foncteur $\iota_{I}$ aux foncteurs hom internes.

Proposition 9.2.9. - Il existe un isomorphisme naturel $\operatorname{Hom}_{\mathcal{G} r, I}\left(\iota_{I}(F), \iota_{I}(G)\right) \simeq$ $\iota_{I}\left(\operatorname{Hom}_{\mathcal{F}}(F, G)\right)$ pour $F, G \in \mathrm{Ob} \mathcal{F}$.

Démonstration. - Comme $\iota_{I}(F)=\mathcal{R}_{\mathbb{N}, I}(\iota(F))$, la proposition 9.1.3 permet de ne traiter que le cas où $I=\mathbb{N}$. La conclusion provient alors des assertions 2 et 4 de la proposition 5.1.7.

Il existe aussi une propriété de commutation relative au foncteur $\sigma_{I}$ :

Proposition 9.2.10. — Il existe dans $\mathcal{F} \otimes \mathcal{F}_{\text {surj }}^{I}$ un isomorphisme

$$
\sigma_{I}\left(\operatorname{Hom}_{\mathcal{G} r, I}\left(\xi_{I}(F), X\right)\right) \simeq \mathbf{H o m}_{\mathcal{F} \otimes \mathcal{F}_{\text {surj }}^{I}}\left(F, \sigma_{I}(X)\right)
$$

naturel en les objets $F$ de $\mathcal{F} \otimes \mathcal{F}_{\text {surj }}^{I}$ et $X$ de $\mathcal{F}_{\mathcal{G} r, I}$.

Démonstration. - Cette propriété s'obtient à partir de l'adjonction entre les foncteurs $\xi_{I}$ et $\sigma_{I}$ (proposition 5.1.17) et de la commutation du foncteur $\xi_{I}$ au produit tensoriel.

La généralisation de la description très simple des foncteurs hom internes de la catégorie $_{\mathbb{k}\left[G L_{n}(\mathbb{k})\right]}$ Mod (cf. [CR90], $§ 10$ D) aux foncteurs pseudo-constants de $\mathcal{F}_{\mathcal{G} r, n}$ est donnée par la proposition suivante, laissée au lecteur (cf. [Dja06d] pour les détails). 
Proposition 9.2.11. - Soient $n \in \mathbb{N}$ et $M$ un $\mathbb{k}\left[G L_{n}(\mathbb{k})\right]$-module fini. Les endofoncteurs $\cdot \otimes \rho_{n}\left(M^{*}\right), \operatorname{Hom}_{\mathcal{G} r, n}\left(\rho_{n}(M), \cdot\right)$ et $\left(\cdot: \rho_{n}(M)\right)$ de $\mathcal{F}_{\mathcal{G} r, n}$ sont naturellement isomorphes.

On en déduit aussitôt la propriété de commutation suivante :

Corollaire 9.2.12. - Soient $n \in \mathbb{N}$ et $M$ un $\mathbb{k}\left[G L_{n}(\mathbb{k})\right]$-module fini. L'endofoncteur $\cdot \otimes \rho_{n}(M)$ de $\mathcal{F}_{\mathcal{G} r, n}$ commute naturellement aux foncteurs hom internes et aux foncteurs de division.

Le foncteur $\operatorname{Ext}_{\mathcal{G} r}^{*}(\iota(F), \cdot)$. — Commençons par introduire une nouvelle notation. Soit $A$ un objet de $\mathcal{E}_{\mathcal{G} r}^{f}$; nous désignerons par $\tau_{A}: \mathcal{F}_{\mathcal{G} r} \rightarrow \mathcal{F}$ le foncteur de précomposition par $\mathcal{E}^{f} \rightarrow \mathcal{E}_{\mathcal{G} r}^{f} \quad E \mapsto E \boxplus A$. Cette construction est fonctorielle en $A$.

Proposition 9.2.13. - Il existe un isomorphisme

$$
\mathbf{E x t}_{\mathcal{G} r}^{*}(\iota(F), X)(A) \simeq \operatorname{Ext}_{\mathcal{F}}^{*}\left(F, \tau_{A}(X)\right)
$$

naturel en les objets $F$ de $\mathcal{F}, X$ de $\mathcal{F}_{\mathcal{G} r}$ et $A$ de $\mathcal{E}_{\mathcal{G} r}^{f}$.

Démonstration. - Il existe des isomorphismes naturels $\tau_{A} \circ \iota \simeq \Delta_{\mathfrak{O}(A)}$ et $\tau_{A}\left(P_{A}^{\mathcal{G} r}\right) \simeq$ $\mathbb{k}\left[\operatorname{End}_{\mathcal{G} r}(A)\right] \otimes P_{\mathfrak{K}(A)}$ (par la proposition 4.1.11). On en déduit une transformation naturelle $F \rightarrow \tau_{A}\left(\iota(F) \otimes P_{A}^{\mathcal{G} r}\right) \simeq \Delta_{\mathfrak{O}(A)}(F) \otimes \mathbb{k}\left[\operatorname{End}_{\mathcal{G} r}(A)\right] \otimes P_{\mathfrak{K}(A)}$ par produit tensoriel des injections canoniques $F \hookrightarrow \Delta_{\mathfrak{O}(A)}(F), \mathbb{k} \hookrightarrow P_{\mathfrak{K}(A)}$ et $\mathbb{k} \hookrightarrow \mathbb{k}\left[\operatorname{End}_{\mathcal{G} r}(A)\right]$ (donnée $\left.\operatorname{par}\left[i d_{A}\right]\right)$.

L'application naturelle $\operatorname{hom}_{\mathcal{G} r}\left(\iota(F) \otimes P_{A}^{\mathcal{G} r}, X\right) \rightarrow \operatorname{hom}_{\mathcal{F}}\left(F, \tau_{A}(X)\right)$ qu'on en déduit est bijective. En effet, il suffit de le voir pour $F$ projectif standard de $\mathcal{F}$, auquel cas c'est une conséquence de la proposition 5.3.4. Cet isomorphisme s'étend, grâce à la proposition A.1.1, en un isomorphisme naturel gradué $\operatorname{Ext}_{\mathcal{G} r}^{*}\left(\iota(F) \otimes P_{A}^{\mathcal{G} r}, X\right) \stackrel{\simeq}{\longrightarrow}$ $\operatorname{Ext}_{\mathcal{F}}^{*}\left(F, \tau_{A}(X)\right)$. La conclusion provient alors de l'isomorphisme naturel (19) de l'appendice $\mathrm{C}$. 



\section{PARTIE III}

\section{PROPRIÉTÉS COHOMOLOGIQUES DU FONCTEUR $\omega$. APPLICATIONS}


Le foncteur d'intégrale en grassmanniennes $\omega: \mathcal{F}_{\mathcal{G} r} \rightarrow \mathcal{F}$ possède un comportement qui diffère notablement de celui des autres foncteurs fondamentaux entre $\mathcal{F}_{\mathcal{G} r}$ et $\mathcal{F}$ ou $\mathcal{F} \otimes \mathcal{F}_{\text {surj }}$. En particulier, il ne préserve pas les objets localement finis; en fait, le foncteur $\omega(X)$ de $\mathcal{F}$ n'est localement fini que si le foncteur $X$ de $\mathcal{F}_{\mathcal{G} r}$ appartient à la sous-catégorie $\mathcal{F}_{\mathcal{G} r, 0} \simeq \mathcal{F}$ - cela résultera du théorème 10.2.1. Une autre façon d'illustrer ce phénomène consiste à étudier la composée $\Delta \circ \omega$ : elle est « nettement plus grosse $»$ que $\omega \circ \Delta-$ cf. section 11.1.

C'est dans ces observations que réside tout l'intérêt du foncteur $\omega$, en vue de l'étude de la structure de la catégorie $\mathcal{F}$. Remarquons que les progrès significatifs obtenus à ce sujet par Powell dans [Pow98c] constituent essentiellement des traductions de propriétés du foncteur $\varpi: \mathcal{F}_{\text {surj }} \rightarrow \mathcal{F}$ (cf. section 2.3) ; il n'est pas étonnant que le foncteur $\omega$, qui généralise $\varpi$, permette d'aller plus loin dans la compréhension de la catégorie $\mathcal{F}$.

L'une des avancées importantes obtenues grâce aux catégories de foncteurs en grassmanniennes réside en le théorème 10.2.1, qui constitue le résultat principal de cette partie. Il donne une propriété d'annulation cohomologique très générale, qui trouve deux applications essentielles.

L'une d'entre elle, traitée dans la section 13.2, concerne le lien entre cohomologie du groupe linéaire $G L(\mathbb{k})$ (ou $K$-théorie stable de $\mathbb{k}$ ) et cohomologie des foncteurs. Le résultat obtenu, qui généralise le théorème de Betley-Suslin donné dans l'appendice de [FFSS99], fournit un isomorphisme entre la cohomologie de $G L(\mathbb{k})$ à coefficients convenables et des groupes d'extensions dans la catégorie $\mathcal{F}_{\mathcal{G} r}(\mathbb{k})$ entre des objets finis, nettement plus faciles d'accès.

Les calculs cohomologiques dans la catégorie $\mathcal{F}(\mathbb{k})$ donnés par le théorème 10.2.1 possèdent également un intérêt intrinsèque. Ainsi, ce théorème suggère une conjecture décrivant la filtration de Krull de la catégorie $\mathcal{F}$, qui renforce toutes les formes antérieurement formulées de la conjecture artinienne; il est l'un des outils fondamentaux de la démonstration des formes partielles que nous en établirons dans la partie IV. Nous discutons ces questions dans le chapitre 12.

L'application du théorème 10.2.1 exposée dans le chapitre 11 traite de propriétés de commutation entre le foncteur $\omega$ et des foncteurs hom internes. Son intérêt est illustré par [Dja06c], comme il est discuté à la fin de la section 11.2. 


\section{CHAPITRE 10}

\section{THÉORÈME D'ANNULATION COHOMOLOGIQUE}

L'objectif de ce chapitre consiste à établir le théorème 10.2.1 et le corollaire 10.2.2 qui s'en déduit. Leur démonstration repose sur des considérations explicites liées à la catégorie $\mathcal{F}_{\text {surj }}$ et des arguments d'adjonction. On emploie également une catégorie de foncteurs auxiliaire, variante de $\mathcal{F}_{\mathcal{G} r}$ obtenue en considérant deux éléments de la grassmannienne d'un espace vectoriel. Hormis l'utilisation de ce nouveau type de catégories, notre démarche procède des mêmes idées conceptuelles que celles inaugurées par Pirashvili (cf. remarque 10.2.3.1).

\subsection{Préliminaires}

On rappelle que $o: \mathcal{F} \rightarrow \mathcal{F}_{\text {surj }}$ désigne le foncteur d'oubli (cf. chapitre 2) et que le foncteur $\mathbb{k}^{\geq i}$ est défini dans la notation 2.3.3 (page 21).

Les résultats de ce chapitre reposent sur la structure du foncteur constant $\mathbb{k}=I_{0}^{\text {surj }}$ de $\mathcal{F}_{\text {surj }}$ donnée par le corollaire 2.3.4. On rappelle que ce foncteur est unisériel.

Lemme 10.1.1. — Il existe dans $\mathcal{F}_{\text {surj }}$ un monomorphisme $\mathbb{k}^{\geq 1} \hookrightarrow o\left(\bar{I}_{\mathbb{k}}\right)$, unique à homothétie près.

Démonstration. - Par l'assertion 3 de la proposition 2.3.6, il existe dans $\mathcal{F}_{\text {surj }}$ un diagramme commutatif

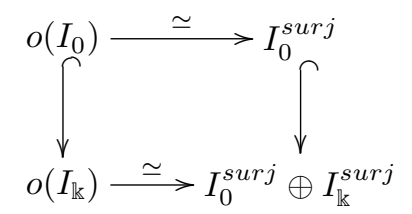

On en déduit un isomorphisme entre $o\left(\bar{I}_{\mathbb{k}}\right)$ et $I_{\mathbb{k}}^{\text {surj }}$.

Par conséquent, hom $\left(\mathbb{k}^{\geq 1}, o\left(\bar{I}_{\mathbb{k}}\right)\right) \simeq \mathbb{k}^{\geq 1}(\mathbb{k})^{*}=\mathbb{k}$. Le morphisme correspondant à 1 est injectif, car sa restriction au socle de $\mathbb{k}^{\geq 1}$, l'objet simple $\mathbb{k}(1)$ (cf. corollaire 2.3.4), 
est non nulle, l'inclusion $\mathbb{k}(1) \hookrightarrow \mathbb{k}^{\geq 1}$ induisant un isomorphisme par évaluation sur $\mathbb{k}$. Cela démontre le lemme.

Remarque 10.1.2. - Le foncteur $\mathbb{k}^{\geq 1}$ est idempotent pour le produit tensoriel : $\left(\mathbb{k}^{\geq 1}\right)^{\otimes 2} \simeq \mathbb{k}^{\geq 1}$. Il induit donc un endofoncteur idempotent $\cdot \otimes \mathbb{k}^{\geq 1}$ de $\mathcal{F}_{\text {surj }}$.

Avant d'exploiter le lemme 10.1.1, nous aurons besoin d'introduire des catégories de foncteurs auxiliaires (qui n'interviendront que dans cette section).

Notation 10.1.3. - (1) Nous désignerons par $\mathcal{E}_{b i-\mathcal{G} r}^{f}$ la catégorie $\left(\mathcal{E}_{\mathcal{G} r}^{f}\right)_{\backslash \mathcal{G} r}$, où l'on note encore $\mathcal{G} r$, par abus de notation, le foncteur composé

$$
\mathcal{E}_{\mathcal{G} r}^{f} \stackrel{\mathfrak{O}}{\longrightarrow} \mathcal{E}^{f} \stackrel{\mathcal{G} r}{\longrightarrow} \text { Ens, }
$$

qui est donc donné sur les objets par $(V, W) \mapsto \mathcal{G} r(V)$.

Autrement dit, les objets de $\mathcal{E}_{b i-\mathcal{G} r}^{f}$ sont les triplets $(V, B, W)$ formés d'un espace vectoriel de dimension finie $V$ et de deux sous-espaces $B$ et $W$ de $V$, et les flèches $(V, B, W) \rightarrow\left(V^{\prime}, B^{\prime}, W^{\prime}\right)$ de $\mathcal{E}_{b i-\mathcal{G} r}^{f}$ sont les applications linéaires $f: V \rightarrow V^{\prime}$ telles que $f(B)=B^{\prime}$ et $f(W)=W^{\prime}$.

La catégorie de foncteurs $\mathbf{F} \operatorname{ct}\left(\mathcal{E}_{b i-\mathcal{G} r}^{f}, \mathcal{E}\right)$ sera notée $\mathcal{F}_{b i-\mathcal{G} r}$.

(2) Nous désignerons par $\mathcal{E}_{2-\mathcal{D} r}^{f}$ la catégorie $\left(\mathcal{E}_{\mathcal{G} r}^{f}\right) \backslash \mathcal{G} r^{\prime}$, où l'on désigne par $\mathcal{G} r^{\prime}$ le foncteur

$$
\mathcal{E}_{\mathcal{G} r}^{f} \stackrel{\mathfrak{B}}{\longrightarrow} \mathcal{E}^{f} \stackrel{\mathcal{G} r}{\longrightarrow} \text { Ens, }
$$

qui est donc donné sur les objets par $(V, W) \mapsto \mathcal{G} r(W)$.

La catégorie de foncteurs $\mathbf{F} \mathbf{c t}\left(\mathcal{E}_{2-\mathcal{D} r}^{f}, \mathcal{E}\right)$ sera notée $\mathcal{F}_{2-\mathcal{D} r}$

(3) L'inclusion naturelle de foncteurs ensemblistes $\mathfrak{B} \hookrightarrow \mathfrak{O}$ identifie $\mathcal{E}_{2-\mathcal{D r}}^{f}$ à une sous-catégorie pleine de $\mathcal{E}_{b i-\mathcal{G} r}^{f}$. Nous noterons $i_{\mathcal{D} r}: \mathcal{F}_{b i-\mathcal{G} r} \rightarrow \mathcal{F}_{2-\mathcal{D} r}$ le foncteur de précomposition par l'inclusion.

Remarque 10.1.4. - L'indice $2-\mathcal{D} r$ utilisé est une abréviation de 2-drapeau. On pourrait naturellement généraliser les considérations de ce paragraphe à des catégories correspondant aux espaces vectoriels munis de drapeaux de longueur fixée arbitraire.

On dispose, conformément aux résultats du chapitre 3, d'un foncteur de plongement $\Upsilon_{\mathcal{G} r}: \mathcal{F}_{\mathcal{G} r} \rightarrow \mathcal{F}_{b i-\mathcal{G} r}$ et d'un foncteur d'intégrale $\Omega_{\mathcal{G} r}: \mathcal{F}_{b i-\mathcal{G} r} \rightarrow \mathcal{F}_{\mathcal{G} r}$. Sur les objets, on a $\Upsilon_{\mathcal{G} r}(X)(V, B, W)=X(V, B)$ et $\Omega_{\mathcal{G} r}(A)(V, B)=\bigoplus_{W \in \mathcal{G} r(V)} A(V, B, W)$.

Définition 10.1.5. - Nous noterons $\mathcal{N}$ la sous-catégorie épaisse de $\mathcal{F}_{b i-\mathcal{G} r}$ noyau du foncteur exact $i_{\mathcal{D} r}$. Explicitement, $\mathcal{N}$ contient les objets $X$ de $\mathcal{F}_{b i-\mathcal{G} r}$ tels que $X(V, B, W)=0$ pour tout objet $(V, B, W)$ de $\mathcal{E}_{b i-\mathcal{G} r}^{f}$ tel que $W \subset B$.

Remarque 10.1.6. - (1) La catégorie $\mathcal{E}_{b i-\mathcal{G} r}^{f}$ s'identifie canoniquement à $\mathcal{E}_{\backslash(\mathcal{G} r, \mathcal{G} r)}^{f}$ : c'est la catégorie des espaces vectoriels de dimension finie munie de deux sous-espaces. Nous avons préféré donner la présentation ci-dessus « dessymétrisant 
les deux bases » de manière à introduire plus naturellement les foncteurs $\Upsilon_{\mathcal{G} r}, \Omega_{\mathcal{G} r}$ et $i_{\mathcal{D} r}$.

(2) La catégorie $\mathcal{F}_{2-\mathcal{D} r}$ n'interviendra pas en elle-même, c'est la sous-catégorie $\mathcal{N}$ de $\mathcal{F}_{b i-\mathcal{G} r}$ qui nous intéressera.

Le lemme suivant donne les faits concrets dont nous aurons besoin pour établir nos résultats d'annulation cohomologique.

Lemme 10.1.7. - (1) Il existe un foncteur $\beta: \mathcal{E}_{b i-\mathcal{G} r}^{f} \rightarrow \mathcal{E}_{\text {surj }}^{f}$ tel que $\beta(V, B, W)=W /(W \cap B)$ pour tout objet $(V, B, W)$ de $\mathcal{E}_{b i-\mathcal{G} r}^{f}$.

(2) Il existe dans $\mathcal{F}_{b i-\mathcal{G} r}$ un épimorphisme du foncteur constant $\mathbb{k}$ vers le foncteur $\beta^{*}\left(\mathbb{k}^{\geq 1}\right)$, où $\beta^{*}: \mathcal{F}_{\text {surj }} \rightarrow \mathcal{F}_{\text {bi-Gr }}$ désigne le foncteur de précomposition par $\beta ; \mathcal{N}$ est la sous-catégorie pleine des objets $X$ de $\mathcal{F}_{b i-\mathcal{G} r}$ tels que la projection canonique $X \rightarrow X \otimes \beta^{*}\left(\mathbb{k}^{\geq 1}\right)$ qui s'en déduit est un isomorphisme. En particulier, $\mathcal{N}$ est un idéal de $\mathcal{F}_{b i-\mathcal{G} r}$ : le produit tensoriel d'un objet de $\mathcal{N}$ et d'un objet de $\mathcal{F}_{b i-\mathcal{G} r}$ appartient $\grave{a} \mathcal{N}$.

(3) Le foncteur $\beta^{*}\left(\mathbb{k}^{\geq 1}\right)$ se plonge dans $\Upsilon_{\mathcal{G} r}\left(\kappa\left(\bar{I}_{\mathbb{k}}\right)\right)$.

Démonstration. - La vérification du premier point est immédiate. Le second point vient de ce que la projection $\mathbb{k} \rightarrow \mathbb{k}^{\geq 1}$ de $\mathcal{F}_{\text {surj }}$ (cf. lemme 2.3.4) induit un épimorphisme $\mathbb{k}=\beta^{*}(\mathbb{k}) \rightarrow \beta^{*}\left(\mathbb{k}_{\mathbb{k}} \geq 1\right)$ dans $\mathcal{F}_{b i-\mathcal{G} r}$ dont l'image est nulle sur l'objet $(V, B, W)$ si et seulement si $\beta(V, B, W)$ est nul, i.e. si $W \subset B$.

On remarque ensuite que le foncteur composé $\mathcal{E}_{b i-\mathcal{G} r}^{f} \stackrel{\beta}{\rightarrow} \mathcal{E}_{\text {surj }}^{f} \hookrightarrow \mathcal{E}^{f}$ se plonge dans le foncteur $\mathcal{E}_{b i-\mathcal{G} r}^{f} \stackrel{\mathcal{O}_{\mathcal{E}_{\mathcal{G} r}^{f}, \mathcal{G}_{r}}}{\longrightarrow} \mathcal{E}_{\mathcal{G} r}^{f} \stackrel{\mathfrak{K}}{\longrightarrow} \mathcal{E}^{f}$ (on rappelle que $\mathcal{O}_{\mathcal{E}_{\mathcal{G} r}^{f}, \mathcal{G} r}$ désigne le foncteur $(V, B, W) \mapsto(V, B)$ - cf. notation 3.1.2), via l'inclusion canonique $\beta(V, B, W)=$ $W /(W \cap B) \hookrightarrow V / B=\mathfrak{K}(V, B)$. Comme les foncteurs de $\mathcal{F}$ préservent les monomorphismes, on en déduit que le foncteur de précomposition $\beta^{*} \circ o: \mathcal{F} \rightarrow \mathcal{F}_{b i-\mathcal{G} r}$ s'injecte dans $\Upsilon_{\mathcal{G} r} \circ \kappa: \mathcal{F} \rightarrow \mathcal{F}_{b i-\mathcal{G} r}$. Le lemme 10.1.1 fournit alors un monomorphisme $\beta^{*}\left(\mathbb{k}^{\geq 1}\right) \hookrightarrow \beta^{*}\left(o\left(\bar{I}_{\mathbb{k}}\right)\right) \hookrightarrow \Upsilon_{\mathcal{G} r}\left(\kappa\left(\bar{I}_{\mathbb{k}}\right)\right)$, ce qui achève la démonstration.

Résultats d'annulation cohomologique « abstraits ». — La première propriété d'annulation cohomologique de ce chapitre, dont toutes les autres se déduiront formellement, s'obtient à l'aide du lemme précédent et du résultat formel donné par le corollaire A.1.2.

Lemme 10.1.8. - Soient $A$ un foncteur analytique de $\mathcal{F}_{\mathcal{G}_{r}}$ et $X$ un objet de $\mathcal{N}$. Le groupe d'extensions $\operatorname{Ext}_{\mathcal{G}_{r}}^{i}\left(A, \Omega_{\mathcal{G} r}(X)\right)$ est nul pour tout $i \in \mathbb{N}$.

Démonstration. - La proposition B.1.14 (page 187) permet de se ramener au cas où $A$ est polynomial : il existe $d \in \mathbb{N}^{*}$ tel que $\Delta^{d} A=0$. 
Notons $F$ l'endofoncteur $\cdot \otimes \kappa\left(\bar{I}_{\mathbb{k}}\right)$ de $\mathcal{F}_{\mathcal{G} r}, G=\Delta^{\mathcal{G} r}, H: \mathcal{N} \rightarrow \mathcal{F}_{\mathcal{G} r}$ la restriction à $\mathcal{N}$ de $\Omega_{\mathcal{G} r}$, et $K$ l'endofoncteur de $\mathcal{N}$ induit par $\cdot \otimes \Upsilon_{\mathcal{G} r}\left(\kappa\left(\bar{I}_{\mathbb{k}}\right)\right.$ ) (on utilise que $\mathcal{N}$ est un idéal de $\mathcal{F}_{b i-\mathcal{G} r}$ ). Alors :

(1) tous ces foncteurs sont exacts ;

(2) $G$ est adjoint à gauche à $F$ par le corollaire 5.3.5;

(3) la proposition 3.1.8 (page 35) fournit un isomorphisme $F \circ H \simeq H \circ K$;

(4) grâce au lemme 10.1.7, il existe un monomorphisme $i d_{\mathcal{N}} \hookrightarrow K$.

Le corollaire A.1.2 (page 181) donne alors la conclusion.

Nous pouvons maintenant énoncer le résultat principal de cette section.

On rappelle que $\mathrm{Is}_{0}$ est une autre écriture pour $\mathbb{k}(0)$ (cf. notation 2.1.4).

Proposition 10.1.9. - Soient $X$ un foncteur analytique de $\mathcal{F}_{\mathcal{G} r}$ et $A$ un objet (quelconque) de $\mathcal{F}_{b i-\mathcal{G} r}$. Le morphisme gradué naturel $\operatorname{Ext}_{\mathcal{G} r}^{*}\left(X, \Omega_{\mathcal{G} r}\left(A \otimes \beta^{*}\left(\operatorname{Is}_{0}\right)\right)\right) \rightarrow$ $\operatorname{Ext}_{\mathcal{G} r}^{*}\left(X, \Omega_{\mathcal{G} r}(A)\right)$ induit par le monomorphisme canonique $A \otimes \beta^{*}\left(\operatorname{Is}_{0}\right) \hookrightarrow A$ fourni par $\mathrm{Is}_{0} \hookrightarrow \mathbb{k}$ (cf. lemme 2.3.4) est un isomorphisme.

Démonstration. - On écrit la suite exacte longue de cohomologie associée à la suite exacte courte $0 \rightarrow A \otimes \beta^{*}\left(\mathrm{Is}_{0}\right) \rightarrow A \rightarrow A \otimes \beta^{*}\left(\mathbb{k}^{\geq 1}\right) \rightarrow 0$ et l'on applique le lemme précédent à l'objet $A \otimes \beta^{*}\left(\mathbb{k}^{\geq 1}\right)$ de $\mathcal{N}$ (cf. lemme 10.1.7).

\subsection{Résultats fondamentaux}

Rappelons que le foncteur $\mathfrak{J}: \mathcal{F}_{\mathcal{G} r} \rightarrow \widetilde{\mathcal{F}}_{\mathcal{G} r}$, l'endofoncteur $\mathcal{I}$ de $\mathcal{F}_{\mathcal{G} r}$ et la transformation naturelle $j^{\omega}: \mathcal{I} \rightarrow \iota \omega$ ont été définis dans la section 6.1 .

Théorème 10.2.1. - Soient $X$ un objet analytique de $\mathcal{F}_{\mathcal{G} r}$ et $Y$ un objet quelconque de $\mathcal{F}_{\mathcal{G} r}$. Le morphisme gradué naturel $\left(j_{Y}^{\omega}\right)_{*}: \operatorname{Ext}_{\mathcal{G} r}^{*}(X, \mathcal{I}(Y)) \rightarrow \operatorname{Ext}_{\mathcal{G} r}^{*}(X, \iota \omega(Y))$ induit par $j_{Y}^{\omega}: \mathcal{I}(Y) \rightarrow \iota \omega(Y)$ est un isomorphisme.

En particulier, les adjonctions des propositions 5.1 .7 et 6.1 .3 fournissent des isomorphismes gradués naturels

$$
\operatorname{Ext}_{\mathcal{F}}^{*}(\omega(X), \omega(Y)) \simeq \operatorname{Ext}_{\mathcal{G} r}^{*}(X, \mathcal{I}(Y)) \simeq \operatorname{Ext}_{\widetilde{\mathcal{F}}_{\mathcal{G} r}}^{*}(\mathfrak{J}(X), \mathfrak{J}(Y)) .
$$

Démonstration. - Notons $\Upsilon_{\mathcal{G} r}^{\prime}: \mathcal{F}_{\mathcal{G} r} \rightarrow \mathcal{F}_{b i-\mathcal{G} r}$ la composée de $\Upsilon_{\mathcal{G} r}$ et du foncteur de précomposition par l'endofoncteur $\mathcal{E}_{b i-\mathcal{G} r}^{f}$ « échangeant les deux bases », i.e. donné par $(V, B, W) \rightarrow(V, W, B)$ sur les objets et sur les morphismes par l'égalité

$$
\operatorname{hom}_{\mathcal{E}_{b i-\mathcal{G} r}^{f}}\left((V, B, W),\left(V^{\prime}, B^{\prime}, W^{\prime}\right)\right)=\operatorname{hom}_{\mathcal{E}_{b i-\mathcal{G} r}^{f}}\left((V, W, B),\left(V^{\prime}, W^{\prime}, B^{\prime}\right)\right) .
$$

Autrement dit, on a $\Upsilon_{\mathcal{G} r}^{\prime}(X)(V, B, W)=X(V, W)$ (tandis que $\Upsilon_{\mathcal{G} r}(X)(V, B, W)=$ $X(V, B))$. 
Alors les endofoncteurs $\Omega_{\mathcal{G} r} \Upsilon_{\mathcal{G} r}^{\prime}$ et $\iota \omega$ de $\mathcal{F}_{\mathcal{G} r}$ coïncident, car il existe des isomorphismes canoniques

$$
\begin{aligned}
& \Omega_{\mathcal{G} r} \Upsilon_{\mathcal{G} r}^{\prime}(X)(V, B) \simeq \bigoplus_{W \in \mathcal{G} r(V)} \Upsilon_{\mathcal{G} r}^{\prime}(X)(V, B, W) \\
& \simeq \bigoplus_{W \in \mathcal{G} r(V)} X(V, W) \simeq \omega(X)(V) \simeq \iota \omega(X)(V, B) .
\end{aligned}
$$

De plus, il existe un isomorphisme $\mathcal{I}(Y) \simeq \Omega_{\mathcal{G} r} \Upsilon_{\mathcal{G}_{r}}^{\prime}\left(Y \otimes \beta^{*}\left(\mathrm{Is}_{0}\right)\right)$ naturel en $Y$, par lequel $j_{Y}^{\omega}$ s'identifie à la transformation naturelle induite par $Y \otimes \beta^{*}\left(\mathrm{Is}_{0}\right) \hookrightarrow Y$. Ainsi, $\left(j_{Y}^{\omega}\right)_{*}: \operatorname{Ext}_{\mathcal{G} r}^{*}(X, \mathcal{I}(Y)) \rightarrow \operatorname{Ext}_{\mathcal{G} r}^{*}(X, \iota \omega(Y))$ est un isomorphisme, par la proposition 10.1.9.

Les autres flèches considérées sont des isomorphismes pour des raisons formelles (corollaire A.1.1).

En utilisant l'adjonction entre foncteurs de restriction et de prolongement par zéro, on en déduit un résultat analogue dans les catégories $\mathcal{F}_{\mathcal{G} r, \leq n}$. Ce même principe est mis en œuvre dans le corollaire suivant.

Corollaire 10.2.2. - Soient $k$ et $n$ deux entiers naturels, $X$ un objet analytique de $\mathcal{F}_{\mathcal{G} r, k}$ et $Y$ un objet quelconque de $\mathcal{F}_{\mathcal{G} r, n}$.

(1) Si $k<n$, alors $\operatorname{Ext}_{\mathcal{F}}^{*}\left(\omega_{k}(X), \omega_{n}(Y)\right)=0$.

(2) Si $k=n$, alors le morphisme naturel $\operatorname{Ext}_{\mathcal{G} r, n}^{*}(X, Y) \rightarrow \operatorname{Ext}_{\mathcal{F}}^{*}\left(\omega_{n}(X), \omega_{n}(Y)\right)$ induit par $\omega_{n}$ est un isomorphisme.

On rappelle que les foncteurs de restriction $\mathcal{R}$ et de prolongement par zéro $\mathcal{P}$ qui interviennent dans la démonstration ci-dessous ont été définis dans la notation 5.1.1.

Démonstration. - Comme $\omega_{k}(X) \simeq \omega \mathcal{P}_{k, \mathbb{N}}(X)$, le théorème 10.2.1 fournit un isomorphisme naturel gradué entre $\operatorname{Ext}_{\mathcal{F}}^{*}\left(\omega_{k}(X), \omega_{n}(Y)\right)$ et $\operatorname{Ext}_{\mathcal{G} r}^{*}\left(\mathcal{P}_{k, \mathbb{N}}(X), \mathcal{I}_{\mathcal{P}_{n, \mathbb{N}}}(Y)\right)$. On remarque que $\mathcal{I}_{\mathcal{P}_{n, \mathbb{N}}}(Y)$ appartient à l'image de la catégorie $\mathcal{F}_{\mathcal{G} r, \geq n}$ dans $\mathcal{F}_{\mathcal{G} r}$ par le foncteur de prolongement par zéro. Comme $\mathcal{E}_{\mathcal{G} r, \geq n}^{f}$ est une sous-catégorie complète à gauche de $\mathcal{E}_{\mathcal{G}_{r}}^{f}$, la proposition C.6.2 (et le corollaire A.1.1) montrent que ce groupe d'extensions est canoniquement isomorphe à

$$
\operatorname{Ext}_{\mathcal{G} r, \geq n}^{*}\left(\mathcal{R}_{\mathbb{N}, \geq n} \mathcal{P}_{k, \mathbb{N}}(X), \mathcal{R}_{\mathbb{N}, \geq n} \mathcal{I} \mathcal{P}_{n, \mathbb{N}}(Y)\right) .
$$

Si $k<n, \mathcal{R}_{\mathbb{N}, \geq n} \mathcal{P}_{k, \mathbb{N}}(X)$ est nul, ce qui établit la première assertion. Si $k=n$, cet objet s'identifie à $\mathcal{P}_{n, \geq n}(X)$. Puisque $\mathcal{E}_{\mathcal{G} r, n}^{f}$ est une sous-catégorie complète à droite de $\mathcal{E}_{\mathcal{G} r, \geq n}^{f}$, la proposition C.6.2 montre cette fois que ce groupe d'extensions est canoniquement isomorphe à $\operatorname{Ext}_{\mathcal{G}_{r, n}}^{*}\left(X, \mathcal{R}_{\mathbb{N}, n} \mathcal{I} \mathcal{P}_{n, \mathbb{N}}(Y)\right)$. On conclut en constatant que le morphisme naturel $Y \rightarrow \mathcal{R}_{\mathbb{N}, n} \mathcal{I} \mathcal{P}_{n, \mathbb{N}}(Y)$ est un isomorphisme.

Remarque 10.2.3. - (1) Les premiers résultats d'annulation cohomologique dans des catégories de foncteurs remontent à Pirashvili. Ainsi, le lemme 0.4 de 
[FLS94] (dû à Pirashvili) s'est avéré l'un des premiers outils efficaces pour simplifier des calculs de groupes d'extensions dans $\mathcal{F}$.

(2) Le cas $k=0$ du corollaire 10.2.2 constitue une généralisation du résultat (énoncé d'ordinaire dans sa variante duale) dû à Franjou selon lequel $\operatorname{Ext}_{\mathcal{F}}^{*}(F, \bar{P})=0$ si $F$ est un foncteur fini de $\mathcal{F}$ (cf. [Pow98a], appendice, pour une démonstration due à Schwartz). Les lemmes techniques de [Pow98a] illustrent l'intérêt de ce genre de résultat cohomologique pour aborder la conjecture artinienne.

Gaudens et Schwartz ont établi dans [GS05] une généralisation du résultat de Franjou que le théorème 10.2.1 recoupe partiellement. 


\section{CHAPITRE 11}

\section{FONCTEUR $\omega$ ET FONCTEURS HOM INTERNES}

Le foncteur $\omega$ étant adjoint à gauche à $\iota$, on dispose pour des raisons formelles de propriétés de compatibilité entre les foncteurs de division et $\omega$ - cf. proposition 9.2.1. En revanche, l'adjoint à gauche à $\omega$ ne se décrivant pas aisément, il est plus délicat d'étudier le comportement mutuel des foncteurs hom internes et $\omega$. Nous utiliserons les foncteurs de décalage, qui sont à la fois des foncteurs hom internes et des foncteurs de division (dans $\mathcal{F}_{\mathcal{G} r}$ comme dans $\mathcal{F}$ ), pour contourner cette difficulté.

\subsection{Scindement de $\Delta_{V} \circ \omega$}

Par les propositions 9.2.1 et 5.1.14, il existe des isomorphismes naturels

$$
\Delta_{V} \omega(X) \simeq\left(\omega(X): \iota\left(I_{V}\right)\right) \simeq \bigoplus_{W \in \mathcal{G} r(V)} \omega\left(X: I_{(V, W)}^{\mathcal{G} r}\right)
$$

où l'on a utilisé l'identification de $\Delta_{V}$ à $\left(\cdot: I_{V}\right)$ (proposition 1.3.1) ; la proposition 9.2.3 décrit explicitement ce scindement, qui sous-tend nombre d'aspects de la structure des catégories $\mathcal{F}_{\mathcal{G} r}$ et $\mathcal{F}$ (cf. partie IV ou [Dja06d]).

Précisons l'effet des foncteurs de division $\left(\cdot: I_{(V, W)}^{\mathcal{G r}}\right)$ qui apparaissent relativement au niveau et aux coniveau (notions introduites dans la définition 5.5.4). On remarque que $\operatorname{niv}\left(X: I_{(V, W)}^{\mathcal{G} r}\right) \leq \operatorname{niv}(X)$ (autrement dit, les foncteurs $\left(\cdot: I_{(V, W)}^{\mathcal{G} r}\right)$ préservent les sous-catégories $\mathcal{F}_{\mathcal{G} r, \leq n}$ de $\left.\mathcal{F}_{\mathcal{G} r}\right)$, mais les foncteurs $\left(\cdot: I_{(V, W)}^{\mathcal{G} r}\right)$ peuvent abaisser strictement le coniveau (donc ils ne préservent pas les sous-catégories $\mathcal{F}_{\mathcal{G} r, n}$, en général). La proposition suivante, que l'on utilisera dans la section suivante, décrit la restriction à $\mathcal{F}_{\mathcal{G} r, 0} \simeq \mathcal{F}$ de $\left(X: I_{(V, W)}^{\mathcal{G} r}\right)$. Elle fait usage des foncteurs $\tau_{A}$ introduits à la fin du chapitre 9 (page 115) et résulte de la proposition 9.2.3 (page 113).

Proposition 11.1.1. - Il existe dans $\mathcal{F}$ un isomorphisme naturel $\mathcal{R}_{\mathbb{N}, 0}\left(X: I_{A}^{\mathcal{G} r}\right) \simeq$ $\tau_{A}(X)$ pour $X \in \mathrm{Ob} \mathcal{F}_{\mathcal{G} r}$ et $A \in \mathrm{Ob} \mathcal{E}_{\mathcal{G} r}^{f}$. 
Signalons enfin que, pour élémentaire qu'il soit, le scindement (11) jouera un rôle essentiel dans les arguments de la partie IV.

\subsection{Le morphisme $h_{X, F}^{*}: \omega\left(\mathbf{E x t}_{\mathcal{G} r}^{*}(\iota(F), X)\right) \rightarrow \mathbf{E x t}_{\mathcal{F}}^{*}(F, \omega(X))$}

Nous donnons d'abord la définition et quelques propriétés générales d'un morphisme gradué $h_{X, F}^{*}$ naturel en un objet $X$ de $\mathcal{F}_{\mathcal{G} r}$ et $F$ de $\mathcal{F}$. Notre objectif principal est d'établir que $h_{X, F}^{*}$ est un isomorphisme lorsque $F$ est localement fini.

Définition 11.2.1. - Soit $h_{X, F}^{0}: \omega\left(\operatorname{Hom}_{\mathcal{G}_{r}}(\iota(F), X)\right) \rightarrow \operatorname{Hom}_{\mathcal{F}}(F, \omega(X))$ le morphisme naturel en les objets $F$ de $\mathcal{F}$ et $X$ de $\mathcal{F}_{\mathcal{G} r}$ adjoint à

$$
\omega\left(\operatorname{Hom}_{\mathcal{G} r}(\iota(F), X)\right) \otimes F \stackrel{\simeq}{\longrightarrow} \omega\left(\operatorname{Hom}_{\mathcal{G} r}(\iota(F), X) \otimes \iota(F)\right) \stackrel{\omega\left(u_{F, X}\right)}{\longrightarrow} \omega(X),
$$

où la première flèche est l'isomorphisme de la dernière assertion de la proposition 5.1.7 et $u_{F, X}$ la coünité de l'adjonction.

Comme le foncteur $\mathbf{H o m}_{\mathcal{G} r}(\iota(F), \cdot)$ est exact lorsque $F$ est un objet projectif de $\mathcal{F}$ (par la proposition 5.3.4) et que le foncteur $\omega$ est exact, ce morphisme naturel s'étend en un morphisme naturel gradué

$$
h_{X, F}^{*}: \omega\left(\mathbf{E x t}_{\mathcal{G} r}^{*}(\iota(F), X)\right) \rightarrow \mathbf{E x t}_{\mathcal{F}}^{*}(F, \omega(X)) .
$$

Notation 11.2.2. - Soit $V$ un espace vectoriel de dimension finie. Dans ce paragraphe, nous noterons $\pi_{V}$ l'épimorphisme canonique $\iota\left(I_{V}\right) \rightarrow \kappa\left(I_{V}\right)$ de $\mathcal{F}_{\mathcal{G} r}$.

Remarque 11.2.3. - L'épimorphisme $\pi_{V}$ peut se lire comme la projection canonique

$$
\iota\left(I_{V}\right) \simeq \bigoplus_{W \in \mathcal{G} r(V)} I_{(V, W)}^{\mathcal{G} r} \rightarrow I_{(V, 0)}^{\mathcal{G} r} \simeq \kappa\left(I_{V}\right)
$$

(cf. propositions 5.1.14 et 5.1.12); il est donc scindé.

Lemme 11.2.4. - Pour tous objets $V$ de $\mathcal{E}^{f}$ et $X$ de $\mathcal{F}_{\mathcal{G} r}$, le diagramme

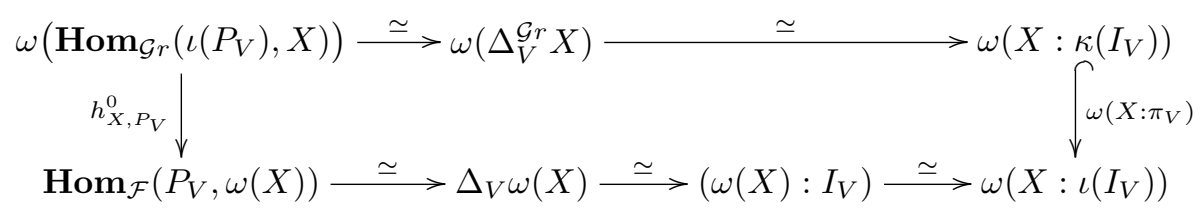

commute, où :

- les flèches supérieures sont les isomorphismes fournis par la proposition 5.3.4;

- les flèches inférieures sont les isomorphismes fournis par les propositions 1.3.1

et 9.2.1. 
Démonstration. - Le morphisme

$$
\omega\left(\Delta_{V}^{\mathcal{G} r} X\right) \simeq \omega\left(\operatorname{Hom}_{\mathcal{G} r}\left(\iota\left(P_{V}\right), X\right)\right) \stackrel{h_{X, P_{V}}^{0}}{\longrightarrow} \operatorname{Hom}_{\mathcal{F}}\left(P_{V}, \omega(X)\right) \simeq \Delta_{V} \omega(X)
$$

est l'adjoint du morphisme $P_{V} \otimes \omega\left(\Delta_{V}^{\mathcal{G} r} X\right) \rightarrow \omega(X)$ donné, sur l'espace vectoriel $A$, par $[f] \otimes a \mapsto X\left(i_{f}\right)(a)$ pour $f \in \operatorname{hom}_{\mathcal{E}}(V, A), B \in \mathcal{G} r(A)$ et $a \in X(A, B)$, où l'on désigne par $i_{f}:(V \oplus A, B) \rightarrow(A, B)$ le morphisme de composante $f$ sur $V$ et $i d_{A}$ sur $A$.

Par conséquent, la flèche

$$
\omega\left(\Delta_{V}^{\mathcal{G} r} X\right) \simeq \omega\left(\operatorname{Hom}_{\mathcal{G} r}\left(\iota\left(P_{V}\right), X\right)\right) \stackrel{h_{X, P_{V}}^{0}}{\longrightarrow} \operatorname{Hom}_{\mathcal{F}}\left(P_{V}, \omega(X)\right) \simeq \Delta_{V} \omega(X)
$$

est donnée sur l'espace $A$ par l'inclusion

$$
\omega\left(\Delta_{V}^{\mathcal{G} r} X\right)(A)=\bigoplus_{B \in \mathcal{G} r(A)} X(V \oplus A, B) \hookrightarrow \bigoplus_{B \in \mathcal{G} r(V \oplus A)} X(V \oplus A, B)=\Delta_{V} \omega(X)(A) .
$$

La conclusion s'obtient alors par la proposition 9.2.3.

Proposition 11.2.5. - Pour tous objets $F$ de $\mathcal{F}$ et $X$ de $\mathcal{F}_{\mathcal{G} r}$, le morphisme $h_{X, F}^{0}$ est injectif.

Démonstration. - Le lemme 11.2.4 montre l'assertion lorsque $F$ est un projectif standard $P_{V}$. On en déduit (par commutation de $\omega$ aux limites et de $\iota$ aux colimites) que $h_{X, P}^{0}$ est également injectif lorsque $P$ est une somme directe de projectifs standard. Le cas général s'en déduit en considérant un épimorphisme $p$ d'un tel projectif $P$ sur $F$ et en considérant le diagramme commutatif

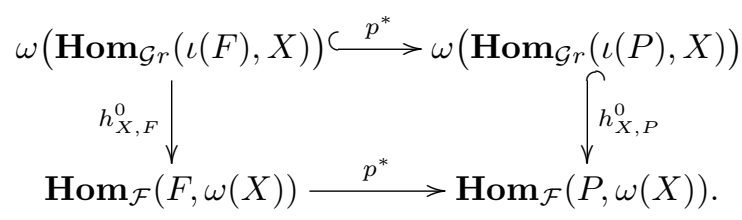

Remarque 11.2.6. - Il existe même un endofoncteur $T_{F}$ de $\mathcal{F}_{\mathcal{G} r}$, un isomorphisme naturel $\operatorname{Hom}_{\mathcal{F}}(F, \omega(X)) \simeq \omega\left(T_{F}(X)\right)$ et une transformation naturelle injective $j_{F}$ : $\operatorname{Hom}_{\mathcal{G} r}(\iota(F), \cdot) \hookrightarrow T_{F}$ telle que $h_{\cdot, F}^{0}$ s'identifie à $\omega\left(j_{F}\right)$. On le voit par un argument analogue, en considérant une présentation de $F$ par des sommes directes de projectifs standard. Ce qui suit précise ces considérations.

Description explicite du morphisme $h_{X, F}^{*} \cdot-$ Soit $V \in \mathrm{Ob}^{f}$. On a d'une part

$\omega\left(\operatorname{Ext}_{\mathcal{G} r}^{*}(\iota(F), X)\right)(V) \simeq \bigoplus_{W \in \mathcal{G} r(V)} \operatorname{Ext}_{\mathcal{G} r}^{*}(\iota(F), X)(V, W) \simeq \bigoplus_{W \in \mathcal{G} r(V)} \operatorname{Ext}_{\mathcal{F}}^{*}\left(F, \tau_{(V, W)}(X)\right)$

par la proposition 9.2.13. 
D'autre part,

$$
\begin{aligned}
\operatorname{Ext}_{\mathcal{F}}^{*}(F, \omega(X))(V) & \simeq \operatorname{Ext}_{\mathcal{F}}^{*}\left(F \otimes P_{V}, \omega(X)\right) \simeq \operatorname{Ext}_{\mathcal{F}}^{*}\left(F, \Delta_{V} \omega(X)\right) \\
& \simeq \bigoplus_{W \in \mathcal{G} r(V)} \operatorname{Ext}_{\mathcal{F}}^{*}\left(F, \omega\left(X: I_{(V, W)}^{\mathcal{G} r}\right)\right)
\end{aligned}
$$

grâce à l'isomorphisme (11) et à la proposition C.3.1.

Proposition 11.2.7. - Via les isomorphismes précédents et la proposition 9.2.3, le morphisme $h_{X, F}^{*}$ est induit par l'inclusion naturelle

$$
\tau_{(V, W)}(X)(E)=X(E \oplus V, W) \hookrightarrow \bigoplus_{\substack{A \in \mathcal{G} r(W) \\ B \in G r(E, A)}} X(E \oplus V, B)=\omega\left(X: I_{(V, W)}^{\mathcal{G} r}\right)(E)
$$

Démonstration. - Par naturalité, il suffit de vérifier cette identification lorsque $F$ est un projectif standard de $\mathcal{F}$, auquel cas elle résulte du lemme 11.2.4.

Les préliminaires précédents nous permettent, à l'aide du théorème fondamental du chapitre précédent, d'établir le résultat principal de cette section.

Proposition 11.2.8. - Si F est un objet localement fini de $\mathcal{F}$, le morphisme naturel gradué

$$
h_{X, F}^{*}: \omega\left(\mathbf{E x t}_{\mathcal{G} r}^{*}(\iota(F), X)\right) \rightarrow \mathbf{E x t}_{\mathcal{F}}^{*}(F, \omega(X))
$$

est un isomorphisme pour tout objet $X$ de $\mathcal{F}_{\mathcal{G} r}$.

Démonstration. - Notons $i_{E}^{X}(V, W)$ l'inclusion naturelle de la proposition 11.2.7 : l'énoncé équivaut à l'annulation des groupes d'extensions $\operatorname{Ext}_{\mathcal{F}}^{*}\left(F, \omega\left(\operatorname{coker}_{E}^{X}\right)\right)$ pour tout $E \in \mathrm{Ob} \mathcal{E}^{f}$. La proposition 11.1.1 montre que l'image par le foncteur de restriction $\mathcal{R}_{\mathbb{N}, 0}$ du foncteur coker $i_{E}^{X}$ de $\mathcal{F}_{\mathcal{G} r}$ est nulle. Le théorème 10.2.1 (ou son corollaire 10.2.2) donne alors la conclusion.

Dans les conséquences qui suivent, nous nous contentons du cas du degré 0 (i.e. des foncteurs hom internes), le plus significatif.

Corollaire 11.2.9. - Soient $F$ un objet localement fini de $\mathcal{F}, n$ et $k$ des entiers naturels et I une partie de $\mathbb{N}$ du type $\leq n$ ou $n$. Les foncteurs $\omega_{I} \circ \operatorname{Hom}_{\mathcal{G} r, I}\left(\iota_{I}(F), \cdot\right)$ et $\operatorname{Hom}_{\mathcal{F}}(F, \cdot) \circ \omega_{I}$ de $\mathcal{F}$ vers $\mathcal{F}_{\mathcal{G} r, I}$ sont isomorphes.

Démonstration. — Par la proposition 11.2.8, il existe un morphisme

$$
\operatorname{Hom}_{\mathcal{F}}(F, \cdot) \circ \omega_{I} \simeq \operatorname{Hom}_{\mathcal{F}}(F, \cdot) \circ \omega \circ \mathcal{P}_{I, \mathbb{N}} \simeq \omega \circ \operatorname{Hom}_{\mathcal{G} r}(\iota(F), \cdot) \circ \mathcal{P}_{I, \mathbb{N}} .
$$

Le corollaire s'en déduit parce que $\operatorname{Hom}_{\mathcal{G} r}(\iota(F), \cdot) \circ \mathcal{P}_{I, \mathbb{N}} \simeq \mathcal{P}_{I, \mathbb{N}} \circ \mathbf{H o m}_{\mathcal{G} r, I}\left(\iota_{I}(F), \cdot\right)$, par la proposition 9.1.3.

Nous terminons ce chapitre en donnant un corollaire des résultats précédents qui décrit notamment l'image par un foncteur $\operatorname{Hom}_{\mathcal{F}}(F, \cdot)$ (où $F \in \mathcal{F}_{\omega}$ ) d'un $\bar{G}(n)$ comodule simple (cf. propositions 5.1.7 et 5.5.10). 
Corollaire 11.2.10. - Soient $n$ et $k$ des entiers naturels, $F$ un objet localement fini de $\mathcal{F}, G$ un objet de $\mathcal{F}$ et $M$ un $\mathbb{k}\left[G L_{n}(\mathbb{k})\right]$-module fini. Il existe dans $\mathcal{F}$ un isomorphisme naturel

$$
\operatorname{Hom}_{\mathcal{F}}\left(F, \omega_{n}\left(\kappa_{n}(G) \otimes \rho_{n}(M)\right)\right) \simeq \omega_{n}\left(\kappa_{n}\left(\operatorname{Hom}_{\mathcal{F}}(F, G)\right) \otimes \rho_{n}(M)\right) .
$$

Démonstration. - Ce résultat s'obtient en combinant les corollaires 9.2.7, 9.2.12 et 11.2.9.

Retour sur la méthode de [Dja06c]. — L'article [Dja06c] étudie, dans le cas $\mathbb{k}=\mathbb{F}_{2}$, la structure de foncteurs de type fini ${ }^{(1)}$ de $\mathcal{F}\left(\mathbb{F}_{2}\right)$ (précisément, le produit tensoriel entre le projectif $P_{\mathbb{F}_{2}{ }^{2}}$ et une puissance extérieure) par un argument de récurrence utilisant le foncteur $\operatorname{Hom}_{\mathcal{F}\left(\mathbb{F}_{2}\right)}\left(\Lambda^{1}, \cdot\right)$, où $\Lambda^{1}$ désigne le foncteur d'inclusion $\mathcal{E}^{f} \hookrightarrow \mathcal{E}$.

Le corollaire 11.2.10 montre, en particulier, que les $\bar{G}(n)$-comodules finis sont nilpotents pour le foncteur $\operatorname{Hom}_{\mathcal{F}\left(\mathbb{F}_{2}\right)}\left(\Lambda^{1}, \cdot\right)$ (cf. proposition 12.3.1 ci-après). Cet énoncé sous-tend toute la démarche de $[\mathbf{D j a 0 6 c}]$; cependant, sans le formalisme des catégories de foncteurs en grassmanniennes, il est malaisé d'en donner une forme et une démonstration générales. La proposition 5.29 de [Dja06c], qui fournit une estimation de l'image par le foncteur $\operatorname{Hom}_{\mathcal{F}\left(\mathbb{F}_{2}\right)}\left(\Lambda^{1}, \cdot\right)$ des foncteurs étudiés dans l'article en question, sert de succédané élémentaire au corollaire 11.2.10. Dans le dernier chapitre de [Dja06d], nous étendons la méthode de [Dja06c] en employant le corollaire 11.2.10 de façon beaucoup plus générale.

\footnotetext{
(1) En fait, cet article travaille sur les objets duaux, de co-type fini. Nous avons traduit, pour la cohérence de l'exposition, ses énoncés en termes de foncteurs de type fini.
} 



\section{CHAPITRE 12}

\section{LA FILTRATION DE KRULL DE LA CATÉGORIE $\mathcal{F}$}

La conjecture que nous introduisons dans ce chapitre, appelée conjecture artinienne extrêmement forte (elle implique la conjecture artinienne très forte 2.3 .24 ), affirme que la restriction aux objets localement finis du foncteur d'intégrale en grassmanniennes $\omega: \mathcal{F}_{\mathcal{G} r} \rightarrow \mathcal{F}$ envoie la filtration de $\mathcal{F}_{\mathcal{G} r}^{l f}$ donnée par les sous-catégories $\mathcal{F}_{\mathcal{G} r, \leq n}^{l f}$ dans la filtration de Krull de $\mathcal{F}$, et induit une équivalence entre les quotients associés. Après des préliminaires présentés dans la section 12.1, nous exposons les différentes formes de la conjecture artinienne extrêmement forte et les cas particuliers qu'on peut en établir (section 12.2) puis en examinons des conséquences importantes (section 12.3).

\subsection{Foncteurs induits}

Nous nous intéresserons à la propriété suivante d'une sous-catégorie pleine $\mathcal{A}$ de $\mathcal{F}$ :

Hypothèse 12.1.1. - Pour toute suite exacte courte $0 \rightarrow A \rightarrow B \rightarrow C \rightarrow 0$ de $\mathcal{F}$, si deux des objets $A, B, C$ appartiennent à $\mathcal{A}$, il en est de même du troisième.

Conjecturalement, les foncteurs induits de hauteur au plus $n$ introduits ci-après sont exactement les foncteurs noethériens de type $n$ de $\mathcal{F}$. L'étude de ceux-là se ramène essentiellement à celle d'objets finis de $\mathcal{F}_{\mathcal{G} r}$, ce qui les rend assez facilement maniables.

Définition 12.1.2. - Soit $n \in \mathbb{Z}$. Nous noterons $\mathcal{F}^{\omega-\operatorname{cons}(n)}$ la plus petite souscatégorie pleine de $\mathcal{F}$ vérifiant 12.1 .1 et contenant l'image de la restriction à la souscatégorie $\mathcal{F}_{\mathcal{G} r, \leq n}^{f}$ des objets finis de $\mathcal{F}_{\mathcal{G} r, \leq n}$ du foncteur $\omega_{\leq n}: \mathcal{F}_{\mathcal{G} r, \leq n} \rightarrow \mathcal{F}$.

Nous dirons que $F$ est induit de hauteur au plus $n$ s'il est objet de $\mathcal{F}^{\omega-\operatorname{cons}(n)}$.

Remarque 12.1.3. - On prendra garde que, dans les versions antérieures de ce travail ([Dja06d], [Dja06a] et $[\mathbf{D j a 0 6 b}])$, les foncteurs induits étaient appelés foncteurs oméga-adaptés, et la sous-catégorie $\mathcal{F}^{\omega-\operatorname{cons}(n)}$ notée $\mathcal{F}^{\omega-a d(n)}$. La notation 
$\mathcal{F}^{\omega-\operatorname{cons}(n)}$ provient de ce que les foncteurs induits sont ceux que l'on peut construire à partir du foncteur $\omega$ et de l'hypothèse 12.1.1.

Proposition 12.1.4. - (1) Le produit tensoriel d'un objet de $\mathcal{F}^{\omega-\operatorname{cons}(n)}$ et d'un objet de $\mathcal{F}^{\omega-\operatorname{cons}(m)}$ est un objet de $\mathcal{F}^{\omega-\operatorname{cons}(n+m)}$.

(2) Les sous-catégories $\mathcal{F}^{\omega-\operatorname{cons}(n)}$ sont stables par le foncteur différence de $\mathcal{F}$.

(3) Tout foncteur induit est $p f_{\infty}$.

Démonstration. - Si $X$ est un objet fini de $\mathcal{F}_{\mathcal{G} r, \leq k}$, alors le produit tensoriel de $\omega_{\leq k}(X)$ et d'un objet induit de hauteur au plus $n$ est induit de hauteur au plus $n+k$. En effet, la sous-catégorie pleine des objets de $\mathcal{F}$ dont le produit tensoriel par $\omega_{\leq k}(X)$ est induit de hauteur au plus $n+k$ contient les $\omega_{\leq n}(A)$, pour $A \in \operatorname{Ob} \mathcal{F}_{\mathcal{G} r, \leq n}^{f}$, par la proposition 5.2.11, et vérifie l'hypothèse 12.1.1. On démontre ensuite par le même raisonnement l'assertion 1.

La proposition 5.1.14 montre, par exactitude de $\omega$, que $\omega$ transforme un objet $\mathrm{pf}_{\infty}$, donc en particulier un objet fini (cf. corollaire 5.5.8), en un objet $\mathrm{pf}_{\infty}$. Comme la sous-catégorie pleine des objets $\mathrm{pf}_{\infty}$ de $\mathcal{F}$ vérifie l'hypothèse 12.1 .1 , par la proposition B.1.6, on en déduit l'assertion 3.

Pour l'assertion 2, on considère la sous-catégorie pleine $\mathcal{C}_{n}$ des objets $F$ de $\mathcal{F}$ tels que $\Delta F$ appartient à $\mathcal{F}^{\omega-\operatorname{cons}(n)}$. Les propositions 9.2.1 et 9.2.3 montrent que $\mathcal{C}_{n}$ contient $\omega_{\leq n}(X)$ pour $X \in \mathrm{Ob} \mathcal{F}_{\mathcal{G} r, \leq n}^{f}$. D'autre part, l'exactitude du foncteur différence montre que $\mathcal{C}_{n}$ vérifie l'hypothèse 12.1 .1 , ce qui achève la démonstration.

Exemple 12.1.5. - Les projectifs standard $P_{V}=\omega\left(P_{(V, V)}^{\mathcal{G} r}\right)=\omega\left(\rho\left(P_{V}^{s u r j}\right)\right)$ de $\mathcal{F}$ sont induits, de hauteur $\operatorname{dim} V$ (le fait que cette hauteur n'est pas strictement inférieure à $\operatorname{dim} V$ découle de la proposition 12.1.6 ci-dessous).

Nous utiliserons, dans cette section, le théorème 10.2.1 par l'intermédiaire du résultat d'annulation cohomologique suivant.

Proposition 12.1.6. - Soient $n \in \mathbb{N}, X$ un objet de $\mathcal{F}_{\mathcal{G} r, \geq n}$ et $F$ un objet de $\mathcal{F}^{\omega-\operatorname{cons}(n-1)}$. On a $\operatorname{Ext}_{\mathcal{F}}^{*}\left(F, \omega_{\geq n}(X)\right)=0$.

Démonstration. - Cette propriété s'obtient à partir du corollaire 10.2.2 et de l'observation que la sous-catégorie pleine de $\mathcal{F}$ formée $\operatorname{des}$ objets $F$ tels que $\operatorname{Ext}_{\mathcal{F}}^{*}\left(F, \omega_{\geq n}(X)\right)=0$ vérifie l'hypothèse 12.1.1.

\subsection{La conjecture artinienne extrêmement forte}

Nous introduisons la conjecture artinienne extrêmement forte sous une forme globale utilisant la notion de foncteur induit.

Conjecture 12.2.1 (Conjecture artinienne extrêmement forte) 
Pour tout $n \in \mathbb{N}$, un quotient d'un foncteur induit de hauteur au plus $n$ est induit de hauteur au plus $n$.

Remarque 12.2.2. - Cet énoncé implique déjà la conjecture artinienne sous sa forme minimale en raison de l'assertion 3 de la proposition 12.1.4.

La proposition suivante lie la conjecture artinienne extrêmement forte à la filtration de Krull de $\mathcal{F}$.

Proposition 12.2.3. - Supposons la conjecture 12.2.1 vérifiée.

(1) Pour tout $n \in \mathbb{N}$, la sous-catégorie $\mathcal{F}^{\omega-\operatorname{cons}(n)}$ de $\mathcal{F}$ est épaisse. Elle est égale à la sous-catégorie $\mathcal{F}_{\mathbf{N T}(n)}$ des objets noethériens de type $n$ de $\mathcal{F}$.

(2) De plus, le foncteur $\omega_{n}: \mathcal{F}_{\mathcal{G} r, n} \rightarrow \mathcal{F}$ induit une équivalence de catégories entre $\mathcal{F}_{\mathcal{G} r, n}^{f}$ et $\mathcal{F}_{\mathbf{N T}(n)} / \mathcal{F}_{\mathbf{N T}(n-1)}$.

(3) Désignons par $\overline{\mathcal{F}^{\omega-c o n s(n)}}$ la plus petite sous-catégorie épaisse de $\mathcal{F}$ stable par colimites contenant $\mathcal{F}^{\omega-\operatorname{cons}(n)}$. C'est aussi la catégorie des foncteurs qui sont colimite de leurs sous-foncteurs noethériens de type $n$. Alors la filtration de Krull de $\mathcal{F}$ est donnée par $\mathcal{K}_{n}(\mathcal{F})=\overline{\mathcal{F} \omega-\operatorname{cons}(n)}$, et le foncteur $\omega_{n}$ induit une équivalence de catégories entre $\mathcal{F}_{\mathcal{G} r, n}^{\text {lf }}$ et $\mathcal{K}_{n}(\mathcal{F}) / \mathcal{K}_{n-1}(\mathcal{F})$.

Démonstration. - Les sous-catégories $\mathcal{F}^{\omega-\operatorname{cons}(n)}$ de $\mathcal{F}$ sont par hypothèse stables par quotients et elles vérifient 12.1.1, elles sont donc épaisses. La description de $\overline{\mathcal{F}^{\omega-\operatorname{cons}(n)}}$ comme sous-catégorie pleine des objets colimite de leurs sous-objets induits de hauteur au plus $n$ vient de ce que les foncteurs induits sont de présentation finie (assertion 3 de la proposition 12.1.4) et de la proposition B.1.11.

On note ensuite que $\mathcal{F}^{\omega-\operatorname{cons}(n)}$ est l'image réciproque par le foncteur canonique $\mathcal{F} \rightarrow \mathcal{F} / \mathcal{F}^{\omega-\operatorname{cons}(n-1)}$ de l'image, notée $\mathcal{C}_{n}$, du foncteur $\mathcal{F}_{\mathcal{G} r, n}^{f} \rightarrow \mathcal{F} / \mathcal{F}^{\omega-\operatorname{cons}(n-1)}$ induit par $\omega_{n}$. En effet, la sous-catégorie des objets de $\mathcal{F}$ dont l'image dans $\mathcal{F} / \mathcal{F}^{\omega-\operatorname{cons}(n-1)}$ appartient à $\mathcal{C}_{n}$ vérifie 12.1 .1 et contient bien les $\omega_{i}(X)$ pour $i \leq n$ et $X \in \mathrm{Ob} \mathcal{F}_{\mathcal{G} r, i}^{f}$, donc elle contient $\mathcal{F}^{\omega-\operatorname{cons}(n)}$. Réciproquement, si $F$ est un objet de $\mathcal{F}$ isomorphe à $\omega_{n}(X)$ dans $\mathcal{F} / \mathcal{F}^{\omega-\operatorname{cons}(n-1)}$, avec $X \in \mathrm{Ob} \mathcal{F}_{\mathcal{G} r, n}^{f}$, la proposition 12.1.6 prouve $^{(1)}$ qu'il existe dans $\mathcal{F}$ un morphisme $F \rightarrow \omega_{n}(X)$ dont le noyau et le conoyau appartiennent à $\mathcal{F}^{\omega-\operatorname{cons}(n-1)}$, ce qui entraîne que $F$ appartient à $\mathcal{F}^{\omega-\operatorname{cons}(n)}$.

On établit les autres résultats par récurrence sur l'entier $n$. Ils sont clairs pour $n=0$. On suppose donc $n>0$ et la proposition vérifiée au rang $n-1$.

Le foncteur $\omega_{n}$ induit une équivalence entre $\mathcal{F}_{\mathcal{G}_{r, n}}^{f}$ et la sous-catégorie épaisse $\mathcal{F}^{\omega-\operatorname{cons}(n)} / \mathcal{F}^{\omega-\operatorname{cons}(n-1)}$ de $\mathcal{F} / \mathcal{F}^{\omega-\operatorname{cons}(n-1)}$, et aussi de $\mathcal{F}_{\mathcal{G} r, n}^{l f}$ vers la sous-catégorie épaisse $\overline{\mathcal{F}^{\omega-\operatorname{cons}(n)}} / \overline{\mathcal{F}^{\omega-\operatorname{cons}(n-1)}}$ de $\mathcal{F} / \overline{\mathcal{F} \omega-\operatorname{cons}(n-1)}$. Cela montre en particulier, compte-tenu de l'hypothèse de récurrence, que ces images sont respectivement incluses dans $\mathcal{F}_{\mathbf{N T}(n)} / \mathcal{F}_{\mathbf{N T}(n-1)}$ et $\mathcal{K}_{n}(\mathcal{F}) / \mathcal{K}_{n-1}(\mathcal{F})$.

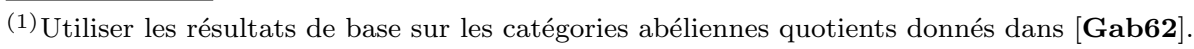


Si $X$ est un objet fini non nul de $\mathcal{F}_{\mathcal{G} r, k}$, avec $k>n$, alors $\omega_{k}(X)$ est limite de ses quotients appartenant à $\mathcal{F}^{\omega-\operatorname{cons}(n)}$ et n'y appartient pas lui-même, par la proposition 12.1.6. On en déduit que son image dans $\mathcal{F} / \mathcal{F}^{\omega-\operatorname{cons}(n-1)}$ est infinie, donc que $\omega_{k}(X)$ n'est pas noethérien de type $n$, puisque $\mathcal{F}^{\omega-\operatorname{cons}(n-1)}=\mathcal{F}_{\mathbf{N T}(n-1)}$ par l'hypothèse de récurrence. Cela entraine qu'il n'y a pas, dans $\mathcal{F}$, d'objet noethérien de type $n$ qui ne soit pas induit de hauteur au plus $n$. On a donc $\mathcal{F}_{\mathbf{N T}(n)}=\mathcal{F}^{\omega-\operatorname{cons}(n)}$.

Comme la catégorie $\mathcal{F}$ est localement noethérienne, par la remarque 12.2.2, la proposition B.4.5 donne $\mathcal{K}_{n}(\mathcal{F})=\overline{\mathcal{F}_{\mathrm{NT}(n)}}=\overline{\mathcal{F} \omega-\operatorname{cons}(n)}$, d'où la proposition.

La proposition suivante, variante tronquée de la proposition 12.2 .3 , se démontre de la même façon.

Proposition 12.2.4. - Soit $n \in \mathbb{N}$ tel que les sous-catégories $\mathcal{F}^{\omega-\operatorname{cons}(i)}$ de $\mathcal{F}$ sont stables par quotient pour $i \leq n$. Alors le foncteur $\omega_{n}$ induit une équivalence entre la sous-catégorie pleine $\mathcal{F}_{\mathcal{G} r, n}^{f}$ des objets finis de $\mathcal{F}_{\mathcal{G} r, n}$ et la catégorie quotient $\mathcal{F}^{\omega-\operatorname{cons}(n)} / \mathcal{F}^{\omega-\operatorname{cons}(n-1)}$. De plus, si $X$ est un objet de $\mathcal{F}_{\mathcal{G} r, n}^{f}$, alors $\omega_{n}(X)$ est un objet noethérien de type $n$ de $\mathcal{F}$.

Les deux énoncés qui suivent constituent les versions « locales » de la conjecture artinienne extrêmement forte.

Conjecture 12.2.5. - Soit $n \in \mathbb{N}$. Le foncteur exact $\mathcal{F}_{\mathcal{G} r, n} \stackrel{\omega_{n}}{\longrightarrow} \mathcal{F} \rightarrow \mathcal{F} / \mathcal{K}_{n-1}(\mathcal{F})$ induit un isomorphisme $G_{0}^{f}\left(\mathcal{F}_{\mathcal{G} r, n}\right) \stackrel{\simeq}{\longrightarrow} G_{0}^{f}\left(\mathcal{F} / \mathcal{K}_{n-1}(\mathcal{F})\right)$ qui préserve les classes des objets simples.

Conjecture 12.2.6. - Soit $n \in \mathbb{N}$. Pour tout objet simple $S$ de $\mathcal{F}_{\mathcal{G} r, n}$, le foncteur $\omega_{n}(S)$ de $\mathcal{F}$ est simple noethérien de type $n$. De plus, un foncteur de $\mathcal{F}$ est simple noethérien de type $n$ si et seulement s'il est isomorphe à un quotient non nul d'un tel objet.

La proposition 12.2.3 fournit l'équivalence entre les différentes conjectures de cette section.

Corollaire 12.2.7. - Les conjectures 12.2.1, 12.2.5 et 12.2.6 sont équivalentes.

Démonstration. - La proposition 12.2.3 montre que la conjecture 12.2.1 implique la conjecture 12.2.6.

La proposition B.4.5 (page 190) montre que la conjecture 12.2.6 entraîne la conjecture 12.2.5.

Supposons maintenant la conjecture 12.2.5 satisfaite. Alors tout foncteur induit de hauteur au plus $n$ appartient à $\mathcal{K}_{n}(\mathcal{F})$. On montre par récurrence sur $n \in \mathbb{N}$ que la sous-catégorie $\mathcal{F}^{\omega-\operatorname{cons}(n)}$ de $\mathcal{F}$ est épaisse et que les objets de $\mathcal{K}_{n}(\mathcal{F})$ sont colimite de sous-objets induits de hauteur au plus $n$. Si cette assertion est vérifiée, tout quotient strict d'un foncteur du type $\omega_{n+1}(S)$, où $S \in \mathrm{Ob} \mathcal{F}_{\mathcal{G} r, n+1}$ est simple, est induit de hauteur au plus $n$ : un tel quotient appartient à $\mathcal{K}_{n}(\mathcal{F})$ (parce que la conjecture 12.2.5 
est satisfaite), et est de type fini. En particulier, tous les quotients de $\omega_{n+1}(S)$ sont dans $\mathcal{F}^{\omega-\operatorname{cons}(n+1)}$. On en déduit que la sous-catégorie $\mathcal{F}^{\omega-\operatorname{cons}(n+1)}$ de $\mathcal{F}$ est épaisse. Son image dans le quotient $\mathcal{F} / \mathcal{K}_{n}(\mathcal{F})$ contient les objets simples de cette catégorie (car la conjecture 12.2.5 est satisfaite); elle est épaisse. Le fait que les foncteurs induits sont de présentation finie implique, par la proposition B.1.11, que la sous-catégorie des foncteurs de $\mathcal{F}$ qui sont colimite de leurs sous-foncteurs induits de hauteur au plus $n+1$ est aussi épaisse. On en déduit que cette sous-catégorie coïncide avec $\mathcal{K}_{n+1}(\mathcal{F})$, par définition de la filtration de Krull. Cela établit la conjecture 12.2.1 et achève la démonstration.

En considérant les objets simples pseudo-constants des $\mathcal{F}_{\mathcal{G} r, n}$ dans la conjecture 12.2.6, on obtient le résultat suivant.

Corollaire 12.2.8. — La conjecture artinienne extrêmement forte implique la conjecture artinienne très forte 2.3.24.

Dans le chapitre 16, nous établirons des formes partielles de la conjecture artinienne extrêmement forte et donnerons une conséquence frappante de cette conjecture en termes de groupes de Grothendieck.

\subsection{Conséquences de la conjecture artinienne extrêmement forte}

Nous donnons des propriétés des foncteurs induits qui montrent que la conjecture artinienne extrêmement forte implique des conjectures profondes sur la structure de la catégorie $\mathcal{F}$ que les formes plus faibles de la conjecture artinienne ne suffisent pas à résoudre.

Proposition 12.3.1. - Soient $k \in \mathbb{N}$ et $F$ un foncteur induit de $\mathcal{F}$. Il existe $N \in \mathbb{N}$ tel que pour tout $n \geq N$ et tout foncteur analytique $A$ de $\mathcal{F}$ tel que $A(0)=0$, on ait $\mathbf{E x t}_{\mathcal{F}}^{*}\left(A^{\otimes n}, F\right)=0$.

Démonstration. - Comme la sous-catégorie pleine des foncteurs $F$ de $\mathcal{F}$ tels que $\mathbf{E x t}^{*}\left(A^{\otimes n}, F\right)=0$ pour $n$ assez grand vérifie l'hypothèse 12.1.1, il suffit de démontrer l'assertion lorsque $F=\omega(X)$, où $X$ est un objet fini de $\mathcal{F}_{\mathcal{G} r}$.

Comme $A(0)=0$, le foncteur $A$ admet une résolution projective dont les termes sont du type $\bar{P}_{\mathbb{k}} \otimes B_{i}$, donc $A^{\otimes n}$ admet une résolution projective dont les termes sont du type $\bar{P}_{\mathbb{k}}^{\otimes n} \otimes C_{i}$. Pour tout entier $n>\operatorname{deg}(X)$ et tout $E \in \operatorname{Ob} \mathcal{E}_{\mathcal{G} r}^{f}$,

$$
\operatorname{hom}_{\mathcal{G} r}\left(\iota\left(\bar{P}_{\mathbb{k}}^{\otimes n} \otimes C_{i}\right) \otimes P_{E}^{\mathcal{G} r}, X\right) \simeq \operatorname{hom}_{\mathcal{G} r}\left(\iota\left(C_{i}\right) \otimes P_{E}^{\mathcal{G} r},\left(\Delta^{\mathcal{G} r}\right)^{n} X\right)=0
$$

(par le corollaire 5.3.5), donc $\operatorname{Ext}_{\mathcal{G} r}^{*}\left(\iota\left(A^{\otimes n}\right) \otimes P_{E}^{\mathcal{G} r}, X\right)=0$, puisque $\left(\iota\left(\bar{P}_{\mathbb{k}}^{\otimes n} \otimes C_{i}\right) \otimes\right.$ $\left.P_{E}^{\mathcal{G} r}\right)_{i}$ est une résolution projective de $\iota\left(A^{\otimes n}\right) \otimes P_{E}^{\mathcal{G} r}$. L'isomorphisme (19) de la proposition C.3.1 fournit alors $\mathbf{E x t}_{\mathcal{G} r}^{*}\left(\iota\left(A^{\otimes n}\right), X\right)=0$ pour $n>\operatorname{deg}(X)$.

La conclusion résulte donc de la proposition 11.2.8. 
Remarque 12.3.2. - (1) L'argument d'annulation cohomologique utilisé dans cette démonstration, analogue à la proposition 1.5.1 de [Fra96], est dû originellement à Pirashvili (cf. remarque 10.2.3.1).

(2) Cette proposition est surtout significative en degré 0, i.e. pour les foncteurs hom internes. En particulier, elle illustre le comportement radicalement différent du foncteur différence $\Delta \simeq \operatorname{Hom}_{\mathcal{F}}\left(\bar{P}_{\mathbb{k}}, \cdot\right)$ et de $\operatorname{Hom}_{\mathcal{F}}(F, \cdot)$ pour $F$ fini.

(3) Pour $\mathbb{k}=\mathbb{F}_{2}$, le cas le plus intéressant est celui où $A=\Lambda^{1}$ (cf. [Dja06c]).

L'énoncé suivant est à comparer à la forme de la conjecture artinienne donnée par l'assertion 9 de la proposition 1.2.1 (page 4).

Proposition 12.3.3. - Si $F$ est un foncteur induit de $\mathcal{F}$, pour tout $i \in \mathbb{N}$, l'ensemble des classes d'isomorphisme d'objets simples $S$ de $\mathcal{F}$ tels que $\operatorname{Ext}_{\mathcal{F}}^{i}(S, F) \neq 0$ est fini.

Démonstration. - Il suffit de montrer la propriété pour $F$ de la forme $\omega_{n}(X)$, où $n \in \mathbb{N}$ et $X \in \operatorname{Ob} \mathcal{F}_{\mathcal{G} r, n}^{f}$. Pour $n=0$, cela vient de ce que les objets finis de $\mathcal{F}$ sont co-pf $\infty_{\infty}$ (cf. [Dja06d] pour les détails); pour $n>0$, tous les groupes $\operatorname{Ext}_{\mathcal{F}}^{i}\left(S, \omega_{n}(X)\right)$ sont nuls par le corollaire 10.2.2, d'où la proposition. 


\section{CHAPITRE 13}

\section{RÉSULTATS D'ANNULATION COHOMOLOGIQUE DANS $\mathcal{F}_{i n j}$}

La comparaison entre les groupes d'extensions dans les catégories $\mathcal{F}$ et des modules sur un groupe linéaire s'opère naturellement par l'intermédiaire de la catégorie $\mathcal{F}_{i n j}$ (ou $\mathcal{F}_{\text {surj }}$ ); on peut l'illustrer par le diagramme commutatif

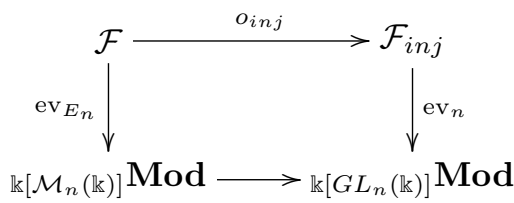

dans lequel la flèche inférieure est le foncteur de restriction : il s'agit d'étudier le comportement cohomologique du foncteur composé $\mathcal{F} \rightarrow{ }_{k}\left[G L_{n}(\mathbb{k})\right]$ Mod de ce diagramme.

Ce principe est implicite dans la démonstration de Suslin, donnée dans l'appendice de [FFSS99], du théorème selon lequel ce foncteur induit un isomorphisme entre les groupes d'extensions entre deux foncteurs finis de la catégorie $\mathcal{F}$, pourvu que $n$ soit assez grand.

Le résultat principal de ce chapitre constitue une généralisation de ce théorème. Il est démontré dans la section 13.2, à partir d'une propriété d'annulation cohomologique déduite du théorème 10.2.1 relatif au foncteur $\omega: \mathcal{F}_{\mathcal{G} r} \rightarrow \mathcal{F}$ et de la proposition 6.2.4 relative à l'auto-dualité du foncteur $\widetilde{\omega}$.

La première section établit, de façon directe, une autre propriété d'annulation cohomologique du foncteur d'oubli $o_{i n j}: \mathcal{F} \rightarrow \mathcal{F}_{\text {inj }}$. Dans la section 13.3, nous formulons une conjecture sur la filtration de Krull de la catégorie $\mathcal{F}_{i n j}$ analogue à la conjecture artinienne extrêmement forte. Nous en démontrons un cas particulier à l'aide des résultats des deux sections précédentes. 


\subsection{Une propriété élémentaire}

Le résultat que nous établissons dans cette section (proposition 13.1.3) repose uniquement sur les résultats du chapitre 2. Pour les appliquer plus commodément, pour traitons d'abord de la catégorie $\mathcal{F}_{\text {surj }}$.

Lemme 13.1.1. - Il existe dans $\mathcal{F}_{\text {surj }}$ un épimorphisme scindé $o(F) \widetilde{\otimes} P_{V}^{\text {surj }} \rightarrow$ $o(F)$ naturel en les objets $V$ de $\mathcal{E}_{\text {surj }}^{f}$ et $F$ de $\mathcal{F}$.

Démonstration. - On définit un morphisme naturel $o(F) \widetilde{\otimes} P_{V}^{\text {surj }} \rightarrow o(F)$ comme l'adjoint de la projection canonique

$$
\varpi\left(o(F) \widetilde{\otimes} P_{V}^{s u r j}\right) \simeq \varpi o(F) \otimes \varpi\left(P_{V}^{s u r j}\right) \simeq \varpi o(F) \otimes P_{V} \rightarrow \varpi o(F) \rightarrow F
$$

déduit de l'épimorphisme $P_{V} \rightarrow \mathbb{k}$ et de la coünité de l'adjonction, via la proposition 2.3.6.

D'autre part, l'épimorphisme $V \rightarrow 0$ induit un morphisme $\mathrm{Is}_{0} \simeq P_{0}^{s u r j} \rightarrow P_{V}^{s u r j}$ dans $\mathcal{F}_{\text {surj }}$, d'où un morphisme naturel $o(F) \simeq o(F) \widetilde{\otimes} \mathrm{Is}_{0} \rightarrow o(F) \widetilde{\otimes} P_{V}^{s u r j}$.

On vérifie aussitôt que le morphisme $o(F) \rightarrow o(F) \widetilde{\otimes} P_{V}^{s u r j} \rightarrow o(F)$ composé des deux flèches précédentes est l'identité.

Proposition 13.1.2. - Soient $X$ un objet fini de $\mathcal{F}_{\text {surj }}$ et $F$ un objet de $\mathcal{F}$. On a

$$
\operatorname{Ext}_{\mathcal{F}_{\text {surj }}}^{*}(o(F), X)=0 .
$$

Démonstration. — Par la proposition 2.2.7, il existe un espace vectoriel de dimension finie $V$ tel que $\delta_{V}(X)=0$. La proposition 2.2.3 et le corollaire A.1.1 montrent alors que $\operatorname{Ext}_{\mathcal{F}_{\text {surj }}}^{*}\left(o(F) \widetilde{\otimes} P_{V}^{s u r j}, X\right) \simeq \operatorname{Ext}_{\mathcal{F}_{\text {surj }}}^{*}\left(o(F), \delta_{V}(X)\right)=0$. Mais $\operatorname{Ext}_{\mathcal{F}_{\text {surj }}}^{*}(o(F), X)$ est facteur direct de $\operatorname{Ext}_{\mathcal{F}_{\text {surj }}}^{*}\left(o(F) \widetilde{\otimes} P_{V}^{\text {surj }}, X\right)$ par le lemme 13.1.1, d'où la proposition.

Revenons maintenant à la catégorie $\mathcal{F}_{i n j}$, plus naturelle pour les considérations suivantes.

Proposition 13.1.3. - Soient $X$ un objet localement fini de $\mathcal{F}_{i n j}$ et $F$ un objet de $\mathcal{F}$. On a

$$
\operatorname{Ext}_{\mathcal{F}_{\text {inj }}}^{*}\left(X, o_{i n j}(F)\right)=0 .
$$

Démonstration. - Le cas où $X$ est fini est dual du précédent. Le cas général s'en déduit par passage à la colimite.

\subsection{Propriétés utilisant le foncteur $\omega$}

Nous présentons maintenant d'autres résultats d'annulation cohomologique dans la catégorie $\mathcal{F}_{i n j}$, reposant sur le théorème 10.2 .1 et la proposition suivante.

Proposition 13.2.1. - Les endofoncteurs $\varpi_{i n j} o_{i n j}$ et $\omega \kappa$ de $\mathcal{F}$ sont isomorphes. 
Démonstration. - Soit $\widetilde{\kappa}: \mathcal{F} \rightarrow \widetilde{\mathcal{F}}_{\mathcal{G} r}$ le foncteur de précomposition par le foncteur de réduction $\widetilde{\mathfrak{K}}: \widetilde{\mathcal{E}}_{\mathcal{G} r}^{f} \rightarrow \mathcal{E}^{f}$. On a des isomorphismes canoniques $\varpi_{i n j} \circ o_{i n j} \simeq \widetilde{\omega}^{\prime} \circ \widetilde{\kappa}$ et $\omega \circ \kappa \simeq \widetilde{\omega} \circ \widetilde{\kappa}$, où $\widetilde{\omega}$ (resp. $\widetilde{\omega}^{\prime}$ ) est le foncteur introduit dans la notation 6.2.1 (resp. 6.2.2), de sorte que la proposition 6.2.4 donne la conclusion.

On introduit à présent un foncteur très analogue à $\mathcal{I}$.

Définition 13.2.2. - On définit un endofoncteur $\mathcal{J}$ de $\mathcal{F}_{\mathcal{G} r}$ par

$$
\mathcal{J}(X)(V, B)=\bigoplus_{W \in \mathcal{G} r(B)} X(V / W, B / W)
$$

l'application linéaire

$$
\mathcal{J}(X)(f): \mathcal{J}(X)(V, B) \rightarrow \mathcal{J}(X)\left(V^{\prime}, B^{\prime}\right)
$$

(où $f:(V, B) \rightarrow\left(V^{\prime}, B^{\prime}\right)$ est une flèche de $\mathcal{E}_{\mathcal{G} r}^{f}$ ) ayant pour composante

$$
X(V / W, B / W) \rightarrow X\left(V^{\prime} / W^{\prime}, B^{\prime} / W^{\prime}\right)
$$

l'application linéaire induite par le morphisme $(V / W, B / W) \rightarrow\left(V^{\prime} / W^{\prime}, B^{\prime} / W^{\prime}\right)$ induit par $f$ si $f(W)=W^{\prime}, 0$ sinon.

La propriété suivante justifie l'apparition du foncteur $\mathcal{J}$ dans cette section.

Proposition 13.2.3. - Il existe un isomorphisme de foncteurs $\omega \kappa \omega \simeq \omega \mathcal{J}$.

Démonstration. - On l'obtient par la suite d'isomorphismes naturels

$$
\begin{aligned}
\omega \kappa \omega(X)(V) & =\bigoplus_{W \in \mathcal{G} r(V)} \omega(X)(V / W) \simeq \bigoplus_{W \subset B \subset V} X(V / W, B / W) \\
& \simeq \bigoplus_{B \in \mathcal{G} r(V)} \mathcal{J}(X)(V, B)=\omega \mathcal{J}(X)(V) .
\end{aligned}
$$

La proposition suivante est la plus importante de ce chapitre. Elle joue dans $\mathcal{F}_{i n j}$ un rôle analogue à celui qu'occupe le théorème 10.2.1 dans la catégorie $\mathcal{F}_{\mathcal{G} r}$.

Proposition 13.2.4. - Soient $X$ un objet localement fini de $\mathcal{F}_{\mathcal{G} r}$ et $Y$ un objet

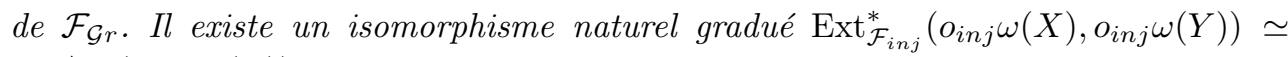
$\operatorname{Ext}_{\mathcal{F}_{\mathcal{G} r}}^{*}(X, \mathcal{I} \mathcal{J}(Y))$.

Démonstration. - Le foncteur $o_{i n j}$ étant adjoint à gauche à $\varpi_{i n j}$ (proposition 2.3.10), le corollaire A.1.1, la proposition 13.2.1, la proposition 13.2.3 puis le théorème 10.2.1 procurent des isomorphismes gradués naturels

$$
\begin{gathered}
\operatorname{Ext}_{\mathcal{F}_{\text {inj }}}^{*}\left(o_{i n j} \omega(X), o_{i n j} \omega(Y)\right) \simeq \operatorname{Ext}_{\mathcal{F}}^{*}\left(\omega(X), \varpi_{i n j} o_{i n j} \omega(Y)\right) \\
\simeq \operatorname{Ext}_{\mathcal{F}}^{*}(\omega(X), \omega \kappa \omega(Y)) \simeq \operatorname{Ext}_{\mathcal{F}}^{*}(\omega(X), \omega \mathcal{J}(Y)) \simeq \operatorname{Ext}_{\mathcal{G} r}^{*}(X, \mathcal{I} \mathcal{J}(Y)) .
\end{gathered}
$$


Remarque 13.2.5. - Ainsi, pour obtenir un énoncé relatif à la catégorie $\mathcal{F}_{\text {inj }}$, nous avons transité par la catégorie $\mathcal{F}_{\mathcal{G} r}$, dont l'étude requiert celle de $\mathcal{F}_{\text {surj }}$. Cela explique pourquoi nous avons dû introduire les deux catégories $\mathcal{F}_{\text {inj }}$ et $\mathcal{F}_{\text {surj }}$.

Le corollaire suivant permet d'utiliser la proposition 13.2.4 tout en s'affranchissant des foncteurs $\mathcal{I}$ et $\mathcal{J}$.

Corollaire 13.2.6. - Soient $k, n \in \mathbb{N}, X$ un objet localement fini de $\mathcal{F}_{\mathcal{G} r, k}$ et $Y$ un objet de $\mathcal{F}_{\mathcal{G} r, n}$.

(1) Si $k<n$, alors $\operatorname{Ext}_{\mathcal{F}_{i n j}}^{*}\left(o_{i n j} \omega_{k}(X), o_{i n j} \omega_{n}(Y)\right)=0$.

(2) Si $k=n$, le foncteur exact $o_{i n j} \omega_{n}$ induit un isomorphisme naturel gradué

$$
\operatorname{Ext}_{\mathcal{G}_{r, n}}^{*}(X, Y) \stackrel{\simeq}{\rightarrow} \operatorname{Ext}_{\mathcal{F}_{\text {inj }}}^{*}\left(o_{i n j} \omega_{n}(X), o_{i n j} \omega_{n}(Y)\right) .
$$

Démonstration. - On déduit ce résultat de la proposition 13.2.4 de la même façon que l'on a déduit le corollaire 10.2.2 du théorème 10.2.1. En effet, si $A$ est un objet de $\mathcal{F}_{\mathcal{G} r}$ de coniveau au moins égal à $n$, l'inclusion canonique $A \hookrightarrow \mathcal{J}(A)$ induit après application du foncteur $\mathcal{R}_{\mathbb{N}, \leq n}$ un isomorphisme.

Application à la $K$-théorie stable. - La démonstration par Suslin (cf. appendice de [FFSS99]) de l'isomorphisme entre la $K$-théorie stable de $\mathbb{k}$ et l'homologie dans $\mathcal{F}(\mathbb{k})$ pour des foncteurs finis se décompose en deux étapes ${ }^{(1)}$.

Rappelons que l'on dispose d'un foncteur exact e : $\mathcal{F}_{i n j} \rightarrow \mathbb{k}[G L(\mathbb{k})]$ Mod, conformément à la section 2.4. Nous noterons $F \mapsto F\left(\mathbb{k}^{\infty}\right)$ le foncteur $\mathcal{F} \rightarrow$ ${ }_{\mathbb{k}[G L(\mathbb{k})]} \operatorname{Mod}$ composé de $o_{i n j}: \mathcal{F} \rightarrow \mathcal{F}_{i n j}$ et du précédent. Il induit un morphisme gradué naturel

$$
\operatorname{Ext}_{\mathcal{F}_{i n j}(\mathbb{k})}^{*}\left(o_{i n j}(F), o_{i n j}(G)\right) \rightarrow \operatorname{Ext}_{\mathbb{k}[G L(\mathbb{k})]}^{*}\left(F\left(\mathbb{k}^{\infty}\right), G\left(\mathbb{k}^{\infty}\right)\right)
$$

pour $F, G \in \mathrm{Ob} \mathcal{F}$. La première étape de la démonstration de Suslin consiste à démontrer que ce morphisme est un isomorphisme lorsque $F$ et $G$ sont finis; c'est vrai en fait lorsque $F$ est $\mathrm{pf}_{\infty}$ et $G$ fini (cf. [Pir02], proposition 4.3). Le lien avec la $K$-théorie stable vient de ce que, à dualisation près, les groupes d'extensions $\operatorname{Ext}_{\mathbb{k}[G L(\mathbb{k})]}^{*}\left(F\left(\mathbb{k}^{\infty}\right), G\left(\mathbb{k}^{\infty}\right)\right)$ sont des groupes de $K$-théorie stable de $\mathbb{k}$ (voir encore [Pir02]).

Corollaire 13.2.7. - Soient $X$ un objet fini de $\mathcal{F}_{\mathcal{G} r}$ et $F$ un objet fini de $\mathcal{F}$. Il existe un isomorphisme naturel gradué

$$
\operatorname{Ext}_{\mathbb{k}[G L(\mathbb{k})]}^{*}\left(\omega(X)\left(\mathbb{k}^{\infty}\right), F\left(\mathbb{k}^{\infty}\right)\right) \simeq \operatorname{Ext}_{\mathcal{G} r}^{*}(X, \mathcal{I} \kappa(F)) .
$$

\footnotetext{
(1) Scorichenko a étendu le résultat de Suslin au cas d'un anneau arbitraire - cf. le dernier chapitre de [FFPS03]
} 
Démonstration. - Comme $X$ est $\mathrm{pf}_{\infty}$ et que le foncteur exact $\omega$ préserve les projectifs de type fini, $\omega(X)$ est $\operatorname{pf}_{\infty}$, de sorte que $\operatorname{Ext}_{\mathcal{F}_{i n j}(\mathbb{k})}^{*}\left(o_{i n j} \omega(X), o_{i n j}(F)\right) \simeq$ $\operatorname{Ext}_{\mathbb{k}[G L(\mathbb{k})]}^{*}\left(\omega(X)\left(\mathbb{k}^{\infty}\right), F\left(\mathbb{k}^{\infty}\right)\right)$. On conclut en appliquant la proposition 13.2.4, puisque $\mathcal{J} \mathcal{P}_{0, \mathbb{N}} \simeq \kappa$.

Lorsque $X$ est de niveau 0, i.e. que $\omega(X)$ est un objet fini de $\mathcal{F}$, on retrouve le résultat de Betley-Suslin affirmant que le morphisme naturel $\operatorname{Ext}_{\mathcal{F}}^{*}(F, G) \rightarrow$ $\operatorname{Ext}_{\mathbb{k}[G L(\mathbb{k})]}^{*}\left(F\left(\mathbb{k}^{\infty}\right), G\left(\mathbb{k}^{\infty}\right)\right)$ est un isomorphisme si $F$ et $G$ sont finis.

\subsection{La filtration de Krull de la catégorie $\mathcal{F}_{i n j}$}

Le corollaire 13.2.6 et la proposition 13.1.3 nous amènent à formuler la conjecture suivante.

Conjecture 13.3.1 (Conjecture artinienne extrêmement forte pour $\mathcal{F}_{\text {inj }}$ )

Pour tout $n \in \mathbb{N}$, le foncteur $o_{i n j} \omega_{n}: \mathcal{F}_{\mathcal{G} r, n} \rightarrow \mathcal{F}_{\text {inj }}$ induit une équivalence entre les catégories $\mathcal{F}_{\mathcal{G} r, n}^{l f}$ et $\mathcal{K}_{n+1}\left(\mathcal{F}_{\text {inj }}\right) / \mathcal{K}_{n}\left(\mathcal{F}_{\text {inj }}\right)$.

Si la conjecture artinienne extrêmement forte est satisfaite, cet énoncé équivaut au suivant.

Conjecture 13.3.2. - Pour tout $n \in \mathbb{N}$, le foncteur $o_{\text {inj }}$ induit une équivalence entre les catégories $\mathcal{K}_{n}(\mathcal{F}) / \mathcal{K}_{n-1}(\mathcal{F})$ et $\mathcal{K}_{n+1}\left(\mathcal{F}_{\text {inj }}\right) / \mathcal{K}_{n}\left(\mathcal{F}_{\text {inj }}\right)$.

Le résultat suivant donne une réponse positive partielle à la conjecture 13.3.1 pour $n=0$.

Proposition 13.3.3. - Le foncteur $o_{i n j}: \mathcal{F} \rightarrow \mathcal{F}_{i n j}$ induit une équivalence entre la sous-catégorie $\mathcal{F}^{f}$ de $\mathcal{F}$ et une sous-catégorie épaisse de $\mathcal{F}_{\text {inj }} / \mathcal{F}_{\text {inj }}^{\text {lf }}$.

Démonstration. - Si $S$ est un objet simple de $\mathcal{F}$, le corollaire 2.3.2 montre, par dualité, que $o_{i n j}(F)$ est un objet simple noethérien de type 1 de $\mathcal{F}_{i n j}$, donc un objet simple de $\mathcal{F}_{i n j} / \mathcal{F}_{i n j}^{l f}$. Le corollaire 13.2.6 (appliqué avec $n=0$ ) et la proposition 13.1.3 établissent par ailleurs que le foncteur $o_{i n j}$ induit un foncteur pleinement fidèle entre $\mathcal{F}^{f}$ et une sous-catégorie de $\mathcal{F}_{i n j} / \mathcal{F}_{\text {inj }}^{l f}$ stable par extensions. Cela prouve la proposition.

Remarque 13.3.4. - (1) Dans [Dja06d], nous établissons ce résultat de façon différente. La méthode suivie, moins élémentaire que celle de la proposition 13.3.3, est une variante moins technique de la démonstration du théorème 14.1.1 ci-après, théorème que l'on ne peut pas en revanche montrer de manière aussi directe que la proposition 13.3.3, le corollaire 2.3.2 n'ayant pas d'équivalent dans la catégorie $\mathcal{F}$. 
(2) La proposition 2.4.16 esquisse une autre approche de la catégorie $\mathcal{F}_{\text {inj }} / \mathcal{F}_{\text {inj }}^{l f}$. Malheureusement, celle-ci ne semble pas suffisante pour établir simplement que tous les objets simples de la catégorie $\mathcal{F}_{i n j} / \mathcal{F}_{i n j}^{l f}$ sont dans l'image du foncteur $\mathcal{F} \stackrel{o_{i n j}}{\longrightarrow}$ $\mathcal{F}_{i n j} \rightarrow \mathcal{F}_{i n j} / \mathcal{F}_{i n j}^{l f}$. 


\section{PARTIE IV}

\section{FONCTEUR $\omega$ ET $\nabla$-NILPOTENCE}


Cette partie est consacrée aux progrès réalisés dans la compréhension de la structure de la catégorie $\mathcal{F}$ grâce au foncteur d'intégrale en grassmanniennes $\omega: \mathcal{F}_{\mathcal{G} r} \rightarrow \mathcal{F}$, suggérés par les considérations du chapitre 12 . Nous montrons d'abord, dans le chapitre 14, comment étudier la catégorie $\mathcal{F} / \mathcal{F}_{\omega}$ à partir du foncteur $\omega_{1}: \mathcal{F}_{\mathcal{G} r, 1} \rightarrow \mathcal{F}$. La généralisation de la partie concrète de la démonstration du théorème principal de ce chapitre nécessite de remplacer le foncteur différence par les foncteurs $\tilde{\nabla}_{n}$ de Powell (introduits dans la section 1.5) : le chapitre 15 montre combien $\tilde{\nabla}_{n}$ est adapté à l'étude de la restriction du foncteur $\omega_{n}: \mathcal{F}_{\mathcal{G} r, n} \rightarrow \mathcal{F}$ aux objets finis de $\mathcal{F}_{\mathcal{G} r, n}$.

Le dernier chapitre présente les résultats obtenus à l'aide des outils introduits auparavant. Il s'agit d'une part de l'injectivité d'un morphisme induit par le foncteur $\omega$ entre des groupes de Grothendieck (théorème 10 de l'introduction), d'autre part du théorème de simplicité généralisé (théorème 12 de l'introduction) et de son application à de nouveaux cas de la conjecture artinienne. Le cas plus général nouvellement obtenu dans [Dja07] repose lourdement, lui aussi, sur le théorème de simplicité généralisé.

Dans cette toute cette partie, on suppose que le corps de base $\mathbb{k}$ est le corps à deux éléments $\mathbb{F}_{2}$. Il sera souvent omis des notations; nous reviendrons au détour de remarques sur les généralisations possibles au cas d'un corps fini arbitraire.

Cette restriction évite des lourdeurs techniques (dues à la décomposition scalaire, essentiellement) mais n'est en rien fondamentale pour la plupart des résultats exposés dans cette partie (au moins en se limitant à un corps fini premier). En revanche, la généralisation du théorème relatif à la structure de $P_{E_{2}} \otimes F$, où $F$ est un foncteur fini de $\mathcal{F}$, donné dans la section 16.4, pose des problèmes théoriques. En effet, ce théorème utilise un résultat dû à Powell qui repose sur des considérations explicites liées à la théorie des représentations, lesquelles dépendent du corps de base. 
CHAPITRE 14

\section{INTRODUCTION : LA CATÉGORIE $\mathcal{F} / \mathcal{F}_{\omega}$}

Ce chapitre démontre une forme partielle de la conjecture artinienne extrêmement forte, pour le quotient $\mathcal{K}_{1}(\mathcal{F}) / \mathcal{K}_{0}(\mathcal{F})$ (section 14.1), qui est ensuite discutée dans la section 14.2. L'essentiel des résultats de cette section ont été déjà établis par un biais différent par Powell, dans [Pow00b], généralisant les théorèmes obtenus par Piriou dans [Pir97] à l'aide de méthodes plus directes.

La démarche présentée dans ce chapitre présente un double intérêt : d'une part, elle précise les résultats de Powell, d'autre part, elle constitue une introduction à la démonstration du théorème de simplicité généralisé (théorème 16.2.7), dont elle contient toutes les idées conceptuelles, hormis l'emploi des foncteurs $\tilde{\nabla}_{n}$, remplacés par le foncteur exact $\Delta$, ce qui en simplifie nettement la partie technique.

\subsection{Le théorème principal}

On rappelle que $\mathcal{F}_{\mathcal{G} r, 1}^{\text {lf }}=\mathcal{K}_{0}\left(\mathcal{F}_{\mathcal{G} r, 1}\right)$ est la sous-catégorie pleine des objets localement finis de $\mathcal{F}_{\mathcal{G} r, 1}$. On sait en effet que la sous-catégorie des objets localement finis de $\mathcal{F}_{\mathcal{G} r, 1}$ est épaisse (cf. corollaire 5.5.8, déduit de ce que les objets finis de $\mathcal{F}_{\mathcal{G} r, 1}$ sont de présentation finie) ; de même, $\mathcal{F}_{\omega}=\mathcal{K}_{0}(\mathcal{F})$ (cf. corollaire 1.3.5).

Le but de cette section est d'établir le résultat suivant :

Théorème 14.1.1. - Le foncteur

$$
\bar{\omega}_{1}: \mathcal{F}_{\mathcal{G} r, 1}^{l f} \hookrightarrow \mathcal{F}_{\mathcal{G} r, 1} \stackrel{\omega_{1}}{\longrightarrow} \mathcal{F} \rightarrow \mathcal{F} / \mathcal{F}_{\omega}
$$

induit une équivalence entre la catégorie $\mathcal{F}_{\mathcal{G} r, 1}^{\text {lf }}$ et une sous-catégorie localisante de $\mathcal{K}_{1}(\mathcal{F}) / \mathcal{K}_{0}(\mathcal{F})=\mathcal{K}_{1}(\mathcal{F}) / \mathcal{F}_{\omega}$. En particulier, il envoie un foncteur simple de $\mathcal{F}_{\mathcal{G} r, 1}$ sur un objet simple de $\mathcal{F} / \mathcal{F}_{\omega}$.

En termes de foncteurs induits, ce théorème prend la forme suivante : 
Corollaire 14.1.2. - La sous-catégorie $\mathcal{F}^{\omega-\operatorname{cons}(1)}$ de $\mathcal{F}$ est épaisse; ses objets sont des foncteurs noethériens de type 1.

De plus, le foncteur $\omega_{1}$ induit une équivalence entre la sous-catégorie pleine des objets finis de $\mathcal{F}_{\mathcal{G} r, 1}$ et la catégorie $\mathcal{F}^{\omega-\operatorname{cons}(1)} / \mathcal{F}^{f}$.

Le théorème 14.1.1 redonne les résultats principaux de l'article [Pow00b] de Powell et les précise. Nous y reviendrons dans la section 14.2.

Remarque 14.1.3. - Il semble en revanche très difficile de prouver que tous les objets simples de $\mathcal{F} / \mathcal{F}_{\omega}$ sont induits de hauteur 1 sans avoir démontré une version forte de la conjecture artinienne.

Avant de démontrer le théorème 14.1.1, nous établissons des résultats préliminaires décrivant le comportement du foncteur différence sur l'image par $\omega_{1}$ d'un foncteur fini de $\mathcal{F}_{\mathcal{G} r, 1}$.

Notation 14.1.4. - Soit $X$ un objet de $\mathcal{F}_{\mathcal{G} r, 1}$. Nous désignerons par $\pi_{X}$ : $\Delta \omega_{1}(X) \rightarrow \omega_{1}(X)$ le morphisme donné sur $V \in \mathrm{Ob} \mathcal{E}^{f}$ par la composée

$$
\begin{aligned}
\Delta \omega_{1}(X)(V) \hookrightarrow \widetilde{\Delta} \omega_{1}(X)(V)=\omega_{1}(X)\left(V \oplus \mathbb{F}_{2}\right)=\bigoplus_{(v, t) \in\left(V \oplus \mathbb{F}_{2}\right) \backslash\{0\}} X\left(V \oplus \mathbb{F}_{2},(v, t)\right) \\
\rightarrow \bigoplus_{v \in V \backslash\{0\}} X\left(V \oplus \mathbb{F}_{2},(v, 1)\right) \rightarrow \bigoplus_{v \in V \backslash\{0\}} X(V, v)=\omega_{1}(X)(V),
\end{aligned}
$$

où la dernière flèche est induite par la projection $V \oplus \mathbb{F}_{2} \rightarrow V$.

Si $k$ est un entier naturel et $X$ un objet de $\mathcal{F}_{\mathcal{G} r, 1}$, nous noterons $\pi_{X}^{k}: \Delta^{k} \omega_{1}(X) \rightarrow$ $\omega_{1}(X)$ le morphisme $\pi_{X} \circ \Delta \pi_{X} \circ \cdots \circ \Delta^{k-1} \pi_{X}$; par convention, $\pi_{X}^{0}=i d_{X}$.

Ces notations seront utilisées uniquement dans cette section.

Dans la suite de cette section, la catégorie $\mathcal{F} \otimes \mathcal{F}_{\text {surj }}^{1}$ but de $\sigma_{1}$ est identifiée à $\mathcal{F}$.

Lemme 14.1.5. - Soit $X$ un objet de $\mathcal{F}_{\mathcal{G} r, 1}$.

(1) On a un isomorphisme naturel $\Delta \omega_{1}(X) \simeq \sigma_{1}(X) \oplus \omega_{1}\left(\Delta^{\mathcal{G} r, 1} X\right) \oplus \omega_{1} \iota_{1} \sigma_{1}(X)$.

(2) À travers cet isomorphisme, $\pi_{X}: \Delta \omega_{1}(X) \rightarrow \omega_{1}(X)$ se lit comme la composée de la projection $\sigma_{1}(X) \oplus \omega_{1}\left(\Delta^{\mathcal{G} r, 1} X\right) \oplus \omega_{1} \iota_{1} \sigma_{1}(X) \rightarrow \omega_{1} \iota_{1} \sigma_{1}(X)$ et du morphisme obtenu en appliquant $\omega_{1}$ à la coünité de l'adjonction $\iota_{1} \sigma_{1}(X) \rightarrow X$.

(3) Le morphisme $\pi_{X}$ est surjectif.

(4) Si $X$ est fini de degré $d \geq 0$, ker $\pi_{X}$ est la somme directe d'un objet fini de $\mathcal{F}$ et de l'image par $\omega_{1}$ d'un objet fini de $\mathcal{F}_{\mathcal{G} r, 1}$ de degré strictement inférieur à $d$.

(5) Soient $V$ un espace vectoriel de dimension finie, $l$ une forme linéaire non nulle sur $V$ et $x=\left(x_{v}\right)_{v \in V \backslash\{0\}}\left(x_{v} \in X(V, v)\right)$ un élément de $\omega_{1}(X)(V)$. Notons $a_{x}$ : $P_{V} \rightarrow \omega_{1}(X)$ le morphisme de $\mathcal{F}$ représenté par $x$. Alors l'élément $\left(y_{v}\right)$ de $\omega_{1}(X)(V)$ représenté par le morphisme

$$
P_{V} \stackrel{u \mapsto[l] \otimes u}{\longrightarrow} \mathbb{F}_{2}\left[V^{*} \backslash\{0\}\right] \otimes P_{V} \simeq \Delta P_{V} \stackrel{\Delta a_{x}}{\longrightarrow} \Delta \omega_{1}(X) \stackrel{\pi_{X}}{\longrightarrow} \omega_{1}(X)
$$


est donné par $y_{v}=l(v) x_{v}$.

Démonstration. — La décomposition ensembliste

$$
\mathcal{G} r_{1}\left(V \oplus \mathbb{F}_{2}\right)=\{(0,1)\} \sqcup\{(v, 0) \mid v \in V \backslash\{0\}\} \sqcup\{(v, 1) \mid v \in V \backslash\{0\}\}
$$

et l'isomorphisme naturel

$$
\bigoplus_{v \in V \backslash\{0\}} X\left(V \oplus \mathbb{F}_{2},(v, 1)\right) \simeq \bigoplus_{v \in V \backslash\{0\}} X\left(V \oplus \mathbb{F}_{2},(0,1)\right)
$$

induit par les isomorphismes $\left(V \oplus \mathbb{F}_{2},(v, 1)\right) \stackrel{\simeq}{\longrightarrow}\left(V \oplus \mathbb{F}_{2},(0,1)\right), \quad(x, t) \mapsto(x+t v, t)$ fournissent un isomorphisme naturel

$$
\widetilde{\Delta} \omega_{1}(X) \simeq \sigma_{1}(X) \oplus \omega_{1}\left(\widetilde{\Delta}^{\mathcal{G} r, 1} X\right) \oplus \omega_{1} \iota_{1} \sigma_{1}(X) .
$$

L'inclusion canonique $\omega_{1}(X) \hookrightarrow \widetilde{\Delta} \omega_{1}(X)$ se lit dans cet isomorphisme comme $\omega_{1}(X) \hookrightarrow \omega_{1}\left(\widetilde{\Delta}^{\mathcal{G} r, 1} X\right) \hookrightarrow \sigma_{1}(X) \oplus \omega_{1}\left(\widetilde{\Delta}^{\mathcal{G} r, 1} X\right) \oplus \omega_{1} \iota_{1} \sigma_{1}(X)$, d'où le premier point.

Pour tout $v \in V \backslash\{0\}$, la composée $X\left(V \oplus \mathbb{F}_{2},(0,1)\right) \stackrel{\simeq}{\longrightarrow} X\left(V \oplus \mathbb{F}_{2},(v, 1)\right) \rightarrow X(V, v)$, où la première flèche est l'inverse de l'isomorphisme utilisé précédemment et la seconde est induite par la projection, est induite par $V \oplus \mathbb{F}_{2} \rightarrow V, \quad(x, t) \mapsto x+t v$. Cela montre l'assertion 2 .

La suite exacte $\iota_{1} \sigma_{1} \Delta^{\mathcal{G} r, 1}(X) \rightarrow \iota_{1} \sigma_{1}(X) \rightarrow X \rightarrow 0$ (début de la résolution canonique de $X$ - cf. proposition 7.2.1), et l'exactitude du foncteur $\omega_{1}$ permettent d'en déduire les assertions 3 et 4 , puisque les foncteurs $\iota_{1}$ et $\sigma_{1}$ respectent le degré.

Pour établir la dernière assertion, notons z l'élément de $\Delta \omega_{1}(X)(V) \subset \omega_{1}(X)(V \oplus$ $\mathbb{F}_{2}$ ) représenté par le morphisme

$$
P_{V} \stackrel{u \mapsto[l] \otimes u}{\longrightarrow} \mathbb{F}_{2}\left[V^{*} \backslash\{0\}\right] \otimes P_{V} \simeq \Delta P_{V} \stackrel{\Delta a_{x}}{\longrightarrow} \Delta \omega_{1}(X) .
$$

On a $z=\left(\omega_{1}(X)(i)\right)(x)+\left(\omega_{1}(X)\left(i_{l}\right)\right)(x)$, où $i: V \rightarrow V \oplus \mathbb{F}_{2}$ est l'inclusion canonique et $i_{l}: V \rightarrow V \oplus \mathbb{F}_{2}$ le morphisme de composantes $i d_{V}$ et $l$.

On en déduit que la composante $z_{v, 1}(v \in V \backslash\{0\})$ de $z$ égale $X\left(i_{l}\right)\left(z_{v}\right)$ si $l(v)=1$, 0 sinon. La conclusion provient donc de ce que la composition $V \stackrel{i_{l}}{\rightarrow} V \oplus \mathbb{F}_{2} \rightarrow V$ est l'identité.

La proposition suivante fournit un argument de stabilisation décisif relatif aux morphismes $\pi_{X}^{k}$.

Proposition 14.1.6. - Soient $X$ un objet de $\mathcal{F}_{\mathcal{G} r, 1}$ et $F$ un sous-objet de $\omega_{1}(X)$. Pour tout entier $k \geq 0$, on note $C_{k}=\pi_{X}^{k}\left(\Delta^{k} F\right)$.

(1) La suite $\left(C_{k}\right)_{k>0}$ de sous-objets de $\omega_{1}(X)$ est croissante; nous noterons $C_{\infty}$ sa réunion. Si $X$ est un objet noethérien de $\mathcal{F}_{\mathcal{G} r, 1}$, cette suite stationne.

(2) Pour tout $k \geq 0$, le foncteur $C_{k}$ est engendré par les éléments du type

$$
\left(l_{1}(v) \ldots l_{k}(v) x_{v}\right)_{v \in V \backslash\{0\}} \in \omega_{1}(X)(V),
$$


où $V$ parcourt les espaces vectoriels de dimension finie, $x=\left(x_{v}\right)_{v \in V \backslash\{0\}}$ les éléments de $F(V)$ et $\left(l_{1}, \ldots, l_{k}\right)$ les $k$-uplets de formes linéaires sur $V$.

(3) Le foncteur $C_{\infty}$ est le plus petit sous- $\bar{P}_{\mathbb{F}_{2}}$-comodule de $\omega_{1}(X)$ contenant $F$. Si $F$ est lui-même un sous- $\bar{P}_{\mathbb{F}_{2}}$-comodule de $\omega_{1}(X)$, on a $C_{k}=F$ pour tout $k \geq 0$.

(4) Si $X$ est localement fini, on a hom $\left(C_{\infty} / F, \omega_{1}(U)\right)=0$ pour tout objet $U$ de $\mathcal{F}_{\mathcal{G} r, 1}$.

Démonstration. - L'assertion 5 du lemme 14.1.5 et la préservation des épimorphismes par le foncteur différence montrent le second point pour $k=1$. Le cas général s'en déduit aussitôt par récurrence.

On en déduit que la suite $\left(C_{k}\right)_{k>0}$ est croissante. Si $v$ est un élément non nul d'un espace vectoriel de dimension finie $V$, soient $l_{1}, \ldots, l_{n}$ les éléments de $V^{*}$ tels que $l_{i}(v)=1$. Alors la fonction polynomiale $l_{1} \ldots l_{n}: V \rightarrow \mathbb{F}_{2}$ est égale à l'indicatrice de $\{v\}$. Si l'on note $F^{g r}(V)$ le plus petit sous-espace vectoriel $V \backslash\{0\}$-gradué de $\omega_{1}(X)(V)$ contenant $F(V)$, ce qui précède montre que $C_{\infty}$ est le plus petit sousfoncteur de $\omega_{1}(X)$ tel que $F^{g r}(V) \subset C_{\infty}(V)$ pour tout $V \in \mathrm{Ob}^{f}$; en particulier, $C_{\infty} \supset F$.

Soit $Y$ le plus petit sous-objet de $X$ tel que $Y(V, v)$ contienne les composantes dans $X(V, v)$ des éléments de $F(V) \subset \omega_{1}(X)(V)$. La proposition 5.1.7 montre d'une part que $\omega_{1}(Y)$ est le plus petit sous- $\bar{P}_{\mathbb{F}_{2}}$-comodule de $\omega_{1}(X)$ contenant $F$. Le paragraphe précédent de la démonstration montre d'autre part que $C_{\infty}=\omega_{1}(Y)$, d'où le troisième point.

Soit $f: C_{\infty} / F \rightarrow \omega_{1}(U)$ un morphisme de $\mathcal{F}$. Si $X$ est localement fini, il en est de même pour $Y$, donc le corollaire 10.2.2 montre que la composée $g: \omega_{1}(Y)=$ $C_{\infty} \rightarrow C_{\infty} / F \stackrel{f}{\rightarrow} \omega_{1}(U)$ est induite par un morphisme $u: Y \rightarrow U$ de $\mathcal{F}_{\mathcal{G} r, 1}$. Comme la composée $F \hookrightarrow \omega_{1}(Y) \stackrel{g}{\rightarrow} \omega_{1}(U)$ est nulle et que $\omega_{1}$ est exact, $F$ est inclus dans $\omega_{1}($ ker $u)$. D'après le troisième point, on en déduit ker $u=Y$, donc $u=0$, puis $g=0$ et $f=0$, d'où la dernière assertion.

Par ailleurs, si $X$ est noethérien, alors $Y$ est de type fini, donc $C_{\infty}=\omega_{1}(Y)$ est de type fini. Cela montre que la suite $\left(C_{k}\right)_{k>0}$ est stationnaire, et achève la démonstration.

Début de la démonstration du théorème 14.1.1. — Le corollaire 10.2.2 entraîne la pleine fidélité de $\bar{\omega}_{1}: \mathcal{F}_{\mathcal{G} r, 1}^{l f} \rightarrow \mathcal{F} / \mathcal{F}_{\omega}$ et la stabilité par extensions de son image. Comme le foncteur $\bar{\omega}_{1}$ commute aux colimites, il suffit donc d'établir qu'un sous-objet d'un objet de l'image $\mathcal{C}$ de sa restriction à la sous-catégorie $\mathcal{F}_{\mathcal{G} r, 1}^{f}$ des objets finis de $\mathcal{F}_{\mathcal{G} r, 1}$ est isomorphe à un objet de $\mathcal{C}$. Pour cela, on procède par récurrence sur le degré polynomial (cf. proposition 5.5.5).

On se donne donc un objet fini $X$ de $\mathcal{F}_{\mathcal{G} r, 1}$ de degré $d \geq 0$ et l'on suppose que l'hypothèse suivante est satisfaite. 
Hypothèse 14.1.7 (Hypothèse de récurrence). - L'image par le foncteur $\bar{\omega}_{1}$ : $\mathcal{F}_{\mathcal{G} r, 1}^{l f} \rightarrow \mathcal{F} / \mathcal{F}_{\omega}$ du théorème 14.1.1 de la sous-catégorie $\left(\mathcal{F}_{\mathcal{G} r, 1}^{f}\right)^{d-1}$ des foncteurs de degré $<d$ de $\mathcal{F}_{\mathcal{G} r, 1}^{f}$ est une sous-catégorie épaisse de $\mathcal{F} / \mathcal{F}_{\omega}$.

Notation 14.1.8. - Dans cette section, nous noterons $\mathcal{A}_{d}$ cette sous-catégorie épaisse de $\mathcal{F} / \mathcal{F}_{\omega}$, et $\mathcal{Q}_{d}$ la catégorie quotient de $\mathcal{F} / \mathcal{F}_{\omega}$ par $\mathcal{A}_{d}$. Nous noterons également $\mathcal{X}$ la sous-catégorie pleine des objets $T$ de $\mathcal{F} / \mathcal{F}_{\omega}$ tels que $\operatorname{hom}\left(T, \bar{\omega}_{1}(U)\right)=0$ pour tout objet $U$ de $\mathcal{F}_{\mathcal{G} r, 1}$.

Lemme 14.1.9. - (1) Le foncteur différence $\Delta$ induit un endofoncteur exact et fidèle de la catégorie $\mathcal{F} / \mathcal{F}_{\omega}$. Il induit également un endofoncteur exact des catégories $\mathcal{A}_{d}$ et $\mathcal{Q}_{d}$.

(2) La sous-catégorie $\mathcal{X}$ de $\mathcal{F} / \mathcal{F}_{\omega}$ est stable par le foncteur induit par le foncteur différence.

(3) L'intersection des sous-catégories $\mathcal{X}$ et $\mathcal{A}_{d}$ de $\mathcal{F} / \mathcal{F}_{\omega}$ est réduite à 0.

(4) Pour tout entier $k>0$, le morphisme $\pi_{X}^{k}$ induit un isomorphisme dans la catégorie $\mathcal{Q}_{d}$.

Démonstration du lemme. - Le foncteur $\Delta$ est exact et conserve $\mathcal{F}_{\omega}$, il induit donc un endofoncteur exact de $\mathcal{F} / \mathcal{F}_{\omega}$. La suite exacte $0 \rightarrow p_{0}(F)=F(0) \rightarrow F \rightarrow \bar{I}_{\mathbb{F}_{2}} \otimes \Delta F$ naturelle en l'objet $F$ de $\mathcal{F}$ montre la fidélité de ce foncteur exact. En effet, le foncteur constant $F(0)$ est analytique, et, comme $\bar{I}_{\mathbb{F}_{2}}$ est analytique, si $\Delta F$ est objet de $\mathcal{F}_{\omega}$, il en est de même pour $\bar{I}_{\mathbb{F}_{2}} \otimes \Delta F$.

Le lemme 14.1.5 montre que $\Delta$ préserve l'image $\mathcal{A}_{d}$ de $\left(\mathcal{F}_{\mathcal{G} r, 1}^{f}\right)^{d-1}$ par $\bar{\omega}_{1}$, il induit donc un endofoncteur exact de $\mathcal{A}_{d}$ et $\mathcal{Q}_{d}=\left(\mathcal{F} / \mathcal{F}_{\omega}\right) / \mathcal{A}_{d}$. Cela achève de prouver le premier point. Le lemme 14.1.5 montre également que le noyau de l'épimorphisme $\pi_{X}$ appartient à $\mathcal{A}_{d}$, puisque $X$ est supposé de degré $d$. Cela établit, par récurrence sur $k$, la dernière assertion.

L'isomorphisme d'adjonction $\operatorname{hom}_{\mathcal{F}}\left(\Delta F, \omega_{1}(U)\right) \simeq \operatorname{hom}_{\mathcal{F}}\left(F, \omega_{1}\left(U \otimes \iota_{1}\left(\bar{I}_{\mathbb{F}_{2}}\right)\right)\right)$ donne le second point, puisque le corollaire 10.2.2 permet de remplacer les ensembles de morphismes considérés dans $\mathcal{F}$ par des ensembles de morphismes analogues dans $\mathcal{F} / \mathcal{F}_{\omega}$.

Le troisième point résulte de la définition de la sous-catégorie $\mathcal{A}_{d}$.

Fin de la démonstration du théorème 14.1.1. - Soit $F$ un sous-objet de $\omega_{1}(X)$; on conserve les notations de la proposition 14.1.6, et l'on se donne $k \in \mathbb{N}^{*}$ tel que $C_{\infty}=C_{k}$ (qui est donc de la forme $\omega_{1}(Y)$ pour un sous-objet $Y$ de $X$ ). Alors $\Delta^{k} F$ et $\Delta^{k} C_{\infty}$ ont la même image $C_{\infty}$ par $\pi_{X}^{k}$, qui induit un isomorphisme dans $\mathcal{Q}_{d}$ (dernière assertion du lemme 14.1.9), donc l'inclusion $\Delta^{k} F \hookrightarrow \Delta^{k} C_{\infty}$ induit un isomorphisme dans $\mathcal{Q}_{d}$. Ainsi, l'image dans $\mathcal{F} / \mathcal{F}_{\omega}$ de $\Delta^{k}\left(C_{\infty} / F\right)$ est objet de $\mathcal{A}_{d}$. Mais c'est aussi un objet de $\mathcal{X}$ par la dernière assertion de la proposition 14.1.6 et la deuxième assertion du lemme 14.1.9. La troisième assertion de ce lemme montre alors que l'image de $\Delta^{k}\left(C_{\infty} / F\right)$ dans $\mathcal{F} / \mathcal{F}_{\omega}$ est nulle, donc aussi l'image de $C_{\infty} / F$ (par la première assertion du lemme). Cela achève la démonstration. 


\subsection{Remarques et conjecture}

- Comme l'article [Pow00b], le théorème 14.1.1 signifie que les foncteurs $P_{\mathbb{F}_{2}} \otimes F$ (avec $F$ fini) sont noethériens de type 1 et donne des renseignements sur leur structure. La méthode qu'emploie Powell dans [Pow00b] repose fortement sur les propriétés des foncteurs $\tilde{\nabla}_{n}$ et certaines considérations explicites sur les représentations des groupes symétriques. La nôtre reste très générale et clarifie les calculs d'algèbre homologique entre les différents objets dont on démontre le caractère simple noethérien de type 1 , entièrement ramenés à des calculs d'algèbre homologique sur des objets finis de $\mathcal{F}_{\mathcal{G} r, 1}$, qui peuvent théoriquement se comprendre à partir des représentations d'algèbres de dimension finie sur $\mathbb{F}_{2}$.

Le théorème 14.1.1 fournit également une construction de l'objet simple noethérien de type 1 et $\mathcal{F}_{\omega}$-parfait associé à une partition régulière $\lambda$ différente de celle de Powell. Nous pouvons aussi montrer, sans calcul, l'égalité $X_{\lambda}=K_{\lambda}$ conjecturée dans [Pow00b], $\S 4.5:$ avec nos notations, elle se réduit à dire que l'image de l'unique morphisme non nul $\iota_{1}\left(S_{\lambda}\right) \rightarrow \iota_{1}\left(S_{\hat{\lambda}}\right)$, où $\hat{\lambda}=\left(\lambda_{1}+1, \ldots, \lambda_{r}+1\right)$ si $\lambda$ est de longueur $r$, est $\kappa_{1}\left(S_{\lambda}\right)$. En effet, cette image contient $\kappa_{1}\left(S_{\lambda}\right)=\operatorname{cosoc} \iota_{1}\left(S_{\lambda}\right)$ (cf. corollaire 5.5.12), et lorsqu'on applique le foncteur exact et fidèle $\sigma_{1}$ au morphisme précédent, on obtient l'unique morphisme non nul (cf. théorème B.3 de [PS98]) $S_{\lambda} \oplus \Delta S_{\lambda} \rightarrow S_{\hat{\lambda}} \oplus \Delta S_{\hat{\lambda}}$, dont l'image est $S_{\lambda}=\sigma_{1} \kappa_{1}\left(S_{\lambda}\right)$.

- On peut généraliser le théorème 14.1.1 au cas d'un corps fini quelconque $\mathbb{k}$. Il convient pour cela de remplacer le foncteur différence par la composée $\mathcal{F}(\mathbb{k}) \stackrel{\Delta}{\longrightarrow}$ $\mathcal{F}(\mathbb{k}) \stackrel{(-)_{i}}{\longrightarrow} \mathcal{F}(\mathbb{k})$, où la dernière flèche provient de la décomposition scalaire, pour tout entier $i \in\{1, \ldots, q-1\}$, où $q=$ Card $\mathbb{k}$.

- Les objets finis de la catégorie $\mathcal{F} / \mathcal{F}_{\omega}$ donnés par le théorème 14.1.1 (il n'y en a pas d'autres si la conjecture artinienne extrêmement forte est vraie) possèdent un représentant $\mathcal{F}_{\omega}$-parfait dans $\mathcal{F}$, du type $\omega_{1}(X)$ (avec $X \in \mathrm{Ob} \mathcal{F}_{\mathcal{G} r, 1}$ ). Il n'en est pas de même pour tous les objets de $\mathcal{F} / \mathcal{F}_{\omega}$. Considérons par exemple le noyau $A$ de l'unique morphisme non nul $\bar{P}_{\mathbb{F}_{2}}^{\otimes 2} \rightarrow \bar{P}_{\mathbb{F}_{2}}$. À partir de la suite exacte

$$
0 \rightarrow A \rightarrow \bar{P}_{\mathbb{F}_{2}}^{\otimes 2} \rightarrow \bar{P}_{\mathbb{F}_{2}} \rightarrow \Lambda^{1} \rightarrow 0
$$

et du corollaire 10.2.2, on obtient un isomorphisme naturel $\operatorname{Ext}^{i}(F, A) \simeq$ $\operatorname{Ext}^{i-2}\left(F, \Lambda^{1}\right)$ pour $F \in \mathrm{Ob} \mathcal{F}_{\omega}$. Ainsi, $A$ est $\mathcal{F}_{\omega}$-fermé, mais non $\mathcal{F}_{\omega}$-parfait.

- L'absence de représentants parfaits pour certains foncteurs équivaut à l'inexactitude du foncteur section $\mathcal{F} / \mathcal{F}_{\omega} \rightarrow \mathcal{F}$. Notons néanmoins la conséquence suivante du corollaire 10.2.2, dans laquelle nous notons $r^{i}$ le $i$-ème foncteur dérivé droit de l'endofoncteur de $\mathcal{F}$ composé du foncteur canonique $\mathcal{F} \rightarrow \mathcal{F} / \mathcal{F}_{\omega}$ et du foncteur section : pour tout foncteur induit $F$, on a $r^{i}(F)=0$ pour $i$ assez grand. Ainsi, la conjecture artinienne extrêmement forte permettrait de contrôler le défaut d'exactitude du foncteur section. 
- Même en admettant la conjecture artinienne extrêmement forte, certains aspects de la structure globale de la catégorie $\mathcal{F} / \mathcal{F}_{\omega}$ demeurent mystérieux. Par exemple, la description de l'enveloppe injective de l'image du foncteur $\bar{P}_{\mathbb{F}_{2}}$ dans cette catégorie quotient, qui en est l'objet simple le plus élémentaire, pose des problèmes très difficiles.

En général, les injectifs indécomposables de $\mathcal{F} / \mathcal{F}_{\omega}$ sont les images par le foncteur section des injectifs indécomposables sans sous-objet fini non nul de $\mathcal{F}$ (cf. [Gab62]), i.e. des injectifs indécomposables « pathologiques ${ }^{(1)} »$ de $\mathcal{F}$.

Nous conjecturons notamment le résultat suivant:

Conjecture 14.2.1. - Tout foncteur injectif indécomposable de la catégorie $\mathcal{F}$ est à valeurs de dimension finie.

Les difficultés inhérentes à ce problème, liées à la compréhension générique des représentations des groupes linéaires, sont conceptuellement analogues à celles que l'on rencontre pour la conjecture artinienne extrêmement forte. Cependant, même en admettant la conjecture artinienne extrêmement forte, l'auteur ignore comment démontrer la conjecture 14.2.1, y compris pour la seule enveloppe injective de $\bar{P}_{\mathbb{F}_{2}}$, notée $I_{\bar{P}}$.

Remarque 14.2.2. - (1) On a $I_{\bar{P}}(0)=0$ et $I_{\bar{P}}\left(\mathbb{F}_{2}\right) \simeq \operatorname{hom}_{\mathcal{F}}\left(\bar{P}_{\mathbb{F}_{2}}, I_{\bar{P}}\right) \simeq \mathbb{F}_{2}$. Les résultats de Powell (cf. [Pow98a]) fournissent $\operatorname{hom}_{\mathcal{F}}\left(Q_{(2)}, I_{\bar{P}}\right) \simeq \mathbb{F}_{2}$ et $\operatorname{hom}_{\mathcal{F}}\left(Q_{(2,1)}, I_{\bar{P}}\right) \simeq \mathbb{F}_{2}$, d'où l'on déduit, compte-tenu de la filtration de $P_{E_{2}}$ donnée dans [Pow98c] (exemple 4.1), que $I_{\bar{P}}\left(E_{2}\right)$ est de dimension 7 .

(2) Si la conjecture artinienne extrêmement forte est vraie au rang 1 (i.e. pour $\mathcal{K}_{1}(\mathcal{F}) / \mathcal{K}_{0}(\mathcal{F})$ ), alors il est facile de décrire l'enveloppe injective $I_{\bar{P}}^{\mathcal{K}, 1}$ de $\bar{P}_{\mathbb{F}_{2}}$ dans la catégorie $\mathcal{K}_{1}(\mathcal{F})$ : c'est l'image par le foncteur $\omega_{1}$ de l'enveloppe injective $I_{\left(\mathbb{F}_{2}, \mathbb{F}_{2}\right)}^{\mathcal{G} r, 1}$ (qui est localement finie) du foncteur constant $\mathbb{F}_{2}$ de $\mathcal{F}_{\mathcal{G} r, 1}$. Le foncteur $I_{\bar{P}}^{\mathcal{K}, 1}$ ainsi obtenu est l'un des deux facteurs indécomposables de $\bar{P}_{\mathbb{F}_{2}} \otimes \bar{I}_{\mathbb{F}_{2}}$, dont le scindement est donné par application du foncteur $\omega_{1}$ au scindement $\iota_{1}\left(\bar{I}_{\mathbb{F}_{2}}\right) \simeq \kappa_{1}\left(\bar{I}_{\mathbb{F}_{2}}\right) \oplus I_{\left(\mathbb{F}_{2}, \mathbb{F}_{2}\right)}^{\mathcal{G} r, 1}$ déduit de la proposition 5.1.14.

Mais le foncteur $I_{\bar{P}}^{\mathcal{K}, 1}$ est beaucoup moins gros que l'enveloppe injective $I_{\bar{P}}$ de $\bar{P}_{\mathbb{F}_{2}}$ dans $\mathcal{F}$, ne serait-ce que parce que cette dernière contient le foncteur $\overline{\mathbb{F}_{2}[\mathcal{G} r]}$ (la proposition 6.3.1 montre en effet que $\overline{\mathbb{F}_{2}[\mathcal{G} r]}$ est une extension essentielle de $\left.\bar{P}_{\mathbb{F}_{2}}\right)$.

La proposition élémentaire suivante va nous aider à discuter la conjecture 14.2.1.

\footnotetext{
$\overline{{ }^{(1)} \text { Remarquons }}$ qu'il n'existe aucun objet projectif " pathologique » dans la catégorie $\mathcal{F}$ : tous les projectifs indécomposables de $\mathcal{F}$ sont de type fini, et tout projectif de $\mathcal{F}$ est somme directe de projectifs indécomposables. Cela provient de la théorie classique de Krull-Schmidt (cf. [Pop73]), parce que les projectifs indécomposables de type fini engendrent $\mathcal{F}$ et ont des anneaux d'endomorphismes locaux.
} 
Proposition 14.2.3. - Le produit tensoriel de deux objets injectifs de la catégorie $\mathcal{F}$ dont l'un est à valeurs de dimension finie est injectif.

Démonstration. - Le produit tensoriel entre un injectif de $\mathcal{F}$ et un injectif standard $I_{V}$ est injectif, car l'adjoint à gauche $\Delta_{V}$ à l'endofoncteur $-\otimes I_{V}$ de $\mathcal{F}$ est exact.

Si $J$ est un injectif à valeurs de dimension finie, le foncteur $-\otimes J$ commute aux produits. Comme tout injectif de $\mathcal{F}$ est facteur direct d'un produit d'injectifs standard, l'injectivité des $J \otimes I_{V}$ établie précédemment donne la conclusion.

Corollaire 14.2.4. - Supposons que la conjecture artinienne et la conjecture 14.2.1 sont satisfaites. Alors le produit tensoriel de deux injectifs de la catégorie $\mathcal{F}$ est injectif.

Démonstration. - Si la catégorie $\mathcal{F}$ est localement noethérienne, tout injectif de $\mathcal{F}$ est isomorphe à une somme directe d'injectifs indécomposables (cf. [Gab62]). La conclusion résulte donc de la proposition 14.2.3 et de la distributivité du produit tensoriel par rapport à la somme directe.

Notons $G_{0}^{i n j}(\mathcal{F})$ le groupe de Grothendieck des objets injectifs de $\mathcal{F}$ à valeurs de dimension finie de $\mathcal{F}$. Sous les hypothèses du corollaire 14.2.4, ce groupe hérite d'une structure d'anneau commutatif induite par le produit tensoriel. Noter que, comme une somme directe infinie non triviale d'objets injectifs à valeurs de dimension finie peut être encore à valeurs de dimension finie, $G_{0}^{i n j}(\mathcal{F})$ est naturellement un anneau topologique non discret. Une autre question naturelle (toujours en admettant la conjecture artinienne et la conjecture 14.2.1) consiste à savoir si le produit tensoriel de deux injectifs indécomposables est une somme directe finie d'injectifs indécomposables, ce qui permettrait essentiellement de réduire l'étude de $G_{0}^{i n j}(\mathcal{F})$ au sous-groupe discret engendré par les classes des injectifs indécomposables (qui en serait alors un sousanneau).

Nous verrons dans la section 16.1 que la conjecture artinienne extrêmement forte donnerait une description assez explicite de l'anneau de Grothendieck $G_{0}^{t f}(\mathcal{F})$ des foncteurs de type fini de $\mathcal{F}$. Même en admettant la conjecture 14.2.1 et la conjecture artinienne extrêmement forte, la structure de l'anneau $G_{0}^{i n j}(\mathcal{F})$ reste obscure. 


\section{CHAPITRE 15}

\section{PRÉLIMINAIRES RELATIFS AUX FONCTEURS $\omega$ ET $\tilde{\nabla}_{n}$}

Nous donnons dans ce chapitre le substrat technique nécessaire à la généralisation des arguments de la section 14.1 au foncteur $\omega_{n}$, où $n$ est un entier supérieur à 1 . Il s'agit d'une part d'étendre le lemme 14.1.5, en remplaçant le foncteur différence par le foncteur $\tilde{\nabla}_{n}$, ce que nous accomplissons dans la section 15.2. Nous aurons besoin d'autre part d'un résultat d'annulation cohomologique relatif aux foncteurs $\tilde{\nabla}_{n}$-nilpotents (généralisant le cas des foncteurs finis) ; c'est l'objet de la section 15.3. Les deux facettes de ce programme reposent sur des arguments élémentaires issus de la structure des injectifs des catégories de foncteurs en grassmanniennes ; le lemme 1.3.6 relatif à la structure de l'injectif $I_{\mathbb{F}_{2}}$ de $\mathcal{F}$, que nous noterons simplement $I$ par la suite, constitue pratiquement leur seul ingrédient non formel.

Convention 15.0.1. - Dans toute ce chapitre, on se donne un entier $n>0$.

\subsection{Les foncteurs $\nabla_{n}^{\mathcal{G} r}$}

Nous introduisons l'outil interne à la catégorie $\mathcal{F}_{\mathcal{G} r}$ qui permettra, dans les sections suivantes, de mener à bien nos investigations relatives aux foncteurs $\omega$ et $\tilde{\nabla}_{n}$.

Définition 15.1.1. — On définit un endofoncteur $\nabla_{n}^{\mathcal{G} r}$ de la catégorie $\mathcal{F}_{\mathcal{G} r}$ par

$$
\nabla_{n}^{\mathcal{G} r}=\operatorname{ker}\left((-: \iota(I)) \rightarrow\left(-: \iota\left(p_{n-1}(I)\right)\right)\right)
$$

où la transformation naturelle est induite par l'inclusion $p_{n-1}(I) \hookrightarrow I$.

Le lien avec le foncteur $\tilde{\nabla}_{n}$ est donné par la proposition suivante.

Proposition 15.1.2. - Il existe un isomorphisme

$$
\omega \circ \nabla_{n}^{\mathcal{G} r} \simeq \tilde{\nabla}_{n} \circ \omega
$$

de foncteurs $\mathcal{F}_{\mathcal{G} r} \rightarrow \mathcal{F}$. 
Démonstration. - Grâce à la proposition 9.2.1 et à l'exactitude du foncteur $\omega$, il existe un diagramme commutatif aux lignes exactes

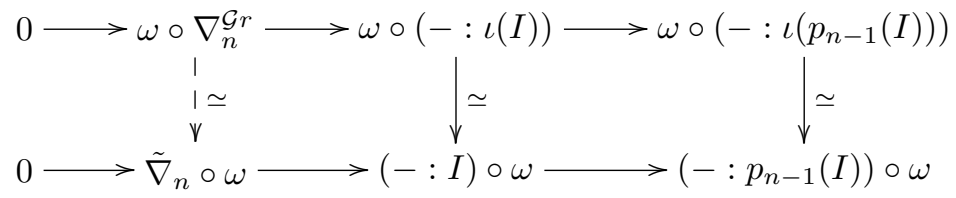

dans lequel les flèches verticales sont des isomorphismes.

Nous donnons maintenant des propriétés formelles du foncteur $\nabla_{n}^{\mathcal{G} r}$, analogues à celles du foncteur $\tilde{\nabla}_{n}$.

La première d'entre elle est une conséquence directe de la suite exacte (2) de la section 1.3. On rappelle que $t_{n}^{*}: I \rightarrow I_{E_{n}}$ (introduit dans cette même section) désigne le morphisme induit par $t_{n} \in \mathbb{F}_{2}\left[\operatorname{hom}\left(E_{n}, \mathbb{F}_{2}\right)\right]$.

Proposition 15.1.3. - Le foncteur $\nabla_{n}^{\mathcal{G} r}$ est l'image de la transformation naturelle $\left(t_{n}\right)_{*}=\left(-: \iota\left(t_{n}^{*}\right)\right):\left(-: \iota\left(I_{E_{n}}\right)\right) \rightarrow(-: \iota(I))$.

Comme les foncteurs $\left(-: \iota\left(I_{E_{n}}\right)\right)$ et $(-: \iota(I))$ sont exacts (cf. proposition 9.2.3), on en déduit :

Corollaire 15.1.4. - Le foncteur $\nabla_{n}^{\mathcal{G} r}$ est additif; il préserve les monomorphismes et les épimorphismes.

Nous utiliserons souvent le foncteur $\nabla_{n}^{\mathcal{G} r}$ sur des foncteurs appartenant à $\mathcal{F}_{\mathcal{G} r, k}$, vue comme sous-catégorie de $\mathcal{F}_{\mathcal{G} r}$ via le prolongement par zéro. Pour ramener son étude à des considérations internes à la catégorie $\mathcal{F}_{\mathcal{G} r, k}$, nous emploierons la proposition suivante.

Proposition 15.1.5. - Soient $k \in \mathbb{N}, X$ un objet de $\mathcal{F}_{\mathcal{G} r, k}$ et $\tilde{X}$ l'objet de $\mathcal{F}_{\mathcal{G} r}$ image de $X$ par le foncteur de prolongement par zéro. Il existe un isomorphisme naturel entre la restriction de $\nabla_{n}^{\mathcal{G} r}(\tilde{X})$ à $\mathcal{F}_{\mathcal{G} r, k}$ et l'image de $\left(t_{n}\right)_{*}=\left(X: \iota_{k}\left(t_{n}^{*}\right)\right)$ : $\left(X: \iota_{k}\left(I_{E_{n}}\right)\right) \rightarrow\left(X: \iota_{k}(I)\right)$.

De plus, le niveau du foncteur $\nabla_{n}^{\mathcal{G} r}(\tilde{X})$ est inférieur ou égal à $k$.

Démonstration. - Cela résulte de la proposition 15.1.3, de l'isomorphisme naturel $\mathcal{R}_{\leq k, k}\left(\mathcal{P}_{k, \leq k}(X): \iota_{\leq k}(F)\right) \simeq\left(X: \iota_{k}(F)\right.$ (pour $F \in$ Ob $\left.\mathcal{F}\right)$ donné par la proposition 9.1.2 et de l'isomorphisme naturel $(\tilde{X}: \iota(F)) \simeq \mathcal{P}_{\leq k, \mathbb{N}}\left(\mathcal{P}_{k, \leq k}(X): \iota_{\leq k}(F)\right)$ qui s'établit par un argument formel d'adjonction analogue.

La proposition suivante découle de la commutativité du produit tensoriel de $\mathcal{F}_{\mathcal{G} r}$ et de ce que ses foncteurs de décalage et son foncteur différence sont isomorphes à des foncteurs de division.

Proposition 15.1.6. - Le foncteur $\nabla_{n}^{\mathcal{G} r}$ commute aux foncteurs de décalage et au foncteur différence de $\mathcal{F}_{\mathcal{G} r}$, ̀̀ isomorphisme naturel près. 
Nous en venons maintenant à des propriétés élémentaires du foncteur $\nabla_{n}^{\mathcal{G} r}$ qui diffèrent du comportement de $\tilde{\nabla}_{n}$. Elles reposent sur la proposition suivante.

Proposition 15.1.7. - Il existe dans $\mathcal{F}_{\mathcal{G} r}$ un diagramme commutatif

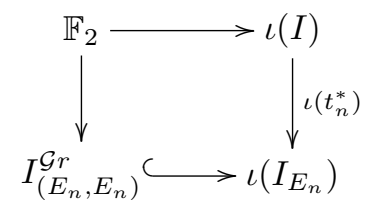

qui induit dans $\mathcal{F}_{\mathcal{G} r, n}$ un diagramme commutatif

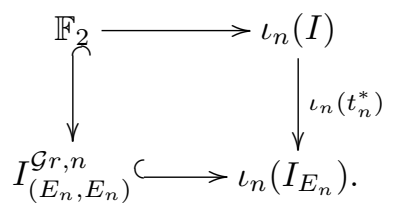

Démonstration. - Le morphisme $\mathbb{F}_{2} \rightarrow I_{\left(E_{n}, E_{n}\right)}^{\mathcal{G r}}$ du diagramme (12) est l'unique flèche non nulle; le morphisme $\mathbb{F}_{2} \rightarrow \iota(I)$ est la composée de l'unique morphisme non nul $\mathbb{F}_{2} \rightarrow I_{\left(\mathbb{F}_{2}, \mathbb{F}_{2}\right)}^{\mathcal{G} r}$ et du monomorphisme scindé $I_{\left(\mathbb{F}_{2}, \mathbb{F}_{2}\right)}^{\mathcal{G} r} \hookrightarrow \iota(I)$ donné par la proposition 5.1.14; le monomorphisme non spécifié provient de cette même proposition.

Comme $\mathbb{F}_{2} \simeq \rho\left(\mathbb{F}_{2}\right)$, par adjonction entre les foncteurs $\rho$ et $\varepsilon$ (proposition 9.2.2), la commutation du diagramme (12) se ramène au lemme 15.1 .8 ci-dessous, puisque $\varepsilon \circ \iota \simeq o$ et $\varepsilon\left(I_{(V, V)}^{\mathcal{G} r}\right) \simeq I_{V}^{\text {surj }}$

Dans le diagramme (13) qu'on déduit de (12) par application du foncteur de restriction, la flèche verticale $\mathbb{F}_{2} \rightarrow I_{\left(E_{n}, E_{n}\right)}^{\mathcal{G} r, n}$ est l'unique morphisme non nul, qui est injectif parce que le foncteur constant $\mathbb{F}_{2}$ de $\mathcal{F}_{\mathcal{G} r, n}$ est simple.

Lemme 15.1.8. - Le diagramme suivant de $\mathcal{F}_{\text {surj }}$ commute

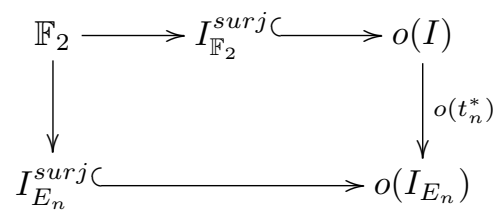

où les deux flèches de source $\mathbb{F}_{2}$ sont les uniques morphismes non nuls et les deux injections non spécifiées les monomorphismes scindés donnés par l'isomorphisme $o\left(I_{V}\right) \simeq \bigoplus_{W \in \mathcal{G} r(V)} I_{W}^{\text {surj }}$ de la proposition 2.3.6 (assertion 3).

Démonstration. - Soit $W$ un sous-espace de $E_{n}$. Le morphisme

$$
\mathbb{F}_{2} \rightarrow I_{\mathbb{F}_{2}}^{\text {surj }} \hookrightarrow o(I) \stackrel{o\left(t_{n}^{*}\right)}{\longrightarrow} o\left(I_{E_{n}}\right) \rightarrow I_{W}^{\text {surj }}
$$

est la somme sur les formes linéaires $l \in E_{n}^{*}$ telles que $l(W) \neq 0$ des composées

$$
\mathbb{F}_{2} \rightarrow I_{\mathbb{F}_{2}}^{\text {surj }} \stackrel{l^{*}}{\rightarrow} I_{W}^{\text {surj }},
$$


qui sont toutes égales à l'unique morphisme non nul. Comme le cardinal de l'ensemble $\left\{l \in E_{n}^{*} \mid l(W) \neq 0\right\}=E_{n}^{*} \backslash W^{\perp}$ est $2^{n}-2^{n-\operatorname{dim} W}$, qui est impair si et seulement si $\operatorname{dim} W=n$, on en conclut que le morphisme $\mathbb{F}_{2} \rightarrow I_{W}^{\text {surj }}$ que l'on étudie est non nul si et seulement si $W=E_{n}$, d'où l'on déduit le lemme.

Notation 15.1.9. - On désigne par $q_{n}^{\nabla}: \nabla_{n}^{\mathcal{G} r} \rightarrow i d$ la transformation naturelle composée de l'inclusion canonique $\nabla_{n}^{\mathcal{G} r} \hookrightarrow(-: \iota(I))$ et de la transformation naturelle $(-: \iota(I)) \rightarrow\left(-: \mathbb{F}_{2}\right) \simeq i d$ induite par le morphisme $\mathbb{F}_{2} \rightarrow \iota(I)$ du diagramme (12).

Ainsi, les propositions 15.1 .7 et 15.1 .3 procurent un diagramme commutatif

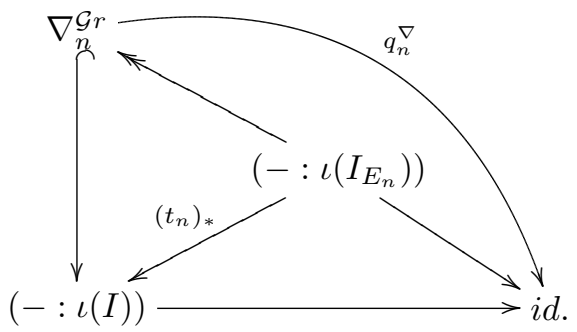

\subsection{Estimation de $\tilde{\nabla}_{n} \omega_{n}$}

Il n'est pas difficile de voir que, pour $i<n$, le foncteur $\tilde{\nabla}_{i}$ a tendance à faire exploser la taille des foncteurs du type $\omega_{n}(X)$. La proposition suivante montre que le comportement de $\tilde{\nabla}_{i}$ sur $\omega_{n}(X)$ est tout-à-fait différent pour $i>n$, lorsque $X$ est un foncteur fini de $\mathcal{F}_{\mathcal{G} r, n}$.

Proposition 15.2.1. - Si F est un foncteur induit de hauteur strictement inférieure à $n$, alors $F$ est $\tilde{\nabla}_{n}$-nilpotent.

Démonstration. - Si $X \in \mathrm{Ob} \mathcal{F}_{\mathcal{G} r, k}$ est simple, il existe un objet simple $F$ de $\mathcal{F}$ et un $G L_{k}$-module simple $S$ tels que $X \simeq \kappa_{k}(F) \otimes \rho_{k}(S)$ (cf. proposition 5.4.13), d'où un épimorphisme

$$
P_{E_{k}} \otimes F \rightarrow \omega_{k} \rho_{k}(S) \otimes F \simeq \omega_{k}\left(\iota_{k}(F) \otimes \rho_{k}(S)\right) \rightarrow \omega_{k}(X) .
$$

Les propositions 1.5 .4 et 1.5.9 montrent que $P_{E_{k}} \otimes F$, donc $\omega_{k}(X)$, est $\tilde{\nabla}_{n}$-nilpotent si $k<n$. On conclut grâce à la proposition 1.5.7.

Nous nous attachons, dans la suite de cette section, à montrer que $\tilde{\nabla}_{n} \omega_{n}(X)$ et $\omega_{n}(X)$ sont en quelque sorte du même ordre de grandeur pour $X$ fini. Dans le cas où $X$ est un foncteur simple pseudo-constant, Powell a calculé exactement, dans [Pow98c], $\tilde{\nabla}_{n} \omega_{n}(X)$ (qui est alors isomorphe à $\omega_{n}(X)$, sauf dans un cas); nous n'en aurons pas usage. 
Notation 15.2.2. - Dans cette section, on note $\tilde{X}$ le prolongement par zéro à $\mathcal{F}_{\mathcal{G} r}$ d'un foncteur $X$ de $\mathcal{F}_{\mathcal{G} r, n}$. On désigne également par $q_{n, X}^{\nabla}$ le morphisme naturel $\left(q_{n}^{\nabla}\right)_{\tilde{X}}: \nabla_{n}^{\mathcal{G} r}(\tilde{X}) \rightarrow \tilde{X}$.

On remarque que la proposition 15.1.2 entraîne l'existence d'un isomorphisme naturel

$$
\tilde{\nabla}_{n} \omega_{n}(X) \simeq \omega \nabla_{n}^{\mathcal{G} r}(\tilde{X}) .
$$

En appliquant le foncteur $\omega$ au morphisme naturel $q_{n, X}^{\nabla}$, on en déduit un morphisme naturel

$$
\pi_{n, X}: \tilde{\nabla}_{n} \omega_{n}(X) \rightarrow \omega_{n}(X) .
$$

Pour $n=1$, on retrouve le morphisme $\pi_{X}$ de la section 14.1.

Une description explicite du morphisme $\pi_{n, X}$ sera donnée dans la section 16.2 (lemme 16.2.3).

Proposition 15.2.3. - Pour tout foncteur $X$ de $\mathcal{F}_{\mathcal{G} r, n}$, le morphisme $\left(q_{n}^{\nabla}\right)_{\tilde{X}}$ : $\nabla_{n}^{\mathcal{G} r}(\tilde{X}) \rightarrow \tilde{X}$ est surjectif.

Démonstration. - Par la proposition 15.1.5, cela découle de la surjectivité de la flèche en pointillé du diagramme

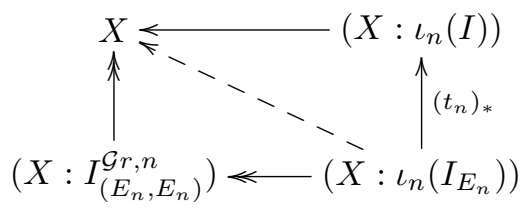

induit par le diagramme (13) de la proposition 15.1.7.

En appliquant le foncteur exact $\omega$, on en déduit l'important résultat suivant.

Corollaire 15.2.4. - La transformation naturelle $\pi_{n}: \tilde{\nabla}_{n} \omega_{n} \rightarrow \omega_{n}$ est surjective.

Lemme 15.2.5. - Si $X$ est un foncteur pseudo-constant de $\mathcal{F}_{\mathcal{G} r, n}$, la restriction à $\mathcal{F}_{\mathcal{G} r, n}$ du morphisme $\left(q_{n}^{\nabla}\right)_{\tilde{X}}: \nabla_{n}^{\mathcal{G} r}(\tilde{X}) \rightarrow \tilde{X}$ est un isomorphisme.

Démonstration. - La proposition 9.2.2 fournit des isomorphismes $\left(\rho_{n}(M): \iota(F)\right) \simeq$ $\rho_{n}\left(M: F\left(E_{n}\right)\right) \simeq \rho_{n}\left(M \otimes F\left(E_{n}\right)^{*}\right)$ naturels en le $G L_{n}$-module $M$ et le foncteur $F$ de $\mathcal{F}^{d f}$. On en déduit, par la proposition 15.1.5, des isomorphismes naturels

$$
\begin{aligned}
\mathcal{R}_{\mathbb{N}, n} \nabla_{n}^{\mathcal{G} r}(\tilde{X}) & \simeq \operatorname{ker}\left(\rho_{n}\left(M \otimes I\left(E_{n}\right)^{*}\right) \stackrel{\left(t_{n}\right)_{*}}{\longrightarrow} \rho_{n}\left(M \otimes p_{n-1}(I)\left(E_{n}\right)^{*}\right)\right) \\
& \left.\simeq \rho_{n}\left(M \otimes\left(I / p_{n-1}(I)\right)\left(E_{n}\right)\right)^{*}\right)
\end{aligned}
$$

où $X=\rho_{n}(M)$ est un foncteur pseudo-constant de $\mathcal{F}_{\mathcal{G} r, n}$. La conclusion provient alors de ce que $\left(I / p_{n-1}(I)\right)\left(E_{n}\right)=\mathbb{F}_{2}$ (lemme 1.3.6). 
Proposition 15.2.6. - Soit $X$ un objet fini de degré $i \geq 0$ de $\mathcal{F}_{\mathcal{G} r, n}$. Il existe dans $\mathcal{F}_{\mathcal{G} r}$ une suite exacte $0 \rightarrow Y \rightarrow \operatorname{ker} q_{n, X}^{\nabla} \rightarrow Z \rightarrow 0$ dans laquelle :

- Y est un foncteur fini de niveau strictement inférieur à $n$ et de degré au plus $i$;

- Z est un foncteur fini de degré strictement inférieur à $i$, de niveau au plus $n$ et de coniveau au moins $n$.

Démonstration. - On pose $Y=\mathcal{P}_{\leq n-1, \mathbb{N}} \mathcal{R}_{\mathbb{N}, \leq n-1} \nabla_{n}^{\mathcal{G} r}(\tilde{X})$ - c'est un sous-foncteur de $\operatorname{ker} q_{n, X}^{\nabla}$ puisque la restriction à $\mathcal{F}_{\mathcal{G} r, \leq n-1}$ de $\tilde{X}$ est nulle - et $Z=\left(\operatorname{ker} q_{n, X}^{\nabla}\right) / Y$. La proposition 15.1.5 montre que la condition de niveau sur $Z$ est vérifiée; la condition de niveau sur $Y$ et celle de coniveau sur $Z$ le sont pour des raisons formelles.

La proposition 15.1.6 montre que le foncteur $\operatorname{ker} q_{n}^{\nabla}: \mathcal{F}_{\mathcal{G} r, n} \rightarrow \mathcal{F}_{\mathcal{G} r}$ commute au foncteur différence à isomorphisme naturel près. On en déduit en particulier $\operatorname{deg} Y \leq$ $\operatorname{deg} \operatorname{ker} q_{n, X}^{\nabla} \leq \operatorname{deg} X=i$, par récurrence sur $i$. L'inégalité $\operatorname{deg} Z<i$ s'obtient de même, par récurrence sur $i$, à partir du lemme 15.2.5. Comme les foncteurs $Y$ et $Z$ sont, comme $\nabla_{n}^{\mathcal{G} r}(X)$, à valeurs de dimension finie, ce qui précède montre qu'ils sont finis, ce qui achève la démonstration.

Notation 15.2.7. - Dans la proposition suivante, $\mathcal{F}_{\tilde{\nabla}_{n},<i}$ désigne la plus petite sous-catégorie épaisse de $\mathcal{F}$ contenant $\mathcal{N} i l_{\tilde{\nabla}_{n}}$ et les objets $\omega_{n}(Y)$, pour $Y$ objet fini de $\mathcal{F}_{\mathcal{G} r, n}$ de degré strictement inférieur à $i$.

Intuitivement, cette catégorie contient tous les objets qui sont « moins gros » que les foncteurs du type $\omega_{n}(A)$, où $A \in \mathrm{Ob} \mathcal{F}_{\mathcal{G} r, n}$ est de degré $i$ (la proposition 15.2.1 montre que $\mathcal{F}_{\tilde{\nabla}_{n},<i}$ contient déjà tous les objets du type $\omega_{k}(X)$ avec $k<n$ et $X \in \operatorname{Ob} \mathcal{F}_{\mathcal{G} r, k}$ fini).

Corollaire 15.2.8. - Soient $i \in \mathbb{N}$ et $X$ un objet fini de degré $i$ de $\mathcal{F}_{\mathcal{G} r, n}$.

Le noyau de $\pi_{n, X}: \tilde{\nabla}_{n} \omega_{n}(X) \rightarrow \omega_{n}(X)$ appartient à $\mathcal{F}_{\tilde{\nabla}_{n},<i}$.

Démonstration. - Soit $0 \rightarrow Y \rightarrow \operatorname{ker} q_{n, X}^{\nabla} \rightarrow Z \rightarrow 0$ la suite exacte donnée par la proposition 15.2.6 : par exactitude du foncteur $\omega$, on en déduit une suite exacte $0 \rightarrow \omega(Y) \rightarrow \operatorname{ker} \pi_{n, X} \rightarrow \omega(Z) \rightarrow 0$. Le foncteur $\omega(Y)$ est $\tilde{\nabla}_{n}$-nilpotent, donc objet de $\mathcal{F}_{\tilde{\nabla}_{n},<i}$, par la proposition 15.2 .1 , et $\omega(Z)$ est objet de $\mathcal{F}_{\tilde{\nabla}_{n},<i}$ parce que $\operatorname{deg} Z<i$, d'où la proposition.

\subsection{Théorème d'annulation cohomologique relatif à la $\nabla$-nilpotence}

La section précédente précisait le comportement du foncteur $\tilde{\nabla}_{n}$ sur l'image du foncteur $\omega_{n}$ en terme de «taille $»$ des objets; nous allons donner l'aspect cohomologique de la comparaison entre $\tilde{\nabla}_{n}$ et $\omega_{n}$. 
Tensorisons le diagramme (13) de la proposition 15.1 .7 par un objet $X$ de $\mathcal{F}_{\mathcal{G} r, n}$ et appliquons le foncteur $\omega_{n}$ : on obtient un diagramme commutatif naturel

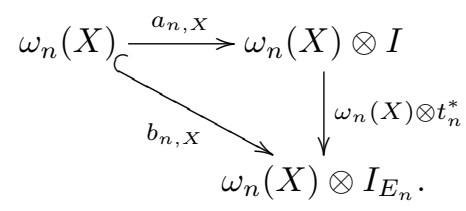

Par adjonction entre les endofoncteurs $\Delta_{V}$ et $-\otimes I_{V}$ de $\mathcal{F}$, on en déduit un diagramme commutatif

$$
\Delta_{E_{n}} \omega_{n}(X) \underset{\mathrm{F}_{2}}{\Delta_{n, X}} \omega_{n}(X)
$$

Le théorème fondamental suivant constitue une variation sur le corollaire 10.2.2 adaptée au contexte de la $\tilde{\nabla}_{n}$-nilpotence. Nous y reviendrons à la remarque 15.3.2.

Théorème 15.3.1. - Pour tout objet $X$ de $\mathcal{F}_{\mathcal{G} r, n}$, le foncteur $\omega_{n}(X)$ est $\overline{\mathcal{N}}_{\bar{\nabla}_{n}}$ parfait : pour tout foncteur $F$ de $\overline{\mathcal{N}}_{\tilde{\nabla}_{n}}$, on a $\operatorname{Ext}_{\mathcal{F}}^{*}\left(F, \omega_{n}(X)\right)=0$.

Démonstration. - Par la proposition B.1.14, il suffit d'établir la nullité des groupes d'extensions $\operatorname{Ext}_{\mathcal{F}}^{i}\left(F, \omega_{n}(X)\right)$ lorsque $F$ est $\tilde{\nabla}_{n}$-nilpotent, assertion que l'on établit par récurrence sur $i$.

La naturalité de la transformation $\left(t_{n}\right)_{*}: \Delta_{E_{n}} \rightarrow \Delta_{\mathbb{F}_{2}}$ se traduit par la commutation des diagrammes

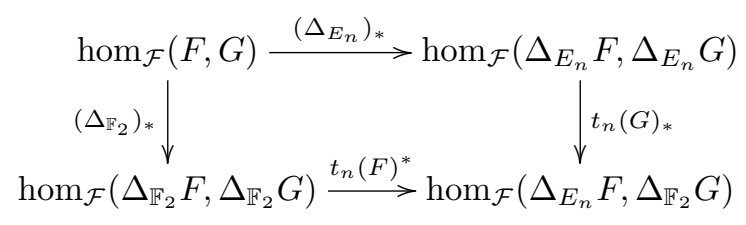

pour tous objets $F$ et $G$ de $\mathcal{F}$, où l'on a noté $t_{n}(F)$ pour $\left(\left(t_{n}\right)_{*}\right)_{F}$, pour simplifier les écritures. Comme les foncteurs $\Delta_{E_{n}}$ et $\Delta_{\mathbb{F}_{2}}$ sont exacts, ce diagramme s'étend en un diagramme commutatif

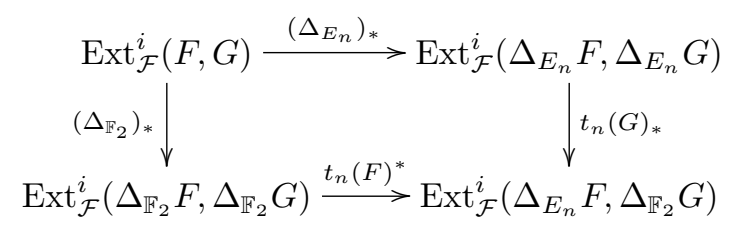

pour tout $i \in \mathbb{N}$. 
On forme alors le diagramme commutatif (18)

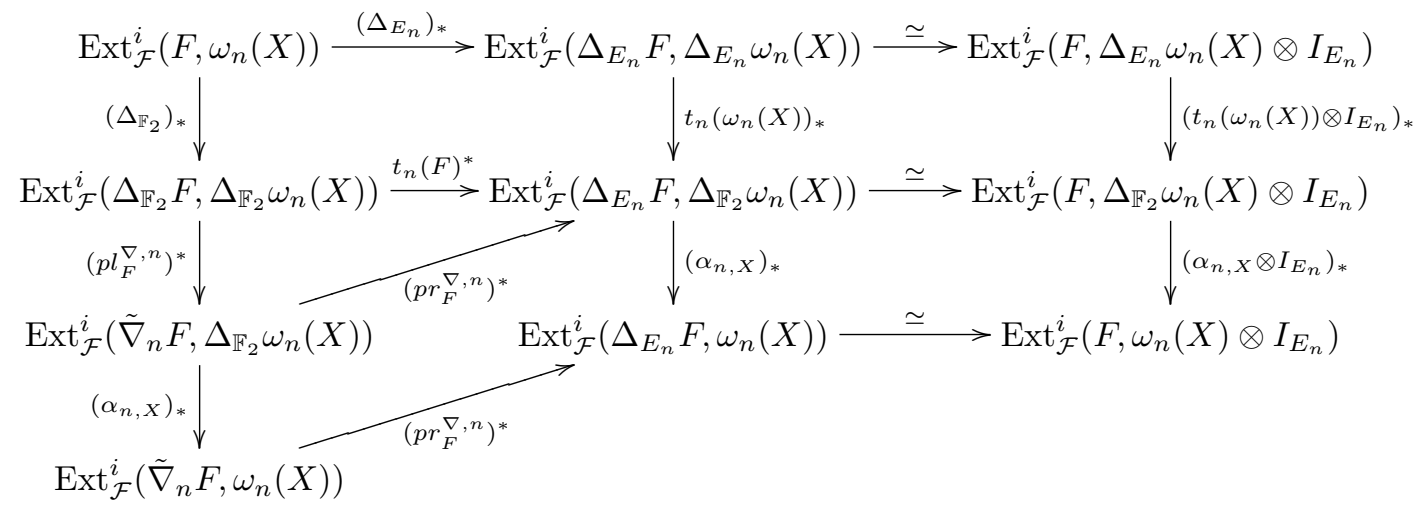

où $\operatorname{pr}_{F}^{\nabla, n}$ désigne la projection canonique $\Delta_{E_{n}} F \rightarrow \tilde{\nabla}_{n} F$, et $p l_{F}^{\nabla, n}: \tilde{\nabla}_{n} F \hookrightarrow \Delta_{\mathbb{F}_{2}} F$ l'inclusion canonique, et où les isomorphismes de droite dérivent de l'adjonction entre les endofoncteurs exacts $\Delta_{V}$ et $-\otimes I_{V}$ de $\mathcal{F}$.

Le morphisme $u: \operatorname{Ext}_{\mathcal{F}}^{i}\left(F, \omega_{n}(X)\right) \rightarrow \operatorname{Ext}_{\mathcal{F}}^{i}\left(F, \omega_{n}(X) \otimes I_{E_{n}}\right)$ obtenu en suivant le diagramme est induit par le monomorphisme $b_{n, X}: \omega_{n}(X) \hookrightarrow \omega_{n}(X) \otimes I_{E_{n}}$. En effet, la composée horizontale supérieure est induite par l'unité $\omega_{n}(X) \rightarrow \Delta_{E_{n}} \omega_{n}(X) \otimes I_{E_{n}}$ de l'adjonction, tandis que la composée verticale de droite est induite par $\beta_{n, X} \otimes I_{E_{n}}$ (par la commutation du diagramme (17)) ; on conclut par adjonction entre les morphismes $b_{n, X}$ et $\beta_{n, X}$.

Comme $b_{n, X}$ provient par application du foncteur $\omega_{n}$ à un monomorphisme $X \hookrightarrow$ $X \otimes \iota_{n}\left(I_{E_{n}}\right)$, il existe un objet $Y$ de $\mathcal{F}_{\mathcal{G} r, n}$ et une suite exacte courte

$$
0 \rightarrow \omega_{n}(X) \stackrel{b_{n, X}}{\longrightarrow} \omega_{n}(X) \otimes I_{E_{n}} \rightarrow \omega_{n}(Y) \rightarrow 0
$$

La suite exacte longue de cohomologie associée et l'hypothèse de récurrence $\operatorname{Ext}_{\mathcal{F}}^{i-1}\left(F, \omega_{n}(Y)\right)=0$ (on rappelle que $F$ est supposé $\tilde{\nabla}_{n}$-nilpotent) montrent que le morphisme $u: \operatorname{Ext}_{\mathcal{F}}^{i}\left(F, \omega_{n}(X)\right) \rightarrow \operatorname{Ext}_{\mathcal{F}}^{i}\left(F, \omega_{n}(X) \otimes I_{E_{n}}\right)$ est injectif. On en déduit, en considérant la colonne de gauche du diagramme (18), un monomorphisme $\operatorname{Ext}_{\mathcal{F}}^{i}\left(F, \omega_{n}(X)\right) \hookrightarrow \operatorname{Ext}_{\mathcal{F}}^{i}\left(\tilde{\nabla}_{n} F, \omega_{n}(X)\right)$, ce pour tous objets $F$ de $\mathcal{N} i l_{\tilde{\nabla}_{n}}$ et $X$ de $\mathcal{F}_{\mathcal{G} r, n}$. Cela fournit, par récurrence sur l'indice de $\tilde{\nabla}_{n}$-nilpotence de $F$, la nullité du groupe d'extensions $\operatorname{Ext}_{\mathcal{F}}^{i}\left(F, \omega_{n}(X)\right)$, ce qui achève la démonstration.

Remarque 15.3.2. - La proposition 15.2.1 montre que le théorème 15.3.1 est une généralisation du résultat d'annulation cohomologique du corollaire 10.2.2 (dont elle ne fournit toutefois pas l'isomorphisme obtenu pour $k=n$ ). Pour $n=1$, ces deux résultats sont identiques. La démonstration du théorème 15.3.1 est une conséquence formelle de l'injectivité du morphisme $b_{n, X}$ du diagramme (16); la seule difficulté technique réside dans l'inexactitude du foncteur $\tilde{\nabla}_{n}$, qui oblige à transiter par les foncteurs exacts $\Delta_{\mathbb{F}_{2}}$ et $\Delta_{E_{n}}$ pour passer aux groupes d'extensions. Conceptuellement, 
cette démonstration procède d'idées très voisines de celles employées pour établir le théorème 10.2.1, reposant sur des adjonctions entre foncteurs décalages et produits tensoriels convenables.

Nous terminons ce chapitre en donnant une nouvelle forme de la conjecture artinienne extrêmement forte, en termes de $\nabla$-nilpotence.

Conjecture 15.3.3. - Un objet de type fini de $\mathcal{F}$ est induit de hauteur au plus $n-1$ si et seulement s'il est $\tilde{\nabla}_{n}$-nilpotent.

Corollaire 15.3.4. - La conjecture 15.3.3 est équivalente à la conjecture artinienne extrêmement forte.

Démonstration. - Cela provient de l'épaisseur de $\mathcal{N} i l_{\tilde{\nabla}_{n}}$ (cf. proposition 1.5.7), de la non $\tilde{\nabla}_{n}$-nilpotence des foncteurs $\omega_{n}(X)$ pour $X \in \mathrm{Ob} \mathcal{F}_{\mathcal{G} r, n}$ non nul (déduite du théorème 15.3.1) et de la $\tilde{\nabla}_{n}$-nilpotence des foncteurs $\omega_{i}(X)$ pour $i<n$ et $X \in$ Ob $\mathcal{F}_{\mathcal{G} r, i}$ fini (cf. proposition 15.2.1). 



\section{CHAPITRE 16}

\section{THÉORÈMES FONDAMENTAUX}

Nous nous consacrons désormais aux applications des résultats du chapitre précédent à la structure de la catégorie $\mathcal{F}$. La première d'entre elle, présentée dans la section 16.1 (théorème 16.1.13), a trait aux groupes de Grothendieck. Des catégories de foncteurs, elle n'utilise les estimations de la section 15.2 que dans le cas des foncteurs pseudo-constants (déjà traité dans [Pow98c]), et les propriétés de base des objets finis des catégories $\mathcal{F}$ et $\mathcal{F}_{\mathcal{G} r}$. Un autre ingrédient essentiel provient de la théorie des représentations : la représentation de Steinberg (modulaire) des groupes linéaires occupe une place privilégiée ${ }^{(1)}$, parce que les facteurs de composition du foncteur de Powell qui lui est associé s'avèrent bien plus faciles à contrôler à l'aide des foncteurs $\tilde{\nabla}_{n}$ que dans le cas général. Outre cette observation assez directe, qui repose uniquement sur le fait que la partition régulière associée à la représentation de Steinberg a une longueur égale à son premier terme, nous emploierons une propriété profonde de la classe de cette représentation dans l'anneau de Grothendieck de l'algèbre du groupe linéaire, qui caractérise l'idéal qu'elle engendre (cf. lemme 16.1.5, dû à Ballard et Lusztig).

Le reste de ce chapitre consiste en la démonstration du théorème de simplicité généralisé (théorème 16.2.7) et de ses conséquences sur la conjecture artinienne extrêmement forte, notamment le théorème 16.4.5, qui donne le caractère noethérien de type 2 de $P^{\otimes 2} \otimes F$, où $F \in \mathrm{Ob} \mathcal{F}$ est fini, et où l'on note $P$ le projectif $P_{\mathbb{F}_{2}}$. Grâce aux préliminaires du chapitre 15, nous pourrons emprunter une voie tout-à-fait analogue à celle du chapitre 14 .

Outre qu'ils reposent sur l'emploi des foncteurs $\omega$ et $\tilde{\nabla}_{n}$, les deux volets principaux de ce dernier chapitre se rejoignent dans l'observation suivante : la compréhension fine de la catégorie $\mathcal{F}$ nécessite un contrôle du comportement générique des représentations des groupes linéaires (ou symétriques). L'application du théorème de simplicité

\footnotetext{
$\overline{{ }^{(1)} \text { Dans [Dja07] }}$, nous fournissons une contribution à la conjecture artinienne qui met en évidence, d'une autre façon, l'importance de cette représentation pour l'étude de la catégorie $\mathcal{F}$.
} 
généralisé à la structure de $P^{\otimes 2} \otimes F$ (pour $F$ fini) comme la propriété d'injectivité du morphisme induit par le foncteur $\omega: \mathcal{F}_{\mathcal{G} r} \rightarrow \mathcal{F}$ entre groupes de Grothendieck ne peuvent s'établir par les seules méthodes fonctorielles; une partie du substrat non formel sous-jacent à la catégorie $\mathcal{F}$ réside manifestement dans des propriétés intrinsèques des groupes linéaires.

\subsection{Le morphisme $\omega_{*}: G_{0}^{f}\left(\mathcal{F}_{\mathcal{G} r}\right) \rightarrow G_{0}^{t f}(\mathcal{F}) \rightarrow \widehat{G}_{0}^{f}(\mathcal{F})$}

On rappelle que les notations relatives aux groupes de Grothendieck sont données dans la section B.3, et que les notations liées aux partitions et facteurs de composition ont été introduites dans la section 1.4.

Notation 16.1.1. - Nous désignerons par $\widehat{G}_{0}^{f}(\mathcal{F})$ le groupe abélien $\mathbb{Z}^{\mathfrak{p}}$ produit de copies de $\mathbb{Z}$ indexées par les partitions régulières.

Pour toute partition régulière $\lambda$, la fonction de multiplicité $m_{\lambda}:$ Ob $\mathcal{F}^{t f} \rightarrow \mathbb{Z}$ est additive en ce sens que si $0 \rightarrow A \rightarrow B \rightarrow C \rightarrow 0$ est une suite exacte courte de $\mathcal{F}$, alors $m_{\lambda}(B)=m_{\lambda}(A)+m_{\lambda}(C)$. Elle induit donc un morphisme de groupes, encore noté $m_{\lambda}$ par abus, de $G_{0}^{t f}(\mathcal{F})$ vers $\mathbb{Z}$.

Notation 16.1.2. - Nous noterons, dans cette section, $j_{G}: G_{0}^{t f}(\mathcal{F}) \rightarrow \widehat{G}_{0}^{f}(\mathcal{F})$ le morphisme de groupes dont les composantes sont données par les $m_{\lambda}(\lambda \in \mathfrak{p})$. Nous l'appellerons morphisme canonique.

Remarque 16.1.3. - La composée $\mathbb{Z}[\mathfrak{p}] \simeq G_{0}^{f}(\mathcal{F}) \rightarrow G_{0}^{t f}(\mathcal{F}) \stackrel{j_{G}}{\longrightarrow} \widehat{G}_{0}^{f}(\mathcal{F})=\mathbb{Z}^{\mathfrak{p}}$ (où le premier morphisme est induit par l'inclusion $\mathrm{Ob} \mathcal{F}^{f} \hookrightarrow \mathrm{Ob} \mathcal{F}^{t f}$ ) coïncide avec l'inclusion canonique. Via cette inclusion, on peut voir le groupe $\widehat{G}_{0}^{f}(\mathcal{F})$ comme un complété convenable de $G_{0}^{f}(\mathcal{F})$.

Le foncteur $\omega: \mathcal{F}_{\mathcal{G} r} \rightarrow \mathcal{F}$ est exact, respecte la structure tensorielle, en prenant à la source le produit tensoriel total $\widetilde{\otimes}$, et transforme un objet fini de $\mathcal{F}_{\mathcal{G} r}$ en un objet de type fini de $\mathcal{F}$. Par conséquent, il induit un morphisme d'anneaux $\omega_{*}$ : $G_{0}^{f}\left(\mathcal{F}_{\mathcal{G}_{r}}\right) \rightarrow G_{0}^{t f}(\mathcal{F})$. Nous noterons encore, par abus, $\omega_{*}: G_{0}^{f}\left(\mathcal{F}_{\mathcal{G} r}\right) \rightarrow \widehat{G}_{0}^{f}(\mathcal{F})$ le morphisme de groupes composé du morphisme précédent et du morphisme canonique $j_{G}: G_{0}^{t f}(\mathcal{F}) \rightarrow \widehat{G}_{0}^{f}(\mathcal{F})$. Notre objectif consiste à démontrer que ce morphisme $\omega_{*}$ est injectif.

Cette section utilise lourdement certaines propriétés de la représentation de Steinberg de $G L_{n}$. Avec nos conventions d'indexation des représentations simples de $G L_{n}$, $R_{<n>}$ est la représentation de Steinberg, où $<n>$ désigne la partition régulière « triangulaire $»(n, n-1, \ldots, 1)$ de $n(n+1) / 2$. On pourra se reporter à [Mit86] pour la démonstration de l'identité entre la définition fonctorielle $R_{<n>}$ et des approches plus classiques de la représentation de Steinberg. 
Le lecteur pourra également consulter [Hum87] pour un survol des propriétés de cette représentation.

La suite de cette section fait usage du symbole $Q_{\lambda}$ introduit dans la notation 2.3.13 pour désigner les foncteurs de Powell, et du symbole $\lambda_{+i}$ (où $\lambda$ est une partition) introduit dans la notation 1.5.5.

Lemme 16.1.4. - Soient $n>0$ et $i \geq 0$ des entiers et $\lambda$ une partition régulière telle que $\lambda_{1}=n$. Si $\lambda=\langle n\rangle$, alors $m_{<n>_{+i}}\left(Q_{\lambda}\right)=1$, sinon $m_{<n>_{+i}}\left(Q_{\lambda}\right)=0$.

Démonstration. - Notons $f_{\lambda}(i)=m_{<n>+i}\left(Q_{\lambda}\right)$. Le lemme découle des quatre points suivants.

(1) La fonction $f_{\lambda}: \mathbb{N} \rightarrow \mathbb{N}$ est décroissante pour toute partition régulière $\lambda$ telle que $\lambda_{1}=n$.

(2) On a $f_{\lambda}(0)=1$ si $\lambda=\langle n>, 0$ sinon.

(3) Pour tout $i \in \mathbb{N}, m_{<n>+i}\left(P_{E_{n}}\right)>0$.

(4) Pour tout $i \in \mathbb{N}, m_{<n>+i}\left(P_{E_{n}}\right)$ est une combinaison linéaire des $f_{\lambda}(i), \lambda$ parcourant les partitions régulières telles que $\lambda_{1}=n$.

On commence par remarquer que si $\mu$ est une partition régulière telle que $\mu_{1}<n$, alors le foncteur de Powell $Q_{\mu}$, qui est un quotient de $P^{\otimes \mu_{1}}$, n'a pas de facteur de composition $S_{\nu}$, si $\nu$ est une partition régulière de longueur $n$.

Comme le noyau de l'épimorphisme (corollaire 15.2.4) $\pi_{n, \rho_{n}\left(R_{\lambda}\right)}: \tilde{\nabla}_{n} Q_{\lambda} \rightarrow Q_{\lambda}$ de la section 15.2 admet une filtration finie dont les quotients sont des foncteurs de ce type (cf. proposition 15.2.6), on a $f_{\lambda}(i)=m_{<n>+i}\left(\tilde{\nabla}_{n} Q_{\lambda}\right)$. Mais $m_{<n>+i}\left(\tilde{\nabla}_{n} Q_{\lambda}\right) \geq$ $m_{<n>+i+1}\left(Q_{\lambda}\right)$ par les propositions 1.5.6 et 1.5.3.1, d'où le premier point.

Le second vient des égalités

$$
f_{\lambda}(0)=\operatorname{dim}_{\operatorname{hom}_{\mathcal{F}}}\left(Q_{<n>}, Q_{\lambda}\right)=\operatorname{dim}_{G o L_{n}}\left(R_{<n>}, R_{\lambda}\right)
$$

\section{(cf. [Pow98c]).}

Quant au troisième, c'est une conséquence du théorème 1.4.7.

Le dernier provient de ce que $P_{E_{n}} \simeq \varpi\left(P_{E_{n}}^{s u r j}\right)$ admet une filtration finie dont les quotients sont des foncteurs de Powell $Q_{\mu}$ avec $\mu_{1} \leq n$ et de la remarque précédente sur les $Q_{\mu}$ pour $\mu_{1}<n$.

Lemme 16.1.5. - Pour tout $n \in \mathbb{N}$, l'endomorphisme du groupe de Grothendieck $G_{0}^{f}\left(\mathbb{F}_{2}\left[G L_{n}\right]\right.$ Mod $)$ induit par le produit tensoriel par la représentation de Steinberg $R_{<n>}$ est injectif.

Démonstration. - D'après un théorème de Ballard et Lusztig, l'idéal de l'anneau $G_{0}^{f}\left(\mathbb{F}_{2}\left[G L_{n}\right]\right.$ Mod) engendré par $R_{<n>}$ est égal à l'image canonique de $K_{0}\left(\mathbb{F}_{2}\left[G L_{n}\right]\right.$ Mod $)$ dans $G_{0}^{f}\left(\mathbb{F}_{2}\left[G L_{n}\right]\right.$ Mod). Pour une démonstration de ce résultat, voir [CR87], chapitre 8 , théorème 72.10 (la proposition 9.3 de cet ouvrage montre que les groupes linéaires sur $\mathbb{F}_{2}$ vérifient les hypothèses dudit théorème). On en déduit, par la théorie 
générale des représentations modulaires (cf. théorème 21.22 de [CR90]), que le conoyau de l'endomorphisme de $G_{0}^{f}\left(\mathbb{F}_{2}\left[G L_{n}\right]\right.$ Mod) induit par le produit tensoriel par $R_{<n>}$ est fini. Comme $G_{0}^{f}\left(\mathbb{F}_{2}\left[G L_{n}\right]\right.$ Mod) est un $\mathbb{Z}$-module libre de rang fini, cet endomorphisme est injectif.

Notation 16.1.6. — Étant donnés des entiers $n, i$ et $d$, nous noterons

$$
\begin{aligned}
& \mathfrak{p}_{n, i}=\left\{\lambda \in \mathfrak{p} \mid \lambda_{n} \geq i\right\}, \\
& \mathfrak{p}_{n, i, \leq d}=\left\{\lambda \in \mathfrak{p}_{n, i}|| \lambda \mid \leq d\right\}, \\
& \mathfrak{p}_{<i}=\left\{\lambda \in \mathfrak{p} \mid \lambda_{1}<i\right\}, \\
& \mathfrak{p}_{n, i}^{\leq n}=\left\{\lambda \in \mathfrak{p}_{n, i} \mid l(\lambda)=n\right\}, \\
& \mathfrak{p}_{n, i, \leq d}^{\leq n}=\mathfrak{p}_{n, i}^{\leq n} \cap \mathfrak{p}_{n, i, \leq d}
\end{aligned}
$$

où l'on convient que $\lambda_{n}=+\infty$ si $n \leq 0$ et considérerons les quotients suivants du groupe $\widehat{G}_{0}^{f}(\mathcal{F})$ :

$$
\begin{aligned}
& \widehat{G}_{0}^{f}(\mathcal{F})_{n, i}=\mathbb{Z}^{\mathfrak{p}_{n, i}}, \\
& \widehat{G}_{0}^{f}(\mathcal{F})_{n, i, \leq d}=\mathbb{Z}^{\mathfrak{p}_{n, i, \leq d}} .
\end{aligned}
$$

On rappelle que si $\lambda$ est une partition régulière, $I_{\lambda}$ désigne l'enveloppe injective de l'objet simple $S_{\lambda}$ de $\mathcal{F}$.

Lemme 16.1.7. - Pour tout $n \in \mathbb{N}$, le morphisme

$$
\alpha: \mathbb{Z}\left[\mathfrak{p}_{<n+1}\right] \rightarrow G_{0}^{f}\left(\mathbb{F}_{2}\left[G L_{n}\right] \text { Mod }\right) \quad[\lambda] \mapsto\left[I_{\lambda}\left(E_{n}\right)\right]
$$

a un conoyau fini.

Démonstration. - Notons $M=\left(M_{\lambda, \mu}\right)_{\lambda, \mu \in \mathfrak{p}_{<n+1}}$ la matrice définie par le fait que $I_{\lambda}$ possède une filtration finie dont les sous-quotients sont les duaux $J_{\mu}$ de $Q_{\mu}$, chacun apparaissant avec la multiplicité $M_{\lambda, \mu}$. L'analyse effectuée dans le $\S 4$ de l'article [Pow98c] (où la matrice $M$ est notée $\left(\alpha_{\lambda, \mu}\right)$ ) montre que $M$ est une matrice inversible sur $\mathbb{Q}$, car il en est de même pour les matrices de Cartan des groupes finis $G L_{i}$.

Les colonnes de la matrice $M^{-1}$ ont pour images par $\alpha \otimes \mathbb{Q}$ les images des $J_{\lambda}\left(E_{n}\right)$ (où $\left.\lambda \in \mathfrak{p}_{<n+1}\right)$ dans $G_{0}^{f}\left(\mathbb{F}_{2}\left[G L_{n}\right]\right.$ Mod $) \otimes \mathbb{Q}$. Comme $J_{\lambda}\left(E_{n}\right) \simeq R_{\lambda}$ si $\lambda_{1}=n$, cela montre que le morphisme $\alpha \otimes \mathbb{Q}$ est surjectif, d'où la conclusion.

Notation 16.1.8. - Dans la suite de cette section, si $\alpha$ est une partition régulière telle que $\alpha_{1}=n$, nous noterons $P_{\alpha}^{G L_{n}}$ la couverture projective de $R_{\alpha}$ dans $\mathbb{F}_{2}\left[G L_{n}\right]$ Mod.

Nous désignerons par $\Delta_{\mu}$, pour $\mu \in \mathfrak{p}$, l'endofoncteur exact $\left(\cdot: I_{\mu}\right)$ de $\mathcal{F}$. C'est un facteur direct de $\Delta_{E_{n}}$, où $n=\mu_{1}$.

Proposition 16.1.9. - Le morphisme

$$
\beta: G_{0}^{f}\left(\mathbb{F}_{2}\left[G L_{n}\right] \text { Mod }\right) \rightarrow \mathbb{Z}^{\mathfrak{p}_{<n+1}} \quad[M] \mapsto\left(m_{<n>+i}\left(\Delta_{\mu} \omega_{n} \rho_{n}(M)\right)\right)_{\mu}
$$

est injectif. 
Démonstration. - Par les propositions 9.2.1 et 9.1.2, il existe un épimorphisme

$$
\Delta_{\mu} Q_{\lambda} \rightarrow \omega_{n}\left(\rho_{n}\left(R_{\lambda}\right): \iota_{n}\left(I_{\mu}\right)\right)
$$

dont le noyau est l'image par $\omega_{\leq n-1}$ d'un foncteur fini pseudo-constant de $\mathcal{F}_{\mathcal{G} r, \leq n-1}$ (cf. démonstration de la proposition 15.1.5). On en déduit (cf. démonstration du lemme 16.1.4) l'égalité

$$
m_{<n>+i}\left(\Delta_{\mu} Q_{\lambda}\right)=m_{<n>+i}\left(\omega_{n}\left(\rho_{n}\left(R_{\lambda}\right): \iota_{n}\left(I_{\mu}\right)\right)\right) .
$$

On a $\left(\rho_{n}\left(R_{\lambda}\right): \iota_{n}\left(I_{\mu}\right)\right) \simeq \rho_{n}\left(R_{\lambda}: I_{\mu}\left(E_{n}\right)\right)$ par la proposition 9.2.2. Le lemme 16.1.4 donne alors

$$
m_{<n>+i}\left(\Delta_{\mu} Q_{\lambda}\right)=m_{R_{<n>}}\left(R_{\lambda} \otimes I_{\mu}\left(E_{n}\right)^{*}\right) .
$$

On a donc, puisque $\mathbb{F}_{2}$ est un corps de décomposition de $\mathbb{F}_{2}\left[G L_{n}\right]$,

$$
m_{<n>+i}\left(\Delta_{\mu} Q_{\lambda}\right)=\operatorname{dim} \operatorname{hom}_{G L_{n}}\left(R_{<n>}, R_{\lambda} \otimes I_{\mu}\left(E_{n}\right)^{*}\right) .
$$

Le lemme 16.1.7 montre alors que le noyau du morphisme $\beta$ est inclus dans le noyau du morphisme

$$
G_{0}^{f}\left(\mathbb{F}_{2}\left[G L_{n}\right] \text { Mod }\right) \rightarrow \mathbb{Z} \quad \operatorname{dim} \operatorname{hom}_{G L_{n}}\left(R_{<n>}, R_{\lambda} \otimes M^{*}\right)
$$

pour tout $G L_{n}$-module $M$.

Or on a

$$
\begin{gathered}
\operatorname{dim} \operatorname{hom}_{G L_{n}}\left(R_{<n>}, R_{\lambda} \otimes\left(P_{\mu}^{G L_{n}}\right)^{*}\right)=\operatorname{dim}_{\operatorname{hom}} L_{L_{n}}\left(R_{<n>} \otimes P_{\mu}^{G L_{n}}, R_{\lambda}\right) \\
=\operatorname{dim} \operatorname{hom}_{G L_{n}}\left(P_{\mu}^{G L_{n}}, R_{\lambda} \otimes R_{<n>}\right)=m_{R_{\mu}}\left(R_{\lambda} \otimes R_{<n>}\right),
\end{gathered}
$$

où l'on a utilisé l'auto-dualité et la projectivité de la représentation de Steinberg (cf. [Jan03], chapitre 10, (10.1) et (10.2)).

L'injectivité du morphisme $\beta$ découle maintenant du lemme 16.1.5

Lemme 16.1.10. - Soient $n$ et $i$ deux entiers positifs. Il existe un entier $d$ tel que le morphisme de groupes

$$
G_{0}^{f}\left(\mathbb{F}_{2}\left[G L_{n}\right] \text { Mod }\right) \stackrel{\left(\omega_{n} \rho_{n}\right)_{*}}{\longrightarrow} G_{0}^{t f}(\mathcal{F}) \stackrel{j_{G}}{\longrightarrow} \widehat{G}_{0}^{f}(\mathcal{F}) \rightarrow \mathbb{Z}^{\mathfrak{p}_{n, i, \leq d}^{\leq n}}
$$

est injectif.

Démonstration. - Soit $\mu \in \mathfrak{p}_{<n+1}$. Pour tout $G L_{n}$-module fini $M$, on a

$$
m_{<n>+i}\left(\Delta_{\mu} \omega_{n} \rho_{n}(M)\right)=\sum_{\lambda \in \mathfrak{p}} m_{<n>+i}\left(\Delta_{\mu} S_{\lambda}\right) m_{\lambda}\left(\omega_{n} \rho_{n}(M)\right)
$$

(somme dont seul un nombre fini des termes sont non nuls). Si $\lambda \in \mathfrak{p}$ est telle que $\lambda \vdash \omega_{n} \rho_{n}(M)$ et $\left\langle n>_{+i} \vdash \Delta_{\mu} S_{\lambda}\right.$, on a $l(\lambda) \leq n$ parce que $\omega_{n} \rho_{n}(M)$ s'obtient par extensions de quotients de $P_{E_{n}}$; en appliquant $\left(\tilde{\nabla}_{n}\right)^{i}$ à la relation $\left\langle n>_{+i} \vdash \widetilde{\Delta}^{n} S_{\lambda}\right.$, on obtient, compte-tenu des propositions 1.5.3 et 1.5.6, $\left(\tilde{\nabla}_{n}\right)^{i} S_{\lambda} \neq 0$, puis $\lambda_{n} \geq i$. Par ailleurs, comme $l(\lambda)=n$, si $\nu \vdash \widetilde{\Delta}^{n} S_{\lambda}$ et $l(\nu)=n$, alors $|\nu| \geq|\lambda|-n^{2}$ grâce à la proposition 4.3.1.2 de [Pow00b] et au théorème 1.4.7, donc $n(n+1) / 2+n i=\mid<$ $n>_{+i}|\geq| \lambda \mid-n^{2}$. 
Par conséquent, si $d \geq n(n+1) / 2+n i+n^{2}$, on peut compléter le diagramme

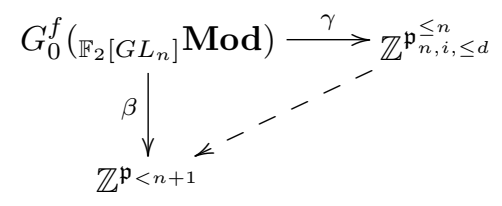

où $\beta$ est le morphisme de la proposition 16.1.9. L'injectivité de $\beta$ donnée par cette proposition implique donc celle de $\gamma$.

Lemme 16.1.11. - Pour tous entiers $n, i \geq 0$, le morphisme de groupes

$$
\mathbb{Z}\left[\mathfrak{p}_{n, i}^{\leq n}\right] \otimes \mathbb{Z}\left[\mathfrak{p}_{<i}\right] \hookrightarrow G_{0}^{f}(\mathcal{F}) \otimes G_{0}^{f}(\mathcal{F}) \rightarrow G_{0}^{f}(\mathcal{F}) \rightarrow \mathbb{Z}\left[\mathfrak{p}_{n, i}\right]
$$

composé de l'inclusion déduite de l'isomorphisme $G_{0}^{f}(\mathcal{F}) \simeq \mathbb{Z}[\mathfrak{p}]$, du produit de l'anneau $G_{0}^{f}(\mathcal{F})$ et de la projection $G_{0}^{f}(\mathcal{F}) \simeq \mathbb{Z}[\mathfrak{p}] \rightarrow \mathbb{Z}\left[\mathfrak{p}_{n, i}\right]$ déduite de l'inclusion $\mathfrak{p}_{n, i} \hookrightarrow$ $\mathfrak{p}$, est injectif.

Démonstration. - Si $\lambda \in \mathfrak{p}_{n, i}^{\leq n}$ et $\mu \in \mathfrak{p}_{<i}$, alors la partition $(\lambda, \mu)$ est régulière et appartient à $\mathfrak{p}_{n, i}$. De plus, la fonction $f: \mathfrak{p}_{n, i}^{\leq n} \times \mathfrak{p}_{<i} \rightarrow \mathfrak{p}_{n, i}$ associant aux partitions $\lambda$ et $\mu$ la partition $(\lambda, \mu)$ est injective, ce qui permet d'identifier cette partition au couple $(\lambda, \mu)$.

Soit $a: \mathfrak{p}_{n, i}^{\leq n} \times \mathfrak{p}_{<i} \rightarrow \mathbb{N}$ une bijection telle que $a(\alpha)<a(\beta)$ si $|\alpha|<|\beta|$ ou $|\alpha|=|\beta|$ et $\alpha>\beta$, et définissons une matrice $\left(u_{i, j}\right)_{(i, j) \in \mathbb{N}^{2}}$ par l'égalité $u_{a(\lambda, \mu), a(\nu)}=m_{\nu}\left(S_{\lambda} \otimes S_{\mu}\right)$ : c'est la matrice dans la base canonique (indexée par $a$ ) du morphisme

$$
\mathbb{Z}\left[\mathfrak{p}_{n, i}^{\leq n} \times \mathfrak{p}_{<i}\right] \simeq \mathbb{Z}\left[\mathfrak{p}_{n, i}^{\leq n}\right] \otimes \mathbb{Z}\left[\mathfrak{p}_{<i}\right] \rightarrow \mathbb{Z}\left[\mathfrak{p}_{n, i}\right] \rightarrow \mathbb{Z}\left[\mathfrak{p}_{n, i}^{\leq n} \times \mathfrak{p}_{<i}\right]
$$

où la flèche centrale est celle de l'énoncé et le dernier épimorphisme est induit par $f$.

Le théorème 1.4.7 montre que la matrice $\left(u_{i, j}\right)_{(i, j) \in \mathbb{N}^{2}}$ est triangulaire, et la proposition 1.4.9 que ses coefficients diagonaux valent 1 , ce qui achève la démonstration.

Lemme 16.1.12. - Soient $n$ et $i$ des entiers naturels. Le morphisme

$$
G_{0}^{f}\left(\mathcal{F}_{\mathcal{G} r, n}\right) \hookrightarrow G_{0}^{f}\left(\mathcal{F}_{\mathcal{G} r}\right) \stackrel{\omega_{*}}{\longrightarrow} \widehat{G}_{0}^{f}(\mathcal{F}) \rightarrow \widehat{G}_{0}^{f}(\mathcal{F})_{n, i}
$$

est injectif.

Démonstration. - Soient $j \in \mathbb{N}$ et $G(j)_{n}$ le sous-groupe de $G_{0}^{f}\left(\mathcal{F}_{\mathcal{G} r, n}\right)$ engendré par les classes d'objets simples de degré au plus $j$; nous noterons simplement $G(j)$ pour $G(j)_{0}$. Comme $G_{0}^{f}\left(\mathcal{F}_{\mathcal{G} r, n}\right)$ est la réunion croissante des sous-groupes $G(j)_{n}$, il suffit de montrer que la restriction à $G(j)_{n}$ du morphisme de l'énoncé est injective pour tout $j$.

Le corollaire 5.5.11 montre que le foncteur $\xi_{n}: \mathcal{F} \otimes \mathbb{F}_{2}\left[G L_{n}\right]$ Mod $\rightarrow \mathcal{F}_{\mathcal{G} r, n}$ induit un isomorphisme

$$
G_{0}^{f}\left(\mathbb{F}_{2}\left[G L_{n}\right] \text { Mod }\right) \otimes G(j) \stackrel{\simeq}{\longrightarrow} G(j)_{n}
$$


Par la proposition 5.1.7.4, on en déduit un diagramme commutatif

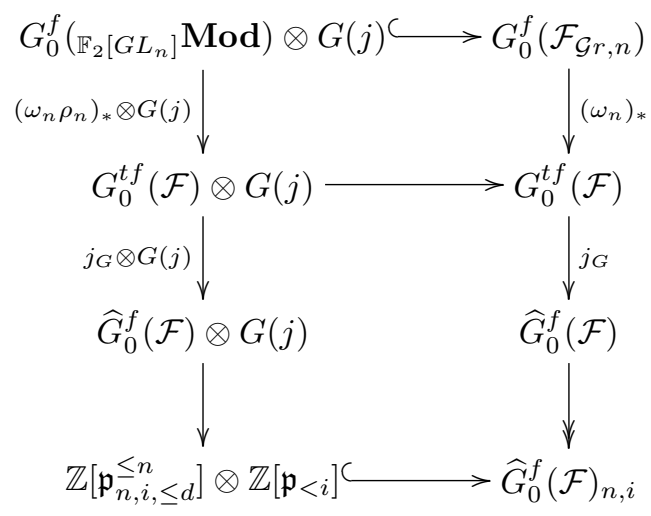

pour $i>j$ et un entier $d$ convenable, dans lequel :

- les flèches horizontales sont induites par le produit tensoriel — celle du bas est injective par le lemme 16.1.11;

- les flèches verticales inférieures sont induites par les projections canoniques et l'inclusion $G(j) \subset \mathbb{Z}\left[\mathfrak{p}_{<i}\right]$ déduite de l'inégalité $i>j$ (on note que $\mathbb{Z}\left[\mathfrak{p}_{n, i, \leq d}^{\leq n}\right] \otimes$ $\mathbb{Z}\left[\mathfrak{p}_{<i}\right] \simeq \mathbb{Z}^{\mathfrak{p}_{n, i, \leq d}^{\leq n}} \otimes \mathbb{Z}^{\mathfrak{p}_{<i}}$ par finitude des ensembles $\mathfrak{p}_{n, i, \leq d}^{\leq n}$ et $\left.\mathfrak{p}_{<i}\right) ;$

- l'entier $d$ est choisi, conformément au lemme 16.1.10, pour que la composée verticale de gauche soit injective.

La composée $G_{0}^{f}\left(\mathbb{F}_{2}\left[G L_{n}\right]\right.$ Mod $) \otimes G(j) \rightarrow \widehat{G}_{0}^{f}(\mathcal{F})_{n, i}$ obtenue en suivant le diagramme est donc injective (suivre la moitié gauche) ; elle s'identifie à la restriction à $G(j)_{n}$ du morphisme de l'énoncé (suivre la moitié droite). Celle-ci est donc injective si $i>j$, donc pour tout $i$, ce qui achève la démonstration.

Nous pouvons désormais établir le résultat principal de cette section.

Théorème 16.1.13. - Le morphisme de groupes $\omega_{*}: G_{0}^{f}\left(\mathcal{F}_{\mathcal{G} r}\right) \rightarrow \widehat{G}_{0}^{f}(\mathcal{F})$ est injectif.

Démonstration. - Il suffit d'établir que pour tout $n \in \mathbb{N}$, la composée

$$
G_{0}^{f}\left(\mathcal{F}_{\mathcal{G} r, \leq n}\right) \hookrightarrow G_{0}^{f}\left(\mathcal{F}_{\mathcal{G} r}\right) \stackrel{\omega_{*}}{\longrightarrow} \widehat{G}_{0}^{f}(\mathcal{F})
$$

est injective, ou encore que pour tout $n$ et tout $j \in \mathbb{N}$, la restriction $f_{n, j}$ de ce morphisme au sous-groupe $G(j)_{\leq n}$ de $G_{0}^{f}\left(\mathcal{F}_{\mathcal{G} r, \leq n}\right)$ engendré par les classes de foncteurs simples de degré au plus $j$ est injective.

Pour $i>j$, la composée $G(j)_{\leq n-1} \hookrightarrow G_{0}^{f}\left(\mathcal{F}_{\mathcal{G} r}\right) \rightarrow \widehat{G}_{0}^{f}(\mathcal{F}) \rightarrow \widehat{G}_{0}^{f}(\mathcal{F})_{n, i}$ est nulle : si $\lambda \vdash P^{\otimes k} \otimes F$ avec $k<n$ et $\operatorname{deg} F<i$, alors $\left.\lambda \notin \mathfrak{p}_{n, i}\right)$. Le lemme précédent permet d'en déduire que le noyau de $f_{n, j}$ est inclus dans $G(j)_{\leq n-1}$, d'où $\operatorname{ker} f_{n, j}=k e r f_{n-1, j}$. On conclut par récurrence sur $n$. 
Corollaire 16.1.14. - (1) Le morphisme d'anneaux $\omega_{*}: G_{0}^{f}\left(\mathcal{F}_{\mathcal{G} r}\right) \rightarrow G_{0}^{t f}(\mathcal{F})$ est injectif.

(2) Si la conjecture artinienne extrêmement forte est vérifiée, c'est un isomorphisme, et le morphisme de groupes $j_{G}: G_{0}^{t f}(\mathcal{F}) \rightarrow \widehat{G}_{0}^{f}(\mathcal{F})$ est injectif.

Démonstration. - Comme la conjecture artinienne extrêmement forte implique que le morphisme $\omega_{*}: G_{0}^{f}\left(\mathcal{F}_{\mathcal{G} r}\right) \rightarrow G_{0}^{t f}(\mathcal{F})$ est surjectif, la conclusion découle du théorème précédent.

\subsection{Théorème de simplicité généralisé}

Le but de cette section est d'établir le théorème 16.2.7, qui constitue un succédané de la conjecture artinienne extrêmement forte, où la filtration de Krull est remplacée par la filtration par $\nabla$-nilpotence de $\mathcal{F}$. Nous suivons la même marche qu'à la section 14.1, où les assertions 3 et 4 du lemme 14.1.5 sont remplacées par les corollaires 15.2.4 et 15.2.8 respectivement, et du corollaire 10.2.2.1 par le théorème 15.3.1.

Convention 16.2.1. - Dans toute cette section, on se donne un entier strictement positif $n$.

On rappelle que le morphisme $\pi_{n, X}: \tilde{\nabla}_{n} \omega_{n}(X) \rightarrow \omega_{n}(X)$ a été défini dans la section 15.2.

Notation 16.2.2. - Si $k$ est un entier naturel et $X$ un objet de $\mathcal{F}_{\mathcal{G} r, n}$, nous désignerons par $\pi_{n, X}^{k}:\left(\tilde{\nabla}_{n}\right)^{k} \omega_{n}(X) \rightarrow \omega_{n}(X)$ le morphisme

$$
\pi_{n, X}^{k}=\pi_{n, X} \circ \tilde{\nabla}_{n} \pi_{n, X} \circ \cdots \circ\left(\tilde{\nabla}_{n}\right)^{k-1} \pi_{n, X} .
$$

Afin de mener des estimations explicites à l'aide de ces morphismes, nous identifions le morphisme $\pi_{n, X}$ sur les objets grâce au lemme suivant, qui emploie le morphisme $\beta_{n, X}$ du diagramme (17) de la section 15.2.

Lemme 16.2.3. - Soit $X \in \mathrm{Ob} \mathcal{F}_{\mathcal{G} r, n}$.

(1) Le morphisme $\beta_{n, X}: \Delta_{E_{n}} \omega_{n}(X) \rightarrow \omega_{n}(X)$ est la composée de la projection canonique $\Delta_{E_{n}} \omega_{n}(X) \rightarrow \tilde{\nabla}_{n} \omega_{n}(X)$ et du morphisme $\pi_{n, X}: \tilde{\nabla}_{n} \omega_{n}(X) \rightarrow \omega_{n}(X)$.

(2) Étant donnés $V \in \mathrm{Ob}^{f}$ et $W \in \mathcal{G} r_{n}(V)$, le morphisme

$$
X\left(V \oplus E_{n}, W\right) \hookrightarrow \omega_{n}(X)\left(V \oplus E_{n}\right)=\Delta_{E_{n}} \omega_{n}(X)(V) \stackrel{\left(\beta_{n, X}\right)_{V}}{\longrightarrow} \omega_{n}(X)(V)
$$

est nul si l'une des deux applications linéaires $W \hookrightarrow V \oplus E_{n} \rightarrow E_{n}$ et $W \hookrightarrow V \oplus E_{n} \rightarrow$ $V$ est de rang $<n$, et est induite par la projection $V \oplus E_{n} \rightarrow V$ sinon.

Démonstration. - La vérification de la première assertion est immédiate. La seconde résulte des observations suivantes, où l'on note $\tilde{X}$ le prolongement par zéro à $\mathcal{F}_{\mathcal{G} r}$ de $X$ : 
(1) le morphisme $\beta_{n, X}$ s'obtient en prenant la restriction à $\mathcal{F}_{\mathcal{G} r, n}$ du morphisme $\left(\tilde{X}: \iota_{n}\left(I_{E_{n}}\right)\right) \rightarrow\left(\tilde{X}: I_{\left(E_{n}, E_{n}\right)}^{\mathcal{G} r}\right) \rightarrow \tilde{X}$ induit par le diagramme (12) de la proposition 15.1.7 puis en appliquant le foncteur $\omega_{n}$;

(2) par la proposition 9.2.3, évalué sur un objet $(V, B)$ de $\mathcal{E}_{\mathcal{G} r}^{f}$, le morphisme $(\tilde{X}$ : $\left.\iota_{n}\left(I_{E_{n}}\right)\right) \rightarrow\left(\tilde{X}: I_{\left(E_{n}, E_{n}\right)}^{\mathcal{G} r}\right)$ est donné par la projection

$$
\bigoplus_{\substack{W \in \mathcal{G} r\left(V \oplus E_{n}\right) \\
i m\left(W \hookrightarrow V \oplus E_{n} \rightarrow V\right)=B}} \tilde{X}\left(V \oplus E_{n}, W\right) \rightarrow \bigoplus_{\begin{array}{c}
W \in \mathcal{G} r\left(V \oplus E_{n}\right) \\
i m\left(W \hookrightarrow V \oplus E_{n} \rightarrow V\right)=B \\
i m\left(W \hookrightarrow V \oplus E_{n} \rightarrow E_{n}\right)=E_{n}
\end{array}} \tilde{X}\left(V \oplus E_{n}, W\right) ;
$$

(3) le morphisme $\left(\tilde{X}: I_{\left(E_{n}, E_{n}\right)}^{\mathcal{G} r}\right) \rightarrow \tilde{X}$, évalué sur un objet $(V, B)$, est donné par l'application linéaire

$$
\begin{aligned}
& \bigoplus_{\substack{W \in \mathcal{G} r\left(V \oplus E_{n}\right) \\
i m\left(W \hookrightarrow V \oplus E_{n} \rightarrow V\right)=B}} \tilde{X}\left(V \oplus E_{n}, W\right) \rightarrow \tilde{X}(V, B) \\
& i m\left(W \hookrightarrow V \oplus E_{n} \rightarrow E_{n}\right)=E_{n}
\end{aligned}
$$

dont chaque composante est induite par la projection $V \oplus E_{n} \rightarrow V$. Cela provient du corollaire 5.2.10 et du lemme de Yoneda.

Lemme 16.2.4. - Soient $V$ un espace vectoriel de dimension finie, $X$ un objet de $\mathcal{F}_{\mathcal{G} r, n}, H$ un sous-espace vectoriel de dimension $n$ de $V^{*}$ et $x$ un élément de $\omega_{n}(X)(V)$. Notons $a_{x}: P_{V} \rightarrow \omega_{n}(X)$ le morphisme de $\mathcal{F}$ représenté par $x$, et $s_{H}=\sum_{h \in H}[h] \in$ $k_{n} P\left(V^{*}\right) \subset \mathbb{F}_{2}\left[V^{*}\right]$.

Le morphisme

$$
P_{V} \stackrel{t \mapsto s_{H} \otimes t}{\longrightarrow} k_{n} P\left(V^{*}\right) \otimes P_{V} \simeq \tilde{\nabla}_{n} P_{V} \stackrel{\tilde{\nabla}_{n}\left(a_{x}\right)}{\longrightarrow} \tilde{\nabla}_{n} \omega_{n}(X) \stackrel{\pi_{n, X}}{\longrightarrow} \omega_{n}(X)
$$

représente l'élément $\Pi_{X, V, H}^{\omega}(x)$ de $\omega_{n}(X)(V)$, où $\Pi_{X, V, H}^{\omega}$ désigne la projection de $\omega_{n}(X)(V)$ sur les facteurs directs $X(V, W)$, où $W \in \mathcal{G} r_{n}(V)$ est tel que $H \cap W^{\perp}=\{0\}$.

Démonstration. - Le diagramme

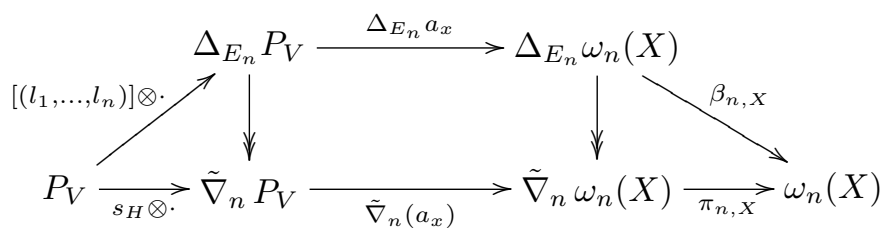

commute, où :

- $\left\{l_{1}, \ldots, l_{n}\right\}$ désigne une base de $H$ (on a noté $\left(l_{1}, \ldots, l_{n}\right)$ l'élément de $\operatorname{hom}_{\mathcal{E}}\left(V, E_{n}\right)$ dont les composantes sont les $\left.l_{i}\right)$;

- l'on a identifié $\Delta_{E_{n}} P_{V}$ à $\mathbb{F}_{2}\left[\operatorname{hom}_{\mathcal{E}}\left(V, E_{n}\right)\right] \otimes P_{V}\left(\right.$ et $\tilde{\nabla}_{n} P_{V}$ à $\left.k_{n} P\left(V^{*}\right) \otimes P_{V}\right)$; 
L'élément $\left[\left(l_{1}, \ldots, l_{n}\right)\right] \otimes\left[i d_{V}\right]$ de $\Delta_{E_{n}} P_{V}(V)$ s'envoie par $\Delta_{E_{n}} a_{x}$ sur l'élément de $\Delta_{E_{n}} \omega_{n}(X)(V)=\omega_{n}(X)\left(V \oplus E_{n}\right)$ image par $f=\left(i d_{V}, l_{1}, \ldots, l_{n}\right): V \rightarrow V \oplus E_{n}$ de $x$.

Soit $W \in \mathcal{G} r_{n}(V)$. Le lemme 16.2.3 montre que la composée

$$
X(V, W) \hookrightarrow \omega_{n}(X)(V) \stackrel{f_{*}}{\longrightarrow} \omega_{n}(X)\left(V \oplus E_{n}\right) \stackrel{\beta_{n, X}}{\longrightarrow} \omega_{n}(X)(V)
$$

est nulle si la projection de $f(W)$ sur $E_{n}$ en est un sous-espace strict, condition équivalente à la non-inversibilité de la restriction à $W$ de $\left(l_{1}, \ldots, l_{n}\right)$, ou encore à $H \cap W^{\perp} \neq\{0\}$, et que sinon elle est égale à l'inclusion $X(V, W) \hookrightarrow \omega_{n}(X)(V)$, puisque la composée de $f: V \rightarrow V \oplus E_{n}$ et de la projection $V \oplus E_{n} \rightarrow V$ est l'identité. Cela établit le lemme.

Notation 16.2.5. - Dans cette section, si $V$ est un espace vectoriel de dimension finie, $W$ un sous-espace de dimension $n$ de $V$ et $H$ un sous-espace de dimension $n$ de $V^{*}$, nous noterons $\langle H, W\rangle$ l'élément de $\mathbb{F}_{2}$ égal à 1 si $H \cap W^{\perp}=\{0\}, 0$ sinon. Autrement dit, si $\left(w_{1}, \ldots, w_{n}\right)$ (resp. $\left.\left(l_{1}, \ldots, l_{n}\right)\right)$ est une base de $W$ (resp. $\left.H\right)$, on a $<H, W>=\operatorname{det}\left(l_{i}\left(w_{j}\right)\right)$.

La propriété de stabilisation suivante, qui fournit la partie « concrète » de la démonstration du théorème 16.2.7, généralise la proposition 14.1.6.

Proposition 16.2.6. - Soient $X$ un objet de $\mathcal{F}_{\mathcal{G} r, n}$ et $F$ un sous-objet de $\omega_{n}(X)$. Pour tout entier $k \geq 0$, on note $C_{k}=\pi_{n, X}^{k}\left(\left(\tilde{\nabla}_{n}\right)^{k} F\right)$.

(1) La suite $\left(C_{k}\right)_{k>0}$ de sous-objets de $\omega_{n}(X)$ est croissante; nous noterons $C_{\infty}$ sa réunion. Si $X$ est un objet noethérien de $\mathcal{F}_{\mathcal{G} r, n}$, cette suite stationne.

(2) Pour tout $k \geq 0$, le foncteur $C_{k}$ est engendré par les éléments du type

$$
\left(<H_{1}, W>\cdots<H_{k}, W>x_{W}\right)_{W \in \mathcal{G} r_{n}(V)} \in \omega_{n}(X)(V),
$$

où $V$ parcourt les espaces vectoriels de dimension finie, $x=\left(x_{W}\right)_{W \in \mathcal{G} r_{n}(V)}$ les éléments de $F(V)$ et $\left(H_{1}, \ldots, H_{k}\right)$ les $k$-uplets d'éléments de $\mathcal{G} r_{n}\left(V^{*}\right)$.

(3) Le foncteur $C_{\infty}$ est le plus petit sous- $\bar{G}(n)$-comodule de $\omega_{n}(X)$ contenant $F$. Si $F$ est lui-même un sous- $\bar{G}(n)$-comodule de $\omega_{n}(X)$, on a $C_{k}=F$ pour tout $k \geq 0$.

(4) Si $X$ est localement fini, alors hom $\left(C_{\infty} / F, \omega_{n}(T)\right)=0$ pour tout objet $T$ de $\mathcal{F}_{\mathcal{G} r, n}$.

Démonstration. - Le lemme 16.2.4 et la préservation des épimorphismes par le foncteur $\tilde{\nabla}_{n}$ montrent le second point pour $k=1$. Le cas général s'en déduit aussitôt par récurrence.

On en déduit que la suite $\left(C_{k}\right)_{k>0}$ est croissante. Si $W$ est un sous-espace de dimension $n$ d'un espace vectoriel de dimension finie $V$, soient $H_{1}, \ldots, H_{k}$ les éléments de $\mathcal{G} r_{n}\left(V^{*}\right)$ tels que $<H_{i}, W>=1$. Alors la fonction $\left\langle H_{1}, \cdot>\cdots<H_{k}, \cdot>\right.$ : $\mathcal{G} r_{n}(V) \rightarrow \mathbb{F}_{2}$ est égale à l'indicatrice de $\{W\}$. Si l'on note $F^{g r}(V)$ le plus petit sousespace vectoriel $\mathcal{G} r_{n}(V)$-gradué de $\omega_{n}(X)(V)$ contenant $F(V)$, ce qui précède montre 
que $C_{\infty}$ est le plus petit sous-foncteur de $\omega_{n}(X)$ tel que $F^{g r}(V) \subset C_{\infty}(V)$ pour tout $V \in \mathrm{Ob} \mathcal{E}^{f}$; en particulier, $C_{\infty} \supset F$.

Soit $Y$ le plus petit sous-objet de $X$ tel que $Y(V, W)$ contienne les composantes dans $X(V, W)$ des éléments de $F(V) \subset \omega_{n}(X)(V)$. La proposition 5.1.7 montre d'une part que $\omega_{n}(Y)$ est le plus petit sous- $\bar{G}(n)$-comodule de $\omega_{n}(X)$ contenant $F$. Le paragraphe précédent montre d'autre part que $C_{\infty}=\omega_{n}(Y)$, d'où le troisième point.

Soit $f: C_{\infty} / F \rightarrow \omega_{n}(T)$ un morphisme de $\mathcal{F}$. Si $X$ est localement fini, il en est de même pour $Y$, donc le corollaire 10.2.2 montre que la composée $g: \omega_{n}(Y)=C_{\infty} \rightarrow$ $C_{\infty} / F \stackrel{f}{\rightarrow} \omega_{n}(T)$ est induite par un morphisme $u: Y \rightarrow T$ de $\mathcal{F}_{\mathcal{G} r, n}$. Comme la composée $F \hookrightarrow \omega_{n}(Y) \stackrel{g}{\rightarrow} \omega_{n}(T)$ est nulle, $F$ est inclus dans $\omega_{n}(k e r u)$. D'après le troisième point, on en déduit ker $u=Y$, puis $f=0$, d'où la dernière assertion.

Par ailleurs, si $X$ est noethérien, alors $Y$ est de type fini, donc $C_{\infty}=\omega_{n}(Y)$ est de type fini. Cela montre que la suite $\left(C_{k}\right)_{k>0}$ est stationnaire, et achève la démonstration.

On rappelle que la notation $\mathcal{F}_{\mathcal{G} r, n}^{l f}$ utilisée dans le théorème fondamental suivant désigne la sous-catégorie localisante des objets localement finis de $\mathcal{F}_{\mathcal{G} r, n}$.

\section{Théorème 16.2.7 (Théorème de simplicité généralisé)}

Le foncteur $\omega_{n}: \mathcal{F}_{\mathcal{G} r, n} \rightarrow \mathcal{F}$ induit une équivalence entre la catégorie $\mathcal{F}_{\mathcal{G} r, n}^{\text {lf }}$ et une sous-catégorie localisante de $\overline{\mathcal{N}}_{\tilde{\nabla}_{n+1}} / \overline{\mathcal{N}}_{\tilde{\nabla}_{n}}$. En particulier, il envoie un foncteur simple de $\mathcal{F}_{\mathcal{G} r, n}$ sur un objet simple de $\mathcal{F} / \overline{\mathcal{N}}_{\tilde{\nabla}_{n}}$.

Démonstration. - Afin de simplifier les notations, nous utiliserons le foncteur suivant

$$
\bar{\omega}_{n}: \mathcal{F}_{\mathcal{G} r, n}^{l f} \hookrightarrow \mathcal{F}_{\mathcal{G} r, n} \stackrel{\omega_{n}}{\longrightarrow} \mathcal{F} \rightarrow \mathcal{F} / \overline{\mathcal{N}}_{\tilde{\nabla}_{n}}
$$

dans cette démonstration.

La proposition 15.2.1 montre que la restriction à $\mathcal{F}_{\mathcal{G} r, n}^{l f}$ du foncteur $\omega_{n}$ est bien à valeurs dans $\overline{\mathcal{N}}_{\tilde{\nabla}_{n+1}}$. Le corollaire 10.2.2 et le théorème 15.3.1 entraînent la pleine fidélité de $\bar{\omega}_{n}$ et la stabilité par extensions de son image. Notons $\mathcal{C}_{n}$ l'image de la restriction de ce foncteur aux objets finis de $\mathcal{F}_{\mathcal{G} r, n}$ : comme $\bar{\omega}_{n}$ commute aux colimites, il suffit d'établir qu'un sous-objet d'un objet de $\mathcal{C}_{n}$ est isomorphe à un objet de $\mathcal{C}_{n}$. On procède par récurrence sur le degré polynomial. On se donne donc un objet fini $X$ de $\mathcal{F}_{\mathcal{G} r, n}$ de degré $d \geq 0$ et l'on suppose que l'hypothèse suivante est satisfaite.

Hypothèse 16.2.8 (Hypothèse de récurrence). — L'image par le foncteur $\bar{\omega}_{n}$ de la sous-catégorie $\left(\mathcal{F}_{\mathcal{G} r, n}^{f}\right)^{d-1}$ des foncteurs de degré $<d$ de $\mathcal{F}_{\mathcal{G} r, n}^{f}$ est une souscatégorie épaisse de $\mathcal{F} / \overline{\mathcal{N}} \boldsymbol{i}_{\tilde{\nabla}_{n}}$.

Notation 16.2.9. - Dans cette section, nous noterons $\mathcal{A}_{n, d}$ cette sous-catégorie épaisse, et $\mathcal{Q}_{n, d}$ la catégorie quotient de $\mathcal{F} / \overline{\mathcal{N}}_{\tilde{\nabla}_{n}}$ par $\mathcal{A}_{n, d}$. Nous noterons également $\mathcal{X}_{n}$ la sous-catégorie pleine des objets $A$ de $\mathcal{F}$ tels que hom $\left(A, \omega_{n}(T)\right)=0$ pour tout objet $T$ de $\mathcal{F}_{\mathcal{G} r, n}$. 
Lemme 16.2.10. - (1) Si F est un objet de $\mathcal{F}$ tel que $\tilde{\nabla}_{n}(F)$ appartienne à $\overline{\mathcal{N}}_{\tilde{\nabla}_{n}}$, alors $F$ appartient à $\overline{\mathcal{N}}_{\tilde{\nabla}_{n}}$.

(2) La sous-catégorie $\mathcal{X}_{n}$ de $\mathcal{F}$ est stable par le foncteur $\tilde{\nabla}_{n}$.

(3) Un objet de $\mathcal{X}_{n}$ dont l'image dans $\mathcal{F} / \overline{\mathcal{N}}_{\tilde{\nabla}_{n}}$ appartient à $\mathcal{A}_{n, d}$ est objet de $\overline{\mathcal{N}}_{\bar{\nabla}} \tilde{\nabla}_{n}$.

(4) Pour tout entier $k>0$, le morphisme $\pi_{n, X}^{k}$ induit un isomorphisme dans la catégorie $\mathcal{Q}_{n, d}$.

Démonstration du lemme. - Soit $F$ est un objet de $\mathcal{F}$ tel que $\tilde{\nabla}_{n} F$ appartienne à $\overline{\mathcal{N} i l}_{\tilde{\nabla}_{n}}$. Si $F$ est de type fini, $\tilde{\nabla}_{n} F$ est aussi de type fini (car c'est un quotient de $\Delta^{n} F$ ), donc $\tilde{\nabla}_{n}$-nilpotent puisqu'objet de $\overline{\mathcal{N}}_{\tilde{\nabla}_{n}}$. Par conséquent, $F$ est $\tilde{\nabla}_{n}$-nilpotent. Dans le cas général, on montre que $F$ est objet de $\overline{\mathcal{N}}_{\bar{\nabla}_{n}}$ en écrivant $F$ comme colimite de ses sous-objets de type fini.

Pour le deuxième point, on remarque que la sous-catégorie $\mathcal{X}_{n}$ est stable par quotient et préservée par le foncteur $\Delta_{E_{n}}$, en raison de l'isomorphisme $\operatorname{hom}_{\mathcal{F}}\left(\Delta_{E_{n}} A, \omega_{n}(T)\right) \simeq \operatorname{hom}_{\mathcal{F}}\left(A, \omega_{n}\left(T \otimes \iota_{n}\left(I_{E_{n}}\right)\right)\right)$.

Le troisième point résulte de la définition de la sous-catégorie $\mathcal{A}_{n, d}$.

La proposition 15.2.8 montre que le noyau de l'épimorphisme $\pi_{n, X}$ (corollaire 15.2.4) appartient à $\mathcal{A}_{n, d}$ (on rappelle que $X$ est de degré $d$ ). Pour en déduire, par récurrence sur $k$, la dernière assertion, il suffit de noter que l'image par le foncteur $\tilde{\nabla}_{n}$ d'un morphisme $f$ de $\mathcal{F}$ qui induit un isomorphisme dans $\mathcal{F} / \overline{\mathcal{N}} \bar{\nabla}_{n}$ vérifie encore la même propriété. Ce résultat s'obtient en notant que les endofoncteurs exacts $\Delta$ et $\Delta^{n}$ de $\mathcal{F}$ préservent $\overline{\mathcal{N}}_{\bar{l}} \tilde{\nabla}_{n}$ (par la dernière assertion de la proposition 1.5.3), de sorte que $\operatorname{ker}(\Delta f) \simeq \Delta(\operatorname{ker} f)$, dont $\operatorname{ker} \tilde{\nabla}_{n}(f)$ est un sous-objet, et coker $\left(\Delta^{n} f\right) \simeq \Delta^{n}($ coker $f)$, dont coker $\tilde{\nabla}_{n}(f)$ est un quotient, sont objets de $\overline{\mathcal{N}}_{\tilde{\nabla}_{n}}$.

Fin de la démonstration du théorème 16.2.7. - Soit $F$ un sous-objet de $\omega_{n}(X)$; on conserve les notations de la proposition 16.2.6, et l'on se donne $k \in \mathbb{N}^{*}$ tel que $C_{\infty}=C_{k}$ (qui est donc de la forme $\omega_{n}(Y)$ pour un sous-objet $Y$ de $X$ ). Alors $\left(\tilde{\nabla}_{n}\right)^{k} F$ et $\left(\tilde{\nabla}_{n}\right)^{k} C_{\infty}$ ont la même image $C_{\infty}$ par $\pi_{X}^{k}$, qui induit un isomorphisme dans $\mathcal{Q}_{n, d}$ (dernière assertion du lemme 16.2.10), donc l'inclusion $\left(\tilde{\nabla}_{n}\right)^{k} F \hookrightarrow\left(\tilde{\nabla}_{n}\right)^{k} C_{\infty}$ induit un isomorphisme dans $\mathcal{Q}_{n, d}$. Ainsi, l'image dans $\mathcal{F} / \overline{\mathcal{N}}_{i} \tilde{\nabla}_{n}$ de $\left(\tilde{\nabla}_{n}\right)^{k} C_{\infty} /\left(\tilde{\nabla}_{n}\right)^{k} F$ est objet de $\mathcal{A}_{n, d}$. Il en est de même pour $\left(\tilde{\nabla}_{n}\right)^{k}\left(C_{\infty} / F\right)$, qui est un quotient de $\left(\tilde{\nabla}_{n}\right)^{k} C_{\infty} /\left(\tilde{\nabla}_{n}\right)^{k} F$ (parce que $\tilde{\nabla}_{n}$ préserve injections et surjections). Mais $\left(\tilde{\nabla}_{n}\right)^{k}\left(C_{\infty} / F\right)$ est aussi un objet de $\mathcal{X}_{n}$ par la dernière assertion de la proposition 16.2.6 et la deuxième assertion du lemme 16.2.10. Il montre alors que $\left(\tilde{\nabla}_{n}\right)^{k}\left(C_{\infty} / F\right)$ appartient à $\overline{\mathcal{N}}_{\tilde{\nabla}_{n}}$ (troisième assertion), donc aussi $C_{\infty} / F$ (première assertion). Cela achève la démonstration.

On démontre de manière similaire la variante suivante du théorème 16.2.7 : 
Théorème 16.2.11. - Le foncteur $\omega_{n}$ induit une équivalence entre la souscatégorie pleine des objets finis de $\mathcal{F}_{\mathcal{G} r, n}$ et une sous-catégorie épaisse de $\mathcal{N} i l_{\tilde{\nabla}_{n+1}} / \mathcal{N} i l_{\tilde{\nabla}_{n}}$.

Remarque 16.2.12. - L'une des conséquences principales de ce théorème est le fait que l'image d'un $\bar{G}(n)$-comodule simple dans la catégorie quotient $\mathcal{F} / \mathcal{N} i l_{\tilde{\nabla}_{n}}$ est simple. Pour un $\bar{G}(n)$-comodule simple associé à un objet simple pseudo-constant de $\mathcal{F}_{\mathcal{G} r, n}$ (i.e. pour un foncteur de Powell), ce résultat est dû à Powell (cf. [Pow98c], théorème 6.0.1, et son corollaire, la proposition 6.1.1), qui l'a nommé théorème de simplicité. Cela justifie la terminologie employée.

La démonstration du théorème 16.2 .7 repose exactement sur le même principe que le théorème de simplicité de Powell, à savoir la considération explicite d'éléments dans les foncteurs, rendue raisonnable par le calcul aisé du foncteur $\tilde{\nabla}_{n}$ sur les projectifs standard, adapté aux catégories de foncteurs en grassmanniennes par les résultats préliminaires de la section 15.2 .

Le théorème 16.2.7 (ou 16.2.11) est un résultat global sur la structure de la catégorie $\mathcal{F}$ (il donne des informations sur tous ses objets de type fini); comme le théorème 14.1.1, qui en constitue le cas particulier $n=1$, il n'utilise pas la théorie des représentations linéaires (la généralisation à tous les $\bar{G}(n)$-comodules simples du théorème de simplicité de Powell fait disparaître les quelques considérations explicites sur les représentations de $G L_{n}$ utilisées dans [Pow98c]).

Remarque 16.2.13. - On peut généraliser le théorème 16.2.7 à un corps fini quelconque, mais il convient de remplacer les foncteurs $\tilde{\nabla}_{n}$ par $\tilde{\nabla}_{(q-1) n}$, où $q$ désigne le cardinal du corps considéré. D'autres variantes sont possibles, en utilisant également la décomposition scalaire (cf. section 14.2, pour le cas $n=1$ ).

\subsection{Foncteurs $\tilde{\nabla}_{n}$-adaptés}

Convention 16.3.1. - Comme dans la section précédente, $n$ désigne un entier strictement positif.

La notion de foncteur $\tilde{\nabla}_{n}$-adapté que nous introduisons ci-dessous est destinée à faciliter certains raisonnements de récurrence pour progresser dans l'étude de la conjecture artinienne extrêmement forte.

Définition 16.3.2. - Soit $F$ un objet de $\mathcal{F}$. On dit que $F$ est un foncteur $\tilde{\nabla}_{n^{-}}$ adapté si tout quotient $\tilde{\nabla}_{n}$-nilpotent de $F$ est induit de hauteur strictement inférieure à $n$ (cf. section 12.1).

Le théorème de simplicité généralisé montre que la conjecture artinienne extrêmement forte équivaut à dire que tout foncteur de type fini est $\tilde{\nabla}_{i}$-adapté pour tout entier $i>0$. 
La définition 16.3.2 est très difficile à vérifier si l'on ignore si un quotient d'un foncteur induit de hauteur strictement inférieure à $n$ est encore induit de hauteur strictement inférieure à $n$ : elle est maniable lorsque l'hypothèse suivante est satisfaite (le but étant de démontrer l'épaisseur de $\left.\mathcal{F}^{\omega-\operatorname{cons}(n)}\right)$.

Hypothèse 16.3.3. - La sous-catégorie $\mathcal{F}^{\omega-\operatorname{cons}(i)}$ de $\mathcal{F}$ est épaisse pour $i<n$.

Proposition 16.3.4. - Soient $A \in \mathrm{Ob} \mathcal{F}$ et $F$ un objet fini de $\mathcal{F}$. On suppose que l'hypothèse 16.3.3 est satisfaite.

(1) Si A est un foncteur $\tilde{\nabla}_{n}$-adapté, il en est de même pour tous ses quotients.

(2) Si $A$ est un foncteur $\tilde{\nabla}_{n}$-adapté, alors $A \otimes F$ est $\tilde{\nabla}_{n}$-adapté.

(3) Si A est $\tilde{\nabla}_{n}$-adapté, alors $(A: F)$ est $\tilde{\nabla}_{n}$-adapté.

Démonstration. - Le premier point est formel.

Pour le second, considérons un épimorphisme $f: A \otimes F \rightarrow Q$, où $Q$ est $\tilde{\nabla}_{n}$-nilpotent. Soit $g: A \rightarrow \operatorname{Hom}_{\mathcal{F}}(F, Q)$ le morphisme adjoint à $f$. Comme $F$ est quotient d'une somme directe finie de projectifs standard, $\operatorname{Hom}_{\mathcal{F}}(F, Q)$ est un sous-foncteur d'une somme directe finie de $\Delta_{V} Q$, il est donc $\tilde{\nabla}_{n}$-nilpotent par la proposition 1.5.3.2. Par conséquent, img est un quotient $\tilde{\nabla}_{n}$-nilpotent de $A$, c'est donc, par hypothèse, un foncteur induit de hauteur strictement inférieure à $n$. Il en est de même pour $i m g \otimes F$, puisque $F$ est fini (cf. proposition 12.1.4).

Le diagramme commutatif

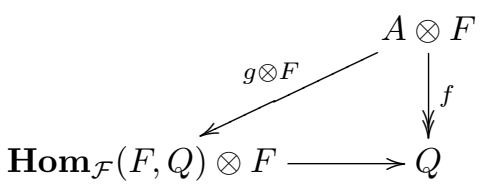

(dont la flèche horizontale est la coünité de l'adjonction) montre que $Q$ est un quotient de $i m g \otimes F ; Q$ est donc objet de $\mathcal{F}^{\omega-\operatorname{cons}(n-1)}$ grâce à ce qui précède et à l'hypothèse 16.3.3.

Le troisième point s'établit par un argument d'adjonction analogue, grâce aux deux remarques suivantes.

(1) Le foncteur $\otimes F$ préserve $\mathcal{N} i l_{\tilde{\nabla}_{n}}$, par la proposition 1.5.9.

(2) Le foncteur de division par $F$ préserve $\mathcal{F}^{\omega-\operatorname{cons}(n-1)}$. En effet, comme $F$ est de co-type fini, ce foncteur est un quotient d'une somme directe finie de foncteurs de décalage, et la sous-catégorie $\mathcal{F}^{\omega-\operatorname{cons}(n-1)}$ de $\mathcal{F}$ est stable par les foncteurs de décalage (cf. proposition 12.1.4).

Avant d'appliquer cette propriété à la proposition 16.3.6, nous mentionnons un lemme élémentaire qui se déduit des résultats de [Kuh94b], §4. 
Lemme 16.3.5. - Soit $M$ un $G L_{n}$-module fini. Il existe un foncteur fini $F$ de $\mathcal{F}$ tel que $F\left(E_{n}\right)$ est isomorphe à $M$ comme $G L_{n}$-module.

La proposition suivante permet de réduire les vérifications nécessaires pour démontrer que $\mathcal{F}^{\omega-\operatorname{cons}(n)}$ est épaisse.

Proposition 16.3.6. - Soit $\lambda$ une partition régulière telle que $\lambda_{1}=n$. On suppose l'hypothèse 16.3.3 vérifiée. Les assertions suivantes sont équivalentes.

(1) La sous-catégorie $\mathcal{F}^{\omega-\operatorname{cons}(n)}$ de $\mathcal{F}$ est épaisse.

(2) Pour tout objet fini $X$ de $\mathcal{F}_{\mathcal{G} r, n}$, le foncteur $\omega_{n}(X)$ est $\tilde{\nabla}_{n}$-adapté.

(3) Pour tout objet simple $S$ de $\mathcal{F}_{\mathcal{G} r, n}$, le foncteur $\omega_{n}(S)$ est $\tilde{\nabla}_{n}$-adapté.

(4) Le foncteur de Powell $Q_{\lambda}$ est $\tilde{\nabla}_{n}$-adapté.

Lorsqu'elles sont vérifiées, pour tout foncteur fini (resp. simple) $X$ de $\mathcal{F}_{\mathcal{G} r, k}$, où $k \leq n$, le foncteur $\omega_{k}(X)$ est noethérien (resp. simple noethérien) de type $k$.

Démonstration. - Supposons l'assertion 4 vérifiée. On commence par montrer que pour tout $G L_{n}$-module simple $S$, le foncteur de Powell $\omega_{n} \rho_{n}(S)$ est $\tilde{\nabla}_{n}$-adapté. Pour cela, on se donne, conformément au lemme 16.3.5, un foncteur fini $F$ de $\mathcal{F}$ tel que le $G L_{n}$-module $F\left(E_{n}\right)$ est isomorphe à $\mathbb{F}_{2}\left[G L_{n}\right]$. Le foncteur $\omega_{n}\left(\rho_{n}\left(R_{\lambda}\right): \iota_{n}(F)\right)$ est $\tilde{\nabla}_{n}$-adapté, car c'est un quotient, par la proposition 9.1 .2 , de

$$
\omega_{\leq n}\left(\mathcal{P}_{n, \leq n} \rho_{n}\left(R_{\lambda}\right): \iota \leq n(F)\right) \simeq\left(\omega_{n} \rho_{n}\left(R_{\lambda}\right): F\right)=\left(Q_{\lambda}: F\right)
$$

(cet isomorphisme venant de la proposition 9.2.1), de sorte que la proposition 16.3.4 prouve ce premier point.

Par ailleurs, $\left(\rho_{n}\left(R_{\lambda}\right): \iota_{n}(F)\right) \simeq \rho_{n}\left(R_{\lambda}: \mathbb{F}_{2}\left[G L_{n}\right]\right)$ par la proposition 9.2 .2 , et

$$
\left(R_{\lambda}: \mathbb{F}_{2}\left[G L_{n}\right]\right) \simeq R_{\lambda} \otimes \mathbb{F}_{2}\left[G L_{n}\right] \simeq \mathbb{F}_{2}\left[G L_{n}\right]^{\oplus i},
$$

où $i=\operatorname{dim}_{\mathbb{F}_{2}} R_{\lambda} \in \mathbb{N}^{*}$. Par conséquent, tout $G L_{n}$-module simple $S$ est quotient de $\left(R_{\lambda}: \mathbb{F}_{2}\left[G L_{n}\right]\right)$, donc le quotient $\omega_{n} \rho_{n}(S)$ de $\omega_{n}\left(\rho_{n}\left(R_{\lambda}\right): \iota_{n}(F)\right)$ est $\tilde{\nabla}_{n}$-adapté.

Si $X$ est un objet simple de $\mathcal{F}_{\mathcal{G} r, n}$, il existe un $G L_{n}$-module simple $S$ et un objet simple $F$ de $\mathcal{F}$ tels que $X \simeq \kappa_{n}(F) \otimes \rho_{n}(S)$ (proposition 5.4.13), donc $\omega_{n}(X)$ est quotient de $\omega_{n}\left(\iota_{n}(F) \otimes \rho_{n}(S)\right) \simeq \omega_{n} \rho_{n}(S) \otimes F$. La proposition 16.3.4 montre à nouveau que ce foncteur est $\tilde{\nabla}_{n}$-adapté.

On a ainsi démontré que 4 implique 3 .

Si l'assertion 3 est vérifiée, le théorème 16.2.7 prouve que tout sous-quotient de $\omega_{n}(S)$, où $S$ est un objet simple de $\mathcal{F}_{\mathcal{G} r, n}$, est objet de $\mathcal{F}^{\omega-\operatorname{cons}(n)}$. Comme la souscatégorie pleine $\mathcal{A}$ des objets de $\mathcal{F}$ dont tous les sous-quotients sont dans $\mathcal{F}^{\omega-\operatorname{cons}(n)}$ vérifie l'hypothèse que pour toute suite exacte courte $0 \rightarrow A \rightarrow B \rightarrow C \rightarrow 0$ de $\mathcal{F}$, si deux des objets $A, B, C$ appartiennent à $\mathcal{A}$, il en est de même du troisième, on en déduit l'assertion 1 .

Il est clair que 2 entraîne 4 . 
Si l'assertion 1 est vérifiée, le foncteur $\omega_{n}$ induit une équivalence entre les catégories $\mathcal{F}_{\mathcal{G} r, n}^{f}$ et $\mathcal{F}^{\omega-\operatorname{cons}(n)} / \mathcal{F}^{\omega-\operatorname{cons}(n-1)}$, donc l'assertion 2 est satisfaite.

Ainsi, les assertions de l'énoncé sont équivalentes.

La fin de la proposition découle de la proposition 12.2.4.

\subsection{Structure de $P^{\otimes 2} \otimes F$ pour un foncteur fini $F$}

Dans l'article [Pow98a] (proposition 7.4) est établi le résultat suivant ${ }^{(2)}$.

Proposition 16.4.1 (Powell). - Soit $F$ un foncteur de type fini de $\mathcal{F}$ tel que $\tilde{\nabla}_{2}(F)=0$ et que $\operatorname{hom}_{\mathcal{F}}(F, \bar{P})=0$. Alors $F$ est un foncteur fini.

En étudiant une filtration explicite du foncteur $\bar{G}(2)$ construite à partir de la filtration co-polynomiale de $\bar{P}$, [Pow98a] déduit de la proposition précédente le corollaire suivant (c'est sa proposition 7.5).

Corollaire 16.4.2 (Powell). - Le foncteur $\bar{G}(2)$ est $\tilde{\nabla}_{2}$-adapté.

Nous aurons également besoin de la conséquence facile suivante de la proposition 16.4.1 (qui est implicite dans [Pow98a]).

Corollaire 16.4.3. - Si F est un foncteur de type fini de $\mathcal{F}$ tel que $\tilde{\nabla}_{2}(F)=0$, alors $F$ est induit de hauteur au plus 1 . Plus précisément, F est isomorphe dans $\mathcal{F} / \mathcal{F}^{f}$ à une somme directe finie de copies de $\bar{P}$.

Démonstration. - Comme $F$ est de type fini, l'ensemble $\operatorname{hom}_{\mathcal{F}}(F, \bar{P})$ est fini. Soient $N$ et $C$ le noyau et le conoyau, respectivement, du morphisme canonique $F \rightarrow$ $\bar{P}^{\operatorname{hom}_{\mathcal{F}}(F, \bar{P})}$. Alors $C$ est fini $\left(\operatorname{car} \operatorname{hom}_{\mathcal{F}}(C, \bar{P})=0\right)$; comme $\bar{P}$ est $\mathcal{F}^{f}$-parfait, on en déduit $\operatorname{hom}_{\mathcal{F}}(N, \bar{P})=0$. Mais $\tilde{\nabla}_{2}(N)=0$, car $N$ est un sous-foncteur de $F$, donc $N$ est fini par la proposition 16.4.1, d'où le corollaire.

Remarque 16.4.4. - La démonstration de la proposition 16.4.1 repose sur des considérations explicites issues de la théorie des représentations - essentiellement, Powell s'appuie sur le fait qu'il existe peu d'extensions non triviales entre les puissances extérieures (cf. [Fra96], proposition 2.3.1), qui sont les seuls foncteurs simples de $\mathcal{F}$ annihilés par $\tilde{\nabla}_{2}$. Malheureusement, la complexité des problèmes posés par la généralisation de cette approche aux foncteurs $\tilde{\nabla}_{n}$ croît très rapidement avec $n$.

Du reste, la seule obstruction sérieuse à la généralisation des résultats présentés dans cette section se trouve concentrée à la proposition 16.4.1.

La satisfaction de l'hypothèse 16.3 .3 de la section précédente pour $n=2$ (corollaire 14.1.2) et le corollaire 16.4.2 permettent de déduire de la proposition 16.3.6 le

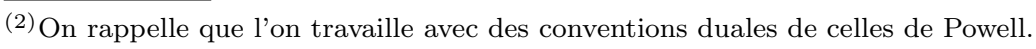


théorème suivant, qui constitue le résultat « concret $»$ le plus important de cette partie.

Théorème 16.4.5. - (1) La sous-catégorie $\mathcal{F}^{\omega-\operatorname{cons}(2)}$ de $\mathcal{F}$ est épaisse.

(2) Tout $\bar{G}(2)$-comodule fidèle fini (resp. simple) est noethérien (resp. simple noethérien) de type 2.

(3) En particulier, pour tout objet fini $F$ de $\mathcal{F}$, le foncteur $P^{\otimes 2} \otimes F$ est noethérien.

Dans le cas où $F$ est constant, ce théorème est dû à Powell (cf. [Pow98a]); nous avons traité le cas où $F$ est une puissance extérieure par d'autres méthodes dans [Dja06c].

Le théorème 16.4.5 a été depuis surpassé par le travail [Dja07], dans lequel l'auteur utilise une analyse approfondie de foncteurs de division pour décrire les foncteurs de type fini $\tilde{\nabla}_{3}$-nilpotents (modulo les foncteurs $\tilde{\nabla}_{2}$-nilpotents).

Nous pouvons maintenant préciser le corollaire 16.4.3 en montrant que la conjecture 15.3.3 est vérifiée pour $n=2$.

Proposition 16.4.6. - Tout foncteur $\tilde{\nabla}_{2}$-nilpotent et de type fini $F$ est induit de hauteur au plus 1 .

Démonstration. - On établit la proposition par récurrence sur l'indice de $\tilde{\nabla}_{2-}$ nilpotence $i$ de $F$. On suppose donc la propriété vérifiée pour tous les foncteurs de type fini annihilés par $\left(\tilde{\nabla}_{2}\right)^{i-1}$.

Il existe une suite exacte

$$
\bar{P} \otimes \tilde{\nabla}_{2}(F) \rightarrow F \rightarrow Q \rightarrow 0
$$

où $\tilde{\nabla}_{2}(Q)=0$ (cf. [Pow98b], $\S 5$ ). Comme $Q$ est de type fini, on en déduit que $Q$ est induit de hauteur au plus 1 par le corollaire 16.4.3. Le foncteur de type fini $\tilde{\nabla}_{2}(F)$ étant annulé par $\left(\tilde{\nabla}_{2}\right)^{i-1}$, l'hypothèse de récurrence montre qu'il est également induit de hauteur au plus 1 , donc $\bar{P} \otimes \tilde{\nabla}_{2}(F)$, puis $F$, sont induits de hauteur au plus 2 (par la proposition 12.1.4 et le théorème 16.4.5). En conséquence, comme le foncteur $\omega_{2}$ induit une équivalence entre les catégories $\mathcal{F}_{\mathcal{G} r, 2}^{f}$ et $\mathcal{F}^{\omega-\operatorname{cons}(2)} / \mathcal{F}^{\omega-\operatorname{cons}(1)}$, il existe une suite exacte

$$
0 \rightarrow A \rightarrow F \rightarrow \omega_{2}(X) \rightarrow B \rightarrow 0
$$

avec $X \in \mathrm{Ob} \mathcal{F}_{\mathcal{G} r, 2}^{f}$ et $A, B \in \mathrm{Ob} \mathcal{F}^{\omega-\operatorname{cons}(1)}$. Vu que la sous-catégorie $\mathcal{N i l}_{\tilde{\nabla}_{2}}$ de $\mathcal{F}$ est épaisse (proposition 1.5.7) et que $A, B$ et $F$ sont $\tilde{\nabla}_{2}$-nilpotents, cela entraîne que $\omega_{2}(X)$ est $\tilde{\nabla}_{2}$-nilpotent, donc que $X=0$ par la proposition 15.3.1.

Par conséquent, $F$ est induit de hauteur au plus 1, ce qu'il fallait démontrer. 



\section{APPENDICE A}

\section{ADJONCTIONS}

\section{A.1. Algèbre homologique}

La propriété immédiate suivante est d'un usage très courant dans les catégories de foncteurs.

Proposition A.1.1. - Supposons que $\mathcal{A}$ et $\mathcal{B}$ sont deux catégories abéliennes possédant suffisamment d'objets injectifs. Si $F: \mathcal{B} \rightarrow \mathcal{A}$ et $G: \mathcal{A} \rightarrow \mathcal{B}$ sont des foncteurs exacts tels que $F$ est adjoint à droite à $G$, alors l'isomorphisme naturel $\operatorname{hom}_{\mathcal{A}}(X, F(Y)) \simeq \operatorname{hom}_{\mathcal{B}}(G(X), Y)$ s'étend en un isomorphisme naturel gradué

$$
\operatorname{Ext}_{\mathcal{A}}^{*}(X, F(Y)) \simeq \operatorname{Ext}_{\mathcal{B}}^{*}(G(X), Y) .
$$

Nous utiliserons parfois cette proposition via le corollaire suivant.

Corollaire A.1.2. - Supposons que:

(1) $\mathcal{A}$ et $\mathcal{B}$ sont deux catégories abéliennes, $\mathcal{A}$ possédant assez d'injectifs;

(2) $F$ et $G$ sont deux endofoncteurs exacts de $\mathcal{A}$, avec $G$ adjoint à gauche à $F$;

(3) $H: \mathcal{B} \rightarrow \mathcal{A}$ est un foncteur exact;

(4) $K$ est un endofonteur de $\mathcal{B}$ tel que $F \circ H \simeq H \circ K$;

(5) il existe une transformation naturelle injective $j: i d_{\mathcal{B}} \hookrightarrow K$.

Soient $X$ un objet de $\mathcal{A}$ tel qu'il existe $n \in \mathbb{N}$ tel que $G^{n}(X)=0$ et $Y$ un objet de B. On a $\operatorname{Ext}_{\mathcal{A}}^{*}(X, H(Y))=0$.

Démonstration. - Quitte à remplacer $F$ par $F^{n}, G$ par $G^{n}$ et $K$ par $K^{n}$, on peut supposer $n=1$.

Pour tout objet $Y$ de $\mathcal{B}$, on a une suite exacte naturelle

$$
0 \rightarrow Y \stackrel{f_{1}}{\longrightarrow} K\left(Y_{1}\right) \stackrel{f_{2}}{\longrightarrow} K\left(Y_{2}\right) \stackrel{f_{3}}{\longrightarrow} \ldots \stackrel{f_{n}}{\longrightarrow} K\left(Y_{n}\right) \stackrel{f_{n+1}}{\longrightarrow} \cdots
$$

où $Y_{1}=Y$ et $f_{1}=j_{Y}$, et pour $n>1, Y_{n}=\operatorname{coker} f_{n-1}$ et $f_{n}$ est la composée $K\left(Y_{n-1}\right) \rightarrow Y_{n} \stackrel{j_{Y_{n}}}{\longrightarrow} K\left(Y_{n}\right)$. En appliquant le foncteur exact $H$ à cette suite exacte 
et en utilisant l'isomorphisme $F \circ H \simeq H \circ K$, on en déduit une résolution de $H(Y)$ par les objets $F\left(H\left(Y_{i}\right)\right)$. Comme $\operatorname{Ext}_{\mathcal{A}}^{*}(X, F(A))=0$ pour tout $A \in \operatorname{Ob} \mathcal{A}$ par la proposition A.1.1, cela donne la conclusion.

\section{A.2. Monades et comonades}

Pour les démonstrations des propriétés rappelées ci-dessous, nous renvoyons à [ML71], chapitre VI.

Définition A.2.1. - Soit $\mathcal{C}$ une catégorie.

(1) Une monade $\operatorname{sur} \mathcal{C}$ est un triplet $(T, \eta, \mu)$ formé d'un endofoncteur $T$ de $\mathcal{C}$ et de transformations naturelles $\eta: i d_{\mathcal{C}} \rightarrow T$ et $\mu: T^{2} \rightarrow T$ telles que $\mu \circ \eta_{T}=i d_{T}=\mu \circ T(\eta)$ et $\mu \circ T(\mu)=\mu \circ \mu_{T}$. La transformation naturelle $\mu$ est appelée multiplication de la monade.

(2) Une comonade sur $\mathcal{C}$ est une monade sur $\mathcal{C}^{o p}$, i.e. un triplet $(T, \gamma, \delta)$ formé d'un endofoncteur $T$ de $\mathcal{C}$ et de transformations naturelles $\gamma: T \rightarrow i d_{\mathcal{C}}$ et $\delta: T \rightarrow T^{2}$ telles que $\gamma_{T} \circ \delta=i d_{T}=T(\gamma) \circ \delta$ et $\delta_{T} \circ \delta=T(\delta) \circ \delta$.

Convention A.2.2. - Dans la suite de cet appendice, nous considérons des catégories $\mathcal{A}$ et $\mathcal{B}$ et des foncteurs $F: \mathcal{B} \rightarrow \mathcal{A}$ et $G: \mathcal{A} \rightarrow \mathcal{B}$ tels que $F$ est adjoint à droite à $G$.

Proposition A.2.3. - L'adjonction entre $F$ et $G$ détermine :

(1) une monade $\left(F G, \eta, F\left(\gamma_{G}\right)\right)$ sur $\mathcal{A}$;

(2) une comonade $\left(G F, \gamma, G\left(\eta_{F}\right)\right)$ sur $\mathcal{B}$.

Définition A.2.4. - (1) Soit $(T, \eta, \mu)$ une monade sur une catégorie $\mathcal{C}$. Un module sur cette monade est la donnée d'un objet $X$ de $\mathcal{C}$ et d'un morphisme $m: T(X) \rightarrow$ $X$ tel que $m \circ \eta_{X}=i d_{X}$ et $m \circ T(m)=m \circ \mu_{X}: T^{2}(X) \rightarrow X$.

(2) Soit $(T, \gamma, \delta)$ une comonade sur une catégorie $\mathcal{C}$. Un comodule sur cette comonade est un module sur la monade de $\mathcal{C}^{o p}$ associée, i.e. un objet $X$ de $\mathcal{C}$ muni d'un morphisme $c: X \rightarrow T(X)$ tel que $\gamma_{X} \circ c=i d_{X}$ et $T(c) \circ c=\delta_{X} \circ c: X \rightarrow T^{2}(X)$.

On définit de façon évidente la notion de morphisme de modules sur une monade, ou de comodules sur une comonade. On prendra garde au fait que nous nommons module (resp. comodule) ce que Mac Lane appelle algèbre (resp. coalgèbre).

Proposition A.2.5. - Il existe un foncteur de $\mathcal{A}$ vers la catégorie des comodules sur la comonade $\left(G F, \gamma, G\left(\eta_{F}\right)\right)$; il s'obtient sur les objets en munissant $G(X)$ (où $X \in \operatorname{Ob} \mathcal{A})$ de la structure de comodule donnée par le morphisme $G\left(\eta_{X}\right): G(X) \rightarrow$ $G F G(X)$. 
Cette proposition possède une variante duale en terme de monade.

Le résultat suivant, qui se déduit de [ML71], chapitre VI, $\S 7$, théorème 1 , est un cas particulier du théorème de Beck.

Proposition A.2.6. - Faisons les hypothèses suivantes :

(1) les catégories $\mathcal{A}$ et $\mathcal{B}$ sont abéliennes;

(2) le foncteur $G$ est exact et fidèle.

Alors le foncteur de la proposition précédente de $\mathcal{A}$ vers la catégorie des comodules sur la comonade déterminée par l'adjonction entre $F$ et $G$ est une équivalence de catégories.

Dualement, on a le résultat suivant.

Proposition A.2.7. — Faisons les hypothèses suivantes :

(1) les catégories $\mathcal{A}$ et $\mathcal{B}$ sont abéliennes;

(2) le foncteur $F$ est exact et fidèle.

Alors la catégorie $\mathcal{B}$ est équivalente à la catégorie des modules sur la monade déterminée par l'adjonction entre $F$ et $G$. 



\section{APPENDICE B}

\section{PROPRIÉTÉS DE FINITUDE DANS LES CATÉGORIES ABÉLIENNES}

Cet appendice donne les définitions, notations et propriétés relatives aux notions de finitude utilisées dans cet article. La plupart des ces notions se trouvent dans [Pop73], [Gab62] ou [CR90]; d'autres références et les démonstrations sont données dans [Dja06d].

Convention B.O.1. - Dans cet appendice, $\mathcal{A}$ désigne une catégorie de Grothendieck.

On rappelle que, par définition, une catégorie de Grothendieck est une catégorie abélienne possédant des colimites, un ensemble de générateurs et dans laquelle les colimites filtrantes sont exactes. Une telle catégorie possède toujours des limites, des enveloppes injectives et un cogénérateur injectif (cf. [Pop73], §3.7 et [Gab62], chapitre II, $\S 6)$.

\section{B.1. Définitions}

Soit $A$ un objet de $\mathcal{A}$.

Définition B.1.1. - On dit que $A$ est :

(1) noethérien si toute suite croissante de sous-objets de $A$ stationne;

(2) artinien si toute suite décroissante de sous-objets de $A$ stationne;

(3) de type fini si toute suite croissante de sous-objets de $A$ de colimite $A$ stationne;

(4) de co-type fini si toute suite décroissante de sous-objets de $A$ de limite nulle stationne.

Notation B.1.2. - Nous désignerons par $\mathcal{A}^{t f}$ la sous-catégorie pleine des objets de type fini de $\mathcal{A}$. 
Définition B.1.3. - On dit que la catégorie $\mathcal{A}$ est localement noethérienne (resp. co-localement artinienne) si elle possède un ensemble de générateurs (resp. cogénérateurs) noethériens (resp. artiniens).

Hypothèse B.1.4. - Il existe dans $\mathcal{A}$ un ensemble de générateurs projectifs de type fini.

Le premier point de la définition suivante est valable sous l'hypothèse B.1.4 : la « bonne » définition d'un objet $\mathrm{pf}_{n}$ est différente lorsque cette hypothèse n'est pas vérifiée.

Définition B.1.5. - Soit $n \in \mathbb{N}^{*}$. On dit que $A$ est :

(1) de $n$-présentation finie, en abrégé $\mathrm{pf}_{n}$, s'il existe une suite exacte

$$
P_{n} \rightarrow P_{n-1} \rightarrow \cdots \rightarrow P_{0} \rightarrow A \rightarrow 0
$$

où les $P_{i}$ sont des objets projectifs de type fini de $\mathcal{A}$.

(2) de $n$-co-présentation finie, en abrégé co-pf $f_{n}$, s'il existe une suite exacte

$$
0 \rightarrow I_{0} \rightarrow \cdots \rightarrow I_{n}
$$

où les $I_{i}$ sont des objets injectifs de co-type fini de $\mathcal{A}$.

On dit que $A$ est de présentation finie s'il est de 1-présentation finie, $\mathrm{pf}_{\infty}$ s'il est $\mathrm{pf}_{n}$ pour tout $n$. On adopte des simplifications terminologiques analogues pour les objets co-pf $f_{i}$ (ainsi, on écrira co-pf pour co-pf $f_{1}$ ).

La manipulation des objets $\mathrm{pf}_{\infty}$ est facilitée par la proposition suivante :

Proposition B.1.6. - Soit $0 \rightarrow A \rightarrow B \rightarrow C \rightarrow 0$ une suite exacte de $\mathcal{A}$. Si deux des objets $A, B$ et $C$ sont $p f_{\infty}$, il en est de même du troisième.

Définition B.1.7. - On dit que $A$ est :

(1) simple s'il est non nul mais que tous ses sous-objets stricts sont nuls;

(2) fini s'il admet une filtration finie de sous-quotients simples;

(3) localement fini s'il est colimite de ses sous-objets finis;

(4) co-localement fini s'il est limite de ses quotients finis.

Notation B.1.8. - On note $\mathcal{A}^{f}$ (respectivement $\mathcal{A}^{l f}$ ) la sous-catégorie pleine des objets finis (resp. localement finis) de $\mathcal{A}$.

Définition B.1.9. - (1) Une sous-catégorie de Serre de $\mathcal{A}$ est une sous-catégorie pleine $\mathcal{C}$ de $\mathcal{A}$ stable par sommes directes finies et par sous-quotients.

(2) Une sous-catégorie épaisse de $\mathcal{A}$ est une sous-catégorie de Serre de $\mathcal{A}$ stable par extensions.

(3) Une sous-catégorie localisante de $\mathcal{A}$ est une sous-catégorie épaisse de $\mathcal{A}$ stable par colimites.

Proposition B.1.10. - (1) La sous-catégorie $\mathcal{A}^{f}$ de $\mathcal{A}$ est épaisse. 
(2) La catégorie $\mathcal{A}^{l f}$ est une sous-catégorie de Serre de $\mathcal{A}$. Si les objets simples de $\mathcal{A}$ sont de présentation finie, elle est épaisse.

La dernière assertion de cette proposition est un cas particulier du résultat suivant.

Proposition B.1.11. - Soit $\mathcal{C}$ une sous-catégorie épaisse de $\mathcal{A}$. Notons $\overline{\mathcal{C}}$ la souscatégorie pleine de $\mathcal{A}$ dont les objets sont les colimites d'objets de $\mathcal{C}$.

Si les objets de $\mathcal{C}$ sont de présentation finie, alors $\overline{\mathcal{C}}$ est une sous-catégorie épaisse de $\mathcal{A}$.

Définition B.1.12. - (1) On appelle socle de $A$ la somme $\operatorname{soc}(A)$ des sous-objets simples de $A$.

(2) On nomme radical de $A$ l'intersection $\operatorname{rad}(A)$ des sous-objets stricts maximaux de $A$. On appelle cosocle de $A$ le quotient $A / \operatorname{rad}(A)$, noté $\operatorname{cosoc}(A)$.

Proposition B.1.13. - Si A est localement fini et que $\operatorname{soc}(A)$ est fini, alors $A$ est de co-type fini.

Catégories abéliennes quotients. - La définition B.1.9 permet également d'introduire la notion classique de catégorie quotient dans le contexte abélien, notion fondamentale dans cet article. Lorsque $\mathcal{C}$ est une sous-catégorie épaisse de $\mathcal{A}$, on peut définir la catégorie abélienne quotient $\mathcal{A} / \mathcal{C}$, et l'on dispose d'un foncteur canonique $\pi: \mathcal{A} \rightarrow \mathcal{A} / \mathcal{C}$, qui est exact. Si $\mathcal{C}$ est de plus localisante, alors $\mathcal{A} / \mathcal{C}$ est une catégorie de Grothendieck, et le foncteur canonique possède un adjoint à droite, appelé foncteur section. Nous renvoyons le lecteur à [Gab62] pour ces considérations.

On utilisera également la terminologie suivante, dans le cas où $\mathcal{C}$ est une souscatégorie épaisse de $\mathcal{A}$.

(1) On dit qu'un objet $X$ de $\mathcal{A}$ est $\mathcal{C}$-fermé si $\operatorname{Ext}_{\mathcal{A}}^{i}(C, X)=0$ pour $C \in \mathrm{Ob} \mathcal{C}$ et $i \in\{0,1\}$.

(2) On dit qu'un objet $X$ de $\mathcal{A}$ est $\mathcal{C}$-parfait si $\operatorname{Ext}_{\mathcal{A}}^{*}(C, X)=0$ pour tout objet $C$ de $\mathcal{C}$. Le cas échéant, et si $\mathcal{C}$ est de plus localisante ${ }^{(1)}$, le foncteur canonique $\pi: \mathcal{A} \rightarrow \mathcal{A} / \mathcal{C}$ induit un isomorphisme $\operatorname{Ext}_{\mathcal{A}}^{*}(A, X) \stackrel{\simeq}{\longrightarrow} \operatorname{Ext}_{\mathcal{A} / \mathcal{C}}^{*}(\pi(A), \pi(X))$ pour tout objet $A \operatorname{de} \mathcal{A}$. Ce fait sera utilisé abondamment dans cet article, sans plus de précision.

Proposition B.1.14. - Soit $\mathcal{C}$ une sous-catégorie épaisse de $\mathcal{A}$ et $\overline{\mathcal{C}}$ la plus petite sous-catégorie localisante de $\mathcal{A}$ contenant $\mathcal{C}$. Tout objet $\mathcal{C}$-parfait est $\overline{\mathcal{C}}$-parfait.

Démonstration. - Soit $X$ un objet $\mathcal{C}$-parfait de $\mathcal{A}$, la sous-catégorie pleine des objets $A$ de $\mathcal{A}$ tels que $\operatorname{Ext}^{*}(B, X)=0$ pour tout sous-objet $B$ de $A$ est épaisse, contient $\mathcal{C}$ par hypothèse, et elle est stable par colimites filtrantes par le théorème 4.2 de [Jen72].

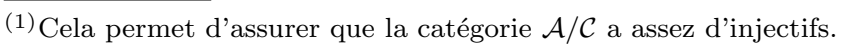




\section{B.2. Effet de foncteurs exacts}

Les propositions énoncées dans cette section s'établissent par des méthodes standard; on en trouvera une démonstration complète dans [Dja06d].

Proposition B.2.1. — Soient $\mathcal{B}$ une catégorie de Grothendieck et $F: \mathcal{A} \rightarrow \mathcal{B}$ un foncteur exact et fidèle.

(1) Si $X$ est un objet de $\mathcal{A}$ tel que $F(X)$ est noethérien (resp. artinien) dans $\mathcal{B}$, alors $X$ est noethérien (resp. artinien) dans $\mathcal{A}$.

(2) Si F commute aux colimites (resp. limites) filtrantes et si $X$ est un objet de $\mathcal{A}$ tel que $F(X)$ est de type fini (resp. de co-type fini), alors $X$ est de type fini (resp. de co-type fini).

(3) Supposons que $F$ est plein et que son image est une sous-catégorie de Serre de B. Si $X$ est un objet noethérien (resp. de type fini, artinien, de co-type fini) de $\mathcal{A}$, alors $F(X)$ est un objet noethérien (resp. de type fini, artinien, de co-type fini) de $\mathcal{B}$.

Proposition B.2.2. - Soient $i \in \mathbb{N}^{*}$ et $F$ un foncteur exact et fidèle de $\mathcal{A}$ dans une catégorie de Grothendieck, commutant aux colimites filtrantes et préservant les objets projectifs de type fini. Si $X$ est un objet de $\mathcal{A}$ tel que $F(X)$ est pf $f_{i}$, alors $X$ est $p f_{i}$.

Proposition B.2.3. - Soient $\mathcal{B}$ une catégorie de Grothendieck et $F: \mathcal{A} \rightarrow \mathcal{B}$ un foncteur exact.

(1) Supposons $F$ fidèle. Si $X$ est un objet de $\mathcal{A}$ tel que $F(X)$ est fini dans $\mathcal{B}$, alors $X$ est fini dans $\mathcal{A}$.

(2) Supposons que $F$ est plein et que son image est une sous-catégorie de Serre de B. Si $X$ est un objet fini de $\mathcal{A}$, alors $F(X)$ est un objet fini de $\mathcal{B}$.

\section{B.3. Groupes de Grothendieck}

Définition B.3.1. - Soit $\mathcal{C}$ une sous-catégorie pleine et petite de $\mathcal{A}$, contenant 0. On appelle groupe de Grothendieck de $\mathcal{C}$ relativement à $\mathcal{A}$ le groupe abélien noté $G_{0}(\mathcal{C} ; \mathcal{A})$ défini par générateurs et relations de la manière suivante.

- Générateurs : un générateur $[A]$ pour chaque objet $A$ de $\mathcal{C}$.

- Relations : $[A]=[B]+[C]$ pour toute suite exacte courte $0 \rightarrow B \rightarrow A \rightarrow$ $C \rightarrow 0$ de $\mathcal{A}$ dont tous les objets $A, B, C$ sont dans $\mathcal{C}$.

On a $[A]=[B]$ dans $G_{0}(\mathcal{C} ; \mathcal{A})$ si $A$ et $B$ sont deux objets isomorphes de $\mathcal{C}$, ce qui permet de définir ce groupe lorsque $\mathcal{C}$ est seulement essentiellement petite.

Lemme B.3.2. - La sous-catégorie $\mathcal{A}^{\text {tf }}$ de $\mathcal{A}$ est essentiellement petite.

Cela permet de donner la notation suivante. 
Notation B.3.3. - Nous noterons respectivement $G_{0}^{f}(\mathcal{A}), G_{0}^{t f}(\mathcal{A})$ et $K_{0}(\mathcal{A})$ les groupes de Grothendieck $G_{0}\left(\mathcal{A}^{f} ; \mathcal{A}\right), G_{0}\left(\mathcal{A}^{t f}, \mathcal{A}\right)$ et $G_{0}(\mathcal{C} ; \mathcal{A})$, où $\mathcal{C}$ est la souscatégorie pleine des objets projectifs de type fini de $\mathcal{A}$.

Le théorème de Jordan-Hölder dit que le groupe abélien $G_{0}^{f}(\mathcal{A})$ est libre, les classes des objets simples de $\mathcal{A}$ en formant une base.

Nous rappelons maintenant la notion de recollement de catégories abéliennes.

Définition B.3.4. - Un diagramme de recollement est un diagramme du type

$$
\mathcal{C} \underset{p}{\stackrel{q}{\rightleftarrows}} \mathcal{A} \underset{r}{\stackrel{l}{\rightleftarrows}} \mathcal{B}
$$

dans lequel :

- $\mathcal{A}, \mathcal{B}$ et $\mathcal{C}$ sont des catégories abéliennes.

- Le foncteur $l$ est adjoint à gauche à $e$ et $e$ est adjoint à gauche à $r$ (en particulier, $e$ est exact).

- L'unité $i d_{\mathcal{B}} \rightarrow e l$ et la coünité $e r \rightarrow i d_{\mathcal{B}}$ sont des isomorphismes.

- Le foncteur $q$ est adjoint à gauche à $i$ et $i$ est adjoint à gauche à $p$ (en particulier, $i$ est exact).

- L'unité $i d_{\mathcal{C}} \rightarrow p i$ et la coünité $q i \rightarrow i d_{\mathcal{C}}$ sont des isomorphismes.

- Le foncteur $i$ est un plongement pleinement fidèle d'image ker e (en particulier, $i$ identifie $\mathcal{C}$ à une sous-catégorie épaisse de $\mathcal{A}$ ).

Dans cette situation, le foncteur $e$ induit une équivalence $\mathcal{A} / \mathcal{C} \cong \mathcal{B}$.

Nous renvoyons à $[\mathbf{K u h 9 4 b}$ ] à ce sujet, ainsi que pour la démonstration de la proposition suivante (et la description explicite de l'isomorphisme).

Proposition B.3.5. - On a un isomorphisme de groupes $G_{0}^{f}(\mathcal{A}) \simeq G_{0}^{f}(\mathcal{B}) \oplus G_{0}^{f}(\mathcal{C})$.

\section{B.4. Filtration de Krull}

Définition B.4.1. - La filtration de Krull de la catégorie $\mathcal{A}$ est la suite croissante de sous-catégories épaisses stables par colimites $\mathcal{K}_{n}(\mathcal{A})$ de $\mathcal{A}$ définie inductivement comme suit.

- La catégorie $\mathcal{K}_{n}(\mathcal{A})$ est réduite à l'objet nul pour $n<0$.

- Pour $n \geq 0, \mathcal{K}_{n}(\mathcal{A})$ est l'image réciproque par le foncteur canonique $\mathcal{A} \rightarrow$ $\mathcal{A} / \mathcal{K}_{n-1}(\mathcal{A})$ de la plus petite sous-catégorie localisante de $\mathcal{A} / \mathcal{K}_{n-1}(\mathcal{A})$ contenant tous les objets simples de cette catégorie.

Remarque B.4.2. - On peut étendre de manière claire la définition de la filtration de Krull à tout ordinal (cf. [Gab62]); nous n'avons introduit que les termes indicés par $\mathbb{N}$ car eux seuls interviendront dans notre contexte. 
Définition B.4.3. - On définit par récurrence sur $n \in \mathbb{N} \cup\{-1\}$ les notions d'objet simple noethérien de type $n$ et noethérien de type $n$ de $\mathcal{A}$ de la façon suivante.

- Un objet est simple noethérien de type -1 s'il est nul.

- Un objet $X$ est noethérien de type $n$ s'il possède une filtration finie $0=F_{0} \subset$ $F_{1} \subset \cdots \subset F_{k}=X$ telle que, pour tout $i \in\{1, \ldots, k\}$, le quotient $F_{i} / F_{i-1}$ est simple noethérien de type $a(i)$, pour un certain entier $a(i) \leq n$.

- Un objet est simple noethérien de type $n$ pour $n \in \mathbb{N}$ si et seulement s'il n'est pas noethérien de type $n-1$ et que tous ses quotients stricts sont noethériens de type $n-1$.

Notation B.4.4. - Nous désignerons par $\mathcal{A}_{\mathbf{N T}(n)}$ la sous-catégorie pleine de $\mathcal{A}$ formée des objets noethériens de type $n$. Elle est épaisse.

Proposition B.4.5. - Soient $n \in \mathbb{N} \cup\{-1\}$ et $X$ un objet noethérien de type $n$ de $\mathcal{A}$. Alors $X$ est noethérien, appartient à $\mathcal{K}_{n}(\mathcal{A})$ et son image dans $\mathcal{A} / \mathcal{K}_{n-1}(\mathcal{A})$ (si $n \geq 0)$ est finie. Elle est même simple si $X$ est simple noethérien de type $n$.

Réciproquement, un objet noethérien de $\mathcal{A}$ qui appartient à $\mathcal{K}_{n}(\mathcal{A})$ est noethérien de type $n$. 


\section{APPENDICE C}

\section{CATÉGORIES DE FONCTEURS}

Nous rappelons ici quelques propriétés d'usage courant des catégories de foncteurs. Bien que standard, elles ne sont pas toujours facilement accessibles dans la littérature — voir [Dja06d] pour plus de détails.

Convention C.0.1. - Dans cet appendice, $\mathcal{I}$ et $\mathcal{J}$ désignent des catégories essentiellement petites, $\mathcal{A}$ et $\mathcal{B}$ des catégories abéliennes et $A$ un anneau.

\section{C.1. Généralités}

Notation C.1.1. - (1) Si $F$ est un foncteur de $\mathcal{J}$ vers $\mathcal{I}$, nous désignerons par $F^{*}: \operatorname{Fct}(\mathcal{I}, \mathcal{A}) \rightarrow \boldsymbol{F} \operatorname{ct}(\mathcal{J}, \mathcal{A})$ le foncteur de précomposition par $F$.

(2) Pour tout foncteur $G: \mathcal{A} \rightarrow \mathcal{B}$, nous désignerons $\operatorname{par} G_{*}: \operatorname{Fct}(\mathcal{I}, \mathcal{A}) \rightarrow$ $\operatorname{Fct}(\mathcal{I}, \mathcal{B})$ le foncteur de postcomposition par $G$.

(3) Soit $E$ un objet de $\mathcal{I}$. Nous noterons $e v_{E}: \operatorname{Fct}(\mathcal{I}, \mathcal{A}) \rightarrow \mathcal{A}$ et ev $E$ : $\operatorname{Fct}\left(\mathcal{I},{ }_{A} \mathbf{M o d}\right) \rightarrow_{A[M]}$ Mod les foncteurs d'évaluation en $E$, donnés par la précomposition par le foncteur $* \rightarrow \mathcal{I}$ d'image $E$ et par le foncteur pleinement fidèle $\operatorname{End}_{\mathcal{I}}(E) \rightarrow \mathcal{I}$ d'image $E$ respectivement.

Proposition C.1.2. - (1) La catégorie $\operatorname{Fct}(\mathcal{I}, \mathcal{A})$ est abélienne.

(2) L'exactitude se teste « argument par argument $»:$ une suite $X \rightarrow Y \rightarrow Z$ de $\operatorname{Fct}(\mathcal{I}, \mathcal{A})$ est exacte si et seulement si la suite $X(E) \rightarrow Y(E) \rightarrow Z(E)$ est exacte dans $\mathcal{A}$ pour tout objet $E$ de $\mathcal{I}$.

(3) Si $\mathcal{A}$ est une catégorie $A$-linéaire, $\operatorname{Fct}(\mathcal{I}, \mathcal{A})$ hérite d'une structure de catégorie A-linéaire.

(4) Supposons que la catégorie abélienne $\mathcal{A}$ est monö̈dale symétrique. Le bifoncteur

$$
\operatorname{Fct}(\mathcal{I}, \mathcal{A}) \times \mathbf{F} \operatorname{ct}(\mathcal{I}, \mathcal{A}) \simeq \operatorname{Fct}(\mathcal{I}, \mathcal{A} \times \mathcal{A}) \stackrel{\otimes_{*}}{\longrightarrow} \operatorname{Fct}(\mathcal{I}, \mathcal{A})
$$

définit une structure monoïdale symétrique $\operatorname{sur} \operatorname{Fct}(\mathcal{I}, \mathcal{A})$. 
On rappelle que les notions de sous-catégorie de Serre et sous-catégorie épaisse sont introduites dans la définition B.1.9.

Proposition C.1.3. - Soit $F: \mathcal{A} \rightarrow \mathcal{B}$ un foncteur.

(1) Si F est exact (resp. additif, exact à gauche, exact à droite), il en est de même pour $F_{*}: \operatorname{Fct}(\mathcal{I}, \mathcal{A}) \rightarrow \operatorname{Fct}(\mathcal{I}, \mathcal{B})$.

(2) Si $F$ est fidèle, alors $F_{*}$ est fidèle.

(3) Si F est pleinement fidèle, alors $F_{*}$ est pleinement fidèle. Si de plus l'image de $F$ est une sous-catégorie de Serre (resp. épaisse) de $\mathcal{B}$, alors l'image de $F_{*}$ est une sous-catégorie de Serre (resp. épaisse) de $\boldsymbol{F} \mathbf{c t}(\mathcal{I}, \mathcal{B})$.

(4) Si $F$ possède un adjoint à gauche $G$, alors $G_{*}$ est adjoint à gauche à $F_{*}$.

Proposition C.1.4. - Soit $F: \mathcal{J} \rightarrow \mathcal{I}$ un foncteur.

(1) Le foncteur $F^{*}: \operatorname{Fct}(\mathcal{I}, \mathcal{A}) \rightarrow \boldsymbol{F} \mathbf{c t}(\mathcal{J}, \mathcal{A})$ commute aux limites et aux colimites ; il est en particulier exact.

(2) Si $\mathcal{A}$ est une catégorie monoïdale symétrique, $F^{*}$ commute au produit tensoriel.

(3) Si F est essentiellement surjectif, $F^{*}$ est fidèle.

(4) Si F est plein et essentiellement surjectif, $F^{*}$ est pleinement fidèle, et son image est une sous-catégorie de Serre de $\operatorname{Fct}(\mathcal{J}, \mathcal{A})$ stable par limites et colimites.

(5) Si F possède un adjoint à gauche $G$, alors $G^{*}$ est adjoint à droite à $F^{*}$.

Dans la suite de cette annexe, on ne s'intéresse plus qu'au cas où la catégorie but est la catégorie d'espaces vectoriels $\mathcal{E}_{\mathbb{k}}$.

\section{C.2. Générateurs projectifs}

Étant donné un objet $E$ de $\mathcal{I}$, nous noterons $P_{E}^{\mathcal{I}}$ l'objet $\mathbb{k}\left[\operatorname{hom}_{\mathcal{I}}(E, \cdot)\right] \operatorname{de} \operatorname{Fct}\left(\mathcal{I}, \mathcal{E}_{\mathbb{k}}\right)$. On rappelle que $\mathbb{k}[$.$] désigne le foncteur de \mathbb{k}$-linéarisation; ainsi, $\mathbb{k}[F]$ désigne la $\mathbb{k}$ linéarisation $\mathbb{k}[\cdot]_{*} F$ d'un foncteur $F: \mathcal{I} \rightarrow$ Ens.

La bifonctoralité de $\operatorname{hom}_{\mathcal{I}}$ permet de considérer $E \mapsto P_{E}^{\mathcal{I}}$ comme un foncteur $\mathcal{I}^{o p} \rightarrow \operatorname{Fct}\left(\mathcal{I}, \mathcal{E}_{\mathbb{k}}\right)$.

Proposition C.2.1 (Lemme de Yoneda linéaire). - Il existe un isomorphisme

$$
\operatorname{hom}_{\mathbf{F c t}\left(\mathcal{I}, \mathcal{E}_{\mathrm{k}}\right)}\left(P_{E}^{\mathcal{I}}, F\right) \simeq F(E)
$$

naturel en les objets $E$ de $\mathcal{I}$ et $F$ de $\boldsymbol{F} \mathbf{c t}\left(\mathcal{I}, \mathcal{E}_{\mathbb{k}}\right)$.

Proposition et définition C.2.2. - Les objets $P_{E}^{\mathcal{I}}$ forment un ensemble de générateurs projectifs de type fini de $\boldsymbol{F} \operatorname{ct}\left(\mathcal{I}, \mathcal{E}_{\mathbb{k}}\right)$ lorsque $E$ parcourt un squelette de $\mathcal{I}$. On les appelle générateurs projectifs standard $\operatorname{de} \operatorname{Fct}\left(\mathcal{I}, \mathcal{E}_{\mathbb{k}}\right)$.

Corollaire C.2.3. - La catégorie de foncteurs $\mathbf{F} \mathbf{c t}\left(\mathcal{I}, \mathcal{E}_{\mathbb{k}}\right)$ est une catégorie de Grothendieck vérifiant l'hypothèse B.1.4. 
Corollaire C.2.4. - Pour tout foncteur $F: \mathcal{J} \rightarrow \mathcal{I}$, le foncteur de précomposition $F^{*}: \operatorname{Fct}\left(\mathcal{I}, \mathcal{E}_{\mathrm{k}}\right) \rightarrow \operatorname{Fct}\left(\mathcal{J}, \mathcal{E}_{\mathrm{k}}\right)$ admet un adjoint à droite et un adjoint à gauche.

Les extensions de Kan (cf. [ML71], ch. X) permettent de donner une construction des adjoints.

Remarque C.2.5. - Si deux objets $V$ et $W$ de $\mathcal{I}$ possèdent une somme, on a un isomorphisme canonique $P_{V}^{\mathcal{I}} \otimes P_{W}^{\mathcal{I}} \simeq P_{V \amalg W}^{\mathcal{I}}$.

Corollaire C.2.6. - Supposons que $\mathcal{I}$ possède des sommes finies. Alors le produit tensoriel de $\boldsymbol{F} \mathbf{c t}\left(\mathcal{I}, \mathcal{E}_{\mathrm{k}}\right)$ préserve les objets projectifs et les objets de type fini.

Proposition et définition C.2.7 (Dualité entre catégories de foncteurs)

Notons $D_{\mathcal{I}, \mathbb{k}}: \mathbf{F c t}\left(\mathcal{I}, \mathcal{E}_{\mathbb{k}}\right)^{o p} \simeq \mathbf{F} \mathbf{c t}\left(\mathcal{I}^{o p}, \mathcal{E}_{\mathbb{k}}^{o p}\right) \rightarrow \mathbf{F c t}\left(\mathcal{I}^{o p}, \mathcal{E}_{\mathbb{k}}\right)$ le foncteur de postcomposition par le foncteur de dualité $(.)^{*}=\operatorname{hom}_{\mathcal{E}}(., \mathbb{k}): \mathcal{E}_{\mathbb{k}}^{o p} \rightarrow \mathcal{E}_{\mathbb{k}}$, et $D_{\mathcal{I}, \mathbb{k}}^{\prime}=D_{\mathcal{I}^{o p}, \mathbb{k}}$ : $\operatorname{Fct}\left(\mathcal{I}^{o p}, \mathcal{E}_{\mathbb{k}}\right)^{o p} \rightarrow \operatorname{F} \operatorname{ct}\left(\mathcal{I}, \mathcal{E}_{\mathbb{k}}\right)$

(1) Les foncteurs $D_{\mathcal{I}, \mathrm{k}}$ et $D_{\mathcal{I}, \mathrm{k}}^{\prime}$ sont exacts et fidèles.

(2) Le foncteur $D_{\mathcal{I}, \mathrm{k}}$ est adjoint à droite à $\left(D_{\mathcal{I}, \mathrm{k}}^{\prime}\right)^{o p}$.

(3) Ces foncteurs induisent des équivalences de catégories réciproques l'une de l'autre entre $\mathbf{F} \mathbf{c t}\left(\mathcal{I}, \mathcal{E}_{\mathbb{k}}^{f}\right)^{o p}$ et $\mathbf{F} \mathbf{c t}\left(\mathcal{I}^{o p}, \mathcal{E}_{\mathbb{k}}^{f}\right)$.

Ces foncteurs seront appelés foncteurs de dualité.

Ce résultat entraîne formellement, via la proposition C.2.1, la propriété suivante.

\section{Proposition et définition C.2.8 (Cogénérateurs injectifs standard)}

Pour tout objet $E$ de $\mathcal{I}$, nous noterons $I_{E}^{\mathcal{I}}$ l'objet $D_{\mathcal{I}, \mathbb{k}}^{\prime}\left(P_{E}^{\mathcal{I}^{o p}}\right) \operatorname{de} \mathbf{F} \mathbf{c t}\left(\mathcal{I}, \mathcal{E}_{\mathbb{k}}\right)$ (ainsi, on a $\left.I_{E}^{\mathcal{I}}(V)=\mathbb{k}^{\operatorname{hom}_{\mathcal{I}}(V, E)}\right)$. Cette construction est fonctorielle contravariante en $E$.

(1) Il existe un isomorphisme $\operatorname{hom}_{\mathbf{F c t}\left(\mathcal{I}, \mathcal{E}_{\mathfrak{k}}\right)}\left(F, I_{E}^{\mathcal{I}}\right) \simeq F(E)^{*}$ naturel en les objets $F$ de $\operatorname{F} \operatorname{ct}\left(\mathcal{I}, \mathcal{E}_{\mathbb{k}}\right)$ et $E$ de $\mathcal{I}$.

(2) Les $I_{E}^{\mathcal{I}}$ forment un ensemble de cogénérateurs injectifs lorsque $E$ décrit un squelette de $\mathcal{I}$. On les appelle cogénérateurs injectifs standard $\operatorname{de} \mathbf{F} \mathbf{c t}\left(\mathcal{I}, \mathcal{E}_{\mathrm{k}}\right)$.

Dans tous les cas que nous considérerons, l'hypothèse suivante sera vérifiée ${ }^{(1)}$.

Hypothèse C.2.9. - Les ensembles $\operatorname{hom}_{\mathcal{I}}(V, W)$ sont finis pour tous $V, W \in \mathrm{Ob} \mathcal{I}$.

Cette hypothèse assure que les foncteurs projectifs standard, donc tous les foncteurs de type fini, prennent des valeurs de dimension finie, de sorte que la dernière assertion de la proposition C.2.7 donne un lien très rigide entre $\operatorname{Fct}\left(\mathcal{I}, \mathcal{E}_{\mathbb{k}}\right)^{o p}$ et $\operatorname{Fct}\left(\mathcal{I}^{o p}, \mathcal{E}_{\mathrm{k}}\right)$.

Corollaire C.2.10. - Lorsque l'hypothèse C.2.9 est satisfaite, les injectifs standard de la catégorie $\mathbf{F} \mathbf{c t}\left(\mathcal{I}, \mathcal{E}_{\mathbb{k}}\right)$ sont de co-type fini.

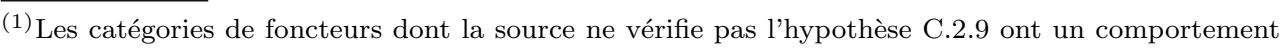
profondément différent de celles que nous étudierons.
} 


\section{C.3. Foncteurs hom internes et foncteurs de division}

Proposition et définition C.3.1. - (1) Pour tout objet $X$ de $\operatorname{Fct}\left(\mathcal{I}, \mathcal{E}_{\mathbb{k}}\right)$, l'endofoncteur $\cdot \otimes X$ de $\mathbf{F c t}\left(\mathcal{I}, \mathcal{E}_{\mathbb{k}}\right)$ admet un adjoint à droite, noté $\operatorname{Hom}_{\mathbf{F c t}\left(\mathcal{I}, \mathcal{E}_{\mathrm{k}}\right)}(X,$.$) ;$ on dispose ainsi d'un foncteur hom interne

$$
\operatorname{Hom}_{\mathrm{Fct}\left(\mathcal{I}, \mathcal{E}_{\mathrm{k}}\right)}: \operatorname{Fct}\left(\mathcal{I}, \mathcal{E}_{\mathrm{k}}\right)^{o p} \times \operatorname{Fct}\left(\mathcal{I}, \mathcal{E}_{\mathrm{k}}\right) \rightarrow \operatorname{Fct}\left(\mathcal{I}, \mathcal{E}_{\mathrm{k}}\right) .
$$

On note $\mathbf{E x t}_{\mathbf{F c t}\left(\mathcal{I}, \mathcal{E}_{\mathrm{k}}\right)}^{*}$ les foncteurs dérivés droits de ce bifoncteur exact à gauche.

(2) On a un isomorphisme naturel gradué

$$
\operatorname{Ext}_{\mathbf{F c t}\left(\mathcal{I}, \mathcal{E}_{\mathfrak{k}}\right)}^{*}(X, Y)(E) \simeq \operatorname{Ext}_{\mathbf{F c t}\left(\mathcal{I}, \mathcal{E}_{\mathbf{k}}\right)}^{*}\left(P_{E}^{\mathcal{I}} \otimes X, Y\right),
$$

où $E$ est un objet de $\mathcal{I}$ et $X$ et $Y$ sont des objets de $\mathbf{F} \mathbf{c t}\left(\mathcal{I}, \mathcal{E}_{\mathbb{k}}\right)$.

(3) Pour tout objet $A$ de $\operatorname{Fct}\left(\mathcal{I}, \mathcal{E}_{\mathrm{k}}^{f}\right)$, l'endofoncteur $\cdot \otimes A \operatorname{de} \boldsymbol{\operatorname { F c t }}\left(\mathcal{I}, \mathcal{E}_{\mathrm{k}}\right)$ admet un adjoint à gauche, noté $(\cdot: A)_{\mathbf{F c t}\left(\mathcal{I}, \mathcal{E}_{\mathrm{k}}\right)}$; on dispose ainsi d'un foncteur de division

$$
(\cdot: \cdot)_{\mathbf{F c t}\left(\mathcal{I}, \mathcal{E}_{\mathrm{k}}\right)}: \operatorname{Fct}\left(\mathcal{I}, \mathcal{E}_{\mathbb{k}}\right) \times \mathbf{F} \operatorname{ct}\left(\mathcal{I}, \mathcal{E}_{\mathbb{k}}^{f}\right)^{o p} \rightarrow \operatorname{Fct}\left(\mathcal{I}, \mathcal{E}_{\mathbb{k}}\right) .
$$

Les indices seront omis dans ces notations quand il n'y a pas d'ambiguïté.

La terminologie de foncteur hom interne est standard (cf. [ML71], ch. VII, §7). Le terme de foncteur de division a quant à lui été introduit par Lannes (cf. [Lan92]) dans le cadre des modules instables sur l'algèbre de Steenrod, voisin de celui des catégories de foncteurs (cf. [Pow98b], §3).

Proposition C.3.2. - Soient $F$ un endofoncteur de $\mathcal{I}$ et $T$ un objet de $\mathbf{F c t}(\mathcal{I}$, Ens $)$ tels qu'il existe une bijection

$$
\operatorname{hom}_{\mathcal{I}}(F(V), W) \simeq \operatorname{hom}_{\mathcal{I}}(V, W) \times T(W)
$$

naturelle en les objets $V$ et $W$ de $\mathcal{I}$.

Alors le foncteur $\mathbf{H o m}_{\mathbf{F c t}\left(\mathcal{I}, \mathcal{E}_{\mathrm{k}}\right)}(\mathbb{k}[T],$.$) est isomorphe au foncteur de précomposition$ $F^{*}$; autrement dit, $F^{*}$ est adjoint à droite $\grave{a} \cdot \otimes \mathbb{k}[T]$.

Proposition C.3.3. - Soient $F$ un endofoncteur de $\mathcal{I}$ et $T$ un objet de $\boldsymbol{F} \operatorname{ct}\left(\mathcal{I}^{o p}\right.$, Ens $\left.^{f}\right)$ tels qu'il existe une bijection

$$
\operatorname{hom}_{\mathcal{I}}(V, F(W)) \simeq \operatorname{hom}_{\mathcal{I}}(V, W) \times T(V)
$$

naturelle en les objets $V$ et $W$ de $\mathcal{I}$.

Alors le foncteur $\left(\cdot: \mathbb{k}^{T}\right)_{\mathbf{F c t}\left(\mathcal{I}, \mathcal{E}_{\mathrm{k}}\right)}$ est isomorphe au foncteur de précomposition $F^{*}$; autrement dit, $F^{*}$ est adjoint à gauche $\grave{a} \cdot \otimes \mathbb{k}^{T}$.

\section{C.4. Décomposition scalaire}

On suppose ici que $\mathcal{I}$ est une catégorie $\mathbb{k}$-linéaire. 
Notation C.4.1. - Étant donné un entier naturel $i$, on désigne par $\operatorname{Fct}\left(\mathcal{I}, \mathcal{E}_{\mathbb{k}}\right)_{i}$ la sous-catégorie pleine $\operatorname{de} \operatorname{Fct}\left(\mathcal{I}, \mathcal{E}_{\mathbb{k}}\right)$ formée des foncteurs $F$ tels que pour tout $\lambda \in \mathbb{k}$ et tout objet $E$ de $\mathcal{I}$, on a $F\left(\lambda . i d_{E}\right)=\lambda^{i} . i d_{F(E)}\left(\right.$ on convient ici que $\left.0^{0}=1\right)$.

Le produit tensoriel induit des foncteurs $\mathbf{F} \mathbf{c t}\left(\mathcal{I}, \mathcal{E}_{\mathbb{k}_{\mathrm{k}}}\right)_{i} \times \mathbf{F} \mathbf{c t}\left(\mathcal{I}, \mathcal{E}_{\mathrm{k}}\right)_{j} \rightarrow \mathbf{F} \mathbf{c t}\left(\mathcal{I}, \mathcal{E}_{\mathrm{k}}\right)_{i+j}$.

Comme le groupe cyclique fini $\mathbb{k}^{\times}$est d'ordre premier à la caractéristique de $\mathbb{k}$, il existe un isomorphisme de $\mathbb{k}$-algèbres $\mathbb{k}\left[\mathbb{k}^{\times}\right] \simeq \mathbb{k}^{q-1}$, et la $\mathbb{k}$-algèbre du monoïde multiplicatif sous-jacent à $\mathbb{k}$ est isomorphe à $\mathbb{k}^{q}$. On en déduit la proposition suivante (cf. [Kuh94a], §3.3).

Proposition et définition C.4.2. - Les inclusions $\operatorname{Fct}\left(\mathcal{I}, \mathcal{E}_{\mathbb{k}}\right)_{i} \hookrightarrow \operatorname{Fct}\left(\mathcal{I}, \mathcal{E}_{\mathbb{k}}\right)$ induisent une équivalence de catégories

$$
\operatorname{Fct}\left(\mathcal{I}, \mathcal{E}_{\mathrm{k}}\right) \simeq \prod_{i=0}^{q-1} \boldsymbol{F} \operatorname{ct}\left(\mathcal{I}, \mathcal{E}_{\mathrm{k}}\right)_{i} .
$$

Nous noterons $F \simeq \bigoplus_{i=0}^{q-1} F_{i}$ la décomposition canonique d'un foncteur $F$ de $\operatorname{F} \operatorname{ct}\left(\mathcal{I}, \mathcal{E}_{\mathbb{k}}\right)$ qu'on en déduit, où $F_{i}$ appartient à $\mathbf{F} \mathbf{c t}\left(\mathcal{I}, \mathcal{E}_{\mathbb{k}}\right)_{i}$. On l'appelle décomposition scalaire de $F$.

\section{C.5. Produit tensoriel extérieur}

C'est le foncteur

$$
\nabla: \operatorname{Fct}\left(\mathcal{I}, \mathcal{E}_{\mathrm{k}}\right) \times \operatorname{Fct}\left(\mathcal{J}, \mathcal{E}_{\mathrm{k}}\right) \rightarrow \operatorname{Fct}\left(\mathcal{I} \times \mathcal{J}, \mathcal{E}_{\mathrm{k}}\right)
$$

défini par la composition

$\operatorname{Fct}\left(\mathcal{I}, \mathcal{E}_{k}\right) \times \mathbf{F} \operatorname{ct}\left(\mathcal{J}, \mathcal{E}_{k}\right) \stackrel{\pi_{\mathcal{I}}^{*} \times \pi_{\mathcal{J}}^{*}}{\longrightarrow} \operatorname{Fct}\left(\mathcal{I} \times \mathcal{J}, \mathcal{E}_{k}\right) \times \mathbf{F} \operatorname{ct}\left(\mathcal{I} \times \mathcal{J}, \mathcal{E}_{k}\right) \stackrel{\otimes}{\longrightarrow} \mathbf{F c t}\left(\mathcal{I} \times \mathcal{J}, \mathcal{E}_{k}\right)$, où l'on note $\pi_{\mathcal{I}}: \mathcal{I} \times \mathcal{J} \rightarrow \mathcal{I}$ et $\pi_{\mathcal{J}}: \mathcal{I} \times \mathcal{J} \rightarrow \mathcal{J}$ les foncteurs de projection. Autrement dit, $(F \otimes G)(A, B)=F(A) \otimes G(B)$.

On a ainsi des isomorphismes canoniques $P_{(A, B)}^{\mathcal{I} \times \mathcal{J}} \simeq P_{A}^{\mathcal{I}} \otimes P_{B}^{\mathcal{J}}$; cette observation et ses conséquences, ainsi que les propriétés que nous rappelons ensuite, justifient la convention de notation suivante.

Notation C.5.1. - Nous désignerons par $\operatorname{Fct}\left(\mathcal{I}, \mathcal{E}_{\mathrm{k}}\right) \otimes \operatorname{Fct}\left(\mathcal{J}, \mathcal{E}_{\mathrm{k}}\right)$, par abus, la catégorie $\operatorname{Fct}\left(\mathcal{I} \times \mathcal{J}, \mathcal{E}_{\mathbb{k}}\right)$.

Proposition C.5.2 (Simples $\left.\operatorname{de} \operatorname{Fct}\left(\mathcal{I} \times \mathcal{J}, \mathcal{E}_{\mathrm{k}}\right)\right)$. - (1) Si $\mathcal{I}$ et $\mathcal{J}$ vérifient l'hypothèse C.2.9, pour tout objet simple $S \operatorname{de} \boldsymbol{F} \mathbf{c t}\left(\mathcal{I} \times \mathcal{J}, \mathcal{E}_{\mathbb{k}}\right)$, il existe un objet simple $S_{1}$ de $\mathbf{F} \operatorname{ct}\left(\mathcal{I}, \mathcal{E}_{\mathrm{k}}\right)$, un objet simple $S_{2}$ de $\mathbf{F c t}\left(\mathcal{I}, \mathcal{E}_{\mathrm{k}}\right)$ et un épimorphisme $S_{1} \otimes S_{2} \rightarrow S$.

(2) Soient $S$ un objet simple de $\mathbf{F} \operatorname{ct}\left(\mathcal{I}, \mathcal{E}_{\mathbb{k}}\right)$ et $S^{\prime}$ un objet simple de $\operatorname{Fct}\left(\mathcal{J}, \mathcal{E}_{\mathbb{k}}\right)$ tel que le corps $\operatorname{End}_{\mathbf{F c t}\left(\mathcal{J}, \mathcal{E}_{\mathbf{k}}\right)}\left(S^{\prime}\right)$ est réduit à $\mathbb{k}$. Alors $S \otimes S^{\prime}$ est un objet simple de $\operatorname{Fct}\left(\mathcal{I} \times \mathcal{J}, \mathcal{E}_{\mathbb{k}}\right)$. 
(3) Soient $S_{1}, S_{2}$ deux objets simples de $\boldsymbol{F} \mathbf{c t}\left(\mathcal{I}, \mathcal{E}_{\mathbb{k}}\right)$ et $S_{1}^{\prime}, S_{2}^{\prime}$ deux objets simples $\operatorname{de} \boldsymbol{F} \operatorname{ct}\left(\mathcal{J}, \mathcal{E}_{\mathbb{k}}\right)$. Si $S_{1} \otimes S_{1}^{\prime} \simeq S_{2} \otimes S_{2}^{\prime}$, alors $S_{1} \simeq S_{1}^{\prime}$ et $S_{2} \simeq S_{2}^{\prime}$.

Corollaire C.5.3. - Supposons que $\mathcal{I}$ et $\mathcal{J}$ vérifient l'hypothèse C.2.9 et que les corps d'endomorphismes des objets simples de $\operatorname{Fct}\left(\mathcal{J}, \mathcal{E}_{\mathbb{k}}\right)$ sont réduits à $\mathbb{k}$.

Le produit tensoriel extérieur induit des isomorphismes de groupes abéliens

$$
\begin{aligned}
& G_{0}^{f}\left(\boldsymbol{F} \operatorname{ct}\left(\mathcal{I} \times \mathcal{J}, \mathcal{E}_{\mathrm{k}}\right)\right) \simeq G_{0}^{f}\left(\boldsymbol{F} \operatorname{ct}\left(\mathcal{I}, \mathcal{E}_{\mathrm{k}}\right)\right) \otimes G_{0}^{f}\left(\operatorname{Fct}\left(\mathcal{J}, \mathcal{E}_{\mathrm{k}}\right)\right), \\
& K_{0}\left(\boldsymbol{F} \operatorname{ct}\left(\mathcal{I} \times \mathcal{J}, \mathcal{E}_{\mathrm{k}}\right)\right) \simeq K_{0}\left(\boldsymbol{F} \operatorname{ct}\left(\mathcal{I}, \mathcal{E}_{\mathrm{k}}\right)\right) \otimes K_{0}\left(\boldsymbol{F} \operatorname{ct}\left(\mathcal{J}, \mathcal{E}_{\mathbb{k}}\right)\right) .
\end{aligned}
$$

Ces propriétés se démontrent de façon analogue à celles du produit tensoriel extérieur usuel en théorie des représentations — cf. [CR90], chapitre 1, §10 E. Une démonstration des énoncés fonctoriels précédents est donnée dans [Dja06d].

\section{C.6. Recollements}

Nous terminons cet appendice avec quelques résultats communs destinés à définir et utiliser commodément le prolongement par zéro dans un cadre assez général. On les trouvera établis dans [Dja06d].

Définition C.6.1. - Soit $\mathcal{C}$ une sous-catégorie pleine de $\mathcal{I}$. Nous dirons que $\mathcal{C}$ est : - une sous-catégorie relativement connexe de $\mathcal{I}$ si pour tous objets $A, B$ et $X$ de $\mathcal{I}$ tels que $A, B \in \operatorname{ObC}$, $\operatorname{hom}(A, X) \neq \varnothing$ et $\operatorname{hom}(X, B) \neq \varnothing$, on a $X \in \mathrm{ObC}$; - une sous-catégorie complète à gauche de $\mathcal{I}$ si pour tout objet $E$ de $\mathcal{I}, E$ est objet de $\mathcal{C}$ dès que $\operatorname{hom}_{\mathcal{I}}(E, X) \neq \varnothing$ pour un objet $X$ de $\mathcal{C}$;

- une sous-catégorie complète à droite de $\mathcal{I}$ si $\mathcal{C}^{o p}$ est une sous-catégorie complète à gauche de $\mathcal{I}^{o p}$.

\section{Proposition et définition C.6.2 (Prolongement par zéro)}

Soit $\mathcal{C}$ une sous-catégorie pleine relativement connexe de $\mathcal{I}$. On note $\mathcal{D}$ la souscatégorie pleine de $\mathcal{I}$ dont la classe d'objets est le complémentaire de celle de $\mathcal{C}$, et $\mathcal{R}: \operatorname{Fct}\left(\mathcal{I}, \mathcal{E}_{\mathbb{k}}\right) \rightarrow \boldsymbol{F} \mathbf{c t}\left(\mathcal{C}, \mathcal{E}_{\mathbb{k}}\right)$ le foncteur de restriction.

(1) On définit un foncteur $\mathcal{P}_{\mathcal{I}, \mathcal{C}}: \operatorname{Fct}\left(\mathcal{C}, \mathcal{E}_{\mathbb{k}}\right) \rightarrow \boldsymbol{F c t}\left(\mathcal{I}, \mathcal{E}_{\mathbb{k}}\right)$ (noté simplement $\mathcal{P}$ lorsqu'il n'y a pas d'ambiguïté) appelé prolongement par zéro en posant $\mathcal{P}(F)(E)=$ $F(E)\left(F \in \operatorname{Ob} \boldsymbol{F c t}\left(\mathcal{C}, \mathcal{E}_{\mathbb{k}}\right)\right)$ si $E \in \mathrm{ObC}, 0$ si $E \in \mathrm{Ob} \mathcal{D} ; \mathcal{P}(F)(t)=F(t)$ sit est une flèche de $\mathcal{C}, 0$ si c'est une autre flèche de $\mathcal{I}$; et $\mathcal{P}(T)_{E}=T_{E}$ si $T$ est une flèche de $\boldsymbol{F} \mathbf{c t}\left(\mathcal{I}, \mathcal{E}_{\mathbb{k}}\right)$ et $E$ un objet de $\mathcal{C}, 0$ sinon.

(2) Supposons $\mathcal{C}$ complète à gauche; $\mathcal{D}$ est donc complète à droite.

(a) Le foncteur $\mathcal{P}$ est adjoint à droite à $\mathcal{R}$.

(b) Le foncteur $\mathcal{P}$ est adjoint à gauche au foncteur $\mathcal{N}: \operatorname{Fct}\left(\mathcal{I}, \mathcal{E}_{\mathbb{k}}\right) \rightarrow$ $\boldsymbol{F} \operatorname{ct}\left(\mathcal{C}, \mathcal{E}_{\mathbb{k}}\right)$ défini comme suit : 
(i) pour tout foncteur $F: \mathcal{I} \rightarrow \mathcal{E}_{\mathbb{k}}$ et tout objet $X$ de $\mathcal{C}$,

$$
\mathcal{N}(F)(X)=k e r\left(F(X) \stackrel{\prod F(f)}{\longrightarrow} \prod_{\substack{X \underset{f}{\longrightarrow} Y \\ Y \in \mathrm{Ob} \mathcal{D}}} F(Y)\right) ;
$$

(ii) si $F: \mathcal{I} \rightarrow \mathcal{E}_{\mathbb{k}}$ est un foncteur, pour toute flèche $X \stackrel{u}{\rightarrow} X^{\prime}$ de $\mathcal{C}, \mathcal{N}(F)(u)$ est induite par $F(u)$;

(iii) si $T: F \rightarrow G$ est une flèche $\operatorname{de} \operatorname{Fct}\left(\mathcal{C}, \mathcal{E}_{\mathbb{k}}\right), \mathcal{N}(T)_{X}$ est induite par $T_{X}$ pour tout objet $X$ de $\mathcal{C}$.

On a un énoncé analogue dans le cas d'une sous-catégorie complète à droite.

Corollaire C.6.3. - On conserve les notations précédentes. Si la sous-catégorie $\mathcal{C}$ de $\mathcal{I}$ est complète à gauche, on a un diagramme de recollement

$$
\operatorname{Fct}\left(\mathcal{C}, \mathcal{E}_{\mathbb{k}}\right) \underset{\mathcal{N}}{\stackrel{\mathcal{R}}{\rightleftarrows}} \operatorname{Fct}\left(\mathcal{I}, \mathcal{E}_{\mathbb{k}}\right) \stackrel{\mathcal{P}}{\underset{\mathcal{N}}{\rightleftarrows}} \operatorname{Fct}\left(\mathcal{D}, \mathcal{E}_{\mathrm{k}}\right)
$$

Dans le cas où $\mathcal{C}$ est complète à droite, on a un diagramme de recollement

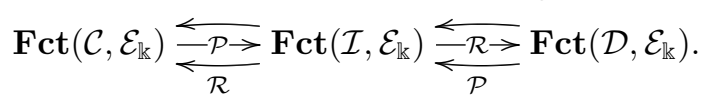





\section{INDEX}

$\tilde{\nabla}_{n}$-adapté, p. 175.

algèbre en grassmanniennes, p. 81. analytique (foncteur), p. 6, 67.

artinien, p. 185.

auto-dual, p. 3, 77.

coanalytique (foncteur), p. 6 .

co-localement artinienne (catégorie), p. 185.

co-localement fini, p. 186.

comodule sur une comonade, p. 182.

comonade, p. 182.

complète à droite, à gauche

(sous-catégorie), p. 196.

coniveau, p. 70.

conjecture artininienne, p. xi, 4.

conjecture artininienne extrêmement

forte, p. 132-134, 141.

conjecture artininienne forte, p. 5.

conjecture artininienne très forte, p. 28.

co-pf, co-pf $f_{n}$, voir co-présentation finie.

co-présentation finie, $n$-co-présentation

finie, p. 186.

corps de décomposition, p. 8.

cosocle, p. 187.

co-type fini (de), p. 185. décalage (foncteur), p. 5, 18, 63, 77, 99.

décomposition scalaire,

p. 4, 15, 59, 77, 99, 195.

degré (d'un foncteur), p. 6, 67.

degré (d'une partition), p. 9.

différence (foncteur), p. 5, 6399.

division (foncteur de), p. 194.

dualité, p. 3, 48, 76, 193.

épaisse (sous-catégorie), p. 186.

facteur de composition, p. 9.

fermé (objet $\mathcal{C}$-fermé), p. 187.

fidèle (comodule), p. 38.

filtration canonique (dans $\mathcal{F}_{\text {surj }}$ ), p. 20.

fini, p. 186.

Grothendieck (catégorie de), p. 185.

Grothendieck (groupe de), p. 188.

hauteur d'un foncteur induit, p. 131.

hom interne (foncteur), p. 194.

induction, p. 4, 16, 59, 77.

induit (foncteur), p. xiii, 131.

injectif standard, p. 3, 14, 193.

intégrale (foncteur), p. 34, 37, 54.

Krull (filtration de), p. 189. 
K-théorie stable, p. 140.

(k-) linéaire (catégorie), p. xix. localement fini, p. 186.

localement noethérienne (catégorie), p. 185.

localisante (sous-catégorie), p. 186. longueur (d'une partition), p. 9.

module sur une monade, p. 182. monade, p. 182.

niveau, p. 70 .

noethérien, p. 185.

noethérien de type $n$, simple noethérien de type $n$, p. 190.

parfait (objet $\mathcal{C}$-parfait), p. 187 . partition, p. 8.

$p f, p f_{n}$, voir présentation finie. polynomial (foncteur), p. 6, 67, 99. polynomiale (filtration), p. 7.

Powell (filtration, foncteur de), p. 26. présentation finie, $n$-présentation finie, p. 186.

produit tensoriel extérieur, p. 195. produit tensoriel total, p. 16, 60. projectif standard, p. 3, 14, 192. prolongement par zéro, p. 196. pseudo-constant (foncteur), p. 66.

radical, p. 187.

recollement (diagramme de), p. 189. régulière (partition), p. 9.

relativement connexe (sous-catégorie), p. 196

résolution canonique, p. 94.

restriction, p. 4, 16, 59, 77.

Serre (sous-catégorie de), p. 186.

simple, p. 186.

socle, p. 187.

système de coefficients, p. 28-32.

tors de Frobenius, p. 4, 16, 59, 77, 99. translation (foncteur), p. 43.

type fini (de), p. 185. 


\section{INDEX DES NOTATIONS}

\section{Catégories}

Comod, catégories de comodules p. xviii.

Comod $^{\text {fid }}$, catégories de comodules fidèles p. 38.

Ens, catégorie des ensembles, p. xviii. Ens $^{f}$ catégorie des ensembles finis, p. xviii.

$\mathcal{E}\left(\right.$ ou $\left.\mathcal{E}_{\mathbb{k}}\right)$, catégorie des $\mathbb{k}$-espaces vectoriels, p. $\mathrm{x}$.

$\mathcal{E}^{f}$ (ou $\mathcal{E}_{\mathbb{k}}^{f}$ ), catégorie des $\mathbb{k}$-espaces vectoriels de dimension finie, $\mathrm{p} . \mathrm{x}$. $\mathcal{E}_{\mathcal{G} r, I}^{f}, \mathcal{E}_{\mathcal{G} r}^{f}$, p. 42.

$\widetilde{\mathcal{E}}_{\mathcal{G} r}^{f}$, p. 41.

$\mathcal{E}_{\text {inj }}^{f}$, p. x.

$\mathcal{E}_{\mathrm{Pl}, n}^{f}$, p. 42

$\mathcal{E}_{\text {surj }}^{f}, \mathcal{E}_{\text {surj }}^{I}$, p. x.

$\boldsymbol{F} \mathbf{c t}(\mathcal{C}, \mathcal{D})$, catégorie des foncteurs d'une catégorie essentiellement petite $\mathcal{C}$ vers une catégorie $\mathcal{D}$, p. xviii.

$\mathcal{F}($ ou $\mathcal{F}(\mathbb{k}))$, catégorie des foncteurs entre espaces vectoriels, p. $\mathrm{x}$.

$\mathcal{F}^{d f}$, sous-catégorie des foncteurs à valeurs de dimension finie de $\mathcal{F}$, p. 3 . $\mathcal{F}^{n}$, p. 6 .

$\mathcal{F}^{\omega-\text { cons }(n)}$, sous-catégorie des foncteurs induits de hauteur $\leq n$ de $\mathcal{F}$, p. 131.
$\mathcal{F}_{\mathcal{G} r}, \mathcal{F}_{\mathcal{G} r, I}$, p. 53 .

$\widetilde{\mathcal{F}}_{\mathcal{G} r}$, p. 75 .

$\mathcal{F}_{i}$, p. 4.

$\mathcal{F}_{\text {inj }}$, p. 13.

$\mathcal{F}_{\omega}$, sous-catégorie des foncteurs analytiques de $\mathcal{F}$, p. 6 .

$\mathcal{F}_{\mathbf{P l}, n}$, p. 97.

$\mathcal{F}_{\text {surj }}, \mathcal{F}_{\text {surj }}^{I}$, p. 13.

$\mathcal{F}_{\tilde{\nabla}_{n},<i}$, p. 158 .

Mod, catégories de modules p. xviii.

$\mathcal{N}_{i l}{\overline{\tilde{\nabla}_{n}}}_{n}, \overline{\mathcal{N}} \boldsymbol{i l}_{\tilde{\nabla}_{n}}$, p. 11.

\section{Constructions générales. -}

$\mathcal{C}^{o p}$, catégorie opposée d'une catégorie $\mathcal{C}$, p. xix.

$\mathcal{C}_{M}$, catégorie des objets d'une catégorie $\mathcal{C}$ munis d'une action d'un monoïde $M$, p. xix.

$\underline{M}$, catégorie à un objet associée à un monoïde $M$, p. xix.

$\mathcal{I}_{\backslash X},(\mathcal{I}$ catégorie, $X: \mathcal{I} \rightarrow$ Ens

foncteur), p. 34 .

$\mathcal{I}_{X, Y}$, p. 36 .

$\mathcal{I}_{/ X}$, p. 39.

$\otimes$, produit tensoriel de catégories de foncteurs, p. 195. 
Constructions à partir d'une catégorie abélienne $\mathcal{A}$. $-\mathcal{A}^{f}$, sous-catégorie des objets finis de $\mathcal{A}$, p. 186.

$\mathcal{A}^{l f}$, sous-catégorie des objets localement finis de $\mathcal{A}$, p. 186.

$\mathcal{A}^{\text {tf }}$, sous-catégorie des objets de type fini de $\mathcal{A}$, p. 185.

$\mathcal{A} / \mathcal{C}$, quotient de $\mathcal{A}$ par une sous-catégorie épaisse $\mathcal{C}$, p. 187.

$\left(\mathcal{K}_{n}(\mathcal{A})\right)$, filtration de Krull de $\mathcal{A}$, p. 189.

\section{Foncteurs}

Objets des catégories de foncteurs. - $\bar{G}(n)$, p. 26.

$I$, injectif standard $I_{\mathbb{F}_{2}}$ de $\mathcal{F}\left(\mathbb{F}_{2}\right)$, p. 153 .

$I_{E}^{\mathcal{I}}$, foncteurs injectifs standard, p. 193.

$I^{\mathcal{G} r}$, injectif standard dans une catégorie de type $\mathcal{F}_{\mathcal{G}_{r}}$, p. 53.

$I_{V}$, injectif standard de la catégorie $\mathcal{F}$, p. 3.

$I_{\lambda}$ ( $\lambda$ partition 2-régulière), enveloppe injective de $S_{\lambda}$, p. 9.

$I_{V}^{\text {surj }}$, injectif standard de la catégorie $\mathcal{F}_{\text {surj }}$, p. 14.

$\mathrm{Is}_{n}$, p. 15.

$\mathbb{k}(n)$, p. 15 .

$\mathbb{k}^{\geq i}$, p. 21 .

$\Lambda$, puissances extérieures, p. 9.

$P$, projectif standard $P_{\mathbb{F}_{2}}$ de $\mathcal{F}\left(\mathbb{F}_{2}\right)$, p. 163 .

$P_{E}^{\mathcal{I}}$, foncteurs projectifs standard, p. 192.

$P^{\mathcal{G} r}$, projectif standard dans une catégorie de type $\mathcal{F}_{\mathcal{G} r}$, p. 53.

$P_{V}$, projectif standard de la catégorie $\mathcal{F}$, p. 3.

$P_{V, i}$, p. 4.

$P_{V}^{\text {surj }}$, projectif standard de la catégorie $\mathcal{F}_{\text {surj }}$, p. 14.

$\widetilde{P}(n)$, p. 27.

$Q_{\lambda}$ ( $\lambda$ partition régulière), p. 26.

$S_{\lambda}$, foncteur simple de $\mathcal{F}\left(\mathbb{F}_{2}\right)$ associé à une partition 2-régulière $\lambda$, p. 9 .

$\bar{F}$, partie sans terme constant d'un foncteur $F$ de $\mathcal{F}$, p. 4.

Foncteurs entre catégories de fonc- $\Delta^{\mathcal{G} r}$, foncteur différence des catégories de teurs. - D, foncteur de dualité de la type $\mathcal{F}_{\mathcal{G} r}$, p. 63.

catégorie $\mathcal{F}$, p. 3.

$\Delta_{V}$, foncteur décalage de la catégorie $\mathcal{F}$ $D_{\mathcal{G} r}$, foncteur de dualité de la catégorie $\widetilde{\mathcal{F}}_{\mathcal{G} r}$, p. 76 . par $V \in \mathrm{Ob}^{f}$, p. 5 .

$\delta^{\text {surj }}, \delta^{\text {inj }}, \delta$, foncteurs de décalage des $\Delta_{V}^{\mathcal{G} r}$, foncteur décalage des catégories de catégories $\mathcal{F}_{\text {surj }}$ ou $\mathcal{F}_{\text {inj }}$, p. 18.

type $\mathcal{F}_{\mathcal{G} r}$, p. 63.

$\Delta$, foncteur différence de la catégorie $\mathcal{F}, \varepsilon, \varepsilon_{I}$, p. 55.

p. 5 .

Ext*, p. 194. 
Hom, foncteur hom interne, p. 194.

$\mathcal{I}$, p. 76.

$\iota, \iota \iota_{I}$, p. 54 .

$\mathfrak{J}$, p. 75 .

$\mathcal{J}$, p. 139.

$k_{n}$, p. 7 .

$\kappa, \kappa_{I}$, p. 54 .

$o$, p. 21.

$o_{i n j}$, p. 24 .

$\Omega$, p. $34,37,39$.

$\omega, \omega_{n}, \omega_{\leq n}$, foncteurs d'intégrale en grassmanniennes, p. 54.

$\widetilde{\omega}$, p. 78 .

$p_{n}$, p. 7 .

$\mathcal{P}$, foncteur de prolobgement par zéro, p. 14, 53, 196.

$q_{n}$, p. 7 .

$\mathcal{R}$, foncteur de restriction, p. 14, 53 .

$\rho, \rho_{I}$, p. 55 .

$\sigma, \sigma_{I}$, p. 56 .

$T_{n}$, p. 20.

$\tau_{A}$, p. 115.

$\theta, \theta_{I}$, p. 56.

$\Upsilon$, p. $34,37,39$.

$\xi, \xi_{I}$, p. 56 .

$\tilde{\nabla}_{n}$, p. 11.

$\nabla_{n}^{\mathcal{G} r}$, p. 153

$\widetilde{\otimes}$, produit tensoriel total, p. 16, 60 .

$\boxplus$, produit tensoriel extérieur, p. 195.

$\varpi$, p. 22 .

$\varpi_{i n j}$, p. 24.

$(\cdot: \cdot)$, foncteur de division, p. 194

Autres foncteurs. - $\mathfrak{B}_{I}$, p. 44 .
$\widetilde{\mathfrak{B}}$, p. 44 .

$\mathfrak{D}_{I}$, p. 45.

$\widetilde{\mathfrak{D}}$, p. 45.

ev, ev, foncteurs d'évaluation, p. 14, 191.

$G r$, p. 50 .

inc $c_{n}^{\mathbf{P l}}$, p. 43.

incl $_{I, J}$, p. 43.

$\widetilde{\text { incl }}_{I}$, p. 42 .

$j_{*}^{n}$, p. 14 .

$\mathfrak{K}_{I}$, p. 44

$\overline{\mathfrak{K}}_{n}$, p. 44.

$\widetilde{\mathfrak{K}}$, p. 44 .

$\mathfrak{L}_{I}$, p. 45 .

$\widetilde{\mathfrak{L}}$, p. 45.

$O_{M}^{\mathcal{C}}$, p. xix.

$\mathcal{O}_{\mathcal{I}, X}$, p. 34 .

$\mathcal{O}^{\mathcal{I}, X}$, p. 39 .

$\mathfrak{O}_{I}$, p. 43.

$\overline{\mathfrak{O}}_{n}$, p. 43.

$\widetilde{\mathfrak{O}}$, p. 43 .

$\mathrm{Pl}$, ensembles de monomorphismes,

p. xix.

$\phi$, automorphisme de Frobenius de $\mathcal{E}_{\mathbb{k}}^{f}$, p. 4.

$\Re_{\bullet}^{\mathcal{G} r, I}$, résolution canonique dans $\mathcal{F}_{\mathcal{G} r, I}$, p. 94 .

$\mathfrak{S}_{n}$, p. 45.

$F^{*}$, foncteur de précomposition par $F$, p. 191.

$F_{*}$, foncteur de postcomposition par $F$, p. 191.

田, p. 43.

$(\cdot)^{\vee}$, foncteur de dualité de $\widetilde{\mathcal{E}}_{\mathcal{G} r}^{f}$, p. 48 .

\section{Autres notations}

$\mathrm{A}_{\mathcal{G} r}, \overline{\mathrm{A}}_{\mathcal{G} r}$, algèbre en grassmanniennes (réduite), p. 81.

coniv, coniveau d'un foncteur, p. 70 . cosoc, cosocle, p. 187.

Aut, groupes d'automorphismes, p. xix. 
$d$ (notation non valable dans la partie IV), degré du corps $\mathbb{k}$ sur son sous-corps premier, p. xviii. deg, degré d'un foncteur polynomial, p. 6 . $E_{n}$, espace vectoriel $\mathbb{k}^{n}$, p. 14.

End, monoïdes d'endomorphismes, p. xix.

Epi, ensembles d'épimorphismes, p. xix. $G_{0}$, groupes de Grothendieck, p. 188.

$G_{0}^{f}, G_{0}^{t f}$, p. 189.

$\widehat{G}_{0}^{f}$, p. 164, 166 .

GL, groupe linéaire infini, p. 30.

$\mathcal{G} r, \mathcal{G} r_{I}$, p. xviii.

$h_{X, F}^{*}$, p. 126.

hom, ensembles de morphismes, p. xix. Iso, ensembles d'isomorphismes, p. xix. $j^{\omega}$, p. 76 .

$j_{G}$, p. 164

$\mathbb{k}$, corps fini de base, p. x.

$K_{0}$, p. 189.

$l(\lambda)$, longueur d'une partition $\lambda$, p. 9 . $m_{\lambda}$ ( $\lambda$ partition 2-régulière), p. 9. $m_{R_{\lambda}}$ ( $\lambda$ partition 2-régulière), p. 10. niv, niveau d'un foncteur, p. 70.

Ob, classe d'objets d'une catégorie, p. xix.

$p$ (notation non valable dans la partie IV), caractéristique du corps $\mathbb{k}$, p. xviii. $\mathfrak{p}$, ensembles des partitions (2-régulières), p. 9, 166. $\pi_{n, X}$, p. 157.

$\mathrm{Pl}$, ensembles de monomorphismes, p. xix.

$\mathbf{P l}_{n}$, p. 42 .

$q$ (notation non valable dans la partie IV), cardinal du corps $\mathbb{k}$, p. xviii. $q_{n}^{\nabla}$, p. 156.

rad, radical, p. 187.

$R_{\lambda}$ ( $\lambda$ partition 2-régulière), p. 10 . $s_{W}$ ( $W$ sous-espace d'un espace vectoriel de dimension finie $V$ sous-entendu), p. 8 . $\Sigma_{n}$, groupe symétrique, p. xix. soc, socle, p. 187.

$\mathcal{T}_{\mathcal{G} r, I}$, p. 87.

$t_{n}$, p. 7 .

[e] (e élément d'un ensemble), p. xviii. $\mathbb{k}[E], \mathbb{k}$-linéarisation d'un ensemble $E$, p. xviii.

$\mathbb{k}[M], \mathbb{k}$-algèbre d'un monö̈de $M$, p. xix $\lambda_{+i}(\lambda$ partition, $i \in \mathbb{N})$, p. 11 .

$\lambda_{-}(\lambda$ partition $)$, p. 11.

$|\lambda|$, degré d'une partition $\lambda$, p. 18. $\leq$, ordre sur les partitions, p. 9 .

$(\lambda, \mu)$, partition concaténée de $\lambda$ et $\mu$, p. 10 .

$<n>$, partition $(n, n-1, \ldots, 1)$, p. 164 .

$(\cdot)^{*}$, dual, contragrédient, p. xix.

$\vdash$, p. 9 . 


\section{BIBLIOGRAPHIE}

[Aus66] M. Auslander - «Coherent functors », in Proc. Conf. Categorical Algebra (La Jolla, Calif., 1965), Springer, New York, 1966, p. 189-231.

[BB69] M. BARR \& J. BECK - «Homology and standard constructions », in Sem. on Triples and Categorical Homology Theory (ETH, Zürich, 1966/67), Springer, Berlin, 1969, p. 245-335.

[Bet99] S. BetLey - «Stable $K$-theory of finite fields », K-Theory 17 (1999), no. 2, p. 103-111.

[CR87] C. W. Curtis \& I. Reiner - Methods of representation theory. Vol. II, Pure and Applied Mathematics (New York), John Wiley \& Sons Inc., New York, 1987, With applications to finite groups and orders, A WileyInterscience Publication.

[CR90] - Methods of representation theory. Vol. I, Wiley Classics Library, John Wiley \& Sons Inc., New York, 1990, With applications to finite groups and orders, Reprint of the 1981 original, A Wiley-Interscience Publication.

[Dja06a] A. Djament - «Catégories de foncteurs en grassmanniennes », arXiv :math.AT/0610598, 2006.

[Dja06b] _ _ Catégories de foncteurs en grassmanniennes et filtration de Krull », arXiv :math.RT/0611861, 2006.

[Dja06c] $\_$Foncteurs de division et structure de $I^{\otimes 2} \otimes \Lambda^{n}$ dans la catégorie $\mathcal{F} »$, à paraître aux Annales de l'Institut Fourier; version préliminaire disponible sur www.math.univ-paris13/〜 djament/, 2006.

[Dja06d] _ « Représentations génériques des groupes linéaires : catégories de foncteurs en grassmanniennes, avec applications à la conjecture artinienne », Thèse, Université Paris 13, 2006, disponible sur http ://tel.archives-ouvertes.fr/tel-00119635. 
[Dja07] « Le foncteur $V \mapsto \mathbb{F}_{2}[V]^{\otimes 3}$ entre $\mathbb{F}_{2}$-espaces vectoriels est noethérien », arXiv :math.AT/0702667, soumis, 2007.

[Dwy80] W. G. DwYER - « Twisted homological stability for general linear groups », Ann. of Math. (2) 111 (1980), no. 2, p. 239-251.

[FFPS03] V. Franjou, E. M. Friedlander, T. Pirashvili \& L. Schwartz - Rational representations, the Steenrod algebra and functor homology, Panoramas et Synthèses [Panoramas and Syntheses], vol. 16, Société Mathématique de France, Paris, 2003.

[FFSS99] V. Franjou, E. M. Friedlander, A. Scorichenko \& A. Suslin «General linear and functor cohomology over finite fields », Ann. of Math. (2) 150 (1999), no. 2, p. 663-728.

[FLS94] V. Franjou, J. LAnnes \& L. Schwartz - « Autour de la cohomologie de Mac Lane des corps finis », Invent. Math. 115 (1994), no. 3, p. 513-538.

[Fra96] V. FRANJOU - « Extensions entre puissances extérieures et entre puissances symétriques », J. Algebra 179 (1996), no. 2, p. 501-522.

[Gab62] P. GABRIEL - «Des catégories abéliennes», Bull. Soc. Math. France 90 (1962), p. 323-448.

[GJ81] L. Gruson \& C. U. JEnsEn - «Dimensions cohomologiques reliées aux foncteurs $\lim ^{(i)}{ }$, in Paul Dubreil and Marie-Paule Malliavin Algebra Seminar, 33rd Year (Paris, 1980), Lecture Notes in Math., vol. 867, Springer, Berlin, 1981, p. 234-294.

[GS05] G. Gaudens \& L. Schwartz - «Un théorème d'annulation en cohomologie de Mac Lane», C. R. Math. Acad. Sci. Paris 341 (2005), no. 2, p. $119-122$.

[HLS93] H.-W. Henn, J. LANNES \& L. SchWARTZ - « The categories of unstable modules and unstable algebras over the Steenrod algebra modulo nilpotent objects », Amer. J. Math. 115 (1993), no. 5, p. 1053-1106.

[HLS95] _ « Localizations of unstable $A$-modules and equivariant mod $p$ cohomology », Math. Ann. 301 (1995), no. 1, p. 23-68.

[Hum87] J. E. Humphreys - « The Steinberg representation», Bull. Amer. Math. Soc. (N.S.) 16 (1987), no. 2, p. 247-263.

[Jam78] G. D. JAMES - The representation theory of the symmetric groups, Lecture Notes in Mathematics, vol. 682, Springer, Berlin, 1978.

[Jan03] J. C. JANTZEN - Representations of algebraic groups, second éd., Mathematical Surveys and Monographs, vol. 107, American Mathematical Society, Providence, RI, 2003. 
[Jen72] C. U. JENSEN - Les foncteurs dérivés de lim et leurs applications en théorie des modules, Springer-Verlag, Berlin, 1972, Lecture Notes in Mathematics, Vol. 254 .

[JP91] M. Jibladze \& T. Pirashvili - « Cohomology of algebraic theories », $J$. Algebra 137 (1991), no. 2, p. 253-296.

[Kra01] H. Krause - « The spectrum of a module category », Mem. Amer. Math. Soc. 149 (2001), no. 707, p. x+125.

[Kuh94a] N. J. KUHN - « Generic representations of the finite general linear groups and the Steenrod algebra. I », Amer. J. Math. 116 (1994), no. 2, p. 327-360.

[Kuh94b] _ « Generic representations of the finite general linear groups and the Steenrod algebra. II », K-Theory 8 (1994), no. 4, p. 395-428.

[Kuh95] _ « Generic representations of the finite general linear groups and the Steenrod algebra. III », K-Theory 9 (1995), no. 3, p. 273-303.

[Kuh02] _ , A stratification of generic representation theory and generalized Schur algebras », K-Theory 26 (2002), no. 1, p. 15-49.

[Lan92] J. LANNES - « Sur les espaces fonctionnels dont la source est le classifiant d'un $p$-groupe abélien élémentaire », Inst. Hautes Études Sci. Publ. Math. (1992), no. 75, p. 135-244, avec un appendice de Michel Zisman.

[Lod98] J.-L. LodAY - Cyclic homology, second éd., Grundlehren der Mathematischen Wissenschaften [Fundamental Principles of Mathematical Sciences], vol. 301, Springer-Verlag, Berlin, 1998, Appendix E by María O. Ronco, Chapter 13 by the author in collaboration with Teimuraz Pirashvili.

[LZ87] J. LANNES \& S. ZARATI - «Sur les foncteurs dérivés de la déstabilisation », Math. Z. 194 (1987), no. 1, p. 25-59.

[LZ95] J. Lannes \& S. Zarati - « Théorie de Smith algébrique et classification des $H^{*} V$-U -injectifs », Bull. Soc. Math. France 123 (1995), no. 2, p. 189 223.

[Mit72] B. MitCheLL - « Rings with several objects », Advances in Math. 8 (1972), p. $1-161$.

[Mit86] S. MitchelL - « On the Steinberg module, representations of the symmetric groups, and the Steenrod algebra », J. Pure Appl. Algebra 39 (1986), no. 3 , p. $275-281$.

[ML57] S. MaC LANE - « Homologie des anneaux et des modules», in Colloque de topologie algébrique, Louvain, 1956, Georges Thone, Liège, 1957, p. 55-80.

[ML63]_ Homology, Die Grundlehren der mathematischen Wissenschaften, Bd. 114, Academic Press Inc., Publishers, New York, 1963. 
[ML71] Categories for the working mathematician, Springer-Verlag, New York, 1971, Graduate Texts in Mathematics, Vol. 5.

[Pir97] L. Piriou - « Sous-objets de $\bar{I} \otimes \Lambda^{n}$ dans la catégorie des foncteurs entre $\mathbf{F}_{2}$-espaces vectoriels », J. Algebra 194 (1997), no. 1, p. 53-78.

[Pir02] T. Pirashvili - « Polynomial functors over finite fields (after Franjou, Friedlander, Henn, Lannes, Schwartz, Suslin)», Astérisque (2002), no. 276, p. 369-388, Séminaire Bourbaki, Vol. 1999/2000.

[Pir03] _ _ André-Quillen homology via functor homology », Proc. Amer. Math. Soc. 131 (2003), no. 6, p. 1687-1694 (electronic).

[Pop73] N. Popescu - Abelian categories with applications to rings and modules, Academic Press, London, 1973, London Mathematical Society Monographs, No. 3.

[Pow98a] G. M. L. PowelL - «The Artinian conjecture for $I^{\otimes 2} »$, J. Pure Appl. Algebra 128 (1998), no. 3, p. 291-310, With an appendix by Lionel Schwartz.

[Pow98b] _ « Polynomial filtrations and Lannes' T-functor », K-Theory 13 (1998), no. 3, p. 279-304.

[Pow98c] _ « The structure of indecomposable injectives in generic representation theory », Trans. Amer. Math. Soc. 350 (1998), no. 10, p. 4167-4193.

[Pow00a]_ «On Artinian objects in the category of functors between $\mathbf{F}_{2^{-}}$ vector spaces », in Infinite length modules (Bielefeld, 1998), Trends Math., Birkhäuser, Basel, 2000, p. 213-228.

[Pow00b] _ between $\mathbf{F}_{2}$-vector spaces », Ann. Inst. Fourier (Grenoble) $\mathbf{5 0}$ (2000), no. 3, p. $781-805$.

[Pow01] _ « The tensor product theorem for $\widetilde{\nabla}$-nilpotence and the dimension of unstable modules », Math. Proc. Cambridge Philos. Soc. 130 (2001), no. 3 , p. $427-439$.

[Pow06]__ « Endomorphisms of $H^{*}\left(K(V, n) ; \mathbb{F}_{2}\right)$ in the category of unstable modules », Math. Z. 254 (2006), no. 1, p. 55-115.

[PR02] T. Pirashvili \& B. Richter - « Hochschild and cyclic homology via functor homology », K-Theory 25 (2002), no. 1, p. 39-49.

[PS98] L. Piriou \& L. SChWARTZ - « Extensions de foncteurs simples », $K$ Theory 15 (1998), no. 3, p. 269-291.

[PW92] T. Pirashvili \& F. WALDhAUSEN - « Mac Lane homology and topological Hochschild homology », J. Pure Appl. Algebra 82 (1992), no. 1, p. 81-98. 
[Sch94] L. SCHWARTZ - Unstable modules over the Steenrod algebra and Sullivan's fixed point set conjecture, Chicago Lectures in Mathematics, University of Chicago Press, Chicago, IL, 1994.

[Ves05] C. Vespa - « La catégorie $\mathcal{F}_{\text {quad }}$ des foncteurs de Mackey généralisés pour les formes quadratiques sur $\mathbb{F}_{2}$ », Thèse, Université Paris 13, 2005, http ://tel.ccsd.cnrs.fr/tel-00011892.

[Ves06]_, « The functor category $\mathcal{F}_{\text {quad }}$ », arXiv :math.AT/0606484, 2006. 